Studien aus dem Max-Planck-Institut

für Sozialrecht und Sozialpolitik

Annemarie Aumann

Arbeitsunfall 4.0

Die Abgrenzung privater und beruflicher Risikosphären in der modernen Arbeitswelt 
Studien aus dem Max-Planck-Institut für Sozialrecht und Sozialpolitik

Band 72 
Annemarie Aumann

\section{Arbeitsunfall 4.0}

Die Abgrenzung privater und beruflicher Risikosphären in der modernen Arbeitswelt 
Die Deutsche Nationalbibliothek verzeichnet diese Publikation in der Deutschen Nationalbibliografie; detaillierte bibliografische Daten sind im Internet über http://dnb.d-nb.de abrufbar.

Zugl.: München, LMU, Diss., 2018

ISBN 978-3-8487-5426-7 (Print)

ISBN 978-3-8452-9596-1 (ePDF)

1. Auflage 2019

( ) Nomos Verlagsgesellschaft, Baden-Baden 2019. Gedruckt in Deutschland. Alle Rechte, auch die des Nachdrucks von Auszügen, der fotomechanischen Wiedergabe und der Übersetzung, vorbehalten. Gedruckt auf alterungsbeständigem Papier. 


\section{Inhaltsübersicht}

$\begin{array}{ll}\text { Inhaltsverzeichnis } & 7\end{array}$

$\begin{array}{ll}\text { Abkürzungsverzeichnis } & 15\end{array}$

$\begin{array}{ll}\text { Einleitung } & 21\end{array}$

$\begin{array}{ll}\text { A. Forschungsfrage } & 21\end{array}$

B. Stand der Forschung 24

C. Gang der Untersuchung 26

Kapitel 1: Die Dogmatik des Arbeitsunfalls der Beschäftigten de lege lata, $\mathbb{8} 8$ Abs. 1 SGB VII 29

A. Der Zuordnungsmechanismus im Überblick 29

B. Der erfasste Personenkreis: Beschäftigte im Sinne des $\mathbb{2}$ Abs. 1 Nr. 1 SGB $\quad 30$

C. Der Unfall als schädigendes Ereignis 31

D. Eröffnung des unfallversicherungsrechtlichen Schutzbereichs 43

E. Die haftungsbegründenden Kausalitäten 78

F. Zwischenergebnis 99

Kapitel 2: Grundprinzipien der Unfallversicherung vor dem Hintergrund gesellschaftlichen Wandels 101

A. Relevanz für die Abgrenzung von Arbeits- und Privatunfall 101

B. Entstehung und Ausgestaltung der gesetzlichen $\begin{array}{ll}\text { Unfallversicherung } & 102\end{array}$

C. Grundprinzipien der Risikoverteilung in der Unfallversicherung 110

D. Die Arbeitswelt des 21. Jahrhunderts 127

E. Entgrenzte Arbeit 135

F. Fazit: Bedeutung der modernen Arbeitswelt für die
Unfallversicherung 


\section{Inhaltsübersicht}

Kapitel 3: Konkrete Auswirkungen entgrenzter Arbeit auf die Reichweite des Unfallversicherungsschutzes

A. Arbeitsunfall und Homeoffice

B. Arbeitsunfall und mobile Arbeit

C. Arbeitsunfall und ständige Erreichbarkeit

D. Lösungs- und Regelungsvorschläge zum

Unfallversicherungsschutz bei entgrenzter Arbeit

Kapitel 4: „Umgekehrte“ Entgrenzung: private Tätigkeiten am Arbeitsplatz

A. Notwendigkeit einer Erweiterung des Unfallversicherungsschutzes auf nicht berufliche Tätigkeiten

B. Beamtenrechtlicher Dienstunfall bei privater Tätigkeit im Vergleich

C. Regelungsvorschlag: Vertragskonformes privates Verhalten während der Arbeit als versicherte Tätigkeit

Schluss

A. Zusammenfassung der Ergebnisse

B. Vorschlag zur Neufassung des $₫ 8$ SGB VII

Literaturverzeichnis 


\section{Inhaltsverzeichnis}

$\begin{array}{ll}\text { Abkürzungsverzeichnis } & 15\end{array}$

$\begin{array}{ll}\text { Einleitung } & 21\end{array}$

A. Forschungsfrage $\quad 21$

B. Stand der Forschung 24

C. Gang der Untersuchung 26

Kapitel 1: Die Dogmatik des Arbeitsunfalls der Beschäftigten de lege lata, $\$ 8$ Abs. 1 SGB VII 29

A. Der Zuordnungsmechanismus im Überblick 29

B. Der erfasste Personenkreis: Beschäftigte im Sinne des $₫ 2$ Abs. 1 Nr. 1 SGB 30

C. Der Unfall als schädigendes Ereignis 31

I. Der Unfall als Ereignis 31

II. Einwirkung von außen $\quad 32$

1. Zweck des Merkmals 32

2. Der Begriff der Einwirkung 32

3. Klassische Abgrenzung von der inneren Ursache 33

4. Weite Auslegung der neueren Rechtsprechung 35

a. Gewillkürte Handlungen als äußere Einwirkungen: das Heben schwerer Gegenstände 35

b. Problem der Identifikation der relevanten Einwirkung: die Zugführer-Fälle 37

c. Das Problem der beherrschbaren, irrtümlichen Herbeiführung des Unfalls 38

III. Scheinmerkmal „auf den Körper“ 40

IV. Das Merkmal „zeitlich begrenzt“ 40

V. Der Primärschaden 41

1. Zur Prüfungsreihenfolge 41

2. Gesundheitsschaden oder Tod, $\$ 8$ Abs. 1 S. 2 SGB VII 42

D. Eröffnung des unfallversicherungsrechtlichen Schutzbereichs 43

I. Terminologie und Funktion des Kriteriums 43 
II. Die zu verbindenden Elemente 45

1. Geschützte Tätigkeit 45

2. Die tatsächliche Verrichtung 45

III. Der sachliche Zusammenhang in $₫ 8$ SGB VII 47

1. Terminologie 47

2. Der sachliche Zusammenhang zwischen tatsächlicher Verrichtung und geschützter Tätigkeit

a. Streit um kausale oder finale Natur der Bestimmungsmodalität 48

b. Dogmatik des sachlichen Zusammenhangs $\quad 50$

c. Abgrenzung zu privaten Tätigkeiten in Gemengelagen

aa. Sogenannte „eigenwirtschaftliche“ Tätigkeiten 53

bb. Unerhebliche Unterbrechung durch private Tätigkeit

cc. Gespaltene Handlungstendenz 56

dd. Gemischte Tätigkeit 57

ee. Besondere Betriebsgefahr 59

d. Kritik am Kriterium des sachlichen Zusammenhangs 61

IV. Vom sachlichen Zusammenhang zur „Erfüllung des

Versicherungstatbestands"

1. Sprachliche Neuetikettierung oder Einschränkung des Versicherungsschutzes?

a. Rein terminologische Änderung 63

b. Einschränkung des Versicherungsschutzes 66

2. Begründung der Änderung durch das BSG 69

3. Kritik am Merkmal „Erfüllung des

Versicherungstatbestands“

4. Resümee 77

$\begin{array}{ll}\text { E. Die haftungsbegründenden Kausalitäten } & 78\end{array}$

I. Kausalität im Recht $\quad 78$

1. Terminologie der haftungsbegründenden Kausalitäten $\quad 78$

2. Kausalität im Recht $\quad 80$

II. Kausalität im Unfallversicherungsrecht: die Theorie der wesentlichen Bedingung

1. Anwendungsbereich 82

2. Entwicklung der Theorie der wesentlichen Bedingung 83

a. Notwendigkeit einer eigenständigen

Kausalitätstheorie für das Unfallversicherungsrecht 
b. Historische Entwicklung und Implementation durch die Sozialgerichte

3. Die heutige Form der Theorie der wesentlichen Bedingung

a. Grundsätze und Begriffe 86

b. Terminologische Unsicherheiten 88

c. Kritik an der Theorie der wesentlichen Bedingung als reiner Wertungsakt

III. Unfallkausalität

1. Abgrenzung zum Schutzbereich und zu anderen Kausalitäten

2. Vermutung im Regelfall 93

3. Problematische Fallgruppen 94

a. Gelegenheitsursache 94

b. Gemischte Tätigkeit 96

c. Eingebrachte Gefahr 96

d. Unerhebliche Unterbrechung 97

IV. Haftungsbegründende Kausalität im engeren Sinne 97

1. Der Zusammenhang zwischen Unfallereignis und Primärschaden

2. Problemfall „Schadensanlage“ 98

F. Zwischenergebnis

Kapitel 2: Grundprinzipien der Unfallversicherung vor dem Hintergrund gesellschaftlichen Wandels

A. Relevanz für die Abgrenzung von Arbeits- und Privatunfall 101

B. Entstehung und Ausgestaltung der gesetzlichen Unfallversicherung

I. Sozialpolitischer Anstoß für die Einführung der gesetzlichen Unfallversicherung: Risikoverteilung vor 1884102

II. Rechtstechnische Ausgestaltung des Unfallversicherungsschutzes

C. Grundprinzipien der Risikoverteilung in der Unfallversicherung

I. Betriebsrisikolehre und Prinzip der Haftungsersetzung $\quad 110$

1. Die Zurechnung von Risiken nach Sphären 110

2. Beherrschbarkeitsargument 113

3. Gewinnerzielungsargument 117

4. Das Prinzip der Haftungsersetzung als Rechtsgrund und Übertragungstechnik 
II. Soziales Schutzprinzip und Fürsorgepflicht des Arbeitgebers

D. Die Arbeitswelt des 21. Jahrhunderts 127

I. Überblick über die Unfallversicherung heute 127

II. Tertiarisierung und Digitalisierung 131

III. Subjektive Modernisierung der Arbeitswelt 133

E. Entgrenzte Arbeit 135

I. Entbetrieblichung: örtliche und zeitliche Flexibilität im Arbeitsverhältnis $\quad 135$

II. Fortschreitende Erosion zwischen Arbeits- und Privatleben 138

1. Eindringen der Arbeit ins Private 138

2. Übergreifen des Privaten in die Arbeit 140

F. Fazit: Bedeutung der modernen Arbeitswelt für die

Unfallversicherung

Kapitel 3: Konkrete Auswirkungen entgrenzter Arbeit auf die

Reichweite des Unfallversicherungsschutzes 144

A. Arbeitsunfall und Homeoffice 144

I. Begriff, Bedeutung und Ausmaß von Arbeit im Homeoffice 144

II. Unfall bei Arbeitstätigkeit am häuslichen Arbeitsplatz 147

III. Verrichtungen mit gespaltener Handlungstendenz bei häuslicher Arbeit $\quad 150$

IV. Betriebswege innerhalb des häuslichen Bereichs 155

1. Widmung des Unfallorts als maßgebendes Kriterium 155

a. Häufigkeit der Nutzung des Unfallortes zu betrieblichen Zwecken als maßgebliches Kriterium 155

b. Keinerlei Unfallschutz für Wege innerhalb eines Wohngebäudes 160

2. Die Handlungstendenz als entscheidendes Kriterium 161

a. Entwicklung in der Rechtsprechung 161

b. Herleitung aus Betriebsrisiko- und sozialem Schutzprinzip

V. Wege zur Nahrungsaufnahme aus dem Homeoffice 166

1. Das Urteil des BSG vom 5.7.2016 166

2. Versicherungsschutz für Wege zur Nahrungsaufnahme innerhalb des häuslichen Bereichs

a. Im Lichte der Betriebsrisikolehre 169

b. Im Lichte des sozialen Schutzprinzips 173

aa. Präventionsaspekt 
bb. Im Lichte der Fürsorgepflicht

176

c. Unter Anwendung des Gleichheitssatzes, Art. 3 Abs. 1 GG

3. Versicherungsschutz für Wege zur Nahrungsaufnahme außerhalb des häuslichen Bereichs

a. Im Lichte der Schutzzwecke der Wegeunfallversicherung

b. Unter Beachtung des Gleichheitssatzes, Art. 3 Abs. 1 GG

4. Bewertung und Fazit

VI. Kindergartenwege aus dem Homeoffice

1. Grundlagen der Versicherung von Wegeunfällen

a. Die Voraussetzungen des $₫ 8$ Abs. 2 Nr. 1 SGB VII

b. Schutzzweck und Rechtsgründe der Versicherung von Wegeunfällen

2. Wegeabweichung zur Unterbringung von Kindern, $\mathbb{\$} 8$ Abs. 2 Nr. 2 lit. a SGB VII

3. Das Verbringen von Kindern in fremde Obhut bei häuslicher Tätigkeit

a. Keine direkte oder analoge Anwendbarkeit des $₫ 8$ Abs. 2 Nr. 2 lit. a SGB VII

b. Bedürfnis einer Korrektur

VII. Unfallversicherungsschutz bei Tätigkeit im Homeoffice und Diskriminierungsverbot

B. Arbeitsunfall und mobile Arbeit 204

I. Begriff, Bedeutung und Umfang mobiler Arbeit 204

II. Mobile Arbeit im Wohnbereich des Beschäftigten 206

a. Meinungsstand in Literatur und Rechtsprechung 206

b. Zurechnung zur Risikosphäre des Unternehmers aufgrund der Betriebsrisikolehre und des sozialen Schutzprinzips

III. Mobile Arbeit außerhalb der Privatwohnung des Beschäftigten

C. Arbeitsunfall und ständige Erreichbarkeit

I. Facetten der Erreichbarkeit: Begriffe und Relevanz 212

II. Unfall während der bloßen Verfügbarkeit 215

1. Rufbereitschaft 215

2. Arbeitsbereitschaft und Bereitschaftsdienst 217

3. Ständige Erreichbarkeit im engeren Sinne 218 
III. Unfall bei tatsächlicher Arbeitserbringung während der Erreichbarkeit

D. Lösungs- und Regelungsvorschläge zum

Unfallversicherungsschutz bei entgrenzter Arbeit

I. Fortentwicklung der Auslegungspraxis und klarstellende Ergänzung des Normtextes hinsichtlich der Bedeutung des Orts der Tätigkeit und der Rufbereitschaft

1. Festschreibung des Unfallorts als bloßes Indiz

2. Klarstellung des Versicherungsschutzes der Rufbereitschaft

II. Erweiterung des Unfallversicherungsschutzes auf Wege von und zu Kinderbetreuungsstätten bei häuslicher oder mobiler Arbeit durch Gesetzesänderung

Kapitel 4: „Umgekehrte“ Entgrenzung: private Tätigkeiten am Arbeitsplatz

A. Notwendigkeit einer Erweiterung des

Unfallversicherungsschutzes auf nicht berufliche Tätigkeiten

B. Beamtenrechtlicher Dienstunfall bei privater Tätigkeit im

Vergleich

I. Überblick über die Dogmatik des $₫ 31$ Abs. 1 BeamtVG

II. Illustration der unterschiedlichen Abgrenzung von Privatund Berufssphäre anhand der aktuellen Rechtsprechung

1. Anhand der Schullandheim-Entscheidungen

a. Relevanz und Kontext der Entscheidungen

b. Entscheidungsgründe im Vergleich

c. Folgerungen

2. Anhand der Garagenfälle 239

3. Zwischenergebnis

III. Die Entwicklung des Dienstbanns aus den Grundprinzipien der Unfallfürsorge

1. Von tätigkeitsbezogenen zu räumlich-zeitlichen Zuordnungskriterien

a. Die Abgrenzung nach der tatsächlichen Verrichtung

b. Ort und Zeit des Unfalls als maßgebliche Kriterien

c. Dogmatische Einordnung des „Dienstbanns“ 
2. Begründung des Fokus auf räumlich-zeitliche Kriterien als Konsequenz des Schutzzwecks der Unfallfürsorge

a. Sinn und Zweck der beamtenrechtlichen Unfallfürsorge

b. Unzulänglichkeiten dieses Begründungsansatzes

3. Begründung des Fokus auf räumlich-zeitliche Kriterien als Konsequenz der Rechtsgründe der Unfallfürsorge und des Beamtenstatus

a. Die Besonderheiten des Beamtenstatus gegenüber dem Arbeitsverhältnis

b. Maßgeblichkeit der Statusunterschiede bei der Einführung der Unfallfürsorge

c. Die Fürsorgepflicht des Dienstherrn im Gegensatz zur Fürsorgepflicht des Arbeitgebers

d. Der Betriebsrisikogedanke im Beamtenrecht

e. Zwischenergebnis: Die physische Gefahrherrschaft des Dienstherrn als ausreichendes Zuordnungskriterium

C. Regelungsvorschlag: Vertragskonformes privates Verhalten während der Arbeit als versicherte Tätigkeit

I. Keine Möglichkeit der Bewältigung mithilfe anerkannter Zuordnungskriterien

1. Keine Subsumtion unter den Versicherungstatbestand

2. Keine Anwendbarkeit von räumlich-zeitlichen Koinzidenzregeln im Sinne eines Betriebsbanns

2. Betriebssport, Gemeinschaftsveranstaltungen, unerhebliche Unterbrechungen

II. Regelungsvorschlag: Privates Verhalten während der Arbeit als versicherte Tätigkeit

B. Vorschlag zur Neufassung des $₫ 8$ SGB VII 274 


\section{Abkürzungsverzeichnis}

a.A.

a.a.O.

a.F.

Abs.

AcP

AJP

AN

AöR

ArbRAktuell

ArbRB

ArbSchG

ArbStättV

ArbuR

ArbZG

Art.

ATSG

ArbuR

Aufl.

AZV

BAnz

BAuA

BayBeamtVG

BB

BBG

BeamtStG

BeamtVG

BeckOK

Begr.

Beil.

Besch.

Beschl.

BG

BGB

BGBl

BGH

BGHSt

BGHZ

BMAS

BMFSJ andere/r Ansicht

am angegebenen Ort

alte Fassung

Absatz

Archiv für die civilistische Praxis $(Z)$

Aktuelle Juristische Praxis ( $\mathrm{Z}$ )

Amtliche Nachrichten des RVA

Archiv des öffentlichen Rechts (Z)

Arbeitsrecht Aktuell (Z)

Arbeitsrecht im Betrieb (Z)

Arbeitsschutzgesetz

Arbeitsstättenverordnung

Arbeit und Recht $(Z)$

Arbeitszeitgesetz

Artikel

Allgemeiner Teil des Sozialversicherungsrechts (Schweiz)

Arbeit und Recht (Z)

Auflage

Arbeitszeitverordnung

Bundesanzeiger

Bundesanstalt für Arbeitsschutz und Arbeitsmedizin

Bayrisches Beamtenversorgungsgesetz

Betriebs-Berater $(Z)$

Bundesbeamtengesetz

Beamtenstatusgesetz

Beamtenversorgungsgesetz

Beck'scher Online-Kommentar

Begründung

Beilage

Bescheid

Beschluss

Die Berufsgenossenschaft, jetzt: BPUVZ (Z)

Bürgerliches Gesetzbuch

Das Bundesgesetzblatt

Bundesgerichtshof

Entscheidungen des Bundesgerichtshofs in Strafsachen

Entscheidungen des Bundesgerichtshofs in Zivilsachen

Bundesministerium für Arbeit und Soziales

Bundesministerium für Familie, Senioren, Frauen und Jugend 


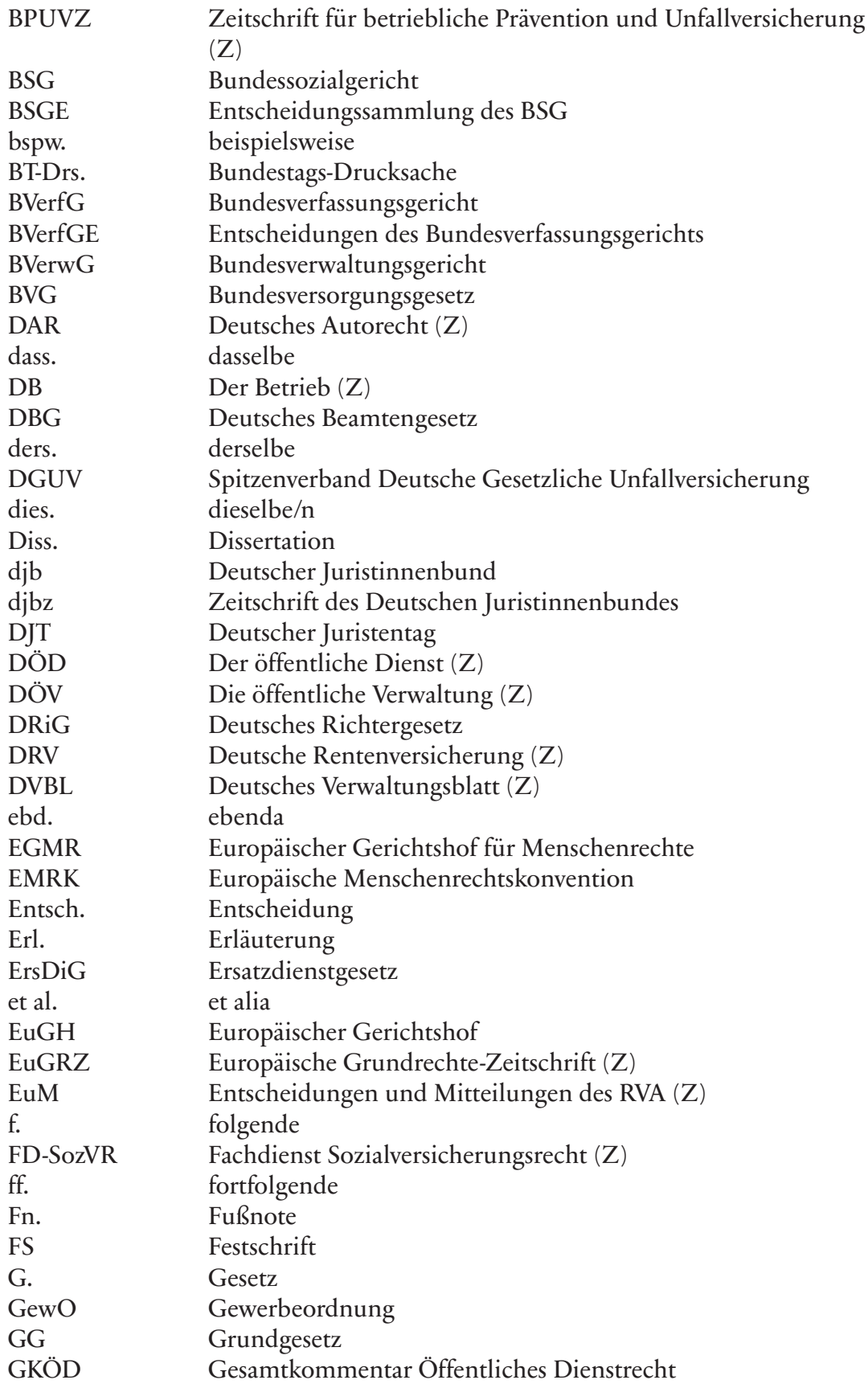


GVBl. Gesetz- und Verordnungsblatt

GWR

Hrsg.

i.E.

i.S.d.

i.V.m.

IAO

IKT

insb.

JA

$\mathrm{jM}$

jurisPR

$\mathrm{JuS}$

JURA

$\mathrm{JZ}$

$\mathrm{KJ}$

$\mathrm{KrV}$

lit.

Ls.

LSG

m.w.N.

MedSach

Mio.

$\mathrm{Nb}$.

NJW

Nr./Nrn.

$\mathrm{NVwZ}$

NVwZ-RR

Nw.

NWVBl.

NZA

NZS

NZV

öAT

$\mathrm{OGH}$

OLG

OVG

PersR

PflegeVG

RBG

$\mathrm{RdNr}$

RG

RGBl.

RGSt
Gesellschafts- und Wirtschaftsrecht $(\mathrm{Z})$

Herausgeber

im Ergebnis

im Sinne des/der

in Verbindung mit

Fraunhofer-Institut für Arbeitswirtschaft und Organisation

Informations- und Kommunikationstechnik

insbesondere

Juristische Ausbildung ( $\mathrm{Z})$

juris Monatszeitschrift $(Z)$

juris PraxisReport $(\mathrm{Z})$

Juristische Schulung $(Z)$

Juristische Ausbildung (Z)

Juristenzeitung (Z)

Kritische Justiz (Z)

Kranken- und Pflegeversicherung $(Z)$

littera

Leitsatz

Landessozialgericht

mit weiteren Nachweisen

Der medizinische Sachverständige $(\mathrm{Z})$

Million/en

Number

Neue juristische Wochenschrift (Z)

Nummer/n

Neue Zeitschrift für Verwaltungsrecht $(Z)$

NVwZ-Rechtsprechungs-Report (Z)

Nachweis(en)

Nordrhein-Westfälische Verwaltungsblätter (Z)

Neue Zeitschrift für Arbeitsrecht (Z)

Neue Zeitschrift für Sozialrecht $(Z)$

Neue Zeitschrift für Verkehrsrecht (Z)

Zeitschrift für das öffentliche Arbeits- und Tarifrecht $(Z)$

Oberster Gerichtshof Österreichs

Oberlandesgericht

Oberverwaltungsgericht

Der Personalrat $(\mathrm{Z})$

Gesetz zur sozialen Absicherung des Risikos der Pflegebedürf-

tigkeit

Reichsbürgergesetz

Randnummer

Reichsgericht

Reichsgesetzblatt

Entscheidungen des Reichsgerichts in Strafsachen 


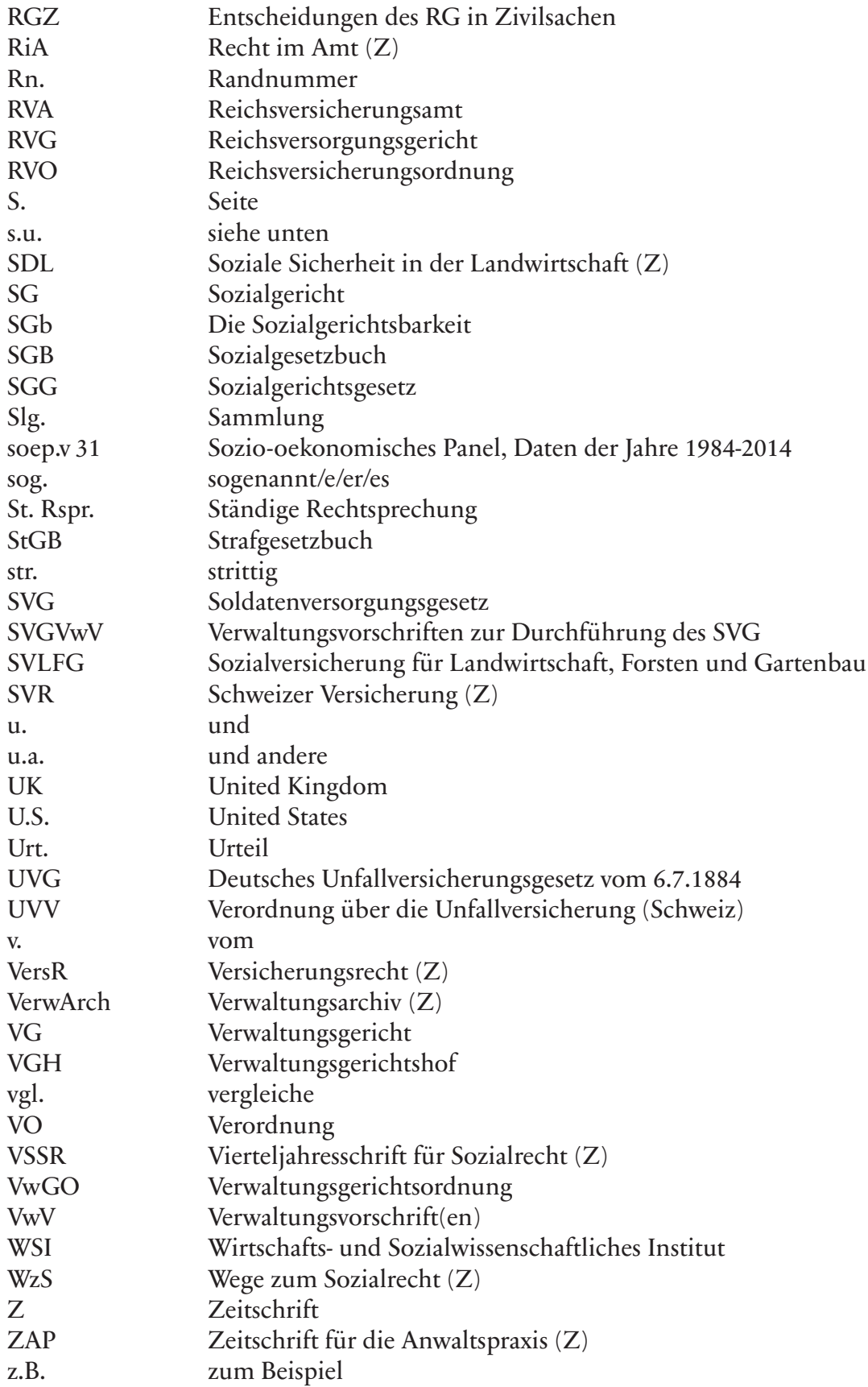


ZBR

ZESAR

ZEuS

ZEW

ZfF

ZfPR

ZfS

ZfSH/SGB

ZIAS

ZLR

ZNR

ZTR
Zeitschrift für Beamtenrecht $(Z)$

Zeitschrift für Europäisches Sozial- und Arbeitsrecht (Z)

Zeitschrift für europarechtliche Studien (Z)

Zentrum für Europäische Wirtschaftsforschung

Zeitschrift für Familienforschung $(Z)$

Zeitschrift für Personalvertretungsrecht $(Z)$

Zentralblatt für Sozialversicherung, Sozialhilfe und Versorgung

(Z)

Zeitschrift für die sozialrechtliche Praxis $(\mathrm{Z})$

Zeitschrift für ausländisches und internationales Arbeits- und

Sozialrecht $(Z)$

Zeitschrift für das gesamte Lebensmittelrecht $(Z)$

Zeitschrift für neuere Rechtsgeschichte $(Z)$

Zeitschrift für Tarifrecht (Z) 


\section{Einleitung}

\section{A. Forschungsfrage}

Seit der Entstehung des Unfallversicherungsrechts wird seine Anwendung von der Frage danach beherrscht, wie die unter Versicherungsschutz stehende Arbeit von der nicht unter Versicherungsschutz stehenden Privatsphäre abzugrenzen ist. ${ }^{1}$ In jüngerer Zeit ist es zu einer deutlichen Verschärfung der Dringlichkeit dieses Problems gekommen. ${ }^{2}$ Die Bereiche, in denen Privatleben und Beruf der Versicherten überlappen und eine Trennung der Risikosphären erschweren, werden im Zuge der Digitalisierung zahlreicher. Arbeitszeit und Arbeitsort der Arbeit $4.0^{3}$ genannten modernen Arbeitswelt sind de-institutionalisiert, die Konturen von Beruf und Freizeit verwässert. Zum einen diffundieren diese Lebensbereiche durch die Entbetrieblichung der Arbeitsverhältnisse: Beschäftigte sind zur Ausübung ihrer beruflichen Tätigkeit nicht mehr an einen bestimmten Arbeitsort oder eine bestimmte Arbeitszeit gebunden. Das Homeoffice verlagert die geschuldete Arbeitsleistung örtlich in das private Umfeld der Arbeitnehmer ${ }^{4}$, das Mobile Office ermöglicht ihre Durchführung an Orten, die aus privaten Gründen für sie vorteilhaft sind. Zum anderen findet eine Erosion der Risikosphären auch auf subjektiver Ebene statt, wenn Beschäftigte einerseits über mobile Internet- und Kommunikationstechnologie auch während ihrer Freizeit ständig für die arbeitsbezogene Kontaktaufnahme zur Verfügung stehen und sie sich umgekehrt während ihrer eigentlichen Arbeitszeit mit Erlaubnis, Duldung oder Billigung des Arbeitgebers

1 Eichenhofer, SGb 2007, S. 742, 746; Köhler, SGb 2006, S. 9, 14.

2 Legerlotz, ArbRB 2011, S. 350, 350; Boemke/Ankersen, BB 2000, S. 1570, 1572; Gitter, BB 1998, Beilage 6 S. 1, 5; LSG München, Urt. v. 5.4.2017 - L 2 U 101/14 -, juris Rn. 86.

3 Dieser Terminus wird zur Bezeichnung von durch die Digitalisierung angetriebenen Veränderungsprozessen der Arbeitswelt genutzt und schließt dabei insbesondere Phänomene der Entgrenzung mit ein, vgl. statt vieler BMAS, Grünbuch Arbeiten 4.0, S. 7; dass., Weißbuch Arbeiten 4.0. S. 18 ff.; Krause, NZA-Beilage 2017, S. 53, 53; Giesen/Kersten, Arbeit 4.0, S. 13 ff., 39 ff.; Grimm, ArbRB 2015, S. 336, 336 ff.; Walser, ArbuR 2016, S. 338, 338.

4 Das hier zur besseren Lesbarkeit vornehmlich verwendete generische Maskulin schließt gleichermaßen Personen jeden Geschlechts ein und soll keine Geschlechterdiskrimierung implizieren. 
auch mit privaten Angelegenheiten befassen. Diese Auflösung der seit der Industrialisierung als Normalfall angenommenen bestehenden Trennung zwischen Arbeits- und Privatsphäre wird als Entgrenzung der Arbeit bezeichnet. $^{5}$

De lege lata gehen die dargestellten Veränderungsprozesse der Arbeitswelt zulasten der betroffenen Beschäftigten. ${ }^{6}$ Der Versicherungsschutz in entgrenzten Arbeitsverhältnissen bleibt hinter dem der klassischen „Betriebsbeschäftigten“ zurück, was weder mit dem Telos der Unfallversicherung noch mit dem Gleichbehandlungsgrundsatz des Verfassungsrechts vereinbar ist. Ziel dieser Arbeit ist daher die notwendige Fortentwicklung des Unfallversicherungsrechts, um die Herausforderungen der zunehmenden Entgrenzung von Arbeit- und Privatsphäre im Interesse von Arbeitnehmern und Arbeitgebern zu bewältigen. ${ }^{7}$

Grund für die Erosion ${ }^{8}$ des Unfallversicherungsschutzes ist, dass seine Reichweite gerade auf der im Rahmen der Prüfung des Versicherungsfalls

5 Der Begriff stammt aus der Soziologie, wo das Phänomen der Entgrenzung als zunehmende Auflösung von für sicher gehaltenen Abgrenzungen zwischen Sphären seit Ende der 90er-Jahre diskutiert wird, vgl. Gottschall/Voß, in: dies. (Hrsg.), Entgrenzung von Arbeit und Leben, S.11. Soziale Entgrenzung allgemein ist demnach der Prozess, „in dem unter bestimmten historischen Bedingungen entstandene gesellschaftliche Strukturen der regulierenden Begrenzung von sozialen Vorgängen ganz oder partiell erodieren (...)" (ebd., S. 18); Sauer, in: Badura et al. (Hrsg.), Fehlzeiten-Report 2012, S. 3, 3; Kratzer, Arbeitskraft in Entgrenzung, S. 38 ff. Auch im juristischen Diskurs hat der Terminus Einzug gehalten, nachdem er von Däubler konkretisiert wurde, SR 2014, S. 45, 45 ff. In der Rechtswissenschaft werden damit wie hier konkret die beiden Aspekte der unabhängig von starren Ortsund Zeitvorgaben stattfindenden Arbeit sowie der Vermengung von Arbeits- und Privatsphäre bezeichnet, vgl. die Verwendung bei Jacobs, NZA 2016, S. 733, 734; Wiebauer, NZA 2016, S. 1430, 1430; Kobte, jurisPR-ArbR 17/2017 Anm. 2; WelskopDeffaa, NZA-Beilage 2017, S. 60, 60; Krause, Digitalisierung der Arbeitswelt, Gutachten B für den 71. DJT, B 18.

6 Krause, Digitalisierung der Arbeitswelt, Gutachten B zum 71. DJT, B 10.

7 Das Sozialrecht hat sich immer wieder nach veränderten tatsächlichen Gegebenheiten auszurichten, vgl. nur Heinze, in: Ständige Deputation des deutschen Juristentages (Hrsg.), Verhandlungen des neunundfünfzigsten deutschen Juristentages, Gutachten E, S. $72 \mathrm{ff}$.

8 Für die betroffenen Versicherten hat die Frage nach dem Vorliegen eines Arbeitsunfalls durchaus praktische Bedeutung. Für sie ist es von Vorteil, wenn ein erlittener Unfall als Arbeitsunfall gilt, Becker, in: Ehlers/Fehling, et al. (Hrsg.), Besonderes Verwaltungsrecht Band 3, $\$ 78$ Rn. 2; Krasney, KrV 2014, S. 102, 102. Denn die Unfallversicherung gewährt über der Krankenbehandlung der andernfalls einstandspflichtigen Krankenversicherung entsprechende Leistungen wie etwa das dem Krankengeld entsprechende Verletztengeld hinaus zusätzlich bspw. medizinische Rehabilitationsleistungen, Leistungen bei Pflegebedürftigkeit sowie Leistun- 
erforderlichen Grenzziehung zwischen Arbeits- und Privatsphäre beruht. Die Prinzipien dieser Abgrenzung basieren auf den der Unfallversicherung bei ihrer Einführung zugrunde gelegten Strukturprinzipien und damit auf den gesellschaftlichen Regeln der Industrialisierung. ${ }^{9}$ Sie sind im Kontext moderner Arbeits- und Lebenswirklichkeiten nicht mehr leistungsfähig, da sich die gesellschaftlichen Verhältnisse zwischenzeitlich grundlegend verändert haben. ${ }^{10}$ Die Spaltung der erlebten Welt - sowohl zeitlich als auch räumlich und sachlich - in einen Bereich der Arbeit und einen Bereich des privaten Lebens ist im engeren Sinne erst mit der Industrialisierung entstanden und wurde lange als irreversibler Vorgang bewertet. ${ }^{11}$ Sie bildet daher die Grundlage der zu dieser Zeit entwickelten Betriebsrisikolehre, welche als primäres Zurechnungsprinzip der Unfallversicherung solche Unfälle als Arbeitsunfälle qualifiziert, die sich innerhalb des Herrschaftsbereichs des Arbeitgebers ereignen. ${ }^{12}$ Die im Rahmen der Betriebsrisikolehre angewandten Parameter der Arbeitszeit und des Arbeitsortes, denen bei der Auslegung des Arbeitsunfallbegriffes historisch bedingt große Bedeutung beigemessen wird, bilden jedoch keine zuverlässigen Abgrenzungskriterien mehr. Stattdessen sind die zum Zeitpunkt des Unfalls ausgeübte Tätigkeit noch stärker zu fokussieren und die Besonderheiten des Nutzens geistiger menschlicher Arbeitskraft als Betriebsmittel zu berück-

gen zur Teilhabe am Arbeitsleben. Außerdem hat der Versicherte im Fall einer dauerhaften Erwerbsunfähigkeit bei Vorliegen eines Arbeitsunfalls Anspruch auf Rentenzahlungen. Diese Privilegierung ist Konsequenz des besonderen Leistungsgrunds der Unfallversicherung, der sie von der Krankenversicherung unterscheidet (Becker, a.a.O., Rn. 2). Zu den Unterschieden zwischen den Leistungen der beiden Versicherungen im Detail ders., a.a.O., Rn. 93 ff; zum Verhältnis der gesetzlichen Unfallversicherung zur gesetzlichen Krankenversicherung vgl. Spinnarke, in: Schulin (Hrsg.), Handbuch des Sozialversicherungsrechts Band 2: Unfallversicherungsrecht, S. $201 \mathrm{ff}$.

9 Das „Normalarbeitskonzept“ (s.u. Kap. 2 B. I.) der Zeit der Industrialisierung bildet die historische Referenzfolie sowohl für die soziologische Diskussion von Entgrenzungsprozessen als auch für die Grenzziehung zwischen Arbeits- und Privatsphäre im Unfallversicherungsrecht, sodass der Begriff der Entgrenzung im vorliegenden Kontext doppelt passend ist, vgl. Kratzer, Arbeitskraft in Entgrenzung, S. 22; Meik, NZA-Beilage 2017, S. 43, 43.

10 Vitu, Die verfassungsrechtlichen Grenzen der Abgabenbelastung des Arbeitgebers, S. 3; Hohmann-Dennhardt, ArbuR 54 (2006), S. 77, 79. Die räumliche Veränderung im Arbeitsverhältnis stellt einen in seiner Bedeutung nicht zu unterschätzenden Paradigmenwechsel dar, Giesen/Kersten, Arbeit 4.0, S. 85.

11 Gottschall/Voß, in: dies. (Hrsg.), Entgrenzung von Arbeit und Leben, S. 19.

12 Becker, Soziales Entschädigungsrecht, S. 44, bezeichnet die spezifische betriebliche Gefährdungssphäre sowie den Unternehmensbezug als traditionelle Anknüpfungspunkte der GUV. 
sichtigen. Dies bedingt eine Neuausrichtung des Betriebsrisikogedankens, welche zu einem systemgerechten - weil am Schutzzweck und den Strukturprinzipien der Unfallversicherung ausgerichteten - Unfallversicherungsschutz auch im Rahmen von „Arbeit 4.0“ führt.

\section{B. Stand der Forschung}

Mit der zunehmenden Anzahl entgrenzter Beschäftigter häufen sich die Fragen zu ihrer unfallversicherungsrechtlichen Behandlung. Sie konnten bisher nicht umfassend geklärt werden. ${ }^{13}$ Dies gilt sowohl für das Eindringen des Beruflichen in die Privatsphäre einerseits als auch für die Diffusion privater Tätigkeiten in den beruflichen Bereich andererseits.

Es liegt bisher keine eingehende Untersuchung darüber vor, wie sich der Zuordnungsmechanismus der gesetzlichen Unfallversicherung angesichts der zunehmenden Erosion der Grenze zwischen Arbeits- und Privatsphäre optimieren lässt. Die Bedeutung der Entgrenzung für das Unfallversicherungsrecht wurde allerdings zwischenzeitlich in Judikatur und Wissenschaft erkannt. In der Literatur sind Bemühungen zur Bewältigung des angesprochenen Problemkreises sichtbar, die sich jedoch auf Beiträge zu einzelnen Themengebieten beschränken. Diese Publikationen konzentrieren sich dabei meist auf einzelne Aspekte von Arbeitsunfällen in Zusammenhang mit der Tätigkeit im Homeoffice; ${ }^{14}$ gelegentlich wird der Problemkreis der mobilen Arbeit thematisiert. ${ }^{15}$ Nur selten findet sich Literatur zu den unfallversicherungsrechtlichen Auswirkungen der ständigen Erreichbarkeit. ${ }^{16}$ Auch die Gerichte bemühen sich um die Entwicklung einer pro-

13 Ricke, in: Körner/Leitherer et al. (Hrsg.), Kasseler Kommentar zum Sozialversicherungsrecht, $\mathbb{8} 8$ SGB VII Rn. 129a; Schwerdtfeger, in: Lauterbach/Watermann/ Breuer (Hrsg.), Unfallversicherung, $\mathbb{\$} 8 \mathrm{Rn} .259 \mathrm{~b}$.

14 Ricke, WzS 2017, S. 9 ff.; Siefert, NZS 2017, S. 766, 769; Schlaeger, jurisPR-SozR 20/2017 Anm. 3; Hollo, jurisPR-SozR 10/2017 Anm. 4; Jung, SGB 2017, S. 408 ff.; Spellbrink, NZS 2016, S. 527 ff.; Häfner-Beil/Nacke, Gute Arbeit 2016, S. 4 ff.; Plagemann, ArbRAktuell 2016, S. 71 f.; Isenhardt, DB 2016, S. 1499, 1502; Brink, FDSozVR 2015, 368217; Leube, NZV 2015, S. 275 ff.; Schur, jM 2014, S. 249 ff.; Brink, ArbRAktuell 2013, S. 632 ff.; Krasney, WzS 2013, S. 67 ff.; Leube, SGb 2012, S. 380 ff.; Mütze, jurisPR-SozR 25/2010, Anm. 5; Schlaeger, NZS 2009, S. 559 ff.; Wagner, jurisPR-SozR 23/2007 Anm. 5; Eichenhofer, SGb 2007, S. 742 ff.; Benz, SGb 2003, S. 12, 18 ff.; Wedde, Telearbeit, S. 91 ff.; Wolber, SozVers 1997, S. 239 ff.

15 Schlaeger, jurisPR-SozR 20/2017 Anm. 3; Eichenhofer, SGb 2007, S. 742, 747; Jung, SGb 2001, S. $398 \mathrm{f}$.

16 Spellbrink, NZS 2016, S. 527, 531 ff.; Nazarek, PersR 2016, S. 28 ff. 
blemorientierten Dogmatik, was sich zum einen an den Begründungen der Urteile zur einschlägigen Thematik zeigt und zum anderen daran erkennbar ist, dass Gerichte der ersten Instanzen Rechtsmittel aufgrund der Bedeutung der Materie zulassen oder das Verfahren aussetzen, um dem Bundessozialgericht entscheidende Fragen vorzulegen. ${ }^{17}$

Bisher vollkommen unbehandelt geblieben ist - soweit ersichtlich - die Frage nach den Konsequenzen privater Tätigkeiten während der Arbeit im Kontext der von der Vermengung von Privat- und Berufsphäre geprägten modernen Arbeitswelt. Während die Ausklammerung eigenwirtschaftlicher Tätigkeiten vom Versicherungsschutz in beinahe jeder Abhandlung zur Unfallversicherung thematisiert wird, ${ }^{18}$ fehlen Untersuchungen darüber, wie die Entgrenzung sich auf diesen Aspekt des Versicherungsschutzes auswirkt, völlig. Der Vergleich mit dem Unfallsicherungsrecht der Beamten, der zur Aufarbeitung des Problems herangezogen wird, wurde dagegen zuletzt in einer Dissertation aus dem Jahr 2016 - als diese Arbeit bereits im Entstehen begriffen war - gezogen, nachdem es seit einer Monographie aus dem Jahr 1970 zunächst still um das Thema geworden war. ${ }^{19}$ Die Untersuchungsansätze der Arbeit von Giesen sind jedoch gänzlich andere. Vorliegend wird der Vergleich mit dem Ziel angestrengt, eine potenzielle Gestaltungsmöglichkeit für den Einbezug privater Tätigkeiten während der Arbeit in der Unfallversicherung zu beleuchten, während es Giesen maßgeblich auf die Beseitigung der Ungleichbehandlung zwischen Beamten und Arbeitnehmern ankommt. ${ }^{20}$ Mit den hier im Zentrum stehen-

17 Vgl. nur BSG, Urt. v. 5.7.2016 - B 2 U 5/15 R -, juris Rn. 25 ff.; v. 18.6.2013 - B 2 U 7/12 -, juris Rn. 11 ff.; v. 9.11.2010 - B 2 U 14/10 R -, juris Rn. 13 ff.; v. 12.12.2006 - B 2 U 28/05 R -, juris Rn. 9 ff.; LSG München, Urt. v. 5.4.2017 - L 2 U 101/14 -, juris Rn. 86 mit Anm. Schlaeger, jurisPR-SozR 20/2017 Anm. 3.

18 Krasney, in: Becker/Burchardt/ders./Kruschinsky, Gesetzliche Unfallversicherung, $\mathbb{} 8$ Rn. 45; Keller, in: Hauck/Noftz/ders. (Hrsg.), SGB VII, $\$ 8$ Rn. 16a; Ricke, in: Körner/Leitherer et al. (Hrsg.), Kasseler Kommentar zum Sozialversicherungsrecht,

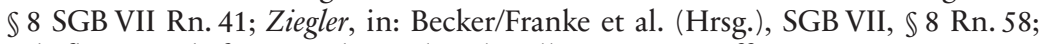
Schafhausen/Schäfer-Kuczinksi, ArbRAktuell 2017, S. 612 ff.; Jung, BPUVZ 2014, S. 202 f.; ders., BPUVZ 2013, S. 623 f.; Triebel, JURA 2007, S. 521, 527; Kunze, VSSR 2005, S. 299, 310; Benz, SGb 1999, S. 83, 84; Krasney, VSSR 1993, S. 81 ff.

19 Giesen, Arbeitsunfall und Dienstunfall, passim; Bulla, Der Dienst- und Arbeitsunfall als Institut des Allgemeinen Verwaltungsrechts, passim. In der Zwischenzeit befassten sich mit dem Vergleich von Aspekten beider Systeme Gontard, in: Deutsche Akademie für Verkehrswissenschaft (Hrsg.), 19. Deutscher Verkehrsgerichtstag 1981, S. 274 ff.; Fieberg, ZTR 2007, S. 421 ff. sowie Leube, ZTR 2012, S. 682 ff.

20 Giesen, Arbeitsunfall und Dienstunfall, S. 20. 
den Entwicklungen der modernen Arbeitswelt befasst sich seine Arbeit nur nebenbei und in geringem Umfang. ${ }^{21}$

\section{Gang der Untersuchung}

Die Untersuchung wird in vier Schritten mit jeweils eigenen Kapiteln vollzogen. Kapitel eins und zwei behandeln die Grundvoraussetzungen zur Erschließung der Problematik, während sich Kapitel drei und vier konkret mit den Auswirkungen der Entgrenzung auf die Reichweite des Unfallversicherungsschutzes auseinandersetzen und Lösungen zur Fortentwicklung des Unfallversicherungsrechts vorschlagen.

Kapitel eins widmet sich einer rechtsdogmatischen ${ }^{22}$ Untersuchung des $\$ 8$ Abs. 1 SGB VII. Diese zielt zum einen auf die Herausarbeitung der Funktionsweise der Zuordnung von Unfällen zur Arbeits- oder Privatsphäre, deren Verständnis Voraussetzung für das Bemühen ist, sie fortzuentwickeln (A.). Mithilfe der Auslegung der einzelnen Tatbestandsmerkmale (B.-E.) wird zum anderen die Reichweite des Unfallversicherungsschutzes der Beschäftigten nach geltendem Recht erarbeitet. Dabei zeigt sich, dass die Abgrenzung zwischen Arbeits- und Privatunfall anhand einer Wertentscheidung vorgenommen wird, welche auf den der Unfallversicherung bei ihrer Einführung zugrunde gelegten Schutzzwecken und Grundprinzipien beruht (F.).

Kapitel zwei befasst sich in der Folge mit den für diese Wertentscheidung notwendigen Grundlagen. Nach der Klärung ihrer Relevanz (A.) werden Entstehungsgeschichte und Ausgestaltung der Unfallversicherung in ihren gesellschaftlichen und sozialpolitischen Kontext eingeordnet (B.), um eine Basis für die historische und genetische Auslegung des $\mathbb{8}$ Abs. 1 SGB VII zu legen. Sodann werden die Zurechnungsprinzipien der Betriebsrisikolehre und der Haftungsersetzung sowie des sozialen Schutzes und der Fürsorgepflicht des Arbeitgebers in ihrer Bedeutung als Grundprinzipien der Unfallversicherung analysiert (C.). Die Auseinandersetzung mit diesen Prinzipien ist unumgänglich für eine teleologische Anwendung der Norm. Um eine gegenwartsbezogene Anwendung des $\$ 8$ Abs. 1 SGB VII zu ermöglichen, werden im nächsten Schritt die Faktoren dargestellt, welche die Arbeitswelt des 21. Jahrhunderts von den bei Einführung

21 Vgl. ebd., S. 62 ff.

22 Zu Begriff und Funktion der Rechtsdogmatik Becker, in: ders. (Hrsg.), Rechtsdogmatik und Rechtsvergleich im Sozialrecht I, S. 11, $14 \mathrm{ff}$. 
der Unfallversicherung herrschenden gesellschaftlichen Verhältnissen unterscheiden (D.). Das Phänomen der entgrenzten Arbeit, dessen Auswirkungen auf die Abgrenzung von Arbeits- und Privatunfall Untersuchungsgegenstand der vorliegenden Arbeit ist, wird im letzten Abschnitt gesondert dargelegt (E.).

Nachdem Kapitel eins und zwei somit das theoretische Fundament für die Beurteilung der Abgrenzung von Arbeits- und Privatunfall im Rahmen entgrenzter Arbeit gelegt haben, widmen sich Kapitel drei und vier den tatsächlichen Auswirkungen solcher Arbeitsgestaltungen auf die Reichweite des Unfallversicherungsschutzes.

Gegenstand von Kapitel drei ist dabei der augenscheinlichere der beiden Aspekte der Entgrenzung. Das Eindringen der Arbeit in die private Sphäre der Beschäftigten wird anhand dreier Fallgruppen - Homeoffice (A.), mobile Arbeit (B.) und ständige Erreichbarkeit (C.) - behandelt, wobei jeweils die konkreten Auswirkungen auf die Reichweite des Unfallversicherungsschutzes de lege lata herausgearbeitet werden. Es stellt sich heraus, dass der Unfallversicherungsschutz der Beschäftigten mit Aufnahme einer entgrenzten Tätigkeit system- und verfassungswidrig deutlich erodiert. Mithilfe der Ergebnisse der ersten beiden Kapitel wird die Betriebsrisikolehre als Zurechnungsprinzip inhaltlich dahingehend aktualisiert, dass ihr Fokus entgegen der bisherigen Auslegungspraxis nicht mehr auf der tatsächlichen Herrschaftsgewalt des Arbeitgebers über die Unfallumgebung, sondern auf der Ausübung der versicherten Tätigkeit zum Unfallzeitpunkt liegt. Dies führt zu einem umfassenderen Versicherungsschutz für entgrenzt Beschäftigte. Das Kapitel schließt mit Vorschlägen zur Anpassung des Normtexts des $₫ 8$ SGB VII, welche die intendierte Fortentwicklung des Unfallversicherungsschutzes festschreiben (D.).

Im vierten Kapitel wird schließlich der zweite Aspekt der Entgrenzung behandelt. Es untersucht die unfallversicherungsrechtlichen Implikationen der Ausübung privater Tätigkeiten während der Arbeitszeit am Arbeitsort. Soweit diese sozial akzeptierte Konsequenz der modernen Lebenswelt und vom Arbeitgeber entweder ausdrücklich erlaubt, gebilligt oder jedenfalls geduldet sind, gehören sie nach der redigierten Betriebsrisikolehre zum Betriebsrisiko des Unternehmers und sind daher in den Schutzbereich der Unfallversicherung aufzunehmen (A.). Mit dem Ziel, eine Gestaltungsmöglichkeit dafür zu eruieren, wird ein Vergleich mit dem beamtenrechtlichen Dienstunfallrecht unternommen, da dieses private Tätigkeiten während der Dienstausübung stets unter Versicherungsschutz stellt (B.). Dabei wird deutlich, dass der Grund für den Einbezug privater Tätigkeiten in den Dienstunfallschutz die im Gegensatz zum Unfallversicherungsrecht als noch stärker empfundene Beherrschbarkeit der tatsächlichen Verhältnisse 
durch den Dienstherrn ist. Im Unterschied zum Arbeitsverhältnis bildet das Beamtenverhältnis eine Basis für die Zugrundelegung der örtlichen Herrschaftssphären, sodass der Einsatz einer räumlich-zeitlichen Koinzidenzregel als Zuordnungsmechanismus im Beamtenrecht sachgerecht ist, im Unfallversicherungsrecht aber - wie Kapitel eins bis drei darlegen - den Strukturprinzipien widerspricht. Da die weitere Untersuchung zeigt, dass keine Möglichkeit besteht, private Tätigkeiten anhand der bisher anerkannten Zuordnungsmechanismen in den Unfallversicherungsschutz einzubeziehen, endet auch Kapitel vier mit einem dahingehenden Gesetzesvorschlag (C.).

Die Arbeit schließt mit einer Zusammenfassung der Ergebnisse und der Darstellung der vorgeschlagenen Gesetzesänderungen in ihrer Gesamtheit. 


\section{Kapitel 1: Die Dogmatik des Arbeitsunfalls der Beschäftigten de lege lata, $\mathbb{} 8$ Abs. 1 SGB VII}

\section{A. Der Zuordnungsmechanismus im Überblick}

Um darzustellen, wie die Abgrenzung zwischen Arbeits- und Privatunfällen in der gesetzlichen Unfallversicherung de lege lata erreicht wird, muss die in $₫ 8$ Abs. 1 SGB VII enthaltene Definition des Arbeitsunfalls in den Blick genommen werden. Aus dieser entwickeln sich die Kriterien, die als Tatbestandsmerkmale des Arbeitsunfalls geprüft werden müssen, um einen Versicherungsfall bejahen zu können. Bevor die einzelnen Tatbestandsmerkmale ausführlich untersucht werden, wird zunächst ein zusammenhängender Überblick über den Zuordnungsmechanismus ${ }^{23}$ insgesamt gegeben, um den Kontext für die detaillierte Analyse zu bieten.

Die Unfallversicherung ist dann einstandspflichtig, wenn der Versicherungsfall eingetreten ist, also gem. $\$ 7$ Abs. 1 SGB VII ein Arbeitsunfall oder eine Berufskrankheit vorliegt. Die Definition des Arbeitsunfalls findet sich in $\$ 8$ Abs. 1 Satz 1 SGB VII. Sie lautet: „Arbeitsunfälle sind Unfälle von Versicherten infolge einer den Versicherungsschutz nach $\mathbb{S} \$ 2,3$ oder 6 begründenden Tätigkeit (versicherte Tätigkeit).“ Diese interpretationsoffene Begriffsbestimmung wird nach herrschender Ansicht dahingehend ausgefüllt, dass ein Unfallereignis immer dann als Arbeitsunfall an-

23 Das Ineinandergreifen aller Elemente, die notwendig sind, um festzustellen, welcher (Risiko-)Sphäre ein Ereignis angehört, wird als Zuordnungsmechanismus bezeichnet. Zuordnung ist damit gleichzeitig der Vorgang, der nötig ist, um einzelne Wertungsbereiche voneinander abzugrenzen. Der Begriff ist verwandt mit dem der Zurechnung, der als die wertende Zuordnung eines Erfolgs zum Verantwortungsbereich eines anderen Rechtssubjekts zu verstehen ist, vgl. Schulin, Der natürliche - vorrechtliche - Kausalitätsbegriff im zivilen Schadensersatzrecht, S. 1. Unter Zuordnung ist also der übergeordnete Begriff zu verstehen, der - am Beispiel des $₫ 8$ Abs. 1 SGB VII gesprochen - die Schutzbereichseröffnung, den finalen Zurechnungszusammenhang und Kausalitätselemente einschließt; Zurechnungsprinzipien dagegen sind einzelne Elemente eines Zuordnungsmechanismus. Sie sind Grundsätze, deren Anwendung die Grenzen der Haftung vorgeben, Marton, AcP 162 (1963), S. 1, 53. 
zusehen ist, wenn ein finaler, sachlicher Zusammenhang ${ }^{24}$ zwischen der zum Unfallzeitpunkt ausgeführten und der versicherten Tätigkeit bejaht werden kann und zusätzlich Kausalität sowohl zwischen der ausgeführten Tätigkeit und dem Unfallereignis als auch zwischen dem Unfallereignis und dem Schaden vorliegt. ${ }^{25}$

\section{B. Der erfasste Personenkreis: Beschäftigte im Sinne des $\mathbb{2}$ Abs. 1 Nr. 1 SGB}

Für die Einordnung eines Unfalls als Arbeitsunfall muss zunächst der persönliche Schutzbereich des $\$ 8$ SGB VII eröffnet sein. Dieser besteht in der gesetzlichen Unfallversicherung aus den Versicherten, welche in den $\$ \$ 2$ bis 6 SGB VII benannt sind. Für die vorliegende Untersuchung ist die Gruppe der Beschäftigten im Sinne des $\$ 2$ Abs. 1 Nr. 1 SGB VII relevant. Diese bilden die zahlenmäßig größte Gruppe in der gesetzlichen Unfallversicherung. ${ }^{26}$ Der Begriff „Beschäftigter" ist die sozialrechtliche Entsprechung des arbeitsrechtlichen Begriffs des „Arbeitnehmers“, wobei inhaltlich höchstens noch geringfügige Unterschiede bestehen. ${ }^{27}$ Eine Beschäftigung übt aus, wer in eine fremde Arbeitsorganisation eingliedert und dem Weisungsrecht des Unternehmers unterworfen ist. ${ }^{28}$ Bei höheren Diensten ist dieses Weisungsrecht eingeschränkt. Hier reicht für die Erfüllung des

24 Zur Relevanz der Rechtsprechungsänderung bzgl. der Bezeichnung dieses Kriteriums als „Erfüllung des Versicherungstatbestands“ in BSG, Urt. v. 15.5.2012, E 111, 37, s. u. Kap. 1 D. IV.

25 Vgl. Köhler, VSSR 2013, S. 47, 48 m.w.N.

26 Marschner, in: Rolfs/Giesen et al. (Hrsg.), BeckOK Sozialrecht, $\mathbb{2}$ SGB VII Rn. 6; zum Beschäftigungsverhältnis als Grundlage des Versicherungsschutzes vgl. Teutsch, Das Beschäftigungsverhältnis als Grundlage des gesetzlichen Unfallversicherungsschutzes, passim.

27 Der mögliche Unterschied bezieht sich auf die sog. fehlerhaften Arbeitsverhältnisse, bei denen strittig ist, ob sie Arbeitsverhältnisse im engeren Sinne sind; jedenfalls aber sind sie Beschäftigungsverhältnisse, vgl. zum Ganzen Held, Die Verfassungsmäßigkeit der Arbeitgeberbeiträge zu den Sozialversicherungen, S. $92 \mathrm{ff}$. m.w.N.; Greiner, SDRSV Bd. 62, S. 9, passim. Zur synonymen Verwendung im Sozialversicherungsrecht Heise, NZA 2017, S. 1571, 1572 m.w.N. Weitere Nachweise zum sozialrechtlichen Begriff der Beschäftigten finden sich bei Giesen, Arbeitsunfall und Dienstunfall, S. 25 ff. In dieser Arbeit werden zur Verbesserung des Leseflusses die Begriffe Beschäftigter und Arbeitnehmer synonym verwendet.

28 Vgl. z.B. BSG, Urt. v. 15.5.2012, E 111, 37, 44; Urt. v. 29.1.1981, E 51, 164, 167; Urt. v. 17.3.1992 - 2 RU 22/91 -, juris Rn. 15. 
Unterordnungskriteriums die „funktionsgerecht dienende Teilhabe am Arbeitsprozess". 29

Abzugrenzen sind Beschäftigte von selbstständig Erwerbstätigen, die im Recht der Unfallversicherung als „Unternehmer“ bezeichnet werden. Die Abgrenzung erfolgt am Einzelfall; entscheidend ist das den Beschäftigten prägende Merkmal der persönlichen Abhängigkeit. Keine Beschäftigten sind etwa Personen, die aus Gefälligkeit Aufgaben übernehmen, sog. EinEuro-Jobber oder Gefangene, die im Gefängnis Arbeit leisten. ${ }^{30}$

\section{Der Unfall als schädigendes Ereignis}

\section{Der Unfall als Ereignis}

Das nächste zu prüfende Tatbestandsmerkmal bei der Frage, ob ein Arbeitsunfall vorliegt, ist, ob das fragliche Geschehen überhaupt als Unfall zu qualifizieren ist. $\mathbb{S} 8$ Abs. 1 S. 2 SGB VII definiert Unfälle als ,zeitlich begrenzte, von außen auf den Körper einwirkende Ereignisse, die zu einem Gesundheitsschaden oder zum Tod führen“.

Ein Ereignis ist nach einhelliger Meinung in Rechtsprechung und Literatur jedes Geschehen, das objektiv wahrnehmbar ist und die Veränderung eines Zustandes bewirkt. ${ }^{31}$ Damit ist nicht lediglich das Aufeinandertreffen zweier physischer Gegenstände gemeint. Vielmehr können sowohl Vorgänge in der Natur als auch Handlungen von Personen, zum Beispiel des Verletzten, Ereignisse sein - entweder in Form einzelner Elementarvorgänge (z.B. Blitzschlag, Sturz oder Hitzschlag) ${ }^{32}$ oder als Zusammentreffen vieler Elementarvorgänge (z.B. Verkehrsunfall $)^{33}$. Auch ungewollte plötzliche Muskelanspannungen oder Bewegungsabläufe können ein Ereignis darstellen. ${ }^{34}$

29 Vgl. BSG, Urt. v. 15.5.2012, E 111, 37, 44; Urt. v. 14.12.1999, E 85, 214, 216 m.w.N.

30 Marschner, in: Rolfs/Giesen et al. (Hrsg.), BeckOK Sozialrecht, \$2 SGB VII Rn. 9.

31 BSG, Urt. v. 17.2.2009 - B 2 U 18/07 R -, juris Rn. 10; v. 29.11.2011 - B 2 U 23/10 -, juris Rn. 15; Giesen, Arbeitsunfall und Dienstunfall, S. 76; Schnellenbach, Beamtenrecht in der Praxis, S. 330; Brockhaus, in: Schütz/Maiwald (Hrsg.), Beam-

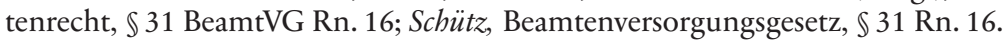

32 RG, Beschl. v. 23.9.1902, Z 52, 197, 199; Urt. v. 11.7.1899, Z 44, 62, 63.

33 Ricke, in: Körner/Leitherer et al. (Hrsg.), Kasseler Kommentar zum Sozialversicherungsrecht, $\mathbb{8}$ SGB VII Rn. 24a; Brockhaus, in: Schütz/Maiwald (Hrsg.), Beamtenrecht, $\mathbb{} 31$ BeamtVG Rn. 16.

34 LSG Sachsen, Urt. v. 10.12.2003 - L 6 V 5/02 -, juris Rn. 30. 
II. Einwirkung von außen

1. Zweck des Merkmals

Gemäß der Definition des $₫ 8$ Abs. 1 S. 2 SGB VII ist ein Geschehen nur dann als Unfall zu qualifizieren, wenn das Ereignis „von außen auf den Körper“ eingewirkt hat. Streit besteht seit dem Jahr 2005 um die Reichweite des den Begriff des Ereignisses beschreibenden Merkmals „,on außen auf den Körper einwirkend“ ( $\mathbb{8} 8$ Abs. 1 S. 2 SGB VII). Das Kriterium der äußeren Einwirkung dient unstreitig der Abgrenzung des Unfallbegriffs von Schäden, die aufgrund innerer, in der Person des Geschädigten selbst liegender Ursachen eingetreten sind. ${ }^{35}$ Dazu gehören etwa persönliche gesundheitliche Anlagen (z.B. Herzinfarkt, Kreislaufkollaps) oder die vorsätzliche Selbstschädigung. ${ }^{36}$ Die genaue Funktionsweise des Tatbestandselements wird im Folgenden dargestellt.

\section{Der Begriff der Einwirkung}

Mit dem Element der „Einwirkung“ haben sich Rechtsprechung und Literatur lange Zeit nicht näher auseinandergesetzt. Das Bundessozialgericht scheint in einem Urteil aus dem Jahr $2012^{37}$ nun erstmalig eine Definition des Merkmals der Einwirkung gegeben zu haben. ${ }^{38}$ Es benennt die Einwirkung dort wie folgt: „Unter ,Einwirkung“ (als Kurzbezeichnung für das von außen kommende, zeitlich begrenzt einwirkende Unfallereignis) ist die durch einen solchen Vorgang ausgelöste Änderung des physiologischen Körperzustandes zu verstehen, die von dem (möglicherweise zeitnah danach eintretenden) Gesundheitserstschaden zu unterscheiden ist. ${ }^{\text {(39 }} \mathrm{Zu}$ dieser Beschreibung wurden drei Kritikpunkte formuliert. Zum einen sei

35 Giesen, Arbeitsunfall und Dienstunfall, S.77; Keller, in: Hauck/Noftz et al. (Hrsg.), SGB VII, $\ 8$ Rn. 11; Becker, SGb 2007, S.721, 726; Kunze, VSSR 2005, S. $299,313$.

36 BSG, Urt. v. 12.4.2005 - B 2 U 27/04 R -, E 94, 269, 271; v. 29.2.1984 - 2 RU 24/ 83 -, juris 1. Orientierungssatz; v. 28.7.1977 - 2 RU 15/76 -, juris 1. Orientierungssatz; Marschner, in: Rolfs/Giesen et al. (Hrsg.), BeckOK Sozialrecht, $\mathbb{} 8$ SGB VII Rn. 7, 32; Ricke, in: Körner/Leitherer et al. (Hrsg.), Kasseler Kommentar zum Sozialversicherungsrecht, $\mathbb{8}$ SGB VII Rn. 24; Becker, SGb 2007, S. 721, 726.

37 BSG, Urt. v. 24.7.2012 - B 2 U 9/11 R -, juris.

38 Ricke, WzS 2013, S. 241, 242.

39 BSG, Urt. v. 24.7.2012 - B 2 U 9/11 R -, juris Rn. 42. 
sie überflüssig, da man bisher auch ohne genaue Präzisierung des Einwirkungsbegriffes ausgekommen sei; der Zweck des Merkmals, die relevanten Einwirkungen von solchen abzugrenzen, die aus innerer Ursache entstehen, würde genügen. ${ }^{40}$ Zum anderen schließe die Wahl des Wortes „physiologisch" psychische Einwirkungen nicht ausdrücklich mit ein, obwohl diese nach gefestigter Rechtsprechung mit umfasst sein sollen. ${ }^{41}$ Zuletzt habe das BSG schon den Grundsatz, man müsse die Änderung des physiologischen Körperzustandes vom Erstschaden trennen, noch in demselben Urteil, in welchem er aufgestellt worden war, missachtet. Denn der entscheidende Senat geht in besagtem Urteil davon aus, dass die Verstauchung der Wirbelsäule den dargelegten Einwirkungsbegriff erfülle. Diese Verstauchung stellt aber gerade den Erstschaden dar. ${ }^{42}$ Folge man der Definition des BSG, so hätte die Einwirkung wohl als „das Auftreffen der Wirbelsäule auf den die Verstauchung einleitenden Widerstand in der Außenwelt" bezeichnet werden müssen. ${ }^{43}$ Weiterhin wird aus der Urteilsbegründung nicht klar, ob überhaupt eine Definition der Einwirkung gegeben werden sollte, da sich hinter dem Begriff in Klammern gesetzt der Hinweis „als Kurzbezeichnung für das von außen kommende, zeitlich begrenzt einwirkende Unfallereignis" findet. Auch dieser Einschub ist jedoch nicht eindeutig, da er zum einen redundant ist, nachdem es sich nur um ein Unfallereignis handelt, wenn es von außen kommt und zeitlich begrenzt eingewirkt hat. Zum anderen ist es unwahrscheinlich, dass das Gericht das Wort Einwirkung mit dem des Unfalls gleichsetzen wollte. Als sicher anzunehmen ist lediglich, dass das Bundessozialgericht betonen wollte, dass der Gesundheitserstschaden vom Unfall zu trennen ist. Insofern enthält die Aussage nichts Neues. ${ }^{44}$

3. Klassische Abgrenzung von der inneren Ursache

Deswegen wird das Merkmal in Rechtsprechung und Lehre negativ bestimmt: Eine äußere Einwirkung liegt immer dann vor, wenn der Unfall

40 Ricke, WzS 2013, S. 241, 242.

41 Ebd.

42 Ebd.

43 Ebd.

44 Ricke bezeichnet die im Urteil getroffene Unterscheidung als „Spitzfindigkeit“, die nicht zu Erkenntnisgewinn führen würde, ebd. 
nicht ausschließlich auf einer inneren Ursache beruht. ${ }^{45}$ Neben diesem Begriff wird auch der Terminus der Gelegenheitsursache verwendet. ${ }^{46}$ Sinnvoll ist, bei der Bestimmung des Unfallereignisses von einer inneren Ursache zu sprechen, während von einer Gelegenheitsursache die Rede sein sollte, wenn es um Probleme der Kausalität zwischen Unfall und Primärschaden geht. ${ }^{47}$ Eine innere Ursache stellen rein körperliche Vorgänge des Geschädigten dar. ${ }^{48}$ Dazu gehören Schwächeanfälle oder Ohnmacht ${ }^{49}$ sowie Selbsttötung. ${ }^{50}$ Sind solche Vorgänge einzige Ursache für das Ereignis, handelt es sich nicht um einen Unfall. Wird ein Ereignis willentlich herbeigeführt, ist das Kriterium der äußeren Einwirkung ebenfalls zu verneinen; die Einwirkung kommt dann aus der Person selbst, damit also von innen und nicht von außen. Denn dem Begriff des Unfalls ist immanent, dass das Geschehen unfreiwillig ist. ${ }^{51} \mathrm{Um}$ eine äußere Einwirkung handelt es sich hingegen, wenn der Verletzte nicht zurechnungsfähig ist ${ }^{52}$ oder das vorsätzliche Verhalten eine angemessene Wahrnehmung dienstlicher oder privater Belange ist. ${ }^{53}$ Unter einer äußeren Einwirkung versteht man somit jeden alltäglichen Vorgang, der seine Ursache nicht in der Person des Geschädigten selbst hat. Es bedarf dafür keines besonderen oder ungewöhnlichen Geschehens. Dies ist auch wichtig, weil sonst riskante Tätigkeiten wenig geschützt wären. ${ }^{54}$ Fünf Gruppen der Tätigkeiten lassen sich unterscheiden: mechanische, akustische, thermische und strahlenausgelöste Einwirkungen sowie überdurchschnittliche Anstrengungen. ${ }^{55}$ Ausreichend sind aber in jedem Fall auch Einwirkungen auf die Psyche des Geschädig-

45 So bereits RVA, Entsch. v. 24.1.1888, AN 1888, 176 Nr. 477; st. Rspr. des BSG, vgl. Urt. v. 14.8.1986 - 2 RU 50/85 -, juris; Bauer, in: Geyer/Grunefeld et al. (Hrsg.),

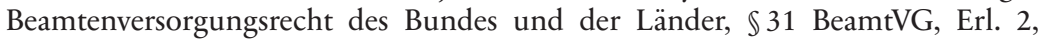
Rn. 2.

46 Z.B. Benz, BB 1982, S. 1860, 1862; Keller, in: Hauck/Noftz/ders. (Hrsg.), SGB VII, $\$ 8$ Rn. 297.

47 So auch Keller, NZS 1995, S. 58, 58.

48 Köhler, VSSR 2013, S. 47, 64; Becker, SGb 2012, S. 691, 694; Kunze, VSSR 2005, S. 299, 313.

49 OVG Nordrhein-Westfalen, Urt. v. 8.12.1976 - VI A 721/75 -, juris.

50 BVerwG, Urt. v. 5.4.1960, E 10, 258.

51 Ein separat zu prüfendes, ungeschriebenes Tatbestandsmerkmal der „Unfreiwilligkeit" ist deswegen nicht anzunehmen, vgl. Giesen, Arbeitsunfall und Dienstunfall, S. 85.

52 BSG, Urt. v. 14.7.1955, E 1, 150.

53 Brockhaus, in: Schütz/Maiwald (Hrsg.), Beamtenrecht, $\$ 31$ BeamtVG Rn.18; Schütz, Beamtenversorgungsgesetz, $\$ 31$ Rn. 17.

54 Kunze, VSSR 2005, S. 299, 313.

55 Kater, in: Kater/Leube, SGB VII, $\mathbb{8}$ Rn. $18 \mathrm{ff}$. 
ten, etwa schwere Beschimpfungen, der Anblick verletzter Personen oder belastende Gespräche. ${ }^{56}$ Auch ein Stolpern beruht auf einer äußeren Einwirkung. ${ }^{57}$

\section{Weite Auslegung der neueren Rechtsprechung}

a. Gewillkürte Handlungen als äußere Einwirkungen: das Heben schwerer Gegenstände

Das BSG hat in mittlerweile gefestigter Rechtsprechung das Merkmal der äußeren Einwirkung ausgeweitet. Im Jahr 2005 hatte das Gericht einen Fall zu entscheiden, in dem ein Arbeitnehmer sich bei dem Versuch, einen festgefrorenen schweren Stein zu heben, eine Verletzung zuzog. ${ }^{58}$ Nach der klassischen Definition der Einwirkung von außen wäre die Prüfung des Arbeitsunfalls bereits beim Merkmal „Unfall“ gescheitert, da es sich beim Anheben eines Gegenstands nicht um eine äußere Einwirkung, sondern um eine freiwillige, willentlich selbst veranlasste Ausübung von Körperkraft handelt. ${ }^{59} \mathrm{Zu}$ Recht nahm das Gericht dennoch einen Unfall an. Der entscheidende Senat argumentierte, dass in Fällen, in denen das schädigende Ereignis ein gewolltes Handeln war, zu differenzieren sei. Gewollte Handlungen seien dann Unfälle, wenn sie eine ungewollte Einwirkung mit sich brächten. Diese sei dann die äußere Einwirkung, welche für den Arbeitsunfall erforderlich ist. Im zu entscheidenden Fall habe der Stein, da er schwer und festgefroren war, eine große Gegenkraft entfaltet. Diese Gegenkraft sei als äußere Einwirkung zu sehen. Es genüge für den Unfallbegriff, wenn „durch eine der versicherten Tätigkeit zuzurechnende außergewöhnliche Kraftanstrengung ein Vorgang im Körperinneren ausgelöst wird, der die gesundheitliche Schädigung bewirkt“.60

Für eine derartige Auslegung des Merkmals spricht, dass äußerlich sichtbare ungewollte Einwirkungen aufgrund gewollten Handelns unbestritte-

56 Keller, SGb 2012, S. 668, 670 m.w.N.

57 BVerwG, Urt. v. 24.10.1963, E 17, 59.

58 BSG, Urt. v. 12.4.2005, E 94, 269.

59 So z.B. das LSG Sachsen noch in seinem Urt. v. 10.12.2003 - L 6 V 5/02 -, juris. Es ging um einen Soldaten, der sich beim Schneeräumen eine Rückenschädigung zugezogen hatte, weil die Last für ihn zu groß war. Eine äußere Einwirkung wurde verneint, da sich der Körper des Soldaten auf die Last eingestellt hatte; dass diese zu groß war, sei unerheblich.

60 BSG, Pressemitteilung Nr. 18/05 vom 13.4.2005 zum Urteil vom 12.4.2005. 
nermaßen den Unfallbegriff erfüllen. Ein anschauliches Beispiel ist der Mitarbeiter eines Sägewerks, der neben einem Stück Holz unabsichtlich auch seinen Daumen mit absägte. Er ist zweifelsohne unfallversicherungsrechtlich geschützt, obwohl er die Säge willentlich betätigt hat, man also nicht von einer äußeren Einwirkung im engeren Sinne sprechen kann. Gleiches muss dann auch für Einwirkungen gelten, deren Folgen äußerlich nicht sichtbar sind - wie etwa die physikalischen Gegenkräfte beim Versuch des Hochhebens eines festgefrorenen Gegenstands. ${ }^{61}$ Entgegen der Ansicht des LSG Bayern geht diese Auslegung auch nicht zu weit. Dieses argumentierte, die Sichtweise begründe einen Unfall im Wesentlichen schon allein mit dem Vorliegen eines Schadens. ${ }^{62}$ Folge man dem Wortlaut der Entscheidung, sei die einzige Korrekturmöglichkeit, zusätzlich eine „außergewöhnliche Kraftanstrengung“ zu verlangen. Dies sei aber kein objektives Kriterium; in der Praxis fiele es schwer, zwischen gewöhnlicher und außergewöhnlicher Kraftaufwendung abzugrenzen. Dagegen ist vorzubringen, dass zur Begründung der äußeren Einwirkung nicht auf den Schaden, sondern auf das den Schaden auslösende Moment abgestellt wird. Vielmehr geht es darum, ein zum Schaden führendes Moment zu isolieren, welches von der betroffenen Person ungewollt war. Auf diese Einwirkung war die Person gerade nicht vorbereitet. ${ }^{63}$ So wird auch verständlich, weshalb beim Umknicken infolge Unachtsamkeit eine Einwirkung von außen vorliegt. Hier wirkt der Fußboden von außen in einer Weise auf den Körper des Versicherten ein, die dieser nicht vorhergesehen und gewollt hat. ${ }^{64}$ Es war deswegen auch nicht nötig, eine Untergrenze für die aufgewendete Kraft festzulegen. ${ }^{65}$ Auch der Wortlaut des $₫ 8$ Abs. 1 S. 2 SGB VII lässt eine weite Auslegung des Merkmals der Einwirkung von auBen zu, da er keine bestimmte Qualität der Einwirkungen fordert. ${ }^{66}$

61 BSG, Urt. v. 29.11.2011 - B 2 U 23/10 R -, juris.

62 LSG Bayern, Urt. v. 19.7.2011 - L 15 VS 7/10 -, juris Rn. 50.

63 Anders, wenn der Körper sich auf die Last bereits eingestellt hatte, vgl. Fn. 79.

64 Keller, SGb 2012, S. 668, 670.

65 LSG Baden-Württemberg, Urt. v. 26.1.2009 - L 1 U 3612/08 -, juris, für das Heben einer $25 \mathrm{~kg}$ schweren Glasscheibe.

66 Keller, SGb 2012, S. 668, 668. 
b. Problem der Identifikation der relevanten Einwirkung: die ZugführerFälle

Ein instruktives Beispiel für die vom Bundessozialgericht vorgenommene Auslegung des Merkmals der äußeren Einwirkung sind die Entscheidungen über einen Triebwagenfahrer eines S-Bahn-Zuges, der eine posttraumatische Belastungsreaktion erlitt, als er eine Schnellbremsung durchführen musste, um einen Zusammenprall mit einem Kraftfahrzeug zu vermeiden. Zwar hatte er den Bremsvorgang selbst ausgelöst. Das Bundessozialgericht sah hier aber die Trägheits- und Scheinkräfte beim Bremsvorgang als von außen auf den Körper des Triebwagenführers einwirkende Ereignisse. ${ }^{67}$ Anders urteilte es an demselben Tag in einem zweiten Rechtsstreit desselben Klägers, in welchem dieser eine posttraumatische Belastungsreaktion als Folge einer Notbremsung geltend machte, die er selbst ausgelöst hatte, weil er irrtümlich annahm, dass sich eine Person auf dem Bahnübergang befände. ${ }^{68} \mathrm{Da}$ sich tatsächlich keine Person dort befand, lag nach Ansicht des Gerichts keine äußere Einwirkung vor. Die Wortlautgrenze des $\mathbb{8} 8$ Abs. 1 Satz 1 SGB VII lasse es nicht zu, eine „bloße Vorstellung“ unter die Formulierung ,äußere Einwirkung “ zu fassen. ${ }^{69}$ Das Gericht scheint dabei davon auszugehen, dass nur physisch nachweisbare bzw. sichtbare Geschehnisse „auf den Körper“ einwirken können. Dabei muss es sich nicht um Kraftentwicklung handeln. Auch Beschimpfungen sind insofern physisch nachweisbar, als sie in Form von gesprochenen Wörtern oder gezeigten Gesten tatsächlich für einen Augenblick in der Welt sind. Bloße Vorstellungen dagegen existieren zu keinem Zeitpunkt physisch.

Die angesprochenen Zugführer-Urteile sind problematisch, weil sie die äußere Einwirkung nicht richtig erfassen. Im ersten Fall wurden die physikalischen Kräfte, die sich beim Bremsvorgang entwickeln, als äußere Einwirkung gewertet. Diese wirkten aber im zweiten Fall genauso, wo sie dem Gericht jedoch nicht als äußere Einwirkung galten. ${ }^{70}$ In beiden Fällen war aber der jeweils geltend gemachte Gesundheitsschaden, das heißt die posttraumatische Belastungsstörung, nicht kausal auf die Bremsung zurückzuführen, sondern im Anlass der Notbremsung begründet. ${ }^{71}$ Richtig wäre es daher gewesen, nicht den Bremsvorgang an sich, sondern gerade den auslösenden Grund für den Bremsvorgang als äußere Einwirkung zu identifizie-

67 BSG, Urt. v. 29.11.2011 - B 2 U 23/10 R -, juris.

68 BSG, Urt. v. 29.11.2011 - B 2 U 10/11 R -, juris.

69 Keller, SGb 2012, S. 668, 670.

70 Krasney, NZS 2014, S. 607, 610.

71 Ebd. 
ren. ${ }^{72} \mathrm{Im}$ ersten, positiv beschiedenen Fall war dies die Anwesenheit des anderen Kraftfahrzeugs auf den Gleisen. Sie zwang den Zugführer zum Handeln, also zum Ziehen der Bremse. Hier lässt sich problemlos begründen, dass die Anwesenheit des anderen Kraftfahrzeugs, mit dem der Zug zu kollidieren drohte, nicht in der Person des Zugführers begründet war und somit eine Einwirkung von außen auf den Zugführer darstellte. Damit kommt man zu einer Bejahung des Unfallbegriffs und letztendlich zu einem Anspruch des Zugführers aus dem Unfallversicherungsrecht.

Im zweiten Fall ist die Einwirkung von außen parallel dazu wieder im Grund des Bremsvorgangs zu suchen. Hier nahm der Zugführer an, eine Person auf dem Gleis zu sehen, und betätigte die Bremse, um eine Kollision mit dieser zu verhindern. Hätte sich tatsächlich eine Person auf dem Gleis befunden, hätte ihre Anwesenheit zweifellos eine Einwirkung von außen auf den Zugführer dargestellt. Da das Gleis allerdings nachweislich leer war, steht der Zugführer vor einem Beweisproblem. Denn ohne äußeren Reiz kann keine Einwirkung von außen angenommen werden, ohne die Wortlautgrenze zu überschreiten. ${ }^{73}$ Handelte es sich um ein bloßes „Hirngespinst", also einen Irrtum des Zugführers, so liegt es in seiner Person selbst begründet. Anders wäre es, wenn beispielsweise ein Schatten, ein Tier o.Ä. eine optische Täuschung bewirkt hätte. Dann wäre wiederum eine Einwirkung von außen zu bejahen gewesen. Teleologisch wäre hier aber eine Bejahung eines Unfallereignisses wünschenswert (dazu sogleich c.).

c. Das Problem der beherrschbaren, irrtümlichen Herbeiführung des Unfalls

An den Zugführer-Fällen zeigt sich auch ein teleologisches Problem, wenn man sich einen dritten Fall vorstellt. Nimmt man an, der Zugführer würde versehentlich den Bremshebel berühren und die Bremse somit unabsichtlich auslösen, wäre dies problemlos als äußere Einwirkung zu qualifizieren.

72 Das Kriterium der Freiwilligkeit kann hier nicht fruchtbar gemacht werden, da der Zugführer jeweils freiwillig und willentlich die Bremse ausgelöst hat. Der Unterschied beider Fälle lag lediglich darin, dass die Gefahr, die der Zugführer durch die Bremsung abwenden wollte, einmal real und einmal nicht real war. Das BSG unterscheidet hier also tatsächlich nicht danach, ob eine Einwirkung von außen stattgefunden hat, sondern danach, warum es eine Einwirkung von außen gegeben hat.

73 Keller, SGb 2012, S. 668, 670. 
Eine nicht beherrschbare körpereigene Bewegung, wie etwa das Stoßen an einen Hebel im Rahmen eines Stolperns, ist schon nach der klassischen, engeren Sichtweise denknotwendig ein Einwirken der Außenwelt auf den Betroffenen (der Betroffene selbst handelt ja gerade nicht willentlich). ${ }^{74}$ Das bedeutet, dass derjenige bessergestellt ist, der im Moment des Unfallereignisses seine Arbeitspflicht weniger gut erfüllt. Denn wer unaufmerksam ist und versehentlich die Bremse auslöst (da die Handlung aus Versehen geschieht, kann sie nicht dem Arbeitgeber zu dienen bestimmt sein), erhält die Leistungen der gesetzlichen Unfallversicherung. Wer dagegen einen Zusammenstoß mit Menschen vermeiden will (und damit seine Arbeitspflicht erfüllt), sich aber über das tatsächliche Vorliegen einer Gefahr irrt, genießt keinen Unfallschutz. Vor dem Hintergrund, dass Zweck der Unfallversicherung ist, gerade solche Risiken, die der Arbeitnehmer zur Erfüllung seiner arbeitsrechtlichen Pflichten auf sich nimmt, abzudecken, ist dieses Ergebnis nicht sachgerecht. Dies ergibt sich auch aus einem Vergleich mit der Konstellation der fahrlässigen Unfallherbeiführung, also des Auslösens des Unfalls auch durch eine Handlung des Beschäftigten, die der im Verkehr erforderlichen Sorgfalt nicht genügt. Ein fahrlässiges Verhalten des Arbeitnehmers schließt, ebenso wie ein vorsätzlich verbotswidriges, gemäß $₫ 7$ Abs. 2 SGB VII die Annahme eines Arbeitsunfalls nicht aus. ${ }^{75}$

Der Grund für die Zurechnung des Risikos der Fahrlässigkeit des Arbeitnehmers zur Risikosphäre des Arbeitgebers besteht darin, dass es in der menschlichen Natur liegt, nicht immer die vollständige Konzentration und Sorgfalt aufwenden zu können. Eine solche Fahrlässigkeit des Beschäftigten zählt daher nach allgemeiner Ansicht zum Betriebsrisiko des Unternehmers. ${ }^{76}$ Genauso ist es aber der menschlichen Natur eigen, sich gelegentlich zu irren. Das Risiko eines Irrtums gehört deswegen ebenso zum Betriebsrisiko des Unternehmers: Wer sich der Arbeitskraft eines Menschen bedient, hat auch das Risiko zu tragen, dass dieser eine Entscheidung trifft, die sich im Nachhinein als irrtümlich herausstellt.

74 Vgl. Keller, SGb 2012, S. 668, 668 m.w.N.

75 Vgl. BSG, Urteil vom 4.6.2002 - B 2 U 11/01 R -, juris 1. Ls.; Wietfeld, in: Rolfs/ Giesen et al. (Hrsg.), BeckOK Sozialrecht, $\$ 7$ SGB VII Rn. 7; Keller, in: Hauck/ Noftz/ders. (Hrsg.), SGB VII, $\$ 7$ Rn. 7; Dahm, NZV 2014, S. 114, 115.

76 Dazu ausführlich s.u. Kap. 2 C. I. 
III. Scheinmerkmal „auf den Körper“

In $\mathbb{8} 8$ Abs. 1 S. 2 SGB VII findet sich in der Definition des Arbeitsunfalls weiterhin das Merkmal, das Ereignis müsse „auf den Körper“ eingewirkt haben. Dies ist jedoch erst beim Primärschaden zu prüfen. Denn jede äußere Einwirkung, die zu einem Gesundheitsschaden oder Tod führt, beeinflusste zwingend den Körper. Die Worte „auf den Körper“ sind in $\$ 8$ Abs. 1 S. 2 SGB nur aufgrund der Satzkonstruktion nötig; die Verbkonstruktion „von außen einwirken“ erfordert zwingend ein Objekt (um die Frage zu beantworten, worauf eingewirkt wird), deswegen muss der Körper erwähnt werden. Es muss also bei Anwendung des $\mathbb{8} 8$ Abs. 1 SGB VII erst im Rahmen des Erstschadens geprüft werden, ob eine Einwirkung auf den Körper vorgelegen hat.

\section{Das Merkmal „zeitlich begrenzt“}

Zur Abgrenzung von Dauerschädigungen ${ }^{77}$ dient das Merkmal der zeitlichen Begrenzung. Die in $\$ 8$ Abs. 1 S. 2 SGB VII enthaltene Formulierung "zeitlich begrenzt" bedeutet zunächst nur, dass das Ereignis einen Anfang und ein Ende haben muss. Darüber hinaus wird der Begriff der zeitlichen Begrenzung dazu verwendet, zu suggerieren, dass es gerade um eine kürzere Zeitspanne geht, das Ereignis also nur einen „verhältnismäßig kurzen Zeitraum" lang dauern darf. ${ }^{78}$ Die von den Gerichten entwickelte Obergrenze dafür ist die Dauer einer einzelnen Arbeitsschicht. ${ }^{79}$ Es ist nicht notwendig, Anfangs- und Endpunkt der Zeitspanne genau benennen zu können. Nicht einmal der genaue Tag, an dem der Unfall sich ereignete, muss bekannt sein. Es genügt, wenn feststeht, dass die Schädigung innerhalb einer einzelnen Arbeitsschicht sowohl begonnen hat als auch abgeschlossen war. ${ }^{80}$ Eine zeitliche Begrenzung liegt zum Beispiel dann nicht

77 Für diese besteht in der Unfallversicherung der Versicherungsfall der Berufskrankheit, vgl. Giesen, Arbeitsunfall und Dienstunfall, S. 79.

78 RVA, Urt. v. 24.11.23, EuM 16, 83; BVerwG, Urt. v. 4.2.1966, E 23, 201.

79 Vgl. nur BSG, Urt. v. 26.9.61, E 15, 112, 112; E 24, 216, 219; Ricke, in: Körner/Leitherer et al. (Hrsg.), Kasseler Kommentar zum Sozialversicherungsrecht, $\mathbb{} 8$ SGB VII Rn. 23; Becker, SGb 2007, S. 721, 726; Kunze, VSSR 2005, S. 299, 313.

80 BSG, Urt. v. 31.1.2012 - B 2 U 2/11 R -, juris Rn. 24; v. 30.5.1985 - 2 RU 17/84 -, juris; Giesen, Arbeitsunfall und Dienstunfall, S. 82; Ricke, in: Körner/Leitherer et al. (Hrsg.), Kasseler Kommentar zum Sozialversicherungsrecht, $₫ 8$ SGB VII Rn. 23. 
vor, wenn der Beschäftigte über Jahre hinweg einem schädigenden Einfluss ausgesetzt war. Ist ein Arbeitnehmer etwa täglich geringen Mengen radioaktiver Strahlung ausgesetzt, die nicht für sich allein, aber über mehrere Tage zusammengenommen gesundheitsschädlich sind, ist das Merkmal der zeitlichen Begrenzung nicht erfüllt. Das Gleiche gilt für eine Infektion, die darauf beruht, dass über mehrere Arbeitsschichten hinweg immer wieder kleinere Mengen von Erregern aufgenommen werden, die erst in ihrer $\mathrm{Ku}-$ mulation zu einer Infektion führen. Anfang und Ende des schädigenden Ereignisses beschränken sich dann nicht auf eine Arbeitsschicht. Ein Arbeitsunfall liegt in diesen Fällen nicht vor. Infektionskrankheiten können aber grundsätzlich auch Arbeitsunfälle sein, und zwar dann, wenn während einer Arbeitsschicht alle Krankheitserreger aufgenommen werden. ${ }^{81}$

\section{Der Primärschaden}

\section{Zur Prüfungsreihenfolge}

Nach dem Wortlaut enthält das Ereignis „Unfall“ bereits per definitionem die Merkmale des Schadens und des Kausalzusammenhangs („die zu einem Gesundheitsschaden führen"). Für die Zwecke der juristischen Prüfung ist das aber ungeschickt, weil damit die einzelnen Glieder der Kausalkette miteinander vermengt werden. Stellt man sich die Frage nach der Gewährung von Unfallhilfe der Anschaulichkeit halber als dreigliedrige Kausalkette vor (1. mit der Arbeit zusammenhängender schädigender Vorgang; 2. primäre Schädigung, 3. Schädigungsfolgen) $)^{82}$, so sind die ersten beiden Glieder miteinander vermengt, obwohl doch zwischen beiden erst ein wichtiger und komplexer Prüfungsschritt zu erfolgen hat, nämlich die Prüfung der haftungsbegründenden Kausalität. Es ist daher sinnvoll, das Unfallereignis, die Unfallkausalität und den Gesundheitsschaden separat zu denken. ${ }^{83}$ Dies ist auch möglich, denn es gibt kein interpretatorisches Verbot, Haupt- und Nebensatz einer Norm getrennt zu betrachten und zu bewerten. ${ }^{84}$

81 Schmitt, SGB VII, $\mathbb{8} 8$ Rn. 142. Vgl. genauer zu diesen Fällen Giesen, Arbeitsunfall und Dienstunfall, S. $83 \mathrm{f}$.

82 Vgl. BSG, Urt. v. 25.3. 2004 - B 9 VS 1/02 R -, juris.

83 So auch LSG Bayern, Urt. v. 19.7.2011 - L 15 VS 7/10 -, juris Rn. 47.

84 Kunze, VSSR 2005, S. 299, 314. 


\section{Gesundheitsschaden oder Tod, $\$ 8$ Abs. 1 S. 2 SGB VII}

Die Primärschädigung ist das Ergebnis des Unfallereignisses. ${ }^{85}$ Laut Gesetzestext kommen dabei Gesundheitsschaden oder Tod in Betracht, $\$ 8$ Abs. 1 S. 2 SGB VII. Bei Ersterem muss es sich um einen Körperschaden handeln, welcher aber auch ein psychischer Schaden sein kann. ${ }^{86}$ Nur ausnahmsweise ist auch ein Sachschaden ausreichend: Der Verlust oder die Beschädigung eines Hilfsmittels wie einer Brille oder Prothese werden in $\$ 8$ Abs. 3 SGB VII als Gesundheitsschaden definiert. Hilfsmittel sind in $\$ 31$ Abs. 1 SGB VII definiert als Sachen, die die Folgen von Gesundheitsschäden mildern oder ausgleichen. Dazu gehören Körperersatzstücke wie Prothesen, orthopädische und sonstige Hilfsmittel wie Brillen oder Rollstühle. ${ }^{87}$ Ein Schaden an diesen Hilfsmitteln ist einem Schaden an der Gesundheit des Beschäftigten gleichzustellen, vorausgesetzt, dass das Hilfsmittel bei Unfalleintritt bestimmungsgemäß in Gebrauch war. ${ }^{88}$ Gesundheitsschäden im engeren Sinne werden in Spiegelung dieses Regel-Ausnahme-Verhältnisses als echte, ersatzfähige Sachschäden dagegen als unechte Schäden bezeichnet. ${ }^{89}$

Die Primärschädigung ist nur die Vorbedingung der Leistungsansprüche, noch nicht aber der Leistungstatbestand selbst. Dies ergibt sich aus der Systematik des SGB VII, denn $\ 8$ SGB VII im ersten Kapitel des dritten Abschnitts des SGB VII regelt nur den Arbeitsunfall als Versicherungsfall, während die Leistungen nach Eintritt eines Versicherungsfalles im dritten Kapitel des dritten Abschnitts des SGB VII geregelt werden. ${ }^{90}$ Die Primärschädigung ist damit in den meisten Fällen eine Tatsachenfrage medizinischer Art. ${ }^{91}$

Als Gesundheitsschaden bezeichnet man jeden regelwidrigen körperlichen, geistigen oder seelischen Zustand. ${ }^{22}$ Dies können körperliche Verletzungen sein, aber auch Schockzustände oder psychische Beeinträchtigun-

85 Becker, SGb 2007, S. 721, 727.

86 Ebd.

87 Heinze/Ricken et al., NZA 2001, S.1376, 1376; Schmitt, SGB VII, $\mathbb{8} 8$ SGB VII Rn. 311.

88 Keller, in: Hauck/Noftz/ders. (Hrsg.), SGB VII, $\mathbb{\int} 8$ Rn. 13.

89 Ricke, in Körner/Leitherer et al. (Hrsg.), Kasseler Kommentar zum Sozialversicherungsrecht, $₫ 8$ SGB VII Rn. 20.

90 Kunze, VSSR 2005, S. 299, $314 \mathrm{f}$.

91 Ebd., S. 299, 315.

92 Nach dem allgemeinen Krankheitsbegriff der Sozialversicherung, vgl. Ricke, in: Körner/Leitherer et al. (Hrsg.), Kasseler Kommentar zum Sozialversicherungsrecht, $₫ 8$ SGB VII Rn. 20; Holtstraeter, in: Knickrehm/Kreikebohm/Waltermann 
gen wie ein Trauma. ${ }^{93}$ Dabei ist zunächst jeder Beschäftigte in dem Gesundheitszustand versichert, in dem er sich bei Eintritt des Versicherungsfalls befunden hat. ${ }^{94}$ Abgestellt wird bei der Bestimmung des Schadens also nicht darauf, ob das Ereignis bei einem durchschnittlich gesunden Menschen einen regelwidrigen Zustand hervorgerufen hat. Es kommt stattdessen auf die individuellen Anlagen des Verletzten an. ${ }^{95}$ Ein Schaden liegt vor, wenn sich dieser Zustand verschlechtert hat, wobei Dauer und Umfang der Verschlechterung für die Definition keine Rolle spielen. ${ }^{96}$ Außerdem ist es unerheblich, ob ein regelwidriger Zustand hervorgerufen oder lediglich verschlimmert wird. ${ }^{97}$ Schmerz allein stellt jedoch noch keinen Gesundheitsschaden dar. ${ }^{98}$

Der Tod des Verunfallten ist dann Primärschaden, wenn er sofort eintritt. Tritt er später ein, handelt es sich um einen Folgeschaden. ${ }^{99}$

\section{Eröffnung des unfallversicherungsrechtlichen Schutzbereichs}

\section{Terminologie und Funktion des Kriteriums}

Nach der Feststellung, dass ein Unfall vorliegt, ist die Eröffnung des unfallversicherungsrechtlichen Schutzbereichs ${ }^{100} \mathrm{zu}$ prüfen. Damit wird bestimmt, ob eine zum Zeitpunkt des Unfalls tatsächlich ausgeführte Ver-

(Hrsg.), Kommentar zum Sozialrecht, $\mathbb{8} 8$ SGB VII Rn. 85; Schmitt, SGB VII, $\mathbb{} 8$ Rn. 145.

93 Holtstraeter, in: Knickrehm/Kreikebohm/Waltermann (Hrsg.), Kommentar zum Sozialrecht, $₫ 8$ SGB VII Rn. 85.

94 Köhler, ZfSH/SGB 2012, S. 383, 388 f.

95 LSG Bayern, Urt. v. 13.10.2011 - L 17 U 159/11 -, juris.

96 Schmitt, SGB VII, $\mathbb{8} 8$ Rn. 145.

97 Ricke, in: Körner/Leitherer et al. (Hrsg.), Kasseler Kommentar zum Sozialversicherungsrecht, $\mathbb{\$} 8$ SGB VII Rn. 20.

98 LSG Baden-Württemberg, Urt. v. 16.1.13 - L 6 U 2874/12 -, juris.

99 Ricke, in: Körner/Leitherer et al. (Hrsg.), Kasseler Kommentar zum Sozialversicherungsrecht, $\mathbb{8} 8$ SGB VII Rn. 22; Holtstraeter, in: Knickrehm/Kreikebohm/ Waltermann (Hrsg.), Kommentar zum Sozialrecht, $\mathbb{\int} 8$ SGB VII Rn. 85.

100 Der Begriff wurde in Anlehnung an die Terminologie in der Grundrechtsprüfung gewählt. Der Terminus „Eröffnung des Schutzbereichs“ bezeichnet in der Grundrechtsdogmatik den Prüfungsschritt, der bestimmt, ob eine bestimmte Tätigkeit generell unter den Schutz eines Grundrechts fällt, bevor in weiteren Schritten ein Eingriff in dieses Grundrecht und dann eine eventuelle Rechtfertigung geprüft werden. Diese vorgelagerte Prüfung, ob ein Handeln überhaupt unter den Schutz einer bestimmten Norm fällt, kann durchaus mit der Prüfung 
richtung überhaupt unter dem generellen Schutz des Unfallsicherungssystems steht. Der Schutzbereich der gesetzlichen Unfallversicherung ist nur dann eröffnet, wenn die tatsächlich ausgeführte Tätigkeit zum Versicherungstatbestand gehört. Dies wird geprüft, indem danach gefragt wird, ob das konkrete Handeln im Unfallzeitpunkt in einem den Versicherungsschutz auslösenden Zusammenhang mit der versicherten Tätigkeit, also der Beschäftigung, steht. Dieser Zusammenhang ist als sachlicher Zusammenhang zu bezeichnen. Es wird also die Grenze zwischen der Sphäre des Privaten und der Sphäre des Beruflichen gezogen, indem der Zurechnungszusammenhang die Risikobereiche voneinander scheidet. ${ }^{101}$ Zwar kann die Frage, ob in einem konkreten Ereignis ein Arbeits- oder Privatunfall zu sehen ist, nicht allein anhand dieses Kriteriums beantwortet werden. Es gibt aber Auskunft darüber, ob die Person bei diesem konkreten Verhalten grundsätzlich überhaupt einen Arbeitsunfall erleiden kann. ${ }^{102}$ Anders formuliert, gilt es, festzustellen, ob die Person zum Unfallzeitpunkt eine versicherte bzw. von der Sicherung umfasste Tätigkeit ausgeübt hat. Das Merkmal ist dann zu bejahen, wenn sich das spezifische mit der geschützten Tätigkeit verbundene Risiko verwirklicht hat. Hintergrund des Kriteriums ist, dass der Unternehmer als alleiniger Träger der Beitragslasten der Unfallversicherung nur für solche Risiken einstehen soll; lediglich dafür kann er schließlich verantwortlich gemacht werden. ${ }^{103}$ In einem $\mathrm{Zu}$ ordnungsmechanismus, in dem jedes Element notwendig ist, lässt sich zwar nicht von einem "wichtigsten“ Merkmal sprechen; fest steht aber, dass, wenn es bei der Bestimmung eines Arbeits- oder Dienstunfalls Probleme gibt, diese sich meist auf der Ebene der Zuordnung der tatsächli-

des Zusammenhangs zwischen der tatsächlichen Verrichtung und der geschützten Tätigkeit verglichen werden. Denn dadurch erfolgt nichts anderes als die Zuordnung einer bestimmten Handlung zum Schutzbereich einer Norm: Nur wenn die tatsächliche Verrichtung der geschützten Tätigkeit zuzuordnen ist, fällt sie unter den Schutzbereich des Unfallversicherungsrechts. Der Begriff „Eröffnung des Schutzbereichs" ist nicht nur inhaltlich passend, sondern auch griffig, zeigt den Zweck des Kriteriums auf und ist dabei abstrakt genug, um im unten in Kap. 4 B. vorzunehmenden Rechtsvergleich auch auf das Unfallsicherungssystem der Beamten angewandt zu werden.

101 Biletzki, NVwZ 2010, S. 688, 689.

102 Nehls, BG 1983, S. 227, 230.

103 Triebel, JURA 2007, S. 521, 527. Zu der dieser Argumentation zugrunde liegenden Betriebsrisikolehre ausführlich s.u. Kap. 2 C. 
chen Verrichtung zur geschützten Tätigkeit abspielen. ${ }^{104}$ Dem Merkmal ist deswegen im Folgenden besondere Aufmerksamkeit zu widmen.

\section{Die zu verbindenden Elemente}

\section{Geschützte Tätigkeit}

Bevor die Natur des Zurechnungszusammenhangs analysiert werden kann, sind zunächst die beiden zu verbindenden Elemente - nämlich geschützte Tätigkeit und tatsächliche Verrichtung - zu bestimmen. Im Recht der gesetzlichen Unfallversicherung ist die geschützte Tätigkeit gem. $\mathbb{\$} 8$ Abs. 1 S. 1 SGB VII jede „den Versicherungsschutz nach $\$ 2,3$ oder 6 begründende Tätigkeit (versicherte Tätigkeit)“. Das Tatbestandsmerkmal grenzt eine solche Tätigkeit von nicht versicherten Tätigkeiten (also eigenwirtschaftlichen oder versicherungsfreien Tätigkeiten, $\mathbb{} 4$ SGB VII) und von Tätigkeiten, die aufgrund eines anderen Versicherungstatbestands versichert sind (vgl. S $\int 121$ ff. SGB VII), ab. ${ }^{105}$ In den hier interessierenden Fällen der Beschäftigten ist die versicherte Tätigkeit allgemein gesprochen grundsätzlich die geschuldete Arbeitsleistung. ${ }^{106}$ Diese hängt vom individuellen Arbeitsvertrag ab. Versichert ist die gesamte arbeitsvertraglich vereinbarte Tätigkeit. Zusätzlich sind gem. $\$ 8$ Abs. 2 SGB VII auch das Zurücklegen von mit der geschuldeten Leistung zusammenhängenden Wegen (Nr. 1-4) und die Sorge für ein Arbeitsgerät (Nr. 5) versicherte Tätigkeiten.

\section{Die tatsächliche Verrichtung}

Unter dem Begriff der tatsächlichen Verrichtung zur Zeit des Unfallereignisses ist die Tätigkeit zu verstehen, die der Versicherte konkret in dem Augenblick ausgeübt hat, in dem sich der Unfall ereignete. ${ }^{107}$ In jüngerer Zeit wird in der Rechtsprechung des Bundessozialgerichts auch auf die Hand-

104 Marschner, in: Rolfs/Giesen et al. (Hrsg.), BeckOK Sozialrecht, $₫ 8$ SGB VII Rn. 11 m.w.N.

105 Becker, SGb 2007, S. 721, 722.

106 Korenke, in: Bontrup/Korenke et al. (Hrsg.), Arbeit - Personal - Soziales, S. 161, 162.

107 Andere Bezeichnungen für diese Handlung wurden vorgeschlagen, können aber nicht in gleicher Weise deutlich machen, dass keine Bewertung einfließen darf, vgl. Becker, SGb 2007, S. 721, 722 ff. 
lung Bezug genommen, die „unmittelbar vor dem Eintritt des Unfallereignisses" 108 ausgeführt wurde; denn das Unfallereignis unterbricht in vielen Fällen diese Handlung. Allerdings gibt es auch Fälle, in denen die Verrichtung nicht durch das Unfallereignis unterbrochen wird. Dies gilt zum Beispiel für Gelegenheiten, in denen die äußere Einwirkung sich über mehrere Stunden erstreckt. Bei Strahlung oder Hitze ist das vorstellbar. Dann wäre es richtig, von der tatsächlichen Verrichtung zum Zeitpunkt des Unfalls zu sprechen. ${ }^{109}$

Die tatsächliche Verrichtung muss genau definiert werden. Es ist notwendig, die ausgeführte Tätigkeit so präzise wie möglich fassen, um feststellen zu können, ob sie in einem sachlichen Zusammenhang zur geschützten Tätigkeit steht. Um dies zu erreichen, bemühte sich das Bundessozialgericht um eine passende Definition. Als tatsächliche Verrichtung bezeichnet es die kleinste, objektiv sichtbare Handlungseinheit, ${ }^{110}$ also ein einzelnes, konkretes, räumlich und zeitlich bestimmtes Verhalten, welches seiner Art nach von Dritten beobachtbar ist. ${ }^{111}$ Die Feststellung, was die tatsächlich ausgeführte Verrichtung ist, ist rein deskriptiver Natur. Es dürfen keine Ursachenüberlegungen in die Beurteilung einfließen. ${ }^{112}$ Als Beispiel wird vielfach die diesem Urteil des Bundessozialgerichts zugrunde liegende Motorradfahrt genannt, die eben eine Fahrt mit dem Motorrad sei und nicht einfach nur eine Fahrt. ${ }^{113}$ Diese Definition wurde als sowohl überflüssig als auch formal falsch kritisiert. ${ }^{114}$ Überflüssig sei sie deshalb, weil der Begriff der tatsächlichen Verrichtung auch ohne weitergehende Definition gar nicht falsch verstanden werden könne. Formal falsch sei sie, weil jede Handlung beobachtbar sei; abstrakte Handlungen (die nur gedacht wären) gibt es nicht, weil sie dann eben keine Handlungen, sondern Gedanken wären. ${ }^{115}$ In der Tat ist ein nicht beobachtbares Verhalten nicht

108 Z.B. BSG, Urt. vom 4.12.2014 - B 2 U 10/13 R -, E (vorgesehen), juris Rn. 11; v. 26.6.2014 - B 2 U 4/13 R -, juris Rn. 21; v. 15.5.2012 - B 2 U 16/11 R -, juris Rn. 10.

109 Schlaeger, jurisPR-SozR 24/2012, Anm. 3.

110 Spellbrink, WzS 2011, S. 351, 353; zustimmend Krasney, NZS 2013, S. 681, 682.

111 BSG, Urt. v. 9.11.2010 - B 2 U 14/10 R -, juris Rn. 24; a.A. Ricke, in: Körner/Leitherer et al. (Hrsg.), Kasseler Kommentar zum Sozialversicherungsrecht, $\mathbb{S} 8$ SGB VII Rn. 9; Ricke, WzS 2013, S. 241, 241.

112 Becker, SGb 2007, S. 721, 722 ff.

113 Gebildet nach BSG, Urt. v. 9.11.2010 - B 2 U 14/10 R -, juris; Spellbrink, WzS 2011, S. 351, 352; Krasney, NZS 2013, S. 681, 682; Köhler, WzS 2011, S. 203 ff.

114 Ricke, WzS 2013, S. 241, 244 f. und ders., WzS 2017, S. 9, 12 f. mit der Bemerkung, dass die Prämisse jedenfalls rechtlich unschädlich sei.

115 Ebd. mit der Bemerkung, dass die Prämisse jedenfalls rechtlich unschädlich sei. 
vorstellbar. Trotzdem hat die vom BSG eingeführte Definition ihre Berechtigung. Denn unabhängig vom Kriterium der Beobachtbarkeit verdeutlicht sie durch das Erfordernis der konkreten, räumlichen und zeitlichen Bestimmbarkeit die Bedeutung der Bestimmung der Verrichtung und zeigt auf, dass es auch um eine kleine Handlungseinheit gehen kann. ${ }^{116}$

\section{Der sachliche Zusammenhang in $\$ 8$ SGB VII}

\section{Terminologie}

Dass ein irgendwie gearteter Zusammenhang zwischen der tatsächlich ausgeführten Tätigkeit und dem Versicherungstatbestand notwendig ist, ist im Wortlaut des $₫ 8$ Abs. 1 S. 1 SGB VII deutlich angelegt. Demnach sind Arbeitsunfälle solche Unfälle, die „infolge“ einer versicherten Tätigkeit geschehen. ${ }^{117}$ Die ausgeführte Tätigkeit muss im Moment des Unfallzeitpunkts der versicherten Tätigkeit zuzuordnen sein. Das BSG gebrauchte für den notwendigen Zurechnungszusammenhang lange Zeit sowohl den Begriff „innerer Zusammenhang“ als auch die Bezeichnung „sachlicher Zusammenhang" synonym. Zwei Termini für denselben Inhalt zu verwenden, ist nicht ideal, da dies zu Verwechslungen und Ungenauigkeiten führen kann. Vorzugswürdig ist es, von einem sachlichen Zusammenhang zu sprechen. Denn das Wort ,innere“ ist schwierig zu interpretieren; es fällt schon nicht leicht, sich im Gegensatz einen „äußeren“ Zusammenhang vorzustellen. „Sachlich“ dagegen ist ein greifbarer Begriff. Er verdeutlicht, dass es nicht um eine ,innere oder mystische Verbindung bzw. dezisionistische Entscheidung geht, sondern es eine Wertung nach sachlichen Gesichtspunkten sein muss“"118 Es hat ein Zusammenhang "in der Sache“ vorzuliegen. Zudem ist das Wort „innerer“ integraler Bestandteil des im Zuordnungsmechanismus der Unfallversicherung wichtigen Begriffes der „in-

116 Häufig werden mehrere Verrichtungen zur selben Zeit ausgeführt, so etwa, wenn eine Zustellerin Zeitungen austrägt und gleichzeitig ihren Hund spazieren führt. Zu solchen sog. gemischten Tätigkeiten Becker, SGb 2007, S. 721, 723; BSG, Urt. v. 2.11.1988, E 64, 159; v. 4.8.1992 - 2 RU 43/91 -, juris sowie genauer Kap. 1 D. III. 2. C. dd.

117 Becker, SGb 2007, S. 721, 722.

118 Becker, SGb 2007, S.721, 724; Sprang, BG 1989, S. 144, 147; BSG, Urt. v. 25.10.1989 - 2 RU 26/88 -, juris; indifferent Ricke, BG 1994, S. 360, 360. 
neren Ursache“; es ist wünschenswert, keine Verwechslungsgefahr mit dieser Materie zu riskieren. ${ }^{119}$

Inzwischen spricht das Bundessozialgericht auch von der „notwendigen Erfüllung des Versicherungstatbestandes". ${ }^{120}$ Dieser Begriff gefällt, da es tatsächlich nicht um zwei verschiedene Handlungen und den Zusammenhang zwischen ihnen geht, sondern darum, zu entscheiden, ob eine Tätigkeit zu den versicherten Tätigkeiten gehört oder nicht. Wann immer Sprache vereinfacht werden kann, ist dies im Sinne der Rechtsklarheit wünschenswert. ${ }^{121}$

2. Der sachliche Zusammenhang zwischen tatsächlicher Verrichtung und geschützter Tätigkeit

a. Streit um kausale oder finale Natur der Bestimmungsmodalität

Das Merkmal des sachlichen Zusammenhangs wurde erst in der neueren Rechtsprechung des BSG entwickelt. Zunächst unterschieden weder das Reichsversicherungsamt noch das Bundessozialgericht oder die juristische Literatur zwischen dem sachlichen Zusammenhang von tatsächlicher Verrichtung und versicherter Tätigkeit gegenüber dem ursächlichen Zusammenhang von der Verrichtung und dem Unfall, der heute als Unfallkausalität bezeichnet wird. ${ }^{122}$ Zur Bestimmung, ob ein Unfall ein Arbeitsunfall ist, wurde darauf abgestellt, ob das unfallbringende Tun mit dem geschützten Bereich in einem „inneren ursächlichen Zusammenhang" stand. ${ }^{123}$ Die Abgrenzung des versicherten Bereichs geschah damit lange Zeit nach Kausalitätsgesichtspunkten. ${ }^{124}$

Dann fand ein Perspektivwechsel statt. ${ }^{125}$ Anstatt sich auf Kausalitätsgesichtspunkte zu berufen, wurde nun darauf abgestellt, welchen Zweck der

119 Sprang, BG 1989, S. 144, 147.

120 BSG, Urt. v. 15.5.2012, E 111, 37 ff. Rz 47; v. 26.6.2014 - B 2 U 4/13 R -, juris Rn. 18; vgl. Keller in Hauck/Noftz/ders. (Hrsg.), SGB VII, $\$ 8$ Rn. 16.

$121 \mathrm{Zu}$ den inhaltlichen Implikationen des Terminus ausführlich s.u. Kap. 1 D. IV. 1.

122 Mit vielen Belegen aus Rechtsprechung und Literatur Ricke, BG 1984, S. 351, 352 f.; vgl. auch Ricke, BG 1994, S. 360, 360; Sprang, BG 1989, S. 144, 146.

123 Sprang, BG 1989, S. 144, 146 m.w.N.

124 Ricke, in: Körner/Leitherer et al. (Hrsg.), Kasseler Kommentar zum Sozialversicherungsrecht, $\mathbb{\int} 8$ SGB VII Rn. 10.

125 Dieser wurde ausgelöst durch die Schrift von Watermann, in: Schimmelpfennig (Hrsg.), Grundsatzfragen der sozialen Unfallversicherung, S. $129 \mathrm{ff}$. 
Geschädigte mit der unfallbringenden Handlung verfolgt hatte. Das Unfallgeschehen wurde also final betrachtet. ${ }^{126}$ Nach Ansicht des Bundessozialgerichts ist es für die Subsumtion der im Unfallzeitpunkt tatsächlich ausgeführten Tätigkeit unter den Versicherungstatbestand notwendig, dass die Tätigkeit ein konkretes Handeln darstellt, welches objektiv seiner Art nach von Dritten beobachtbar und subjektiv zumindest auch auf die Erfüllung des Tatbestandes der versicherten Tätigkeit ausgerichtet ist. ${ }^{127}$ Der sachliche Zusammenhang soll dabei gerade nicht als Ursachenzusammenhang gesehen werden. ${ }^{128}$

Anderer Ansicht nach habe der Wechsel zur finalen Beurteilungsweise in der Praxis nicht zu einer anderen Art der Beurteilung des notwendigen Zusammenhangs geführt. ${ }^{129}$ Kausale Begründungen seien auch weiterhin zum Teil nötig. ${ }^{130}$ Dies sei nur natürlich, denn während Kausalität retrospektiv das Warum eines Handelns betrachte, frage Finalität prospektiv nach dessen Wozu. ${ }^{131}$ Daraus folge, dass jede finale Handlungstendenz ein kausales Motiv habe. Wer beispielsweise handelt, um seine arbeitsvertragliche Pflicht zu erfüllen (finale Betrachtungsweise), tue dies nur, weil er in einem Beschäftigungsverhältnis steht (kausale Betrachtungsweise). 132

Der diese Argumentation widerlegende Beweis, dass der unfallrechtlich geschützte Bereich nicht durch die Anwendung der Kausalitätslehre abgrenzbar ist, wurde anhand eines Beispielkataloges geführt. ${ }^{133}$ Viele Geschehensabläufe hängen ursächlich mit dem Beruf zusammen, können aber nicht in den Schutzbereich der Unfallversicherung fallen. Als Beispie-

126 Statt vieler Jung, WzS 2011, S. 227, 228.

127 BSG, Urt. v. 15.5.2012, E 111, 37 ff., Rn. 21 f.

128 So schon Watermann, Die Ordnungsfunktionen von Kausalität und Finalität im Recht, passim; Watermann, in: Schimmelpfennig (Hrsg.), Grundsatzfragen der sozialen Unfallversicherung, S. 129 ff.; Nehls, BG 1983, S. 227, 227 ff.; aktuell vgl. nur: Kunze, VSSR 2005, S. 299, 310; Kater, in: ders./Leube, SGB VII, Vor. \$\$ 7-13 Rn. 8; Marschner, in: Rolfs/Giesen et al. (Hrsg.), BeckOK Sozialrecht, $\mathbb{S} 8$ SGB VII Rn. 11; a.A. Ricke, BG 1984, S. 351, 351.

129 Schwerdtfeger, in: Lauterbach/Watermann/Breuer (Hrsg.), Unfallversicherung, $\mathbb{} 8$ Rn. 20; Ricke, in: Körner/Leitherer et al. (Hrsg.), Kasseler Kommentar zum Sozialversicherungsrecht, $\mathbb{8}$ SGB VII Rn. 10.

130 Schwerdtfeger, in: Lauterbach/Watermann/Breuer (Hrsg.), Unfallversicherung, $\mathbb{S} 8$ Rn. 20; Ricke, in: Körner/Leitherer et al. (Hrsg.), Kasseler Kommentar zum Sozialversicherungsrecht, $\mathbb{\int} 8$ SGB VII Rn. 10.

131 Ricke, BG 1984, S. 351, 351.

132 Ricke, in: Körner/Leitherer et al. (Hrsg.), Kasseler Kommentar zum Sozialversicherungsrecht, $\$ 8$ SGB VII Rn. 17; ders., BG 1994, S. 360, 361; ders., BG 1984, S. 351, 351.

133 Sprang, BG 1989, S. 144, 146. 
le werden etwa ein Apotheker genannt, der sich aus Liebeskummer mit apothekeneigenen Medikamenten tötet, oder ein Beschäftigter, der sich bei dem Versuch, ein wertvolles Instrument aus dem Lager zu stehlen, verletzt. ${ }^{134}$ Die unfallbringenden Handlungen beider können kausal auf das jeweilige Arbeitsverhältnis zurückgeführt, jedoch nicht final mit den arbeitsvertraglichen Pflichten verknüpft werden. Nur die finale Betrachtungsweise kann also die Zuordnung zur Risikosphäre der Unfallversicherung leisten.

\section{b. Dogmatik des sachlichen Zusammenhangs}

Zur Prüfung des Vorliegens des sachlichen Zusammenhangs nutzte das Bundessozialgericht lange Zeit eine Standardformulierung. Demnach war wertend ${ }^{135}$ danach zu entscheiden, ob die tatsächliche Verrichtung „innerhalb der Grenze liegt, bis zu der nach dem Gesetz der Unfallversicherungsschutz reicht. Maßgebend ist dabei, ob der Versicherte eine dem Beschäftigungsunternehmen dienende Tätigkeit ausüben wollte und ob diese Handlungstendenz durch die objektiven Umstände des Einzelfalls bestätigt wird“.136 Diese Wertentscheidung muss den Schutzzweck des Versicherungstatbestandes und die Grundprinzipien der Unfallversicherung, vor allem die Haftungsablösung der Unternehmer, den Gedanken des Betriebsrisikos, die alleinige Finanzierung durch die Unternehmer sowie die Fürsorgepflicht des Arbeitgebers für Beschäftigte berücksichtigen. ${ }^{137}$ Entscheidend ist, ob der Geschädigte subjektiv der Auffassung sein konnte, die Tätigkeit sei geeignet, wesentlich den Interessen des Arbeitgebers zu dienen. ${ }^{138}$ Der Handlungstendenz des Beschäftigten kommt dabei zentrale Bedeutung zu. Das sperrige Wort „Handlungstendenz“ stammt aus der Psychologie und beschreibt die Motivation, die ein Individuum zu einer Handlung bewegt. ${ }^{139}$ Ein gebräuchlicherer Begriff wäre „Absicht“. Inzwi-

134 Ebd.

135 BSG, Urt. v. 30.1.1970, E 30, 278, 281; Becker, SGb 2007, S. 721, 724.

136 Um Fundstellen gekürzte Standardformulierung des BSG, z.B. Urt. v. 10.10.2006 - B 2 U 20/05 R -, juris Rn. 14; v. 30.4.1985, E 58, 76, 77; v. 20.1.1987, E 61, 127, 128; v. 28.6.1988, E 63, 273, 274.

137 Becker, SGb 2007, S. 721, 724.

138 Marschner, in: Rolfs/Giesen et al. (Hrsg.), BeckOK Sozialrecht, $\mathbb{} 8$ SGB VII Rn. 12.

139 Reisenzein, in: Wirtz/Strohmer (Hrsg.), Lexikon der Psychologie, Stichwort: Handlungstendenz. 
schen benutzt das Bundessozialgericht vermehrt den Begriff „Intention“140 Die Wortverbindung „Handlungstendenz“ hat gegenüber den Begriffen Motivation, Intention und Absicht allerdings noch den Vorteil, Handeln und Handlungsgrund sprachlich direkt miteinander zu verknüpfen. ${ }^{141}$

Es geht bei der Frage nach der Handlungstendenz also darum, ob die tatsächliche Verrichtung des Geschädigten dadurch motiviert war, dem Betrieb dienen zu wollen. Dies muss laut der Rechtsprechung nach den objektiven Umständen des Einzelfalls beurteilt werden - die tatsächliche Verrichtung muss also von außen betrachtet bereits die Absicht erkennen lassen, dem Betrieb dienen zu wollen. ${ }^{142}$ Dabei wird auf einen fiktiven objektiven Dritten abgestellt. Das ist sinnvoll. Denn die Alternative wäre, die (ggf. übereinstimmende) Vorstellung von Arbeitgeber und Arbeitnehmer als Maß dessen heranzuziehen, was betriebsdienlich ist. Dies würde aber bedeuten, dass Arbeitgeber und Arbeitnehmer die Grenzen des Versicherungsschutzes bestimmen würden. ${ }^{143}$ In einem anschaulichen Beispiel zum objektiven Dritten wird beschrieben, bei der Bestimmung des sachlichen Zusammenhangs sei es sinnvoll, zunächst den Unfall aus der Überlegung auszuklammern und sich einen Begleiter der geschädigten Person vorzustellen. ${ }^{144}$ Dieser Begleiter beobachtet das Verhalten der Person und kann ihr in jedem Augenblick mitteilen, ob sie gerade grundsätzlich einen Arbeitsunfall erleiden könne. Während eines dienstlichen Telefonats zum Beispiel lautete die Antwort ja, während eines illegalen Glücksspiels mit Kollegen nein. ${ }^{145}$

Wichtig ist, die Handlung auf ihrem „kleinsten und konkretesten Beschreibungsniveau" 146 zu betrachten. Dies wird durch die oben besprochene Definition der tatsächlichen Verrichtung geleistet. ${ }^{147}$ Denn oft wird die Handlung auch ein eigenmotiviertes Ziel haben, etwa, dem Arbeitgeber positiv aufzufallen und so seine eigenen Chancen auf eine Beförderung zu erhöhen. Dies kann aber nicht für den Zuordnungsmechanismus der Un-

140 Schlaeger, jurisPR-SozR 24/2012, Anm. 3, der sich auf BSG, Urt. v. 15.5.2012, E 111, 37 ff., Rn. 22, 48, 76 bezieht.

141 Der Streit um die korrekte Bezeichnung ist nachlesbar bei Krasney, NZS 2013, S. 681, 681 f.; Spellbrink, WzS 2011, S.351, 351 ff.; Köhler, WzS 2011, S. 203, $203 \mathrm{ff}$.

142 Krasney, NZS 2000, S. 373, $373 \mathrm{ff}$.

143 BSG, Urt. v. 4.6.2002 - B 2 U 24/01 R -, juris; v. 13.12.2005 - B 2 U 29/04 R -, juris.

144 Nehls, BG 1983, S. 227, 230.

145 Ebd.

146 Spellbrink, WzS 2011, S. 351, 352.

147 S.o. Kap. 1 D. II. 2. 
fallversicherung ausschlaggebend sein. Die tatsächliche Tätigkeit ist deshalb auf ihre gleichsam an der obersten Schicht liegende Handlungstendenz hin zu untersuchen.

Es ist auch richtig, nur auf die Motivation des Arbeitnehmers abzustellen und nicht darauf, ob die Tätigkeit dem Arbeitgeber tatsächlich diente. Denn den rechtlichen Rahmen des Arbeitsverhältnisses zwischen Arbeitgeber und Arbeitnehmer bestimmt $\$ 611$ BGB. Der Arbeitnehmer schuldet demnach gerade keinen Erfolg, sondern lediglich die Erbringung der Arbeitsleistung. ${ }^{148}$ Es muss also genügen, dass der Versicherte davon ausgehen konnte, dass es sich um eine dem Arbeitgeber dienende Tätigkeit handelte. ${ }^{149}$ Das Erfordernis der Bestätigung durch die objektiven Umstände des Einzelfalls ist also ein Korrektiv, um die Angaben des Beschäftigten dahingehend zu überprüfen, ob eine solche Handlungstendenz plausibel gemacht wurde oder nachvollziehbar ist. ${ }^{150}$ Jedenfalls die Erfüllung von Vertragspflichten sollte stets darunter fallen. ${ }^{151}$ Dabei lässt sich aber keine Regel dergestalt aufstellen, dass der Arbeitnehmer immer betriebsdienlich handelt, wenn er eine Weisung des Arbeitgebers befolgt; denn der Arbeitgeber kann auch Weisungen erteilen, die lediglich das eigenwirtschaftliche Verhalten im Betrieb regeln. ${ }^{152}$

Ist die Handlungstendenz des Beschäftigten nicht aufklärbar, so wird zu seinen Lasten davon ausgegangen, dass keine versicherte Tätigkeit vorliegt, da der Beschäftigte die Beweislast für das Vorliegen der Voraussetzungen des $₫ 8$ Abs. 1 SGB VII trägt. ${ }^{153}$

Weiterhin verlangt die Rechtsprechung, dass die Tätigkeit dem Unternehmen nicht nur auf irgendeine, sondern auf wesentliche Weise dienen muss. Für die Bestimmung dieser Wesentlichkeit werden wiederum Anleihen bei der Theorie der wesentlichen Bedingung genommen. ${ }^{154}$ Dieses Kriterium wird sogleich bei der Fallgruppe der gemischten Tätigkeiten näher untersucht (s.u. Kap. 1 D. III. 2. C. dd.).

148 Maschmann, NZA-Beil. 2006, S. 13, $14 \mathrm{ff}$.; ebenso Giesen, Arbeitsunfall und Dienstunfall, S. 34 Fn. 62 m.w.N.

149 Becker, SGb 2007, S. 721, 724; std. Rspr. des BSG, vgl. Urt v. 28.2.1964, E 20, 215, 218; v. 30.1.1970, E 30, 282, 283; v. 24.6.1981, E 52, 57, 59.

150 Becker, SGb 2007, S. 721, 724; BSG, Urt. v. 12.4.2005 - B 2 U 5/04 R -, juris.

151 Krasney, NZS 2013, S. 681, 682.

152 BSG, Urt. v. 19.1.1995 - 2 RU 3/94 -, juris.

153 BSG, Urt. v. 20.12.2016 - B 2 U 16/15 R -, juris, Orientierungssatz; Schafhausen/ Schäfer-Kuchzinksi, ArbRAktuell 2017, S. 612, 613.

154 Holstraeter, in: Knickrehm/Kreikebohm/Waltermann (Hrsg.), Kommentar zum Sozialrecht, $\mathbb{8} 8$ SGB VII Rn.11; zur Theorie der wesentlichen Bedingung s.u. Kap. 1 E. II. 
c. Abgrenzung zu privaten Tätigkeiten in Gemengelagen

aa. Sogenannte „eigenwirtschaftliche“ Tätigkeiten

Zentrale Bedeutung für die vorliegende Untersuchung hat die Abgrenzung der versicherten Tätigkeiten von privaten Tätigkeiten. Sie findet ebenfalls auf der Ebene des sachlichen Zusammenhangs statt. Da dessen Bestimmung anhand der objektivierten Handlungstendenz des Beschäftigten stark von den Wertungen abhängt, die der einzelne Rechtsanwender in die Prüfung einbringt, hat sich in der Rechtsprechung eine Fülle an Fallgruppen herausgebildet, zu denen sich Grundsätze zur Behandlung des sachlichen Zusammenhangs entwickelt haben. ${ }^{155}$ Die Fallgruppen, welche die Systematik des Zuordnungsmechanismus des $₫ 8$ Abs. 1 SGB VII sowie die Herausforderungen entgrenzter Arbeit besonders deutlich zu illustrieren vermögen, werden im Folgenden dargestellt.

Dies betrifft zunächst die sogenannten „eigenwirtschaftlichen“ Verrichtungen. Damit werden Tätigkeiten bezeichnet, die rein privat veranlasst sind. Das Adjektiv „eigenwirtschaftlich“ ist dabei missverständlich, weil es suggeriert, dass die Tätigkeit zum Gelderwerb außerhalb des Berufes dienen soll, ${ }^{156}$ was indes nicht gemeint ist. Vielmehr sollen sämtliche Handlungen erfasst werden, die ihren Grund nicht in der beruflichen Sphäre des Beschäftigten finden. Sprachlich vorzugswürdig ist es daher, von einem

155 Aufgrund der Fülle des dazu bereits vorhandenen Materials bleibt es anderen Publikationen überlassen, die Vielzahl typischer Anwendungsfälle des sachlichen Zusammenhangs in ihrer Gesamtheit ausführlich darzustellen. Übersichtliche, umfangreiche und verständliche Darstellungen finden sich in großer Zahl in Aufsätzen und in Kommentaren, die laufend aktualisiert und um aktuelle Rechtsprechungsbeispiele ergänzt werden, z.B. Marschner, in: Rolfs/Giesen et al. (Hrsg.), BeckOK Sozialrecht, $\ 8$ SGB VII Rn. 10-25; Rolfs, in: Müller-Glöge/ Preis et al. (Hrsg.), Erfurter Kommentar zum Arbeitsrecht, $\mathbb{} 8$ SGB VII Rn. 511; Holstraeter, in Knickrehm/Kreikebohm/Waltermann (Hrsg.), Kommentar zum Sozialrecht, $\mathbb{8}$ SGB VII Rn. 17-72; Krasney, in: Becker/Burchardt/ders./ Kruschinsky, Gesetzliche Unfallversicherung, $\$ 8$ Rn. 44 ff.; Bereiter-Hahn (Begr.)/ Mehrtens, Gesetzliche Unfallversicherung, $\$ 8$ Anm.7; Wagner, in: Schlaeger/ Voelzke et al., SGB VII, $\mathbb{} 8 \mathrm{Rn} .31 \mathrm{ff}$; Schwerdtfeger, in: Lauterbach/Watermann/ Breuer (Hrsg.), Unfallversicherung, $\mathbb{8} 8$ Rn. 87 ff.; Schulin, in: ders. (Hrsg.), Handbuch des Sozialversicherungsrechts Band 2: Unfallversicherungsrecht, S. 550, 570 Rn. 12 ff.; Krasney, KrV 2014, S. 102 ff.

156 Krasney, in: Becker/Burchardt/ders./Kruschinsky, Gesetzliche Unfallversicherung, $\mathbb{} 8$ Rn. 45; Keller, in: Hauck/Noftz/ders. (Hrsg.), SGB VII, $\mathbb{8}$ Rn. 16a; Ricke, in: Körner/Leitherer et al. (Hrsg.), Kasseler Kommentar zum Sozialversicherungsrecht, $\mathbb{\int} 8$ SGB VII Rn. 41; Kunze, VSSR 2005, S. 299, 310. 
Gegenverantwortungsbereich zur betrieblichen Sphäre ${ }^{157}$, von einer nichtberuflichen ${ }^{158}$ oder einfach von einer privaten ${ }^{159}$ Tätigkeit zu sprechen. Bei solchen privaten Verrichtungen liegt der sachliche Zusammenhang zur versicherten Tätigkeit - nämlich zur Beschäftigung - augenscheinlich nicht vor; deswegen unterfallen sie nicht dem Schutz der gesetzlichen Unfallversicherung. So gilt etwa ein bei der Einnahme des Mittagessens in der betriebseigenen Kantine erlittener Unfall als Privatsache, weil die Nahrungsaufnahme als originär private Handlung angesehen wird und daher keine betriebsdienliche Handlungstendenz aufweist. ${ }^{160}$ Andere klassische Beispiele für private Tätigkeiten sind Telefonate nicht-beruflichen Inhalts mit Familie oder Freunden, die Medikamenteneinnahme, Zigarettenpausen, das Schlafen, das Waschen, das Benutzen der Toilette oder die Freizeitgestaltung auf Dienstreisen. ${ }^{161}$

Versicherte Tätigkeit ist allerdings das Zurücklegen der Wege, die der Beschäftigte auf sich nimmt, um die genannten privaten Tätigkeiten verrichten zu können; denn sie sind, wenn die Arbeit im Anschluss fortgesetzt wird, betrieblich veranlasst. ${ }^{162}$

bb. Unerhebliche Unterbrechung durch private Tätigkeit

Wird der private Zweck aber innerhalb einer lediglich sehr kurzen Unterbrechung einer versicherten Tätigkeit verfolgt, kann die Verrichtung dennoch vom Versicherungsschutz umfasst sein. ${ }^{163}$ Die Rechtsprechung unter-

157 So Barta, Kausalität im Sozialrecht, S. 474.

158 So Kunze, VSSR 2005, S. 299, 310.

159 So Keller, in: Hauck/Noftz/ders. (Hrsg.), SGB VII, $\mathbb{8} 8$ Rn. 16a m.w.N.; Ziegler, in: Becker/Franke et al. (Hrsg.), SGB VII, $\$ 8$ Rn. 58.

160 Std. Rspr., z.B. BSG, Urt. v. 10.10.2002 - B 2 U 6/02 R -, juris; Urt. v. 7.8.1991 - 8 RKnU 1/90 -, juris; Urt. v. 23.6.1982 - 9b/8 RU 18/81 -, juris; BSG, Urt. v. 7.3.1969 - 2 RU 264/66 -, juris; LSG Baden-Württemberg, Urt. v. 22.11.2012 L 6 U 1735/12 -, juris.

161 Triebel, JURA 2007, S. 521, 527 mit Beispielen aus der Rechtsprechung.

162 Vgl. vgl. BSG, Urt. v. 6.12.1989 - 2 RU 5/89 -, juris; v. 25.11.1992 - 2 RU 1/92 -, juris. Besonders verwirrend ist es, wenn vom versicherten „Gang zur Toilette“ gesprochen wird; dabei ist nur das Gehen zu dem Toilettenraum gemeint, das tatsächliche Benutzen der Toilette ist nicht umfasst. „Wege zur Nahrungsaufnahme" werden unten gesondert behandelt, s.u. Kap. 3 A. VI. Zur Widersprüchlichkeit dieser Grundsätze s.u. Kap. 4 C. II.

163 BSG, Urt. v. 9.12.2003, E 91, 293; v. 12.4.2005, E 94, 262; Becker, SGb 2007, S. 721,725 . 
scheidet zwischen erheblichen und unerheblichen Unterbrechungen. Bei einer unerheblichen Unterbrechung besteht der Versicherungsschutz fort. Unerheblichkeit wird angenommen, wenn die Verrichtung zeitlich und räumlich so geringfügig ist, dass sie "quasi im Vorbeigehen“ und "ganz nebenher" erledigt wird. ${ }^{164}$ Die Argumentation für das Fortbestehen des sachlichen Zusammenhangs in solchen Fällen ist, dass die versicherte Tätigkeit der Grund dafür ist, dass die private Handlung so beiläufig erledigt werden kann. Der Beschäftigte würde sich ohne seine versicherte Tätigkeit nicht in einer vergleichbaren Situation befinden. Da der Mensch nicht wie ein Roboter ohne jegliche kurze Abschweifung im eigenen Interesse arbeiten kann, gehören diese Unterbrechungen zum Betriebsrisiko desjenigen, der sich für seinen Betrieb des arbeitenden Menschen als Betriebsmittel bedient. ${ }^{165}$ Dabei kommt es nicht darauf an, ob die sich im konkreten Unfall realisierende Gefahr privater oder beruflicher Natur ist. ${ }^{166}$

Eine feste Grenze für die maximale Dauer einer solchen Unterbrechung ist nicht anerkannt, wobei einer Ansicht nach fünf Minuten die Höchstgrenze darstellen sollen, ${ }^{167}$ anderer Ansicht nach aber bereits ein dreiminütiges Telefonat als zu lange angesehen wurde. ${ }^{168}$ Auch die räumliche Entfernung vom Arbeitsplatz wird als eine die Erheblichkeit indizierende Zäsur zur versicherten Tätigkeit gesehen, wobei auch hier keine Maximaldistanz anerkannt ist. ${ }^{169}$ Beispiele für als unerheblich anerkannte Unterbrechungen aus der Rechtsprechung sind das Pflücken von Kirschen durch

164 BSG, Urt. v. 12.4.2005, E 94, 262; LSG Baden-Württemberg, Urt. v. 16.6.2015 - L 9 U 1534/14 -, juris Rn. 28.

165 BSG, Urt. v. 18.12.1974 - 2 RU 37/73 -, juris Rn. 18; LSG Bayern, Urt. v. 24.4.2007 - L 3 U 447/04 -; Keller, in: Hauck/Noftz/ders. (Hrsg.), SGB VII, $\mathbb{} 8$ Rn. 38; Schulin, in: ders. (Hrsg.), Handbuch des Sozialversicherungsrechts Band 2, $\$ 30$ Rdnr. 46. Zur Einordnung in das Zurechnungsprinzip der Betriebsrisikolehre s.u. Kap. 2. C. I. .

166 BSG, Urt. v. 9.12.2003, E 91, 293. Kritisch dazu Ricke, in: Körner/Leitherer et al. (Hrsg.), Kasseler Kommentar zum Sozialversicherungsrecht, $\$ 8$ SGB VII Rn. 43, sowie zur unerheblichen Unterbrechung im Rahmen der Wegeunfallversicherung Schur/Spellbrink, SGb 2014, S. 589, 593. Das Verlangen einer betriebsspezifischen Gefahr würde die Fallgruppe der unerheblichen Unterbrechung jedoch insgesamt redundant machen, da die erfassten Fälle deckungsgleich mit der Fallgruppe der „besonderen Betriebsgefahr“ wären, s.u.

167 Benz, SGb 1999, S. 83, 84; Ricke, in: Körner/Leitherer et al. (Hrsg.), Kasseler Kommentar zum Sozialversicherungsrecht, $\$ 8$ SGB VII Rn. 42.

168 LSG Hessen, Beschl. v. 16.10.2013 - L 3 U 33/11 -, juris; LSG Baden-Württemberg, Beschl. v. 27.3.2017 - L 3 U 4821/16 -, juris.

169 „Wenige Schritte“ bei Ricke, in: Körner/Leitherer et al. (Hrsg.), Kasseler Kommentar zum Sozialversicherungsrecht, $\mathbb{8} 8$ SGB VII Rn. 42 m.w.N. 
einen Dachdecker von einem Ast, der unmittelbar über dem zu deckenden Dach hängt, ${ }^{170}$ sowie das Hereinheben eines fremden Kinderwagens in ein öffentliches Verkehrsmittel auf dem versicherten Weg ${ }^{171}$ und der Kauf einer Zeitschrift an einem Kiosk während eines versicherten Weges ${ }^{172}$. Als erheblich galten dagegen eine zwanzigminütige Pause im Mitarbeitercasino ${ }^{173}$ sowie das Aussteigen aus einem Pkw, um einen Brief in einen ca. $5 \mathrm{~m}$ entfernten Briefkasten einzuwerfen, was voraussichtlich weniger als $5 \mathrm{Mi}$ nuten gedauert hätte. ${ }^{174}$

\section{cc. Gespaltene Handlungstendenz}

Interessant sind besonders die Fälle der gespaltenen Handlungstendenz, die - wegen der begrifflichen Ähnlichkeit zur sogleich zu erläuternden gemischten Tätigkeit nicht zur Klarheit beitragend - auch Fälle der gemischten Motivationslage ${ }^{175}$ genannt werden. Hier liegt nur eine einzige Verrichtung vor, mit der aber mehrere Zwecke verfolgt werden. ${ }^{176}$ Die Handlung weist also mindestens zwei Handlungstendenzen auf. Ein viel zitiertes Beispiel ist die Autofahrt eines versicherten Vertreters, der Waren ausliefern und in der Nähe des Bestimmungsortes seine Tochter besuchen möchte. Er verunglückt auf einer Wegstrecke der Autobahn, die zu beiden Zwecken befahren werden musste. ${ }^{177}$ Ein anderer Fall ist derjenige eines Beschäftigten, der eine Fahrt unternehmen muss, um zu einem anderen Arbeitsort zu wechseln, und dabei gleichzeitig sein privates Motorrad von der Werkstatt nach Hause bringt, indem er mit dem Motorrad fährt. ${ }^{178}$ Die Frage nach dem sachlichen Zusammenhang zwischen der Motorradfahrt bzw. der Autofahrt und der versicherten Tätigkeit wird hier danach entschieden,

170 BSG, Urt. v. 26.10.2004, E 93, 279; vorgehend LSG Baden-Württemberg, Urt. v. 17.4.2003 - L 7 U 5003/01 -, juris.

171 Ricke, in: Körner/Leitherer et al. (Hrsg.), Kasseler Kommentar zum Sozialversicherungsrecht, $\$ 8$ SGB VII Rn. 42 m.w.N.

172 BSG, Urt. 12.4.2005, E 94, 262.

173 LSG Baden-Württemberg, Urt. v. 16.6.2015 - L 9 U 1534/14 -, juris.

174 LSG Bayern, Urt. v. 27.3.2013 - L 2 U 284/12 -, juris.

175 Z.B. BSG, Urt. v. 12.5.2009 - B 2 U 12/08 R -, juris. Spellbrink, WzS 2011, S. 351, 352 Fn. 11 bevorzugt diesen Ausdruck mit dem Argument, er sei präziser am subjektiven Erleben ausgerichtet.

176 Z.B. aus der jüngeren Zeit SG Karlsruhe, Urt. v. 27.10.2015 - S 4 U 1189/15 -, juris, Rn. 41.

177 BSG, Urt. v. 5.5.1994 - 2 RU 26/93 -, juris.

178 BSG, Urt. v. 9.11.2010 - B 2 U 14/10 R -, juris. 
ob die Verrichtung hypothetisch auch dann vorgenommen worden wäre, wenn die privaten Gründe des Handelns nicht vorgelegen hätten. ${ }^{179}$ Ein sachlicher Zusammenhang zwischen der Verrichtung und der versicherten Tätigkeit wird also dann bejaht, wenn die Verrichtung nach den objektiven Umständen in ihrer konkreten Ausgestaltung ihren Grund in der betrieblichen Handlungstendenz findet. ${ }^{180}$ Auf der Ebene des sachlichen Zusammenhangs ist gerade nicht zu fragen, ob der Unfallerfolg eingetreten wäre, wenn die private Verrichtung entfallen wäre. ${ }^{181}$ Lässt die Verrichtung objektiv die versicherungsbezogene Handlungstendenz erkennen, liegt ein sachlicher Zusammenhang vor. Im Fall der Motorradfahrt verneinte das Bundessozialgericht die objektive Erkennbarkeit einer betriebsbezogenen Handlungstendenz. Der Geschädigte hätte die Motorradfahrt ohne die private Intention nicht vorgenommen. ${ }^{182}$ Auf hypothetische Ausführungen darüber, was vermutlich geschehen wäre, wenn der Geschädigte nicht mit dem Motorrad gefahren wäre, darf es - anders als das vorinstanzliche LSG $^{183}$ meinte - demnach nicht ankommen. ${ }^{184}$ Anders liegt der Fall eines Vaters, der seine Tochter besuchen wollte, weil er sich wegen eines beruflichen Termins in der Nähe ihrer Wohnung befand. Seine tatsächliche Verrichtung, nämlich das Zurücklegen einer Wegstrecke auf der Autobahn, wäre von ihm genauso durchgeführt worden und hätte aus Sicht eines objektiven Dritten genauso ausgesehen, wenn er den Familienbesuch nicht hätte machen wollen. Damit wäre auch hier ein sachlicher Zusammenhang zu bejahen gewesen.

\section{dd. Gemischte Tätigkeit}

Von den soeben behandelten Fällen der gespaltenen Handlungstendenz streng zu unterscheiden ${ }^{185}$ sind Sachverhalte, in denen ein Beschäftigter mehrere Verrichtungen unterschiedlicher Natur gleichzeitig und unmittel-

179 BSG, Urt. v. 12.5.2009 - B 2 U 12/08 R -, juris.

180 Spellbrink, WzS 2011, S. 351, 352; Krasney, KrV 2014, S. 102, 104.

181 Für denkbar hält ein solches Vorgehen allerdings Spellbrink, WzS 2011, S. 351, 353.

182 Vgl. Jung, WzS 2011, S. 227, 231.

183 LSG Nordrhein-Westfalen, Urt. v. 13.4.2010 - L 15 U 11/09 -, juris.

184 Spellbrink, WzS 2011, S. 351, 352.

185 Das BSG führte diese scharfe Trennung mitsamt Terminologie in seinem Urteil v. 9.11.2010 - B 2 U 14/10 R -, juris, ein. Deutlich auch in Urt. v. 26.6.2014 - B 2 U 4/13 R -, juris Rn. 20 ff. Vorher wurden diese Sachverhalte vermischt, vgl. Urt. v. 5.5.1994 - 2 RU 26/93 -, juris. 
bar vor dem Unfallzeitpunkt ausführt. Sind diese Verrichtungen so miteinander verbunden, dass sie nicht in zwei nacheinander liegende Teile getrennt werden können, sondern nur in zwei parallel stattfindende, so spricht man von einer gemischten Tätigkeit. ${ }^{186}$ Ein Beispiel, in dem eine private und eine versicherte Tätigkeit zugleich ausgeführt wurden, ist der Fall der Altenpflegerin in Rufbereitschaft, die während des Spazierenführens ihres Hundes ein berufliches Telefonat annimmt und führt, dabei stolpert und verunfallt. ${ }^{187}$ Diese beiden Handlungen lassen sich nicht in nacheinander liegende Teile zerlegen, sie finden gleichzeitig statt und bilden eine einzige gemischte Tätigkeit. Unter Anwendung der Maßstäbe des sachlichen Zusammenhangs prüfte die Rechtsprechung früher in diesen Fällen, ob die konkrete Tätigkeit - das telefonierende Herumgehen mit Hund - betrieblichen Interessen „auch wesentlich zu dienen bestimmt war". 188

Um das Kriterium der Wesentlichkeit zu bestimmen, wurde dabei auf die Maßstäbe zur Wesentlichkeit aus der Kausalitätstheorie der wesentlichen Bedingung abgestellt. Kurz zusammengefasst hat sie zum Inhalt, dass nur diejenige Ursache rechtserheblich ist, die den Erfolg wesentlich mitbeeinflusst hat. Sie muss weder überwiegender noch einziger, aber wertend betrachtet wesentlicher Grund des Erfolgseintritts sein. ${ }^{189}$ Bei der Bestimmung der Wesentlichkeit wird ex negativo vorgegangen. Dies geschieht, indem gedanklich geprüft wird, ob die Tätigkeit hypothetisch auch ohne den privaten Zweck vorgenommen worden wäre. ${ }^{190}$ Es wird hier die aus der Literatur zur Theorie der wesentlichen Bedingung stammende sogenannte Krasney'sche Prozentrelation angewendet, welche besagt, dass eine Tätigkeit dann unter Unfallversicherungsschutz steht, wenn sie zumindest zu 10 Prozent dem Unternehmen dient. ${ }^{191}$

Es ist allerdings bedenklich, hier die Theorie der wesentlichen Bedingung fruchtbar zu machen. Beim sachlichen Zusammenhang handelt es sich ja gerade nicht um die Feststellung eines Kausalzusammenhangs. Verlangt man aber, dass der betriebliche Zweck wesentlich für die unfallbringende Verrichtung gewesen ist, liegt der (Trug-)Schluss nahe, der betriebli-

186 Becker, SGb 2012, S. 691, 695; Schwerdtfeger, in: Lauterbach/Watermann/Breuer (Hrsg.), Unfallversicherung, $\mathbb{8}$ Rn. 219, 224 m.w.N.

187 BSG, Urt. v. 26.6.2014 - B 2 U 4/13 R -, juris.

188 Z.B. BSG, Urt. v. 22.8.2000 - B 2 U 18/99 R -, juris; so noch Köhler, WzS 2011, S. 203, 203.

189 Ausführlich s.u. Kap. 1 E. II.

190 Becker, SGb 2007, S. 721, 725.

191 Köhler, ZfSH/SGB 2012, S. 383, 386. 
che Zweck müsse überhaupt ursächlich für die unfallbringende Verrichtung gewesen sein. Eine Prüfung in diesem Sinne - nämlich, ob der Unfall entfallen wäre, wenn der betriebliche Zweck nicht gewesen wäre - ist aber gerade nicht gefordert. ${ }^{192}$ So sieht es auch die jüngere Rechtsprechung des Bundessozialgerichts. ${ }^{193}$ Es muss bei der gemischten Tätigkeit also keine hypothetische Prüfung vorgenommen werden, ob die versicherte Tätigkeit auch ohne die private Motivation durchgeführt worden wäre. Handelt es sich um eine gemischte Tätigkeit, so liegt ein sachlicher Zusammenhang mit der versicherten Tätigkeit stets vor, denn die Tätigkeit war zumindest auch dem Unternehmen zu dienen bestimmt. ${ }^{194}$ Auf der Ebene der Kausalität ist dann zu prüfen, ob der beruflich motivierte Teil der gemischten Tätigkeit auch wesentlich für den Erfolgseintritt war. ${ }^{195}$ Die gemischte Tätigkeit wird daher vornehmlich auf der Ebene der Unfallkausalität problematisch. ${ }^{196}$

\section{ee. Besondere Betriebsgefahr}

Eine weitere Fallgruppe, in der private Tätigkeiten vom Unfallversicherungsschutz erfasst werden können, sind die Fälle der sogenannten „besonderen Betriebsgefahr" ${ }^{\text {“197 }}$ oder "spezifischen Gefahr der versicherten Tätigkeit“. ${ }^{198}$ Nach dem unter diesen Stichworten von der Rechtsprechung entwickelten Grundsatz ist ein Unfall, der sich am Arbeitsplatz und in zeitlichem Zusammenhang mit der Arbeitsausführung, aber während einer privaten Handlung ereignet, als Arbeitsunfall anzusehen, wenn dieser durch eine besondere Betriebsgefahr wesentlich (mit)verursacht wurde. ${ }^{199}$ Die

192 Kunze, VSSR 2005, S. 299, 311; a.A. Becker, SGb 2012, S. 691, 695.

193 BSG, Urt. v. 26.6.2014 - B 2 U 4/13 R -, juris Rn. 22.

194 Ebenso Mühlheims, DGUV Forum 2014, S.41, 41; Kruschinsky, jurisPR-SozR 6/2015, Anm. 4.

195 Ebenso Kruschinsky, jurisPR-SozR 6/2015, Anm. 4.

196 BSG, Urt. v. 26.6.2014 - B 2 U 4/13 R -, juris Rn. 22.

197 Becker, in: Ehlers/Fehling et al. (Hrsg.), Besonderes Verwaltungsrecht Band 3, $\$ 78$ Rn 48; Winter, Sozialversicherung 2003, S. 317, 317.

198 Keller, in: Hauck/Noftz/ders. (Hrsg.), SGB VII, $\mathbb{} 8$ Rn. 45.

199 Vgl. nur BSG, Urt. v. 19.1.1995 - 2 RU 3/94 -, juris; v. 20.2.2001 - B 2 U 6/00 R -, juris; v. 23.6.1982 - 9b/8 RU 18/81 -, juris Rn. 15 f.; Becker, in: Ehlers/ Fehling et al. (Hrsg.), Besonderes Verwaltungsrecht Band 3, $\$ 78$ Rn 48; Winter, NZS 2009, S. 199, 201; Ziegler, in: Becker/Franke et al. (Hrsg.), SGB VII, $\mathbb{} 8$ Rn. 178 ff. In der früheren Rechtsprechung kam es dabei auf die Benutzung einer objektiv gefährlichen Betriebseinrichtung an, vgl. Winter, NZS 2009, 
Beschäftigten sollen jedenfalls gegen die typischen Gefahren des Betriebes geschützt sein. ${ }^{200}$ Es ist gerade Zweck der Unfallversicherung, typische Betriebsgefahren zu umfassen, Beschäftigte vor deren Folgen zu schützen und Unternehmen von Schadensersatzansprüchen freizustellen. ${ }^{201}$ Davon wird ausnahmsweise abgewichen, wenn die ausgeführte private Tätigkeit selbst rechtlich wesentlich für den Unfalleintritt war. ${ }^{202}$

Es geht bei dieser Fallgruppe um Fälle, in denen sich eine Gefahr realisiert, welcher der Versicherte gerade durch sein Beschäftigungsverhältnis ausgesetzt ist. ${ }^{203}$ Der durch die spezifische Gefahr wesentlich mitverursachte Unfall muss sich in zeitlichem Zusammenhang mit der Arbeit und in räumlichem Zusammenhang mit dem Arbeitsplatz ereignet haben. ${ }^{204}$ Es genügt aber für die Bejahung eines zeitlichen Zusammenhangs, dass der Unfall während der normalen Arbeitszeit, unmittelbar davor oder danach stattgefunden hat; auch Arbeitspausen sind umfasst. ${ }^{205}$ Der örtliche $\mathrm{Zu}$ sammenhang erfordert, dass sich der Beschäftigte am Arbeitsplatz im weiteren Sinne aufhält, er also den zusammenhängenden Betriebsbereich nicht verlassen hat. ${ }^{206}$ Auf eine kleine Entfernung zum unmittelbaren Arbeitsplatz - es wurde ein Abstand von maximal sechs Metern vorgeschlagen ${ }^{207}$ - kann es nicht ankommen, weil Sinn der Unfallversicherung gerade ist, betriebsspezifische Gefahren zu umfassen, und eine solche Gefahr sich auch außerhalb dieses engen Umkreises auswirken kann.

Beispielsweise hat sich eine besondere betriebsbedingte Gefahr in dem Fall verwirklicht, in welchem ein Beschäftigter während eines privaten Telefonats an seinem Arbeitsplatz durch die Explosion eines Betriebsmittels

S. 199, 201 mit Hinweis auf BSG, Urt. v. 22.6.1976 - 8 RU 146/75 -, juris; v. 18.2.1987 - 2 RU 222/86 -, juris; Mutschler, NZS 2014, S. 647, 648.

200 Becker, in: Ehlers/Fehling et al. (Hrsg.), Besonderes Verwaltungsrecht Band 3, $\$ 78 \mathrm{Rn} 48$; darin liegt eine ausnahmsweise Anerkennung der Figur des Betriebsbanns. Zu dieser s.u. Kap. 4 C. I. 2.

$201 \mathrm{Zu}$ den damit angesprochenen Grundprinzipien der Unfallversicherung ausführlich s.u. Kap. 2 B. und C.

202 Köhler, SGb 2005, S. 655, 657; BSG, Urt. v. 22.1.1976 - 2 RU 101/75 -, juris; v. 19.1.1995 - 2 RU 3/94 -, juris.

203 Ziegler, in: Becker/Franke et al. (Hrsg.), SGB VII, $\mathbb{} 8$ Rn. 178.

204 St. Rspr., vgl. BSG, Urt. v. 12.5.2009 - B 2 U 12/08 R -, juris; Keller, in: Hauck/ Noftz/ders. (Hrsg.), SGB VII, $\$ 8$ Rn. 45 b.

205 Keller, in: Hauck/Noftz/ders. (Hrsg.), SGB VII, $\mathbb{8} 8$ Rn. 45b; a.A. Winter, Sozialversicherung 2003, S. 317, 319.

206 Keller, in: Hauck/Noftz/ders. (Hrsg.), SGB VII, $\$ 8$ Rn. 45b.

207 Winter, Sozialversicherung 2003, S. 317, 319. 
verletzt wurde. ${ }^{208}$ Dagegen fanden die Grundsätze der besonderen betriebsbedingten Gefahr keine Anwendung auf den Fall, in dem ein Beschäftigter eines Kaufhauses nach Ende seiner Arbeitszeit im Kaufhaus verunglückte, während er auf dem Weg in ein anderes Stockwerk einen Privateinkauf abholen wollte. Die private Tätigkeit, einen persönlichen Einkauf abzuholen, wurde hier als rechtlich wesentlich für den Unfalleintritt angesehen, sodass die Grundsätze der betriebsbedingten Gefahr keine Anwendung fanden. ${ }^{209}$

Ausnahmsweise wird auch außerhalb des räumlich-zeitlichen Zusammenhangs mit der Beschäftigung ein Unfall der Berufssphäre zugeordnet. Dies ist der Fall, wenn das betriebliche Geschehen gegenüber allen anderen Ursachen klar im Vordergrund steht. Diese Fallgruppe betrifft Überfälle und Racheakte, die gegen den Beschäftigten gerade aufgrund seines Beschäftigungsverhältnisses verübt werden. ${ }^{210}$

\section{d. Kritik am Kriterium des sachlichen Zusammenhangs}

Die Figur des sachlichen Zusammenhangs ist als überflüssig kritisiert worden. ${ }^{211}$ Zum einen sei die Prüfung desselben ein Zirkelschluss. Mit der Frage, ob ein sachlicher Zusammenhang besteht, solle geprüft werden, ob Versicherungsschutz besteht. Dieser sachliche Zusammenhang könne aber nur dann bejaht werden, wenn Versicherungsschutz bestünde, was aber gerade zu untersuchen sei. ${ }^{212}$ Bei dieser Argumentation wird übersehen, dass sich der sachliche Zusammenhang aus mehreren Elementen zusammensetzt. Zunächst ist zu prüfen, welche tatsächliche Verrichtung im Unfallzeitpunkt ausgeführt wurde. In der Folge ist in einer Wertentscheidung abzuschätzen, ob diese mit der Handlungstendenz ausgeführt wurde, dem Unternehmen zu dienen. Ist dies der Fall, so steht die Verrichtung in einem solchen Zusammenhang mit der versicherten Tätigkeit, dass es gerechtfertigt ist, sie unter Versicherungsschutz zu stellen. Es muss also zwi-

208 Ähnlich BSG, Urt. v. 19.1.1995 - 2 RU 3/94 -, juris Rn. 24; v. 22.1.1976 - 2 RU 191/75 -, juris; Mutschler, NZS 2014, S. 647, 648; Mutschler, SGb 2011, S. 684, 685; Becker, SGb 2007, S.721, 723; Keller, in: Hauck/Noftz/ders. (Hrsg.), SGB VII, $₫ 8$ Rn. 22.

209 BSG, Urt. v. 19.1.1995 - 2 RU 3/94 -, juris Rn. 24.

210 Keller, in: Hauck/Noftz/ders. (Hrsg.), SGB VII, $\mathbb{\$} 8$ Rn.45d; Ricke, NZS 2011, S. 213, 215; Mutschler, NZS 2014, S. 647, 648.

211 Kunze, VSSR 2005, S. 299, 310.

212 Ebd. 
schen versicherter und tatsächlich ausgeführter Tätigkeit unterschieden werden. Unter dieser Voraussetzung liegt kein Zirkelschluss vor.

Weiterhin wurde die Relevanz der Handlungstendenz des Verunfallten an sich in Zweifel gezogen. ${ }^{213}$ Bei Fragen der Bestimmung eines sozialversicherungspflichtigen Beschäftigungsverhältnisses, etwa in Abgrenzung zu selbstständigen Tätigkeiten, käme es immer auf objektive Verhältnisse an. Der Wille der Vertragsparteien spiele dabei keine Rolle. Dass es bei der Bestimmung der Reichweite des Versicherungstatbestandes nun auf den subjektiven Willen des Beschäftigten ankomme, sei ein Systembruch. Er ermögliche es den Beschäftigten, ihre gemischten Tätigkeiten stets so einzurichten, dass sie der Unfallversicherung unterfallen. Sie könnten beispielsweise auf jede private Spazierfahrt Akten mitnehmen, um den Schutz der Unfallversicherung auszulösen. In dieser Dramatik entsprechen diese Aussagen wohl nicht der Realität. Das bloße Mitnehmen von Akten auf eine beliebige Fahrt vermag noch keinen sachlichen Zusammenhang mit der versicherten Tätigkeit auszulösen. Dazu müsste schon wenigstens der arbeitstechnische Zweck kommen, Akten von einem Ort zum anderen zu transportieren - und zwar ohne größere Um- und Abwege.

Als Alternative zur subjektiven Betrachtungsweise wurde vorgeschlagen, die Zugehörigkeit einer Tätigkeit zum Versicherungstatbestand nach objektiven Kriterien zu prüfen. ${ }^{214}$ Eine solche objektive Bestimmungsmodalität würde dieser Ansicht nach Beweisschwierigkeiten und Missbrauchsgefahr vorbeugen. Eine Handlungstendenz sei als subjektives Kriterium schwer zu beweisen; vor allem, wenn der Verunfallte verstorben ist, kann er zu seiner Intention keine Aussage machen. Auch lebende Unfallopfer könnten ihre Handlungstendenz nachträglich in Richtung ihrer versicherten Tätigkeit „nachbessern“. Dies wäre nicht mehr der Fall, wenn nach objektiven Kriterien entschieden werden würde. Konkret solle der Bezug der tatsächlichen Verrichtung zur versicherten Tätigkeit nur anhand von Fallgruppen überprüfen sein. ${ }^{215}$ Solche Fallgruppen sollten dieser Ansicht nach möglichst konkret gefasst sein und sich auf die typischen Merkmale beschränken. Da rein privat motivierte Tätigkeiten von vornherein unproblematisch aus dem Versicherungsschutz ausgeschieden und eindeutig beruflich motivierte Verrichtungen ohne Weiteres zugeordnet werden könnten, seien nur für die problematischen Fälle sogenannter gemischtwirt-

213 Ebd., S. 299, $311 \mathrm{f}$.

214 Kunze, VSSR 2005, S. 299, $310 \mathrm{ff}$.

215 Ebd. 
schaftlicher Tätigkeiten Fallgruppen nötig. ${ }^{216}$ Außerdem müssten noch Typisierungen bei Unterbrechungen von Wegen und Auswirkungen von besonderen betrieblichen Gefahren getroffen werden. Abstrakte Rechtsgrundsätze würden sich nicht dazu eignen, die gesetzliche Regelung zu konkretisieren. Dafür halte das Leben zu viele Möglichkeiten von Fallgestaltungen bereit. ${ }^{217}$ Es dürfe nur darauf ankommen, ob die Arbeit die unfallbringende Tätigkeit objektiv wesentlich geprägt hat. ${ }^{218}$

Offen bleibt bei dieser Kritik, wie diese objektive Abgrenzung genau aussehen könnte. Schon in dem angeführten Beispielsfall einer „Sonntagsfahrt mit Akten“ ist es objektiv schwierig zu entscheiden, ob die Fahrt wesentlich von der Arbeit geprägt war. Die Methode der Rechtsprechung, danach zu fragen, ob der Geschädigte die Fahrt auch ohne die Akten hypothetisch vorgenommen hätte, ist deutlich greifbarer. Immerhin: Auch das Bundessozialgericht betont regelmäßig, dass es auf die Handlungstendenz in der Form ankomme, wie sie insbesondere durch objektive Umstände des Einzelfalls bestätigt wird. ${ }^{219}$ Gerade die angeführte Vielgestaltigkeit des Lebens verbietet aber eine reine Unterteilung in Fallgruppen. Solange auf die Tätigkeit an sich abgestellt wird, verspricht eine von gesicherten Kriterien abhängige Wertentscheidung eine größere Einzelfallgerechtigkeit.

\section{Vom sachlichen Zusammenhang zur „Erfüllung des Versicherungstatbestands“}

1. Sprachliche Neuetikettierung oder Einschränkung des Versicherungsschutzes?

a. Rein terminologische Änderung

Für Verwirrung sorgt das Bundessozialgericht seit dem Jahr 2012, als es erstmalig die Prüfung des sachlichen Zusammenhangs durch die Prüfung der „Erfüllung des Versicherungstatbestands“ ersetzte. ${ }^{220} \mathrm{Ob}$ es sich dabei

216 Ebd., S. 299, 310.

217 Ebd.

218 Ebd., S. 299, 299, 312.

219 Z.B. BSG, Urt. v. 4.6.02 - B 2 U 11/01 R -, juris.

220 BSG, Urt. v. 15.5.2012, E 111, 37. Die neue Formulierung hat das Gericht seither beibehalten, vgl. Urt. v. 13.11.2012 - B 2 U 27/11 R -, juris Rn. 23 f.; v. 4.7.2013 B 2 U 5/12 R -, juris Rn. 18; v. 14.11.2013 - B 2 U 15/12 R -, juris Rn. 13; v. 26.6.2014 - B 2 U 4/13 R -, juris Rn. 16; v. 26.4.2014 - B 2 U 7/13 R -, juris 
um ein völlig neues Tatbestandsmerkmal oder um eine rein terminologische Änderung handelt, wird noch immer kontrovers diskutiert. $221 \mathrm{Ob}-$ wohl sich die relevanten Entscheidungen durchaus als dogmatischer Überwurf lesen lassen, hat das Gericht bereits 2013 klargestellt, dass gerade keine inhaltlichen Unterschiede zum „sachlichen Zusammenhang“ gewünscht waren.222

Zum ersten Mal findet sich die Bezeichnung „Erfüllung des Versicherungstatbestands“ in einem Urteil aus dem Jahr 2012, in dem es um eine wegen Altersteilzeit bereits von der Arbeit freigestellte Klägerin ging, die auf dem Betriebsgelände stolperte, während sie ihrem Arbeitgeber ein Formular des Rentenversicherungsträgers überbrachte. ${ }^{223}$ Die entscheidende Frage war, ob die Klägerin zum Unfallzeitpunkt eine versicherte Tätigkeit verrichtet hatte. Statt wie in seinen vorherigen Entscheidungen den sachlichen Zusammenhang zwischen dem Überbringen eines solchen Formulars und der geschützten Tätigkeit zu überprüfen, betonte das Gericht zunächst, Versicherungsschutz bestehe generell dann, wenn eine Verrichtung unter einen den Versicherungsschutz begründenden Tatbestand subsumiert werden könne. Eine solche versicherte Tätigkeit als Beschäftigte im Sinne des $\mathbb{2}$ Abs. 1 Nr. 1 SGB VII setze voraus, dass die Geschädigte in Eingliederung in das Unternehmen eine eigene Tätigkeit einer anderen Person zu dem Zweck verrichte, dass die Ergebnisse nicht der Geschädigten, sondern der anderen Person unmittelbar zum Vorteil oder Nachteil gereichten. Dies sei „nur“ in drei Fallgruppen gegeben. Erstens dann, wenn die tatsächliche Handlung der Beschädigten zumindest dazu ansetzt und darauf gerichtet ist, eine eigene objektiv bestehende Haupt- oder Ne-

Rn. 12; v. 4.12.2014 - B 2 U 14/13 R -, juris Rn. 19; v. 5.7.2016 - B 2 U 19/14 R -, juris Rn. 12 (zur Veröffentlichung in der Entscheidungssammlung vorgesehen); v. 15.11.2016 - B 2 U 12/15 R -, juris Rn. 17.

221 Während Ricke, in: Körner/Leitherer et al. (Hrsg.), Kasseler Kommentar zum Sozialversicherungsrecht, $₫ 8$ SGB VII Rn. 10c sowie Schlaeger, jurisPR-SozR 23/2015 12.11.2015, Anm. 3, es als inhaltliche Änderung verstehen, halten Keller, in: Hauck/Noftz/ders. (Hrsg.), SGB VII, $\$ 8$ Rn. 16, Köhler, VSSR 2016, S. 31, 35 und Krasney, in: Becker/Burchardt/ders./Kruschinsky, Gesetzliche Unfallversicherung, $\mathbb{S} 8$ Rn. 34a; ders., NZS 2013, S. 681, 682, die Änderung für rein terminologisch. Offen gelassen wird die Frage bei Giesen, Arbeitsunfall und Dienstunfall, S. 38 .

222 „Wenn der erkennende Senat neuerdings den Aspekt in den Vordergrund rückt, dass die konkrete Betätigung nach dem Schutzbereich des Versicherungstatbestands zu den versicherten Tätigkeiten gehören muss (...), so ist hiermit keine inhaltliche Änderung gegenüber den soeben aufgezeigten früheren Formulierungen verbunden." BSG, Urt. v. 4.7.2013 - B 2 U 5/12 R -, juris Rn. 18. 
benpflicht aus ihrem Beschäftigungsverhältnis zu erfüllen. Zweitens, wenn die Beschädigte eine objektiv nicht geschuldete Handlung vornimmt, um eine vermeintliche Pflicht aus dem Beschäftigungsverhältnis zu erfüllen, sofern sie nach den besonderen Umständen ihrer Beschäftigung zur Zeit der Verrichtung annehmen durfte, sie treffe eine solche Pflicht. Drittens dann, wenn die Beschädigte eigene, unternehmensbezogene Rechte aus der Beschäftigung ausübt. ${ }^{224}$ Dass nur bei Vorliegen einer dieser drei Fallgruppen Versicherungsschutz gewährt werden könne, ergebe sich aus dem "Schutzzweck“ der Beschäftigtenversicherung. ${ }^{225}$ Könne nicht eindeutig subsumiert werden, sei auf die „objektivierte Intention“ abzustellen. ${ }^{226}$ Sodann prüfte das Gericht, ob entweder eine Haupt- oder eine Nebenpflicht der Klägerin dahingehend bestand, ihrem Arbeitgeber das fragliche Formular zu überbringen. Da es dies verneinte und weiter zu dem Schluss kam, dass die Geschädigte auch nicht hätte annehmen dürfen, einer derartigen Pflicht zu unterliegen, verneinte es das Vorliegen einer versicherten Tätigkeit.

Schon hier bezeichnete das Gericht den "sachlichen Zusammenhang“ als „früheren Sprachgebrauch“ 227 und deutete damit an, eine rein terminologische Fortentwicklung zu bezwecken. Zudem hat es in folgenden Urteilen seine Aufzählung von umfassten Fallgruppen nicht mehr durch das Wort „nur" eingeleitet. ${ }^{228}$ Es wird daher teilweise davon ausgegangen, das Bundessozialgericht habe die Beschränkung auf Haupt- und Nebenpflichten in dem Urteil229, in dem es die neue Standardformulierung erstmalig einsetze, irrtümlich vorgenommen und sie noch vor der eigentlichen Klarstellung des BSG durch die Auslassung des Adverbs in einem weiteren Urteil ${ }^{230}$ korrigiert. ${ }^{231}$ Dadurch sei klargestellt, dass es sich bei der Erfüllung von Pflichten aus dem Arbeitsverhältnis um einen Hauptanwendungsfall, nicht aber um den einzigen Anwendungsfall handele. Nach Lektüre des

224 Z.B. BSG, Urt. v. 15.5.2012, E 111, 37 Rn. 27. Solche unternehmensbezogenen Rechte sind etwa die Teilnahme an einer Betriebswahl oder die Tätigkeit als Betriebsrat, Schlaeger, jurisPR-SozR 24/2012, Anm. 3.

225 BSG, Urt. v. 15.5.2012, E 111, 37 Rn. 35.

226 Ebd. Rn. 22.

227 Ebd. Rn. 47.

228 Und zwar nur in dem, in dem es die Zuordnungsregel „Erfüllung des Versicherungstatbestandes“ zum ersten Mal aufstellte, BSG, Urt. v. 15.5.2012, E 111, 37.

229 Ebd.

230 BSG, Urt. v. 13.11.2012 - B 2 U 27/11 R -, juris Rn. 28 ff.

231 Krasney, in: Becker/Burchardt/ders./Kruschinsky, Gesetzliche Unfallversicherung, \8 Rn. 34a; ebenso Krasney, NZS 2013, S. 681, 682. 
letztgenannten Urteils ist dem aber nicht zuzustimmen. ${ }^{232}$ In der Tat wird der Katalog der Tatbestände dort nicht durch das Wort „nur“ eingeleitet, und es ist schwer vorstellbar, dass diese Auslassung versehentlich erfolgte. Aber das Fehlen des Adverbs ändert nichts daran, dass die Aufzählung abschließend formuliert ist. Außerdem sprechen - speziell im zitierten Urteil - die ganze Prüfungsreihenfolge, der Aufbau der Argumentation und die Konklusion des Urteiles dafür, dass die Aufzählung abschließend verstanden werden soll. Es wird dort zunächst geprüft, ob der Geschädigte eine Hauptpflicht erfüllt hat, dann, ob er eine Nebenpflicht erfüllte. Schließlich heißt es: „Da der Kläger durch sein Sportklettern weder eine Haupt- noch eine Nebenpflicht aus der Beschäftigung als Berufseishockeyspieler erfüllte, war die Verrichtung ,Klettern' nicht aufgrund des Versicherungstatbestands nach $\$ 2$ Abs. 1 Nr. 1 SGB VII versichert. ${ }^{\star 233}$

Auch Spellbrink, der seit 2016 vorsitzender Richter des für die Unfallversicherung zuständigen II. Senats des Bundessozialgerichts ist, nennt die Änderung rein terminologisch. Er prüft in einem jüngst veröffentlichten Beitrag im Rahmen eines Beispielsfalles, „ob der Verletzte durch eine Verrichtung vor dem fraglichen Unfallereignis den gesetzlichen Tatbestand einer versicherten Tätigkeit erfüllt hat", und erläutert, dass das jeweilige Handeln zumindest auch „auf die Erfüllung des Tatbestandes der jeweiligen Tätigkeit ausgerichtet sein (sog. Handlungstendenz)“ müsse. ${ }^{234} \mathrm{Als} \mathrm{Be-}$ leg für dieses Erfordernis der Handlungstendenz führt er gerade die Urteile an, in denen die Etablierung der „Erfüllung des Versicherungstatbestands“ als Tatbestandsmerkmal erfolgte. ${ }^{235}$

\section{b. Einschränkung des Versicherungsschutzes}

Obwohl das BSG in dem bereits erwähnten Urteil mitteilt, dass es die „Erfüllung des Versicherungstatbestands" lediglich als sprachliche Neuetikettierung verstanden wissen will, spricht vieles dafür, den Prüfungsschritt als neues Tatbestandsmerkmal zu sehen. Die Judikatur des Gerichts ist inso-

232 Ebenso Ricken, NZS 2013, S. 774.

233 BSG, Urt. v. 13.11.2012 - B 2 U 27/11 R -, juris Rn. 42.

234 Spellbrink, NZS 2016, S. 527, 528.

235 Namentlich Urt. v. 24.7.2012 - B 2 U 9/11 R sowie Urt v. 26.6.2014 - B 2 U 4/13 R, vgl. Spellbrink, NZS 2016, S. 527, 528 Fn. 11. 
weit widersprüchlich. ${ }^{236}$ Denn im Urteil vom 15.5.2012 hat das BSG die Beschränkung des Versicherungsschutzes auf die genannten Fallgruppen ausführlich begründet. ${ }^{237}$ Aus Wortlaut und Entstehungsgeschichte der Norm, aus dem Schutzzweck der Beschäftigtenversicherung sowie aus der Entwicklung der Rechtsprechung ergäbe sich zwingend, dass nur die vom Gericht erwähnten drei Fallgruppen von Verrichtungen versicherte Tätigkeiten darstellen würden. Dies sind zunächst solche Verrichtungen, die darauf gerichtet sind, eine Haupt- oder Nebenpflicht aus dem Beschäftigungsverhältnis zu erfüllen. Zum zweiten sollen Verrichtungen umfasst sein, die objektiv nicht geschuldete Handlungen darstellen, während derer der Beschäftigte aber annehmen durfte, er schuldete sie. Die dritte Fallgruppe stellen Verrichtungen in Ausübung unternehmensbezogener Rechte dar. Diese Begründung wird sogleich näher untersucht (s.u. Kap. 1 D. IV. 2.).

Zudem mehrten sich bereits in früheren Urteilen Andeutungen, dass das Bundessozialgericht den Umfang der versicherten Tätigkeit auf die Erfüllung der Haupt- und Nebenpflichten begrenzen will. In seinem Urteil vom 7.12.2004 beispielsweise erläuterte das Gericht, der Inhalt der versicherten Tätigkeit ergebe sich aus dem Arbeitsverhältnis, nach dem der Beschäftigte zur Leistung der versprochenen Dienste verpflichtet sei, und folgerte: „Diese Dienste sind die versicherte Tätigkeit“.238 Im MotorradfahrtUrteil vom 9.11.2010 prüfte es innerhalb des sachlichen Zusammenhangs den Tatbestand der versicherten Tätigkeit ausschließlich danach, ob der Beschäftigte eine arbeitsvertragliche Haupt- oder Nebenpflicht erfüllte. ${ }^{239}$ Zudem hätte das Gericht seine Aufzählung deutlich als offene, beispielhafte Reihung formulieren können. Eine sprachliche Abkehr von dem als abschließend formulierten Katalog ist jedoch auch in jüngeren Urteilen nicht zu erkennen. ${ }^{240}$ Zwar wird in diesen Urteilen stets auf das Wort „nur“ verzichtet. Es finden sich aber nie Andeutungen, die auf eine Offenheit des Kataloges hinweisen könnten (kein „zum Beispiel“, kein „unter anderem“).

236 Dies dürfte auch der Grund dafür sein, warum das Problem noch drei Jahre nach erfolgter Klarstellung als ungeklärt angesehen wird, vgl. Giesen, Arbeitsunfall und Dienstunfall, S. 38.

237 BSG, Urt. v. 15.5.2012, E 111, 37 Rn. 27-60.

238 BSG, Urt. v. 7.12.2004 - B 2 U 47/03 R -, juris Rn. 26.

239 BSG, Urt. v. 9.11.2010 - B 2 U 14/10 R -, juris Rn. 19.

240 Zuletzt BSG, Urt. v. 15.11.2016 - B 2 U 12/15 R -, juris Rn. 17; v. 5.7.2016 - B 2 U 19/14 R -, juris Rn. 12 (zur Veröffentlichung in der Entscheidungssammlung vorgesehen); v. 23.4.2015 - B 2 U 5/14 R -, juris Rn. 14; v. 26.6.2014 - B 2 U 4/13 $\mathrm{R}-$, juris Rn. 16. 
Die Aufzählung lautet nun wiederholt: „Eine Tätigkeit als Beschäftigter wird verrichtet, wenn der Verletzte zur Erfüllung eines von ihm begründeten Rechts- und damit Beschäftigungsverhältnisses, insbesondere eines Arbeitsverhältnisses eine eigene Tätigkeit in Eingliederung in das Unternehmen eines anderen zu dem Zweck verrichtet, dass die Ergebnisse seiner Verrichtung diesem und nicht ihm selbst unmittelbar zum Vorteil oder Nachteil gereichen. Dabei kommt es objektiv auf die Eingliederung des Handelns des Verletzten in das Unternehmen eines anderen und subjektiv auf die zumindest auch darauf gerichtete Willensausrichtung an, dass die eigene Tätigkeit unmittelbare Vorteile für das Unternehmen des anderen bringen soll. Eine Beschäftigung iS des $₫ 2$ Abs 1 Nr 1 SGB VII wird daher ausgeübt, wenn die Verrichtung zumindest dazu ansetzt und darauf gerichtet ist, eine eigene objektiv bestehende Haupt- oder Nebenpflicht aus dem zugrunde liegenden Rechtsverhältnis zu erfüllen, der Verletzte eine objektiv nicht geschuldete Handlung vornimmt, um einer vermeintlichen Pflicht aus dem Rechtsverhältnis nachzugehen, sofern er nach den besonderen Umständen seiner Beschäftigung zur Zeit der Verrichtung annehmen durfte, ihn treffe eine solche Pflicht, oder er unternehmensbezogene Rechte aus dem Rechtsverhältnis ausübt (BSG vom 15.5.2012 - B 2 U 8/11 $\mathrm{R}$ - BSGE 111, 37 = SozR 4-2700 $\$ 2 \mathrm{Nr} 20, \mathrm{RdNr} 27 \mathrm{ff} ;[\ldots .$.$] ). 241$ Dabei nimmt das Gericht stets auf das Urteil vom 15.5.2012 Bezug, in dem sich eine Passage findet, die nur als Wille zur Änderung der Prüfungsdogmatik gelesen werden kann: „[A]uch die Entwicklung der Rechtsprechung [...] führt zu dem Ergebnis, dass nur unter den drei oben genannten Voraussetzungen eine Beschäftigung verrichtet wird“242. Wenn das Gericht die Erfüllung des Versicherungstatbestands lediglich als terminologische Weiterentwicklung des sachlichen Zusammenhangs verstanden haben will, wäre es wünschenswert, dass es sich bei der Formulierung auf das Urteil bezöge, in dem es dies klargestellt hat.

In den niedereren Instanzen der Sozialgerichtsbarkeit sowie in der Literatur wird der Änderung in vielen Fällen keinerlei Beachtung geschenkt. ${ }^{243}$

241 BSG, Urt. v. 26.6.2014 - B 2 U 4/13 R -, juris Rn. 16.

242 BSG, Urt. v. 15.5.2012, E 111, 37 Rn. 42.

243 Sie bleibt unerwähnt z.B. bei LSG Sachen, Urt. v. 7.10.2015 - L 6 U 183/13 -, juris Rn. 12; LSG Bayern, Urt. v. 18.6.2015 - L 2 U 298/14 -, juris; LSG BadenWürttemberg, Urt. v. 16.6.2015 - L 9 U 1534/14 -, juris; LSG Hessen, Urt. v. 29.5.2015 - L 9 U 41/13 -, juris sowie bei Plagemann/Radtke-Schwenzer, NJW 2013, S. 1924, 1924; Osing, jurisPR-SozR 2013, Anm. 3; Höller, in: Udsching/ Rolfs (Hrsg.), Jahrbuch des Sozialrechts 34 (2013), S. 211, 211 f. So resümiert 
2. Begründung der Änderung durch das BSG

Das BSG hat in seinem Urteil vom 15.5.2012 dargelegt, die vorgenommene Änderung sei zwingend aus Wortlaut und Entstehungsgeschichte des $\mathbb{} 2$ Abs. 1 Nr. 1 SGB VII sowie aus den Schutzzwecken der Unfallversicherung abzuleiten. ${ }^{244}$

Zunächst leitete es aus Wortlaut und Entstehungsgeschichte des $\mathbb{} 2$ Abs. 1 Nr. 1 SGB VII her, dass eine Tätigkeit als Beschäftigter nur dann ausgeübt wird, wenn die handelnde Person objektiv in das Unternehmen eingegliedert ist und subjektiv dem Unternehmen mit der Handlung unmittelbar einen Vorteil bringen möchte. ${ }^{245}$ Das folge daraus, dass der Beschäftigtenbegriff des $\mathbb{} 2$ Abs. 1 Nr. 1 SGB VII dem des $\mathbb{7}$ Abs. 1 SGB IV entspreche. ${ }^{246}$ Dieser beschreibt Beschäftigung als nicht-selbstständige Arbeit, welche durch Unterordnung unter ein Weisungsrecht und Eingliederung in die Arbeitsorganisation gekennzeichnet ist. Eine Beschäftigung im Sinne des $\$ 2$ Abs. 1 Nr. 1 SGB VII könne deswegen nur eine Handlung sein, die diese Voraussetzungen erfüllt. Um diesen Argumentationsstrang zu überprüfen, muss danach gefragt werden, ob eine Handlung vorstellbar ist, die nicht zu den vom Gericht aufgestellten Fallgruppen gehört, aber dennoch die Voraussetzungen des $₫ 7$ Abs. 1 SGB IV erfüllt. Zunächst ist an überobligatorische Leistungen zu denken. Als Beispiel diene ein Buchhalter, der über seine Arbeitspflicht hinaus eine defekte Steckdose des Arbeitgebers repariert. ${ }^{247}$ Unzweifelhaft ist dessen Handlung dem Unternehmen unmittelbar zu dienen bestimmt. Die konkrete Verrichtung - das Reparieren der Steckdose - ist allerdings nicht in der Unterordnung unter ein Weisungsrecht begründet. Sein Arbeitgeber könnte ihn gar nicht anweisen, eine Steckdose zu reparieren, da dies nicht zu den arbeitsvertraglich geschuldeten Leistungen des Buchhalters gehört. Der Geschädigte ist zwar als Buchhalter in die Organisation des Unternehmens eingegliedert. Sein Platz in dieser Organisation ist aber nicht derjenige eines Elektrikers, sondern der eines Buchhalters. Er handelt damit gerade nicht innerhalb seiner Eingliederung in die Arbeitsorganisation. Auch wer arbeitsunfähig ist und trotzdem arbeitet, ist keiner Weisungspflicht unterworfen; dasselbe gilt für

Krasney, dem BSG werde insoweit schlicht nicht gefolgt, ders., KrV 2014, S. 102, 103.

244 BSG, Urt. v. 15.5.2012, E 111, 37 Rn. 27-33.

245 Ebd.

246 BT-Drs. 13/2204, S. 74 zu $\$ 2$ Abs. 1 SGB VII.

247 Beispiel bei Krasney, NZS 2013, S. 681, 682; ähnlich Krasney, KrV 2014, S. 102, 103. 
freiwillige Überstunden. Werden solche Überstunden aber faktisch vom Arbeitnehmer erwartet, so sind sie als Teil der geschuldeten Arbeitsleistung zu sehen. Auch bei der Teilnahme am Betriebssport oder an betrieblichen Gemeinschaftsveranstaltungen liegt keine Weisungsgebundenheit vor. Die Argumentation des Bundessozialgerichts ist soweit nachvollziehbar.

Allerdings lässt sich an der Prämisse dieser Argumentation rütteln, nach welcher nur die tatsächliche Beschäftigung versichert ist. $\$ 8$ Abs. 1 S. 1 SGB VII, der den Versicherungsfall definiert, lautet: „Arbeitsunfälle sind Unfälle von Versicherten infolge einer den Versicherungsschutz nach $\$ 2,3$ oder 6 begründenden Tätigkeit (versicherte Tätigkeit)“. Die Argumentation des Bundessozialgerichts geht davon aus, dass sich die in Klammern gesetzte Legaldefinition nur auf den Teil „einer den Versicherungsschutz nach $\$ 2,3$ oder 6 begründenden Tätigkeit" bezieht und damit lediglich die in diesen Paragraphen genannten Handlungen versicherte Tätigkeiten sind. Genauso gut lässt sich jedoch annehmen, dass sich die Legaldefinition auf den gesamten Satzteil ,infolge einer den Versicherungsschutz nach $\$ 2,3$ oder 6 begründenden Tätigkeit" bezieht und damit nicht nur Handlungen, die zur Beschäftigung zählen, sondern auch Handlungen, die ,infolge“ der Beschäftigung ausgeführt werden, versicherte Tätigkeiten sind. Welcher Zusammenhang mit dem Wort „infolge“ gemeint ist, bedarf dann der Auslegung. Für diese Interpretation des $\$ 8$ Abs. 1 S. 1 SGB VII spricht, dass das Wort „infolge“ im Gesetzestext sonst falsch gewählt wäre. Es bedeutet „als Folge (eines Geschehens); aufgrund von“248, sodass sich $\$ 8$ Abs. 1 S. 1 SGB VII umformulieren lässt in „Arbeitsunfälle sind Unfälle von Versicherten aufgrund von einer den Versicherungsschutz nach $\$ 2,3$ oder 6 begründenden Tätigkeit“. Dies schließt auch Tätigkeiten ein, die nicht selbst den Versicherungsschutz nach $\$ 2,3$ oder 6 SGB VII begründen, aber durch den Versicherungsschutz begründende Tätigkeiten veranlasst worden sind. Hätte man ausdrücken wollen, lediglich die tatsächliche Beschäftigung sei versichert, so wäre das Wort „durch“ anstelle von „infolge“ die bessere Wahl gewesen. Der Argumentation des Bundessozialgerichts, Wortlaut und Entstehungsgeschichte der Norm würden gebieten, dass lediglich die genannten Fallgruppen - also in der Hauptsache die (vermeintliche) Erfüllung von Haupt- und Nebenpflichten - in den Versicherungsschutz einzubeziehen sein dürften, wird daher hier nicht gefolgt.

248 Klappenbach/Steinitz (Hrsg.), Wörterbuch der deutschen Gegenwartssprache, Digitale Edition, Stichwort: infolge, abrufbar unter http://www.dwds.de/ressour cen/woerterbuecher/wdg/ (Stand: 23.10.2018). 
Das Bundessozialgericht führt weiterhin aus, die „Schutzzwecke der Beschäftigtenversicherung und ihre Stellung im Rechtssystem“ würden den Anwendungsbereich des $\mathbb{} 2$ Abs. 1 Nr. 1 SGB VII auf die von ihm herausgearbeiteten Fallgruppen begrenzen. ${ }^{249}$ Um beurteilen zu können, ob eine Beschränkung auf diese drei Verrichtungstypen aus den Schutzzwecken der gesetzlichen Unfallversicherung imperativ ist, müssen Letztere zunächst definiert werden. Sie werden unten ausführlich behandelt;, 250 ein Vorgriff in der gebotenen Kürze ist hier jedoch unerlässlich, um die Argumentation des BSG nachvollziehen zu können. Gesellschaftspolitischer Zweck der Einführung der Unfallversicherung war es, die bis dahin ungeschützten Beschäftigten von dem Risiko zu befreien, infolge eines Arbeitsunfalls erlittene Gesundheitsschäden alleine bewältigen zu müssen. Dabei sollen die Beschäftigten einerseits gegen die unmittelbar aus der Verrichtung der Arbeitstätigkeit resultierenden Gefahren, andererseits aber auch gegen die Gefahren abgesichert werden, denen sie nach Ort und Zeit aufgrund der versicherten Tätigkeit ausgesetzt sind. ${ }^{251}$ Zudem sollte der Betriebsfrieden gesichert werden, indem man verhinderte, dass Beschäftigte und Arbeitgeber vor Gericht gegeneinander vorgehen mussten. Dies sieht wohl auch das Bundessozialgericht im zitierten Urteil so. Zweck seien der umfassende Unfallversicherungsschutz aller Beschäftigten vor und bei Gesundheitsschäden infolge der Verrichtung der Beschäftigung, die Erhaltung des Betriebsfriedens sowie die gerechte Lastenverteilung unter den beitragszahlenden Unternehmern. ${ }^{252}$ Daraus folge, dass die gesetzliche Unfallversicherung die Beschäftigten nur gegen solche Gesundheits- und Lebensgefahren versichert, die "sich spezifisch daraus ergeben, dass sie Tätigkeiten für einen anderen unter Eingliederung in dessen Tätigkeit und nur zu dessen unmittelbare[m] Vorteil verrichten ${ }^{\text {" } 253}$. Anders gewendet bedeutet das, dass sich die Gefahr daraus ergeben muss, dass der Geschädigte Beschäftigter ist. Auch hier ist aber - wie bei der Analyse des Wortlauts bereits bemerkt - zu bedenken, dass das Beschäftigungsverhältnis nicht nur für die vertraglich geschuldete Leistung, sondern auch für andere Verhaltensweisen wesentliche Ursache ist. Der bezweckte Schutz der Beschäftigten gegen Gefahren, die nicht direkt aus der Tätigkeit, sondern aus örtlichen und zeitlichen Gegebenheiten aufgrund der Arbeitstätigkeit resultieren, ist

249 BSG, Urt. v. 15.5.2012, E 111, 37 Rn. 35; ebenso versteht es Schlaeger, jurisPRSozR 24/2012, Anm. 3.

250 S.u. Kap. 2 B. und C.

251 Mutschler, SGb 2011, S. 684, 684.

252 BSG, Urt. v. 15.5.2012, E 111, 37 Rn. 36-39.

253 Ebd. Rn. 41. 
nach den drei Fallgruppen nur unvollkommen verwirklicht - nämlich nur während der Wahrnehmung unternehmensbezogener Rechte. Dieser Schutzzweck wäre besser verwirklicht, wenn - wie bisher unter dem Prüfungsregime des sachlichen Zusammenhangs - alle Handlungen, die im Zusammenhang mit der Beschäftigung vorgenommen wären, vom Unfallversicherungsschutz umfasst wären. Insbesondere gilt dies für die Fälle, in denen sich eine besondere Betriebsgefahr verwirklicht, während der grundsätzlich Versicherte einer privaten Tätigkeit nachgeht. Die sich in diesen Fällen verwirklichende Gefahr stammt gerade aus dem spezifischen Beschäftigungsverhältnis, welches das Bundessozialgericht als Maßstab angelegt hat. Es handelt sich hierbei oft um Fälle, in denen - gäbe es keine gesetzliche Unfallversicherung - der Arbeitgeber dem Beschäftigten für den entstandenen Schaden haften müsste. Ausgenommen sind nur Fälle, in denen der Arbeitgeber nicht haften würde, die Gefahr sich also zufällig verwirklicht oder der Arbeitnehmer sie durch seine private Tätigkeit erst geschaffen hat. Dass die Fälle der betriebsbedingten Gefahr umfasst sein sollten, lässt sich deswegen aus dem Prinzip der Haftungsablösung der Unternehmerhaftpflicht ableiten. ${ }^{254}$ In diesen Fällen ist der Arbeitnehmer auf die Geltendmachung privatrechtlicher Ansprüche gegen den Arbeitgeber verwiesen - wie vor der Einführung der gesetzlichen Unfallversicherung und mit allen dazugehörigen Nachteilen. ${ }^{255}$

Auch die Maxime, den Betriebsfrieden zu erhalten, wäre bei einer solch breiteren Auslegung erfolgreicher. Geht man davon aus, dass nur solche Unfälle versichert sein sollen, für die auch eine Gefährdungs- oder Deliktshaftung oder eine Haftung aus Verletzung von arbeitsrechtlichen Schutz- oder Fürsorgepflichten konstruiert werden könnte, wie das Urteil das andeutet, ist ebenfalls keine Beschränkung auf die Erfüllung von Haupt- und Nebenpflichten zwingend. Eine Gefährdungshaftung kann sich beispielsweise auch während einer Betriebsveranstaltung in vom Arbeitgeber zur Verfügung gestellten Räumen ergeben.

Für die gerechte Verteilung der Lasten der Unternehmer untereinander dürfte dies dagegen kein beeinflussender Faktor sein.

Im Ergebnis ist es nicht zwingend, die Gesetzestexte des gesetzlichen Unfallversicherungsrechts in der Weise auszulegen, dass die versicherte Tätigkeit in einem derartigen Umfang beschränkt wird. Die Schutzzwecke der gesetzlichen Unfallversicherung wären besser erfüllt, wenn von der Einschränkung abgesehen würde.

254 Ziegler, in: Becker/Franke et al. (Hrsg.), SGB VII, $\mathbb{} 8$ Rn. 179.

255 S.u. Kap. 2. B. I. 


\section{Kritik am Merkmal „Erfüllung des Versicherungstatbestands“}

Die Rechtsprechung zur Erfüllung des Versicherungstatbestands ist sowohl im Hinblick darauf, dass sie als inhaltliche Modifikation verstanden wurde, als auch als rein terminologische Änderung kritisiert worden. ${ }^{256}$

Vor allem wurde moniert, dass durch den abschließenden Katalog einige Fallgruppen nicht mehr als zur versicherten Tätigkeit angehörig gesehen werden könnten, die bisher von der ständigen Rechtsprechung anerkannt waren. Angeführt wurden vor allem die Fallgruppen der betrieblichen Gemeinschaftsveranstaltung ${ }^{257}$ und des Betriebssports ${ }^{258}$, welche bisher unter bestimmten Bedingungen als zum Schutzbereich der Unfallversicherung gehörend angesehen wurden. ${ }^{259}$

Zwar waren sowohl die Gewährung von Unfallschutz bei betrieblichen Gemeinschaftsveranstaltungen als auch beim Betriebssport richterliche Interpretationen des $\$ 2$ Abs. 1 Nr. 1 SGB VII, die deswegen auch zurückgezogen werden können. Jedoch wird die Entscheidung, von dieser Rechtsprechung abzukehren, im vorliegenden Urteil nicht erwähnt, sodass unklar war, ob das Gericht dies tatsächlich beabsichtigt hat. ${ }^{260}$ Die Teilnahme an einer betrieblichen Gemeinschaftsveranstaltung war nach bisheriger Praxis dann einer versicherten Tätigkeit gleichzusetzen, wenn sie im Einvernehmen mit der Unternehmensleitung stattfand, die Teilnahme allen Betriebsangehörigen offenstand und die Unternehmensleitung persönlich an der Veranstaltung teilnahm. ${ }^{261}$ In einem jüngeren Urteil hat das Bundessozialgericht das Problem dahingehend gelöst, dass es die Teilnahme an einer betrieblichen Gemeinschaftsveranstaltung als Teil der im Rahmen des Arbeitsvertrags geschuldeten Tätigkeit und damit als originär versicherte Tätigkeit angesehen hat. ${ }^{262}$ Denn eine von der Unternehmensleitung gewünschte Teilnahme zeige das Unternehmensinteresse, die betriebliche

256 Ricke, WzS 2013, S. 241, 243 f.; Ricke, in: Körner/Leitherer et al. (Hrsg.), Kasseler Kommentar zum Sozialversicherungsrecht, $\mathbb{8}$ SGB VII Rn. 10c; Krasney, NZS 2013, S. 681, 681 ff. Schlaeger, jurisPR-SozR 24/2012, Anm.3, spricht gar von einer „Devolution des Unfallversicherungsrechts“.

257 Vgl. BSG, Urt. v. 22.9.2009 - B 2 U 27/08 R -, juris.

258 Vgl. Schlaeger/Lenger, DB 2010, S. 2280, 2280 m.w.N.

259 Schlaeger, jurisPR-SozR 24/2012, Anm. 3.

260 Ebd.

261 Std. Rspr., vgl. BSG, Urt. v. 22.8.1955 - 2 RU 49/54 - E 1, 179, 182 f.; Urt. v. 9.12.2003 - B 2 U 52/02 R -, juris Rn. 14; v. 7.12.2004 - B 2 U 47/03 R -, juris Rn. 13. Vgl. dazu Krasney, KrV 2014, S. 104, 106 f.

262 BSG, Urt. v. 5.7.2016 - B 2 U 19/14 R -, juris Rn. 13 (zur Veröffentlichung in Entscheidungssammlung vorgesehen). Die Klägerin hatte sich während einer 
Verbundenheit zu fördern. Das Gericht lässt deshalb unter Hinweis auf den „Schutzzweck der Beschäftigtenversicherung“ eine „auf die Teilnahme an der betrieblichen Gemeinschaftsveranstaltung gerichtete Handlungstendenz des Versicherten" ausreichen, um eine Eröffnung des unfallversicherungsrechtlichen Schutzbereichs anzunehmen. ${ }^{263}$ Wenig zur Klarheit beitragend formulierte das BSG einige Monate später in einem weiteren Urteil zum Thema betriebliche Gemeinschaftsveranstaltung, eine Verrichtung, „die nicht der Erfüllung einer Pflicht aus dem Beschäftigungsverhältnis dient oder dienen soll“, könne „nur dann im sachlichen Zusammenhang mit der versicherten Tätigkeit stehen, wenn der Beschäftigte sie wegen des Beschäftigungsverhältnisses vornimmt, um durch sie zumindest auch dem Unternehmen in nicht offensichtlich untauglicher Weise zu dienen“, und kehrt damit zum Tatbestandsmerkmal des sachlichen Zusammenhangs zurück. ${ }^{264}$ Jedoch resümiert das Gericht noch in derselben Randnummer, dieses „unternehmensdienliche Verhalten" rechtfertige es, „die Teilnahme an einer betrieblichen Gemeinschaftsveranstaltung als Bestandteil der aufgrund des Beschäftigungsverhältnisses geschuldeten versicherten Tätigkeit iS des $₫ 2$ Abs. 1 Nr. 1 SGB VII zu betrachten“. Insgesamt scheint das Bundessozialgericht an seiner Prüfung der Erfüllung des Versicherungstatbestands festhalten zu wollen, ohne dass es innerhalb der Fallgruppe der betrieblichen Gemeinschaftsveranstaltung zu anderen Ergebnissen kommen möchte.

Auch die Fälle der kurzfristigen Unterbrechung der versicherten Tätigkeit, in denen die Rechtsprechung den sachlichen Zusammenhang mit der versicherten Tätigkeit bejahte, ${ }^{265}$ sind bei wortgetreuer Anwendung der Definition der „Erfüllung des Versicherungstatbestands“ nicht mehr umfasst. ${ }^{266}$ Die ausgeführte Tätigkeit kann in diesen Fällen - unabhängig von ihrer Dauer - nicht zum arbeitsvertraglichen Pflichtenkreis gerechnet werden.

Aus dem Versicherungsschutz heraus fielen bei Annahme einer inhaltlichen Änderung durch die Einführung der Zuordnungsfigur „Erfüllung

Wanderung im Rahmen einer Weihnachtsfeier eine Verletzung zugezogen. Mit dieser Entscheidung hat das Gericht auch das Kriterium der persönlichen Teilnahme der Unternehmensleitung aufgegeben, a.a.O. Rn. 16. Vgl. dazu die Anmerkung von Waltermann, NZS 2017, S. 25, 28.

263 BSG, Urt. v. 5.7.2016 - B 2 U 19/14 R -, juris Rn. 13.

264 BSG, Urt. v. 15.11.2016 - B 2 U 12/14 R -, juris Rn. 19.

265 S.o. Kap. 1 D. III. 2. C. bb.

266 Ricke, in: Körner/Leitherer et al. (Hrsg.), Kasseler Kommentar zum Sozialversicherungsrecht, $\mathbb{8} 8$ SGB VII Rn. 10c. 
des Versicherungstatbestands“ zudem alle Fälle des Grundsatzes der „betriebsbedingten Gefahr". ${ }^{267}$ Die Versicherung der aus der besonderen Betriebsgefahr erwachsenden Risiken erscheint so essenziell, dass laut dem Schrifttum „trotz der absoluten Formulierung“ nicht davon ausgegangen werden könne, dass das Bundessozialgericht diese Fallgruppen ausschlieBen wollte. ${ }^{268}$

Die Prüfung der „Erfüllung des Versicherungstatbestands“, wie sie das BSG in seinen letzten Urteilen durchführt, schließt zudem auch solche Handlungen aus, die Versicherte über ihre Arbeitspflichten hinaus vornehmen, die aber dennoch dem Unternehmen zu dienen bestimmt sind. Überobligatorische Leistungen, ${ }^{269}$ freiwillige und unbezahlte Überstunden $^{270}$ oder arbeitsunfähig geschriebene Beschäftigte, die dennoch arbeiten $^{271}$, sind in diesem Zusammenhang zu nennen. Ein Unternehmen profitiert aber gerade von solchen Arbeitnehmern, die mehr zu leisten bereit sind als den bloßen „Dienst nach Vorschrift“272 Mitarbeiter, die mehr für das Unternehmen tun wollen als die Erfüllung ihrer Pflichten aus dem Beschäftigungsverhältnis, tragen maßgeblich zum Erfolg eines Unternehmens bei. ${ }^{273}$ Bisher waren solche Handlungen vom Schutz der Unfallversicherung umfasst, da es für die Bejahung des Zurechnungszusammenhangs darauf ankam, ob der Beschäftigte annehmen durfte, die Verrichtung würde dem Unternehmer dienen (s.o. Kap. 1 D. III.). ${ }^{274}$

Einer Ansicht nach umfasst die „Erfüllung des Versicherungstatbestands" auch solche Fälle nicht, in denen spezifische Gefahren aus dem versicherten Risikobereich den Unfall beeinflussten. ${ }^{275}$ Als Beispiel wird ein

267 Ziegler, in: Becker/Franke et al. (Hrsg.), SGB VII, $\mathbb{S} 8$ Rn. 178; zur Fallgruppe der besonderen Betriebsgefahr oder „betriebsbedingten Gefahr“s.o. Kap. 1 D. III. 2. C. ee.

268 Ziegler, in: Becker/Franke et al. (Hrsg.), SGB VII, $\mathbb{} 8$ Rn. 178.

269 Beispiel bei Krasney, NZS 2013, S. 681, 682; ähnlich Krasney, KrV 2014, S. 102 , 103.

270 Ricke, WzS 2013, S. 241, 244.

271 Krasney, KrV 2014, S. 102, 103.

272 Krasney, NZS 2013, S. 681, 682.

273 Miller, business-netz.com 2012, www.business-netz.com/Mitarbeiterfuehrung/U nternehmenserfolg-steigern-Engagierte-Mitarbeiter-sind-ein-Muss/ (Stand: 23.10.2018); Wolf, business-wissen.de Magazin 2007, www.business-wissen.de/art $\mathrm{ikel} /$ motivation-der-engagierte-mitarbeiter-als-zentraler-wettbewerbsfaktor/ (Stand: 23.10.2018).

274 Z.B. BSG, Urt. v. 17.2.1972 - 7/2 RU 27/69 -, juris zu einem Kraftfahrer, der sein Dienstfahrzeug gründlicher reinigte, als er hätte müssen, und dabei verunfallte.

275 Ricke, in: Körner/Leitherer et al. (Hrsg.), Kasseler Kommentar zum Sozialversicherungsrecht, $\mathbb{8} 8$ SGB VII Rn. 10c; Ricke, WzS 2013, S. 241, 244. 
Beschäftigter genannt, der etwas trinken muss, um seinen durch die Arbeit ausgelösten Durst zu löschen, und sich dabei verletzt. Wenn getrunken wird, um die Arbeitspflicht weiter erfüllen zu können, wäre die Tätigkeit jedoch von der neuen Definition umfasst. Sie gehört dann zu der ersten Fallgruppe der „Erfüllung einer Haupt- oder Nebenpflicht", da die Verrichtung mit der Intention durchgeführt wird, die Arbeitskraft zu erhalten. Die Gesunderhaltungspflicht ist anerkannte Nebenpflicht des Arbeitnehmers und leitet sich aus $\$ 241$ Abs. 2 BGB her. ${ }^{276}$

Auffällig ist, dass das Bundessozialgericht im Rahmen seiner Argumentation zur Erfüllung des Versicherungstatbestands sehr genau zwischen Haupt- und Nebenpflichten aus dem Arbeitsverhältnis differenziert. ${ }^{277}$ Dies ist verwunderlich, weil es bis dato nicht erfolgt ist - und zwar berechtigterweise, da es für die sozialrechtliche Prüfung des Vorliegens eines Arbeitsunfalls keinen Unterschied macht, ob eine Haupt- oder eine Nebenpflicht erfüllt werden sollte. Auch nach dem neuen Prüfschema des Bundessozialgerichts ist diese Unterscheidung folgenlos. Dennoch wurde auf diese Unterscheidung im Urteil vom 15.5.2012 besonderer Wert gelegt und gleichzeitig explizit angesprochen, dass die Unterscheidung bisher nicht getroffen wurde. ${ }^{278}$ Dies ist wohl so zu verstehen, dass sie zukünftig getroffen werden soll. ${ }^{279}$ Zwar ist die Unterscheidung folgenlos für das Urteil. ${ }^{280}$ Aus Gründen der rechtlichen Vorhersehbarkeit wäre es jedoch zu begrüßen, wenn möglichst präzise dargelegt wird, warum eine Verrichtung den Versicherungstatbestand erfüllt. Außerdem bedeutet diese Unterscheidung, dass in Zukunft Sozialgerichte mit arbeitsrechtlichen Fragen befasst werden. Es wäre jedoch wünschenswert, wenn sozialrechtliche Normen so wenig wie möglich von Begriffen aus anderen Rechtsgebieten abhängig wären. ${ }^{281}$ Zudem ist grundsätzlich nur in ein Urteil aufzunehmen, was entscheidungsrelevant ist. ${ }^{282}$ In einem anderen Urteil vom 13.11.2012 283 sprach das Bundessozialgericht dann nicht mehr nur von Pflichten aus dem Beschäftigungsverhältnis, sondern von Pflichten sowohl aus dem Beschäftigungsverhältnis als auch aus dem Arbeitsvertrag. Dabei handelt es

276 Seel, JA 2009, S. 131, 135.

277 BSG, Urt. v. 15.5.2012, E 111, 37 Rn. 47 ff.

278 Ebd.

279 Krasney, NZS 2013, S. 681, 682.

280 Ricke, in: Körner/Leitherer et al. (Hrsg.), Kasseler Kommentar zum Sozialversicherungsrecht, $\mathbb{8}$ SGB VII Rn. 10c.

281 Krasney, NZS 2013, S. 681, 683.

282 Ricke, WzS 2013, S. 241, 245 m.w.N.

283 BSG, Urt. v. 13.11. 2012 - B 2 U 27/11 R -, juris. 
sich um unterschiedliche Dinge: Das Beschäftigungsverhältnis schließt gem. $\$ 7$ Abs. 1 S. 1 SGB VII das Arbeitsverhältnis ein, sodass es nicht notwendig ist, neben dem Beschäftigungsverhältnis noch andere mögliche Quellen für Pflichten zu prüfen. Da die Auslegung des Arbeitsverhältnisses eine speziell arbeitsrechtliche Frage ist, gilt wiederum, dass es wünschenswert wäre, sie nicht zum entscheidenden Kriterium im Sozialversicherungsrecht zu machen, wenn dies nicht erforderlich ist. ${ }^{284}$

Dazu kommt, dass insbesondere die Nebenpflichten, die aus einem Arbeitsvertrag stammen, sehr schwierig einzugrenzen und $\mathrm{zu}$ bestimmen sind. ${ }^{285}$

Zuletzt wurde auch kritisiert, dass das Gericht ausdrücklich auf den Schutzzweck des Unfallversicherungsrechts abstellte. ${ }^{286}$ Es suggeriere damit eine objektive Entscheidungsfindung an einer Stelle, wo eine solche nicht möglich sei. Die Frage nach dem Schutzzweck der Norm ist nicht eindeutig zu beantworten, sondern bedarf der rechtlichen Abwägung, wobei die Absichten des Gesetzgebers beachtet werden müssen. Es handelt sich also auch bei der Bestimmung des Schutzzwecks der Norm um eine subjektive Wertentscheidung. ${ }^{287}$ Wenn eine solche zu treffen ist, ist es vorzugswürdig, dies auch deutlich zu machen.

\section{Resümee}

Insgesamt ist die neue Rechtsprechung des Bundessozialgerichts nicht als Aufgabe der Dogmatik des sachlichen Zusammenhangs zu sehen, da es selbst ausdrücklich betont hat, keine inhaltliche Änderung zu beabsichtigen. Festzuhalten bleibt aber, dass das Gericht die Prüfung des Schutzbereichs der Unfallversicherung nun anders durchführt. Durch die stetige Nennung des Fallgruppenkatalogs betont es, dass es die Zugehörigkeit der ausgeführten Tätigkeit zum Kreis der versicherten Tätigkeiten als das relevanteste Kriterium sieht. Es wirkt, als wolle das BSG deutlich machen, dass nur in Ausnahmefällen außerhalb dieses Katalogs Arbeitsunfälle vorliegen können, deren Vorliegen dann anhand der Kriterien des „sachlichen Zu-

284 Krasney, NZS 2013, S. 681, 683.

285 Joussen, in: Rolfs/Giesen et al. (Hrsg.), BeckOK Arbeitsrecht, $\$ 611$ BGB Rn. 384; Preis, in: Müller-Glöge/Preis et al. (Hrsg.), Erfurter Kommentar zum Arbeitsrecht, Rn. 708.

286 Schlaeger, jurisPR-SozR 24/2012, Anm. 3.

287 Becker, MedSach 2011, S. 32; Becker, MedSach 2007, S. 92, 94; Schlaeger, jurisPRSozR 24/2012, Anm. 3. 
sammenhangs" geprüft werden muss. Dies zeigt sich auch im bereits oben erwähnten Urteil zur Gemeinschaftsveranstaltung, in dem das BSG unter Zuhandnahme seines Fallgruppenkataloges argumentiert hat, dass die Teilnahme an der Veranstaltung zu den arbeitsvertraglichen Pflichten der Geschädigten gehörte. ${ }^{288}$ In früheren Urteilen zum Themenkomplex der Gemeinschaftsveranstaltungen hatte es den Versicherungsschutz mit dem sachlichen Zusammenhang der Veranstaltung zur versicherten Tätigkeit begründet. ${ }^{289}$

\section{E. Die haftungsbegründenden Kausalitäten}

\section{Kausalität im Recht}

\section{Terminologie der haftungsbegründenden Kausalitäten}

Ist der Schutzbereich des $₫ 8$ Abs. 1 SGB VII eröffnet, folgt im nächsten Schritt die Prüfung des Vorliegens der haftungsbegründenden Kausalitäten. Die Dogmatik des $\$ 8$ Abs. 1 SGB VII beinhaltet zwei dahingehende Tatbestandsmerkmale. Diese werden teilweise unterschiedlich bezeichnet. Lange Zeit wurde von einer „doppelten Kausalität“ gesprochen, die sich aus haftungsbegründender (dem Ursachenzusammenhang zwischen Verrichtung und Unfallereignis) und haftungsausfüllender Kausalität (dem Ursachenzusammenhang zwischen Unfallereignis und Gesundheitsschaden bzw. Tod) zusammensetzte. ${ }^{290}$ Mittlerweile hat das BSG seine Nomenklatur geändert und bezeichnet nun die zu prüfende Kausalität zwischen Verrichtung und Unfallereignis als Unfallkausalität. ${ }^{291}$ Demgegenüber wird die Kausalität zwischen Unfallereignis und Schaden haftungsbegründende Kausalität genannt, womit klargestellt ist, dass auch dies noch ein notwendiges Tatbestandsmerkmal für die Bejahung eines Arbeitsunfalls

288 BSG, Urt. v. 5.7.2016 - B 2 U 19/14 R -, juris Rn. 13 (zur Veröffentlichung in Entscheidungssammlung vorgesehen).

289 Grundlegend BSG, Urt. v. 22.8.1955 - 2 RU 49/54 -, E 1, 179, 182 f.; aus jüngerer Zeit z.B. Urt. v. 9.12.2003 - B 2 U 52/02 R -, juris Rn. 14.

290 Z.B. Schöpf, Multikausale Schäden in der gesetzlichen Unfallversicherung, S. 11.

291 Std. Rspr. seit BSG, Urt. v. 9.5.2006 - B 2 U 1/05 R -, E 96, 196, 198. Das Schrifttum zur Diskussion um die Natur der Kausalitäten vor der Änderung reflektierend Köhler, SdL 2006, S. 185, 191 ff.; zur Kritik am Begriff „Unfallkausalität“ m.w.N. vgl. Giesen, Arbeitsunfall und Dienstunfall, S. 87 Fn. 381. 
ist. ${ }^{292}$ Als haftungsausfüllende Kausalität wird der Ursachenzusammenhang zwischen Gesundheitserstschaden und weiteren, längerfristigen Unfallfolgen (z.B. Spätfolgen) bezeichnet. ${ }^{293}$ Dies ist folgerichtig, denn der Versicherungsfall der gesetzlichen Unfallversicherung begründet erst die Haftung, sagt aber noch nichts über die Entschädigungshöhe aus, sodass die beiden Kausalzusammenhänge, die zur Bejahung des Versicherungsfalls führen, als haftungsbegründend zu bezeichnen sind. ${ }^{294}$ Haftungsausfüllend ist dann der Kausalzusammenhang, der vom Versicherungsfall zum Leistungsfall führt. ${ }^{295}$ Zudem ermöglicht diese Terminologie eine genauere Differenzierung im Schadensbereich und führt zu einer einheitlicheren Verwendung der Begriffe, da die Bezeichnungen in Anlehnung an das $\mathrm{Zi}$ vilrecht gewählt wurden. ${ }^{296}$ Sie wird daher auch in dieser Arbeit verwendet.

Bevor die Merkmale der Unfall- und der haftungsbegründenden Kausalität im engeren Sinne untersucht werden, muss im Folgenden die dem Unfallversicherungsrecht eigene Kausalitätstheorie, die Theorie der wesentlichen Bedingung, in den Blick genommen worden. Wie stets im Recht ist auch im Unfallversicherungsrecht nicht nur eine naturgesetzliche Verbundenheit zweier Ereignisse ${ }^{297}$ gemeint, wenn von Kausalität gesprochen wird. Vielmehr kommt ein wertendes Element hinzu. Im Folgenden wird nach einer kurzen Einführung in die Signifikanz der Kausalität im Recht (E. I. 2.) zunächst die Theorie der wesentlichen Bedingung in ihrer Bedeutung, Entwicklung und Anwendung dargestellt (E. II.), bevor die Tatbestandsmerkmale Unfallkausalität (E. III.) und haftungsbegründende Kausalität im engeren Sinne (E. IV.) untersucht werden.

292 BSG, Urt. v. 12.4.2005, E 94, 262; v. 9.5.2006, E 96, 196; v. 5.9.2006 - B 2 U 24/05 R -, juris; v. 12.12.2006 - B 2 U 28/05 R -, juris; Schütz, Beamtenversorgungsgesetz, $\mathbb{S} 31 \mathrm{Rn} .40$ erkannte dies bereits 1980 auf dem Gebiet des Unfallfürsorgerechts.

293 Vgl. Köhler, VSSR 2013, S. 47, 48. Ausführlich zur Kritik an der herkömmlichen Unterscheidung mit Darstellung der Literaturmeinungen Köhler, SdL 2006, S. $185,185$.

294 Kunze, VSSR 2005, S. 299, 305.

295 Ebd.

296 Becker, SGb 2007, S. 721, 722; Sprang, BG 1989, S. 144, 145 argumentiert aber, dass haftungsbegründende Kausalität eine treffende Bezeichnung sei, da sie deutlich mache, dass die Haftung der Berufsgenossenschaften auf der ursächlichen Verbindung zwischen einem geschützten Bereich und einem Unfallereignis beruht.

297 So die vorrechtliche Bedeutung des Begriffes, vgl. Planck, Der Kausalbegriff in der Physik, S. 3; Schulin, Der natürliche - vorrechtliche - Kausalitätsbegriff im zivilen Schadensersatzrecht, S. 1. 


\section{Kausalität im Recht}

Kausalität - von lateinisch causa, „Ursache“ - spielt in der gesamten erfahrungswissenschaftlichen Theorie eine wichtige Rolle. Sie ist der Versuch, in der Realität zu beobachtende Tatbestände oder Vorgänge auf ihre Ursachen zurückzuführen. Nötig sind dazu Aussagen („wenn p, dann q“), die eine logische Beziehung zwischen Ursache $\mathrm{p}$ und Wirkung q herstellen. Unter Kausalität versteht man also zunächst einmal - den juristischen Sprachgebrauch außen vor lassend - ganz allgemein die Beziehung zwischen Ursache und Wirkung, Aktion und Reaktion. Für die Naturwissenschaften formulierte Max Planck eingängig, ein Kausalzusammenhang sei eine „gewisse gesetzmäßige Verkettung zweier Ereignisse, wobei das frühere Ereignis als Ursache, das spätere als Wirkung bezeichnet wird“.298 Auch in den Geisteswissenschaften wird mit Kausalitätstheorien gearbeitet. ${ }^{299}$ Für Kant gilt dieses Prinzip a priori und ist notwendig, um unsere Erfahrung zu ordnen..$^{300}$

In der Rechtswissenschaft sind Kausaltheorien ebenfalls unerlässlich. ${ }^{301}$ Hier wird das Prinzip der Kausalität vor allem dazu genutzt, Verantwortungszusammenhänge aufzudecken. Zur Verknüpfung von Ursache und Wirkung tritt dabei ein zusätzliches, wertendes Element hinzu, welches je nach angewandter Kausalitätstheorie differiert. ${ }^{302} \mathrm{Im}$ Strafrecht etwa ist im

298 Planck, in: Max-Planck-Gesellschaft (Hrsg.), Max Planck, Vorträge und Reden, S. 19, 21.

$299 \mathrm{Zu}$ den unterschiedlichen Bedeutungen von Kausalität in beiden Feldern und im Recht lesenswert Kelsen, Österreichische Zeitschrift für öffentliches Recht und Völkerrecht 1953/55, S. 125, $125 \mathrm{ff}$.

300 Kant, Kant's gesammelte Schriften. Herausgegeben von der (Königlich) preussischen Akademie der Wissenschaften, AA III, 166/KrV B 232. Hume dagegen hält das Kausalitätsprinzip für eine ungerechtfertigte Verallgemeinerung unserer Gewohnheit, Ereignisse in Abhängigkeit voneinander zu sehen, vgl. ders., Untersuchung in Betreff des menschlichen Verstandes, S. $108 \mathrm{f}$. In der modernen Wissenschaftstheorie behilft man sich, indem man das Kausalitätsprinzip durch einen funktionalen Zusammenhang ersetzt, der die Ereignisse miteinander verbindet, ihre Aufeinanderfolge aber nur beschreibt und nicht erklärt. Vgl. insgesamt zur Wissenschaftstheorie von Kausalität Stegmüller, Wissenschaftliche Erklärung und Begründung, S. $501 \mathrm{ff}$.

301 Schaer betont die Relevanz des Kausalitätsprinzips im Besonderen, wenn er schreibt: „Bekanntlich gibt es zwei Arten von Juristen [...]: diejenigen, die sich noch nicht mit der natürlichen und adäquaten Kausalität befasst haben, und diejenigen, die dies schon hinter sich haben, aber zu keinem befriedigenden Resultat gekommen sind.", SVR 1993, S. 25.

302 Kahrs, Kausalität und überholende Kausalität im Zivilrecht, S. 12 ff. 
Rahmen der Prüfung eines Erfolgsdelikts zu untersuchen, ob die dem Täter vorgeworfene Handlung den tatbestandlichen Erfolg herbeigeführt hat. Dabei bedient man sich der Bedingungstheorie, die auch Äquivalenztheorie genannt wird: Kausal ist jede Handlung, die nicht hinweggedacht werden kann, ohne dass der Erfolg in seiner konkreten Gestalt entfiele (conditio sine qua non). Wenn es im Zivilrecht um Haftung geht, ist die Prüfung der Kausalität zumeist erforderlich. Hier wird die soeben dargestellte Bedingungstheorie von der Adäquanztheorie ergänzt, nach der nur solche Bedingungen kausal sind, die im Allgemeinen und nicht nur unter besonders eigenartigen, unwahrscheinlichen und nach dem gewöhnlichen Lauf der Dinge außer Betracht zu bleibenden Umständen geeignet sind, einen Erfolg dieser Art herbeizuführen. Ein Beispiel dafür sind Schadensersatzansprüche: Sie erlauben es, von einer anderen Person Ausgleich für eine erlittene Einbuße zu verlangen, wenn diese kausal auf ein in der Verantwortung der in Anspruch genommenen Person liegendes Element zurückzuführen ist (beispielsweise vorsätzliches Handeln oder das Eröffnen einer Gefährdungssituation). Auch im öffentlichen Recht existieren unterschiedliche Kausalitätsprüfungen. Im Polizeirecht etwa wird nach dem Verursacher einer Gefahr mithilfe der Theorie der unmittelbaren Verursachung gesucht, nach der nur diejenige Person verhaltensverantwortlich ist, die die Gefahr unmittelbar herbeigeführt hat. In Gesetzestexten wird die Notwendigkeit einer Kausalitätsprüfung etwa durch Schlüsselwörter angezeigt (bspw. „hierdurch entstehend“ in $\$ 280$ Abs. 1 BGB, ,daraus entstehend“ in $\$ 823$ Abs. 1 S. 1 BGB, ,durch “ in $\$ 833$ S. 1 BGB und $\$ 836$ Abs. 1 S. 1 BGB, „verursacht“ in $\$ 840$ Abs. 2 BGB) $)^{303}$ oder aber in der Formulierung durch die Nutzung eines aktiven Verbes deutlich gemacht: „Wer einen Menschen tötet ...“ ( $\mathbb{2} 212$ Abs. 1 StGB) setzt voraus, dass die Person den Tod des anderen Menschen kausal verursacht hat.

Kausalität tritt im gesamten Sozialversicherungsrecht und sozialen Entschädigungsrecht als wesentliches Zuordnungselement auf. Speziell auf dem Gebiet der Unfallversicherung ist sie systemtheoretisch stark verwurzelt. ${ }^{304}$ Hier bestimmt sie die Grenze der Einstandspflichten der Unfallver-

303 Das Wort „anlässlich“ dagegen verlangt zwar eine engere Verbindung verschiedener Umstände als nur ein zufälliges Nebeneinander, erreicht aber nicht die Stärke eines ursächlichen Zusammenhangs, BSG, Urt. v. 26.2.1958, E 7, 19, 19 ff. Rn. 23.

304 Köhler, SdL 2006, S. 185, 187. 
sicherungsträger und grenzt diese somit ab von den Einstandspflichten anderer Haftungssubjekte, etwa der Kranken- und Rentenversicherung. ${ }^{305}$

II. Kausalität im Unfallversicherungsrecht: die Theorie der wesentlichen Bedingung

\section{Anwendungsbereich}

Im Unfallversicherungsrecht wird zur Bestimmung der rechtlich relevanten Kausalität die sog. „Theorie der wesentlichen Bedingung“, die präziser, aber umständlicher auch „Theorie der rechtlich wesentlich mitwirkenden Ursache" genannt wird, ${ }^{306}$ angewandt. Dieser im gesamten Sozial- und Entschädigungsrecht genutzte Kausalitätsbegriff ${ }^{307}$ wurde von der Sozialrechtsprechung im und für das Unfallversicherungsrecht geschaffen ${ }^{308}$ und auf diesem Gebiet weiterentwickelt ${ }^{309}$. Sie besagt, dass von allen im Sinne der Conditio-sine-qua-non-Formel gleichwertigen Ursachen eines Ereignisses nur diejenigen rechtlich bedeutend sind, die nach den Erfahrungen und Anschauungen des praktischen Lebens unter Berücksichtigung ihrer besonderen Beziehung zum eingetretenen Erfolg als wesentlich für dessen Eintritt zu werten sind. Die Theorie fragt also danach, ob die Bedingung im Einzelfall und im Verhältnis zu allen anderen Bedingungen wesentlich für den Erfolg war. ${ }^{310}$

305 Schöpf, Multikausale Schäden in der gesetzlichen Unfallversicherung, S. 3. In diesem Zusammenhang wurde Kausalität von Watermann als „einzig mögliches rechtliches Element der risikomäßigen Zuordnung“ bezeichnet. Das Kausalitätsprinzip sei „als konstituierendes und zugleich essentielles Element im Recht der GUV unlöslich verankert", ders., in: Gitter/Thieme, et al. (Hrsg.), Im Dienst des Sozialrechts, S. 661, 661. Allerdings darf Kausalität nicht mit Zuordnung gleichgesetzt werden. Sie ist nur ein bestimmter - wenn auch wesentlicher - Ausschnitt des Zuordnungsprozesses, vgl. Wallerath, VSSR 1974, S. 233, 234.

306 Z.B. bei Nehls, BG 1983, S. 227, 227.

307 Schudt, Kausalitätsprobleme im Verwaltungsrecht, S. 1; Kunze, VSSR 2005, S. 299, 305. Auch im Unfallfürsorgerecht der Beamten sowie im Soldatenversorgungsrecht wird die Theorie der wesentlichen Bedingung angewandt, vgl. BVerwG, Urt. v. 20.5.1958, E 7, 48, 49 f.; v. 20.4.2967, E 26, 332, 337 bzw. Keller, NZS 1994, S. 161, 164; Wallerath, VSSR 1974, S. 233, $238 \mathrm{f}$.

308 Peltzer, Die Theorie der wesentlichen Bedingung, S. 45 f.

309 Ausführlich Barta, Kausalität im Sozialrecht, passim. Kunze hält die Theorie dennoch nicht für umfassend durchdrungen, vgl. ders., VSSR 2005, S. 299, 305.

310 Haueisen, JZ 1961, S. 9, 9 f. 
Da die Entwicklung der Theorie instruktiv für das Verständnis der Strukturprinzipien der Unfallversicherung ist, wird diese zunächst untersucht, bevor die Theorie selbst genauer dargestellt wird.

\section{Entwicklung der Theorie der wesentlichen Bedingung}

a. Notwendigkeit einer eigenständigen Kausalitätstheorie für das Unfallversicherungsrecht

Die herkömmlichen, aus dem Zivil- und Strafrecht bekannten Kausalitätslehren wurden dem Zweck der Unfallversicherung nicht gerecht, sodass ein eigenständiger ${ }^{311}$ Kausalitätsbegriff entwickelt werden musste. ${ }^{312}$ Denn im Unfallversicherungsrecht geht es darum, zu unterscheiden, ob ein erlittener Schaden die Verwirklichung eines privaten, allgemeinen Lebensrisikos darstellt oder ob er der Sphäre des Erwerbslebens des Geschädigten zuzuordnen ist. Dies folgt aus den der Versicherung zugrunde liegenden sozialpolitischen Zielen, Gesundheitsschäden, die durch betriebliche Einwirkungen bedingt sind, abzusichern, sowie den Betriebsfrieden zu wahren. ${ }^{313}$ Eine Abgrenzung zwischen privatem und beruflichem Risiko ist daher unumgänglich, und sie muss notwendigerweise nach der Art und Weise der schädigenden Einwirkung erfolgen. ${ }^{314}$

311 Die Theorie der wesentlichen Bedingung ist eine selbstständige Ursachenlehre, nicht lediglich eine Modifikation der Adäquanztheorie, vgl. Schlegel, DVBl. 1962, S. 8, 9.

312 BSG, Urt. v. 28.6.1988 - 2/9b RU 28/87 -, E 63, 277, 279; Gitter, Schadensausgleich im Arbeitsunfallrecht, S. 110 f.; Keller, in: Hauck/Noftz/ders. (Hrsg.), SGB VII, $\mathbb{8} 8$ Rn. 6b; Köhler, ZfSH/SGB 2012, S. 383, 383 f.; ders., SdL 2006, S. 185, 185 ff.; Giesen, Arbeitsunfall und Dienstunfall, S. 89; Krasney, in: Wannagat (Hrsg.), Entwicklung des Sozialrechts, S. 361 m.w.N. Zu den Auswirkungen der Anwendung unterschiedlicher Kausalitätstheorien im Zivil- und Sozialrecht vgl. exemplarisch Becker, MedSach 2011, S. $32 \mathrm{ff}$.

313 Es ging darum, die zivilrechtliche Unternehmerhaftung durch die Einführung eines öffentlich-rechtlichen Anspruches gegen den Versicherungsträger abzulösen. Um die dem Arbeitgeber im Zivilrecht zustehenden Einwendungsmöglichkeiten abzubilden, war ein Zurechnungskorrektiv nötig, welches man als Verlangen nach der Wesentlichkeit der Bedingung ausgestaltete, vgl. Bultmann, SGb 2016, S. 143, 144. Zu den sozialpolitischen Hintergründen der Einführung der Unfallversicherung und den damit zusammenhängenden Grundprinzipien ausführlich s.u. Kap. 2 B.

314 Köhler, SdL 2006, S. 185, 186 f. 
Im Straf- und Zivilrecht dagegen sind die verfolgten Zwecke der Kausalitätstheorien andere. Die im Strafrecht anzuwendende Äquivalenztheorie ${ }^{315}$ - conditio sine qua non - bewertet alle Ursachen als in gleichem Maße kausal, sodass der tödliche Schuss genauso ursächlich für den Tod des Opfers ist wie die Zeugung des Täters durch seine Eltern. ${ }^{316}$ Die dadurch entstehende unendliche Anzahl von Ursachen wird erst durch die weiteren Voraussetzungen der objektiven Zurechenbarkeit, der Rechtswidrigkeit und der persönlichen Schuld wertend auf die für eine Strafbarkeit relevanten reduziert. Für die Bestimmung der Ursache eines Arbeitsunfalls ist die reine Äquivalenztheorie im selben Maße ungeeignet, da auch hier eine unendliche Anzahl an Ereignissen als ursächlich für den Arbeitsunfall bewertet werden müsste (z.B. Sturz beim Einkauf von Bürokleidung; Verletzung bei der morgendlichen Rasur vor der Arbeit, um gepflegt auszusehen). Die eingrenzenden Parameter des Strafrechts zielen auf die persönliche Verantwortung des Schädigers ab und sind nicht auf das Unfallversicherungsrecht übertragbar, da es hier nicht um eine individuelle Zuweisung von Verantwortung an eine Person, sondern um die Einstandspflicht öffentlich-rechtlicher Körperschaften geht. ${ }^{317}$

Auch die zivilrechtliche Adäquanztheorie ist nicht zur Bewältigung der im Unfallversicherungsrecht nötigen Fragen geeignet. Nach dieser Theorie sind vollkommen unwahrscheinliche Kausalverläufe nicht zu betrachten; die Haftung beschränkt sich daher auf die Fälle, in denen das zum Schaden führende Ereignis im Allgemeinen und nicht nur unter besonders eigenartigen, unwahrscheinlichen und nach dem gewöhnlichen Verlauf der Dinge außer Acht zu lassenden Umständen geeignet ist, die Wirkung herbeizuführen. ${ }^{318}$ Es sollen nur allgemein vorhersehbare Risiken der Haftung unterliegen, denn die zivilrechtliche Haftung knüpft an das Verhalten des Schädigers an. Darin liegt ein Vorwurf eines Missverhaltens, der sich im Falle gänzlich unvorhersehbarer Folgen nicht aufrechterhalten lässt. Im Unfallversicherungsrecht dagegen kommt es gerade nicht darauf an, wer der Schädiger war, sondern nur darauf, wodurch die schädigende Einwirkung bedingt war. Sogar eine eigene Verursachung des Schadens durch den Geschädigten selbst schließt die Einstandspflicht der Unfallsicherungsträger nicht aus (vgl. $\$ 7$ Abs. 2 SGB VII). Hier ist eine abstrahierende Be-

315 Statt aller vgl. nur RG, Urt. v. 30.9.1941, St 75, 374; BGH, Urt. v. 29.9.1951, St 1, $332,333$.

316 Watermann, Die Ordnungsfunktionen von Kausalität und Finalität im Recht, S. $76 \mathrm{f}$.

317 Köhler, SdL 2006, S. 185, 188.

318 St. Rspr., vgl. nur BGH, Urt. v. 25.9.1952, Z 7, 204, 204 ff. Rn. 15. 
trachtung nicht maßgeblich; vielmehr soll gerade das verwirklichte Risiko abgesichert werden, auch wenn sein Eintritt unwahrscheinlich war. ${ }^{319} \mathrm{Im}$ Gegensatz zur abstrakten Betrachtungsweise des Zivilrechts kommt es dabei auf das konkrete Augenmerk an. ${ }^{320} \mathrm{Zu}$ diesem Zweck wurde die heute unter dem Namen der "Theorie der wesentlichen Bedingung" bekannte Kausalitätstheorie entwickelt. Sie ist eine speziell-konkrete Ex-post-Betrachtungsweise, während die Adäquanztheorie eine generell-abstrakte Ex-anteSichtweise darstellt. ${ }^{321}$

b. Historische Entwicklung und Implementation durch die Sozialgerichte

In der rechtshistorischen Literatur zur Theorie der wesentlichen Bedingung gilt ihr geistiger Schöpfer als unbekannt; nach ihm oder ihr wird auch nicht erschöpfend gesucht. ${ }^{322}$ Richtigerweise war es Birkmeyer, der mit seiner 1885 formulierten Kausallehre ${ }^{323}$ den Anstoß zur dieser Theorie gab. ${ }^{324}$ Sie erhielt ihre heutige Gestalt sodann durch die Rechtsprechung des Reichsversicherungsamtes. ${ }^{325}$ Das 1954 eröffnete Bundessozialgericht schloss sich der Kausalitätstheorie - ebenfalls wie davor das Reichsversorgungsgericht ${ }^{326}$ - an. In seinem ersten einschlägigen Urteil vom 10.6.1955 begründete der X. Senat dies damit, dass keine Veranlassung dazu besteht,

319 Erlenkämper, Brennpunkte des Sozialrechts 1996, S. 1, 6.

320 Dies gilt auch im Versorgungsrecht der Soldaten und im Unfallfürsorgerecht der Beamten. Diese Rechtsgebiete haben ebenfalls nicht die Zurechnung eines Schadens zu einem Schädiger, sondern die Übernahme von Schäden durch den Staat zum Gegenstand, vgl. Wallerath, VSSR 1974, S. 233, 240.

321 BSG, Urt. v. 7.11.2011, E 89, 75.

322 Peltzer, Die Theorie der wesentlichen Bedingung, S. 45.

323 Birkmeyer, Über Ursachenbegriff und Kausalzusammenhang im Strafrecht, passim.

324 Vgl. Roemer, ZfS 1962, S. 305, 345; Peltzer, Die Theorie der wesentlichen Bedingung, S. $45 \mathrm{f}$.

325 RVA, Urt v. 24.5.1912, AN 1912, 930 f.; Haueisen, JZ 1961, S. 9 f.; Gitter, SGb 1993, S. 297, 299; Bultmann, SGb 2016, S. 143, 144; Giesen, Arbeitsunfall und Dienstunfall, S. 88; Becker, Soziales Entschädigungsrecht, S. 163 f. Zu Schaffung, Organisation und Zuständigkeit des Reichsversicherungsamts vgl. Ayaß, in: Masuch/Spellbrink/Becker/Leibfried (Hrsg.), Grundlagen und Herausforderungen, Bd. 1, S. 269, 271 ff.; zur Entwicklung der Sozialgerichtsbarkeit und ihrer Vorgängerinstitutionen insgesamt Knörr, Die Entstehung einer eigenständigen Sozialgerichtsbarkeit unter besonderer Berücksichtigung Bayerns, passim.

326 Vgl. RVG, Urt. v. 1.4.1921, E 2, 11; v. 20.6.1923, E 3, 197. 
davon abzuweichen..$^{327}$ In einem weiteren Urteil vom 14.7.1955 $5^{328}$ bekräftigte der entscheidende VIII. Senat dies und fasste den Inhalt der Theorie der wesentlichen Bedingung, ohne diese so zu nennen, prägnant zusammen: „Danach sind nur solche Ursachen als adäquat und damit rechtserheblich anzusehen, die wegen ihrer besonderen Beziehungen zum Erfolg zu dessen Eintritt wesentlich mitgewirkt haben, während die sonstigen Glieder der Kausalreihe, die nur rein philosophisch, nicht aber als Ursachen im Rechtssinne in Betracht kommen, auszuscheiden sind (vgl. AN. 1912 S. 930; Entsch. des RVGer. Bd. 3 S. 197). Haben mehrere Umstände zu einem Erfolg beigetragen, so sind sie rechtlich nur dann nebeneinanderstehende Mitursachen, wenn sie in ihrer Bedeutung und Tragweite für den Eintritt des Erfolges annähernd gleichwertig sind. Kommt einem der Umstände gegenüber dem anderen eine überragende Bedeutung zu, so ist der betreffende Umstand allein Ursache im Rechtssinne. An diesem Begriff der wesentlichen Ursache haben die genannten Gerichte in ständiger Rechtsprechung festgehalten (vg. AN. 1914 S. 411, 1926 S. 480; EuM. Bd. 39 S. 265; Entsch. des RVGer. Bd. 9 S. 161). Auch der erkennende Senat hat keine Bedenken, an diesem Ursachenbegriff festzuhalten. "329 Aufgrund dieser Entstehungsgeschichte, die rein innerhalb der sozialrechtlichen Rechtsprechung unabhängig von jeglichem Anhaltspunkt im Gesetzestext erfolgte, gilt die Theorie der wesentlichen Bedingung als Musterbeispiel für Rechtsfortbildung durch Richterrecht. ${ }^{330}$

3. Die heutige Form der Theorie der wesentlichen Bedingung

a. Grundsätze und Begriffe

Die heute anerkannten Grundsätze der Theorie der wesentlichen Bedingung legt das Bundessozialgericht in seiner Entscheidung vom 9.5.2006 anschaulich dar. ${ }^{331}$ Die Theorie verlangt eine der Prüfung des natürlichen Kausalzusammenhangs im Sinne der Conditio-sine-qua-non-Formel nachgelagerte zweite Prüfungsstufe, die aus allen naturwissenschaftlich-philosophischen Ursachen diejenigen herausfiltern soll, die als rechtlich unerheb-

327 BSG, Urt. v. 10.6.1955, E 1, 72, 75 f.

328 BSG, Urt. v. 14.7.1955, E 1, 150, 156.

329 Ebd., 150, $156 \mathrm{f}$.

330 Wannagat, in: Deutscher Sozialgerichtstag (Hrsg.), SDRSV Bd. 10, S. 27; Krasney, in: Wannagat (Hrsg.), Entwicklung des Sozialrechts, S. 361.

331 BSG, Urt. v. 9.5.2006, E 96, 196, 199 ff. 
lich zu bewerten sind. Rechtserheblich kausal sind danach nur solche Ursachen, die wegen ihrer besonderen Beziehung zum Erfolg an dessen Eintritt wesentlich mitgewirkt haben. ${ }^{332}$ Welche Ursachen dabei als wesentlich anzusehen sind, ist im Einzelfall im Rahmen einer Wertungsentscheidung "aus der Auffassung des praktischen Lebens" 333 abzuleiten. Die Beurteilung des Ursachenzusammenhangs hat im Einzelfall mit Blick auf den individuellen Geschädigten, aber auf der Basis des aktuellen wissenschaftlichen Erkenntnisstandes über die Möglichkeit von Ursachenzusammenhängen zu erfolgen. ${ }^{334} \mathrm{Zu}$ berücksichtigen sind dabei das Unfallereignis als solches, die Art und das Ausmaß der Einwirkung, evtl. konkurrierende Ursachen, der zeitliche Geschehensablauf, Rückschlüsse aus dem Verhalten des Verletzten nach dem Unfall, Befunde und Diagnosen des erstbehandelnden Arztes, die gesamte Krankengeschichte und der Schutzzweck der Norm. 335

Im Rahmen der Theorie der wesentlichen Bedingung können auch mehrere Ursachen gleichzeitig als rechtlich wesentlich für den Erfolg anzusehen sein (konkurrierende Kausalität). Ein Arbeitsunfall liegt bereits dann vor, wenn eine dieser Mitursachen die Zuordnung zur Unfallversicherung auslöst. Dabei ist es für den Begriff der rechtlichen Wesentlichkeit nicht notwendig, dass die arbeitsbezogene Ursache die anderen Ursachen überwiegt. Eine Ursache kann auch dann rechtlich wesentlich sein, wenn sie rechnerisch verhältnismäßig weniger zum Erfolg beigetragen hat als eine andere Ursache. ${ }^{336}$ Dies gilt allerdings nur, solange die andere (privat konnotierte) Ursache keine überragende Bedeutung hat. ${ }^{337}$ In einem solchen Fall ist nur die überragende Ursache - die sich auch aus mehreren Ursachen zusammensetzen kann - als wesentlich anzusehen. ${ }^{338}$ Kann praktisch nicht festgestellt werden, ob eine Kausalreihe eine andere überwiegt oder

332 St. Rspr., vgl. nur BSG, Urt. v. 9.5.2006, E 96, 196, 199 m.w.N.; vgl. RVA, Urt. v. 24.5.1912, AN 1912, 930 f.; BSG, Urt. v. 10.6.1955, E 1, 72, 76; v. 14.7.1955, E 1, $150,156 \mathrm{f}$.

333 BSG, Urt. v. 10.6.1955, E 1, 72, 76. Als „Leerformel“ kritisiert diese Formulierung Keller, in: Hauck/Noftz/ders. (Hrsg.), SGB VII, $\mathbb{} 8$ Rn. 9.

334 BSG, Urt. v. 9.5.2006, E 96, 196, 200 f. m.w.N.

335 BSG, Urt. v. 9.5.2006, E 96, 196, 200; v. 19.9.1974, E 38, 127, 129; vgl. auch Becker, SGb 2006, S. 449, 450.

336 BSG, Urt. v. 11.12.1963 - 5 RKn 31/60 -, juris.

337 BSG, Urt. v. 11.12.1963 - 5 RKn 31/60 -, juris; v. 12.2.1970 - 7/2 RU 262/67 -, juris.

338 BSG, Urt. v. 30.6.1960, E 12, 242, 245. 
welche die überragende Ursache ist, so sind alle Ursachen als annähernd gleichwertig zu bewerten. ${ }^{339}$

Umgekehrt kann auch eine Ursache, die im Rahmen einer versicherten Tätigkeit kausal für den einen Gesundheitsschaden verursachenden Unfall geworden ist und damit formal alle soeben gestellten Voraussetzungen an die Bezeichnung „Arbeitsunfall“ erfüllt, als nicht rechtlich wesentlich bewertet werden. Dies ist bei sogenannten „Gelegenheitsursachen“ oder „Auslösern“ der Fall. ${ }^{340}$ Als solche bezeichnet man eine konkrete Verrichtung, die nur zufällig der versicherten Tätigkeit zuzurechnen ist. Damit ist gemeint, dass sich der Unfall aufgrund einer beim Geschädigten bereits bestehenden Schadensanlage auch bei jeder anderen, austauschbaren, nicht versicherten Tätigkeit hätte ereignen können. ${ }^{341}$ Hätte jedes andere alltäglich vorkommende Ereignis zu derselben Zeit den Erfolg ausgelöst, ist die versicherte Tätigkeit nur Gelegenheitsursache und löst keine Zuordnung zum Schutzbereich der Unfallversicherung aus. ${ }^{342}$ Bei der vorzunehmenden Abwägung kann die Schwere des Unfallereignisses eine Rolle spielen. ${ }^{343}$

Da es für die Beurteilung der wesentlichen Bedingung gerade auf den konkreten Schadensablauf ankommt, spielt die Fallgruppe der hypothetischen Kausalität im Unfallversicherungsrecht keine Rolle. Es ist für die Beurteilung der Wesentlichkeit eines Faktors gleichgültig, ob sich nachträglich feststellen lässt, dass der unfallbedingte Erfolg zu einem späteren Zeitpunkt auch durch eine andere Bedingung und einen anderen Kausalablauf ausgelöst worden wäre. ${ }^{344}$

\section{b. Terminologische Unsicherheiten}

Das Bundessozialgericht verwendet im Rahmen der Kausalitätsprüfung unterschiedliche Bezeichnungen. ${ }^{345}$ Mit „Ursache“ oder „Mitursache“ war

339 Köhler, ZfSH/SGB 2012, S. 383, 385.

340 BSG, Urt. v. 9.5.2006, E 96, 196, 199 ff. m.w.N. Ob dies ein noch notwendiges Konstrukt ist, wird bezweifelt; dargestellt wird dies bei Köhler, VSSR 2013, S. 47, 73, der die Frage bejaht.

341 Köhler, VSSR 2013, S. 47, 49.

342 BSG, Urt. v. 9.5.2006, E 96, 196, 200 m.w.N.

343 Ebd. m.w.N.

344 BSG, Urt. v. 28.6.1988, E 63, 277, 280; v. 25.4.1961, E 14, 172, 176; Tomandl, Sozialversicherung 1975, S. 137, 141.

345 Ricke, BG 1994, S. 360, 360 f.; Sprang, BG 1989, S. 144, 144 f. 
teilweise jede Bedingung im Sinne der Äquivalenzformel, teilweise aber auch nur jede im Sinne der Theorie der wesentlichen Bedingung rechtlich erheblich kausale Ursache gemeint. Im letzteren Fall wurden im Sinne der Äquivalenzformel kausale, im Sinne der Theorie der wesentlichen Bedingung nicht-kausale Bedingungen als „Gelegenheitsursachen“ bezeichnet. ${ }^{346}$ Dieselbe Bedeutung wird auch der Formulierung „(rechtlich) allein wesentliche Bedingung" zugemessen. ${ }^{347}$

In seiner neueren Rechtsprechung spricht das BSG von der „(Wirk-)Ursache “348 und sorgte damit für erhebliche Missverständnisse. ${ }^{349}$ Inzwischen ist geklärt, dass mit der Bezeichnung eine rein terminologische Präzisierung des Ursachenbegriffs intendiert und keinerlei dogmatische Änderung bezweckt war. ${ }^{350}$ Die teilweise weit darüber hinausgehende Interpretation dieser Rechtsprechung in der Literatur zeigt - wie schon die Konfusion um die „Erfüllung des Versicherungstatbestandes“ - einmal mehr, dass sprachliche Inkonsistenzen im Wissenschaftsbetrieb zu Verwirrung führen und deswegen tunlichst zu vermeiden sind. ${ }^{351}$

c. Kritik an der Theorie der wesentlichen Bedingung als reiner Wertungsakt

Die Theorie der wesentlichen Bedingung ist nicht unangegriffen geblieben. In seiner umfangreichen Habilitationsschrift von 1983 kritisiert Barta vor allem, dass sie völlig durch Richterrecht ausgefüllt werden muss, wodurch sie dem Missbrauch Tür und Tor öffne. ${ }^{352}$ In der Tat findet sich in

346 Z.B. BSG, Urt. v. 12.3.1958, E 7, 53, 55.

347 Köhler, ZfSH/SGB 2012, S. 383, 385.

348 Diese Bezeichnung findet sich, noch als „Wirkursache“, zum ersten Mal in BSG, Urt. v. 24.7.2012 - B 2 U 23/11 R -, juris Rn. 26 ff.; v. 24.7.2012 - B 2 U 9/11 R -, juris Rn. 36 ff.; v. 13.11.2012, E 112, 177.

349 Kritisch zum Begriff Ricke, WzS 2013, S. 241, 241 f.; Leube, SGb 2014, S. 392, 396 Fn. 14; Keller, in: Hauck/Noftz/ders. (Hrsg.), SGB VII, $\mathbb{8} 8$ Rn. 6b. Teilweise wurde der Begriff - genährt durch die unklare Ausdrucksweise des BSG - als Aufgabe der bisherigen Dogmatik und Einführung einer neuen Kausalitätsprüfung verstanden, vgl. Bultmann, SGb 2016, S. 143, $143 \mathrm{ff}$.

350 Deutlich Spellbrink, SGb 2017, S. 1, 5. Ebenso Giesen, Arbeitsunfall und Dienstunfall, S. 89; Ricke, WzS 2013, S. 241, 241 f.; Siefert, NZW 2017, 766, 770.

351 Insofern (selbst-)kritisch Spellbrink, SGb 2017, S. 1, 5 mit Hinweis auf einen entsprechenden Appell zur Klarheit des BSG-Präsidenten Schlegel, abgedruckt in SGb 2016, S. 605, 605.

352 Barta, Kausalität im Sozialrecht, passim. 
keinem Gesetzestext eine Erwähnung oder gar Definition der Theorie der wesentlichen Bedingung. Indem diese maßgeblich darauf abstellt, ob eine von allen Ursachen als „wesentlich“ zu betrachten ist, ist sie inhaltlich vollkommen von der Einschätzung des Rechtsanwenders abhängig. Dieser kann seine Entscheidung nur anhand der individuellen Fallgestaltung treffen. Generalisierungen oder Fallgruppenbildungen sind hier fehl am Platz, denn jeder Schadensfall hat eine originäre, nicht zu wiederholende Zusammensetzung von Ursachen. Die Kritik, dass der Geschädigte von der Beurteilung des entscheidenden Richters abhängig ist, ist deswegen nicht von der Hand zu weisen. Auch anderorts wird diese Unschärfe bemängelt. In der Theorie der wesentlichen Bedingung liege keinerlei Logik; die Zuordnung erfolge lediglich über den Begriff der Wesentlichkeit, welcher sich einer genaueren Definition entzöge. Es handele sich deswegen nicht um eine konditionale Verknüpfung von Ursache und Wirkung im Sinne einer Kausalitätstheorie, sondern um einen reinen Wertungsakt. ${ }^{353}$ Wenn dies der Fall ist, streiten die Prinzipien der Rechtssicherheit, der Vorhersehbarkeit und der Einfachheit der Rechtsanwendung gegen die Implementation dieser Theorie.

Ein Wertungselement ist allerdings notwendigerweise allen juristischen Kausalitätstheorien immanent. Eine rein naturwissenschaftliche Definition von Kausalität, die auf Gesetzen der Logik basierte, würde in der Anwendung im Rechtssystem zu unerträglichen Ergebnissen führen. Die Vielfalt menschlicher Handlungen, Betätigungen der Willensfreiheit und gesellschaftlicher Situationen erlaubt es gerade nicht, sie im Sinne der UrsacheWirkungs-Relation festen Strukturen zu unterwerfen. Sachgerechte Entscheidungen werden gerade durch die Erlaubnis, jeden Einzelfall für sich zu betrachten, ermöglicht. In allen Rechtsgebieten, die Kausalität anwenden, sei es Zivil-, Straf- oder öffentliches Recht, wird deswegen die zur empirischen Kausalitätsermittlung genutzte Bedingungstheorie durch wertende Zurechnungskriterien ergänzt ${ }^{354}$ - etwa durch die Lehre der Adäquanz, durch den Schutzzweck der Norm, durch das Erfordernis von Unmittelbarkeit oder durch die Lehre von der objektiven Zurechnung. Keine dieser Möglichkeiten ist für den Bereich des Unfallversicherungsrechts besser geeignet als die Theorie der wesentlichen Bedingung. ${ }^{355}$ Sie vermag es, den Zweck des Unfallversicherungsrechts in die Kausalitätsprüfung zu integrie-

353 Tomandl, Sozialversicherung 1975, S. 137, $140 \mathrm{f}$.

354 Rönnau/Faust et al., JuS 2004, S. 113, 118.

355 Dass die Theorie der wesentlichen Bedingung von allen bekannten Theorien die für das Unfallversicherungsrecht geeignetste sei, ist das Hauptargument im Schrifttum, vgl. Keller, in: Hauck/Noftz/ders. (Hrsg.), SGB VII, $\mathbb{8}$ Rn. 8; Köhler, 
ren. ${ }^{356}$ Denn das Wesentlichkeitskriterium bezieht sich gerade auf die ausgeübte Tätigkeit, nicht aber auf die Person eines Schädigers. So wird deutlich, dass der Versicherte nicht gegen Schädiger, sondern gegen aufgrund seiner versicherten Tätigkeit erlittene Schäden geschützt werden soll. In der Unfallversicherung sollen die Schäden Ansprüche auslösen, die dem Geschädigten gerade in engem Zusammenhang mit seiner beruflichen Tätigkeit entstanden sind. Es geht darum, zu vermeiden, dem Arbeitgeber das allgemeine Lebensrisiko seines Arbeitnehmers aufzubürden, sondern es soll nur das spezifische, mit der Erwerbstätigkeit verknüpfte Risiko ihm aufgebürdet werden. Um dies zu erreichen, ist die Frage danach, ob die Erwerbstätigkeit (also die geschützte Tätigkeit) eine nicht nur unwesentliche Rolle in der Verursachung des Schadens gespielt hat, zielführend.

Es ist auch nicht unangebracht, den Schutzzweck des Unfallversicherungsrechts bereits als Kriterium der wertenden Bestimmung der Wesentlichkeit zu betrachten. Denn bei der Suche nach einer relevanten Ursache ist es unumgänglich, vom Ziel dieser Suche her zu denken. Nur so lassen sich aus der unendlichen Anzahl von Ursachen diejenigen herausfiltern, die überhaupt als relevant in Betracht gezogen werden können. ${ }^{357}$

Um dem Problem der Unvorhersehbarkeit zu entgegnen, wurde vorgeschlagen, den Begriff der „Wesentlichkeit“ schärfer zu konturieren. Krasney schlug eine mathematische Definition des Kriteriums vor. Die Schwelle einer wesentlichen Bedingung solle immer dann als überschritten gelten, wenn ihr Anteil an dem erfolgten Ereignis mindestens 10 Prozent beträgt. ${ }^{358}$ Solch eine Zahl vermag jedoch das Problem nicht zu lösen. Denn zu oft lassen sich menschliches Verhalten und verschiedene Ursachen nicht quantifizieren. ${ }^{359}$ Dafür fehlt es zudem an einem Maßstab, gleichsam an einem Umrechnungsschlüssel. Dem medizinischen Sachverständigen, der die konkrete Sachlage zu beurteilen hat, fällt es genauso schwer, eine Ursa-

ZfSH/SGB 2012, S. 383, 385; Krasney, in: Wannagat (Hrsg.), Entwicklung des Sozialrechts, S. 361 f.; Wilhelm, in: Franke/Weiß (Hrsg.), GKÖD, $\$ 31$ BeamtVG Rn. 18, 19.

356 Insofern besteht Ähnlichkeit zur Theorie des Schutzzwecks der Norm, die im Anschluss an eine Äquivalenz- und Adäquanzprüfung die Beantwortung der Frage verlangt, ob die einschlägige haftungsbegründende Norm gerade vor genau dem Schaden schützen soll, zu welchem das geprüfte Ereignis kausal ist, vgl. Oetker, in: Säcker/Rixecker (Hrsg.), Münchner Kommentar zum BGB, $\mathbb{} 249$ Rn. 120-126.

357 Vgl. dazu anschaulich Analyse und Beispiel bei Stegmüller, Wissenschaftliche Erklärung und Begründung, S. $509 \mathrm{f}$.

358 Woitowitz/Krasney, Der Betriebsarzt 1996, S. 209, 211 m.w.N.

359 Keller, in: Hauck/Noftz/ders. (Hrsg.), SGB VII, $\$ 8$ Rn. 9a. 
che mit mindestens 10 Prozent zu beziffern, wie sie als wesentlich zu begründen. ${ }^{360}$ Immerhin aber verleiht eine solche Zahl Rechtsanwendern eine ungefähre Vorstellung davon, was „wesentlich“ zu bedeuten hat. Aus der Zahl „10 Prozent“" wird etwa deutlich, dass die infrage kommende Ursache bei Weitem nicht die überwiegende oder einzelne Ursache sein muss. Kann man die Zahl also auch nicht mathematisch nutzen und die Wesentlichkeit einer Ursache nicht formelhaft berechnen, so ist Krasneys als „Tabubruch“ bezeichneter ${ }^{361}$ Vorstoß dennoch zu begrüßen. ${ }^{362}$ Denn auch ohne tatsächliche Berechenbarkeit geht von einer Zahl, so man sich ihrer bewusst ist, eine Regulationswirkung aus.

Die Kritik an der mangelnden Vorhersehbarkeit der Theorie der wesentlichen Bedingung wird im Kontext des Unfallversicherungsrechts dadurch entschärft, dass im Rahmen des Tatbestandsmerkmals der Unfallkausalität das Vorliegen des Ursachenzusammenhangs im Sinne der Theorie der wesentlichen Bedingung regelmäßig vermutet wird. ${ }^{363}$ Der Geschädigte ist damit von der objektiven Beweislast bezüglich des Vorliegens der Wesentlichkeit befreit. Liegt nicht ausnahmsweise eine Fallgestaltung vor, in der die Unfallkausalität nicht vermutet wird, ist also die Vorhersehbarkeit der Beurteilung dieses Tatbestandsmerkmals gegeben.

\section{Unfallkausalität}

\section{Abgrenzung zum Schutzbereich und zu anderen Kausalitäten}

Die Unfallkausalität ${ }^{364}$ benennt den ursächlichen Zusammenhang zwischen der Verrichtung zur Zeit des Unfallereignisses und dem Unfallereignis selbst. Dies ist nicht mit der Prüfung des Schutzbereichs zu verwechseln, bei der es darum geht, den Zusammenhang zwischen der Verrichtung zur Zeit des Unfallereignisses und dem vom Unfallsicherungssystem geschützten Tätigkeitsbereich zu bestimmen. Ein Beispiel verdeutlicht die Differenz zwischen den Tatbestandsmerkmalen. Geht es etwa darum, dass die Hand einer Beschäftigten beim Tackern abrutscht und sie sich den Finger verletzt, würde zunächst im Rahmen des Schutzbereichs geprüft wer-

360 Kunze, VSSR 2005, S. 299, 306.

361 Ebd., S. 299, 308.

362 Ebenso Keller, in: Hauck/Noftz/ders. (Hrsg.), SGB VII, $\$ 8$ Rn. 9 b.

363 Dazu näher sogleich s.u. Kap. 1 E. III. 2.

364 Zum Begriff vgl. Fn. 291. 
den, ob das Tackern eine Verrichtung war, die zur Erfüllung der Pflichten aus dem Arbeitsverhältnis dienen sollte. Dies wäre etwa zu bejahen, wenn die Beschäftigte Dokumente verklammerte, um Rechercheergebnisse zur Übergabe an ihren Vorgesetzten vorzubereiten. Der unfallversicherungsrechtliche Schutzbereich ist eröffnet. Im Rahmen der Unfallkausalität wird anschließend geprüft, ob die Tätigkeit des Verklammerns kausal für den Unfall, also das Treiben der Klammer in den Finger, war. Hier wird die Theorie der wesentlichen Bedingung angewandt. Zweifellos war das Verklammern äquivalent kausal. Zu fragen bleibt dann, ob es auch wesentlich an der Herbeiführung des Unfalls war. Als wesentliche Ursache könnte neben der Arbeitstätigkeit zum Beispiel eine durch ein gleichzeitig geführtes privates Telefongespräch bedingte Ablenkung in Betracht kommen.

Die sogleich (s.u.) zu behandelnde haftungsbegründende Kausalität im engeren Sinne bezeichnet dagegen den Ursachenzusammenhang zwischen dem Unfall und dem eingetretenen Gesundheitsschaden. Im Beispiel wäre dies der Zusammenhang zwischen dem Treiben der Klammer in den Finger und der blutenden Wunde an der Fingerkuppe. ${ }^{365}$

\section{Vermutung im Regelfall}

Die Notwendigkeit eines Kausalzusammenhangs zwischen der tatsächlichen Verrichtung zur Zeit des Unfallereignisses und dem Unfallereignis selbst wird aus dem Wort "infolge“ in $\mathbb{8} 8$ Abs. 1 Satz 1 SGB VII hergeleitet. ${ }^{366}$ Diese Präposition, welche „als Folge eines Geschehens; aufgrund von “ 367 bedeutet, zeigt die Forderung nach einer Ursachenbeziehung an. Über die genaue Art dieser Ursachenbeziehung ist damit noch keine Aussage getroffen. Aus den oben dargelegten Gründen wird für den Kausalzusammenhang die Theorie der wesentlichen Bedingung verwendet. Demnach besteht Unfallkausalität, wenn die versicherte Tätigkeit wesentliche Ursache im Sinne der Theorie der wesentlichen Bedingung für den Eintritt des Unfalls ist.

365 Als haftungsausfüllende Kausalität wird schließlich der Ursachenzusammenhang zwischen dem Gesundheitsschaden (der sogenannten Primärschädigung) und den weiteren Unfallfolgen bezeichnet. Für die Subsumtion unter den Versicherungsfall spielt diese Kausalität keine Rolle; sie bestimmt nach erfolgter Bejahung eines Versicherungsfalls den Umfang des Leistungsfalls, vgl. Köhler, SdL 2006, S. 185, 204.

366 Köhler, VSSR 2013, S. 47, 48.

367 Wissenschaftlicher Rat der Dudenredaktion (Hrsg.), Duden, S. 1934. 
Dies wird grundsätzlich vermutet. ${ }^{368}$ Mit der Vermutung werden die Versicherten von der objektiven Beweislast befreit, da der Versicherungsschutz aufgrund von Beweisschwierigkeiten sonst vielfach leerzulaufen droht. ${ }^{369}$ Denn häufig ist kein Grund erkennbar, warum sich der Unfall gerade in dieser Form zugetragen hat. ${ }^{370}$ Die Unfallkausalität ist daher stets zu bejahen, außer es bestehen zusätzlich zum kausalen Anknüpfungspunkt der versicherten Tätigkeit noch andere Tatsachen, die Konkurrenzursachen gewesen sein könnten. ${ }^{371}$ Diese Fallgestaltungen, in denen die Unfallkausalität nicht vermutet werden kann, sind Fälle einer möglichen inneren Ursache, einer gemischten Tätigkeit, einer unerheblichen Unterbrechung oder einer eingebrachten Gefahr (s.u. Kap. 1 E III. 3. a.-d.). Steht eine konkurrierende Ursache im Sinne der Äquivalenztheorie fest, ist die Vermutung der Unfallkausalität erschüttert und es ist eine Kausalitätsprüfung im Sinne der Theorie der wesentlichen Bedingung durchzuführen. Dabei muss nach den oben erläuterten Kriterien wertend entschieden werden, ob die versicherte Ursache wesentlich im Sinne der Theorie der wesentlichen Bedingung für den Eintritt des Unfallereignisses war. ${ }^{372}$

\section{Problematische Fallgruppen}

\section{a. Gelegenheitsursache}

Wie bereits dargelegt, schließt das Vorliegen einer inneren Ursache grundsätzlich bereits die Einordnung eines schädigenden Ereignisses als Unfall aus. ${ }^{373}$ Nur, wenn eine grundsätzlich versicherte Tätigkeit wesentlich zum Vorhandensein oder zum Ausprägungsgrad der inneren Ursache beigetragen hat, ist die Unfallkausalität zu bejahen. ${ }^{374}$ Dies ist beispielsweise der

368 Std. Rspr., vgl. nur BSG, Urt. v. 30.1.2007, E 98, 79, 81; v. 17.2.2009 - B 2 U 18/07 R -, juris Rn. 13; Keller, in: Hauck/Noftz/ders. (Hrsg.), SGB VII, $\mathbb{\$} 8$ Rn. 342; Becker, SGb 2007, S. 721, 726; ders., SGb 2012, S. 691, 693.

369 BSG, Urt. v. 30.1.2007, E 98, 79, 82.

370 Ebd.

371 Korenke, in: Bontrup/Korenke et al. (Hrsg.), Arbeit - Personal - Soziales, S. 161, 167; Becker, SGb 2007, S. 721, 726; ders., SGb 2012, S. 691, 693.

372 Vgl. BSG, Urt. v. 30.1.2007, E 98, 79.

373 S.o. Kap. 1 C II. 3.

374 BSG, Urt. v. 15.2.2005 - B 2 U 1/04 R -, juris Rn. 23. 
Fall, wenn ein Sturz durch einen infolge der Anstrengungen bei der Arbeit erlittenen körpereigenen Kreislaufkollaps erfolgt. ${ }^{375}$

Ein Arbeitsunfall kann weiterhin vorliegen, wenn neben der dann als Gelegenheitsursache zu bezeichnenden körpereigenen Teilursache eine äuBere, betriebliche Einwirkung als rechtlich wesentlich zu beurteilen ist. ${ }^{376}$ Dafür ist maßgeblich, ob jedes andere alltäglich vorkommende Ereignis zu derselben Zeit die Erscheinung ausgelöst hätte. ${ }^{377}$ Hätte sich der Unfall ohne die versicherte Tätigkeit nicht in derselben Art oder Schwere ereignet, ist die versicherte Tätigkeit wesentliche Ursache im Sinne der Theorie der wesentlichen Bedingung und Unfallkausalität liegt vor. ${ }^{378}$ Dies betrifft etwa Fälle, in denen der Versicherte während eines Kreislaufkollapses von einem hohen Betriebsgerät stürzt und der Gesundheitsschaden deswegen besonders groß ist. Bei der vorzunehmenden Abwägung kann die Schwere des Unfallereignisses eine Rolle spielen. ${ }^{379}$ Ein anderes Beispiel bildet die Versicherte, die auf einem versicherten Weg einen epileptischen Anfall erleidet, aufgrund dessen sie stürzt und sich den Arm bricht. ${ }^{380}$ Steht der epileptische Anfall im Vollbeweis (mit an Gewissheit grenzender Wahrscheinlichkeit) als naturwissenschaftlich-kausale Ursache für den Sturz fest, ist zu prüfen, ob diese Ursache im Gegensatz zum Zurücklegen des versicherten Weges überragende Bedeutung hat. ${ }^{381}$ Dann ist sie im Sinne der Theorie der wesentlichen Bedingung einzig wesentliche Ursache für den Sturz und die Unfallkausalität ist zu verneinen. Die versicherte Ursache des Zurücklegens des Weges bleibt unbeachtlich und würde als bloße Gelegenheitsursache bezeichnet. ${ }^{382}$

375 Keller, in: Hauck/Noftz/ders. (Hrsg.), SGB VII, $\$ 8$ Rn. 273 d.

376 Köhler, VSSR 2013, S. 47, 64.

377 St. Rspr., z. B. BSG, Urt. v. 15.2.2005 - B 2 U 1/04 R -, juris Rn. 23; v. 9.5.2006, E 96, 196, 200 m.w.N. Kritisch dazu Benz, SGb 2001, S. 220, 224, der den Aspekt der mitwirkenden besonderen Betriebsgefahr als Einfallstor für einen über den Schutzzweck der Unfallversicherung hinausgehenden und daher unerwünschten Betriebsbann sieht.

378 Keller, in: Hauck/Noftz/ders. (Hrsg.), SGB VII, $\$ 8$ Rn. 273.

379 BSG, Urt. v. 9.5.2006, E 96, 196, 200.

380 Becker, SGb 2012, S. 691, 694, gebildet nach BSG, Urt. v. 17.2.2009 - B 2 U 18/07 $\mathrm{R}-$, juris.

381 Für die Beurteilung spielen meist medizinische Gesichtspunkte eine tragende Rolle.

382 Becker, SGb 2012, S. 691, 694. 


\section{b. Gemischte Tätigkeit}

Die Fallgruppe der gemischten Tätigkeit wurde bereits beim Zurechnungszusammenhang angesprochen, ${ }^{383}$ gehört aber ebenso zur Problematik der Unfallkausalität. Es handelt sich um Fälle, in denen das gesamte Verhalten der versicherten Person in mehrere Verrichtungen trennbar ist, von denen mindestens zwei gleichzeitig ausgeführt werden und die sich nicht in sich zeitlich nacheinander ereignende Teile aufspalten lassen. Ist jeweils eine dieser Verrichtungen dienstlich, die andere privat konnotiert, muss auf der Ebene der Unfallkausalität geprüft werden, ob der beruflich motivierte Teil der gemischten Tätigkeit auch wesentlich für den Erfolgseintritt war. ${ }^{384}$ Wie gesehen kommt es dabei darauf an, ob - nach lebenspraktischen und naturwissenschaftlich-technischen Gesichtspunkten sowie juristischer Wertbeurteilung - der versicherten Ursache ein hinreichend gewichtiger Anteil an der Verursachung insgesamt zugesprochen werden kann oder ob die konkurrierende private Ursache von überragender Bedeutung ist. ${ }^{385}$

\section{c. Eingebrachte Gefahr}

Von einer eingebrachten Gefahr spricht man, wenn der Versicherte unbewusst eine Gefahr aus seiner Privatsphäre mit in den Bereich des Arbeitgebers gebracht hat, er sich beispielsweise beim Griff nach dem Schlüssel zu seinem Dienstwagen in seiner Hosentasche an einem Messer verletzt, welches sein Sohn dort versteckt hatte. ${ }^{386}$ Auch hier kommt es darauf an, ob das Vorhandensein des Messers in der Hosentasche als private Ursache für den Unfall im Gegensatz zu der versicherten Ursache nach einer vom Rechtsanwender zu fällenden Wertentscheidung von überragender Bedeutung war. ${ }^{387}$ Ist dies zu bejahen, liegt keine Unfallkausalität vor, da die beruflich konnotierte Ursache dann nicht als wesentlich im Sinne der Theorie der wesentlichen Bedingung anzusehen ist.

383 S.o. Kap. 1 D. III. 2. c. dd.

384 Ebenso Kruschinsky, jurisPR-SozR 6/2015, Anm. 4.

385 Becker, SGb 2012, S. 691, 697.

386 Ebd., gebildet nach BSG, Urt. v. 26.1.1978 - 2 RU 39/77 -, juris.

387 Keller, in: Hauck/Noftz/ders. (Hrsg.), SGB VII, $\mathbb{\$} 8$ Rn. 290 d. 


\section{d. Unerhebliche Unterbrechung}

Auch in den beim Tatbestandsmerkmal der Erfüllung des Versicherungstatbestands ebenfalls bereits angesprochenen Fällen, ${ }^{388}$ in denen die versicherte Tätigkeit kurz durch Verfolgung eines privaten Zweckes unterbrochen wird und der Zurechnungszusammenhang zwischen versicherter Tätigkeit und Unfall aufgrund der Unerheblichkeit der Unterbrechung bejaht wird, ist die Unfallkausalität genauer zu erörtern. Die versicherte Tätigkeit muss auch hier grundsätzlich als wesentliche Ursache angesehen werden. Jedenfalls darf der Unfall nicht die Verwirklichung einer originär aus dem privaten Bereich stammenden, sog. selbstgeschaffenen Gefahr darstellen, da sonst der Ausnahmecharakter des Versicherungsschutzes während geringfügiger Unterbrechungen nicht berücksichtigt werden würde. ${ }^{389}$

IV. Haftungsbegründende Kausalität im engeren Sinne

\section{Der Zusammenhang zwischen Unfallereignis und Primärschaden}

Für die Bejahung eines Arbeitsunfalls ist weiterhin ein ursächlicher $\mathrm{Zu}$ sammenhang zwischen dem Unfallereignis und dem Primärschaden notwendig. Dieser Ursachenzusammenhang wird der sprachlichen Einfachheit halber in Literatur und Rechtsprechung als haftungsbegründende Kausalität bezeichnet, ${ }^{390}$ wenn auch „haftungsbegründende Kausalität im engeren Sinne“ der präzisere Ausdruck wäre. ${ }^{391}$

Während es sich beim Unfall um einen Vorgang handelt, ist die Primärschädigung ein Ergebnis, auch wenn beides oft gleichzeitig auftritt. ${ }^{392} \mathrm{Um}$ sie miteinander zu verbinden, ist ein Ursachenzusammenhang zwischen Unfallereignis und Gesundheitsschaden notwendig. Dieser ist nach der Theorie der wesentlichen Bedingung zu beurteilen. ${ }^{393}$ Dabei handelt es

388 S.o. Kap. 1 D. III. 2. c. aa.

389 Keller, in: Hauck/Noftz/ders. (Hrsg.), SGB VII, $\$ 8$ Rn. 290 e.

390 Anders aber Wallerath/Rühr, NZS 2007, S. 63, 63; Marschner, in: Rolfs/Giesen et al. (Hrsg.), BeckOK Sozialrecht, $\mathbb{8} 8$ SGB VII Rn. 33.

391 „Haftungsbegründende Kausalität im engeren Sinne“ ist deswegen präziser, weil auch die Unfallkausalität haftungsbegründender Natur ist.

392 Becker, SGb 2007, S. 721, 727.

393 Ebd. 
sich notwendigerweise um eine medizinische Frage. ${ }^{394}$ Häufig bereitet dieser Prüfungsschritt keine Probleme. Ist der Unfall etwa das Abschneiden eines Daumens, ist dessen wesentliche Ursächlichkeit für den Gesundheitsschaden „abgeschnittener Daumen“ offensichtlich. ${ }^{395}$

\section{Problemfall „Schadensanlage“}

Näherer Erläuterung bedarf die haftungsbegründende Kausalität im engeren Sinne in Fällen, in denen das Unfallereignis nicht die einzige Ursache des Schadenseintritts ist. Dies gilt insbesondere, wenn zusätzlich zu dem Unfallereignis noch eine bereits in der Person des Geschädigten bestehende krankhafte Veranlagung, eine sogenannte „Schadensanlage“ oder „innere Ursache“, vorliegt. ${ }^{396}$ Eine solche Schadensanlage darf die Bedeutung der Ursächlichkeit des Unfalls nicht überwiegen, da der Unfall sonst nicht mehr wesentlich im Sinne der Theorie der wesentlichen Bedingung ist. Der Unfall ist dann wiederum nur noch eine sogenannte „Gelegenheitsursache“.397 Unwesentlich ist der Unfall als Ursache dann, wenn der Schaden auch ohne den Unfall eingetreten wäre - und zwar ungefähr zur selben Zeit, ungefähr im selben Umfang und entweder ohne Mitwirkung eines äußeren Ereignisses oder unter Mitwirkung eines äußeren Ereignisses, welches der normalen alltäglichen Belastung entspricht. ${ }^{398}$ Sinn ist, dass sich der Unfall nur zufällig in Zusammenhang mit dem Beschäftigungsverhältnis ereignet hat und genauso gut im privaten Bereich hätte eintreten können. ${ }^{399}$ Der Schaden ist dann dem allgemeinen Lebensrisiko des Geschädigten und nicht dem Betriebsrisiko des Arbeitgebers zuzuordnen. ${ }^{400}$ Ein

394 Kunze, VSSR 2005, S. 299, 320.

395 Korenke, in: Bontrup/Korenke et al. (Hrsg.), Arbeit - Personal - Soziales, S. 161, 168.

396 Marschner, in: Rolfs/Giesen et al. (Hrsg.), BeckOK Sozialrecht, $\mathbb{8} 8$ SGB VII Rn. $34 \mathrm{f}$.

397 Ricke, in: Körner/Leitherer et al. (Hrsg.), Kasseler Kommentar zum Sozialversicherungsrecht, $\$ 8$ SGB VII Rn. 26; Köhler, VSSR 2013, S. 47, 47. Der Begriff wird als missverständlich kritisiert, vgl. Keller, in: Hauck/Noftz/ders. (Hrsg.), SGB VII, $\$ 8$ Rn. 297 m.w.N. Alternative Bezeichnungen sind „Gelegenheitsanlass" oder „unwesentliche Mitursache“, a.a.O.

398 BSG, Urt. v. 27.11.1980 - 8a RU 12/79 -, juris Rn. 22. Ricke, in: Körner/Leitherer et al. (Hrsg.), Kasseler Kommentar zum Sozialversicherungsrecht, $\mathbb{} 8$ SGB VII Rn. 27; Keller, in: Hauck/Noftz/ders. (Hrsg.), SGB VII, $\$ 8$ Rn. 298 ff., insb. 301.

399 Köhler, VSSR 2013, S. 47, 49.

400 Zur Betriebsrisikolehre, auf der diese Argumentation beruht, s.u. Kap. 2. C. 
häufiges Beispiel für solche Schadensanlagen sind degenerative Veränderungen der Bandscheibe, die beim Bücken am Arbeitsplatz wesentliche Ursache für einen Bandscheibenvorfall sind. ${ }^{401}$

Für den Fall, dass der Arbeitsunfall einen bereits bestehenden krankhaften Zustand so verschlechtert, dass der Versicherte stirbt, wendet die Rechtsprechung eine besondere Regel an. Der Tod ist demnach nur als Folge des Versicherungsfalles anzusehen, wenn der Versicherte ohne den Arbeitsunfall höchstwahrscheinlich noch länger als ein Jahr gelebt hätte. ${ }^{402}$

\section{F. Zwischenergebnis}

Für die hier zentrale Frage nach der Abgrenzung von Arbeits- und Privatunfall hat die dogmatische Analyse des $\$ 8$ Abs. 1 SGB VII ergeben, dass sie maßgeblich auf den Ebenen des sachlichen Zusammenhangs und der Unfallkausalität stattfindet. Für die Subsumtion eines konkreten Unfallgeschehens unter diese Tatbestandsmerkmale kommt es jeweils auf eine wertende Betrachtung ihrer Umstände an. Letztendlich wird die Reichweite der beruflichen respektive der privaten Sphäre dadurch bestimmt, welche Bedeutung der Rechtsanwender vorhandenen Indizien zumisst. Als rechtliche Entscheidung muss die Würdigung dieser konkreten Tatsachen mit den geltenden Normen in Bezug gesetzt werden. ${ }^{403}$ Es ist insbesondere notwendig, die Tatbestandsmerkmale im Rahmen der Auslegung in ihren historischen Kontext einzuordnen und nach ihrem Telos zu fragen. Daraus folgt, dass sich die Beurteilung des Sachverhalts an den Schutzzwecken und Grundprinzipien der Unfallversicherung orientieren muss. Die in die Entstehung der Norm einfließenden Wertungen bilden die Interpretationsfolien, vor deren Hintergrund die Einordnung von Unfällen in Arbeitsund Privatunfälle stattzufinden hat. ${ }^{404} \mathrm{Im}$ nächsten Kapitel werden daher die für diese Wertentscheidungen ausschlaggebenden historischen Gegebenheiten, Zielsetzungen und Grundprinzipien der Unfallversicherung untersucht. Ihre Einordnung in den Kontext der tatsächlichen Arbeitswirk-

401 Dazu Balke, SVR 2009, S. 58, 58; Marschner, in: Rolfs/Giesen et al. (Hrsg.), BeckOK Sozialrecht, $\mathbb{\$} 8$ SGB VII Rn. 35 .

402 BSG, Urt. v. 27.10.1987, E 62, 220.

403 Jørgensen, Recht und Gesellschaft, S. 9.

404 Spellbrink, NZS 2016, S. 527, 527. So erklärt sich auch, dass bereits für die Darstellung der Reichweite des Unfallversicherungsschutzes immer wieder auf die Grundprinzipien der Unfallversicherung verwiesen werden musste. 
Kapitel 1: Die Dogmatik des Arbeitsunfalls der Beschäftigten de lege lata

lichkeit ermöglicht sodann ihre Anwendung auf entgrenzte Arbeitsverhältnisse in den Kapiteln drei und vier. 


\section{Kapitel 2: Grundprinzipien der Unfallversicherung vor dem Hintergrund gesellschaftlichen Wandels}

\section{A. Relevanz für die Abgrenzung von Arbeits- und Privatunfall}

Aus dem ersten Kapitel ergibt sich für die Abgrenzung von Arbeits- und Privatunfall die Notwendigkeit, Wertentscheidungen zu treffen, die auf der Auslegung des $₫ 8$ Abs. 1 SGB VII basieren. Das vorliegende Kapitel eruiert daher die für diese Auslegung maßgeblichen Faktoren in vier Abschnitten.

Gegenstand der Auslegung ist nach allgemeiner Ansicht der in der konkreten Norm zum Ausdruck kommende objektivierte Wille des Gesetzgebers, so wie er sich aus dem Wortlaut der Gesetzesbestimmung in ihrem Sinnzusammenhang ergibt. ${ }^{405}$ Dabei ist die Entstehungsgeschichte der Norm als Hilfsmittel zum Verständnis der mit ihr verfolgten Ziele heranzuziehen. Um dies zu ermöglichen, wird im Folgenden in einem ersten Schritt die Lebenswirklichkeit zur Zeit der Einführung der Unfallversicherung untersucht (B.). Die Darstellung des sozialpolitischen Anstoßes für die Einführung der Unfallversicherung und die Begründung ihrer rechtstechnischen Ausgestaltung ermöglichen es, bei der Wertentscheidung zur Reichweite des Schutzbereichs auf die sich aus der genetischen und historischen Betrachtung ergebenden teleologischen Aspekte zu achten. Aus diesen Schutzzwecken ergeben sich die in der Unfallversicherung wirkenden Zurechnungsprinzipien der Betriebsrisikolehre, der Haftungsersetzung, des sozialen Schutzes und der Fürsorgepflicht, welche die Basis jeder Argumentation im Rahmen der Schutzbereichseröffnung darstellen und als solche sowohl in ihrem historischen als auch in ihrem aktuellen Kontext untersucht werden (C.). Das Verständnis dieser Grundgedanken und der daraus folgenden Zurechnungsprinzipien ist unbedingt notwendig, um Auslegungsfragen systemgerecht beantworten zu können. ${ }^{406}$

405 Vgl. nur Rüthers/Fischer/Birk, Rechtstheorie, Rn. 725 f.; Wank, Die Auslegung von Gesetzen, S.37; Zippelius, Juristische Methodenlehre, S. 17; Schweigler, Das Recht auf Anhörung eines bestimmten Arztes, S. 30 ff. m.w.N.

406 Gitter/Nunius, in: Schulin (Hrsg.), Handbuch des Sozialversicherungsrechts Band 2: Unfallversicherungsrecht, S. 98, 98, Rn. 4; Schweigler, Das Recht auf Anhörung eines bestimmten Arztes, S. 32 . 
Bei der Anwendung einer auszulegenden Norm darf nicht bei einer rückwärtsgerichteten Betrachtung stehengeblieben werden. ${ }^{407}$ Vielmehr ist auf ihren Schutzzweck zum Zeitpunkt der Auslegung abzustellen. So wird berücksichtigt, dass die sozialen und gesellschaftlich-politischen Verhältnisse, auf welche das Gesetz wirken soll, mit zunehmendem zeitlichem Abstand zwischen dem Gesetzesbefehl und seiner Anwendung Veränderungen unterliegen. ${ }^{408}$ Die Ergebnisse des entstehungsgeschichtlichen Kontextes sind daher zu modifizieren, wenn sich die tatsächlichen Rahmenbedingungen der Anwendung der Norm verändert haben. ${ }^{409}$ In Abschnitt D. werden daher die Aspekte der modernen Arbeitswelt dargelegt, die eine solche relevante Modifikation der erlebten Welt bewirkt haben. Neben einem Überblick über die heutige Ausgestaltung der Unfallversicherung (D. I.) werden dabei die Aspekte Digitalisierung, Tertiarisierung und subjektive Modernisierung der Arbeitsverhältnisse beleuchtet, da diese maßgeblich für den Anpassungsbedarf der Unfallversicherung verantwortlich sind (D. II. und III.) Auf das Phänomen der entgrenzten Arbeit, welches die hier konkret im Mittelpunkt stehende Veränderung auslöst, wird im Anschluss speziell eingegangen (E.).

\section{B. Entstehung und Ausgestaltung der gesetzlichen Unfallversicherung}

I. Sozialpolitischer Anstoß für die Einführung der gesetzlichen Unfallversicherung: Risikoverteilung vor 1884

Zum Verständnis der in die gesetzliche Unfallversicherung einfließenden Wertungen ist es unverzichtbar, sich die gesellschaftliche Gesamtsituation in der zweiten Hälfte des 19. Jahrhunderts vor Augen zu führen. ${ }^{410}$ Sie war

407 Std. Rspr., vgl. BVerfG, Urt. v. 17.5.1960, E 11, 126, 130; v. 11.6.1980, E 54, 277, 298 f.; v. 19.9.2007, E 119, 247, 274.

408 BVerfG, Urt. v. 14.2.1973, E 34, 269, 288; Schweigler, Das Recht auf Anhörung eines bestimmten Arztes, S. 32 .

409 Rüthers/Fischer/Birk, Rechtstheorie, Rn. 795; Schweigler, a.a.O., S. 33.

410 Kohte, ArbuR 31 (1983), S. 229, 229; zur Gesamtsituation und zu ihrer Auswirkung auf die sogenannte „Arbeitergesetzgebung“ des Deutschen Kaiserreichs insgesamt vgl. Hänlein/Tennstedt, in: Maydell/Ruland et al. (Hrsg.), Sozialrechtshandbuch, S. 67, 67 ff.; Breuer, in: Schulin (Hrsg.), Handbuch des Sozialversicherungsrechts Band 2: Unfallversicherungsrecht, S. 1, 2, Rn. 1 ff. Da es dazu auch in Bezug auf die Unfallversicherung bereits eine Vielfalt an vertiefender Literatur gibt, kann und soll hier ein kurzer Abriss der sozialen Lage genügen. Ausführlich zum geschichtlichen Hintergrund z.B. Baldschun, Solidarität und sozia- 
der sozialpolitische Impuls für die Entstehung der gesetzlichen Unfallversicherung. ${ }^{411}$ Ausgangspunkt ist die auf die erste Hälfte des 19. Jahrhunderts zu datierende industrielle Revolution in Deutschland, ${ }^{412}$ die zum Entstehen der unter dem Schlagwort "Soziale Frage“ bekannt gewordenen Missstände führte. Im hiesigen Kontext interessiert vor allem die wirtschaftliche Notlage der sozial schwachen Gruppe der Arbeiterschaft, die infolge der Landflucht durch ein Überangebot von Arbeitskräften geprägt war und dadurch bedingt ein sehr niedriges Lohnniveau hinnehmen musste, welches die Menschen zu Nacht- und Sonntagsarbeit und überlangen Arbeitsschichten von 12 bis zu 18 Stunden zwang.

Zudem entwickelte sich erstmalig eine klare Ausdifferenzierung zwischen Arbeits- und Privatsphäre. ${ }^{413}$ In der bis dahin agrarisch und handwerklich geprägten Bevölkerung waren Arbeit und Privatleben zum größten Teil miteinander verschmolzen und fanden unter einem Dach statt. ${ }^{414}$ Landwirtschaftliche Arbeiter und Handwerksgesellen der vorindustriellen Zeit waren in den sozialen Verband der arbeitgebenden Familie eingegliedert gewesen, welche Kost und Logis am eigenen Gut und im Falle von Unfälle und Krankheiten Fürsorge gewährte. ${ }^{415}$ Nun, da die Erwerbsarbeit in Fabriken an Maschinen und Fließbändern geleistet wurde, war sie an den Betriebsort gekoppelt und fand nur dort statt. Wohn- und Arbeitsplatz wurden damit getrennt. Auch zeitlich gab die Produktion den Ablauf des Arbeitstages vor. Arbeitszeiten waren genau festgelegt und standardisiert, sodass auch insoweit eine klare Konturierung zwischen Arbeitszeit und

les Schutzprinzip in der gesetzlichen Unfallversicherung, S. $20 \mathrm{ff}$; Gitter, in: Hauptverband der gewerblichen Berufsgenossenschaften e.v./Bundesverband der landwirtschaftlichen Berufsgenossenschaften e.V. et al. (Hrsg.), 100 Jahre gesetzliche Unfallversicherung, S. 22, 22 ff.; Reiff, Die Lösung des ursächlichen Zusammenhangs zwischen Unfall und Tätigkeit in der Unfallversicherung der RVO, S. 6 ff.; Wickenhagen, Geschichte der gewerblichen Unfallversicherung, S. 5 ff.; Wicke, Soziale Sicherung bei Arbeitsunfällen und Berufskrankheiten, S. $71 \mathrm{ff}$.

411 Rohrbeck, in: Bogs (Hrsg.), Gegenwartsfragen sozialer Versicherung, S. 17, 17.

412 Die soziale Lage, die zur Einführung eines Unfallsicherungssystems führte, war in vielen Ländern vergleichbar. Siehe z.B. für eine ausführliche Analyse der Gründe der Einführung der workers' compensation laws in den USA Fishback/ Kantor, A Prelude to the Welfare State, 2000, S. $28 \mathrm{ff}$.

413 Kleemann, Zur Re-Integration von Arbeit und Leben in Teleheimarbeit, in: Gottschall/Voß (Hrsg.), Entgrenzung von Arbeit und Leben, S. 59, 61, 66.

414 Gitter, SGb 1993, S. 297, 297; Däubler, SR 2014, S. 45, 46; Hillmann, Wörterbuch der Soziologie, S. 12. Zu den Arbeitsbedingungen während des Wandels von der Agrar- zur Industriegesellschaft überblicksartig auch Hromadka, Zukunft des Arbeitsrechts, NZA 1998, S. 1, $1 \mathrm{f}$.

415 Gitter, SGb 1993, S. 297, 297. 
Freizeit entstand. Die industrielle Arbeitskultur bedeutete mithin eine deutliche räumlich-zeitliche Abgrenzung von Erwerbsarbeit und Privatleben. ${ }^{416} \mathrm{Da}$ der Arbeiter nun nicht mehr in einem familiären Solidarverband mit seinem Arbeitgeber lebte, erhielt er auch nicht mehr dessen Schutz in Unglücksfällen. ${ }^{417}$

Zur Existenzerhaltung einer Familie war es nötig, dass mehrere Familienmitglieder arbeiteten; auf Gesundheits- oder Unfallschutz wurde wegen des Überschusses an billigen Arbeitskräften, die wegen ihrer mangelnden Spezialisierung leicht austauschbar waren, kein Wert gelegt. ${ }^{418}$ Unfälle während der Arbeitsausübung kamen immer häufiger vor, da die neu entwickelten industriellen Maschinen bisher unbekannte Verletzungsrisiken bargen und es weder Unfallverhütungs- noch Schutzvorschriften bei gefährdender Tätigkeit gab. ${ }^{419}$ Die Tätigkeiten in der Fabrik waren meist weitaus risikobehafteter als die Verrichtung von Arbeit im landwirtschaftlichen und handwerklichen Betrieb. ${ }^{420}$ Verlor ein Werktätiger bei einem solchen Arbeitsunfall die Fähigkeit zu arbeiten, fiel er als Arbeitskraft für das Unternehmen aus, erhielt aber weder eine Entgeltfortzahlung noch andere soziale Leistungen. Damit verlor er nicht nur seine eigene Existenzgrundlage, sondern auch die Möglichkeit, seine Familie zu ernähren. Denn ausschließliche Haftungsgrundlage im damaligen Schadensersatzrecht war das Verschuldensprinzip. ${ }^{421}$ Nur wenn der Arbeitgeber den Unfall in seiner Person selbst verschuldet hatte und der Arbeitnehmer dies beweisen konn-

416 Diese sog. „fordistisch-tayloristische Normalarbeit“ bildet die historische Referenzfolie für die Diskussion von Entgrenzungsprozessen, vgl. ausführlich Kratzer/Sauer, Entgrenzung von Arbeit, in Gottschall/Voß (Hrsg.), Entgrenzung von Arbeit und Leben, S. 87, 94 ff. Zum Ganzen Deutschmann, Der Weg zum Normalarbeitstag, passim; Ritter/Tenfelde, Arbeiter im Deutschen Kaiserreich, S. $263 \mathrm{ff}$.

417 Gitter, SGb 1993, S. 297, 297.

418 Vgl. zum Ganzen Stolleis, Geschichte des Sozialrechts in Deutschland, S. 23 ff., 58 ff.; Frerich/Frey, Handbuch der Geschichte der Sozialpolitik in Deutschland Band 1: Von der vorindustriellen Zeit bis zum Ende des Dritten Reiches, S. 85 ff.; Tennstedt, Sozialgeschichte der Sozialpolitik in Deutschland, S. 51 ff.; Wehler, Deutsche Gesellschaftsgeschichte: Dritter Band, S. $772 \mathrm{ff}$.

419 Tennstedt/Winter, ZSR 1993, S. 362, 366; Tennstedt, in: Hänlein/Roßnagel (Hrsg.), Wirtschaftsverfassung in Deutschland und Europa, S. 483, 483; mit Zahlenmaterial Wickenhagen, Geschichte der gewerblichen Unfallversicherung, S. $21 \mathrm{f}$.

420 Gitter, SGb 1993, S. 297, 297.

421 Zacharias, Wesen und Rechtsnatur der Ansprüche der Versicherten aus der gesetzlichen Unfallversicherung, S. 4; Rümelin, Der Zufall im Recht., S. $6 \mathrm{ff}$. 
te, standen dem Geschädigten Ansprüche gegen den Arbeitgeber zu. ${ }^{422}$ Die häufigen Fälle, in denen etwa eine Maschine versagte oder auf sonstige Weise ohne das Verschulden eines Einzelnen ein Unfall geschah oder in denen neben dem Verschulden des Arbeitgebers noch andere Faktoren eine Rolle gespielt hatten, waren somit nicht erfasst.

Der Fortschritt der Industrie hatte folglich Verhältnisse geschaffen, denen die bestehenden Regelungen des Schadensersatzrechts nicht mehr gerecht wurden. ${ }^{423} \mathrm{Im}$ Jahr 1871 wurde daher versucht, dieser Situation durch die Einführung des Reichshaftpflichtgesetzes ${ }^{424}$ Abhilfe zu verschaffen. Darin wurden für Unternehmer einiger "gefahrgeneigter" Branchen der strenge Verschuldensgrundsatz ausgehebelt und die Verantwortung des Unternehmers auf das Verschulden seiner Repräsentanten und höhergestellten Angestellten ausgedehnt. Einige Arbeitnehmer hatten nun die Möglichkeit, im Rahmen der Bestimmungen des Reichshaftpflichtgesetzes gegen den Unternehmer zu klagen. Dazu mussten sie nachweisen, dass den Unternehmer das Verschulden an der Verletzung traf. ${ }^{425}$ Vor diesem Unterfangen schreckten viele aus Angst, gekündigt zu werden, zurück. Durch die Anstrengung eines solchen Prozesses setzte sich der Beschäftigte dem reellen Risiko aus, seinen Arbeitsplatz und eventuelle freiwillige Zuwendungen seines Arbeitgebers zu verlieren. ${ }^{426}$

Selbstverschuldete sowie durch Zufall oder das Verschulden anderer Personen (etwa anderer Arbeitnehmer) ausgelöste Unfälle führten noch immer nicht zu Entschädigungsansprüchen gegen den Arbeitgeber. ${ }^{427}$ Selbst wenn theoretisch ein deliktsrechtlicher Anspruch gegen den Arbeitgeber bestand, war es für den Arbeitnehmer beinahe unmöglich, diesen gerichtlich durchzusetzen. Zum einen konnte der „typische“ Arbeiter sich die

422 Tennstedt/Winter, ZSR 1993, S.362, 367; Schmitt, in: Maydell/Ruland et al. (Hrsg.), Sozialrechtshandbuch, S. 802, 803 Rn. 2.

423 Kohte, ArbuR 31 (1983), S. 229, 231 f. Ausführlich zum historischen Prozess des Umdenkens sowie zu den moralisch-philosophischen Hintergründen Stegner, Zwischen Souveränität und Ökonomie, S. 62 ff.

424 Gesetz v. 7.6.1871 betreffend die Verbindlichkeit zum Schadensersatz für die bei dem Betriebe von Eisenbahnen, Bergwerken usw. herbeigeführten Tödtungen und Körperverletzungen, RGBl. 1871, S. 207.

425 Baldschun, Solidarität und soziales Schutzprinzip in der gesetzlichen Unfallversicherung, S. 21.

426 Ebd., S. 20.

427 Ausführlich Bernstein, Schadensausgleich bei Arbeitsunfällen, S. 42 ff. 
Prozesskosten nicht leisten; ${ }^{428}$ zum anderen lag die Beweislast für das Verschulden des Unternehmers beim Arbeitnehmer. ${ }^{429}$ Beweisschwierigkeiten und die existenzielle Bedeutung des Ausgangs eines solchen Gerichtsstreits für den Geschädigten resultierten in einer erheblichen Arbeitsbelastung für die Gerichte. ${ }^{430} \mathrm{Zu}$ guter Letzt war die Situation auch für Unternehmer nicht optimal. Bei Unfällen größeren Umfangs mit vielen Verletzten mussten sie immerhin fürchten, dass hohe Forderungen zahlreicher Inhaber deliktischer Ansprüche sie finanziell überlasteten. 431

Das Reichshaftpflichtgesetz genügte somit nicht, um die rechtliche Lage signifikant zu verbessern. Bevor die Verteilung des Risikos eines Gesundheitsschadens im Beruf durch die Einführung der gesetzlichen Unfallversicherung im Jahr 1884 gesetzlich geregelt wurde, trug der Beschäftigte das Risiko faktisch selbst und allein. Mit dem Argument, der Arbeiter habe seinen Arbeitsvertrag freiwillig geschlossen, sich also der durch die Arbeit entstehenden Risiken bewusst ausgesetzt, und erhielte als Gegenleistung den Lohn, gab sich eine Mehrheit der zeitgenössischen Juristen zunächst mit diesen Gegebenheiten zufrieden. ${ }^{432}$

Vor dem Hintergrund der geschilderten Situation wurde die von Reichskanzler Otto von Bismarck angestoßene Diskussion um die Einführung einer gesetzlichen Unfallversicherung geführt. Ziel war es, das Risiko eines Arbeitsunfalls auf mehrere Schultern zu verteilen. Es sollte ein umfassender und insolvenzfester Schutz der bisher schutzlosen Beschäftigten bei Arbeitsunfällen bewirkt werden; ${ }^{433}$ das Risiko, durch Gesundheitsschäden in der Erwerbsfähigkeit beeinträchtigt zu sein, sollte aufgefangen werden.

428 Bulla, Der Dienst- und Arbeitsunfall als Institut des Allgemeinen Verwaltungsrechts, S. 11, 13 mit Hinweis darauf, dass es zwar ein Armenrechtsgesuch gab, das aber wegen Verfahrensmängeln meist nicht funktionierte.

429 Reichshaftpflichtgesetz v. 7.6.1871, RGB1. S. 207; Gitter, in: Hauptverband der gewerblichen Berufsgenossenschaften e.v./Bundesverband der landwirtschaftlichen Berufsgenossenschaften e.V. et al. (Hrsg.), 100 Jahre gesetzliche Unfallversicherung, S. 22, 22. Detailliert zu den damaligen Anforderungen an die Beweisführung Wicke, Soziale Sicherung bei Arbeitsunfällen und Berufskrankheiten, S. $78 \mathrm{f}$.

430 Hippel, in: Fleming/Hellner et al. (Hrsg.), Haftungsersetzung durch Versicherungsschutz, S. 40, 43.

431 Baldschun, Solidarität und soziales Schutzprinzip in der gesetzlichen Unfallversicherung, S. 20.

432 Andersen, in: Machtan (Hrsg.), Bismarcks Sozialstaat, S. 207, 211; Endemann, Vierteljahresschrift für Volkswirtschaft und Kulturgeschichte 1872, $58 \mathrm{f}$.

433 Becker, Soziales Entschädigungsrecht, S. 38 f. 
Zudem wünschte Bismarck, ein „soziales Band“ zwischen Arbeitnehmer und Arbeitgeber zu knüpfen.

\section{Rechtstechnische Ausgestaltung des Unfallversicherungsschutzes}

Zur erwünschten Absicherung der Beschäftigten standen bei den Überlegungen zur Einführung einer gesetzlichen Ausgestaltung Ende des 19. Jahrhunderts zwei Modelle im Raum: ${ }^{434}$ die Ausgestaltung einer gesetzlichen, sozialen Versicherung zum Zwecke der Absicherung des Arbeitsunfallrisikos sowie alternativ eine Verschärfung der zivilrechtlichen Haftung des Arbeitgebers. Letztere Überlegung sah vor, das bereits bestehende Reichshaftpflichtgesetz zu erweitern, indem die Haftung des Arbeitgebers auf verschuldensunabhängige Tatbestände ausgedehnt und/oder Beweisregeln zugunsten der Verletzten hätten eingeführt werden sollen. ${ }^{435}$ Gegen eine solche verschärfte zivilrechtliche Haftung hätten sich die Arbeitgeber dann durch den Abschluss einer privatrechtlichen Versicherung absichern können. Die juristische Alternative, statt der Einführung einer Versicherung die zivilrechtliche Haftung des Arbeitgebers zu verschärfen, lehnte Bismarck mit dem Argument ab, dass sie die beiden Parteien eines Arbeitsvertrags in eine „feindliche Stellung“ zueinander hätten treten lassen.436 Denn wie zuvor hätten auch nach der Einführung einer Haftungsverschärfung Arbeitnehmer und Arbeitgeber nach Eintritt eines Arbeitsunfalls gerichtlich gegeneinander vorgehen müssen. Der Reichskanzler erwartete sich von der Einführung einer Unfallsicherung in Form einer Sozialversicherung nicht zuletzt eine Befriedung der Betriebe und damit einen Beitrag zum sozialen Frieden in der Gesellschaft insgesamt. ${ }^{437}$ Es setzte sich

434 Vgl. nur Tennstedt, in: Hänlein/Roßnagel (Hrsg.), Wirtschaftsverfassung in Deutschland und Europa, S. 483, 487 f.; Tennstedt/Winter, ZSR 1993, S. 362, 378; Wicke, Soziale Sicherung bei Arbeitsunfällen und Berufskrankheiten, S. $31 \mathrm{ff}$.

435 Diese Alternative wurde z.B. in Großbritannien (vgl. Ricke, ZESAR 2008, S. 13, 13 ff.) und in Tschechien (vgl. Wicke, Soziale Sicherung bei Arbeitsunfällen und Berufskrankheiten, S. 156 ff.) gewählt; zu Sicherungssystemen im Ausland vgl. Gitter, Schadensausgleich im Arbeitsunfallrecht, S. 82 ff. sowie die Literaturhinweise bei Ricke, in Körner/Leitherer et al. (Hrsg.), Kasseler Kommentar zum Sozialversicherungsrecht, Vor $\$ 1$ SGB VII Rn. 11.

436 Gitter, Schadensausgleich im Arbeitsunfallrecht, S. $26 \mathrm{ff}$.

437 Vgl. statt vieler nur Krasney, ArbuR 49 (2001), S. 423, 423 ff. Bismarck verfolgte darüber hinaus noch andere politische Ziele, wie bei Kaltenborn, JZ 1998, S. 770, 773 nachzulesen ist. 
daher die Variante einer öffentlich-rechtlich ausgestalteten Pflichtversicherung $^{438}$ der Arbeitnehmer auf Rechnung der Arbeitgeber durch. ${ }^{439}$

Als zweiter Zweig der Sozialversicherung wurde die gesetzliche Unfallversicherung durch das Unfallversicherungsgesetz vom 6. Juli $1884^{440}$ in Deutschland eingeführt. In seinem $\mathbb{1} 1$ normierte es einen vom Verschulden des Unternehmers unabhängigen Entschädigungsanspruch für in bestimmten gewerblichen Unternehmen beschäftigte Arbeiter, die einen „Betriebsunfall“ erlitten: „Alle in Bergwerken, Salinen, Aufbereitungsanstalten, Steinbrüchen, Gräbereien (Gruben), auf Werften und Bauhöfen, sowie in Fabriken und Hüttenwerken beschäftigten Arbeiter und Betriebsbeamten, letztere sofern ihr Jahresarbeitsverdienst an Lohn oder Gehalt zweitausend Mark nicht übersteigt, werden gegen die Folgen der bei dem Betriebe sich ereignenden Unfälle nach Maßgabe der Bestimmungen dieses Gesetzes versichert."

Die versicherungspflichtigen Unternehmer hatten die Finanzierung alleine zu stemmen ( $\mathbb{1 0}$ UVG). Dazu wurden die Unternehmen in Gefahrenklassen eingeteilt; sie zahlten diesen entsprechend unterschiedliche Beitragssätze. Von einem Reichszuschuss oder Arbeitnehmerbeitrag wurde abgesehen. ${ }^{441}$ Als Träger der Unfallversicherung wurden statt einer zentralen Trägerorganisation in Form einer Reichsversicherungsanstalt Berufsgenossenschaften eingerichtet. Diese sind Zwangszusammenschlüsse der einzelnen Unternehmen einer Branche, die sich selbst verwalten. Dadurch erhielten die Beschäftigten im Falle eines Arbeitsunfalls einen sicheren, durchsetzungsfähigen Anspruch gegen eine stets leistungsfähige öffentlichrechtliche Solidargemeinschaft. ${ }^{442} \mathrm{Im}$ Gegenzug wurden die Unternehmer

438 Die Auseinandersetzung mit der lange Zeit geführten Diskussion um die Rechtsnatur der gesetzlichen Unfallversicherung - Daseinsvorsorge, Fürsorge oder, richtigerweise, Versicherung - würde den Rahmen dieser Arbeit sprengen und ist für den Verlauf der Untersuchung entbehrlich. Folgende Literaturhinweise zu dieser Problematik mögen genügen: Rohrbeck, in: Bogs (Hrsg.), Gegenwartsfragen sozialer Versicherung, S. 17, 17 ff.; Bulla, SGb 2007, S. 653, 659; Zacharias, Wesen und Rechtsnatur der Ansprüche der Versicherten aus der gesetzlichen Unfallversicherung, jeweils m.w.N.

439 Baldschun, Solidarität und soziales Schutzprinzip in der gesetzlichen Unfallversicherung, S. $20 \mathrm{f}$.

440 Unfallversicherungsgesetz v. 6.7.1884, bekanntgemacht am 9.7.1884, in Kraft getreten zum 1.10.1884, RGBl. 1884 Nr. 19, S. 69-111. Zum Entstehungsprozess des Gesetzes über all seine Entwürfe hinweg eingehend Tennstedt/Winter, ZSR 1993, S. 362.

441 Gitter, Schadensausgleich im Arbeitsunfallrecht, S. $46 \mathrm{ff}$.

442 Kaltenborn, JZ 1998, S. 770, $771 \mathrm{f}$. 
durch $\$ 95$ UVG von ihrer individuellen zivilrechtlichen Haftung befreit, sofern weder der Unternehmer selbst noch seine Bevollmächtigten oder Repräsentanten den Unfall vorsätzlich herbeigeführt hatten. Auf ein Selbst- oder Mitverschulden des Verletzten oder des Unternehmers kam es nicht an. Die gesetzliche Unfallversicherung wurde das erste öffentlichrechtliche Entschädigungssystem, das auf dem Gefährdungsgedanken beruhte. ${ }^{443}$ Diese Übertragung des Schadensrisikos musste nicht nur sozialpolitisch, sondern auch rechtlich begründbar sein; ohne Beziehung zwischen Finanzierung und Leistung findet ein Sozialsystem bei seinen Adressaten keine Akzeptanz. ${ }^{444}$ Aufgrund der deutlich sicht- und fühlbaren Unterscheidung zwischen Arbeits- und Privatsphäre war es naheliegend, den Schutz der Unfallversicherung von der Zugehörigkeit eines schädigenden Ereignisses zu der beruflichen Sphäre abhängig zu machen.

Die genannten Erwägungen brachten die zwei als Grundprinzipien der Unfallversicherung bezeichneten Zurechnungsprinzipien hervor, die Prinzip der Haftungsersetzung und soziales Schutzprinzip genannt werden. ${ }^{445}$ Als Basis aller Auslegung von Zuordnungsfragen und Fortentwicklung der Unfallversicherung ist ihre genaue Untersuchung unumgänglich. Ihre Her-

443 Bulla, SGb 2007, S. 653, 655 mit Aufzählung der heutigen hinzugekommenen Entsprechungen, etwa im Atom- oder Arzneimittelhaftungsrecht.

444 von Maydell, NJW 1992, S. 2195, 2197. Die Diskussion um die Rechtfertigung der Risikotragung der Arbeitgeber ist nicht allein in der Unfallversicherung relevant. Auch bei der Frage der Haftung der Arbeitgeber für das Fehlverhalten ihrer Arbeitnehmer ist sie virulent (dazu z.B. Schelp, Die Haftungsbelastung des Arbeitnehmers bei Schädigung Dritter, passim; Diem, Die theoretischen Grundlagen der Beschränkung der Haftung des Arbeitnehmers gegenüber dem Arbeitgeber bei schadensgeneigter Arbeit, passim; Reinhardt, Die dogmatische Begründung der Haftungsbeschränkung des Arbeitnehmers, passim; Kohte, Arbeitnehmerhaftung und Arbeitgeberrisiko, passim). Ein Blick auf Lösungsansätze, die zu diesem Problemkreis entwickelt wurden, ist daher lohnend, vgl. ebenso Kohte, ArbuR 31 (1983), S. 229, 229 ff., 236; Frey, ArbuR 7 (1959), S. 193, 203 f. Auch bei der Frage nach den Arbeitgeberbeiträgen in der Sozialversicherung allgemein wird die Rechtfertigung der Risikotragung diskutiert, vgl. z.B. Vitú, Die verfassungsrechtlichen Grenzen der Abgabenbelastung des Arbeitgebers, passim; Weingarten, Die Beitragspflicht der Arbeitgeber und Arbeitnehmer zur sozialen Pflegeversicherung unter grundrechtlichem Blickwinkel, passim; Friauf, DB 1991, S. 1773 ff.; Isensee, Umverteilung durch Sozialversicherungsbeiträge, passim; Zacher (Hrsg.), Die Rolle des Beitrags in der sozialen Sicherung, passim.

445 Vgl. nur Ricke, in: DGUV (Hrsg.), 125 Jahre gesetzliche Unfallversicherung, S. 18 f.; Gitter, BB 1998, Beilage 6 S. 1, 3; ders./Nunius, in: Schulin (Hrsg.), Handbuch des Sozialversicherungsrechts Band 2, $\mathbb{5} \mathrm{Rn} .28 \mathrm{ff}$. Passender ist es, statt vom Prinzip der Haftungsersetzung vom Prinzip der Betriebsrisikozuweisung zu sprechen, s.u. Kap. 2 C I. 
kunft und Bedeutung sind daher Gegenstand des folgenden Abschnitts (Kap. 2 C).

\section{Grundprinzipien der Risikoverteilung in der Unfallversicherung}

\section{Betriebsrisikolehre und Prinzip der Haftungsersetzung}

\section{Die Zurechnung von Risiken nach Sphären}

Tragendes Argument für die Absicherung der Arbeitsunfälle in der arbeitgeberfinanzierten Unfallversicherung war bei ihrer Einführung die Betriebsrisikolehre. ${ }^{446}$ Die Analyse des Zuordnungsmechanismus des $\$ 8$ Abs. 1 SGB VII in Kapitel 1 zeigte, dass der Gedanke des Unternehmerwagnisses - wie die Betriebsrisikolehre auch bezeichnet wird - in der kontemporären Rechtsprechung und Literatur ein gewichtiges Argument innerhalb der wertenden Auslegung des Arbeitsunfalls darstellt. Die Betriebsrisikolehre ist untrennbar mit dem in der Unfallversicherung gewählten Prinzip der Haftungsersetzung verbunden. Ob die Haftungsersetzung der zivilrechtlichen Haftung der Unternehmer lediglich Mittel zur Umsetzung der Betriebsrisikolehre oder eigenständiger Rechtsgrund ist, ${ }^{447}$ ist, wie die folgenden Ausführungen deutlich machen, letztlich aufgrund ihrer Verwobenheit ineinander nicht entscheidend.

Die Betriebsrisikolehre ist eine Ausprägung der Zurechnung nach Risikosphären, welche als „Risikoprinzip“ im Deliktsrecht entwickelt wurde. ${ }^{448}$ Unter einer Sphäre in diesem Sinne ist der Lebensbereich einer Partei zu verstehen, aus dem heraus Gefahren entstehen oder abgewehrt wer-

446 Inzwischen wird der Begriff der „Betriebsrisikolehre“ zumeist für die hier nicht gemeinte, nunmehr in $\$ 615$ BGB geregelte Frage gebraucht, ob der Arbeitgeber auch dann Lohn zu zahlen hat, wenn der Betrieb aus von ihm unverschuldeten Gründen stillsteht, z.B. Buchner, in: Buchner/Becker, Mutterschutzgesetz und Bundeselterngeld- und Elternzeitgesetz, Rn. 12; Preis, in: Müller-Glöge/Preis et al. (Hrsg.), Erfurter Kommentar zum Arbeitsrecht, BGB $\$ 615$ Rn. 120, 123 ff. Die rechtliche Figur des Betriebsrisikos ist bereits im römischen Recht sowie in den Glossen des Mittelalters nachweisbar, vgl. Söllner, AcP 1967, S. 132, 143, m.w.N. Zum eigenständigen Zurechnungsgrund wurde sie im Unfallversicherungsrecht entwickelt, siehe Kohte, ArbuR 31 (1983), S. 229, 232, sodass der Begriff hier weiterhin genutzt werden sollte.

447 Für Ersteres Ricke, WzS 2017, S.9, 11 f.; Letzteres befürwortend Waltermann, VSSR 2005, S. 103, 107.

448 Kümper, Risikoverteilung im Staatshaftungsrecht, S. $144 \mathrm{ff}$. 
den können. ${ }^{449}$ Der Risiko- oder Sphärengedanke ist ein eigenständiger Zurechnungsgrund. ${ }^{450}$ Er besagt, dass derjenige den Schaden zu tragen hat, von dem man annimmt, dass er besser als der andere eine mögliche Schädigung des Vertragsgegenstandes verhindern kann. ${ }^{451}$ Auf eine tatsächliche Beherrschungsmöglichkeit kommt es dabei nicht an. ${ }^{452}$ Tragend ist der Gedanke, dass der Schaden von einer Seite eher hätte verhindert werden können als von der anderen. ${ }^{453}$ Das Risiko trifft damit diejenige Vertragspartei, die näher am Schaden ist. ${ }^{454}$ Das Risikoprinzip bezweckt dadurch eine möglichst gerechte Verteilung von Lasten in Fällen, in denen es sich nicht um Unrecht, sondern um Unglück handelt. ${ }^{455}$ Diese Zuordnung erscheint so logisch, dass sie als „vorgegebene Struktur, die der Natur der Sache entspringt und gar nicht erfunden, sondern nur gefunden werden konnte ${ }^{\star 456}$ bezeichnet wurde. Tatsächlich lässt sich der Gedanke in der

449 Erman, JZ 1965, S. 657, 657.

450 Gamillscheg/Hanau, Die Haftung des Arbeitnehmers, S. 48.

451 Ebd.; Diem, Die theoretischen Grundlagen der Beschränkung der Haftung des Arbeitnehmers gegenüber dem Arbeitgeber bei schadensgeneigter Arbeit, S. 74. Darin unterscheidet sich die Risikozurechnung nach Sphären von dem aus dem römischen Recht stammenden und im 19. Jahrhundert noch durchgängig angewandten Prinzip casum sentit dominus, vgl. Meder, Schuld, Zufall, Risiko, S. 20, 39-75. Demnach soll die Sphäre, in der das Risiko eintritt, zugleich Risikosphäre des Geschädigten sein. Das Prinzip bezeichnet also die „natürliche“ Schadenszuständigkeit des Rechts- und Sachinhabers und teilt diesem auch das allgemeine soziale Risiko, das die Sache betrifft, zu, Faude, Selbstverantwortung und Solidarverantwortung im Sozialrecht, S. 96; Hübner, Schadenszurechnung nach Risikosphären, S. 57. Es gilt als genereller Zurechnungsgrund noch immer dann, wenn keine ausdrückliche Zurechnung zu anderen Sphären erfolgt, Zacher, ZfS 1983, S. 171, 172; Esser, Grundlagen und Entwicklung der Gefährdungshaftung, S. 69 ff. Dies zeigt sich etwa in der generellen Regel der Beweislastverteilung: Derjenige, der den Schaden hat, braucht ihn nur dann nicht zu tragen, wenn er beweisen kann, dass jemand anders zur Übernahme der Last verpflichtet ist, Prütting, Gegenwartsprobleme der Beweislast, S. 240.

452 Die gesetzliche Unfallversicherung ist damit eine sozialrechtliche Gefährdungshaftung, vgl. dazu Becker, Soziales Entschädigungsrecht, S. 75 f.; Keller, in: Hauck/Noftz, et al. (Hrsg.), SGB VII, Einführung, E 010, S. 3; Fuchs, SGb 1995, S. 421, 422; Triebel, JURA 2007, S. 521, 522.

453 Müller-Erzbach, AcP 106 (1910), S. 309, 413; Diem, Die theoretischen Grundlagen der Beschränkung der Haftung des Arbeitnehmers gegenüber dem Arbeitgeber bei schadensgeneigter Arbeit, S. $74 \mathrm{f}$.

454 Erman, JZ 1965, S. 657, 657.

455 Kümper, Risikoverteilung im Staatshaftungsrecht, S. 15, 144.

456 Gamillscheg/Hanau, Die Haftung des Arbeitnehmers, S. 49; auch in vielen ausländischen Rechtsordnungen sei der Sphärengedanke eigenständiger Zurechnungsgrund, S. 50 Fn. 173. 
Rechtsprechung und in der wissenschaftlichen Literatur immer wieder als eines von mehreren begründenden Elementen von Haftungserleichterungen nachweisen. ${ }^{457}$ Begründet wird die Zurechnung nach dem Sphärenprinzip damit, dass derjenige den Schaden zu tragen hat, der durch diese Last dazu gebracht werden soll, das Entstehen des Schadens überhaupt zu verhindern, weil dieser am ehesten in seiner Vermeidbarkeitssphäre liegt. ${ }^{458}$ Die Verwirklichung des Schadenseintritts wann immer möglich zu vermeiden, ist erhoffter Effekt dieser Theorie; wenn derjenige haftet, der am ehesten in der Lage war, die Realisierung der Gefahr zu verhindern, dann wird dieser alles ihm Mögliche tun, um das Risiko gering zu halten. 459

Die Betriebsrisikolehre konkret bezeichnet den Gedanken, dass Schäden, die infolge betrieblicher Arbeit entstehen, zum Betrieb gehören und damit dem Inhaber des Betriebes - dem Arbeitgeber - zuzuordnen sind. ${ }^{460}$ Diese Sphärentheorie - auch: Lehre der Gefahrtragung - wurde vornehmlich in Literatur und Rechtsprechung zu der Frage der Arbeitnehmerhaftung bei schadensgeneigter Arbeit weiterentwickelt, wird aber auf vielen Gebieten fruchtbar gemacht. ${ }^{461}$ Unter einem Betrieb wird die organisatorische Einheit verstanden, innerhalb derer bestimmte arbeitstechnische Ziele fortgesetzt verfolgt werden. ${ }^{462} \mathrm{Im}$ alltäglichen Sprachgebrauch ist damit vor allem der Ort, an dem die Beschäftigten zusammen arbeiten, gemeint. ${ }^{463}$ Derjenige, der die für diese Zusammenarbeit notwendigen Arbeitsmittel, also Material, Räume, Maschinen etc., bereitstellt und durch seine Weisungen die Arbeitsleistung nach Zeit, Ort und Modus bestimmt, muss nach der Betriebsrisikolehre für die Fehler und Störungen in diesem Bereich einstehen und trägt das in diesem Bereich entstehende Risiko. ${ }^{464}$ Dies liegt an der unterschiedlichen wirtschaftlichen und sozialen Stellung von Arbeitgeber und Arbeitnehmer. ${ }^{465}$ Auch ökonomisch wurde argumentiert, dass Schäden, die ein Unternehmen seiner Umgebung zufügt, zu des-

457 Dargestellt bei Gamillscheg/Hanau, Die Haftung des Arbeitnehmers, S. 50.

458 Diem, Die theoretischen Grundlagen der Beschränkung der Haftung des Arbeitnehmers gegenüber dem Arbeitgeber bei schadensgeneigter Arbeit, S. 74.

459 Müller-Erzbach, AcP 106 (1910), S. 309, 414; zum Präventionsprinzip in der Haftung vgl. instruktiv Marton, AcP 162 (1963), S. 1, 45 ff.

460 Ebenso für den Bereich der Arbeitnehmerhaftung Kohte, Arbeitnehmerhaftung und Arbeitgeberrisiko, S. 226.

461 Söllner, AcP 167 (1967), S. 132, 141, Fn. 39.

462 Vgl. Giesen/Kersten, Arbeit 4.0, S. 125 m.w.N.

463 Ebd. m.w.N.

464 Söllner, AcP 167 (1967), S. 132, 143.

465 Jorns, Das Betriebsrisiko, S. $47 \mathrm{ff}$. 
sen Betriebskosten hinzuzurechnen sind; alles andere wäre eine Quasi-Subvention des Unternehmens auf Kosten der Geschädigten, die durch eine reine Geltung des Verschuldensprinzips nicht ausreichend geschützt wären. ${ }^{466} \mathrm{Im}$ entscheidenden Entwurf des Unfallversicherungsgesetzes von 1884 wurde dies so formuliert: „Wie dem Betriebsunternehmer die an dem Anlage- und Betriebskapital entstehenden Schäden und Verluste zur Last fallen, so soll derselbe auch die Verluste an persönlicher Arbeitskraft, welche durch die seinem Industriezweig eigentümlichen Gefahren veranlaßt wurden, tragen und für beide in dem Gesamtertrage des Unternehmens Deckung finden."467

Diese Idee stützte sich in der Diskussion zur Einführung der gesetzlichen Unfallversicherung im Wesentlichen auf zwei Wertungsaspekte, nämlich zum einen auf den Gedanken, dass der Arbeitgeber die Herrschaftsmacht über die beruflichen Sphäre des Arbeitnehmers hat (Beherrschbarkeitsargument); zum anderen auf den Aspekt, dass der Arbeitgeber, da er die Gewinnchancen des Betriebes hat, auch das Risiko des Betriebes tragen muss (Gewinnerzielungsargument). ${ }^{468}$ Beide Wertungsaspekte werden im Folgenden näher dargestellt.

\section{Beherrschbarkeitsargument}

Dominierender Rechtsgrund für die alleinige Finanzierung der Unfallversicherung durch die Arbeitgeber war bei der Einführung der gesetzlichen Unfallversicherung der Gedanke, dass die berufliche Sphäre eines Beschäftigten von seinem Arbeitgeber beherrscht wird. Der Arbeitnehmer wird schon definitionsgemäß in den vom Arbeitgeber organisierten und gesteuerten Arbeitsprozess integriert. ${ }^{469}$ Er soll dabei den arbeitstechnischen

466 Mataja, Das Recht des Schadenersatzes vom Standpunkte der Nationalökonomie, S. 28, 57; Marton, AcP 162 (1963), S. 1, 48 f.

467 Zitiert nach Kohte, ArbuR 31 (1983), S. 229, 232. Die Tatsache, dass die Eröffnung des Betriebes den Arbeitnehmern durch die Schaffung ihrer Arbeitsplätze zum Vorteil gereicht (vgl. Eich, NZA 1984, S. 65, 67), ändert nichts daran, dass der Unternehmer auch die mit dem Betrieb verbundenen Risiken geschaffen hat.

468 Diese beiden Aspekte finden sich auch in der Rechtsprechung des BAG zur Arbeitnehmerhaftung, vgl. Kohte, Arbeitnehmerhaftung und Arbeitgeberrisiko, S. $226 \mathrm{ff}$.

469 „Arbeitnehmer ist, wer aufgrund eines privatrechtlichen Vertrages zur Arbeit im Dienst eines anderen verpflichtet ist“; vgl. Thüsing, NZA 2015, S. 1478, 1478 m.w.N.; inzwischen ohne inhaltliche Änderung legaldefiniert in $\$ 611 \mathrm{a}$ Abs. 1 
Zweck durch weisungsgebundene Arbeit verwirklichen. Der Arbeitgeber erteilt die Weisungen und legt den Zweck der Arbeit sowie die technische und taktische Betriebsorganisation fest; er gestaltet die gesamten Arbeitsbedingungen des Arbeitnehmers. Das Umfeld, in dem sich ein Arbeitsunfall zutragen kann, liegt also insoweit unter der Kontrolle des Arbeitgebers.

Da der Arbeitgeber die Betriebsorganisation kontrolliert, hat er es in der Hand, Unfallrisiken seiner Arbeitnehmer zu minimieren. Er kann in Ausübung seiner Organisationsbefugnis Risiken sowohl schaffen als auch vernichten, verringern, erhöhen, beibehalten oder verlagern; es liegt an ihm, Gefahren durch die Veränderung von Arbeitsabläufen, durch die Installation von Schutzvorrichtungen und durch Schulung und Überwachung seiner Mitarbeiter zu mindern. ${ }^{470}$ Der Arbeitnehmer dagegen kann die bestimmenden Faktoren seiner Arbeitsaufgaben und -umgebung weder tatsächlich noch rechtlich beeinflussen. ${ }^{471}$ Die Arbeitsorganisation des Unternehmers bestimmt also im Wesentlichen das Schadensrisiko des Beschäftigten. ${ }^{472} \mathrm{Als}$ der für das Entstehen dieser Risiken Verantwortliche soll der Arbeitgeber deshalb auch die aus ihnen erwachsenden Lasten tragen. ${ }^{473}$ Hintergrund dieser Überlegungen war die bereits dargestellte Situation der Arbeiter in Fabriken, die zur Arbeit an fehleranfälligen Maschinen angewiesen waren. Ein Versagen der noch jungen Technik konnte schnell zu schwerwiegenden Verletzungen des Personals führen. Durch das Treffen von Schutzvorkehrungen wie beispielsweise der Einstellung der Produktionsgeschwindigkeit, dem von der Maschine zu haltenden Abstand, der Vermeidung von Überhitzung oder der Vorschrift zum Tragen von Schutzkleidung war der Arbeitgeber unmittelbar in der Lage, die vom Arbeitsort ausgehenden Risiken zu verringern.

Die alleinige Zuweisung des Betriebsrisikos an den Unternehmer ist auch angesichts weiterer Entwicklungen in der Arbeitswelt gerechtfertigt. ${ }^{474}$ Zwar ist der Arbeitgeber bei der Gestaltung des Betriebsgeländes nunmehr an Sicherheitsvorschriften und technische Vorschriften gebun-

BGB. Zur Bestimmung der Arbeitnehmereigenschaft spielt der Gesichtspunkt der Eingliederung in den Betrieb eine wesentliche Rolle, vgl. nur Reiserer, in: Moll (Hrsg.), Münchener Anwaltshandbuch Arbeitsrecht, $\$ 6$ Rn. $12 \mathrm{f}$.

470 BAG, Beschl. v. 27.9.1994, E 78, 56, 57.

471 Baldschun, Solidarität und soziales Schutzprinzip in der gesetzlichen Unfallversicherung, S. 20; Gitter, Schadensausgleich im Arbeitsunfallrecht, S. 18.

472 Zänker, Die Haftung der Unternehmer, der Unternehmensangehörigen und anderer Personen in der gesetzlichen Unfallversicherung, S. $24 \mathrm{f}$.

473 Schelp, Die Haftungsbelastung des Arbeitnehmers bei Schädigung Dritter, S. 38 m.w.N.; Kohte, ArbuR 31 (1983), S. 229, 230.

474 Schelp, Die Haftungsbelastung des Arbeitnehmers bei Schädigung Dritter, S. 41. 
den, die ihn in seiner diesbezüglichen Freiheit beschränken. In Betrieben, in denen ein Betriebsrat besteht, können Arbeitnehmer zudem über diesen an der Gestaltung ihrer unmittelbaren Arbeitsumgebung mitwirken: Über

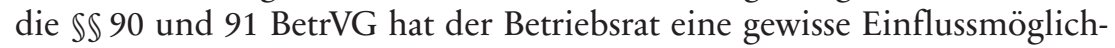
keit, was die Gestaltung der Arbeitsplätze angeht. Diese ist jedoch gering; das Mitbestimmungsrecht des $\$ 91$ BetrVG gilt nur, wenn die Arbeitnehmer durch eine Veränderung der Gestaltung des Arbeitsplatzes offensichtlich besonders belastet werden. Das Ausmaß dieser Mitbestimmungsrechte ist nicht umfassend genug, um eine teilweise Zuordnung des Betriebsrisikos an die Arbeitnehmer zu gebieten. Sicherheitsvorschriften und technische Vorgaben führen nicht zu einer gefahrbehafteteren Umgebung, sondern intendieren gerade die Minimierung von Arbeitsrisiken und stellen für den Unternehmer daher keine Verschlechterung, sondern eine Erleichterung seiner Risikolast dar.

Von vornherein war die Unfallversicherung auch dann einstandspflichtig, wenn der Arbeitgeber den konkreten Arbeitsunfall auch durch eine andere Betriebsorganisation nicht hätte verhindern können. Auch das Risiko unverschuldeter, fremdverschuldeter und zufälliger Unfälle wurde auf die Arbeitgebergemeinschaft übertragen. Dies liegt daran, dass sich der Beschäftigte nicht in der zum Unfall führenden Situation befunden hätte, wenn er nicht Arbeitnehmer seines Unternehmers gewesen wäre. Dieses Argument kann nicht durch den Hinweis entkräftet werden, dass Arbeitnehmer den sie zur Arbeit verpflichtenden Vertrag privatautonom eingegangen seien und es in ihrer Hand gelegen hätte, einen anderen Arbeitsplatz zu wählen. ${ }^{475}$ Es ist unbestritten, dass der Abschluss eines Arbeitsvertrages aufgrund seiner gravierenden Folgen für den Beschäftigten nicht als gänzlich frei anzusehen ist: Zur Sicherung seiner Existenzgrundlage ist (beinahe) jeder Mensch auf Erwerbstätigkeit angewiesen - nicht nur des Lohnes wegen, auch viele Sozialleistungen werden an das Beschäftigungsverhältnis geknüpft. ${ }^{476}$ Darüber hinaus ist Arbeit das wesentliche gesellschaftliche Identifikationselement, mit dem soziale Anerkennung und Teilhabe an der Gesellschaft einhergehen. ${ }^{477}$ Die Tatsache, dass der Beschäftigte dem Arbeitgeber die Arbeit schuldet, in deren Zusammenhang er den Unfall erlitt, lässt diesen in die Verantwortungssphäre des Arbeitgebers

475 Beckers, Die Außenhaftung des Arbeitnehmers, S. 25.

476 Becker, in: Benvenisti/Nolte (Hrsg.), The Welfare State, Globalization, and International Law, S. 1, 9; Zacher, in: Gitter/Schulin/ders. (Hrsg.), FS Krasney, S. 807, $807 \mathrm{f}$.

477 Negt, Arbeit und menschliche Würde, S. 10 f.; Körner, Das internationale Menschenrecht auf Arbeit, S. 12. 
übertreten. Denn macht sich der Unternehmer die Arbeitskraft seiner Beschäftigten zunutze und tragen diese dabei Verletzungen davon, so sind diese Gesundheitsschäden Auswirkungen der besonderen Zwangslage, in der sich die Arbeitnehmer aufgrund ihres Arbeitsverhältnisses befinden: dass sie zur Erwirtschaftung ihres Lebensunterhalts darauf angewiesen sind, dem Arbeitgeber ihre körperliche oder geistige Arbeitskraft zur Verfügung zu stellen. ${ }^{478}$ Dass der Arbeitnehmer sich selbst durch den Abschluss des Arbeitsvertrages in die Gefahrenzone begibt, bleibt als Verantwortungsgrund daher hinter der Schaffung der Gefahrenquelle zurück.

Der Gedanke der Betriebsgefahr geht so weit, dass auch die Beschäftigten des Betriebes als Betriebsmittel angesehen werden. Weder fahrlässiges noch vorsätzlich verbotswidriges oder risikoerhöhendes Verhalten des Beschäftigten hindert die Zurechnung der konkreten Tätigkeit zur Betriebsrisikosphäre. ${ }^{479}$ Handelt ein Arbeitnehmer fahrlässig und verursacht dadurch einen Arbeitsunfall, so ist das Ausdruck der menschlichen Natur: Jeder lässt gelegentlich die erforderliche Sorgfalt außer Acht. ${ }^{480}$ Der Arbeitsstil des Beschäftigten - sei er ruhig und bedächtig, überlegt und risikobewusst oder unruhig, impulsiv, risikofreudig und unüberlegt - hat keinen Einfluss auf die unfallversicherungsrechtliche Beurteilung eines Unfallereignisses, da die Individualität des Arbeitsstils der menschlichen Arbeitskraft immanent ist. ${ }^{481}$ Diese Argumentationslinie basierte zwar zunächst auf einem speziellen Bild des Beschäftigten, das für die Zeit, in der die Unfallversicherung entstand, typisch war. Im Mittelpunkt der Überlegungen stand der in seiner Leistung jederzeit austauschbare, weil nicht mit Denk-, sondern mit rein körperlicher Arbeit befasste Fabrikarbeiter, welcher nur

478 Baldschun, Solidarität und soziales Schutzprinzip in der gesetzlichen Unfallversicherung, S. 20; Gitter, SGb 1993, S. 297, 298 f.

479 Ausführlich Köhler, VSSR 2017, S. 111, 122 ff.; Gitter, BB 1998, Beilage 6 S. 1, 8 f. So ist etwa auch die strafbare alkoholisierte Teilnahme am Straßenverkehr nach den Grundsätzen der inneren Ursache zu behandeln und schließt einen Unfallversicherungsschutz nicht von vornherein aus, vgl. Sandbiller, SGb 2012, S. 576, passim; Gitter, a.a.O. S. 1, 9; Krasney, VSSR 1993, S. 81, 109 f. Ausdrücklich geregelt in $\$ 7$ Abs. 2 SGB VII, der weit ausgelegt wird, vgl. Krasney, in: Devetzi/ Janda (Hrsg.), FS Eichenhofer, S. 389, 394 m.w.N.

480 Vgl. v.a. die Beiträge der Abgeordneten Winterer und Hertling in den Verhandlungen des Reichstags, abgedruckt bei Kohte, ArbuR 31 (1983), S. 229, 232; ausführlich Gamillscheg/Hanau, Die Haftung des Arbeitnehmers, S. 54 ff. Siehe auch Canaris, RdA 1966, S. 41, 45; Schelp, Die Haftungsbelastung des Arbeitnehmers bei Schädigung Dritter, S. 33 f. m.w.N.

481 Krasney, in: Devetzi/Janda (Hrsg.), FS Eichenhofer, S. 389, 390, der den individuellen Arbeitsstil in seiner konkreten Ausprägung zur Würde des Menschen zählt. 
in seiner Funktion als Beschäftigter existiert und dessen Handlungen ausschließlich durch die Weisungen seines Arbeitgebers bestimmt werden. Aber auch für Beschäftigte, die nicht nur mit rein körperlichen Aufgaben betraut sind, beansprucht der Aspekt Gültigkeit. Ermüdung und individuelle Leistungsschwankungen im Tagesablauf treffen jeden arbeitenden Menschen. ${ }^{482}$ Kein Beschäftigter wird im Laufe seines Arbeitslebens fehlerfrei bleiben. ${ }^{483}$ Bedient man sich der menschlichen Arbeitskraft, so sind menschliche Unzulänglichkeiten wie Arbeiterfahrlässigkeit, schwankende Arbeitsgeschwindigkeit und -qualität, Missachtung von Vorgaben, risikoerhöhendes Verhalten, Fehleinschätzungen oder Irrtümer der Betriebsgefahr zuzurechnen; der Arbeitgeber hat daher auch das daraus entstehende Risiko zu tragen. ${ }^{484}$

\section{Gewinnerzielungsargument}

Der zweite Aspekt der Betriebsrisikolehre ist das sog. „Gewinnerzielungsargument“. Aus der Stellung des Arbeitgebers als Unternehmensinhaber resultiert, dass er die Möglichkeit hat, aus seinem Betrieb Gewinne zu ziehen. Seine finanzielle Situation verbessert sich mit steigendem Gewinn des Unternehmens, während die Beschäftigten unabhängig von der Höhe des wirtschaftlichen Erfolgs des Unternehmens einen gleichbleibenden Lohn erhalten. Der Unternehmer übernimmt damit das Risiko, dass sich sein Betrieb nicht als Gewinn bringend erweist. ${ }^{485}$ Ein möglicher Gewinn des Unternehmers schöpft sich nicht nur aus der Arbeitsleistung der Beschäftigten, sondern notwendigerweise auch aus den Risiken, die für die Beschäftigten mit der Betätigung verbunden sind. ${ }^{486}$ Als Inhaber der Gewinnchancen muss der Arbeitgeber umgekehrt auch die Chancen des Ver-

482 Reinhardt, Die dogmatische Begründung der Haftungsbeschränkung des Arbeitnehmers, S. $136 \mathrm{ff}$.

483 Schuffelen, Die verschuldensunabhängige Haftung des Arbeitgebers, S. 21, m.w.N.

484 Kohte, Arbeitnehmerhaftung und Arbeitgeberrisiko, S. 272. Deswegen schuldet ein Arbeitnehmer auch nicht den erwünschten Erfolg, sondern nur die Leistung, vgl. Fuchs, in: Bamberger/Roth/Hau/Poseck (Hrsg.), BeckOK BGB, $\mathbb{6} 611$ Rn. 10.

485 Bulla, DAR 1942, S. 19, 21.

486 Zänker, Die Haftung der Unternehmer, der Unternehmensangehörigen und anderer Personen in der gesetzlichen Unfallversicherung, S. 24. 
lustes, also die Risiken seines Betriebes, tragen. ${ }^{487}$ Dieses Argument lässt sich mit dem lateinischen Sinnspruch cuius commodum, eius periculum (wessen Sache, dessen Gefahr) umschreiben. ${ }^{488}$ Es sei, so einer der Entwürfe zum Unfallversicherungsgesetz, ein „die ganze Güterproduktion beherrschender Satz, daß jedes Unternehmen seine eigenen Kosten zu tragen hat"; zu diesen Kosten gehöre das Risiko der Arbeitsunfälle. ${ }^{489}$

Der Arbeitnehmer übernimmt die mit der Berufsausübung verbundenen Verletzungsrisiken aufgrund des Arbeitsvertrags zugunsten des Arbeitgebers. Dies ist der Grund dafür, warum dem Arbeitgeber auch die Haftung für solche Schäden der Beschäftigten auferlegt werden kann, die der Arbeitnehmer entweder selbst (mit-)verschuldet hat oder die durch Zufall, also ohne Verschulden des Arbeitnehmers oder Arbeitgebers, entstanden sind. ${ }^{490}$ Denn - in den Worten des Abgeordneten des Reichstags Winterer „ein einfacher Lohnarbeiter kann nicht für einen Betrieb, der nicht der seine ist, verantwortlich gemacht werden" ${ }^{491}$

487 Schadensrisiko und Gewinnchance zusammen bilden den Begriff „Unternehmerwagnis“, so Herschel, Iherings Jahrbücher für die Dogmatik des bürgerlichen Rechts 1943, S. 145, $151 \mathrm{f}$. Zu der Debatte des Reichstags zusammenfassend Gitter, Schadensausgleich im Arbeitsunfallrecht, S. 18 f.; Kohte, Arbeitnehmerhaftung und Arbeitgeberrisiko, S. 226; Kobte, ArbuR 31 (1983), S. 229, 230. Zur Verbindung von Gewinn und Risiko als rechtlichem Prinzip Diem, Die theoretischen Grundlagen der Beschränkung der Haftung des Arbeitnehmers gegenüber dem Arbeitgeber bei schadensgeneigter Arbeit, S. 81 ff. Vgl. auch Rümelin, Dienstvertrag und Werkvertrag, S. $96 \mathrm{ff}$.

488 Gamillscheg/Hanau, Die Haftung des Arbeitnehmers, S. 48; Kritik am Zurechnungsprinzip des Vorteil-Nachteil-Gedankens als rein tatsächlichem Zusammenhang übt Diem, Die theoretischen Grundlagen der Beschränkung der Haftung des Arbeitnehmers gegenüber dem Arbeitgeber bei schadensgeneigter Arbeit, S. $83 \mathrm{ff}$.

489 Tennstedt/Winter, ZSR 1993, S. 362, 370 m.w.N.

490 Verhandlungen des Reichstags, Bd. 62, S. 727, im Wortlaut bei Kohte, ArbuR 31 (1983), S. 229, 232.

491 Verhandlungen des Reichstags, Bd. 63, S.1530, im Wortlaut bei Kohte, aa.O. S. 229, 232. Zwar existieren mittlerweile Modelle der Lohnzahlung, nach denen Arbeitnehmer am Gewinn ihres Unternehmens teilhaben können. Möglich sind zum Beispiel Belegschaftsaktien, Genussrechte oder GmbH-Anteile für die Mitarbeiter. Eine derartige Mitarbeiterkapitalbeteiligung ist politisch erwünscht und wird staatlich gefördert, vgl. BMAS (Hrsg.), Mitarbeiterkapitalbeteiligung Modelle und Förderwege, http://www.bmas.de (Stand: 18.11.2018). Sie bildet dennoch die Ausnahme: Nur 10 Prozent der deutschen Betriebe beteiligten ihre Beschäftigten im Jahr 2011 am Gewinn und nur 2 Prozent am Kapital des Unternehmens, vgl. Möller, IAB-Forum 2013, S. 48, 49. In größeren Betrieben mit mehr als 500 Beschäftigen ist die Mitarbeiterbeteiligung mit 36 Prozent dabei häufiger als in kleineren Betrieben mit bis zu 49 Mitarbeitern (9 Prozent). In 


\section{Das Prinzip der Haftungsersetzung als Rechtsgrund und Übertragungstechnik}

Die gesetzliche Unfallversicherung wurde von Anfang an als Zwangs-Haftpflichtversicherung der Unternehmer zugunsten der Beschäftigten ausgestaltet. Das bedeutet, dass die Arbeitgeber im Gegenzug für die Übernahme der vollen Beitragslast eine Freistellung von jeglichen zivilrechtlichen Schadensersatzansprüchen des Arbeitnehmers, die sich auf individuelle Haftung des Arbeitgebers für eigenes oder fremdes Verschulden gestützt hätten, erhalten (anfangs in $\$ 95$ UVG, heute in den $\$ \$ 104 \mathrm{ff}$. SGB VII geregelt). ${ }^{492}$ Ausnahmen bestehen gemäß $₫ 104$ Abs. 1 S. 1 SGB VII für Wegeunfälle sowie dann, wenn der Arbeitgeber vorsätzlich gehandelt hat.

Dieses Prinzip der Haftungsersetzung ist sowohl ein Rechtsgrund für die Risikoübertragung auf die Unternehmer als auch ein rechtstechnisches (und politisches) ${ }^{493}$ Mittel, um diese Übertragung zu verwirklichen. ${ }^{494}$

Da die Arbeitgeberschaft durch die Unfallversicherung von ihren Haftungsrisiken befreit wird, hat sie die Kosten für diese Risikobefreiung tragen. ${ }^{495}$ Die Haftungsablösung kann deswegen als Rechtsgrund für die Übertragung der Risiken, die aus der zivilrechtlichen Verantwortung des

großen europäischen Unternehmen ist die Mitarbeiterbeteiligung dagegen inzwischen die Regel: Im Jahr 2014 hatten 94 Prozent aller großen europäischen Firmen irgendeine Art der Arbeitnehmerbeteiligung, Mathieu, Annual Economic Survey Of Employee Ownership In European Countries 2014, http://www.e fesonline.org/AnnualEconomicSurvey/2014/Survey2014.pdf (Stand: 18.11.2018). Beschäftigte sind daher nur in sehr eingeschränktem Maß als Mitunternehmer zu bezeichnen. Soweit ein Beschäftigter tatsächlich Mitunternehmer ist, so ist er das im Vergleich zum Unternehmer selbst nur in sehr geringem Maße. Das Gewinnerzielungsargument, nach dem das Risiko demjenigen zuzuordnen ist, der aus ihm Vorteile zieht, führt deswegen auch in diesem Kontext noch zu einer alleinigen Risikotragung des Arbeitgebers. Für die Unfallversicherung zwischen Arbeitnehmern mit und Arbeitnehmern ohne Gewinn- und Verlustbeteiligung am Unternehmen zu unterscheiden, wäre zudem impraktikabel.

492 Vgl. nur Becker, in: Ehlers/Fehling et al. (Hrsg.), Besonderes Verwaltungsrecht Band 3, $\$ 78$ Rn. 1, 111 ff.; Burmann/Jahnke, NZV 2014, S. 5, 5; Möller, SGb 2014, S. 435, 435; Waltermann, VSSR 2005, S. 103, $107 \mathrm{ff}$.

493 Ricke, WzS 2017, S. 9, 12.

494 Zur Vereinbarkeit des in $\$ 104$ SGB VII enthaltenen Ausschlusses von Schmerzensgeldansprüchen mit dem Verfassungsrecht vgl. nur BGH, Urt. v. 4.6.2009 III ZR 229/07 -, juris; Ricke, in: Körner/Leitherer et al. (Hrsg.), Kasseler Kommentar zum Sozialversicherungsrecht, $\mathbb{} 105$ SGB VII Rn. 2 m.w.N.; zum Ganzen eingehend Lepa, Haftungsbeschränkungen bei Personenschäden nach dem Unfallversicherungsrecht, passim.

495 Möller, SGb 2014, S. 435, 435. 
Arbeitgebers herrühren, bezeichnet werden. Bedenken gegen die Einordnung der Haftungsersetzung als Rechtsgrund wurden vorgetragen, weil die Leistungen der Unfallversicherung nicht in jedem Fall deckungsgleich mit denen des zivilrechtlichen Schadensersatzes sind. ${ }^{496}$ Soweit die Unfallversicherung für mehr Schäden einsteht als für solche, die durch zivilrechtlich ahndbares Verhalten des Arbeitgebers entstanden sind, kann sie nicht auf der Beschränkung der Haftung der Unternehmer basieren. ${ }^{497}$ Insoweit gründet die Unfallversicherung auf dem Gedanken des Betriebsrisikos sowie dem sozialen Schutzprinzip (dazu sogleich Kap. 3 C. II.).

Als Übertragungstechnik löst das Haftungsprivileg mehrere Probleme: Erstens erhalten die Beschäftigten mit der Übertragung auf die Unfallkassen Ansprüche gegen diese Körperschaften. Deren Solvenz ist im Normalfall derer ihres Arbeitgebers überlegen, sodass die Arbeitnehmer in jedem Fall einen leistungsfähigen Schuldner beanspruchen können. ${ }^{498}$ Das Insolvenzrisiko des Arbeitgebers entfällt damit. Zudem können die Ansprüche des Arbeitnehmers schneller festgestellt werden, da langwierige Streitigkeiten über Verschulden und Mitverschulden unterbleiben. ${ }^{499}$ Zweitens wird durch die Ablösung aller individuellen Haftungsgründe sichergestellt, dass sich Arbeitnehmer und Arbeitgeber nicht doch als Feinde gegenüberstehen. Der Verletzte hat sich nunmehr mit der Unfallkasse, nicht aber mit seinem Arbeitgeber auseinanderzusetzen. Der Betriebsfrieden, der durch einen öffentlichen Streit zwischen den Parteien des Beschäftigungsverhältnisses gefährdet wäre, ${ }^{500}$ ist damit gesichert. ${ }^{501}$ Dies gilt allerdings nicht für entstandene Sachschäden. Da diese nicht von $\$ 104$ SGB VII umfasst sind,

496 von Koppenfels-Spies, SDSRV Bd. 62, S. 87, $96 \mathrm{f.;} \mathrm{Waltermann,} \mathrm{in:} \mathrm{von} \mathrm{Wulffen/}$ Krasney (Hrsg.), FS 50 Jahre BSG, S. 571, passim; Rolfs, in: Müller-Glöge/Preis et al. (Hrsg.), Erfurter Kommentar zum Arbeitsrecht, $\$ 104$ SGB VII Rn. 2 m.w.N.

497 Waltermann, in: von Wulffen/Krasney (Hrsg.), FS 50 Jahre BSG, S. 571, 589 nennt für diese Fälle den Gedanken der Gefahrengemeinschaft als tragenden Rechtsgrund für die Risikoübertragung.

498 Stelljes, in: Rolfs/Giesen et al. (Hrsg.), BeckOK Sozialrecht, $\$ 104$ SGB VII Rn. 6.

499 Ebd.

500 Vgl. Krasney, ArbuR 49 (2001), S. 423, 424.

501 Sog. „Friedensargument“, vgl. Gitter, Schadensausgleich im Arbeitsunfallrecht, S. 238 ff.; ders., BB 1998, Beilage 6 S. 1, 3; Stelljes, in: Rolfs/Giesen et al. (Hrsg.), BeckOK Sozialrecht, $\mathbb{1} 104$ SGB VII Rn. 2; von Koppenfels-Spies, in: Knickrehm/ Kreikebohm/Waltermann (Hrsg.), Kommentar zum Sozialrecht, $\mathbb{1 0 4}$ SGB VII Rn. 2 m.w.N.; Ricke, in: Körner/Leitherer et al. (Hrsg.), Kasseler Kommentar zum Sozialversicherungsrecht, $\mathbb{S} 104$ SGB VII Rn. 2. Historisch: Verhandlungen des Reichstags, 5. Legislaturperiode, VI. Session, Stenogr. Berichte S. 89, im Wortlaut bei Kohte, ArbuR 31 (1983), S. 229, 232. 
bleiben gerichtliche Auseinandersetzungen um sie möglich. ${ }^{502}$ Insgesamt ist das Haftungsprivileg dennoch dazu geeignet, Konflikte im Betrieb zu verringern. ${ }^{503}$ Drittens löst die Einführung der Haftungsersetzung das Problem, dass sich Unternehmer durch die Regelungen der Unfallversicherung ungerechtfertigterweise als benachteiligt ansehen könnten. Da sie durch ihre Beitragszahlung von der zivilrechtlichen Haftung frei werden, erhalten sie eine spürbare Gegenleistung für ihren finanziellen Einsatz. ${ }^{504}$ Dieser äußert sich auch darin, dass das Risiko von Arbeitsunfällen durch die Haftungsersetzung für den Arbeitgeber kalkulierbar wird. ${ }^{505}$

Das Prinzip der Haftungsersetzung ist somit Rechtsgrund für die Übertragung des Arbeitsunfallrisikos auf die Unternehmer sowie Mittel zur Durchsetzung der Unfallversicherung zugleich. Die Haftungsablösung kann nur im Zusammenhang mit der Betriebsrisikolehre gesehen und verstanden werden. Schutzzweck und Finanzierungsgrundlage bedingen sich dabei gegenseitig. ${ }^{506}$ Allerdings begründet die Betriebsrisikolehre eine $\mathrm{Zu}$ ordnung von Risiken deutlich über die bis dahin angenommene zivilrechtliche Verantwortlichkeit hinaus, sodass das Prinzip der Haftungsersetzung bei der Auslegung der Zuordnungsmechanismen der Unfallversicherung mit Bedacht angewendet werden muss. ${ }^{507}$ Die zwei tragenden Grundprinzipien der Unfallversicherung sind daher korrekterweise nicht als Prinzip

502 von Koppenfels-Spies, in: Knickrehm/Kreikebohm/Waltermann (Hrsg.), Kommentar zum Sozialrecht, $\mathbb{\$} 104$ SGB VII Rn. 2.

503 Vgl. BVerfG, Beschl. v.7.11.1972, E 34, 118; Stelljes, in: Rolfs/Giesen et al. (Hrsg.), BeckOK Sozialrecht, $\$ 104$ SGB VII Rn. 5 f.

504 Sog. Finanzierungsargument, vgl. Ricke, in: Körner/Leitherer et al. (Hrsg.), Kasseler Kommentar zum Sozialversicherungsrecht, $\mathbb{1} 104$ SGB VII Rn. 2; von Koppenfels-Spies, in: Knickrehm/Kreikebohm/Waltermann (Hrsg.), Kommentar zum Sozialrecht, $\$ 104$ SGB VII Rn. 2.

505 Stelljes, in: Rolfs/Giesen et al. (Hrsg.), BeckOK Sozialrecht, $\$ 104$ SGB VII Rn. 6.

506 Ricke, in Körner/Leitherer et al. (Hrsg.), Kasseler Kommentar zum Sozialversicherungsrecht, Vor $\$ 1$ SGB VII Rn. 3.

507 In diesem Sinne auch Ricke, WzS 2017, S.9, 12, der die Absicherung der Beschäftigten als Zweck der Unfallversicherung, die Haftungsablösung dagegen nur als Mittel zur Finanzierung bezeichnet sehen will, ihr aber dadurch nicht ihr Wesen als Strukturprinzip absprechen möchte; ders., in: Körner/Leitherer et al. (Hrsg.), Kasseler Kommentar zum Sozialversicherungsrecht, Vor $₫ 1$ SGB VII Rn. 3a. Vgl. dazu Waltermann, VSSR 2005, S. 103, 112, der betont, dass die Haftungsfreistellung der Unternehmer selbst über die Haftungsablösung hinausgeht, also nicht die gesamte Befreiung der Arbeitgeber von ihrer Haftung mit deren alleiniger Finanzierung der Unfallversicherung begründet werden kann. Vielmehr kommen hier wiederum das Friedensargument und zusätzlich der Gedanke der Gefahrengemeinschaft der Unternehmer untereinander zum Tragen. 
der Haftungsersetzung und Prinzip des sozialen Schutzes, sondern genauer als Betriebsrisikolehre und soziales Schutzprinzip zu bezeichnen.

\section{Soziales Schutzprinzip und Fürsorgepflicht des Arbeitgebers}

Gleichsam ergänzend und auch als Gegenpol zu Betriebsrisikolehre und Prinzip der Haftungsersetzung wirkt das soziale Schutzprinzip. ${ }^{508}$ Als solches wird das primäre Ziel der Einführung der Unfallversicherung bezeichnet, Arbeitnehmer gegen die Risiken, denen sie sich im Berufsleben ausgesetzt sahen, abzusichern. ${ }^{509}$ Dieser Schutz durch die Gewährung von Leistungen im Schadensfall sollte ihnen unabhängig von einem etwaigen eigenen Verschulden zustehen und durch eine leistungsfähige Versicherung gewährleistet werden. ${ }^{510}$ Angelehnt an die Strukturen der zivilrechtlichen Gefährdungshaftung greift der Schutz der Unfallversicherung nur dann nicht, wenn der Verunfallte den Unfall vorsätzlich herbeigeführt hat. ${ }^{511}$ Selbst Handeln gegen ein ausdrückliches betriebliches, behördliches oder gesetzliches Verbot hindert die Einordnung als Arbeitsunfall nicht. ${ }^{512}$ Das soziale Schutzprinzip äußert sich darüber hinaus darin, dass auch die Prävention von Arbeitsunfällen schon seit ihrer Einführung als wichtige Aufgabe der gesetzlichen Unfallversicherung angesehen wird. ${ }^{513}$

508 Zum Zusammenhang beider Prinzipien Gitter, BB 1998, Beilage 6, S. 1, 3.

509 Waltermann, VSSR 2005, S. 103, 105; Gitter/Nunius, in: Schulin (Hrsg.), Handbuch des Sozialversicherungsrechts Band 2, $\$ 5$ Rn. 43; Krohn, in: Schimmelpfennig (Hrsg.), Grundsatzfragen der sozialen Unfallversicherung, S. 23, 30.

510 Gitter/Nunius, in: Schulin (Hrsg.), Handbuch des Sozialversicherungsrechts Band 2, \$ 5 Rn. 43; Gitter, SGb 1993, S. 297, 299.

511 Gitter/Nunius, in: Schulin (Hrsg.), Handbuch des Sozialversicherungsrechts Band 2, 55 Rn. 45. Eine vorsätzliche Selbstschädigung entspringt vollkommen der Privatsphäre des Beschäftigten und kann dem Arbeitgeber nicht zugerechnet werden, vgl. nur Ricke, in: Körner/Leitherer et al. (Hrsg.), Kasseler Kommentar zum Sozialversicherungsrecht, $\mathbb{} 7$ SGB VII Rn. 15 f. m.w.N. Hier fehlt es bereits am Vorliegen eines Unfalls, da ein bewusst herbeigeführtes schädigendes Ereignis dessen Definition nicht zu erfüllen vermag (s.o. Kap. 1 C.).

512 Heute normiert in $₫ 7$ Abs. 2 SGB VII (vorher: $₫ 548$ Abs. 3 RVO). Vgl. dazu Ricke, in: Körner/Leitherer et al. (Hrsg.), Kasseler Kommentar zum Sozialversicherungsrecht, $\mathbb{} 7$ SGB VII Rn. 14. Relevant ist die Regelung inzwischen vor allem bei Unfällen, die sich im Straßenverkehr ereignen und während derer der Beschäftigte gegen $\$ 315 \mathrm{c}$ StGB verstößt, vgl. Marschner, in: Rolfs/Giesen et al. (Hrsg.), BeckOK Sozialrecht, $\$ 7$ SGB VII Rn. 4.

513 Waltermann, VSSR 2005, S. 103, 105; vgl. die heutige Normierung der Präventionsaufgabe in $₫ 1$ SGB VII. 
Argumentativ speist sich das soziale Schutzprinzip im historischen Kontext aus der Fürsorgepflicht, welche dem Unternehmer für die Arbeitnehmer und ihre Familien obliegt. ${ }^{514}$ Diese Fürsorgepflicht war nach der allgemeinen Vorstellung bei der Einführung der Unfallversicherung den Arbeitgebern öffentlich-rechtlich auferlegt, ${ }^{515}$ knüpfte aber an das Arbeitsvertragsverhältnis an und sollte sich aus dem Abhängigkeitsverhältnis des Arbeitnehmers vom Arbeitgeber ergeben. ${ }^{516}$ Den Motiven zum Unfallversicherungsgesetz von 1884 lässt sich entnehmen, dass diese Fürsorgepflicht als tragendes Prinzip der alleinigen Beitragslast der Unternehmer gesehen wurde. ${ }^{517}$ Die Argumentation ging dahin, dass der Unternehmer eigentlich nur für Verschulden einzustehen habe, wegen der ihm obliegenden Fürsorgepflicht allerdings auch das Risiko der zufälligen Unfälle tragen müsse. ${ }^{518}$ Da im Arbeitsleben der Industrialisierung häufig schon ein leicht fahrlässiges Verhalten eines Werktätigen, wie es alle Menschen gelegentlich an den Tag legen, zu einer erheblichen Verletzung seiner selbst oder anderer Personen führen konnte, war das Absehen vom Verschuldenskriterium auch sozial gerechtfertigt. ${ }^{519}$ Die Fürsorgepflicht manifestiert den sozial-ethischen Gedanken, dass der Unternehmer noch immer ${ }^{520}$ auch dann für seinen Arbeitnehmer und dessen Familie zu sorgen hat, wenn dieser im Dienste des Unternehmens seine Gesundheit eingebüßt hat. ${ }^{521}$ Das soziale Schutzprinzip dient außerdem dem bei Einführung der Unfallversicherung gewünschten Zweck, den Betriebsfrieden zu erhalten, da der Verzicht auf das Verschuldenskriterium Ermittlungen innerhalb des Betriebes ent-

514 Baldschun, Solidarität und soziales Schutzprinzip in der gesetzlichen Unfallversicherung, S. 22; Gitter, Schadensausgleich im Arbeitsunfallrecht, S. 18; ders., SGb 1993, S. 297, 299; Pesch, DVBl. 1959, S. 43, 46; a.A. Schnapp, in: Söllner/Gitter et al. (Hrsg.), Gedächtnisschrift für Meinhard Heinze, S. 815 ff.

515 Gitter, Schadensausgleich im Arbeitsunfallrecht, S. 18; Baldschun, Solidarität und soziales Schutzprinzip in der gesetzlichen Unfallversicherung, S. 22.

516 Schrader, ZSR 1958, S. 682, 684.

517 Stenographische Berichte über die Verhandlungen des Reichstags, 5. Legislaturperiode, IV. Session 1884, Bd. 77, Berlin 1884, S. 66; abrufbar unter http://www. reichstagsprotokolle.de (Stand: 18.11.2018); ebenso Gitter, Schadensausgleich im Arbeitsunfallrecht, S. 18.

518 Koch, ZSR 1956, S. 207, 208.

519 Gitter/Nunius, in: Schulin (Hrsg.), Handbuch des Sozialversicherungsrechts Band 2, 55 Rn. 48; Gitter, BB 1998, Beilage 6 S. 1, 3.

520 So wie dies bereits vor der Industrialisierung üblich war, vgl. Gitter, SGb 1993, S. 297, 297 s.o. Kap. 2 B. I.

521 Schrader, ZSR 1958, S. 682, 683; Koch, ZSR 1956, S. 207, 208. 
behrlich macht, welche beinahe zwangsläufig zu Vorhalten und Misstrauen zwischen Kollegen und Vorgesetzten führen würden. ${ }^{522}$

Unter dem Stichwort des „emanzipierten Arbeitnehmers“ wurde in jüngerer Zeit allerdings in Zweifel gezogen, ob überhaupt noch Bedarf für die Annahme einer Fürsorgepflicht des Arbeitgebers gegenüber dem Arbeitnehmer besteht. ${ }^{523} \mathrm{Im}$ Ergebnis ist die Fürsorgepflicht - wenn auch ihre dogmatische Herleitung im Einzelnen strittig ist - auch heute noch integraler Bestandteil des Arbeitsrechts. ${ }^{524}$ Jeder privatrechtliche gegenseitige Vertrag birgt nicht nur Leistungs-, sondern auch Verhaltenspflichten, die früher aus dem Grundsatz von Treu und Glauben $(\$ 242$ BGB a.F.) hergeleitet wurden und inzwischen in $\$ 241$ Abs. 2 BGB ausdrücklich genannt sind. Diese Nebenpflichten verlangen die Rücksichtnahme auf Rechte, Rechtsgüter und Interessen des Vertragspartners. Da das Verhältnis zwischen Arbeitnehmer und Arbeitgeber aufgrund des arbeitgeberseitigen Weisungsrechts und der Eingliederung des Arbeitnehmers in den Betrieb besonders eng ist, sind diese Nebenpflichten im Arbeitsverhältnis besonders ausgeprägt und verdichten sich zur Fürsorgepflicht des Arbeitgebers sowie zur Treuepflicht des Arbeitnehmers. ${ }^{525}$ Dies ist gerechtfertigt, da der Arbeitnehmer in einem stärkeren Maße von seinem Arbeitgeber abhängig ist, als das in einem anderen Vertragsverhältnis der Fall wäre. ${ }^{526}$ Beschäftigte sind auch in ihrer Persönlichkeitsentfaltung und finanziellen Existenz der Bestimmung des Arbeitgebers unterworfen, wodurch persönliche und wirtschaftliche Dispositionsmöglichkeiten verloren gehen. ${ }^{527}$ Daraus folgt eine Mitverantwortung des Arbeitgebers für seinen Arbeitnehmer. ${ }^{528}$ Die Fürsorgepflicht umfasst die Verantwortung des Arbeitgebers, Betriebsab-

522 Gitter/Nunius, in: Schulin (Hrsg.), Handbuch des Sozialversicherungsrechts Band 2, $\$ 5$ Rn. 49.

523 Diem, Die theoretischen Grundlagen der Beschränkung der Haftung des Arbeitnehmers gegenüber dem Arbeitgeber bei schadensgeneigter Arbeit, S. $60 \mathrm{ff}$. m.w.N.

524 Vgl. Nachweise bei Gitter, in: Hauptverband der gewerblichen Berufsgenossenschaften e.v./Bundesverband der landwirtschaftlichen Berufsgenossenschaften e.V. et al. (Hrsg.), 100 Jahre gesetzliche Unfallversicherung, S. 22; ders., Schadensausgleich im Arbeitsunfallrecht, S. 18; Link, in: Schaub, Arbeitsrechts-Handbuch, $\mathbb{S} 108 \mathrm{Rn} .2$.

525 Koch, in: Schaub, Arbeitsrechts-Handbuch, $\mathbb{1} 106$ Rn. 1; Edenfeld, NZA 2009, S. $938,939$.

526 Schelp, Die Haftungsbelastung des Arbeitnehmers bei Schädigung Dritter, S. 36.

527 Wiedemann, Das Arbeitsverhältnis als Austausch- und Gemeinschaftsverhältnis, S. 14.

528 Schelp, Die Haftungsbelastung des Arbeitnehmers bei Schädigung Dritter, S. 36 m.w.N. 
läufe möglichst risikoarm zu gestalten, Gesundheit und Leben des Arbeitnehmers möglichst zu schützen, das jeweils Gebotene zu tun, um betriebsbedingte Schäden vom Arbeitnehmer abzuhalten sowie dennoch erlittene Schäden auszugleichen. Sie erstreckt sich auf Gefahren und Umstände, die ihren Ursprung in der betrieblichen Sphäre haben. ${ }^{529}$ Diese Schutzpflicht ist dem Unternehmer zwar öffentlich-rechtlich auferlegt, knüpft aber an das privatrechtliche Verhältnis des Arbeitsvertrages an. ${ }^{530}$ Konkretisierun-

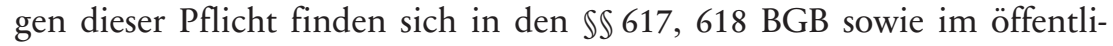
chen Arbeitnehmerschutzrecht. ${ }^{531}$ Teilweise wird sogar von einem personen- oder gemeinschaftsrechtlichen Einschlag 532 des Arbeitsverhältnisses gesprochen, also eine Verwandtschaft des Arbeitsverhältnisses mit solchen Rechtsverhältnissen angenommen, die die Sorge um das Wohl eines anderen zum Inhalt haben. ${ }^{533}$

Es ist realitätsfern, ein persönliches Verhältnis zwischen Arbeitnehmer und Arbeitgeber als Norm anzunehmen, um daraus eine besondere Schutzpflicht zu begründen. In den meisten Fällen arbeiten Arbeitnehmer in Großbetrieben, in denen sie keinerlei persönliches Verhältnis zu ihrem Arbeitgeber haben. Das Arbeitsverhältnis ist im Regelfall ein rein wirtschaftliches, nicht gefühlsbestimmtes Verhältnis. ${ }^{534}$ Was aber bleibt, ist das starke Machtgefälle zwischen Arbeitgeber und Arbeitnehmer, in dem Letzterer das eindeutig schwächere Glied ist. Daran ändern auch die Vielzahl an Arbeitnehmerschutzgesetzen und die Organisation der Arbeitnehmer in Gewerkschaften nichts. Diese Veränderungen basieren aber gerade auf der Schutzpflicht der Arbeitgeber. Sie ist deswegen weiterhin notwendig, denn an der ihnen zugrunde liegenden Abhängigkeit des Arbeitnehmers vom Arbeitgeber hat sich dem Grunde nach nichts geändert. ${ }^{535} \mathrm{Nach}$ wie vor ist der Arbeitgeber dem Arbeitnehmer überlegen, weil Letzterer zur Sicherung seiner wirtschaftlichen und persönlichen Existenzgrundlage auf Ersteren angewiesen ist. Verletzt der Unternehmer diese Schutzpflicht und erleidet der Beschäftigte dadurch einen Arbeitsunfall, wird der Arbeitgeber

529 LSG Hessen, Urt. v. 15.05.2011 - L 9 U 154/09 -, juris.

530 Baldschun, Solidarität und soziales Schutzprinzip in der gesetzlichen Unfallversicherung, S. 20; Gitter, Schadensausgleich im Arbeitsunfallrecht, S. 18, 55.

531 Koch, in: Schaub, Arbeitsrechts-Handbuch, $\$ 106$ Rn. 12.

532 Ebd. Rn. 1.

533 Schuster, Die rechtliche Stellung des mehrköpfigen Betriebsrats, S. 84.

534 Diem, Die theoretischen Grundlagen der Beschränkung der Haftung des Arbeitnehmers gegenüber dem Arbeitgeber bei schadensgeneigter Arbeit, S. 57; Schelp, Die Haftungsbelastung des Arbeitnehmers bei Schädigung Dritter, S. 37.

535 Schelp, aa.O., S. $36 \mathrm{f}$. 
durch die gesetzliche Unfallversicherung für Beschäftigte von der Haftung dafür frei; 536 im Umkehrschluss ist daher das Bestehen dieser Schutzpflicht auch heute noch ein Grund dafür, warum es rechtfertigbar ist, das Risiko auf den Arbeitgeber zu übertragen.

Insgesamt ergänzt das soziale Schutzprinzip die soeben dargestellte historische Argumentation um das Betriebsrisiko bzw. Unternehmerwagnis herum, welche ebenso zeigt, dass man vom Verschuldensprinzip Abstand genommen hatte und eine Gefährdungshaftung entstanden war. ${ }^{537}$ Die Fürsorgepflicht muss nicht separat, sondern verstärkend als Rechtsgrund herangezogen werden: Nimmt man an, dass das Risiko sowieso in den Gefahrenbereich des Arbeitgebers fällt, bedarf es keiner Fürsorgepflicht mehr, um es ihm zu übertragen. ${ }^{538}$

Gleichzeitig stellt eine stärkere Ausbildung des sozialen Schutzprinzips auch eine gewisse Loslösung vom Prinzip der Haftungsersetzung dar. Durch die Fortentwicklung der Unfallversicherung hinsichtlich Aspekten, welche nur teilweise durch die Haftungsersetzung erklärt werden können - etwa die Erweiterung der Versicherungsfälle um Wegeunfälle und Berufskrankheiten, ${ }^{539}$ eine Ausweitung des versicherten Personenkreises ${ }^{540}$ sowie die Verbesserung der Leistungen und die Betonung des Präventionsprinzips $^{541}$-, hat das soziale Schutzprinzip im Laufe der Entwicklung der Unfallversicherung eine zentralere Bedeutung gewonnen. ${ }^{542}$ So rückte vor allem Ricke in jüngerer Zeit verstärkt den Aspekt des sozialen Schutzes und der Fürsorge wieder in den Fokus. ${ }^{543}$ Seiner Ansicht nach ist der Gedanke der Betriebsrisikolehre für die Unfallversicherung unbeachtlich und es dürfe aus der Tatsache, dass der Unternehmer Einflussmöglichkeiten auf die

536 BSG, Urt. v. 19.12.2000, E 87, 224, 224; LSG Hessen, Urt. vom 15.05.2011 - L 9 U 154/09 -, juris.

537 Vgl. Gitter, Schadensausgleich im Arbeitsunfallrecht, S. 18.

538 Gamillscheg/Hanau, Die Haftung des Arbeitnehmers, S. 50, die auch nachweisen, dass in der Rechtsprechung dennoch Betriebsrisikolehre und Fürsorgegedanke zusammen als Gründe angeführt werden.

539 Dazu sogleich Kap. 2 D. I.

540 Ebd.

541 Waltermann, VSSR 2005, S. 103, 106.

542 Das soziale Schutzprinzip als vorrangiges Grundprinzip betonend Krohn, in: Schimmelpfennig (Hrsg.), Grundsatzfragen der sozialen Unfallversicherung, S. 23, 32; zur zunehmenden Bedeutung Gitter/Nunius, in: Schulin (Hrsg.), Handbuch des Sozialversicherungsrechts Band 2, $\$ 5$ Rn. 50.

543 Ricke, WzS 2017, S. 9, 12; ders., in: Körner/Leitherer et al. (Hrsg.), Kasseler Kommentar zum Sozialversicherungsrecht, Vor $₫ 1$ SGB VII Rn.3. Ebenso bereits Krohn, in: Schimmelpfennig (Hrsg.), Grundsatzfragen der sozialen Unfallversicherung, S. 23, $30 \mathrm{f}$. 
sich im Unfall verwirklichten Risiken hatte, keinerlei Argument für oder gegen das Bestehen eines Unfallversicherungsschutzes gewonnen werden. Es käme bei der Beurteilung eines Unfalls rein auf ,autochthon unfallversicherungsrechtliche Merkmale des sozialen Schutzbedürfnisses" an. ${ }^{544}$ Während Ricke eine konkrete Definition dieser Merkmale schuldig bleibt, so ist aus einer Zusammenschau seiner diesbezüglichen Veröffentlichungen doch herauszulesen, dass er für die Einordnung eines Unfalls als Arbeitsunfall dessen Zusammenhang mit der Arbeitstätigkeit, die Berufsbezogenheit der verwirklichten Risiken und die konkrete Schutzbedürftigkeit des Beschäftigten für maßgeblich erachtet. ${ }^{545}$ Zur Beurteilung des Vorliegens eines rechtsfolgenauslösenden Zusammenhangs zwischen Unfall und versicherter Tätigkeit wird aber auch von Ricke die Betriebsrisikolehre herangezogen, da die Beherrschbarkeit des Geschehens durch den Unternehmer ein starker Indikator für die Berufsbezogenheit ist. ${ }^{546}$

\section{Die Arbeitswelt des 21. Jabrhunderts}

I. Überblick über die Unfallversicherung heute

Die eben umrissene, während der Industrialisierung geltende gesellschaftliche Lage bildet noch immer den Hintergrund der rechtlichen Regelungen der gesetzlichen Unfallversicherung. ${ }^{547}$ Ihre Strukturprinzipien sind unangetastet geblieben. Veränderungen bezogen sich vor allem auf den Normtext und die Reichweite des persönlichen Schutzbereichs. Im Folgenden werden sie kurz dargestellt.

544 Ricke, WzS 2017, S. 9, 12.

545 Vgl. Ricke, in: Körner/Leitherer et al. (Hrsg.), Kasseler Kommentar zum Sozialversicherungsrecht, Vor $₫ 1$ SGB VII Rn. $3 \mathrm{ff}$.

546 So z.B. in Ricke, WzS 2017, S. 9, 11.

547 Meik, NZA-Beilage 2017, S. 43, 43. Vgl. zu der Argumentation mit den oben genannten, „historischen“ Rechtsgründen z.B. Keller, in: Hauck/Noftz/ders. (Hrsg.), SGB VII, Einführung, E 100, S. 5; Köhler, VSSR 2013, S. 47, 51 f.; Zänker, Die Haftung der Unternehmer, der Unternehmensangehörigen und anderer Personen in der gesetzlichen Unfallversicherung, S. 24. 
$\mathrm{Zu}$ den wichtigsten Neuerungen zählt die Einführung von Berufskrankheiten ${ }^{548}$ und Wegeunfällen ${ }^{549}$ als Versicherungsfälle im Jahr 1925. ${ }^{550}$

Konzeptionell wurde die zunächst als Betriebsversicherung ausgestaltete Unfallversicherung zwischenzeitlich in eine umfassende Personenversicherung umgewandelt, als ihr personeller Schutzbereich im Jahr 1942 erheblich erweitert 551 und später auf Schüler, Studenten und Kindergartenkinder $(1971)^{552}$, Pflegepersonen $(1994)^{553}$ sowie ehrenamtlich Tätige (2004) $)^{554}$ ausgedehnt wurde.

548 Verordnung über die Ausdehnung der Unfallversicherung auf gewerbliche Berufskrankheiten vom 12.5.1925, RGBl. I S. 69, die am 1.7.1925 in Kraft trat. Bereits bei Einführung der Reichsunfallversicherung hatte man darüber nachgedacht, dass Arbeitnehmer auch Gesundheitsschäden erlitten, die zwar in direktem Zusammenhang mit ihrer Erwerbstätigkeit standen, aber den Begriff des „Unfalls“ nicht erfüllten, da es am Kriterium der Plötzlichkeit scheiterte. Sehr bald, nämlich bereits am 19.7.1911, erhielt das Gesetz eine Ermächtigungsgrundlage, $\$ 547$ RVO (RGBl. S. 509), den Schutz der Unfallversicherung auf sog. Gewerbekrankheiten auszudehnen, also Krankheiten, die „als das Endergebnis der eine längere Zeit andauernden, der Gesundheit nachteiligen Betriebsweise bei bestimmten Gewerbetätigkeiten aufzutreten pflegen“, vgl. Reichsversicherungsamt (Hrsg.), Handbuch der Unfallversicherung Band 1, $\mathbb{} 1$ Anm. 26, S.70. Grund für die Risikozuweisung sind auch für den Versicherungsfall der Berufskrankheiten das soziale Schutzprinzip und die Betriebsrisikolehre: Der Arbeitgeber bestimmt, welchen schädigenden Einwirkungen der Beschäftigte ausgesetzt ist: Sei es hohe Lärmbelastung oder der Umgang mit giftigen Stoffen, immer liegt es am Unternehmer, seinen Betrieb so zu organisieren, dass der Arbeitnehmer keinen Schaden davonträgt. Auch im Falle der Berufskrankheiten profitiert der Arbeitgeber von der Haftungsablösung durch die Unfallversicherung. Ob der Gesundheitsschaden im Zusammenhang mit der Arbeitsausübung durch ein plötzliches Ereignis oder durch lang andauernden Kontakt mit dem schädlichen Einfluss entsteht, macht für die Verantwortlichkeit des Arbeitgebers keinen Unterschied. Zum Einbezug des Berufskrankheitsrisikos vgl. Gitter, BB 1998, Beilage 6 S. 1, 7 f.

549 2. Änderungsgesetz zur Unfallversicherung v. 14.7.1925, RGBl. I S. 97. Die Erwägungen, die zum Einbezug des Wegeunfallrisikos führten, werden unten (Kap. 3 A. VI. 3. a.) dargestellt.

550 Zur Situation vor der Eingliederung des Berufskrankheitsrisikos lesenswert Andersen, in: Machtan (Hrsg.), Bismarcks Sozialstaat, S. 207 ff.

551 6. Änderungsgesetz vom 9.3.1942, RGBl. I, S. 107.

552 Gesetz vom 18.3.1971, BGBl. I, S. 237.

553 Durch das PflegeVG vom 26.5.1994, BGBl. I, S. 1014.

554 Gesetz zur Verbesserung des unfallversicherungsrechtlichen Schutzes bürgerschaftlich Engagierter und weiterer Personen vom 9.12.2004, BGBl. I, S. 3299. 
Mit dem Unfallversicherungseinordnungsgesetz vom 7.8.1997 erfolgte die Eingliederung der gesetzlichen Unfallversicherung als siebtes Buch in das SGB. ${ }^{555}$

Die Aufgaben der gesetzlichen Unfallversicherung werden heute in $₫ 1$ SGB VII prägnant aufgezählt: Sie hat mit allen geeigneten Mitteln Arbeitsunfälle, Berufskrankheiten sowie arbeitsbedingte Gesundheitsgefahren zu verhüten und, so solche eintreten, die Leistungsfähigkeit der Versicherten mit allen geeigneten Mitteln wiederherzustellen und sie oder ihre Hinterbliebenen durch Geldleistungen zu entschädigen. Man spricht von einer Aufgabentrias: Prävention, Rehabilitation und Entschädigung. ${ }^{556}$ Das Gesetz stellt klar, dass die Priorität dieser Aufgaben in dieser Reihenfolge besteht. Nach dem Grundsatz „besser verhüten als vergüten“ ist die Verhinderung von Schadenseintritten das oberste Gebot der gesetzlichen Unfallversicherung. Dies dient sowohl der Verwirklichung der Menschenwürde aus Art. 1 Abs. 1 GG und des Schutzes der körperlichen Integrität aus Art. 2 Abs. 2 GG $^{557}$ als auch der Finanzierbarkeit der gesetzlichen Unfallversicherung, da dadurch das versicherte Risiko begrenzt wird. ${ }^{558}$

Die Einweisungsvorschrift $\mathbb{2} 22$ Abs. 1 SGB I benennt, für welche Leistungen die gesetzliche Unfallversicherung in Anspruch genommen werden kann. Dies sind demnach präventive Maßnahmen zur Verhütung von Arbeitsunfällen und Berufskrankheiten, Heilbehandlungen, Teilhabeleistungen am Arbeitsleben, Erwerbsminderungs- und Hinterbliebenenrenten sowie Rentenabfindungen, Sterbegeld, Haushalts- und Betriebshilfen. Die Datenschutzvorschrift des $₫ 199$ SGB VII bietet einen Katalog aller Aufgaben der gesetzlichen Unfallversicherung. Dazu gehören neben dem Erbringen der Leistungen an die Versicherten auch organisatorische Aufgaben wie die Feststellung der Zuständigkeit und des Versicherungsstatus oder die Berechnung und Erhebung von Beiträgen. Außerdem ist auch die Erforschung von Risiken und Gesundheitsgefahren für die Versicherten Aufgabe der gesetzlichen Unfallversicherung.

Zuständig für die Durchführung der gesetzlichen Unfallversicherung sind die nach Branchen gegliederten Berufsgenossenschaften und die Un-

555 BGBl. I, S. 1254. Zu den Entwicklungstendenzen über die Historie hinweg vgl. Becker, in: ders./Burchardt/Krasney/Kruschinsky, Gesetzliche Unfallversicherung, S. 9 Rn. $28 \mathrm{ff}$.

556 Reyels, in: Schlegel/Voelzke et al. (Hrsg.), SGB VII, $\mathbb{1} 1$ Rn. 23.

557 Ebd. Rn. 29.

558 Kranig, in: Hauck/Noftz/ders. (Hrsg.), SGB VII, $\mathbb{} 1$ Rn. 6. 
fallkassen der öffentlichen Hand ( $\$ 22$ Abs. 2 SGB I). ${ }^{559}$ Die Unternehmer sind Zwangsmitglieder dieser Körperschaften. ${ }^{560}$ Im Jahr 2007 schlossen sich diese zu einem gemeinsamen Spitzenverband zusammen, dem Verband „Deutsche Gesetzliche Unfallversicherung - DGUV“. Lediglich die landwirtschaftlichen Berufsgenossenschaften gehören nicht dazu; sie sind in der Sozialversicherung für Landwirtschaft, Forsten und Gartenbau (SVLFG) organisiert. Die gesetzliche Unfallversicherung wird paritätisch durch Arbeitnehmer und Arbeitgeber selbstverwaltet. Als Teil der unmittelbaren Staatsverwaltung unterliegt sie gem. Art. 87 Abs. 2 GG, $\$ 90$ SGB IV staatlicher Aufsicht. ${ }^{561}$ Die ausschließliche Finanzierung der Unfallversicherung durch die Arbeitgeber ist in $₫ 150$ Abs. 1 SGB VII geregelt. Dies geschieht nach dem Prinzip der nachträglichen Bedarfsdeckung, indem der Finanzbedarf des abgelaufenen Kalenderjahres auf alle Beitragspflichtigen umgelegt wird ( $\$ 152$ Abs. 2 SGB VII). Wie viel der Unternehmer bezahlt, hängt von der Vergütung seiner Arbeitnehmer und von der Gefahrenklasse des Unternehmens ab. ${ }^{562}$ Die als Gegenleistung zu verstehende Ablösung der zivilrechtlichen Haftungsansprüche des Arbeitnehmers gegen den Arbeitgeber findet sich in $₫ 104 \mathrm{f}$. SGB VII (Ausnahmen bestehen gem. $\$ 104$ Abs. 1 SGB VII bei vorsätzlichem Handeln und Unfällen auf versicherten Wegen im Sinne des $\$ 8$ Abs. 2 Nr. 1 bis 4 SGB VII). $\$ 7$ Abs. 2 SGB VII bestimmt, dass das Verschulden des Versicherten einen Versicherungsfall nicht ausschließt.

Im Jahr 2013 waren 3,9 Mio. Unternehmen und Einrichtungen Mitglieder des Spitzenverbands der gesetzlichen Unfallversicherung (DGUV). Rund 78,1 Mio. Personen waren damit gegen Arbeits-, Wege- und Schülerunfälle sowie Berufskrankheiten versichert. ${ }^{563} 2012$ wurden bei den Berufs-

559 Zur Organisation der Unfallversicherung vgl. Becker, in: Maydell/Ruland et al. (Hrsg.), Sozialrechtshandbuch, S. 673, 682, Rn. 29 f.; Brock, in: Doetsch (Hrsg.), Handbuch zum Sozialrecht, Rn. $453 \mathrm{ff}$.

560 Zur Frage der Vereinbarkeit des faktischen Unfallversicherungsmonopols der Berufsgenossenschaften mit dem Verfassungs- und Europarecht vgl. BSG, Urt. v. 9.5.2006 - B 2 U 34/05 R -, juris, Keller, in: Hauck/Noftz/ders. (Hrsg.), SGB VII, Einführung, E 010, S. 6-8 u. 10-12.

561 Triebel, JURA 2007, S. 521, 522.

562 Ebd., S. 521, 523; zum Finanzausgleich in der gesetzlichen Unfallversicherung vgl. Becker, Der Finanzausgleich in der gesetzlichen Unfallversicherung, S. $35 \mathrm{ff}$.

563 Spitzenverband Deutsche Gesetzliche Unfallversicherung, http://www.dguv.de/ de/Zahlen-und-Fakten/Versicherte-und-Unternehmen/index.jsp (Stand: 18.11.2018). Das Bundesministerium für Arbeit und Soziales gibt in seinem Sozialbericht 2017, S. 128, an, abzüglich der Schülerunfallversicherung stünden et- 
genossenschaften und Unfallkassen 885.009 Arbeitsunfälle gemeldet. Das Unfallrisiko betrug 23,32 Arbeitsunfälle je 1000 Vollarbeiter. ${ }^{564}$

\section{Tertiarisierung und Digitalisierung}

Die Arbeitswirklichkeit des 21. Jahrhunderts unterscheidet sich maßgeblich von den bei der Einführung der Unfallversicherung herrschenden soziokulturellen Gegebenheiten. Sie wird vor allem durch die Digitalisierung und die Metamorphose der Industriegesellschaft zur Dienstleistungsgesellschaft, die Tertiarisierung genannt wird, geprägt. Körperliche Arbeit in der Fabrik weicht in vielen Fällen der kundenorientierten Dienstleistungsarbeit. ${ }^{565}$ Dabei wird die wirtschaftliche Wertschöpfung immer stärker durch den Umgang mit Informationen und weniger durch die Bearbeitung materieller Gegenstände erreicht. ${ }^{566}$ Man spricht deswegen auch von der "Informationsgesellschaft“. Der bisher als "Grundtatbestand des Arbeitsrechts ${ }^{“ 567}$ anerkannte Arbeitnehmer, der Leistung nach den Weisungen des Leistungsberechtigten unter Eingliederung in dessen Betrieb erbringt, ist im Rückgang begriffen. ${ }^{568}$ An seiner Stelle entwickeln sich unterschiedliche Arbeitskulturen, die nach dem Grad der Verselbstständigung der Arbeitnehmer unterschieden werden. Sie werden als „Prozessarbeit" (weitgehend bezüglich Arbeitsort und Arbeitszeit vorbestimmte, vollständig weisungsgebundene Tätigkeiten), „Projektarbeit“ (vollständig dem Weisungsrecht unterworfene Tätigkeiten, die aufgrund der technischen Möglichkeiten mobiler Internet-Kommunikationsmittel bezüglich Arbeitszeit und Arbeitsort in größerem Umfang durch die Arbeitnehmer selbst festgelegt werden können, etwa im Einkauf, im Anwaltsberuf, im Personalbereich) und „Pionierarbeit“ (Tätigkeiten, bei denen der Arbeitge-

wa 62,6 Millionen Menschen unter dem Schutz der gesetzlichen Unfallversicherung, von denen der Hauptteil durch Beschäftigte gebildet wird. Abzurufen unter www.bmas.de (Stand: 18.11.2018).

564 Geschäfts- und Rechnungsergebnisse der Berufsgenossenschaften und Unfallkassen, veröffentlicht am 1.8.2013, zitiert nach FD-ArbR 2013, 349005 - beck-online.

565 Welche Veränderungen sich im unmittelbaren Produktionsbetrieb im Rahmen der Digitalisierung ergeben werden, ist noch nicht genau vorhersehbar; Stichworte dazu sind „Industrie 4.0“ bzw. „connected industry“, siehe Schirmer, NZABeilage 2016, S. 85, 86.

566 Krause, Digitalisierung der Arbeitswelt, Gutachten B zum 71. DJT, B 16.

567 Mestitz, ZNR 1980, S. 47, 47.

568 Krause, Digitalisierung der Arbeitswelt, Gutachten B zum 71. DJT, B 20. 
ber sein inhaltliches Weisungsrecht sowie sein Recht, Arbeitsort und -zeit zu bestimmen, vollkommen an den Mitarbeiter übertragen hat, etwa im Forschungs- und Entwicklungsbereich) bezeichnet. ${ }^{569}$

Mit der Entstehung der Informationsgesellschaft einher geht die sogenannte Digitalisierung der Arbeitswelt. Erst die technischen Fortschritte digitaler Informations- und Kommunikationstechnologie ermöglichen den örtlich und zeitlich unabhängigen Austausch von Informationen. Dass die fortschreitende Digitalisierung rechtlichen Anpassungsbedarf auslöst, ist längst als drängendes Thema erkannt und Gegenstand nicht nur wissenschaftlicher, sondern auch gesamtgesellschaftlicher kontroverser Debatten. ${ }^{570}$ Unter dem Stichwort „Arbeiten 4.0“ wird die berufliche Tätigkeit in einer digital vernetzten und flexiblen Arbeitswelt diskutiert. ${ }^{511}$ Krause hat das Topos in seinem Gutachten für den 71. Deutschen Juristentag auch auf tatsächlicher Ebene durchdrungen und so eine Grundlage für die rechtliche Auseinandersetzung mit ihren Auswirkungen geschaffen. ${ }^{572}$ Mit dem Begriff der Digitalisierung der Arbeitswelt sind, ihm folgend, die zunehmende Durchdringung des Arbeitslebens mit digitaler Informations- und

569 Schirmer, NZA-Beilage 2016, S. 85, 86 f. Arbeitsrechtlich stellen sich hier Probleme der Abgrenzung von Arbeitsverhältnis und freiem Dienstverhältnis, ebd.

570 Krause, NZA-Beilage 2017, S. 53, 53, nennt die Digitalisierung der Arbeitswelt das „nationale und internationale ,Megathema der arbeitsrechtlichen und arbeitsrechtspolitischen Debatte“"; bei Schirmer, NZA-Beilage 2016, S. 85, 86 ist vom „Megatrend Digitalisierung/Vernetzung“ die Rede. Welskop-Deffaa sieht die Wichtigkeit der Digitalisierung als „gefestigte Überzeugung der meisten Akteure der arbeitswissenschaftlichen und sozialpolitischen Debatte des Jahres 2016“" an, NZA-Beilage 2017, S. 60, 60. Der 71. Deutsche Juristentag widmete sich in seiner arbeits- und sozialrechtlichen Abteilung diesem Thema, vgl. Krause, Digitalisierung der Arbeitswelt, Gutachten B zum 71. DJT, B 7 ff., und das Bundesministerium für Arbeit und Soziales stieß mit einem Grünbuch einen gesamtgesellschaftlichen Dialog an und brachte schließlich ein Weißbuch mit Ergebnissen heraus, vgl. BMAS, Grünbuch Arbeiten 4.0, passim; dass., Weißbuch Arbeiten 4.0, passim. In der rechtswissenschaftlichen Literatur finden sich außerdem zahlreiche Debattenbeiträge, z.B. Meik, NZA-Beilage 2017, S. 43, 43 ff.; Heise, NZA 2017, S. 1571, 1571 ff.; Uffmann, NZA 2016, S.977, 977 f. m.w.N.; Eichendorf, Sicherheitsingenieur 2016, S. 20, $20 \mathrm{ff}$.

571 I.E. Krause, Digitalisierung der Arbeitswelt, Gutachten B zum 71. DJT, B 11 Rn. 27; vgl. zum Begriff auch Giesen/Kersten, Arbeit 4.0, S. 13 ff.

572 Krause, Digitalisierung der Arbeitswelt, Gutachten B zum 71. DJT, B 7 ff.; vgl. auch So auch Uffmann, NZA 2016, S. 977, 981. Krause betont insbesondere, dass sich die Veränderungen der Arbeitswelt nicht alleine durch den technischen Fortschritt vollziehen, sondern maßgeblich durch vorhandene ökonomischen Interessen, den institutionellen Rahmenbedingungen und den zur Verfügung stehenden Machtsressourcen bedingt werden, ebd. B 9. 
Kommunikationstechnologie und ihre Auswirkungen gemeint. ${ }^{573}$ Für die vorliegende Untersuchung relevant sind insbesondere die technischen Entwicklungen, die ein von der klassischen Betriebsstätte unabhängiges Arbeiten ermöglichen. Anzusprechen sind dabei insbesondere Software, die den Zugriff auf firmeneigene Datenverarbeitungsnetzwerke von auswärtigen Rechnern zulässt, sowie Endgeräte wie Smartphones, Laptops und Tablets, die ein örtlich ungebundenes Arbeiten ermöglichen können. ${ }^{574}$ Studien darüber, wie viele Arbeitnehmer mit diesen Technologien arbeiten, kommen zu zwar voneinander abweichenden, aber stets hohen Prozentzahlen. ${ }^{575}$

\section{Subjektive Modernisierung der Arbeitswelt}

Auch Mentalitätsveränderungen der Arbeitnehmer und Arbeitgeber haben Einfluss auf die Arbeitswelt (sog. subjektive Modernisierung). ${ }^{576}$ Die Machtverhältnisse zwischen Arbeitgebern und Arbeitnehmern sind nicht dieselben geblieben. Beschäftigte sind zu immer größerem Anteil nicht mehr wirtschaftlich und intellektuell unterlegene Fabrikarbeiter, wie sie den Sozialreformen Bismarcks zugrunde lagen, sondern mündige Fachleute. ${ }^{577}$ Der ungelernte, schnell ersetzbare Arbeiter, für dessen Auswahl der Arbeitgeber kaum Zeit aufwenden musste, wird inzwischen durch Maschinen ersetzt. Dafür steigt die Anzahl von Angestellten, die wegen der für ihren Aufgabenbereich notwendigen Vorbildung weniger austauschbar

573 Mit ausführlicher Darstellung verschiedener Technologien und deren Einsatzbereiche Krause, Digitalisierung der Arbeitswelt, Gutachten B zum 71. DJT, B 7 ff. Zustimmend Uffmann, NZA 2016, S. 977, 977 f.

574 Schirmer, NZA-Beilage 2016, S. 85, 86.

575 Das BMAS etwa spricht von 83 Prozent der Beschäftigten, die an ihrem Arbeitsplatz mit einem PC arbeiten, und von 82 Prozent der Führungskräfte in Betrieben mit mehr als 500 Beschäftigten, die ein mobiles Endgerät zu Arbeitszwecken besäßen, BMAS (Hrsg.), Digitalisierung am Arbeitsplatz, www.bmas.de/Sh aredDocs/.../a875-monitor-digitalisierung-am-arbeitsplatz.pdf (Stand: 18.11.2018), S. 6, 8. Nach dem IKT-Report der ZEW waren im Jahr 201449 Prozent aller Beschäftigten überwiegend am ortsfesten PC tätig, und 23 Prozent aller Arbeitnehmer wurden von ihrem Arbeitgeber mit mobilen Endgeräten ausgestattet, was eine erhebliche Steigerung zum Stand des Jahres 2010 bedeutete, vgl. ZEW (Hrsg.), IKT-Report 2015, www.zew.de/fileadmin/FTP/div/IKTRep/ IKT_Report_2015.pdf (Stand: 18.11.2018), S. 3.

576 Krause, Digitalisierung der Arbeitswelt, Gutachten B zum 71. DJT, B 20.

577 Latzel/Picker, in: dies. (Hrsg.), Neue Arbeitswelt, S. V. 
sind. ${ }^{578}$ Durch die Verschmelzung einzelner Unternehmen zu immer gröBeren Organisationen mehren sich die Arbeitsplätze der leitenden Angestellten, die Arbeitgeberfunktionen übernehmen und mit der Rolle des unterlegenen Arbeiters nicht mehr vergleichbar sind. ${ }^{579}$ Ebenfalls zunehmend ist die Anzahl von beruflichen Tätigkeiten, die Kreativität erfordern. Dazu gehören etwa Tätigkeiten in der Softwareentwicklung. Mit kreativen Aufgaben betraute Arbeitnehmer benötigen Freiräume, um bestmöglich arbeiten zu können, und sind deswegen häufig zeitlich und örtlich wenig gebunden. ${ }^{580}$ Beschäftigte wünschen sich zudem mehr Handlungsautonomie, um ihre Arbeit im Hinblick auf Zeit und Ort der Ausführung selbstbestimmt erledigen zu können. ${ }^{581}$ Sie wollen damit eine verbesserte „WorkLife-Balance" erreichen. ${ }^{582}$ Auch deswegen wird davon ausgegangen, dass sich die bis dato als prägend angesehenen Kennzeichen der Arbeitnehmereigenschaft - Weisungsbindung und Eingliederung in eine feste Arbeitsorganisation zu festen Zeiten - verflüchtigen werden. ${ }^{583}$

Nicht nur im Berufsleben, sondern auch privat nutzt ein großer Anteil der Beschäftigten ständig Informations- und Kommunikationstechnologien. Wer beispielsweise ein Smartphone hat, ${ }^{584}$ nimmt dieses einer Studie zufolge im Durchschnitt alle sechs Minuten zur Hand, um mit anderen in

578 Walter/Böhm, in: Paulssen/Richter et al. (Hrsg.), Sozialpolitik, Arbeits- und Sozialrecht, S. 181, $185 \mathrm{ff}$.

579 Ebd., S. 181, 189.

580 Schirmer, NZA-Beilage 2016, S. 85, $86 \mathrm{f}$.

581 Krause, Digitalisierung der Arbeitswelt, Gutachten B zum 71. DJT, B 20. Dies gilt vor allem für Beschäftigte mit Kindern unter 14 Jahren, solche, die Überstunden machen, unter hohem Termindruck stehen oder mit Multitasking-Aufgaben befasst sind, vgl. BMAS, Mobiles und entgrenztes Arbeiten, S.17. Der Wertewandel hin zu Individualität und Autonomie (vom „Berufsautomaten“ zum „flexiblen Mitarbeiter“) begann in den 1970er Jahren, vgl. Bernet, in: Dietz/ Neuheiser (Hrsg.), Wertewandel in der Wirtschaft und Arbeitswelt, S. $32 \mathrm{f}$.

$582 B M A S$, Mobiles und entgrenztes Arbeiten, S. 17. $\mathrm{Zu}$ den Hintergründen und Studien bzgl. der motivierenden Faktoren der sog. "Generation Y“ vgl. BMAS, Wertewelten Arbeiten 4.0, passim; Krause, Digitalisierung der Arbeitswelt, Gutachten B zum 71. DJT, B 20 m.w.N.

583 Uffmann, NZA 2016, S. 977, 979.

584 Nach einer Erhebung des Interessenverbandes Bitkom waren das im Januar 201778 Prozent aller Deutschen, https://www.bitkom.org/Presse/Anhaenge-anPIs/2017/02-Februar/Bitkom-Pressekonferenz-Smartphone-Markt-Konjunktur-u nd-Trends-22-02-2017-Praesentation.pdf (Stand: 23.11.2018). 
Kontakt zu treten. ${ }^{585}$ Die Digitalisierung ist damit bei jeder rechtspolitischen Überlegung mitzudenken. ${ }^{586}$

\section{E. Entgrenzte Arbeit}

I. Entbetrieblichung: örtliche und zeitliche Flexibilität im Arbeitsverhältnis

Mit der Digitalisierung unmittelbar zusammenhängend ist das für die Unfallversicherung besonderes relevante Phänomen der Entgrenzung. ${ }^{587}$ Strukturen, die bisher als betrieblich wahrgenommen wurden, sind in Auflösung begriffen. ${ }^{588}$ Grenzen zwischen Arbeits- und Privatleben verschwimmen nicht nur zeitlich und räumlich, sondern auch inhaltlich. Diese Entwicklung steht den Strukturprinzipien der Unfallversicherung diametral entgegen, da diese, wie gezeigt werden konnte, gerade die Grenzziehung zwischen privater und beruflicher Sphäre als Basis für die Einordnung von Versicherungsfällen nutzt. ${ }^{589}$

Treiber dieses Aspekts der Entgrenzung ist unter anderem die Natur dienstleistungsbezogener Arbeit, welche erhöhte Anforderungen an die zeitliche und örtliche Flexibilität der Arbeitnehmer stellt. Beschäftigte im Dienstleistungsbereich sollen dann dort verfügbar sein, wo der Kunde sie wünscht. Der technische Fortschritt ermöglicht diesen Beschäftigten, örtlich und zeitlich immer flexibler zu arbeiten. ${ }^{590}$ Anders als körperlich geprägte Arbeit, die nach der Schicht am Werktor endet, kann dienstleistungsbezogene Arbeit - durch moderne Technologien unterstützt - von vielerlei Orten aus verrichtet werden. ${ }^{591}$ Diese Art entgrenzten Arbeitens

585 Bitkom (Hrsg.), Crowdsourcing für Unternehmen, https://www.bitkom.org/noi ndex/ Publikationen/2014/Leitfaden/Crowdsourcing/140917-Crowdsourcing.pd f (Stand: 18.11.2018), S. 6.

586 Krause, Digitalisierung der Arbeitswelt, Gutachten B zum 71. DJT, B 15 m.w.N.

587 Uffmann, NZA 2016, S. 977, 979; zum Begriff der Entgrenzung vgl. Fn. 5.

588 Dies gilt in unterschiedlichem Maße, da Entgrenzung sehr stark vom Grad der Digitalisierung abhängt, welche wiederum branchen- und betriebsabhängig ist („Digitale Ungleichzeitigkeit“), vgl. Giesen/Kersten, Arbeit 4.0, S. 128. Die stetige Verstärkung der Entgrenzung belegend Däubler, SR 2014, S. 45, 58 ff.

589 S.o. Kap. 2 C.

590 Latzel/Picker, in: dies. (Hrsg.), Neue Arbeitswelt, S. V, V.

591 Giesen/Kersten, Arbeit 4.0, S. 86. 
gewinnt immer mehr an Bedeutung. ${ }^{992}$ Eine Erosion des Betriebs als stabile räumliche und organisatorische Einheit ist die Folge. ${ }^{593}$ Denn örtlich flexibles Arbeiten bedeutet, dass sich der Beschäftigte nicht in einem vom Arbeitgeber zur Verfügung gestellten Gebäude aufhalten muss, um seine Arbeit zu verrichten. Stattdessen kann er sogenannte Telearbeit ${ }^{594}$ erbringen, indem er ganz oder teilweise außerhalb des Betriebs arbeitet, zum Beispiel in Nachbarschafts- und Satellitenbüros, unterwegs oder von zu Hause aus (Homeoffice oder Teleheimarbeit, zu den Begriffen s.u. Kap. 3 A. I.). Laut einer Erhebung aus dem Jahr 2016 bietet bereits ein Drittel der Betriebe in Deutschland seinen Beschäftigten die Möglichkeit, von zu Hause zu arbeiten; bei Betrieben mit über 500 Beschäftigten ist es die Hälfte. ${ }^{595}$ Nicht nur in der Dienstleistungsbranche, sondern selbst in der Industrieproduktion hat zeitlich und örtlich entgrenztes Arbeiten bereits Einzug gehalten Produktionsmitarbeiter können die Fertigung etwa über Tablets steuern, wenn ihnen Videobilder über den Maschinenzustand übermittelt werden. ${ }^{596}$ Damit ist der - ursprünglich zugleich als Arbeitsort verstandene Betrieb in der entgrenzten Arbeitswelt keine räumliche Einheit mehr, sondern umfasst die Verfolgung des gemeinschaftlichen Arbeitszwecks von verschiedenen Örtlichkeiten aus. ${ }^{597}$ Auch zeitlich sind Beschäftigte häufig nicht mehr an strikte Vorgaben gebunden. Gleitzeitmodelle oder das Konzept der Vertrauensarbeit ermöglichen es Arbeitnehmern, ihre Arbeitszeit innerhalb gewisser Vorgaben selbst festzulegen. Hanau bezeichnet diesen Prozess der Lösung der Konzentration von Arbeitserbringung an einem physischen Ort als „Entbetrieblichung“.598 Richtig ist, dass der Betrieb in seinem ursprünglichen Sinne als physischer Ort, an dem Arbeitnehmer

592 So auch das BSG, welches darauf hinweist, dass Arbeitnehmern im Zuge moderner Entwicklungen mehr Freiheiten bezüglich der Bestimmung von Arbeitsort und Arbeitszeit eingeräumt werden, vgl. Urt. v. 18.11.2015 - B 12 KR 16/13 R -, juris Rn. 36 m.w.N. Vgl. auch Uffmann, NZA 2016, S.977, 979; dies., in: Giesen/ Junker/Rieble (Hrsg.), Industrie 4.0 als Herausforderung des Arbeitsrechts, S. $31,37 \mathrm{f}$.

593 Krause, Digitalisierung der Arbeitswelt, Gutachten B zum 71. DJT, B 19; Giesen/ Kersten, Arbeit 4.0, S. 128.

594 Begriff, Entwicklung, Anwendungsbereiche und arbeitsrechtliche Aspekte der Telearbeit werden eingehend dargelegt bei Wedde, Telearbeit, passim.

595 BMAS, Mobiles und entgrenztes Arbeiten, S. 7, 8 f.;.

596 Unter Nennung eines konkreten Beispiels Uffmann, in: Giesen/Junker/Rieble (Hrsg.), Industrie 4.0 als Herausforderung des Arbeitsrechts, S. 31, 34.

597 Giesen/Kersten, Arbeit 4.0, S. 129, die die Schwierigkeiten der Definition des Betriebes im Hinblick auf das Betriebsverfassungsrecht behandeln. Zur verringerten Eingliederung am Beispiel von IT-Kräften Heise, NZA 2017, S. 1571, 1574.

Hanau, NJW 2016, S. 2613, 2614. 
gleichzeitig ihre Arbeitsleistung erbringen, für diese Fälle der Entgrenzung nicht mehr besteht. Als organisatorische Einheit ist er jedoch im selben Maße existent: Bei raum- und zeitübergreifendem Zusammenarbeiten auf einen arbeitstechnischen Zweck hin ist lediglich der physische Ort einem virtuellen Raum gewichen. ${ }^{599}$

Insgesamt ist davon auszugehen, dass die Tendenz weiterhin zu zeitlich und örtlich ungebundenerer Arbeit geht. ${ }^{600}$ Gerade die im Zunehmen begriffene Gruppe der Arbeitnehmer, die kreativ denken müssen, benötigt Freiraum in zeitlicher und örtlicher Hinsicht. Die meisten Ideen entstehen schon jetzt im privaten Umfeld, nicht im Büro. ${ }^{601}$ Dies bestätigt auch eine Studie des Fraunhofer-Instituts für Arbeitswirtschaft und Organisation (IAO), welcher zufolge der feste Arbeitsplatz in naher Zukunft immer mehr zugunsten von Smart Working Centers, öffentlichen Cafés, TransitLounges, Verkehrsmitteln, Wohnungen von Privatpersonen und Ähnlichem verschwinden wird. ${ }^{602}$

Diese Entwicklung scheint im Interesse sowohl der Arbeitnehmer- als auch der Arbeitgeberseite zu sein. ${ }^{603}$ Die Beschäftigten begrüßen ihre gröBere zeitliche und örtliche Flexibilität, um Privat- und Berufsleben besser in Einklang bringen zu können. ${ }^{604}$ Das gesellschaftliche Problem der Vereinbarkeit gerade von familiärer Sorgearbeit und beruflicher Tätigkeit bedingt die in diesem Zusammenhang stehende anhaltende Diskussion darüber, ob es ein Recht auf „Wahlarbeitszeit“ geben soll. Hier befindet sich vieles im Umbruch. Der 71. Deutsche Juristentag forderte im Herbst 2016 in seinen Beschlüssen sogar ein Recht der Beschäftigten auf örtliche und

599 Giesen/Kersten, Arbeit 4.0, S. 131, 133; Franzen, in: Giesen/Junker/Rieble (Hrsg.), Industrie 4.0, S. 107, 112 ff.; Thüsing, SR 2016, S. 87, 102 f.

600 Vgl. etwa den Vorschlag zur Einführung einer "Wahlarbeitszeit” des Deutschen Juristinnenbundes, djb-Kommission für Arbeits-, Gleichstellungs- und Wirtschaftsrecht, djbz 2015, S. 121, 188; näher dazu Aumann/Hack, ZESAR 2016, S. 266 ff. Insgesamt zu den diesbezüglichen Gestaltungsvorschlägen Uffmann, NZA-Beilage 2017, S. 45, $45 \mathrm{ff}$.

601 Schirmer, NZA-Beilage 2016, S. 85, 88.

602 Bauer/Rief et al., Arbeitswelten 4.0, S. 27.

603 Den Vorteilen stehen gerade für die Beschäftigten allerdings auch gravierende Nachteile gegenüber, wie z.B. das erhöhte Risiko der Überarbeitung, eines Burnouts etc., vgl. nur Giesen/Kersten, Arbeit 4.0, S. 75 ff., 86.

604 BMAS, Mobiles und entgrenztes Arbeiten, S. 17. Allerdings besteht weiterhin der Wunsch nach einer Trennung von Beruf und Privatem, was durch eine Beschäftigung im Homeoffice als gefährdet angesehen wird, vgl. BMAS, Mobiles und entgrenztes Arbeiten, S. 16. 
zeitliche Souveränität im Hinblick auf die zu leistende Arbeit. ${ }^{605}$ Auch für Arbeitgeber bringt das örtlich und zeitlich entgrenzte Arbeiten Vorteile. Beschäftigte, die räumlich und/oder zeitlich entgrenzt arbeiten, fühlen sich etwa enger mit ihrem Betrieb verbunden als solche, die dies nie tun. ${ }^{606}$ Arbeitnehmer, die gelegentlich im Homeoffice arbeiten, arbeiten zudem insgesamt deutlich mehr als Angestellte, die nur im Betrieb selbst arbeiten. ${ }^{607}$

\section{Fortschreitende Erosion zwischen Arbeits- und Privatleben}

\section{Eindringen der Arbeit ins Private}

Die zweite Facette der Entgrenzung der Arbeit ist die vermehrte zeitliche, räumliche und inhaltliche Vermischung von Berufs- und Privatleben. Der Begriff der Entgrenzung bezieht sich dabei auf die Erosion der Trennlinie beider Lebensbereiche. Diese ist kein einseitiger Prozess. Nicht nur die berufliche Sphäre dringt in den privaten Lebensbereich ein, sondern auch Privates greift auf das Berufsleben über. Zum einen handelt es sich auch hier um eine Begleiterscheinung des digitalisierten Arbeitens. ${ }^{608}$ Denn Tätigkeiten, die überall zu jeder Zeit ausgeübt werden können, eröffnen den Arbeitnehmern Freiräume für ein in stärkerem Maße selbstbestimmteres Arbeiten, das zu beliebigen Zeiten an beliebigen Orten ausgeführt werden kann. ${ }^{609}$ Die vielzitierte „ständige Erreichbarkeit“, die durch Mobiltelefone, Tablets und Internet bedingt ist, betrifft eine große Anzahl von Beschäftig-

605 Vgl. Beschlüsse des 71. DJT, Arbeits- und sozialrechtliche Abteilung, Beschluss III.1 (abrufbar unter http://www.djt.de/fileadmin/downloads/71/Beschluesse_ge samt.pdf, Stand: 18.11.2018).

606 BMAS, Mobiles und entgrenztes Arbeiten, S. 7, 11.

607 Ebd., S. 10 (43,5 Stunden pro Woche im Gegensatz zu 39,4 Stunden pro Woche).

608 Schirmer, NZA-Beilage 2016, S. 85, 85. Die Hälfte der Beschäftigten, die gelegentlich im Homeoffice arbeiten, empfinden dadurch eine stärkere Vermischung von Arbeit und Privatem, vgl. BMAS, Mobiles und entgrenztes Arbeiten, S. 14. Virulent ist die Frage vor allem in Bezug auf das Arbeitszeitrecht, wenn es darum geht, ob die gesetzlich vorgeschriebenen Ruhezeiten eingehalten werden, vgl. m.w.N. Krause, Digitalisierung der Arbeitswelt, Gutachten B zum 71. DJT, B 39 ff.

609 Schirmer, NZA-Beilage 2016, S. 85, 85. 56 Prozent derjenigen, die von zu Hause arbeiten, tun dies außerhalb der Zeit, die sie selbst als Arbeitszeit betrachten, vgl. BMAS, Mobiles und entgrenztes Arbeiten, S. 12. 
ten. ${ }^{610}$ Jedenfalls 90 Prozent aller Arbeitnehmer sind zumindest außerhalb ihrer Arbeitszeit erreichbar. ${ }^{611}$ Zwei Drittel aller Beschäftigten werden zumindest gelegentlich in der Freizeit dienstlich kontaktiert; lediglich 35 Prozent sind nie in der Freizeit dienstlich erreichbar. ${ }^{612}$ Aber auch unabhängig von der Digitalisierung greift die Arbeitswelt durch sogenannte „Freizeittätigkeit“ auf den privaten Lebensbereich über; etwa 15 Prozent der Beschäftigten nehmen regelmäßig Arbeit mit nach Hause. ${ }^{613}$ Verbreitet ist auch das zielgerichtete abendliche oder morgendliche Überprüfen des dienstlichen E-Mail-Accounts. ${ }^{614}$ Kurze Kontaktaufnahmen von Vorgesetzten mit Beschäftigten auch in den eigentlich nach der vereinbarten Arbeitszeit liegenden Abendstunden werden als sozialadäquat angesehen. ${ }^{615}$

Schon auf tatsächlicher Ebene - ohne den Zurechnungsmechanismus der Unfallversicherung mitzudenken - ist es gerade in informationsbezogenen Berufen wegen der ständigen Möglichkeit, auf seine Arbeitsmaterialien zuzugreifen, schwierig, zwischen Arbeit und Freizeit zu unterscheiden. Schirmer nennt als Beispiel einen Arbeitnehmer, der im Homeoffice arbeitet, eine Stunde Joggen geht und während des Joggens eine kreative Idee entwickelt, die ihm im Anschluss dabei hilft, ein schwieriges Problem am Schreibtisch besser zu lösen. ${ }^{616}$ Es sei unmöglich, hier trennscharf zwischen Arbeitszeit und Freizeit zu unterscheiden, da auch nicht nachweisbar sei, ob der Arbeitnehmer nur Sport gemacht oder auch über ein betriebliches Problem nachgedacht habe. ${ }^{617}$ Selbst wenn der Arbeitgeber keine Kenntnis davon hat, dass der Arbeitnehmer eine vertragsgemäße Tätigkeit entfaltet, liegt nach überwiegender Meinung in der Literatur Arbeit vor, solange der Arbeitgeber sich ein solches Tätigwerden aus Eigeninitiati-

610 Das extrem verbreitete, „kurze“ „Checken der E-Mails“ am Smartphone aus einem inneren Drang des Beschäftigten heraus wird als „Morbus Blackberry“ bezeichnet, vgl. von Steinau-Steinrück, NJW-Spezial 6/2012, S. 178, 178; Tamm, ZfPR 2011, S. 67, 67.

611 Schuchart, in: Latzel/Picker (Hrsg.), Neue Arbeitswelt, S. 175, 178.

$612 B M A S$, Mobiles und entgrenztes Arbeiten, S. 11.

613 Krause, Digitalisierung der Arbeitswelt, Gutachten B zum 71. DJT, B 28 m.w.N.; $B M A S$, Mobiles und entgrenztes Arbeiten, S. 9 f.

614 Krause, Digitalisierung der Arbeitswelt, Gutachten B zum 71. DJT, B 46.

615 Giesen/Kersten, Arbeit 4.0, S. 102 m.w.N.

616 Schirmer, NZA-Beilage 2016, S. 85, 87, im Zusammenhang mit der Frage nach der Anpassung des Arbeitszeitrechts im Rahmen der Digitalisierung der Arbeitswelt.

617 Schirmer, NZA-Beilage 2016, S. 85, 87. 
ve nicht ausdrücklich verbeten hat. ${ }^{618}$ Nur, wenn sich der Beschäftigte ausschließlich im eigenen Interesse während seiner Freizeit mit dienstlichen Belangen beschäftigt, etwa in seinem dienstlichen E-Mail-Account liest, sei diese Tätigkeit dieser Ansicht nach nicht als „Arbeit“ zu qualifizieren. ${ }^{619}$

\section{2. Übergreifen des Privaten in die Arbeit}

Selbst bei der Verrichtung der Beschäftigung am Arbeitsort zur Arbeitszeit geht die Trennung von Privat- und Berufssphäre, wie sie im Rahmen der Prüfung des Unfallversicherungsschutzes durch die Bestimmung der wesentlichen Handlungstendenz der tatsächlichen Verrichtung notwendig gemacht wird, in vielen Fällen an der Lebenswirklichkeit der Beschäftigten vorbei. ${ }^{620}$ Wo Arbeitgeber verlangen und erwarten, dass Arbeitnehmer auch außerhalb der Bürozeiten erreichbar sind und sich selbst, wenn nötig, in den „Dienst“ versetzen, sehen Arbeitnehmer die Grenze zwischen Privatem und Beruflichem selbst ebenfalls nicht mehr so scharf und nehmen sich deswegen umgekehrt die Freiheit, während ihrer Arbeitszeit am Arbeitsort private Dinge zu erledigen. ${ }^{621}$ Die Hälfte aller Arbeitnehmer tut dies bereits, ${ }^{622}$ obwohl sie die arbeitsvertraglich vereinbarte Arbeitszeit ohne Abzug von privat genutzten Zeiten schulden. ${ }^{623}$ Eine Studie aus dem Jahr 2016 kommt zu dem Ergebnis, dass 66 Prozent der Arbeitnehmer gelegentlich während der Arbeitszeit Privatem nachgehen, wobei es sich vor allem um private Telefonate, das Surfen im Internet, den Besuch von Social-Media-Portalen und Online-Shopping handelt. ${ }^{624}$ Unter den 18- bis 34jährigen Beschäftigten erledigen sogar 82 Prozent zumindest eine private

618 Krause, Digitalisierung der Arbeitswelt, Gutachten B zum 71. DJT, B 36 m.w.N. sowie Giesen/Kersten, Arbeit 4.0, S. 98 m.w.N.

619 Krause, Digitalisierung der Arbeitswelt, Gutachten B zum 71. DJT, B 36; von Steinau-Steinrück, NJW-Spezial 6/2012, S. 178, 178.

620 Vgl. nur Giesen/Kersten, Arbeit 4.0, S. 77 f.

621 Falder, NZA 2010, S. 1150, 1150; Krause, Digitalisierung der Arbeitswelt, Gutachten B zum 71. DJT, B 43, mit dem Hinweis auf eine australische Studie, nach der dortige Arbeitnehmer das Internet durchschnittlich in einem größeren zeitlichen Umfang während der Arbeit für private Zwecke als während der Freizeit für dienstliche Zwecke nutzen (Wajcman et. al 2010).

622 Schuchart, in: Latzel/Picker (Hrsg.), Neue Arbeitswelt, S. 175, 178. Dieses Phänomen wird unter dem Schlagwort „Work-Life-Integration“ behandelt.

623 Gem. \611a Abs. 1 BGB, vgl. Giesen/Kersten, Arbeit 4.0, S. 99 m.w.N.

624 Die Daten stammen aus einer Umfrage von YouGov und Statista, für die im August 2016543 Arbeitnehmer befragt wurden, abrufbar unter https://de.statista.c 
Tätigkeit täglich am Arbeitsplatz. ${ }^{625}$ Dieser Aspekt der Entgrenzung hängt maßgeblich auch mit der Omnipräsenz von Smartphones und ähnlichen mobilen Endgeräten sowohl im Privat- als auch im Berufsleben der Beschäftigten zusammen. So wird etwa vom Arbeitsplatz aus das Familienleben organisiert, indem über das Mobiltelefon mit den Mitgliedern des eigenen Haushalts in Kontakt getreten wird und Einkäufe, Kinderbetreuung und Ähnliches koordiniert werden. ${ }^{626}$ Häufig nutzen Arbeitnehmer ein und dasselbe mobile Endgerät sowohl für berufliche als auch private Zwecke („Bring Your Own Device“), ${ }^{627}$ was - wenn man bedenkt, dass diese Geräte von ihren Nutzern im Durchschnitt alle sechs Minuten ${ }^{628}$ auf Neuigkeiten überprüft werden und das Gerät dabei unterschiedslos berufliche und private Meldungen weitergibt - eine Scheidung der beiden Lebenssphären weiter lebensfremd macht. Die kurze Konsultation des Mobiltelefons, etwa um digital Beziehungen zu pflegen, wird inzwischen auch während der Arbeitszeit in den meisten Fällen als sozialadäquat akzeptiert. ${ }^{629}$ Umgekehrt werden auch vom Arbeitgeber zur Verfügung gestellte Arbeitsmittel, wie etwa das „Dienst“-Handy oder -Hardware, schon aus Praktikabilitätsgründen privat genutzt. 630

In einigen Fällen ist diese Verquickung von Privat- und Berufsleben vom Arbeitgeber sogar ausdrücklich erwünscht. Eine wachsende Anzahl von Unternehmen bemüht sich bewusst darum, das Privatleben ihrer Mitarbeiter mitzugestalten, und wird dies nach Einschätzung des Fraunhofer-Instituts für Arbeitswirtschaft und Organisation in immer stärkerem Maße tun. ${ }^{631}$ Ziel dieser Arbeitgeber ist es, Privat- und Berufsleben möglichst po-

om/infografik/6432/private-taetigkeiten-waehrend-der-arbeit/ (Stand: 23.11.2018).

625 So das Ergebnis der Studie „Mobilelron Gen M Survey“, für die das Meinungsforschungsinstitut Harris Poll im Auftrag des Herstellers Mobilelron 3.500 Vollund Teilzeitbeschäftigte in sechs Ländern befragt hat, abrufbar unter https:/ww w.mobileiron.com/en/company/press-room/press-releases/global-study-finds-58generation-mobile-suffering-mobile-guilt (Stand: 18.11.2018).

626 Giesen/Kersten, Arbeit 4.0, S. 98.

627 Krause, Digitalisierung der Arbeitswelt, Gutachten B zum 71. DJT, B 15; Günther/Böglmüller, NZA 2015, S. 1025, 1030.

628 Bitkom (Hrsg.), Crowdsourcing für Unternehmen, https:/www.bitkom.org/noi ndex/ Publikationen/2014/Leitfaden/Crowdsourcing/140917-Crowdsourcing.pd f (Stand: 18.11.2018), S. 6.

629 Giesen/Kersten, Arbeit 4.0, S. 100.

630 Günther/Böglmüller, NZA 2015, S. 1025, 1030 m.w.N.

631 So das Ergebnis einer Studie, die ,aus der Befragung ausgewählter Experten sowie Auswertung zahlreicher Quellen zu Trend- und Zukunftsstudien“ entstanden ist, Bauer/Rief et al., Arbeitswelten 4.0, S. 45. 
sitiv miteinander zu vermengen. Dieses Konzept wird auch „Corporate Life" genannt. Die Unternehmen wollen als "Caring Companies“ ihren Mitarbeitern Wohnangebote, Ausbildungsmöglichkeiten, gesundheitsund vorsorgebezogene Maßnahmen sowie Freizeitangebote bieten, um zum einen die wegen des fortschreitenden Fachkräftemangels immer notwendigere Attraktivität des Arbeitsplatzes zu steigern und zum anderen ihre Mitarbeiter körperlich fit und leistungsfähig zu halten. ${ }^{632}$ Eine gelungene Work-Life-Integration wird für die Beschäftigten als Statussymbol gelten. ${ }^{633}$

Das in der Industrialisierung angenommene Gesellschaftsmodell einer zeitlichen, räumlichen und inhaltlichen Trennung von Arbeits- und Privatleben - auf welchem das System der Unfallversicherung beruht - ist bereits weniger Realität denn je und wird immer weiter zurückgedrängt werden. 634

\section{F. Fazit: Bedeutung der modernen Arbeitswelt für die Unfallversicherung}

Dem System der Unfallversicherung liegt die gesellschaftliche Realität der Industrialisierung zugrunde. Für die Eröffnung des unfallversicherungsrechtlichen Schutzbereichs kommt es darauf an, dass der Unfall als Arbeitsunfall qualifiziert wurde, was der Fall war, wenn er mittels der Zurechnungsprinzipien der Betriebsrisikolehre oder des sozialen Schutzprinzips der Risikosphäre des Arbeitgebers zugeordnet wurde. Der Fokus dieser Wertentscheidung lag im Rahmen der Betriebsrisikolehre auf der Eingliederung des Beschäftigten in den Betrieb des Unternehmers. Dessen Möglichkeit, die örtliche Umgebung und den zeitlichen Ablauf der betrieblichen Tätigkeit kraft seiner Organisationsgewalt und Weisungsbefugnis zu beherrschen, stellte das primäre Argument für die Zuordnung eines Unfalls zum Betriebsrisiko dar. Dies erklärt sich aus der Entstehungsgeschichte der Unfallversicherung, da ihre Notwendigkeit aus der neuartigen, gefahrträchtigen Arbeit an Maschinen in Fabriken im Zuge der Industrialisierung geboren wurde.

Bereits mit dem Übergang zur Dienstleistungsgesellschaft sank die Bedeutung ortsbezogener Unfallrisiken, da Beschäftigte häufiger an Schreib-

632 Ebd., S. 18.

633 Ebd., S. 27.

$634 \mathrm{Ob}$ die Mehrheit der Arbeitnehmerschaft dies begrüßt, kann noch nicht nachgewiesen werden, vgl. Krause, Digitalisierung der Arbeitswelt, Gutachten B zum 71. DJT, B 44 m.w.N. 
tischen als in der Fertigung arbeiten. Mit der mit der Entgrenzung der Arbeitsverhältnisse einhergehenden zunehmenden Entbetrieblichung sind die traditionell zur Abgrenzung von Arbeit und Freizeit genutzten Parameter von Arbeitsort und Arbeitszeit endgültig nicht mehr in dem Maße aussagekräftig, wie sie im Rahmen der Betriebsrisikolehre traditionell für die Bestimmung eines Arbeitsunfalls angewandt wurden. Die Abgrenzung von Arbeit und Freizeit kann in diesen Fällen nicht mehr nach räumlich-zeitlichen Kriterien vorgenommen werden, was dazu führt, dass das Kriterium der physischen Beherrschbarkeit des Arbeitgebers für die Zuordnung des Arbeitsunfalls nicht mehr zeitgemäß ist. Es liegt daher die Vermutung nahe, dass sich die geänderte Lebenswirklichkeit der Beschäftigten negativ auf die Reichweite ihres Unfallversicherungsschutzes auswirkt. Dies zu bestätigen und einen Lösungsvorschlag für die dadurch entstandene Erosion des Unfallversicherungsschutzes der Beschäftigten zu entwickeln, ist Ziel der Kapitel drei und vier. 


\section{Kapitel 3: Konkrete Auswirkungen entgrenzter Arbeit auf die Reichweite des Unfallversicherungsschutzes}

\section{A. Arbeitsunfall und Homeoffice}

\section{Begriff, Bedeutung und Ausmaß von Arbeit im Homeoffice}

Die teilweise oder gänzliche Auslagerung der Erwerbstätigkeit aus dem Betrieb in den eigenen Wohnbereich des Beschäftigten wird unter mehreren Begriffen diskutiert, die verschiedene Unzulänglichkeiten aufweisen, wie folgend deutlich wird. Besonders häufig ist von „Teleheimarbeit“ oder „Telearbeit“ die Rede. Damit waren, wie das Präfix „Tele-“ zeigt, zunächst nur solche Arbeiten gemeint, die unter Zuhilfenahme von Fernkommunikationsmitteln ausgeführt werden. ${ }^{635}$ Die Anwendungsbreite außerbetrieblicher Arbeitsstätten beschränkt sich jedoch nicht auf den Bereich der Bildschirmarbeit. Viele Tätigkeiten können außerhalb des Unternehmens erledigt werden und setzen keine ständige telekommunikative Vernetzung mit dem Betrieb voraus. In vielen Branchen ist Arbeitszeit nicht primär betriebliche Anwesenheitszeit, sondern „ortsunabhängige Denkzeit““636 „Telearbeit" bezieht sich zudem allgemein auf ortsunabhängige Arbeit und beschränkt sich nicht auf die Arbeit im häuslichen Bereich; die Unterscheidung nach Ausführungsorten ist aber, wie die nächsten Abschnitte zeigen werden, für die Unfallversicherung von Relevanz und sollte sich daher auch begrifflich widerspiegeln. „Heimarbeit“ wiederum ist keine glückliche Wortwahl, weil sie einen Bezug zum Heimarbeitsgesetz suggeriert, welches aber nur Personen betrifft, die keinem Direktionsrecht unterliegen und somit nicht abhängig beschäftigt sind. Etabliert hat sich zudem der Scheinanglizismus Homeoffice, welcher zwar zu Recht aufgrund seiner fal-

635 Wedde, Telearbeit, S. 2 Rn. 6; Kleemann, in: Gottschall/Voß (Hrsg.), Entgrenzung von Arbeit und Leben, S. 59, 61. Eine Definition des Telearbeitsplatzes findet sich in $₫ 2$ Abs. 7 ArbStättV, nach dem Telearbeitsplätze vom Arbeitgeber fest eingerichtete Bildschirmarbeitsplätze im Privatbereich der Beschäftigten sind, für die der Arbeitgeber eine mit dem Beschäftigten vereinbarte wöchentliche Arbeitszeit und die Dauer der Einrichtung festgelegt hat.

636 Albrecht, NZA 1996, S. 1240, 1240. 
schen Nutzung im Deutschen kritisiert wird, ${ }^{637}$ jedoch von zutreffenden inhaltlichen Prämissen ausgeht, im deutschen Sprachraum als Erwerbsarbeit im Wohnbereich verstanden wird und damit den geeignetsten Begriff für die vorliegende Untersuchung darstellt. ${ }^{638}$ Gemeint ist hier die Ausführung einer arbeitsvertraglich geschuldeten Tätigkeit aus dem häuslichen Bereich des Beschäftigten heraus, gleichgültig, ob lediglich im Homeoffice oder abwechselnd zu Hause und in der Betriebsstätte gearbeitet wird (sog. alternierende Telearbeit). Häusliche Arbeitsplätze kamen vermehrt Mitte der 1970er Jahre auf, als die Verringerung der Pendlerströme und ein Plus an individuellen Gestaltungsmöglichkeiten des Alltags als Vorteile dieser Art von Tätigkeit erkannt wurden. ${ }^{639}$ Heute ist primäres Motiv der Beschäftigten für eine Arbeit von zuhause die bessere Vereinbarkeit von Familie und Beruf, die man sich dadurch verspricht. ${ }^{640}$ Tatsächlich ist belegt, dass häusliche Erwerbsarbeit die Work-Life-Balance der betroffenen Personen verbessern kann. ${ }^{641}$ Frauen, die auch im Homeoffice arbeiten, sind insgesamt mehr Stunden erwerbstätig und seltener prekär beschäftigt; Männer übernehmen unter diesen Bedingungen mehr Sorgearbeit im Rahmen der Kinderbetreuung. ${ }^{642}$ In einer repräsentativen Längsschnittstudie für sozialversicherungspflichtig Beschäftigte in Deutschland konnte gezeigt werden, dass rund $40 \%$ der Befragten zumindest gelegentlich im Homeoffice arbeiten wollen. ${ }^{643}$ Andere Quellen geben an, dass dies sogar für die Hälfte aller Befragten gilt. ${ }^{644}$ Tatsächlich arbeiten in Deutschland derzeit etwa 31 Prozent aller Erwerbstätigen und 12 Prozent aller abhängig Beschäftigten auch

637 Der Begriff „Home Office“ ist die ursprüngliche Bezeichnung für das englische Innenministerium; die Erbringung von Arbeitsleistung von zu Hause könnte im Englischen korrekt als „working from home“ bezeichnet werden, häusliche Arbeitsplätze als „offices at home“, vgl. Ricke, WzS 2017, S. 9, 10.

638 Aus Gründen der verbesserten Lesbarkeit werden auch die anderen Begriffe beizeiten genutzt, um Wiederholungen zu vermeiden.

639 Angermaier, jM 2017, S. 69, 69 f. Selbstverständlich gab es bereits früher Menschen, die wohnten, wo sie arbeiteten; dazu mit Beispielen aus der unfallversicherungsrechtlichen Rechtsprechung Ricke, WzS 2017, S. 9, 10.

640 BMAS, Mobiles und entgrenztes Arbeiten, S. 17.

641 Lott, Stressed despite or because of flexible work arrangements?, Hans Böckler Stiftung Working Paper Nb. 46/2017, S. 8 m.w.N.

642 Boll, Die Arbeitsteilung im Paar, S. 50.

643 Arnold/Steffes/Wolter (Hrsg.), Mobiles und entgrenztes Arbeiten, S. 17.

644 So etwa die Expertise der Roland Berger GmbH, BMFSJ (Hrsg.), Digitalisierung - Chancen und Herausforderungen, https://www.bmfsfj.de/blob/75934/433b3a0 $5 \mathrm{df5} 43 \mathrm{f} 87 \mathrm{bd} 2 \mathrm{cce} 88 \mathrm{ae} 6 \mathrm{c} 7 \mathrm{cf} 6 /$ digitalisierung-chancen-und-herausforderungen-dat a.pdf (Stand: 18.11.2018), S. 21, sowie eine im Auftrag von Thompson Reuters News Service durchgeführte marktforschungsorientierte Umfrage von Ipsos 
von zu Hause. ${ }^{645}$ Die Diskrepanz zwischen Nachfrage und tatsächlicher häuslicher Arbeit erklärt sich dadurch, dass Arbeitgeber in Deutschland dem Arbeiten im Homeoffice zögerlich gegenüberstehen. ${ }^{646}$ Die Möglichkeit, im Homeoffice zu arbeiten, ist für deutsche Arbeitnehmer aufgrund des vom Arbeitgeber zu leistenden Vertrauensvorschusses ein Statusmerkmal und findet sich vor allem in höherqualifizierten Berufen. ${ }^{647}$ Unter abhängig Beschäftigten mit Hochschulabschluss arbeitet bereits jetzt ein Drittel zuhause. ${ }^{648}$ Ein Blick auf die Nachbarländer, in denen bereits deutlich mehr Menschen von zu Hause arbeiten, ${ }^{649}$ sowie immer lauter werdende Rufe nach einem Umdenken der Unternehmer lassen indes vermuten, dass die Zahlen weiter steigen werden. ${ }^{650}$ Arbeitgeber profitieren bei alternierender Telearbeit insbesondere von der erhöhten Qualität der Ar-

2011 in 24 Ländern mit ca. 19.000 Befragten, http://www.innovations-report.de/ html/berichte/studien-analysen/zweite-deutsche-wuerde-hause-arbeiten-duerfte-1 89208.html (Stand: 18.11.2018).

645 Bundesverband Informationswirtschaft, Telekommunikation und Neue Medien (Bitkom), Arbeiten in der digitalen Welt, Berlin 2013, zitiert nach Günther/Böglmüller, NZA 2015, S. 1025, 1029; Brenke, DIW 5/2016, S.96. Der Anteil der zu Hause arbeitenden Personen ist damit wieder im Steigen begriffen, nachdem er im Jahr 2014 als rückgängig angesehen wurde, vgl. Brenke, DIW 8/2014, S. 131; einen weiteren Rückgang der Homeoffice-Zahlen vermuten Giesen/Kersten, Arbeit 4.0, S. $103 \mathrm{f}$. Nach Zahlen des BMAS nehmen allerdings sogar 15 Prozent der Arbeitnehmer regelmäßig Arbeit mit nach Hause, vgl. Krause, Digitalisierung der Arbeitswelt, Gutachten B zum 71. DJT, B 28 m.w.N.; BMAS, Mobiles und entgrenztes Arbeiten, S. $9 \mathrm{f}$.

646 Expertise der Roland Berger GmbH, BMFSJ (Hrsg.), Digitalisierung - Chancen und Herausforderungen, https:/www.bmfsfj.de/blob/75934/433b3a05df543f87b d2cce88ae6c7cf6/digitalisierung-chancen-und-herausforderungen-data.pdf (Stand: 18.11.2018), S. 21.

647 Lott, Selbstorganisiertes Arbeiten als Ressource für Beschäftigte nutzen, S. 5 f.

648 Brenke, DIW 5/2016, S. 99.

649 Ebd., S. 98. Spitzenreiter der Studie ist Island mit einem Anteil von mehr als 30 Prozent aller abhängig Beschäftigten.

650 Über 96 Prozent der befragten Unternehmen stimmten der Aussage zu, sie wollten das Angebot für digital gestützte flexible Arbeitsformen erweitern, vgl. Expertise der Roland Berger GmbH, BMFSJ (Hrsg.), Digitalisierung - Chancen und Herausforderungen, https://www.bmfsfj.de/blob/75934/433b3a05df543f87b d2cce88ae6c7cf6/digitalisierung-chancen-und-herausforderungen-data.pdf (Stand: 23.11.2018), S. 20. Gründe für die im Gegensatz zu anderen Ländern zögerliche Entwicklung des Homeoffices in Deutschland liegen wohl auch in der hierzulande noch stark ausgeprägten Präsenzkultur, vgl. Institut für Demoskopie Allensbach, Zu Hause arbeiten, https:/www.bmfsfj.de/blob/75938/508d01a6051f ae61633ce098b84f58d3/bericht-zuhause-arbeiten-data.pdf (Stand: 23.11.2017), S. 32 . 
beit sowie deutlich längeren Arbeitszeiten der im Homeoffice Beschäftigten. ${ }^{651}$

\section{Unfall bei Arbeitstätigkeit am häuslichen Arbeitsplatz}

Arbeitnehmer, die ihre Arbeitsleistung teilweise oder vollständig von zu Hause erbringen, sind ohne Weiteres Beschäftigte im Sinne der $\mathbb{} 7$ Abs. 1 SGB IV, $\mathbb{2}$ Abs. 1 Nr. 1 SGB VII. ${ }^{652}$ Für sie gelten daher die in Kapitel 1 dargelegten allgemeinen Grundsätze des Versicherungsschutzes. ${ }^{653}$ Die im Folgenden dargestellten Besonderheiten für Personen, die von zu Hause arbeiten, ergeben sich erst aus der Anwendung dieser Grundsätze. Sie finden sich insbesondere auf der Ebene des sachlichen Zusammenhangs zwischen tatsächlicher Verrichtung im Unfallzeitpunkt und versicherter Tätigkeit, also bei der Frage nach der Erfüllung des Versicherungstatbestandes.

Der Grundfall eines Unfalls im Homeoffice ist unproblematisch zu lösen. Verunfallt ein Beschäftigter während und wegen einer eindeutig und ausschließlich seiner versicherten Tätigkeit zuzurechnenden Verrichtung direkt an seinem vertraglich vereinbarten häuslichen Arbeitsplatz, liegt unstrittig ein Arbeitsunfall vor. ${ }^{654}$ Fügt sich beispielsweise ein Mitarbeiter einer Personalabteilung, der in Absprache mit seiner Arbeitgeberin an seinem häuslichen Schreibtisch Bewerbungsunterlagen sortiert, beim Heften dieser Dokumente eine Wunde zu, indem er sich die Heftklammer versehentlich in den Finger treibt, liegt ein Arbeitsunfall vor, da die tatsächliche Verrichtung - das Heften - darauf gerichtet war, eine eigene, objektiv bestehende Hauptpflicht aus seinem Beschäftigungsverhältnis zu erfüllen und das Heften auch wesentliche Bedingung für das Unfallereignis war. Dieser Grundfall fügt sich unproblematisch in das Grundgefüge der Schutzzwecke und Rechtsgründe der Unfallversicherung ein. Das sich im

651 Wedde, Telearbeit, S. 8 f.; Brenke, DIW 5/2016, S. $101 \mathrm{f}$.

652 Albrecht, NZA 1996, S. 1240, 1241; Boemke/Ankersen, BB 2000, S. 1570, 1572.

653 Wolber, SozVers 1997, S. 239, 239; Wedde, Telearbeit, Rn. 574.

654 Leube, SGb 2012, S. 380, 381; Wolber, SozVers 1997, S. 239, 239; Ricke, in: Körner/Leitherer et al. (Hrsg.), Kasseler Kommentar zum Sozialversicherungsrecht,

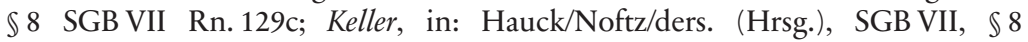
Rn. $116 \mathrm{f}$. Weniger eindeutig gelingt die Einordnung des Unfalls, wenn er sich nicht direkt am eingerichteten häuslichen Arbeitsplatz, sondern an einem anderen Ort in der Wohnung ereignet hat, wenn eine gemischte Tätigkeit vorliegt oder der Unfall während eines Weges innerhalb der Wohnung oder von der Wohnung aus stattfindet, s.u. Hier gehen die Meinungen über den anzulegenden Entscheidungsmaßstab auseinander. 
Unfallschaden verwirklichende Risiko ist nach der Betriebsrisikolehre der Herrschaftssphäre der Arbeitgeberin zuzuordnen. Denn obwohl sich der häusliche Arbeitsplatz in der privaten Umgebung des Arbeitnehmers befindet, dessen Gefahren dieser aufgrund der Integration in seinen persönlichen Lebensbereich primär selbst einschätzen, beherrschen und regulieren kann, reicht die Organisations- und Verfügungshoheit der Arbeitgeberin deutlich in das Homeoffice hinein. Zunächst einmal obliegt es der Arbeitgeberin, überhaupt eine Beschäftigung von zu Hause zuzulassen. Kraft ihres Weisungsrechts aus $\$ 106$ GewO bestimmt sie einseitig, wo der Versicherte tätig wird. ${ }^{655}$ Jedenfalls bisher gibt es kein „Recht auf Homeoffice ${ }^{“ 656}$, das der Beschäftigte in Anspruch nehmen könnte. Indem sie häusliche Arbeit anweist, übt die Arbeitgeberin ihre Organisationshoheit über den Ablauf der betrieblichen Tätigkeit aus. Sie nimmt die durch die Ausübung von Tätigkeiten im Homeoffice zusätzlich entstehenden Risiken bewusst in die Risikosphäre ihres Unternehmens auf, um im Gegenzug den mit Telearbeit verbundenen Nutzen zu genießen, was die Zuordnung nach dem der Betriebsrisikolehre immanenten Gewinnerzielungsargument nach sich zieht. ${ }^{657}$

Der häusliche Arbeitsplatz ist als Teil des Betriebs zu qualifizieren, sodass für die Zuordnung zum Betriebsrisiko dieselben Grundsätze wie für solche auf dem Betriebsgelände gelten. ${ }^{658}$ Folgerichtig bestehen für Arbeitgeber nicht nur Möglichkeiten, sondern auch Pflichten, die Einrichtung des häuslichen Arbeitsplatzes seines Beschäftigten zu beeinflussen und zu kontrollieren. Für die Einhaltung der Arbeitsschutzvorschriften sind auch

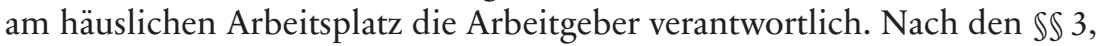
4 ArbSchG i.V.m. $\$ 618$ BGB trifft die Arbeitgeber die Pflicht zur gefahrfreien Gestaltung des Arbeitsplatzes. ${ }^{659}$ Nach der das Arbeitsschutzgesetz konkretisierenden Arbeitsstättenverordnung richtet der Arbeitgeber Telear-

655 Imping, in: Kilian/Heussen (Hrsg.), Computerrechts-Handbuch, 70.11 Rn. 17 und 19.

656 Bisher besteht lediglich ein Recht des Arbeitnehmers auf ermessensfehlerfreie Entscheidung des Arbeitgebers, ob Homeoffice möglich ist, vgl. Isenhardt, DB 2016, S. 1499, 1499; die Einführung eines gesetzlichen Anspruchs wird diskutiert, vgl. BMAS, Arbeiten 4.0, S. 120 f.; Beschlüsse des 71. DJT, Arbeits- und sozialrechtliche Abteilung, Beschluss III.1 (abrufbar unter http://www.djt.de/filea dmin/downloads/71/Beschluesse_gesamt.pdf, Stand: 18.11.2018). Zur Ausgestaltung des Rechts auf Homeoffice in den Niederlanden Walser, ArbuR 2016, S. $338 \mathrm{ff}$.

657 Wedde, Telearbeit, S. 91 f., Rn. 382 ff.

658 Ebd.

659 Oberthür, NZA 2013, S. 246, 247. 
beitsplätze als fest eingerichtete Bildschirmarbeitsplätze im Privatbereich der Beschäftigten ein. ${ }^{600}$ Die Arbeitsmittel, etwa Mobiliar und Kommunikationseinrichtungen, stellt dabei der Unternehmer zur Verfügung. Er hat dafür zu sorgen, dass der Arbeitsbereich professionell und ergonomisch ausgestattet ist. Freilich ist dies nur in gegenüber den Möglichkeiten an seiner eigenen Betriebsstätte eingeschränkter Form möglich. Konkret hat der Arbeitgeber den häuslichen Arbeitsplatz einzurichten und dabei eine Gefährdungsbeurteilung nach $\$ 3$ ArbStättV vorzunehmen, entsprechend deren Ergebnisse Maßnahmen zum Schutz der Beschäftigten zu ergreifen sind, $\mathbb{} 3$ Abs. 1 S. 4 ArbStättV. Bei Telearbeitsplätzen ist lediglich bei der erstmaligen Beurteilung der Arbeitsbedingungen und des Arbeitsplatzes eine Gefährdungsprognose zu erstellen, $\mathbb{\$} 1$ Abs. 3 S. 1 Nr. 1 ArbStättV. Zudem muss der Arbeitgeber die Arbeitnehmer gemäß $\$ 6$ ArbStättV hinsichtlich des Arbeitsschutzes unterweisen, wobei er Informationen über das bestimmungsgemäße Betreiben der Arbeitsstätte sowie alle gesundheits- und sicherheitsrelevanten Fragen in Zusammenhang mit ihrer Tätigkeit zu geben hat. In der Praxis hat der Arbeitgeber also vor erstmaliger Einrichtung des häuslichen Arbeitsplatzes eine Gefährdungsbeurteilung zu erstellen, für die er entweder vorher den häuslichen Arbeitsplatz besichtigen muss oder die häuslichen Umstände beim Beschäftigten zu erfragen hat. ${ }^{661}$ Für eine Besichtigung benötigt der Arbeitgeber die Zustimmung des Arbeitnehmers, welcher allerdings nach $\mathbb{1} 15$ Abs. 1 S. 1 ArbSchG zum Eigenschutz und nach $\mathbb{1} 16$ Abs. 2 S. 1 ArbSchG zur Mitwirkung verpflichtet ist. ${ }^{662}$ Insgesamt beherrscht der Arbeitgeber kraft des Arbeitsschutzrechts auch den häuslichen Arbeitsplatz in erheblichem Maße. ${ }^{663}$ Seine

660 „Telearbeitsplätze sind vom Arbeitgeber fest eingerichtete Bildschirmarbeitsplätze im Privatbereich der Beschäftigten, für die der Arbeitgeber eine mit den Beschäftigten vereinbarte wöchentliche Arbeitszeit und die Dauer der Einrichtung festgelegt hat. Ein Telearbeitsplatz ist vom Arbeitgeber erst dann eingerichtet, wenn Arbeitgeber und Beschäftigte die Bedingungen der Telearbeit arbeitsvertraglich oder im Rahmen einer Vereinbarung festgelegt haben und die benötigte Ausstattung des Telearbeitsplatzes mit Mobiliar, Arbeitsmitteln einschließlich der Kommunikationseinrichtungen durch den Arbeitgeber oder eine von ihm beauftragte Person im Privatbereich des Beschäftigten bereitgestellt und installiert ist.“ $\mathbb{} 2$ Abs. 7 ArbStättV, eingefügt mit Novelle v. 3.12.2016, BGBl I, S. 2681.

661 Wiebauer, in: Landmann/Rohmer (Begr.), Gewerbeordnung, $\mathbb{1}$ ArbSchG Rn. 71; Wissenschaftliche Dienste des Deutschen Bundestags, Sachstand Telearbeit und Mobiles Arbeiten, WD 6 - 3000 - 149/16, S. 8.

662 Wiebauer, in: Landmann/Rohmer (Begr.), Gewerbeordnung, $\mathbb{1}$ ArbSchG Rn. 72; Wissenschaftliche Dienste des Deutschen Bundestags, Sachstand Telearbeit und Mobiles Arbeiten, WD 6 - 3000 - 149/16, S. 8.

663 Ebenso Häfner-Beil/Nacke, Gute Arbeit 2016, S. 4. 
Verantwortung für das Arbeitsumfeld spricht auch bei Telearbeitsplätzen im Sinne der Betriebsrisikolehre für eine Zuordnung zum versicherten Bereich. ${ }^{664}$

Gelegentlich werden allerdings Bedenken hinsichtlich der Beweisbarkeit der Erfüllung des Versicherungstatbestands vorgebracht. Es wird befürchtet, Beschäftigte im Homeoffice würden wahrheitswidrig angeben, zum Unfallzeitpunkt eine berufliche Tätigkeit verrichtet zu haben, um in den Genuss der höheren Leistungen der gesetzlichen Unfallversicherung zu kommen. ${ }^{665}$ Bisher wurden derartige Versuche nicht dokumentiert. ${ }^{666}$ Ein solches Missbrauchsrisiko besteht im Übrigen ebenso bei Unfällen, die sich auf dem Betriebsgelände ereignen. Genauso wie im Homeoffice kann es auch hier zu Geschehnissen kommen, die weder durch Zeugen beobachtet noch aufgezeichnet oder im Detail nachvollzogen werden können. Auch hier kann der Versicherungsträger praktisch kaum abschließend überprüfen, ob die Angaben zum Unfallereignis der Wahrheit entsprechen. ${ }^{667} \mathrm{Be}-$ weisschwierigkeiten treffen zudem die Beschäftigten, welche die Beweislast hinsichtlich des Vorliegens eines Arbeitsunfalls tragen. Zu begrüßen ist daher die Vorgehensweise des BSG, welches - jedenfalls im Bereich von zuhause tätiger Selbstständiger - betont, die Schwierigkeit der Beweisführung hinsichtlich der Handlungstendenz im häuslichen Bereich müsse berücksichtigt werden. ${ }^{668}$

III. Verrichtungen mit gespaltener Handlungstendenz bei häuslicher Arbeit

Uneinigkeit über die für die Zurechnung zur Risikosphäre des Arbeitgebers anzulegenden Maßstäbe bei der Einordnung von Unfällen als Arbeitsunfälle besteht bei Tätigkeiten mit gemischter Motivationslage im Zusammenhang mit häuslicher Arbeit.

Wenn die zum Unfallzeitpunkt tatsächlich verrichtete Tätigkeit sowohl privat als auch beruflich motiviert ist, wird der für die Erfüllung des Versi-

664 A.A. Spellbrink, NZS 2016, S. 527, 530.

665 Wedde, Telearbeit, Rn. 588.

666 Ricke, WzS 2017, S. 9, 13.

667 Vgl. zu Möglichkeiten der Berücksichtigung von Angaben der Versicherten im Rahmen der Beweiswürdigung Keller, in: Hauck/Noftz/ders. (Hrsg.), SGB VII, $\$ 8$ Rn. 330a.

668 Vgl. BSG, Urt. v. 31.8.2017 - B 2 U 9/16 R -, juris Rn. 17 (zur Veröffentlichung in BSGE vorgesehen). 
cherungstatbestands erforderliche sachliche Zusammenhang bei Tätigkeiten mit gespaltener Handlungstendenz in der Betriebsstätte dann bejaht, wenn die Verrichtung hypothetisch auch dann vorgenommen worden wäre, wenn die privaten Gründe des Handelns nicht vorgelegen hätten. ${ }^{669}$

Im Gegensatz dazu wird vertreten, dass bei einer gespaltenen Handlungstendenz während der Arbeit von zu Hause grundsätzlich kein Unfallversicherungsschutz besteht. ${ }^{670}$ Es sei vielmehr ohne tatsächliche Prüfung der Annahme davon auszugehen, dass die Handlung ohne private Motivation nicht ausgeführt worden wäre und ein sachlicher Zusammenhang zwischen der verrichteten Tätigkeit und dem Unfallereignis daher nicht vorliegt. ${ }^{671}$ Als Grund für diese als Vermutung einzuordnende Regel werden die häusliche Umgebung und der gewöhnliche Lebensrhythmus des Beschäftigten angegeben, aufgrund derer die konkrete Durchführung der Verrichtung nicht durch betriebliche Umstände vorgegeben sei. ${ }^{672} \mathrm{Im}$ Fall einer freiwillig versicherten Selbstständigen, die auf dem Weg zum Empfang von möglicherweise privater, möglicherweise beruflicher Post zwischen Arbeitszimmer und Haustür auf der Treppe verunfallte, entschied das LSG Baden-Württemberg mit dieser Begründung, dass kein Arbeitsunfall vorlag. ${ }^{673}$

Jedoch sind Unfälle auch im häuslichen Bereich bei Vorliegen einer gemischten Tätigkeit nicht anders zu behandeln als Unfälle, die an der Betriebsstätte stattfinden. Auch hier ist danach zu fragen, ob die tatsächliche Verrichtung zum Unfallzeitpunkt hypothetisch auch dann vorgenommen worden wäre, wenn die private Motivation entfallen wäre. ${ }^{674}$ Dieses Ergebnis ergibt sich aus den Grundprinzipien der Unfallversicherung. Deutlich

669 S.o. Kap. 1 D. III. 2. c. cc.

670 LSG Baden-Württemberg, Urt. v. 9.2.2015 - L 1 U 1882/14 -, juris; Leube, SGb 2012, S. 380, 381; Keller, in: Hauck/Noftz/ders. (Hrsg.), SGB VII, $\$ 8$ Rn. 116a.

671 Leube, SGb 2012, S. 380, 381, der dies als allgemeine Ansicht darstellt und dazu Ricke, in: Körner/Leitherer et al. (Hrsg.), Kasseler Kommentar zum Sozialversicherungsrecht, $\mathbb{S} 8$ SGB VII Rn. 130 zitiert, den er in seinem Sinne versteht. Letzterer schreibt a.a.O. jedoch lediglich, im häuslichen Bereich (nicht im Arbeitsbereich zu Hause) bestünde nur dann Versicherungsschutz, wenn der Unfall „sich klar als tätigkeitsbedingt unabhängig vom Wohnumfeld zeigt“. Eine Positionierung zur Frage nach der gemischten Tätigkeit ist daraus jedoch nicht eindeutig ableitbar. Denn wäre die Tätigkeit hypothetisch auch ohne private Motivation ausgeführt worden, ist sie ebenso als unabhängig vom Wohnumfeld zu qualifizieren.

672 Leube, SGb 2012, S. 380, 381; Keller, in: Hauck/Noftz/ders. (Hrsg.), SGB VII, $\mathbb{} 8$ Rn. 116a.

673 LSG Baden-Württemberg, Urt.v. 9.2.2015 - L 1 U 1882/14 -, juris.

674 Ebenso Brink, FD-SozVR 2015, 368217; Spellbrink, NZS 2016, S. 527, 528. 
wird es an folgendem Beispiel:675 Ein Beschäftigter, der von zu Hause arbeitet, unterbricht seine am Schreibtisch stattfindende berufliche Tätigkeit, um seinen Briefkasten zu leeren. Dies wollte er, wie jeden Tag, routinemäßig tun, ohne sicher zu wissen, welche Sendungen sich darin befanden. Es sammelt sich dort regelmäßig gleichermaßen berufliche wie auch private Post. Während des Aufsperrens des Briefkastens verunfallt der Beschäftigte. Nun ist fraglich, ob es sich bei dem erlittenen Unfall um einen Arbeits- oder einen Privatunfall handelt. Zweifellos ist das Ausleeren des Briefkastens sowohl privat als auch beruflich motiviert, da der Beschäftigte nie vollkommen ausschließen können wird, dass sich neben beruflicher auch private Sendungen darin befinden - und umgekehrt. Es liegt deswegen eine gemischte Tätigkeit vor, sodass die erstgenannte Ansicht das Vorliegen eines sachlichen Zusammenhangs zwischen der tatsächlichen Verrichtung und der versicherten Tätigkeit verneinen würde. Gestützt wird dies durch den Gedanken, dass das Ausleeren des Briefkastens auch ohne berufliche Konnotation in genau derselben Art und Weise stattgefunden hätte; es wird durch die tägliche Routine des Versicherten bestimmt und stellt eine in das private Lebensumfeld des Verunfallten eingeordnete Tätigkeit dar, die keinen vom Arbeitgeber beherrschbaren betrieblichen Zwängen unterliegt. ${ }^{676}$ Damit sei sie, so die Vertreter dieser Ansicht, auch nicht in der gesetzlichen Unfallversicherung zu schützen. Diese Argumentation basiert auf der Annahme, dass der für die Risikoverteilung in der Unfallversicherung tragende Gedanke der Betriebsrisikolehre hier nicht greife, weil der Arbeitgeber keinen Einfluss auf die Organisation des Ausleerens des Briefkastens hat und den Ablauf deswegen nicht beherrscht, sodass sich daraus ergebende Risiken nicht in seinen Verantwortungsbereich einordnen ließen. Diese Argumentation greift allerdings zu kurz, denn sie bezieht sich lediglich auf einen Teilaspekt der Betriebsrisikolehre und lässt die anderen Rechtsgründe, aus denen Unfallversicherungsschutz gewährt wird, außer Betracht. Wie bereits dargestellt wurde, umfasst der Versicherungsschutz nicht nur Unfälle, die der Arbeitgeber durch eine andersartige Organisation der Betriebs- und Arbeitsabläufe verhindern hätte können, sondern gerade auch unverschuldete, fremdverschuldete oder zufällige Unfälle, die dem Betriebsrisiko des Arbeitgebers deswegen angehören, weil

675 Von Leube, SGb 2012, S. 380, 381 angedeutet und wohl auf LSG Baden-Württemberg, Urt. v. 9.2.2015 - L 1 U 1882/14 -, juris, basierend. In diesem Urteil ging es allerdings nicht um eine Beschäftigte, sondern um eine freiwillig versicherte selbstständige Person.

676 Leube, SGb 2012, S. 380, 381; Keller, in: Hauck/Noftz/ders. (Hrsg.), SGB VII, $\mathbb{} 8$ Rn. 116a. 
sich der Beschäftigte gerade in seiner Eigenschaft als Arbeitnehmer in der zum Unfall führenden Situation befand. Der Arbeitgeber muss also das Unfallgeschehen gerade nicht unmittelbar faktisch beherrschen. Vielmehr genügt es für die Bejahung einer Zuordnung zum Betriebsrisiko des Arbeitgebers, wenn der Arbeitnehmer infolge seines Beschäftigungsverhältnisses verunfallte. Handelt es sich - wie hier - bei der zum Unfallzeitpunkt ausgeführten Tätigkeit um eine Verrichtung, die dieser sowohl als Beschäftigter als auch als Privatperson tätigt, wird das Zuordnungsproblem in bewährter Weise sachgerecht mit der hypothetischen Frage nach dem Entfallen der privaten Motivation gelöst. Nur wenn die Tätigkeit in derselben Weise ebenso vorgenommen wäre, wenn die private Motivation hinweggedacht wird, ist der sachliche Zusammenhang der Verrichtung mit der versicherten Tätigkeit zu bejahen. Im konkreten Beispiel ist dies der Fall. Die hinwegzudenkende private Handlungstendenz ist der Wille, private Post aus dem Postkasten zu entnehmen. Auch, wenn der Beschäftigte sich sicher gewesen wäre, an diesem Tag keine private Post zu erhalten, hätte er den Briefkasten in derselben Art und Weise geleert, wenn er es für möglich gehalten hätte, dass dienstliche Sendungen eingeworfen wurden. Das Entfallen der privaten Motivation ändert nichts an der Ausführung der Verrichtung. Damit ist der sachliche Zusammenhang zwischen der tatsächlichen Verrichtung und der versicherten Tätigkeit zu bejahen.

Dies ist vor dem Hintergrund der Schutzzwecke der Unfallversicherung auch sachgerecht. Der Beschäftigte ist eben auch wegen der Möglichkeit beruflicher Post mit der Leerung des Briefkastens befasst. Daran ändert die Tatsache, dass es sich um die häusliche Umgebung des Arbeitnehmers handelt, nichts. Der für die Zuordnung nach den Regeln der Fallgruppe der gemischten Tätigkeit eigentlich notwendige Umkehrschluss, dass der Gang zum Briefkasten ohne private Gründe nicht ausgeführt worden wäre, lässt sich aus der Tatsache, dass der Beschäftigte den Briefkasten auch privat leeren würde, wenn er keine dienstliche Post erwartet, gerade nicht ziehen. Dienstliche Sendungen wiederum empfängt er nur, weil er sich im Arbeitsvertrag mit dem Arbeitgeber dazu verpflichtet hat, eine Arbeitsleistung zu erbringen, zu der auch das Entgegennehmen von beruflicher Post gehört. Mit diesem Argument ist der Bezug zur Herrschaftssphäre des Arbeitgebers nach der Betriebsrisikolehre hergestellt. Auch die für die Betriebsrisikolehre zweite maßgebliche Komponente, das Gewinnerzielungsargument, greift für den Empfang dienstlicher Post im Wohnumfeld des Beschäftigten genauso wie für deren Entgegennahme in der Betriebsstätte. 
Nicht notwendig ist es, zwischen kommunikationsbezogenen und anderen Berufsfeldern zu unterscheiden. ${ }^{677}$ Brink schlug vor, Wege zur Empfangnahme von Post in Berufen, in denen Kommunikation wichtig ist, immer zu versichern, und implizierte damit, diese in anderen Berufsfeldern als unversichert anzusehen zu wollen. Als Beispiele für „kommunikative“ Branchen nannte er die Rechtsanwaltschaft und den Journalismus. Für eine solche Trennung in Sparten liegen allerdings keine Sachgründe vor. Es ist nicht rechtfertigbar, eine berufliche Verrichtung in einem Berufsfeld nicht unter Versicherungsschutz zu stellen, nur weil sie weniger häufig als in anderen Branchen zu erledigen ist. Sobald ein Beschäftigter eine Verrichtung vornimmt, um eine Pflicht aus seinem Beschäftigungsverhältnis zu erfüllen, handelt es sich um eine Verrichtung, die den Versicherungstatbestand erfüllt, gleichgültig, wie häufig diese Pflicht auftritt. Maßgeblich ist allein die Handlungstendenz des Beschäftigten.

Der genannte Vorschlag enthielt weiterhin die Empfehlung, den Versicherungsschutz von Unfällen im Zusammenhang mit Postsendungen entfallen zu lassen, soweit die konkrete Lieferung privater Natur sei. ${ }^{678}$ Auch darauf kann es aber nicht ankommen. Solange der Beschäftigte davon ausgeht, dass eine berufliche Sendung möglich ist, handelt er auch mit beruflicher Handlungstendenz, sodass der sachliche Zusammenhang zwischen tatsächlicher Verrichtung und versicherter Tätigkeit gegeben ist. Ansonsten entschiede der Zufall, nämlich welche Post sich genau im Briefkasten befindet, darüber, ob Versicherungsschutz besteht oder nicht. Dies wäre nicht nur unbefriedigend ${ }^{679}$, sondern auch mit den Grundsätzen der Unfallversicherung unvereinbar, nach denen jeder Unfall, der infolge einer versicherten Tätigkeit eintritt, als Arbeitsunfall zu qualifizieren ist. Denn dass sich nur private Sendungen im Briefkasten befanden, ändert nichts daran, dass er mit der auch nach außen erkennbaren Zielrichtung, auch berufliche Post herauszuholen, geleert worden ist.

677 So Brink, FD-SozVR 2015, 368217.

678 Ebd.

679 Ebd. 
IV. Betriebswege innerhalb des häuslichen Bereichs

1. Widmung des Unfallorts als maßgebendes Kriterium

a. Häufigkeit der Nutzung des Unfallortes zu betrieblichen Zwecken als maßgebliches Kriterium

Schwierigkeiten bereitet weiterhin die Einordnung von Unfällen, die sich auf Betriebswegen innerhalb des Lebensbereichs des Beschäftigten ereignen. Dazu werden im Wesentlichen drei Meinungen vertreten, welche im Folgenden kritisch beleuchtet werden.

Nach der bisherigen höchstrichterlichen Rechtsprechung sowie Teilen der Literatur soll es für die Bejahung eines sachlichen Zusammenhangs darauf ankommen, wie häufig der konkrete Unfallort zu betrieblichen Zwecken genutzt wird (dazu sogleich); anderer Ansicht nach kann es für Wege innerhalb eines Wohngebäudes von vornherein keinen Unfallversicherungsschutz geben (dazu b.). Zu folgen ist dagegen der in der Literatur verbreiteten und wohl auch vom Bundessozialgericht angenommenen Sichtweise, dass es auch für die Beurteilung von Betriebswegen im Wohngebäude maßgeblich auf die Handlungstendenz des Versicherten zum Unfallzeitpunkt ankommen muss (dazu 2.).

Unter Betriebswegen werden im Unfallversicherungsrecht Wege verstanden, deren Zurücklegen selbst eine versicherte Tätigkeit darstellt. ${ }^{600} \mathrm{Ge}-$ meint sind also Wege, die nicht zurückgelegt werden, um am Bestimmungsort eine versicherte Tätigkeit zu verrichten, sondern Wege, die selbst als Arbeitsleistung geschuldet sind. ${ }^{681}$ Anders als Wegeunfälle sind Betriebswege folgerichtig bereits vom Schutzbereich des $\mathbb{} 8$ Abs. 1 SGB VII umfasst. Sie sind nicht auf das Betriebsgelände beschränkt, sondern können auch außerhalb der Betriebsstätte anfallen. ${ }^{682}$ Beispiele sind der Gang vom Büro zum allgemeinen Firmendrucker am anderen Ende des Flures oder die Fahrt eines Handwerkers vom Betrieb zum Kunden. Diese Strecken werden unmittelbar mit betriebsdienlicher Handlungstendenz zu-

680 BSG, Urt. v. 7.9.2004 - B 2 U 35/03 R -, juris Rn. 16; v. 9.11.2010 - B 2 U 14/10 R -, juris Rn. 20; Benz, SGb 2003, S. 12, 14.

681 BSG, Urt. v. 7.11.2000 - B 2 U 39/99 R -, juris Rn. 20; v. 9.11.2010 - B 2 U 14/10 R -, juris Rn. 20; Urt. v. 18.6.2013 - B 2 U 7/12 R -, juris Rn. 13 (zur Veröffentlichung in Entscheidungssammlung vorgesehen); Keller, in: Hauck/Noftz/ders. (Hrsg.), SGB VII, $\mathbb{} 8$ Rn. 32.

682 BSG, Urt. v. 28.2.1990 - 2 RU 34/89 -, juris Rn. 16; v. 5.7.2016 - B 2 U 5/15 R -, juris Rn. 20. 
rückgelegt und sind daher selbst versicherte Tätigkeit. ${ }^{683} \mathrm{Da}$ diese Wege Arbeitsleistung darstellen, bedarf es für ihren Schutz im Unfallversicherungsrecht keiner besonderen Begründung; ihr sachlicher Zusammenhang mit dem Versicherungstatbestand ergibt sich ohne Weiteres aus den bereits dargelegten Grundsätzen. Eine gesonderte Bezeichnung für diese Art von Tätigkeiten wäre mithin gar nicht notwendig, um sie unter $\mathbb{8} 8$ Abs. 1 SGB VII subsumieren zu können.

Umso mehr überrascht es, dass in weiten Teilen der Rechtsprechung und Literatur davon ausgegangen wird, dass im Unterschied zu örtlich im Betrieb stattfindenden Strecken innerhalb einer Wohnung zurückgelegte Wege nur im Ausnahmefall als Betriebswege gelten können. ${ }^{684}$ Nach der bisherigen Entscheidungspraxis des BSG kommen Betriebswege grundsätzlich nur außerhalb des Wohngebäudes in Betracht. Werden sie direkt von der Wohnung aus angetreten - wie etwa eine Dienstreise -, so beginnen sie deswegen erst mit dem Durchschreiten der Außentür des Gebäudes, in dem sich die Wohnung des Versicherten befindet. ${ }^{685}$ Das Gericht zieht diese Grenze analog zur Rechtsprechung zu den Wegeunfällen nach $₫ 8$ Abs. 2 Nr. 1 SGB VII bewusst starr, um objektive, leicht feststellbare Merkmale für die Abgrenzung von unversichertem Lebensbereich und versichertem Betriebsweg zur Verfügung zu haben. ${ }^{686}$ Zudem soll so berücksichtigt werden, dass Rechtsgrund für den Schutz der versicherten Tätigkeit in der Unfallversicherung auch die Freistellung des Unternehmers von der Haftung für Betriebsgefahren ist. ${ }^{687} \mathrm{Da}$ der Unternehmer innerhalb des Wohngebäudes des Versicherten keinerlei Einfluss auf die Betriebsgefahren habe (zu dieser Annahme näher s.u. Kap. 3 B. II. b.), sei hier eine Haftungsfreistellung des Unternehmers nicht notwendig und im Gegenzug auch kein Versicherungsschutz indiziert. Selbst angesichts der Tatsache, dass inzwischen viele betriebsdienliche Verrichtungen im häuslichen Bereich stattfin-

$683 \mathrm{Zu}$ den von ebenfalls als Betriebswegen bezeichneten Wegen mit eigenwirtschaftlichem Ziel, etwa zur Nahrungsaufnahme oder zur Toilette, s. u. Kap. 3 A. VI.

684 BSG, Urt. v. 12.12.2006, E 98, S. 20 ff., 24; LSG Nordrhein-Westfalen, Urt. v. 19.9.2007, juris 2. Ls; LSG Baden-Württemberg, Urt. v. 9.2.2015, juris Rn. 23; Benz, SGb 2003, S. 12, 20; Wagner, jurisPR-SozR 23/2007 Anm. 5; Schlaeger, jurisPR-SozR 20/2017 Anm. 3; Leube, SGb 2012, S. 380, 383, 385; Ricke, in: Körner/Leitherer et al. (Hrsg.), Kasseler Kommentar zum Sozialversicherungsrecht, $\mathbb{8} 8$ SGB VII Rn. 129e.

685 BSG, Urt. v. 7.11.2000 - B 2 U 39/99 -, juris Rn. 21; Keller, in: Hauck/Noftz/ders. (Hrsg.), SGB VII, $\$ 8$ Rn. 33b; Benz, SGb 2003, S. 12, 18.

686 BSG, Urt. v. 5.7.2016 - B 2 U 5/15 R -, juris Rn. 21.

687 Ebd. 
den, betont das Bundessozialgericht, die Außentür als Grenze im Interesse der Rechtssicherheit aufrechterhalten zu wollen. ${ }^{688}$ Betriebswege kommen innerhalb eines Wohngebäudes nach der Rechtsprechung des Bundessozialgerichts nur in Betracht, wenn der konkrete Unfallort Betriebszwecken wesentlich diente. ${ }^{689}$ Dies wird bejaht, wenn eine „ständige und nicht nur gelegentliche Nutzung des Unfallorts für betriebliche Zwecke“ erfolgte. 690 Als ausreichend angesehen wurde etwa das arbeitsbezogene Nutzen einer Treppe an jedem Werktag. ${ }^{61}$ Dagegen genügte es nicht, dass das Betreten des Unfallortes unbedingt notwendig war, um den häuslichen Arbeitsplatz zu erreichen. ${ }^{692}$ Für den reinen Wohnbereich wird davon ausgegangen, dass dieser nicht regelmäßig betriebsdienlich genutzt wird. ${ }^{693}$

Bei gemischt genutzten Räumen - etwa einer Treppe, die nicht nur Arbeitszimmer und Küche, sondern auch Wohnzimmer und Schlafzimmer miteinander verbindet - kommt es dieser Ansicht nach darauf an, ob der Ort hauptsächlich betrieblichen Zwecken dient. ${ }^{694}$ Es wird beispielsweise danach gefragt, wie häufig eine Treppe aus betrieblichen und wie häufig sie aus privaten Gründen betreten wird. ${ }^{695}$ Nur wenn die betriebliche Nutzung im Verhältnis zur privaten Nutzung deutlich überwiegt, kommt eine Einordnung des Geschehens als versicherte Tätigkeit in Betracht. Besteht in der Privatwohnung des Beschäftigten ein getrenntes Arbeitszimmer, so beginne der Versicherungsschutz eines Weges mit dem Durchschreiten der zu diesem Raum führenden Tür. ${ }^{696}$ Wege im Arbeitsraum selbst werden als

688 Ebd.; BSG, Urt. v. 12.12.2006, E 98, S. 20 ff., 24; v. 7.11.2000 - B 2 U 39/99 -, juris Rn. 21.

689 BSG, Urt. v. 12.12.2006, E 98, S. 20 ff., 24; LSG Nordrhein-Westfalen, Urt. v. 19.9.2007, juris 2. Ls.; LSG Baden-Württemberg, Urt. v. 9.2.2015, juris Rn. 23; Benz, SGb 2003, S. 12, 20; Wagner, jurisPR-SozR 23/2007 Anm.5; Schlaeger, jurisPR-SozR 20/2017 Anm. 3.

690 Aus der Rechtsprechung: BSG, Urt. v. 12.12.2006, E 98, S. 20 ff., 24; v. 27.10.1987 - 2 RU 32/87 -, juris 1. Ls.; v. 29.1.1960, E 11, 267, 270; vgl. Zusammenfassung dieser Rechtsprechung in Urt. v. 5.7.2016 - B 2 U 5/15 R -, juris Rn. 23, in welchem sich die Abkehr von dieser Rechtsprechung ankündigte (Rn. 24), welche schließlich mit Urt. v. 31.08.2017 - B 2 U 9/16 R -, juris Rn. 16 (zur Veröffentlichung in BSGE vorgesehen), erfolgte. Aus der Literatur: Pattar/Sartorius, ZAP Fach 18, S. 1503, $1511 \mathrm{f}$.

691 LSG Berlin-Brandenburg, Urt. v. 7.2.2013 - L 3 U 288/11 -, juris 2. Ls.

692 LSG Bayern, Urt. v. 5.4.2017 - L 2 U 101/14 -, juris 3. Ls.

693 BSG, Urt. v. 25.2.1993 - 2 RU 12/92 -, juris Rn. 20.

694 LSG Nordrhein-Westfalen, Urt. v. 19.9.2007 - L 17 U 106/07 -, juris Rn 25.

695 BSG, Urt. v. 12.12.2006, E 98, 20 ff., 24.

696 LSG Bayern, Urt. v. 5.4.2017 - L 2 U 101/14 -, juris Rn. 69; Wedde, Telearbeit, Rn. 576; Leube, SGb 2012, S. 380, 382, nennt dies die „einhellige Ansicht“. 
unfallversichert angesehen, wenn sie der versicherten Tätigkeit dienen sollten, eine Beschäftigte also beispielsweise auf dem Weg zwischen Zimmertür und Schreibtischstuhl stürzt. Als unversicherte Tätigkeit gelten dagegen Wege innerhalb der Wohnung zur oder von der Arbeitszimmertür aus. ${ }^{697}$

Für den Fall, dass der Beschäftigte nicht über einen fest eingerichteten häuslichen Arbeitsplatz verfügt, sondern seine Arbeit zu Hause an verschiedenen Orten erledigt - etwa unter Zuhilfenahme eines mobilen Rechners alternierend von Küchen- und Esszimmertisch, von der Couch oder vom Bett aus -, sollen Wege grundsätzlich nicht als Betriebswege eingeordnet werden können. ${ }^{698}$ Einzig bei bestehender außerordentlicher Dringlichkeit soll ein versicherter Betriebsweg außerhalb eines fest eingerichteten Arbeitsplatzes bejaht werden können: ${ }^{699}$ War die verrichtete Tätigkeit durch eine der Rufbereitschaft ähnelnde Notwendigkeit sofortigen Handelns geprägt, wird von einem sachlichen Zusammenhang zur versicherten Tätigkeit ausgegangen. ${ }^{700}$ Dies wird etwa angenommen, wenn der Arbeitnehmer sich bei einem Sprint durch die Wohnung verletzt, der notwendig geworden war, um sein Kind davon abzuhalten, sein Arbeitsgerät zu zerstören. ${ }^{701}$

Die von dieser Ansicht vorgenommene Anknüpfung an den Unfallort anstatt an die Handlungstendenz der Tätigkeit ist für die bestehende $\mathrm{Zu}$ ordnungsdogmatik vollkommen untypisch und widerspricht dem allgemein anerkannten Grundsatz, im Unfallversicherungsrecht gäbe es keinen Betriebsbann, da es für die Bejahung des sachlichen Zusammenhangs nicht auf den Unfallort, sondern allein auf die Handlungstendenz der zum Unfallzeitpunkt verrichteten Tätigkeit ankäme. ${ }^{702}$ Die Rechtsprechung löst dieses Dilemma scheinbar, indem sie das Abstellen auf den Unfallort als

697 BSG, 25.2.1993 - 2 RU 12/92 -, juris Rn. 20 m.w.N.; SG Karlsruhe, Urt. v. 30.9.2010 - S 4 U 675/10 -, juris Rn. 23; Mütze, jurisPR-SozR 25/2010, Anm. 5; Leube, SGb 2012, S. 380, 382.

698 Keller, in: Hauck/Noftz/ders. (Hrsg.), SGB VII, $\$ 8$ Rn. 33c; Mütze, jurisPR-SozR 25/2010, Anm. 5; Leube, SGb 2012, S. 380 ff., 383.

699 Keller, in: Hauck/Noftz/ders. (Hrsg.), SGB VII, $\mathbb{} 8$ Rn. $33 \mathrm{c}$ und d.

700 Ebd. Rn.33b; Benz, SGb 2003, S.12, 18; Leube, SGb 2012, S.380, 383; Schwerdtfeger, in: Lauterbach/Watermann/Breuer (Hrsg.), Unfallversicherung, $\mathbb{} 8$ Rn. 258, 259; BSG, Urt. v. 12.12.2006, E 98, 20 ff.; v. 26.6.1970 - 2 RU 126/68 -, juris Rn. 16; v. 11.11.1971 - 2 RU 133/68 -, juris Rn. 23.

701 Leube, SGb 2012, S. 380, 383.

702 Zuletzt BSG, Urt. v. 5.7.2016 - B 2 U 5/15 R -, juris Rn. 25 (zur Veröffentlichung in Entscheidungssammlung vorgesehen); v. 26.6.2014 - B 2 U 4/13 R -, juris Rn. 28. Vgl. aus der Literatur nur Giesen, Arbeitsunfall und Dienstunfall, S. 32 m.w.N; Köhler, WzS 2011, S. 203, 203; Becker, SGb 2007, S. 721, 722; Kras- 
Anwendung des Kriteriums der Handlungstendenz begreift: ${ }^{703}$ Der Aufenthaltsort des Beschäftigten zum Unfallzeitpunkt stelle einen objektiven Hinweis auf die Handlungstendenz des Versicherten dar. ${ }^{704}$ Augenscheinlich wird der Unfallort allerdings als so starker Anhaltspunkt für die Handlungstendenz des Verunfallten gesehen, dass auf eine Prüfung weiterer Gegebenheiten verzichtet wird. Tatsächlich handelt es sich somit nicht um eine Prüfung des sachlichen Zusammenhangs im Sinne der objektivierten Handlungstendenz, sondern um eine pauschale Zuordnung des Unfallrisikos nach ausschließlich räumlichen Kriterien. Obwohl anerkannt wird, dass auch das soziale Schutzprinzip ein Grundpfeiler des Unfallversicherungsrechts ist, wird die Ablösung der Unternehmerhaftung als argumentativ gewichtiger angesehen und vermeintlich in ihrem Sinne entschieden, wenn sich in der häuslichen Lebensphäre des Beschäftigten ereignende Unfälle pauschal dem persönlichen Lebensrisiko des Versicherten zugewiesen werden. ${ }^{705}$

Begründet wird dieser Bruch mit dem Zuordnungsmechanismus damit, dass eine Einbeziehung von örtlich im Privatbereich des Versicherten stattfindenden Unfällen dem Strukturprinzip der Ablösung der Unternehmerhaftpflicht widerspräche. ${ }^{706}$ Räume, die nicht wesentlich betrieblich genutzt werden, seien jedoch dem Geschädigten besser bekannt als dem Unternehmer, sodass nicht der Arbeitgeber, sondern der Versicherte selbst die dortigen Gefahren beherrsche. ${ }^{707}$ Der Versicherte sei für diese Gefahrenquellen selbst verantwortlich, da er sie durch entsprechende Einrichtung und Pflege der Wohnung und durch angemessenes eigenes Verhalten zum Unfallzeitpunkt selbst beeinflussen kann. ${ }^{708}$ Sie seien daher nicht unter das Betriebsrisiko des Unternehmers zu fassen.

ney, NZS 2000, S.373, 373. Paradox deswegen die Anmerkung Schlaegers, es könne schon deswegen nur im Arbeitszimmer Arbeitsunfälle geben, da ansonsten „der Weg zu einem Wohnungsbann“ nicht mehr weit wäre, ders., jurisPRSozR 20/2017 Anm. 3.

703 BSG, Urt. v. 12.2.2006 - B 2 U 1/06 R -, juris Rn. 22.

704 Ebd.

705 Vgl. ebd. Rn. 21; Ebenso bereits BSG, Urt. v. 13.3.1956, E 2, 239.

706 Schlaeger, jurisPR-SozR 20/2017 Anm. 3.

707 BSG, Urt. v. 13.3.1956, E 2, 239, 244; v. 7.11.2000 - B 2 U 39/99 R -, juris Rn. 22.

708 BSG, Urt. v. 7.11.2000 - B 2 U 39/99 R -, juris Rn. 22; Ricke, in: Körner/Leitherer et al. (Hrsg.), Kasseler Kommentar zum Sozialversicherungsrecht, $\mathbb{} 8$ SGB VII Rn. 129e. 
b. Keinerlei Unfallschutz für Wege innerhalb eines Wohngebäudes

Vereinzelt wird darüber hinausgehend auch bei Bestehen eines getrennten Arbeitszimmers Versicherungsschutz auf Wegen innerhalb dieses Raumes verneint. Leube argumentiert, auch Wege in ausschließlich beruflich genutzten Räumen seien nicht in sachlichem Zusammenhang mit der versicherten Tätigkeit zu sehen, da die Räumlichkeit trotz ihrer Nutzung als Arbeitsplatz immer noch privaten Charakter habe und der Arbeitnehmer sich in seiner Privatsphäre aufhalte. ${ }^{709}$ Als Beispiel nennt er einen Beschäftigten, der in Reaktion auf ein Klingeln seines gemischt genutzten Telefonapparats von seinem häuslichen Schreibtisch zum im selben Raum befindlichen Telefon läuft, um einen betrieblichen Anruf entgegenzunehmen. Es sei nicht durch äußerliche Umstände erkennbar, ob er mit betrieblicher oder privater Intention handle; die betriebsdienliche Zweckrichtung trete nach außen nicht zutage, sodass auch das Zurücklegen dieses Weges unversichert bleiben müsse. ${ }^{710}$ Dieser Ansicht ist ebenfalls zu widersprechen. ${ }^{711}$ Denn sie misst der Tatsache, dass sich das Arbeitszimmer in der Wohnung des Versicherten befindet, zu große Bedeutung bei. Auch sie nimmt eine Zuordnung zur Risikosphäre ausschließlich nach örtlichen Kriterien vor, was angesichts der Bedeutung der versicherten Tätigkeit für das Unfallversicherungsrecht systemwidrig ist. ${ }^{712}$ Die Handlungstendenz eines Weges kann, während er zurückgelegt wird, in den meisten Fällen nicht objektiv beurteilt werden. Er wird bestimmt durch seinen Ausgangs- und seinen Endpunkt sowie die Route und das Beförderungsmittel, das gewählt wird, weiterhin durch eventuell mitgeführte Dinge. Zu einem Zeitpunkt vor Erreichen des Zieles lässt sich von außen nicht erkennen, ob eine sich mit leeren Händen an das andere Ende des Flures begebende Person vorhat, etwa den Bürodrucker, das Telefon oder die danebenstehende Kaffeemaschine zu erreichen. Auch in der Betriebsstätte ist die Zweckrichtung eines solchen Weges nicht für einen (hypothetischen) objektiven Dritten erkennbar. Der Schutz dieser Wege als Betriebswege ist allerdings unstrittig. ${ }^{713} \mathrm{Al}-$ lein die Tatsache, dass sich der Versicherte in einem Raum seiner häuslichen Umgebung befindet, rechtfertigt keine Vermutung auf die Privatheit seines Weges hin. Im Gegenteil sprechen die äußeren Anhaltspunkte im

709 Leube, SGb 2012, S. 380, 383, 385.

710 Ebd.

711 Ablehnend ebenfalls Keller, in: Hauck/Noftz/ders. (Hrsg.), SGB VII, $\mathbb{} 8$ Rn. 116a.

712 S.o. Kap. 3 V. 1. a.

713 Ebd. 
Arbeitszimmer des Beschäftigten eher für eine betriebliche Intention des Weges.

\section{Die Handlungstendenz als entscheidendes Kriterium}

\section{a. Entwicklung in der Rechtsprechung}

Vor dem Hintergrund der Strukturprinzipien der Unfallversicherung - der Betriebsrisikolehre und des sozialen Schutzprinzips - ist es dagegen auch hier richtig und notwendig, auf die objektivierte Handlungstendenz des Beschäftigten bei der tatsächlichen Verrichtung zum Unfallzeitpunkt abzustellen. Diese in Teilen der Literatur bereits seit einiger Zeit vertretene Ansicht wurde jüngst auch vom Bundessozialgericht anerkannt: Der erst im Februar 2018 erschienen Entscheidungsbegründung zum Fall einer selbständigen Friseurmeisterin ist zu entnehmen, dass das BSG den Unfallort nunmehr lediglich als ein Indiz ansieht, welches bei der vorzunehmenden wertenden Beurteilung des sachlichen Zusammenhangs neben weiteren Faktoren zu beachten ist. ${ }^{714}$ Demnach kommt es bei allen Unfällen im häuslichen Bereich, unabhängig davon, welcher Nutzung der konkrete Unfallort gewidmet ist, auf die konkrete Handlungstendenz des Verunfallten an. Virulent wurde die Frage im genannten Prozess, da sich der Arbeitsunfall der Friseurmeisterin in einem Gebäude ereignete, in welchem sich sowohl ihre Wohnung als auch ihr Friseursalon befand. In der Waschküche des Hauses befand sich eine Waschmaschine, welche die Verunfallte sowohl privat als auch beruflich nutzte. Auf dem Weg zu dieser stürzte sie auf dem Wohnungsflur, welcher sowohl die privaten Wohnräume als auch die Geschäftsräume mit dem Waschraum verband, und zog sich eine Sprunggelenksverletzung zu. Die Friseurmeisterin hatte die Strecke zurückgelegt, um die Geschäftswäsche aus der Waschmaschine zu holen. Das in zweiter Instanz damit befasste LSG ${ }^{715}$ ging nach der oben dargelegten bisherigen Rechtsprechung des BSG davon aus, dass der Unfall als Arbeitsun-

714 Urt. v. 31.8.2017 - B 2 U 9/16 R -, juris Rn. 18 (zur Veröffentlichung in BSGE vorgesehen). So verstand auch Schlaeger, jurisPR-SozR 20/2017 Anm. 3 den entsprechenden BSG-Terminbericht Nr. 38/17, Ziff. 4. Bereits im Urt. v. 5.7.2016 - B 2 U 5/15 R -, juris Rn. 24, erwähnte das Gericht Zweifel an dieser Rechtsprechung, hatte das Ergebnis aber noch offengelassen.

715 LSG Baden-Württemberg, Urt. v. 25.2.2016 - L 10 U 1241/14 -, juris Rn. 26, bestätigte dabei die vorinstanzliche Entscheidung des SG Heilbronn, Urt. v. 5.2.2014 - S 4 U 2935/12 -, juris. 
fall anzusehen ist, da der Wohnungsflur ständig betrieblich genutzt wurde. In der Revisionsentscheidung stellte das BSG klar, dass entgegen der Argumentation des LSG und in Abkehr von seiner früheren Rechtsprechung die Häufigkeit der betrieblichen Nutzung des Unfallorts nicht entscheidend für die Anerkennung eines Versicherungsfalls sei. Das notwendige betriebliche Interesse an der tatsächlichen Verrichtung bestimme sich ausschließlich nach der objektivierten Handlungstendenz des Versicherten. ${ }^{716}$ Der Unfallort und seine Zweckbestimmung sind allenfalls noch als Indizien heranzuziehen. ${ }^{717}$

Ähnlich hatte das Bundessozialgericht bereits 1994 im Fall eines Außendienstmitarbeiters einer Versandfirma entschieden, welcher sein häusliches Arbeitszimmer renovierte und zu diesem Zweck private Tapetenreste vom Dachboden in seinen Arbeitsbereich transportierte. ${ }^{718}$ Seinen Sturz auf der Trittleiter zum Dachboden sah der entscheidende Senat als Arbeitsunfall an, da die Erhaltung des Arbeitszimmers betrieblichen Interessen zu dienen bestimmt war und das Besteigen der Leiter damit in sachlichem $\mathrm{Zu}$ sammenhang zur versicherten Tätigkeit stand. ${ }^{719}$ Auch hier kam es dem entscheidenden Gericht maßgeblich auf die Handlungstendenz und nicht auf den Widmungszweck des Unfallortes an. ${ }^{720}$

\section{b. Herleitung aus Betriebsrisiko- und sozialem Schutzprinzip}

Dass es für die Zuordnung von auf Betriebswegen erlittenen Unfällen innerhalb der Privatwohnung des Arbeitnehmers nur auf die zum Zeitpunkt des Unfalls vorliegende objektivierte Handlungstendenz ankommen kann, folgt wiederum aus den Grundprinzipien der Unfallversicherung. Sowohl die Betriebsrisikolehre als auch das soziale Schutzprinzip sprechen, wie die folgenden Ausführungen zeigen, für diese Zuordnung.

Zum einen ordnet die Betriebsrisikolehre auch das Risiko, im privaten Wohnbereich bei Ausübung einer arbeitsvertraglich geschuldeten Tätigkeit

716 Urt. v. 31.8.2017 - B 2 U 9/16 R -, juris Rn. 16 (zur Veröffentlichung in BSGE vorgesehen).

717 Der Terminbericht nennt als Beispiel den Sturz eines Beschäftigten im privaten Weinkeller weit nach Mitternacht.

718 BSG, Urt. v. 8.12.1994 - 2 RU 41/93 -, juris.

719 Ebd. Rn. 15. Auch hier handelte es sich um ein Problem des sachlichen Zusammenhangs, und nicht - wie von Isenhardt, DB 2016, S. 1499, 1502 angenommen - um eine Kausalitätsfrage.

720 Zustimmend Wolber, SozVers 1997, S. 239, 239. 
zu verunfallen, dem Arbeitgeber zu. Korrekterweise kann es für die Zuordnung zum Betriebsrisiko nur auf die objektivierte Handlungstendenz der zum Unfallzeitpunkt ausgeführten Tätigkeit ankommen. Zwar ist zuzugeben, dass der Aspekt der Kontrolle über die physische Unfallumgebung, der in der Betriebsrisikolehre bisher eine große Rolle gespielt hat, im privaten Wohnbereich nicht für eine Zuordnung dort befindlicher Risiken zur Arbeitgebersphäre spricht. Denn anders als im Falle eines eingerichteten Telearbeitsplatzes kann der Unternehmer auf die Privaträume des Beschäftigten auch nicht nach den Vorschriften des Arbeitsschutzgesetzes und der Arbeitsstättenverordnung Einfluss ausüben. Insofern ist seine tatsächliche Organisationsherrschaft über den Unfallort des Beschäftigten gegenüber einem Unfall am häuslichen Arbeitsplatz geringer. Jedoch ist die argumentative Fokussierung auf die Herrschaft über die physische Umgebung angesichts der in Bezug genommenen Gestaltungsmodelle kontemporärer Arbeitsverhältnisse nicht gerechtfertigt. Zum einen war sie schon zu Zeiten der Einführung der Unfallversicherung nicht das einzige zu beachtende Indiz, das für eine Zuordnung zur Arbeitgebersphäre sprach. Zum anderen ist es angesichts des Wandels der Arbeitsgesellschaft geboten, diesem Aspekt weniger Gewicht zuzuschreiben. Denn während zu Zeiten der Industrialisierung die meisten Unfallrisiken zweifellos aus örtlichen Gesichtspunkten - etwa der Einrichtung und Auswahl der Produktionsmaschinen - stammten und es daher auf der Hand lag, mit der Macht des Arbeitgebers, diese Faktoren zu minimieren, zu argumentieren, unterliegen Arbeitnehmer, die mit der Arbeit an Computern oder mobilen Endgeräten bzw. mit reiner Denkarbeit befasst sind, kaum vergleichbaren ortsgebundenen Gefahren.

Dagegen muss stärker in den Blick genommen werden, dass die menschliche Arbeitskraft an sich das wesentliche Betriebsmittel dieser Unternehmen darstellt. Die menschlichen Faktoren - bspw. Fahrlässigkeit, Ermüdung, Fehleinschätzung -, die seit jeher als Folge der Bemühung menschlicher Arbeitskraft als zum Betriebsrisiko gehörig angesehen werden, sind nicht an einen physischen Ort gebunden. Wie oben gezeigt wurde, ist mit zunehmender Digitalisierung für immer mehr Beschäftigte Realität geworden, ihre Arbeit jederzeit an jedem Ort ausführen zu können. Zur Verquickung von Arbeits- und Privatleben gehört auch die Ausführung beruflicher Tätigkeiten in privater Umgebung, die im Übrigen schon lange als sozialadäquat akzeptiert wird (was sich unter anderem an den genannten hohen Zahlen von Arbeitnehmern zeigt, die auch außerhalb der Arbeitszeit für ihre Vorgesetzten, Kunden und Kollegen erreichbar sind). Wenn aber die arbeitsweltliche Realität dergestalt ist, dass die Arbeitsaufnahme durch den Beschäftigten auch in privater Umgebung akzeptiert und sogar ge- 
wünscht ist, so muss auch zum Betriebsrisiko gehören, dass der Beschäftigte sich bei Ausführung einer versicherten Tätigkeit in einer vom Arbeitgeber physisch nicht beherrschbaren Umgebung befindet. Aus diesem Grund ist eine Reduzierung des Unfallversicherungsschutzes auf Unfälle, die sich als Realisierung tätigkeitsbezogener Gefahren erweisen, abzulehnen.

Die Einordnung als Betriebsrisiko wird ferner durch die zum Unfallzeitpunkt verrichtete unternehmensdienliche Tätigkeit geboten. Unbestritten stellt die objektivierte Handlungstendenz des Versicherten den notwendigen sachlichen Zusammenhang zur beruflichen Sphäre her. Die Ausführung einer versicherten Tätigkeit in privater Umgebung ändert nichts daran, dass der Beschäftigte die Verrichtung aufgrund seiner arbeitsvertraglichen Verpflichtung und damit aufgrund der besonderen Zwangslage, in der er sich qua seiner Stellung als Arbeitnehmer befindet, durchführt. Seine berufliche Handlungstendenz wird durch seinen Aufenthaltsort nicht infrage gestellt. Ist seine zum Unfallzeitpunkt ausgeführte Handlung darauf gerichtet, dem Arbeitgeber zu dienen, so ist der Versicherungstatbestand erfüllt. ${ }^{721}$ Dies gilt ohne Abstriche sowohl auch für Betriebswege, die gerade selbst die Erfüllung der versicherten Tätigkeit darstellen.

Dem Arbeitgeber stehen darüber hinaus Möglichkeiten zur Verfügung, die Arbeitsausführung des Beschäftigten im Sinne der Gefahrvermeidung zu beeinflussen. So liegt es etwa in seiner Hand, Mitarbeiter hinsichtlich im jeweiligen Betätigungsfeld üblicher Gefahren zu schulen. ${ }^{722} \mathrm{Er}$ ist ihm außerdem unbenommen, die Ausführung von Arbeit an anderen Orten als dem betrieblichen Arbeitsplatz ausdrücklich untersagen. Eine dennoch ausgeführte Tätigkeit anderswo vermag dann keinen sachlichen Zusammenhang im Sinne des $\$ 8$ Abs. 1 SGB VII mehr mit der beruflichen Tätigkeit herzustellen, da sie nicht mehr arbeitsvertraglich geschuldet ist.

Dies wird schließlich auch durch das sog. Gewinnerzielungsargument bestärkt: Der Arbeitgeber genießt auch die Vorteile der örtlich flexiblen Arbeit. Die daraus folgende höhere Motivation der Mitarbeiter und ihre im Schnitt längeren Arbeitszeiten wurden bereits genannt, zudem ist ein

721 Dies führt, entgegen etwa von Jung, SGb 2001, S. 398, 399, geäußerter Befürchtungen, nicht zu einer den Schutzzwecken der Unfallversicherung widersprechenden unkontrollierten Ausweitung der Unfallversicherung. Schließlich muss auf der Ebene der Unfallkausalität noch der ursächliche Zusammenhang zwischen der verrichteten Tätigkeit und dem Unfall abgeprüft werden, bevor ein Arbeitsunfall angenommen werden kann. Sich rein aus dem Ort ergebende Risiken werden dadurch häufig nicht als Arbeitsunfall gelten. Vgl. BAG, Beschl. v. 27.9.1994, E 78, S. 56, 57. 
Arbeitnehmer, der sich an Ort und Stelle der beruflichen Tätigkeit widmen kann, schneller verfügbar als einer, der sich dazu zunächst an einen vereinbarten Arbeitsort begeben muss. Die ständige Erreichbarkeit des Beschäftigten machte wenig Sinn, wenn dieser den Anruf seines Vorgesetzten nicht an seinem momentanen Aufenthaltsort entgegennehmen kann. Da die Vorteile und Chancen dieser Tätigkeitsergreifung aus der physischen Privatsphäre heraus für den Arbeitgeber bestimmt sind, fallen ihm auch die Risiken und Lasten derselben anheim. ${ }^{723}$

Zum anderen streitet auch das als soziales Schutzprinzip bezeichnete Hauptanliegen der Unfallversicherung, Beschäftigte vor den Folgen von Risiken zu bewahren, denen sie sich im Zusammenhang mit ihrer beruflichen Tätigkeit ausgesetzt sehen, für einen Einbezug von in privaten Räumen erlittenen Unfällen in den Schutzbereich des $\$ 8$ Abs. 1 SGB VII. ${ }^{724}$ Die Fürsorgepflicht des Arbeitgebers kennt keine räumlichen Grenzen, sondern ist an das Arbeitsverhältnis und damit an die Ausübung einer unternehmensdienlichen Tätigkeit geknüpft. Sie geht ausdrücklich über die vom Arbeitgeber beherrschbaren Umstände, zu denen die physische Gewalt über die unmittelbare Umgebung des Unfallortes gehört, hinaus. Der sozial-ethische Gedanke der Verantwortung des Arbeitgebers für die Gesundheit seiner Beschäftigten erstreckt sich auf alle Gefahren, deren diese in Zusammenhang mit der Erfüllung seiner arbeitsvertraglichen Pflichten ausgesetzt sind. Im Sinne des sozialen Schutzprinzips sind betriebsdienliche Verrichtungen, die in privater Umgebung ausgeführt werden, in sachlichem Zusammenhang mit der versicherten Tätigkeit zu sehen und damit der Risikosphäre des Arbeitgebers zuzurechnen.

Die Betriebsrisikolehre verlangt entgegen der beiden zuerst genannten Ansichten eben gerade keine vollständige Herrschaft des Arbeitgebers über den Unfallort, sondern misst der betrieblichen Intention des Beschäftigten eine deutlich höhere Bedeutung zu. ${ }^{725}$ Dies führt zu der Erkenntnis, dass Betriebswege im privaten Wohnbereich im selben Maße zum Schutzbereich der Unfallversicherung zählen wie Betriebswege, die in der Betriebsstätte stattfinden - nämlich immer dann, wenn sie eine Ausübung der versicherten Tätigkeit darstellen, da sie dadurch zum Betriebsrisiko des Unternehmers zählen.

723 Ähnlich Wedde, Telearbeit, S. 91 f., Rn. 382 ff.

724 Zum sozialen Schutzprinzip s.o. Kap. 2 C. II.

725 Insofern missversteht Hollo die Konsequenzen der Rechtsprechungsänderung, indem er zur Begründung dieser seiner Ansicht nach verschärften Anforderungen wiederum auf die fehlenden Gefahrherrschaft des Arbeitgebers über die örtliche Umgebung abstellt, vgl. Hollo, jurisPR-SozR 10/2017 Anm. 4. 


\section{Wege zur Nahrungsaufnahme aus dem Homeoffice}

\section{Das Urteil des BSG vom 5.7.2016}

Umfangreiches Echo in der Literatur erfuhr ein Urteil des BSG aus dem Jahr 2016. ${ }^{726}$ Es ging um die Zuordnung eines Unfalls einer Arbeitnehmerin, die in ihrer Wohnung stürzte, als sie sich auf dem Weg von ihrem arbeitsvertraglich vereinbarten häuslichen Arbeitsplatz in ihre Küche befand, wo sie sich Trinkwasser zum Verzehr während der Weiterarbeit am Schreibtisch holen wollte. ${ }^{727}$ Das Gericht äußerte sich hier zur Frage der Gleichbehandlung von Versicherten mit häuslichen Arbeitsplätzen und Versicherten, die ausschließlich in der Betriebsstätte tätig sind, und vertrat die Ansicht, die beiden Personengruppen seien im Lichte von Art. 3 Abs. 1 GG als „ungleich“ anzusehen, sodass eine Gleichbehandlung nicht geboten sei. Die Frage stellte sich, da es sich - denkt man sich das Geschehen in ein arbeitgebereigenes Bürogebäude - um einen Sachverhalt handelt, zu dem eine gefestigte Rechtsprechung existiert. „Wege zur Nahrungsaufnahme“, also auch der Weg zur Kantine, um Wasser zu holen, gelten nach dieser grundsätzlich als gem. $\$ 8$ Abs. 1 SGB VII versicherte Betriebswege. ${ }^{728} \mathrm{Au}$ Berhalb des Betriebes handelt es sich um nach $₫ 8$ Abs. 2 Nr. 1 SGB VII ver-

726 BSG, Urt. v. 5.7.2016 - B 2 U 5/15 R -, juris. Eine knappe und präzise Darstellung von Sachverhalt und Entscheidung findet sich bei Ricken, NZA 2017, S. 371, 371. Besprochen wurde das Urteil in der Literatur u.a. von Angermaier, jM 2017, S. 69 ff.; Boewer/Gaul, Aktuelles Arbeitsrecht 2016, S. 629 ff.; HäfnerBeil/Nacke, Gute Arbeit 2016, S. 4; Hollo, jurisPR-SozR 10/2017 Anm. 4; Pattar/ Sartorius, ZAP 2017, S. 421 ff.; Ricke, in: Körner/Leitherer et al. (Hrsg.), Kasseler Kommentar zum Sozialversicherungsrecht, $\mathbb{8}$ SGB VII Rn. 129 f.; Schur, jM 2014, S. 249 ff.; Schwirz, BGHM-Aktuell 2017, S. 31; Spellbrink, NZS 2016, S. 527, 530; Wietfeld, in: Rolfs/Giesen et al. (Hrsg.), BeckOK Sozialrecht, $\mathbb{} 1$ Rn. 9.

727 BSG, Urt. v. 5.7.2016 - B 2 U 5/15 R -, juris.

728 BSG, v. 5.7.2016 - B 2 U 5/15 R -, juris Rn. 19, 25; v. 18.6.2013 - B 2 U 7/12 R -, juris Rn. 20 (zur Veröffentlichung in Entscheidungssammlung vorgesehen); v. 2.12.2008 - B 2 U 17/07 R -, juris Rn. 30; v. 2.7.1996 - 2 RU 34/95 -, juris Rn. 15; v. 6.12.1989 - 2 RU 5/89 -, juris Rn. 16; v. 24.6.2003 - B 2 U 24/02 R -, juris Rn.14; Keller, in: Hauck/Noftz/ders. (Hrsg.), SGB VII, $\mathbb{8} 8$ Rn. 91 . Dieser Schutz kommt ebenso Wegen zur Verrichtung der Notdurft zugute, vgl. nur BSG, Urt. v. 6.12.1989 - 2 RU 5/89 -, juris Rn. 16. Das Essen und Trinken bzw. die Verrichtung der Notdurft selbst sind dagegen nach std. Rspr. dem persönlichen Bereich zuzuordnende nicht versicherte Betätigungen, vgl. nur BSG, Urt. v. 24.6.2003 - B 2 U 24/02 -, juris Rn. 14 m.w.N., kritisch dazu s.u. Kap. 4 C. II. 
sicherte Wegeunfälle. ${ }^{729}$ Als Grund für den Versicherungsschutz dieser Strecken wird angegeben, dass sie in ihrem Ausgangs- und Zielpunkt durch die Notwendigkeit, persönlich im Beschäftigungsbetrieb zur Ausübung betrieblicher Tätigkeit anwesend zu sein, geprägt würden. ${ }^{730}$ Ein sachlicher Zusammenhang zwischen der Verrichtung und der versicherten Tätigkeit sei daher zu bejahen. ${ }^{731}$ Außerdem diene die beabsichtigte Nahrungsaufnahme der Aufrechterhaltung der Arbeitsfähigkeit und damit der Fortsetzung der betrieblichen Tätigkeit, sodass Wege zur Nahrungsaufnahme unmittelbar im betrieblichen Interesse vorgenommen würden. ${ }^{732}$ Dies gilt jedoch nur, wenn die Handlungstendenz des Beschäftigten auf die Stillung von Hunger und Durst gerichtet ist, nicht also beispielsweise beim Spaziergang zur Eisdiele oder beim Holen von Kaffee. ${ }^{733}$ Versichert sind dabei sowohl Wege in die betriebseigene Kantine im selben Gebäude oder in ein Restaurant als auch in eine Privatwohnung oder zum Supermarkt, und es ist unerheblich, ob die Lebensmittel dort oder später am Arbeitsplatz konsumiert werden sollen. Der Versicherungsschutz endet jeweils an der Außentür des Zielgebäudes und lebt bei deren Durchtreten auf dem Rückweg wieder auf.734

Hätte sich die Beschäftigte aus dem genannten Urteil im Bürogebäude ihres Arbeitgebers befunden und wäre dort auf dem Weg von ihrem Arbeitsplatz zur Mitarbeiterküche verunfallt, als sie sich ein Glas Wasser holen wollte, läge nach den soeben dargestellten Grundsätzen unproblematisch ein als Betriebsweg einzuordnender Weg zur Nahrungsaufnahme vor, sodass die tatsächliche Verrichtung zum Unfallzeitpunkt den Versicherungstatbestand des $₫ 8$ Abs. 1 SGB VII erfüllt hätte. Der Unfall wäre als Arbeitsunfall zu qualifizieren gewesen. Der vorliegende Unfall wurde vom BSG jedoch nicht als Arbeitsunfall angesehen, da er sich innerhalb der Wohnung der Versicherten zugetragen hat. Im Gegensatz zu Wegen zur Nahrungsaufnahme, die von der Betriebsstätte aus angetreten werden,

729 Keller, in: Hauck/Noftz/ders. (Hrsg.), SGB VII, $\mathbb{} 8$ Rn. 91.

730 BSG, Urt. v. 5.7.2016 - B 2 U 5/15 R -, juris Rn. 19, 25; Keller, in: Hauck/Noftz/ ders. (Hrsg.), SGB VII, $\mathbb{} 8$ Rn. 91.

731 BSG, Urt. v. 5.7.2016 - B 2 U 5/15 R -, juris Rn. 19, 25.

732 Ebd.; Urt. v. 18.6.2013 - B 2 U 7/12 R -, juris Rn. 21 (zur Veröffentlichung in Entscheidungssammlung vorgesehen); Keller, in: Hauck/Noftz/ders. (Hrsg.), SGB VII, $\mathbb{} 8$ Rn. 91. Andernorts ist davon die Rede, dass das Gericht hier ausnahmsweise einen mittelbaren betrieblichen Nutzen ausreichen lässt, vgl. BSG, Urt. v. 2.7.1996 - 2 RU 34/95 -, juris Rn. 15.

733 BSG, Urt. v. 25.11.1992 - 2 RU 1/92 -, juris Rn. 20; Keller, in: Hauck/Noftz/ders. (Hrsg.), SGB VII, $\mathbb{} 8$ Rn. 91.

734 Zum Ganzen Keller, in: Hauck/Noftz/ders. (Hrsg.), SGB VII, $\mathbb{} 8$ Rn. 92a, 93. 
herrschten - so die Argumentation des Gerichts ${ }^{735}$ - im Privatgebäude der Beschäftigten keine betrieblichen Vorgaben oder Zwänge. Die zurückzulegende Strecke sei nicht durch einen räumlich außerhalb der Wohnung gelegenen Betriebsort vorgegeben und nicht innerhalb eines durch den Betriebsablauf diktierten zeitlichen Rahmens zu erledigen gewesen. Insgesamt stünde der Weg in keinem Zusammenhang mit bereits erbrachter Arbeit. Vielmehr hätte es der Versicherten freigestanden, zu welchem Zeitpunkt und von welchem Ort sie ihr Getränk holte. Aus diesem Grund sei die Sachverhaltslage nicht mit einem Unfall auf einem Weg zur Nahrungsaufnahme von Versicherten, die außerhalb ihrer Wohnung arbeiteten, vergleichbar. Ein sachlicher Zusammenhang der tatsächlichen Verrichtung mit der versicherten Tätigkeit sei daher zu verneinen, sodass kein Betriebsweg und auch kein Arbeitsunfall vorlägen. Dies sei wegen der grundsätzlichen Verschiedenheit von Arbeit im Homeoffice und Arbeit in der Betriebsstätte auch kein Verstoß gegen den allgemeinen Gleichheitsgrundsatz aus Art. 3 Abs. 1 GG. Darin, so formuliert das BSG in seinem zweiten Leitsatz, liege „keine verfassungswidrige Ungleichbehandlung gegenüber Versicherten, die außerhalb des Wohngebäudes ihre Beschäftigung ausüben und auf dem Weg zur Nahrungsaufnahme oder zum Einkauf von Lebensmitteln für den alsbaldigen Verzehr am Arbeitsplatz geschützt sind “736 Daraus folgt, dass das BSG nicht nur keine Wege zur Nahrungsaufnahme innerhalb des Wohngebäudes, sondern auch solche, die außerhalb - etwa vom häuslichen Arbeitsplatz zu einem Restaurant - zurückgelegt werden, als nicht von $\mathbb{8} 8$ Abs. 1 bzw. Abs. 2 Nr. 1 SGB VII geschützt ansieht. Für die folgende Untersuchung der Fallgruppe „Wege zur Nahrungsaufnahme aus dem Homeoffice" ist es daher notwendig, beide Sachverhaltsalternativen getrennt zu betrachten, da sie nicht nur auf unterschiedlichen Rechtsnormen, sondern auch auf unterschiedlichen Strukturprinzipien fußen. Zur Beantwortung der Frage nach dem Versicherungsschutz von Wegen zur Nahrungsaufnahme im Homeoffice arbeitender Personen einerseits innerhalb und andererseits mit Zielorten außerhalb des häuslichen Bereichs sind wiederum die oben dargestellten Grundprinzipien der Unfallversicherung - Betriebsrisikolehre und soziales Schutzprinzip - als „Interpretationsfolien" anzuwenden. ${ }^{737}$ Das Ergebnis muss mit diesen im Einklang stehen. Zudem müssen Anwendbarkeit und Einschlägigkeit des vom Bundes-

735 BSG, Urt. v. 5.7.2016 - B 2 U 5/15 R -, juris Rn. 19, 25. Zustimmend Ricke, in: Körner/Leitherer et al. (Hrsg.), Kasseler Kommentar zum Sozialversicherungsrecht, $\mathbb{8} 8$ SGB VII Rn. $129 \mathrm{f}$.

736 BSG, Urt. v. 5.7.2016 - B 2 U 5/15 R -, juris.

737 Spellbrink, NZS 2016, S. 527, 527. 
sozialgericht selbst ins Spiel gebrachten Art. 3 Abs. 1 GG überprüft werden.

2. Versicherungsschutz für Wege zur Nahrungsaufnahme innerhalb des häuslichen Bereichs

a. Im Lichte der Betriebsrisikolehre

Für die positive Beurteilung des sachlichen Zusammenhangs von Wegen zur Nahrungsaufnahme in den klassischen Fällen von Betriebsbeschäftigten sind nach unbestrittener Rechtsprechung zwei Aspekte notwendig, die kumulativ vorliegen müssen. ${ }^{738}$ Diese beiden - die Prägung von Ausgangsund Zielpunkt des Weges durch die Notwendigkeit der persönlichen Anwesenheit im Betrieb zur Ausübung betrieblicher Tätigkeit ${ }^{739}$ sowie das Vorliegen einer betriebsdienliche Intention, namentlich das Erhalten der Arbeitsfähigkeit durch Stillen von Hunger und Durst ${ }^{740}$ - fußen wiederum auf der Betriebsrisikolehre. Durch diese Kriterien wird nach Ansicht der Rechtsprechung sichergestellt, dass der Unfall eine Verwirklichung eines der Unternehmenssphäre zugehörigen Risikos darstellt. Selbiges müsse für Wege zur Nahrungsaufnahme innerhalb des häuslichen Bereichs gelten, wenn sie ebenfalls von betriebsdienlicher Intention geleitet und in ihrem Ausgangs- und Zielort von betrieblichen Erwägungen geprägt wären.

Hinsichtlich des Handlungszweckes besteht beim Gang eines Beschäftigten von seinem häuslichen Arbeitszimmer aus zu seiner Küche, um dort während einer Arbeitspause zu Mittag zu essen, kein Unterschied zur oben dargestellten Rechtsprechungspraxis zu Betriebsbeschäftigten. Für die Beurteilung der Zweckrichtung des Essens ist es nicht von Bedeutung, wo die Nahrungsaufnahme stattfindet. Auch im Homeoffice arbeitende Personen handeln auf dem Weg zur Einnahme ihres Mittagessens im Sinne ihres Arbeitgebers, da sie ebenso wie Betriebsbeschäftigte zur Aufrechterhaltung

738 Ebd., S. 527, $529 \mathrm{f}$.

739 BSG, v. 5.7.2016 - B 2 U 5/15 R -, juris Rn. 19, 25; v. 18.6.2013 - B 2 U 7/12 R -, juris Rn. 20 (zur Veröffentlichung in Entscheidungssammlung vorgesehen); v. 2.12.2008 - B 2 U 17/07 R -, juris Rn. 30; v. 2.7.1996 - 2 RU 34/95 -, juris Rn. 15; v. 6.12.1989 - 2 RU 5/89 -, juris Rn. 16; v. 24.6.2003 - B 2 U 24/02 R --, juris Rn. 14; Keller, in: Hauck/Noftz- et al. (Hrsg.), SGB VII, $\$ 8$ Rn. 20a, 91.

740 BSG, v. 5.7.2016 - B 2 U 5/15 R -, juris Rn. 19, 25; v. 18.6.2013 - B 2 U 7/12 R -, juris Rn. 21 (zur Veröffentlichung in Entscheidungssammlung vorgesehen); Keller, in: Hauck/Noftz/ders. (Hrsg.), SGB VII, $\$ 8$ Rn. 91. 
der Arbeitsfähigkeit auf das Stillen von Hunger und Durst angewiesen sind. ${ }^{71}$ Die Fortsetzung der Betriebstätigkeit nach Abschluss der Nahrungsaufnahme ist auch hier intendiert. An diesem Aspekt des Zusammenhangs mit der versicherten Tätigkeit ändert sich durch die Tätigkeit im Homeoffice nichts, sodass daraus kein Argument für ein Entfallen des Versicherungsschutzes der Wege zur Nahrungsaufnahme im häuslichen Bereich gewonnen werden kann.

Lediglich der zweite von der Rechtsprechung geforderte Aspekt stellt sich für Telearbeitnehmer unterschiedlich dar. Er ist örtlicher Natur. Da sie nicht persönlich im Betrieb anwesend sind, sei - so das zitierte Urteil auch ihr Weg zur Nahrungsaufnahme nicht durch eine Notwendigkeit der Anwesenheit „im Betrieb“ geprägt. Die eingefügten Anführungszeichen sind hier unentbehrlich: Denn während sich die Arbeitnehmer nicht im Betrieb im klassischen Wortsinn aufhalten, so befinden sie sich doch in der Betriebsstätte, so wie der unfallversicherungsrechtliche Begriff des Betriebes vor dem Hintergrund entgrenzter Arbeit zu verstehen ist. Das Homeoffice des Beschäftigten ist ebenfalls Teil des Betriebes des Unternehmers, so wie die in Telearbeit beschäftigten Personen Teil der Belegschaft sind. ${ }^{742}$ Der Betrieb ist kein Gebäude mehr, in dem sich Unternehmer und Beschäftigte zum gemeinschaftlichen Tätigwerden treffen. ${ }^{743}$ Die notwendige organisatorische Einheit kann ebenso durch die Nutzung von Kommunikationsmitteln hergestellt werden. ${ }^{74}$ Der häusliche Arbeitsplatz ist als Betriebsort lediglich physisch vom Fabrik- oder Bürogebäude des Arbeitgebers entkoppelt, stellt aber genauso einen Ort dar, an dem der Arbeitnehmer weisungsgebunden unter der organisatorischen Anleitung des Unternehmers auf dessen arbeitstechnisches Ziel hinwirkt. Da die Betriebsrisiko-

741 Die Prämisse, Essen und Trinken geschähen, um die Arbeitskraft zu erhalten, kann mit Recht kritisiert werden, da die Nahrungsaufnahme zuvorderst in den meisten Fällen der Erhaltung der eigenen Lebenskraft dienen wird und kaum ein Beschäftigter während des Essens bewusst daran denken dürfte, dass er im Sinne seines Arbeitgebers seine Leistungsfähigkeit erhalten möchte, vgl. Krasney, VSSR 1993, S. 81, 95 f.; Ricke, WzS 2017, S.9, 12. Tatsächlich aber wäre die Qualität der Arbeitsleistung ohne Nahrungsaufnahme nach einer gewissen Zeit mit Sicherheit eingeschränkt, sodass die Betriebsdienlichkeit zu bejahen ist, auch wenn sie - wie Ricke a.a.O. treffend formuliert - weniger als bewusste Intention als vielmehr als Reflex auftritt.

742 Giesen/Kersten, Arbeit 4.0, S. 131.

743 Ebd., S. 129. Im Betriebsverfassungsrecht ringt man daher bereits um einen neuen Betriebsbegriff, der die Anwendung des BetrVG zeitgerecht ermöglicht, vgl. ebd. m.w.N.

744 Giesen/Kersten, Arbeit 4.0, S. 130. 
lehre seit jeher darauf abzielt, den Zusammenhang zwischen Tätigkeiten des Arbeitnehmers mit seiner beruflichen Sphäre herzustellen, ist es unumgänglich, nicht auf eine rein örtliche Definition des Betriebes abzustellen, sondern diesen funktional zu begreifen. Vor diesem Hintergrund zeigt sich, dass Ausgangs- und Endpunkt eines innerhäuslichen Weges zur Nahrungsaufnahme sehr wohl betrieblich geprägt sind.

Beide örtlichen Fixpunkte befinden sich innerhalb der Wohnung des Beschäftigten. Damit findet der Weg zwar zweifellos in einer Umgebung statt, welche der Versicherte selbst am besten kennt. Jedoch gelten auch hier die Ergebnisse der obenstehenden Untersuchung des Versicherungsschutzes von Unfällen im häuslichen Bereich: Die Organisations- und Verfügungshoheit des Arbeitgebers reicht - insbesondere durch dessen Weisungsrecht sowie die Vorgaben des Arbeitsschutzrechtes - weit in den häuslichen Bereich hinein. Den Arbeitsplatz beherrscht der Arbeitgeber rechtlich in erheblichem Maße; seine Verantwortung für das Arbeitsumfeld streitet auch bei Telearbeitsplätzen im Sinne der Betriebsrisikolehre für eine Zuordnung zum versicherten Bereich ${ }^{75}$ und insbesondere für die Einordnung des Telearbeitsplatzes als Betriebsstätte. Dies bedeutet freilich, dass der Ausgangspunkt des infrage stehenden Weges zur Nahrungsaufnahme - nämlich der häusliche Arbeitsplatz - ebenso wie der Arbeitsplatz im arbeitgebereigenen Bürogebäude durch die Notwendigkeit, im Betrieb anwesend zu sein, geprägt ist.

Soweit argumentiert wird, Betriebsbeschäftigte seien durch die Notwendigkeit ihrer Anwesenheit im Betrieb wegen ihrer Einbindung in den zeitlichen Betriebsablauf stärker gebunden als zu Hause Arbeitende, ist auch dies keineswegs ein pauschal gültiges Argument. ${ }^{746}$ Denn einerseits gibt es Betriebsbeschäftigte, die zeitlich vollkommen unabhängig arbeiten, weil sie etwa in Gleitzeit ohne Kernarbeitszeit ihre Tätigkeit verrichten. Andererseits steht es dem Arbeitgeber frei, auch den im Homeoffice arbeitenden Beschäftigten zu bestimmten (Kern-)Arbeitszeiten anzuweisen und seine Pausenzeiten festzuschreiben. Sein Weisungsrecht nach $\$ 106 \mathrm{GewO}$ gilt ohne Abstriche. Muss der Beschäftigte etwa zu gewissen Zeiten telefonisch erreichbar sein und kann deswegen zu diesen nicht in die Mittagspause gehen, ist er ebenso wie ein Beschäftigter in der Betriebsstätte in den zeitlichen Unternehmensablauf eingegliedert und von ihm abhängig. Da Bürokommunikation meist automatisch umgeleitet wird, gilt dies in der Regel. Präsenz- und Pausenzeiten von Beschäftigten im Homeoffice sind häufig

745 S.o. Kap. 3 A. II.

746 Spellbrink, NZS 2016, S. 527, 530. 
wie bei den in der Betriebsstätte tätigen Arbeitnehmern arbeitsvertraglich geregelt. ${ }^{747}$

Im Ergebnis fällt aufgrund der Organisationsherrschaft des Arbeitgebers über den Telearbeitsplatz jedenfalls der Ausgangspunkt des Weges zur Nahrungsaufnahme nach der Betriebsrisikolehre in den Verantwortungsbereich des Arbeitgebers. Der Weg selbst ist deswegen ebenso wie der von Betriebsbeschäftigten in seinem Ausgangspunkt durch die Notwendigkeit, sich am jeweiligen Arbeitsplatz zu befinden, geprägt. Lediglich das Ziel des Weges liegt in der Privatsphäre des Beschäftigten. Auch dieses hängt aber unmittelbar mit der vom Arbeitgeber angewiesenen Tätigkeit im Homeoffice zusammen. Zumindest zum Unfallzeitpunkt befindet sich der Beschäftigte nicht nur aus privaten, sondern wesentlich auch aus beruflichen Gründen auf dem Weg in die Küche.

Ein Verneinen des sachlichen Zusammenhangs des Weges zur Nahrungsaufnahme kann im Ergebnis unter Zugrundelegung der Betriebsrisikolehre lediglich auf die Tatsache gestützt werden, dass der Zielort des Weges ein Raum in der Privatwohnung des Beschäftigten ist und die Strecke selbst ebenso in der Privatwohnung des Beschäftigten liegt. Alle anderen von der Rechtsprechung zur Bejahung des sachlichen Zusammenhangs verlangten Kriterien, namentlich der betriebsdienliche Handlungszweck, die Notwendigkeit der Anwesenheit am Arbeitsort sowie die Prägung des Weges durch den Ausgangsort durch die berufliche Tätigkeit, sind gegeben. Es muss daher wertend entschieden werden, ob die Tatsache, dass der Zielort des Weges innerhalb der Privatwohnung liegt, Grund genug ist, den gesamten Weg zur Nahrungsaufnahme nicht unter Unfallversicherungsschutz zu stellen. Ein Argument für die Zuordnung zum Betriebsrisiko kann daraus gewonnen werden, dass ein Weg zur Nahrungsaufnahme aus dem arbeitgebereigenen Bürogebäude heraus mit dem Ziel der privaten Wohnung ebenfalls unter Versicherungsschutz gestanden hätte. In diesem Fall erstreckt sich der Versicherungsschutz auf den Weg vom Arbeitsplatz bis zur Außentür des Wohngebäudes des Versicherten. ${ }^{748}$ Zwar ist auch in diesen Fällen die Zurücklegung eines Weges innerhalb der privaten Wohnung nicht vom Unfallversicherungsschutz umfasst. Jedoch wird deutlich, dass das Sichbefinden des Zielortes im privaten Bereich der Arbeitnehmer für die Zuordnung des Weges zum Betriebsrisiko des Unternehmers zunächst unerheblich ist. Für nicht entgrenzt arbeitende Personen ist das Ende des Versicherungsschutzes an der Außentür des Wohnge-

747 Vgl. Schur, jM 2014, S. 249, 251.

748 Dazu s.u. Kap. 3 A. VI. 3. 
bäudes auch deswegen folgerichtig, weil ihr Arbeitgeber - anders als der Arbeitgeber eines im Homeoffice Beschäftigten - keinerlei Einflussmöglichkeiten auf die Wohnumgebung des Beschäftigten hat.

Diese Betrachtungen zeigen, dass eine Zuordnung des Weges der Nahrungsaufnahme vom häuslichen Arbeitsplatz bis zur räumlichen Abgrenzung des Bereichs, in dem die Nahrungsaufnahme stattfinden soll, zum Betriebsrisiko des Unternehmers unter Zugrundelegung der Betriebsrisikolehre möglich ist. ${ }^{749} \mathrm{Da}$ die objektivierte Handlungstendenz des Beschäftigten zum Zeitpunkt des Unfalls als das maßgebliche Kriterium der $\mathrm{Zu}$ ordnung zum Schutzbereich der Unfallversicherung gilt, ist ein Unterschutzstellen der mit betriebsdienlicher Intention zurückgelegten Wege innerhalb der Wohnung des Versicherten insgesamt sachgerecht. Dass das Bundessozialgericht im zitierten Urteil anders entschieden hat, ${ }^{750}$ zeigt, dass es den Unfallort als maßgeblichen Faktor für die Bestimmung der Handlungstendenz ansieht. Obwohl es noch im selben Urteil anmerkt, dass es daran zweifelt, ob an der Rechtsprechung, die Unfälle in der Wohnung der Beschäftigten nur dann als Arbeitsunfälle ansieht, wenn sie sich an regelmäßig wesentlich zu betrieblichen Zwecken genutzten Orten ereignen, festzuhalten ist, greift es - wie aus der Analyse ersichtlich wurde auf das Kriterium des Ortes als entscheidenden Faktor zurück. Es erscheint allerdings möglich, dass angesichts der Tatsache, dass das Gericht in einem jüngeren Urteil das Kriterium der Örtlichkeit des Unfalls in ausdrücklicher Abkehr von seiner früheren Entscheidungspraxis auf einen weit geringer zu gewichtenden Platz verwiesen hat, seine Meinung zu Wegen zur Nahrungsaufnahme innerhalb des häuslichen Bereichs ebenfalls revidieren wird.

b. Im Lichte des sozialen Schutzprinzips

aa. Präventionsaspekt

Neben der Betriebsrisikolehre wurden für eine Ausklammerung von Wegen zur Nahrungsaufnahme im häuslichen Bereich auch Aspekte des sozialen Schutzprinzips in Feld geführt. So wurde argumentiert, eine Versiche-

749 Ebenso Häfner-Beil/Nacke, Gute Arbeit 2016, S. 4; Schur, jM 2014, S. 249, 251; im Ergebnis auch LSG Rheinland-Pfalz, Urt. v. 27.1.2015 - L 3 U 171/14 -, juris Rn. 21.

750 BSG, Urt. v. 5.7.2016 - B 2 U 5/15 R -, juris Rn. 27. 
rung von Unfällen im Wohnbereich des Arbeitnehmers würde dem Strukturprinzip der Prävention widersprechen. ${ }^{751}$ Die Prävention ist eine zentrale Aufgabe der Unfallversicherung, $\mathbb{1} 1$ Abs. 1 SGB VII, $\$ 14 \mathrm{ff}$. SGB VII. Durch Maßnahmen der General- und Individualprävention sollen Arbeitsunfälle so weit als möglich vermieden werden. ${ }^{752}$ Damit wird der Schutz der Beschäftigten vor gesundheitlichen Schäden genauso bezweckt wie der Schutz der Unternehmerschaft vor der wirtschaftlichen Belastung, die durch eine Leistungspflicht der Unfallversicherung eintritt. ${ }^{753}$ Für die Durchführung der Präventionsmaßnahmen sind Unternehmer nach $\$ 21$ Abs. 1 SGB VII verantwortlich. Da die Arbeitsschutzpflichten der Unternehmer hier mit dem Recht der Beschäftigten auf Unverletzlichkeit der Wohnung aus Art. 13 GG kollidieren, sind ihre Einflussmöglichkeiten im Hinblick auf präventive Maßnahmen gegenüber der Betriebsstätte eingeschränkt. Die Pflicht zur Durchführung von Präventionsmaßnahmen beschränkt sich auf den häuslichen Arbeitsplatz an sich und gilt nicht für andere häusliche Räumlichkeiten wie den Flur oder die Küche. ${ }^{754}$ Die Unfallversicherungsträger trifft ebenfalls eine Präventionspflicht aus $₫ 14$ Abs. 1 SGB VII. Sie haben deswegen die Durchführung der Präventionsmaßnahmen durch den Arbeitgeber zu überwachen. In der Betriebsstätte sind ihre Aufsichtspersonen deswegen befugt, sie zu den Betriebs- und Geschäftszeiten zu besichtigen und zu prüfen. In der Wohnung des Beschäftigten ist eine solche Maßnahme jedoch wegen Art. 13 Abs. 7 Alt. 2 GG nur möglich, wenn dies zur Verhütung dringender Gefahren geboten ist, $\mathbb{} 19$ Abs. 2 S. 3 und 4 SGB VII, oder wenn sich der Beschäftigte damit einverstanden erklärt. ${ }^{755}$ Dabei ist umstritten, ob die Aufnahme von Telearbeit bereits ein konkludentes Einverständnis mit dem Betreten seiner Wohnung zur Präventionsausübung darstellt oder eine separate arbeitsvertragli-

751 BSG, Urt. v. 5.7.2016 - B 2 U 5/15 R -, juris Rn. 28; Spellbrink, NZS 2016, S. 527, 530; Leube, SGb 2012, S. 380, 385.

752 Tiemann, SGb 1996, S. 405, 408.

753 Wietfeld, in: Rolfs/Giesen et al. (Hrsg.), BeckOK Sozialrecht, $\mathbb{1} 1$ Rn. 9; ebenso wohl Boewer/Gaul, Aktuelles Arbeitsrecht 2016, S. 632, die als Grund für eine ihrer Meinung nach gerechtfertigte möglichst restriktive Anerkennung von Arbeitsunfällen im Homeoffice aus der gesetzlichen Unfallversicherung wenig konkret den "Schutz der Versichertengemeinschaft" angeben.

754 BSG, Urt. v. 5.7.2016 - B 2 U 5/15 R -, juris Rn. 28; Leube, SGb 2012, S. 380, 384.

755 Leube, SGb 2012, S. 380, 384; BSG, Urt. v. 5.7.2016 - B 2 U 5/15 R -, juris Rn. 28. $\mathrm{Zu}$ bedenken ist zudem die Tangierung der Grundrechte evtl. Mitbewohner, vgl. a.a.O. S. 385 . 
che Regelung notwendig ist. ${ }^{756}$ Angekündigt werden muss eine Besichtigung nicht. ${ }^{757}$

Da Arbeitgeber und Unfallversicherungsträger aus diesen Gründen häusliche Arbeitsplätze in weniger umfangreichem Maße als Betriebsarbeitsplätze präventiv sicher gestalten könnten, sei es „sachgerecht und nicht unbillig“, das vom häuslichen Lebensbereich ausgehende Unfallrisiko vom Schutzbereich der Unfallversicherung auszunehmen. ${ }^{78}$ Denn durch die verringerte Verantwortung des Arbeitgebers für die konkrete Gestaltung des Arbeitsumfelds sei eine Zuordnung zur Betriebssphäre nicht mehr möglich. ${ }^{759}$

Was unter dem Titel der Prävention diskutiert wird, entpuppt sich damit bei näherer Betrachtung wiederum als eine Argumentation auf Basis der Betriebsrisikolehre. Dabei wird davon ausgegangen, dass durch die geringeren Einflussmöglichkeiten des Unternehmers auch seine Verantwortung für Unfälle sinke. Dagegen ist einmal mehr anzuführen, dass die Ablösung der Unternehmerhaftung gerade nicht ausschließlicher Rechtsgrund, sondern vor allem Mittel zur Umsetzung der gesetzlichen Unfallversicherung ist und von vornherein auch vom Unternehmer nicht gänzlich zu verhindernde und sogar durch den Beschäftigten selbstverschuldete Unfälle versichert sind. Zudem handelt es sich auch bei Unfällen auf dem Weg zur Nahrungsaufnahme innerhalb des häuslichen Bereichs nicht stets um die Verwirklichung von Risiken, die von der persönlichen Lebenssphäre ausgehen, ${ }^{760}$ sondern häufig auch um Risiken, die sich lediglich zufällig dort verwirklichen. Im Übrigen gilt das zur Betriebsrisikolehre bereits Gesagte:

756 Richtigerweise kann keine stillschweigende generelle Betretungserlaubnis der Wohnung angenommen werden, da der Beschäftigte über den konkreten Zeitpunkt, Anlass und Umfang einer solchen Besichtigung im Normalfall informiert wollen sein dürfte. Indem er einer fremden Person Zutritt zu seiner Wohnung eröffnet, gibt der Beschäftigte weitaus mehr Daten als nur die Beschaffenheit seines Arbeitsplatzes preis. So können selbst durch nur flüchtig im Vorbeigehen gewonnene Eindrücke Informationen über Lebensstil, Beziehungsstatus, finanzielle Situation, Gesundheit, Ordnungsaffinität u.v.m. des Beschäftigten gewonnen werden. Für die Annahme eines konkludenten Einverständnisses allerdings Wedde, Telearbeit, Rn. 512; dagegen: Leube, SGb 2012, S.380, 384; Wiese, RdA 1999, S. 344, 351.

757 Leube, SGb 2012, S. 380, 384; Ricke, in: Körner/Leitherer et al. (Hrsg.), Kasseler Kommentar zum Sozialversicherungsrecht, $\$ 19$ SGB VII Rn. 10; Schmitt, SGB VII, $₫ 19$ Rn. 15.

758 BSG, Urt. v. 5.7.2016 - B 2 U 5/15 R -, juris Rn. 28.

759 Spellbrink, NZS 2016, 527, 530.

760 Ein tatsächlich von der persönlichen Lebenssphäre ausgehendes Risiko wären etwa Unfälle aufgrund eines Einsturzes einer „nachträglich in Heimarbeit einge- 
Der Arbeitgeber hat es in der Hand, dem Beschäftigten die Arbeit von zu Hause aus zu verwehren. Ebenso kann er auf dem regelmäßigen Zugang zum Zwecke der Gefahrprävention beharren. Der Bezug zur betrieblichen Risikosphäre wird weiterhin durch die Notwendigkeit eines sachlichen $\mathrm{Zu}$ sammenhangs mit der versicherten Tätigkeit hergestellt.

\section{bb. Im Lichte der Fürsorgepflicht}

Wie bereits dargestellt, beruht das Unterschutzstellen von Arbeitsunfällen nicht ausschließlich auf der Betriebsrisikolehre, sondern auch maßgeblich auf dem Gedanken des sozialen Schutzprinzips und der Fürsorgepflicht der Unternehmer. ${ }^{761}$ So werden nicht nur Unfälle, die der Arbeitgeber kraft seiner Risikoherrschaft durch andere Organisation des Arbeitsablaufs hätte verhindern können, umfasst, sondern gerade auch solche Unfälle, die dem Zufall geschuldet sind. ${ }^{762}$ Diese Art von Unfällen ist aber häufig vollkommen unabhängig von einer etwaigen Einflusssphäre des Unternehmers. Als Beispiel möge eine Beschäftigte dienen, die beim Aufstehen vom Bürostuhl mit dem Fuß umknickt und sich diesen dabei verstaucht. Das Umknicken hätte ebenso beim Aufstehen von einem Stuhl zu Hause, von einer Sitzgelegenheit im Restaurant oder von einer Parkbank geschehen können. Obwohl der Unternehmer für letztere Unfälle nicht hätte haftbar gemacht werden können, sind sie als Arbeitsunfälle vom Versicherungsschutz der Unfallversicherung umfasst, wenn das Aufstehen anlässlich einer betrieblichen Tätigkeit geschah (etwa, weil die Beschäftigte die Bürotür öffnen wollte, um eine Kollegin hereinzubitten). Geschieht ein solches Umknicken auf dem Weg zur Nahrungsaufnahme, wäre es in der Betriebsstätte als Arbeits-, auf dem Weg von häuslichem Arbeitszimmer zur Küche jedoch als Privatunfall einzuordnen. Der Schutz von im Homeoffice Arbeitenden ist damit im Hinblick auf Unfälle, die durch andersartige Organisation von Abläufen und Einrichtung nicht hätten verhindert werden können, verkürzt. An der sozialen Schutzbedürftigkeit der verunfallten Person ändert sich durch die örtlich abweichende Konstellation jedoch nichts. Zudem obliegt dem Arbeitgeber für beide Personengruppen in gleichem Maße eine Fürsorgepflicht. Der Bereich des Versicherungsumfangs, der für Be-

bauten Wendeltreppe (...), die sämtlichen Bauvorschriften widerspricht", vgl. Spellbrink, NZS 2016, 527, 530.

761 Zuletzt Ricke, WzS 2017, S. 9, 12.

762 S.o. Kap. 2 C. 
triebsbeschäftigte aus diesen Grundprinzipien der Unfallversicherung hergeleitet wird, entfällt für Telearbeitnehmer. Dies stellt einen Bruch mit dem sozialen Schutzprinzip dar. Seine Anwendung würde auch für zu Hause Beschäftigte einen Schutz vor zufälligen Unfällen bei Wegen zur Nahrungsaufnahme gebieten.

\section{c. Unter Anwendung des Gleichheitssatzes, Art. 3 Abs. 1 GG}

Das eben Ausgeführte wird noch durch die Prüfung des Gleichheitssatzes bestärkt. Art. 3 Abs. 1 GG verlangt, ,weder wesentlich Gleiches willkürlich ungleich, noch wesentlich Ungleiches willkürlich gleich“763 zu behandeln. Als willkürlich ist anzusehen, was tatsächlich und eindeutig unangemessen in Bezug auf den zu ordnenden Gesetzgebungsgegenstand ist. ${ }^{764}$ Zwei Gruppen von Normadressaten sind also dann gleich zu behandeln, wenn zwischen ihnen keine Unterschiede von solcher Art und solchem Gewicht bestehen, dass sie eine ungleiche Behandlung rechtfertigen können. ${ }^{765}$ Der sachliche Grund für die Ungleichbehandlung muss sich dabei gerade aus dem Sachverhalt ergeben, der geregelt werden soll. ${ }^{766}$ Dies bedeutet für

763 Std. Rspr., vgl. nur BVerfGE 4, 144, 155 und aus neuerer Zeit E 50, 177, 186; 51, 295, 300; 60, 16, 42; 86, 81, 87. Starck, in: v. Mangoldt/Klein/ders. (Hrsg.), GG, Art. 3 Rn. 10. Umfangreiche Literaturhinweise zu Art. 3 GG finden sich insb. bei Kirchhof, in: Maunz/Dürig et al. (Hrsg.), Grundgesetz, Art. 3 vor Rn. 1.

764 Std. Rspr., vgl. nur BVerfGE 1, 14, 52; 4, 144, 155. Kirchhof, in: Maunz/Dürig et al. (Hrsg.), Grundgesetz, Art. 3 Rn. 432; Starck, in: v. Mangoldt/Klein/ders. (Hrsg.), GG, Art. 3 Rn. 10 m.w.N.

765 Sog. „neue Formel“, vgl. nur BVerfG, Beschl. v. 30.3.2007 - 1 BvR 3144/06 -, juris Rn. 18 m.w.N.; Kirchhof, in: Maunz/Dürig et al. (Hrsg.), Grundgesetz, Art. 3 Rn. 75 m.w.N.; Starck, in: $v$. Mangoldt/Klein/ders. (Hrsg.), GG, Art. 3 Rn. 11 m.w.N. Es ist dabei nicht nötig, eine „Vergleichbarkeit“ der Gruppen festzustellen, vgl. Kischel, in: Epping/Hillgruber (Hrsg.), BeckOK GG, Art. 3 Rn. 18; ders., AöR 124 (1999), S. 174, 184; Kempny/Reimer, Die Gleichheitssätze, S. 41 f., 130; a.A. Koenig, JuS 1995, S. 313, 314 f. Auch die Feststellung einer Benachteiligung ist entbehrlich, vgl. BVerfGE 18, 38, 46; Kischel, in: Epping/Hillgruber (Hrsg.), BeckOK GG, Art. 3 Rn. 19; Sachs, DÖV 1984, S. 411, 416 f.; a.A. Huster, in: Friauf/Höfling (Hrsg.), GG, Art. 3 Rn. 46; Jarass/Pieroth, GG, Art. 3 Rn. 10 f.; Osterlob/Nußberger, in: Sachs (Hrsg.), GG, Art 3 Rn. 84.

766 Vgl. BVerfGE 46, 299, 312 f.; Kirchhof, in: Maunz/Dürig et al. (Hrsg.), Grundgesetz, Art. 3 Rn. 392, 433. Der Maßstab für die Rechtfertigung ist dabei dem Grad der Ungleichbehandlung angepasst: Die Kontrolldichte reicht von einer reinen Willkürkontrolle bis zur Verhältnismäßigkeitsprüfung, sog. „neueste Formel“, vgl. BVerfGE 88, 87, 96; Herzog, in: Maunz/Dürig (Hrsg.), Anh. Art. 3 Rn. 10, wobei der Maßstab stufenlos an Regelungsgegenstand und Differenzierungs- 
den vorliegenden Sachverhalt, dass die Strukturprinzipien des Unfallversicherungsrechts selbst den Sachgrund für eine Ungleichbehandlung liefern müssten.

Bei seiner Anwendung des Gleichheitssatzes im Urteil vom 5.7.2016 kommt das Bundessozialgericht zu dem Schluss, dass eine Ungleichbehandlung von Betriebsbeschäftigten und Telearbeitnehmern im Hinblick auf Wege zur Nahrungsaufnahme gerechtfertigt sei. Dies ist allerdings nur teilweise richtig. Zu kritisieren ist nicht nur der durch den II. Senat gewählte Argumentationsweg, sondern auch das damit gefundene Ergebnis. Denn das Gericht rechtfertigt seine Ungleichbehandlung von Beschäftigten, die ihren Weg zur Nahrungsaufnahme innerhalb ihrer Wohnung zurücklegen, im Vergleich zu Beschäftigten, deren Weg innerhalb der Betriebsstätte liegt, mit der Tatsache, dass sich nur Letztere örtlich im Unternehmen selbst befinden. Der wesentliche Unterschied bestünde darin, dass lediglich die sich in der Betriebsstätte befindenden Arbeitnehmer betrieblichen Zwängen unterlägen. ${ }^{767}$ Diese Begründung kann indes keine Allgemeingültigkeit beanspruchen. Denn während in vielen Fällen auch Betriebsbeschäftigte selbstständig über Ort und Zeit ihrer Mittagspause entscheiden können, sind andererseits auch die meisten im Homeoffice Arbeitenden an zeitliche und örtliche Vorgaben gebunden. Die geforderte „besondere Beziehung zur Betriebstätigkeit" liegt in diesen Fällen auch bei Telearbeitnehmern problemlos vor. So kann etwa eine Umleitung der betrieblichen Kommunikation an den häuslichen Arbeitsplatz, wie sie häufig der Fall ist, eine persönliche Anwesenheit im Homeoffice zu den telefonischen Geschäftszeiten des Unternehmens erfordern. Ist dies der Fall, so sind auch Küche und Toilette im räumlichen Bezug zu diesem Beschäftigungsort vorgegeben. Der entscheidende Unterschied zwischen Betriebsbeschäftigten und zu Hause arbeitenden Personen liegt mithin nicht in der Organisationshoheit des Arbeitgebers, sondern in der Beherrschbarkeit der örtlichen Umgebung durch den Beschäftigten selbst begründet. Während der Arbeitgeber, wie gesehen, in beiden Fällen nach der Betriebsrisikolehre die Arbeitsabläufe beherrscht, haben jedoch lediglich Telearbeitnehmer ebenfalls die Gefahrherrschaft über die Örtlichkeit. Über Flur und Kantine im Bürogebäude üben Beschäftigte keinerlei Organisationshoheit aus, über Korridor und Küche in der eigenen Wohnung dagegen schon. Selbst wenn

merkmale anzupassen ist, sog. „Stufenlos-Formel“, BVerfGE 129, 49, 49. Zu Entwicklung und Meinungsstand in der Literatur Kischel, in: Epping/Hillgruber (Hrsg.), BeckOK GG, Art. 3 Rn. 24 ff. m.w.N.

767 BSG, Urt. v. 5.7.2016 - B 2 U 5/15 R -, juris Rn. 31; zustimmend Spellbrink, NZS 2016, S. 527, 530 . 
sie nicht zeitlich über die Lage ihrer Pause bestimmen können bzw. durch Anwesenheitspflicht am häuslichen Arbeitsplatz darauf verwiesen sind, die eigenen Räumlichkeiten für ihre Pause zu nutzen, so können sie doch durch Ausgestaltung dieser deren Gefahrquellen beherrschen. Zudem kennen sie die Eigenheiten ihrer Einrichtung selbst besser als der Unternehmer. Darin unterscheidet sich die Situation der im Homeoffice Tätigen deutlich von derjenigen der am Unternehmenssitz beschäftigten Personen. Da nur Telearbeitnehmer zusätzlich zum Arbeitgeber die Risiken der Wegstrecke beherrschen können, sind sie in Bezug auf Wege zur Nahrungsaufnahme innerhalb des Gebäudes nicht im Sinne des Art. 3 Abs. 1 GG mit Betriebsbeschäftigten vergleichbar. Ihre unterschiedliche Behandlung durch die Auslegung des $\$ 8$ Abs. 1 SGB VII ist insoweit gerechtfertigt.

Dies kann aber nur für solche Unfälle gelten, welche tatsächlich mit den örtlichen Risikofaktoren zusammenhängen. Die Prüfung der Fallgruppe vor den Interpretationsfolien der Betriebsrisikolehre und des sozialen Schutzprinzips bzw. der Fürsorgepflicht der Arbeitgeber hat gezeigt, dass ein Unterschied zwischen beiden Beschäftigtengruppen jedenfalls bei Unfällen, welche aus Gründen des sozialen Schutzes heraus vom Versicherungsschutz umfasst sind, nicht an der Frage nach der Einflussnahmemöglichkeit des Arbeitgebers festgemacht werden kann. Bei solchen dem $\mathrm{Zu}$ fall geschuldeten Unfällen besteht damit kein eine unterschiedliche Behandlung rechtfertigender Sachgrund. Art. 3 Abs. 1 GG gebietet es daher, Telearbeitnehmer und Betriebsbeschäftigte unfallversicherungsrechtlich höchstens dann unterschiedlich zu behandeln, wenn der auf dem Weg zur Nahrungsaufnahme erlittene Unfall tatsächlich eine durch die äußeren, vom Arbeitgeber beeinflussbaren Faktoren verursachte Gefahrverwirklichung darstellt.

3. Versicherungsschutz für Wege zur Nahrungsaufnahme außerhalb des häuslichen Bereichs

a. Im Lichte der Schutzzwecke der Wegeunfallversicherung

Führt der Weg zur Nahrungsaufnahme Beschäftigte von der unternehmereigenen Betriebsstätte aus in ein Restaurant, einen Supermarkt oder einen anderen Ort außerhalb des Betriebsgeländes, so steht das Zurücklegen dieses Weges nach gefestigter Rechtsprechung ebenfalls in sachlichem Zusam- 
menhang mit der versicherten Tätigkeit. ${ }^{768}$ Unfälle auf diesen Wegen werden als nach $₫ 8$ Abs. 2 Nr. 1 SGB VII versicherte Wegeunfälle angesehen. Grund dafür ist auch hier die doppelte Verknüpfung des Weges mit der Betriebstätigkeit, die über die auf die Aufrechterhaltung der Arbeitsfähigkeit gerichtete Handlungstendenz und die Prägung des Weges durch die Notwendigkeit, persönlich im Beschäftigungsbetrieb anwesend zu sein, hergestellt wird. ${ }^{769}$ Der Versicherungsschutz auf solchen Wegen außerhalb des Betriebes endet nach den allgemeinen Grundsätzen für Wegeunfälle an der Außentür des Zielorts. ${ }^{770}$ Da es sich hierbei um Wegeunfälle handelt, sind als für die Auslegung der Reichweite des Versicherungsschutzes maßgebliche Kriterien die Schutzzwecke des Einbezugs der Wegeunfälle heranzuziehen. Wie oben dargelegt, handelt es sich dabei ebenfalls um die Betriebsrisikolehre und das soziale Schutzprinzip; zusätzlich aber ist zu beachten, dass wesentliches Ziel der Erweiterung des Unfallversicherungsschutzes auf Wegeunfälle war, die Beschäftigten speziell vor den Gefahren des öffentlichen Verkehrsraums zu schützen. ${ }^{771}$

Für Beschäftigte, die vom häuslichen Arbeitsplatz aus einen Weg zu einem außerhalb der eigenen Wohnung liegenden Ziel unternehmen, um dort zu essen, verneinte die Rechtsprechung in zwei jüngeren Urteilen dagegen den sachlichen Zusammenhang mit der beruflichen Tätigkeit. ${ }^{772} \mathrm{Im}$ Fall eines leitenden Angestellten, der abends zu Hause seiner beruflichen Tätigkeit nachging, diese für eine Fahrt zum Restaurant unterbrach, wo er etwas essen und währenddessen weiterarbeiten wollte, und auf dem Rückweg verunfallte, prüfte das BSG ausführlich das Vorliegen eines Betriebsweges, eines Wegeunfalls und eines Unfalls im Rahmen der Fallgruppe "Wege zur Nahrungsaufnahme“. Die detailreiche Argumentation dieses Urteils zeigt deutlich, wie schwierig es dem Gericht fällt, den häuslichen Arbeitsplatz versicherungsrechtlich einzuordnen. ${ }^{773}$

Es verneinte zunächst den Versicherungsschutz unter dem Gesichtspunkt eines Betriebsweges für das Zurücklegen des Weges zwischen Restaurant und Privatwohnung mit dem Argument, dass der Beschäftigte mit gemischter Motivationslage handelte. Die Intention zur Nahrungsaufnahme sei als privatwirtschaftlich, der Wille zum Fortführen der Arbeit im Re-

768 Vgl. nur BSG, Urt. v. 2.12.2008 - B 2 U 17/07 R -, juris Rn. 25; v. 27.4.2010 - B 2 U 23/09 -, juris Rn 15; v. 18.6.2013 - B 2 U 7/12 R -, juris Rn. 20 mwN.

769 Keller, in: Hauck/Noftz/ders. (Hrsg.), SGB VII, $\mathbb{8} 8$ Rn. 91.

770 BSG, Urt. v. 24.6.2003 - B 2 U 24/02 R -, juris 2. Ls.

771 Kap. 3 A. VI. 3. a.

772 BSG, Urt. v. 5.7.2016 - B 2 U 5/15 R -, juris; v. 18.6.2013 - B 2 U 7/12 R -, juris.

773 BSG, Urt. v. 18.6.2013 - B 2 U 7/12 R -, juris. 
staurant als betriebsdienlich einzuordnen. Nach der Rechtsprechungspraxis zu dieser Fallgruppe beurteilte das Gericht das Zurücklegen des Weges anschließend danach, ob der Weg hypothetisch auch dann zurückgelegt worden wäre, wenn die private Motivation des Handelns entfallen wäre. ${ }^{774}$ Geht man davon aus, dass der Versicherte nichts essen wollte, sei anzunehmen, dass er nicht in ein Restaurant gefahren wäre, sodass die Verrichtung nach der Betriebsrisikolehre nicht der Unternehmensphäre zuzuordnen sei und daher kein sachlicher Zusammenhang vorliege. ${ }^{775}$ Dass die Nahrungsaufnahme auf die Aufrechterhaltung der Arbeitsfähigkeit zielte und der Beschäftigte im Restaurant gedachte, während des Essens weiter betrieblichen Tätigkeiten nachzugehen, hätte hier allerdings zum gegenteiligen Ergebnis führen sollen. ${ }^{776}$ Es ist anzunehmen, dass das Gericht diese Erwägungen erst im Rahmen der Prüfung der Fallgruppe „Wege zur Nahrungsaufnahme" anbringen wollte.

Im Anschluss prüfte das Gericht den Versicherungsschutz unter dem Gesichtspunkt eines klassischen Wegeunfalls nach $₫ 8$ Abs. 2 Nr. 1 SGB VII. Nach dieser Norm ist das Zurücklegen des mit der versicherten Tätigkeit zusammenhängenden unmittelbaren Weges nach und von dem Ort der Tätigkeit vom Versicherungsschutz umfasst. Als Rechtsgrund dafür wird angegeben, dass diese Wege nicht aus privaten Interessen, sondern mit einer auf die versicherte Tätigkeit bezogenen Handlungstendenz unternommen werden. ${ }^{777} \mathrm{Im}$ genannten Urteil konnte das Bundessozialgericht das Homeoffice des Beschäftigten nicht als Ort der Tätigkeit anerkennen, da in der Vorinstanz nichts dazu festgestellt worden war. Ob der Unfall als Wegeunfall anzuerkennen sei, blieb daher offen. Das Gericht stellte aber fest, dass jeder Arbeitsort Ort der Tätigkeit im Sinne des $\$ 8$ Abs. 2 Nr. 1 SGB VII ist, wenn er arbeitsvertraglich festgelegt ist und der Beschäftigte dort mit Billigung seines Arbeitgebers tätig wird. Nicht umfasst seien „lediglich zusätzliche Arbeitsgelegenheiten“, welche der Beschäftigte sich in seinem privaten Lebensbereich eingerichtet hat, um dort außerhalb seiner aus dem Beschäftigungsverhältnis resultierenden Pflichten Arbeiten zu erbringen. ${ }^{778}$ Nur wenn der Arbeitgeber um den häuslichen Arbeitsplatz des Beschäftigten weiß, ist er also Ort der Tätigkeit im Sinne des $\$ 8$ Abs. 2 Nr. 1 SGB VII, was im Lichte der Betriebsrisikolehre folgerichtig ist.

774 Ebd. Rn. 15.

775 Ebd. Rn. 16.

776 Ebenso Brink, ArbRAktuell 2013, S. 632, 632.

777 BSG, Urt. v. 2.12.2008 - B 2 U 17/07 R -, juris Rn. 14 m.w.N.

778 BSG, Urt. v. 18.6.2013 - B 2 U 7/12 R -, juris Rn. 19. 
Als dritten Ansatzpunkt ordnete das Gericht nun den Unfall des Angestellten in die Fallgruppe der "Wege zur Nahrungsaufnahme“ ein, die - soweit sie vom Ort der Tätigkeit aus in einen anderen Bereich führen - in ständiger Rechtsprechung als Wegeunfälle anerkannt sind, wenn das Essen zur Aufrechterhaltung der Arbeitsfähigkeit dienen soll und der Weg durch eine Eingliederung in den betrieblichen Ablauf geprägt ist. ${ }^{779} \mathrm{Zu}$ verlangen seien demnach eine arbeitsvertragliche Regelung, nach der die geschuldete Arbeitsleistung auch vom häuslichen Arbeitsplatz aus erbracht werden kann, und eine direkte örtliche und zeitliche Einbindung der Nahrungsaufnahme in eine objektiv bestehende betriebliche Ablauforganisation. ${ }^{780} \mathrm{Da}$ der fragliche Beschäftigte in der Organisation seines Arbeitstages vollkommen frei war, verneinte das Gericht die erforderliche Beziehung zur Betriebstätigkeit. Es argumentierte, dass Beschäftigte ansonsten vollkommen selbst bestimmen könnten, wann und wie sie den Versicherungsschutz der Wegeunfallversicherung begründen könnten, was zu einem „Versicherungsschutz rund um die Uhr" führen würde. ${ }^{781}$ Dies ist freilich kein überzeugender Aspekt. Denn da der sachliche Zusammenhang zur beruflichen Sphäre gerade durch eine berufliche Tätigkeit hergestellt wird, ist selbstverständlich, dass das Ausüben einer arbeitsvertraglich geschuldeten Tätigkeit auch dann unter Unfallversicherungsschutz zu stehen hat, wenn dies tatsächlich „rund um die Uhr“ ist. Andauerndes Arbeiten kann, wenn es geschuldet ist, nicht zu einer Einschränkung des Versicherungsschutzes führen.

Vor allem aber trägt die Betriebsrisikolehre die vorgebrachten Überlegungen nicht. Die Argumentation der zitierten Rechtsprechung beruht auf zwei Pfeilern: Zum einen wird vorgebracht, der Arbeitgeber beherrsche die am häuslichen Arbeitsplatz bestehenden Risiken nicht in gleichem Maße wie die Gefahrenquellen seines eigenen Gebäudes; zum anderen wird betont, der Versicherte selbst habe die Gefahrherrschaft über diese Räumlichkeiten inne. Dieser Gedankengang verfängt jedoch nur dann, wenn der Weg zur Nahrungsaufnahme komplett in den Grenzen des eigenen Wohnbereichs des Beschäftigten stattfindet. Begibt sich der Versicherte auf einen Weg, der aus dem häuslichen Arbeitszimmer in den öffentlichen Verkehrsraum führt, bleibt zwar gleich, dass der Unternehmer die sich auf dem

779 BSG, Urt. v. 2.12.2008 - B 2 U 17/07 R -, juris Rn. 30; v. 27.4.2010 - B 2 U 23/09 -, juris Rn 15; v. 18.6.2013 - B 2 U 7/12 R -, juris Rn. 20 m.w.N.

780 BSG, Urt. v. 2.12.2008 - B 2 U 17/07 R -, juris Rn.30; zustimmend Schur, jM 2014, S. 249, 251.

781 BSG, Urt. v. 18.6.2013 - B 2 U 7/12 R -, juris Rn. 22; zustimmend Keller, in: Hauck/Noftz/ders. (Hrsg.), SGB VII, $\$ 8$ Rn. 20b; Schur, jM 2014, S. 249, 251. 
Weg befindlichen Gefahrenquellen nicht beherrscht; andererseits hat auch der Arbeitnehmer keinerlei Organisationshoheit über weite Teile dieser Strecke. Er beeinflusst die bestehenden Risiken durch die Wahl des Restaurants, des Verkehrsmittels und der Route dorthin. Diese Parameter setzen aber auch am Unternehmenssitz Beschäftigte selbst, wenn sie von dort aus zur Mittagspause aufbrechen. Lediglich in der Bestimmung des Ausgangsortes besteht ein Unterschied: Während der zu Hause Arbeitende von seinem Wohnort aus startet, beginnt der Betriebsbeschäftigte seinen Weg an der Betriebsstätte. Während die Adresse des Wohnorts vom Beschäftigten selbst gewählt wurde, wurde die Betriebstätte vom Unternehmer gewählt. Dieser Unterschied ist nicht signifikant genug, um eine unterschiedliche Zuordnung des Unfalls zu rechtfertigen. Denn in beiden Fällen obliegt es letztendlich dem Arbeitgeber, den Arbeitsort des Beschäftigten zu bestimmen. Seine Entscheidung, sein Weisungsrecht aus $\$ 106$ GewO dahingehend auszuüben, dass er den Beschäftigten gerade nicht zeitlichen und örtlichen Zwängen unterwirft, ist eine unternehmerische Entscheidung, die ebenso wie die entgegengesetzte zu seiner betrieblichen Sphäre zählt und deren Risiken er als Konsequenz zu übernehmen hat. Dass dem Ausgangsort eines Weges im Sinne des $₫ 8$ Abs. 2 Nr. 1 SGB VII keine große Bedeutung zugemessen werden darf, wird dadurch bestärkt, dass in ständiger Rechtsprechung bei der Anerkennung eines Wegeunfalls kein Unterschied darin besteht, ob der Weg von der Wohnung des Beschäftigten oder einem sogenannten „dritten Ort“ angetreten wurde. Das Zurücklegen der Strecke zum Ort der Tätigkeit steht immer unter Versicherungsschutz, wenn der Ausgangsort nicht dazu führt, dass Länge und Dauer des Arbeitsweges in unangemessenem Verhältnis zum unmittelbaren Weg von der Wohnung aus stehen. ${ }^{782}$ Der Ausgangsort spielt argumentativ hinsichtlich der Risikoerweiterung insoweit keine Rolle. ${ }^{783}$

$\mathrm{Zu}$ einem anderen Ergebnis kann auch nicht die mehrfach vorgebrachte Argumentation, der Arbeitnehmer habe für die für ihn vorteilhafte Situation der zeitlichen und örtlichen Flexibilität mit verringertem Unfallversicherungsschutz zu bezahlen, kommen. ${ }^{784}$ Denn wie bereits dargestellt wurde und durch Studien belegt ist, profitiert nicht nur der Arbeitnehmer, sondern auch der Arbeitgeber erheblich davon, seine Mitarbeiter im Homeoffice tätig sein zu lassen: Im Schnitt arbeiten Beschäftigte am häusli-

782 Ricke, in: Körner/Leitherer et al. (Hrsg.), Kasseler Kommentar zum Sozialversicherungsrecht, $\mathbb{8} 8$ SGB VII Rn. 210.

783 Ebd.

784 So Schur, jM 2014, S. 249, 251; Spellbrink, NZS 2016, S. 527, 530. 
chen Arbeitsplatz mehr und länger als Beschäftigte am Unternehmensstandort; zudem „bezahlen“ Erstere bereits mit einem erheblich höheren Risiko der (unbezahlten) Mehrarbeit und des erhöhten Stresses. ${ }^{785}$

Da zudem ohnehin anerkannt ist, dass der Wegeunfallschutz lediglich bis zur Außentür des Restaurants bzw. des Supermarkts oder Wohngebäudes reicht und damit der Privatheit des gewählten Zieles bereits Rechnung getragen ist, ist es unbillig, die Wege zur Nahrungsaufnahme von Telearbeitnehmern nicht unter Versicherungsschutz zu stellen.

$\mathrm{Zu}$ diesem Ergebnis muss man auch gelangen, wenn man die Besonderheit der hier im Fokus stehenden Wegeunfallversicherung als Maßstab anlegt. Wie oben dargelegt wurde, war ein wesentlicher Schutzzweck der Wegeunfallversicherung die Erwägung, Beschäftigte gerade vor den Folgen der Gefahren des öffentlichen Verkehrsraums zu schützen, da dieser als besonders risikoreich angesehen wurde. ${ }^{786}$ Die Risiken des öffentlichen Verkehrs treffen aber gerade jeden Teilnehmer daran in gleicher Weise, unabhängig davon, ob er von seiner Privatwohnung oder vom betrieblichen Arbeitsplatz aus an den konkreten Unfallort gelangt ist.

Keiner Wiederholung bedürfen die hier ebenso einschlägigen Überlegungen zum sozialen Schutzprinzip: Da der Arbeitgeber Telearbeitnehmern gegenüber ebenso fürsorgepflichtig ist, streitet dieses ebenfalls für eine Zurechnung von Wegen zur Nahrungsaufnahme zur betrieblichen Sphäre.

Auch Wege zur Nahrungsaufnahme, die vom häuslichen Bereich ausgehen, sollten bei ansonsten vorliegender betrieblicher Motivation vom Versicherungsschutz erfasst sein. ${ }^{787}$

\section{b. Unter Beachtung des Gleichheitssatzes, Art. 3 Abs. 1 GG}

Eine Bejahung des sachlichen Zusammenhangs von Wegen zur Nahrungsaufnahme aus dem Homeoffice heraus ist auch gemäß Art. 3 Abs. 1 GG geboten. ${ }^{788}$ Die zitierte Rechtsprechung verletzt den allgemeinen Gleichheitssatz, da sie eine Gruppe von Normadressaten im Vergleich zu anderen Normadressaten anders behandelt, obwohl zwischen beiden Gruppen keine Unterschiede von solcher Art und solchem Gewicht bestehen, dass sie

785 Dunkel/Kratzer/Menz, WSI Mitteilungen 2010, S. 357, 357 ff.; Däubler, SR 2014, S. $45,61$.

786 S.o. Kap. 3 A. VI. 3. a.

787 Ebenso Brink, ArbRAktuell 2013, S. 632, 632.

788 Vgl. Fn. 763. 
die ungleiche Behandlung rechtfertigen können. Zu vergleichen sind hier wiederum in Telearbeit Beschäftigte mit Beschäftigten, welche am Unternehmenssitz tätig sind. Wie die soeben im Lichte der Betriebsrisikolehre durchgeführte Analyse gezeigt hat, besteht im Hinblick auf deren Wege zur Nahrungsaufnahme vom eigenen Arbeitsplatz hinaus in den öffentlichen Verkehrsraum zwischen beiden Personengruppen lediglich der Unterschied, dass die zu Hause arbeitenden Personen ihren Wohnort einmalig selbst gewählt haben. Die für das Unfallversicherungsrecht maßgeblichen Parameter jedoch sind für beide Personengruppen nahezu identisch: Auch der häusliche Arbeitsplatz steht unter der Organisationshoheit des Arbeitgebers und ist diesem nach der Betriebsrisikolehre zuzuordnen. Der Unterschied im Ausgangsort des Weges stellt keinen Grund dar, der gravierend genug wäre, um eine Ungleichbehandlung der Personengruppen zu rechtfertigen. ${ }^{789}$ Ein solcher Grund läge nur vor, wenn nach den Schutzzwecken der Unfallversicherung eine abweichende Behandlung der Personengruppen möglich wäre. Dass dies nicht der Fall ist, wurde soeben ausführlich dargelegt.

Tatsächlich führt die Versagung des Versicherungsschutzes für Wege zur Nahrungsaufnahme, die vom häuslichen Arbeitsplatz aus angetreten werden, zu einer gravierenden Benachteiligung von Telearbeitnehmern gegenüber Arbeitnehmern, die an der Betriebsstätte arbeiten. Denn nach den aufgestellten Maßstäben sind die Wege der Ersteren zur Nahrungsaufnahme niemals als Wegeunfälle im Sinne des $₫ 8$ Abs. 2 Nr. 1 SGB VII anzuerkennen, egal, wie stark der Zusammenhang zur betrieblichen Tätigkeit selbst ausgeprägt ist.

Im zitierten Urteil führte das Gericht denn auch in einem obiter dictum aus, dass es „möglicherweise aus Gleichheitsgründen zu fordern sei“, dass „jedenfalls ein Weg täglich zur Nahrungsaufnahme unter Versicherungsschutz stehen muss“, wenn ein Beschäftigter in Vollzeit von zu Hause tätig ist. ${ }^{790}$ Konsequenterweise erkennt auch Spellbrink eine ungerechtfertigte Ungleichbehandlung der Telearbeitnehmer vorsichtig an. ${ }^{791}$ Allerdings ist kein sachlicher Grund dafür ersichtlich, eine Gleichbehandlung lediglich auf in Vollzeit tätige Arbeitnehmer zu erstrecken, die ohne Ausnahme im Homeoffice tätig sind. Auch für in Teilzeit und alternierender Telearbeit

789 Im Ergebnis auch Spellbrink, NZS 2016, S. 527, 530.

790 BSG, Urt. v. 18.6.2013 - B 2 U 7/12 R -, juris Rn. 22.

791 Spellbrink, NZS 2016, S. 527, 530; a.A. Keller, in: Hauck/Noftz/ders. (Hrsg.), SGB VII, $\mathbb{8}$ Rn. 20b; Ricke, WzS 2017, S. 9, 14. 
beschäftigte Personen gelten die vorgebrachten Erwägungen ohne Abstriche.

\section{Bewertung und Fazit}

Während die Rechtsprechung zu Wegen zur Nahrungaufnahme aus der Sicht der Rechtssicherheit positiv gesehen wurde, weil eine grundsätzliche Verweigerung von Unfallversicherungsschutz auf Wegen innerhalb einer häuslichen Arbeitsstätte Klarheit und Vorhersehbarkeit bringe, ${ }^{792}$ ist sie im Lichte der Strukturprinzipien der Unfallversicherung nicht sachgerecht. Betriebsrisikolehre und soziales Schutzprinzip streiten im Kontext entgrenzter Arbeit insgesamt für eine Zuordnung auch der Wege zur Nahrungsaufnahme im häuslichen Bereich zum Betriebsrisiko. Für die Fallgruppe von Unfällen, die nicht aus ortsgebundenen Risiken resultieren, verbietet Art. 3 Abs. 1 GG sogar die Zuordnung zur privaten Risikosphäre, da ansonsten eine ungerechtfertigte Schlechterstellung von im Homeoffice beschäftigten Personen gegenüber am Unternehmenssitz tätigen Arbeitnehmern vorläge. ${ }^{793}$

Auch Wege zur Nahrungsaufnahme eines im Homeoffice tätigen Arbeitnehmers, die diesen aus der Wohnung heraus etwa in ein Restaurant führen, sind ebenso wie entsprechende Wege von Betriebsbeschäftigten als vom Unfallversicherungsschutz des $₫ 8$ Abs. 2 SGB VII umfasst anzusehen. Eine andere Behandlung dieser Fallgruppe ist aufgrund der jeder Auslegung des $₫ 8$ SGB VII zugrunde liegenden Betriebsrisikolehre sowie im Hinblick auf Art. 3 Abs. 1 GG nicht möglich. Entgegen der Ansicht Rickes $^{794}$ ist die der Argumentation mit der Betriebsrisikolehre zugrunde liegende Annahme des Bundessozialgerichts, die Risikosphären von Unternehmer und Beschäftigtem ließen sich nach örtlichen Kriterien trennen, gerade nicht zeitlos gültig, sondern angesichts räumlich flexibler Arbeitsverhältnisse nicht mehr tragfähig.

792 Hollo, jurisPR-SozR 10/2017 Anm. 4.

793 Ebenso Häfner-Beil/Nacke, Gute Arbeit 2016, S. 4; Siefert, NZS 2017, S. 766, 769; a.A. Schur, jM 2014, S. 249, 251.

794 Ricke, WzS 2017, S. 9, 12. 
VI. Kindergartenwege aus dem Homeoffice

1. Grundlagen der Versicherung von Wegeunfällen

\section{a. Die Voraussetzungen des $₫ 8$ Abs. 2 Nr. 1 SGB VII}

Ein weiterer Fall der Ungleichbehandlung von Telearbeitnehmern und Betriebsbeschäftigten offenbart sich, wenn man den Unfallversicherungsschutz auf dem Weg des Beschäftigten zu Kinderbetreuungseinrichtungen betrachtet. Während Arbeitnehmer, die von ihrer Wohnung aus ein Kind fremder Obhut anvertrauen und anschließend am Unternehmenssitz die Arbeit aufnehmen, während des gesamten Weges unter Unfallversicherungsschutz stehen, sind Telearbeitnehmer auf dem Weg zur Unterbringung ihrer Kinder nicht unfallversicherungsrechtlich geschützt. ${ }^{795} \mathrm{Um}$ die Gründe dieser Differenzierung nachvollziehen und ihre Rechtfertigung beurteilen zu können, müssen zunächst die Grundlagen des Wegeunfallschutzes dargelegt werden.

$\int 8$ Abs. 2 SGB VII bestimmt, dass die in seinen Nrn. 1-5 genannten Verrichtungen als spezielle Unterfälle des $\$ 8$ Abs. 1 SGB VII ebenfalls versicherte Tätigkeiten sind. ${ }^{796}$ In $\ 8$ Abs. 2 Nr. 1 SGB VII ist geregelt, dass „das Zurücklegen des mit der versicherten Tätigkeit zusammenhängenden unmittelbaren Weges nach und von dem Ort der Tätigkeit“ eine versicherte Tätigkeit darstellt. Unfälle, die sich zum Zeitpunkt einer solchen Verrichtung ereignen, werden „Wegeunfälle“ genannt. ${ }^{797}$ Notwendig für die Zuordnung zum Schutzbereich der Unfallversicherung ist, dass das Zurücklegen des Weges in sachlichem Zusammenhang mit der Berufstätigkeit steht. ${ }^{798}$ Dieser liegt vor, wenn die Strecke mit der Intention zurückgelegt wird, die Arbeit aufzunehmen oder von ihr zurückzukehren. ${ }^{799}$

795 SG Hannover, Urt. v. 17.12.2015 - S 22 U 1/15 -, juris Rn. 16.

796 Die geregelten Tatbestände stellen keine rechtlichen Fiktionen, sondern Spezialfälle des Arbeitsunfalls dar, vgl. Marschner, in: Rolfs/Giesen et al. (Hrsg.), BeckOK Sozialrecht, $\mathbb{8}$ SGB VII Rn. 46; Giesen, Arbeitsunfall und Dienstunfall, S. 113 m.w.N.

797 Diese gängige Bezeichnung findet sich nicht im Gesetz, vgl. Marschner, in: Rolfs/Giesen et al. (Hrsg.), BeckOK Sozialrecht, $\$ 8$ SGB VII Rn. 46.

798 Marschner, in: Rolfs/Giesen et al. (Hrsg.), BeckOK Sozialrecht, $\mathbb{8}$ SGB VII Rn. 48; Ricke, in: Körner/Leitherer et al. (Hrsg.), Kasseler Kommentar zum Sozialversicherungsrecht, $\mathbb{\$} 8$ SGB VII Rn. 189; Schlaeger, NZS 2009, S. 559, 560.

799 BSG Urt. v. 4.7.2013 - B 2 U 3/13 R -, juris Rn. 12; v. 31.1.1974, E 37, 98; Ricke, in: Körner/Leitherer et al. (Hrsg.), Kasseler Kommentar zum Sozialversicherungsrecht, $\mathbb{8} 8$ SGB VII Rn. 189. 
Die geschützte Strecke beginnt nach der ständigen Rechtsprechung mit der Außentür des Wohngebäudes des Beschäftigten (bzw. mit der Außentür eines anderen, sog. „dritten“ Ortes ${ }^{800}$ und endet an der Außentür des Orts der Tätigkeit. ${ }^{801}$ Die Konsequenz des Außentür-Kriteriums ist, dass es innerhalb des Wohngebäudes des Versicherten keine Wegeunfälle geben kann. ${ }^{802}$ Ort der Tätigkeit ist der Ort, an dem der Beschäftigte seine arbeitsvertraglich geschuldete Tätigkeit verrichtet. ${ }^{803}$ Dies muss nicht der Unternehmenssitz sein: Jeder Ort, an dem der Arbeitnehmer in Übereinstimmung mit dem Arbeitgeber tätig wird, ist Arbeitsstätte im Sinne des $\mathbb{8} 8$

800 Das Gesetz legt nur den Ort der Tätigkeit als Grenzpunkt fest, der notwendige andere Grenzpunkt wird nicht bestimmt. Im Allgemeinen handelt es sich um die Privatwohnung des Beschäftigten; andere Orte werden als „dritter Ort“ bezeichnet und sind dann als Ausgangs- bzw. Zielort maßgeblich, wenn der Versicherte sich dort länger als zwei Stunden aufgehalten hat, BSG, Urt. v. 2.5.2001 B 2 U 33/00 -, juris Rn. 16; Krasney, SGb 2013, S. 313, 315 f.; ders., NZV 1989, S. 369, 370 f.; Marschner, in: Rolfs/Giesen et al. (Hrsg.), BeckOK Sozialrecht, $\mathbb{} 8$ SGB VII Rn. 59; Ricke, in: Körner/Leitherer et al. (Hrsg.), Kasseler Kommentar zum Sozialversicherungsrecht, $\mathbb{8} 8$ SGB VII Rn. $209 \mathrm{ff}$.

801 „Außentür“ ist dabei funktional zu verstehen. Gemeint ist damit der Gebäudeabschluss. Am Privatgebäude kann es sich neben einer Außenhaustür dabei auch um eine Keller- oder Terrassentür handeln, auf dem Betriebsgelände etwa um ein Werkstor oder die Eingangstür des Betriebsgebäudes, vgl. Ricke, in: Körner/Leitherer et al. (Hrsg.), Kasseler Kommentar zum Sozialversicherungsrecht, \8 SGB VII Rn. 182; Giesen, Arbeitsunfall und Dienstunfall, S. 116 f.; Marschner, in: Rolfs/Giesen et al. (Hrsg.), BeckOK Sozialrecht, $\mathbb{} 8$ SGB VII Rn. 52; Leube, ZTR 2012, S. 682, 686. Mit dem räumlichen Kriterium der Außentür als einzigem maßgeblichem Faktor wurde die Grenze zwischen privater und beruflicher Sphäre hier absolut gezogen, um Abgrenzungsschwierigkeiten zu vermeiden. Die Rechtsprechung ist deswegen immer wieder der Kritik ausgesetzt, vgl. Ricke, a.a.O., Rn. 182 sowie die Literaturnachweise bei Giesen, a.a.O., S. 117 Fn. 555. Für eine Aufgabe des Kriteriums der Außentür in Homeoffice-Fällen zugunsten einer rein tätigkeitsbezogenen Betrachtung andeutungsweise Marschner, in: Rolfs/Giesen et al. (Hrsg.), BeckOK Sozialrecht, $\mathbb{\$} 8$ SGB VII Rn. 54.

802 Vgl. nur BSG, Urt. v. 5.7.2016 - B 2 U 5/15 R -, juris Rn. 32. Gäbe es einen Wegeunfall im häuslichen Bereich, würden Versicherte mit häuslichem Arbeitsplatz besser stehen als Versicherte, die ausschließlich im Betrieb arbeiten, da ihr Versicherungsschutz erst bei der Außentür beginnt, selbst wenn es von dieser bis zu ihrem Arbeitsplatz nur wenige Meter sind, obgleich beide Wege auf den Beginn einer beruflichen Tätigkeit gerichtet sind, BSG, Urt. v. 25.2.1993 - 2 RU 12/92 -, juris Rn. 20; zustimmend Benz, SGb 2003, S. 12, 18.

803 Vgl. BSG, Urt. v. 18.6.2013 - B 2 U 7/12 R -, juris Rn. 19 (zur Veröffentlichung in Entscheidungssammlung vorgesehen); v. 8.7.1980 - 2 RU 17/9 -, juris Rn. 23; Krasney, SGb 2013, S. 313 ff., 315 m.w.N.; Keller, in: Hauck/Noftz/ders. (Hrsg.), SGB VII, $\$ 8$ Rn. 192. 
Abs. 2 Nr. 1 SGB VII. ${ }^{804}$ Dazu gehört auch das Homeoffice. ${ }^{805}$ Versichert ist grundsätzlich der unmittelbare Weg, wobei die Beschäftigten Verkehrsmittel und Strecke im Rahmen vernünftiger Erwägungen selbst wählen können. ${ }^{806}$ Der Versicherungsschutz entfällt, wenn Umwege oder Unterbrechungen, die nicht geringfügig sind, aus privaten Gründen gewählt werden. ${ }^{807}$ Ausnahmen zu diesem Grundsatz finden sich in $\$ 8$ Abs. 2 Nrn. 24 SGB VII.

b. Schutzzweck und Rechtsgründe der Versicherung von Wegeunfällen

Das Zurücklegen des Arbeitsweges wurde im Jahr 1925 in den Unfallversicherungsschutz einbezogen. ${ }^{808}$ Ziel dieser Regelung war es, Arbeitnehmer vor den spezifischen Gefahren des öffentlichen Verkehrsraums zu schützen. ${ }^{809}$ Dies ist Ausdruck des sozialen Schutzprinzips. ${ }^{810}$ Dass die alleinige Beitragslast für die Unfallversicherung weiterhin bei den Unternehmern verblieb und somit das Risiko, im öffentlichen Raum einen Unfall zu erleiden, der Arbeitgebersphäre zugeordnet wurde, wurde damit begründet, dass Beschäftigte sich nur wegen ihrer Arbeitstätigkeit den Risiken des

804 BSG, Urt. v. 18.12.1969 - 2 RU 19/68 -, juris Rn. 16; v. 8.7.1980 - 2 RU 17/9 -, juris Rn. 23; Keller, in: Hauck/Noftz/ders. (Hrsg.), SGB VII, $\mathbb{8}$ Rn. 192; Giesen, Arbeitsunfall und Dienstunfall, S. 117; Krasney, SGb 2013, S. 313, 315 m.w.N. Maßgeblich ist nicht der tatsächliche Arbeitsplatz, sondern das Gelände, auf dem sich dieser befindet, vgl. Ricke, in: Körner/Leitherer et al. (Hrsg.), Kasseler Kommentar zum Sozialversicherungsrecht, $\mathbb{\$} 8$ SGB VII Rn. 185.

805 Keller, in: Hauck/Noftz/ders. (Hrsg.), SGB VII, $\$ 8$ Rn. 192a.

806 Näher Ricke, in: Körner/Leitherer et al. (Hrsg.), Kasseler Kommentar zum Sozialversicherungsrecht, $₫ 8$ SGB VII Rn. 201.

807 Krasney, NZV 1989, S. 369, 372 m.w.N. Zum Sonderfall des Inobhutbringens von Kindern sogleich Kap. 3 A. VII.

808 2. Änderungsgesetz zur Unfallversicherung v. 14.7.1925, RGBl. I S. 97.

809 BSG, Urt. v. 13.11.2012 - B 2 U 19/11 R -, E 112, 177, 186 f.; Spellbrink, NZS 2016, S. 527, 529; a.A. die frühere Rechtsprechung des BSG, vgl. Urt. v. 13.3.1956 - 2 RU 124/54 -, juris Rn. 14, 17, 18. Zur zahlenmäßigen Bedeutung von Verkehrsunfällen im Unfallversicherungsrecht vgl. Krasney, NZV 1989, S. 369, 369 sowie die jährliche erscheinende Statistik des DGUV, abrufbar unter http://publikationen.dguv.de/ (Stand: 18.11.2018). A.A. Ricke, nach dem es ein „spezifisches Wegerisiko“ nicht gibt, SGb 2016, S. 705, 710.

810 Gitter, Schadensausgleich im Arbeitsunfallrecht, S. 61; Keller, in: Hauck/Noftz/ ders. (Hrsg.), SGB VII, $\mathbb{8} 8$ Rn. 190. 
Straßenverkehrs aussetzen. ${ }^{811}$ Es ist deswegen nicht möglich, alleine mit dem Argument des genannten Schutzzwecks die Einbeziehung sämtlicher Verkehrsunfälle in den Unfallversicherungsschutz zu begründen. Vielmehr ist es notwendig, zwischen privaten und beruflichen Wegen abzugrenzen und Letztere der betrieblichen Sphäre zuzuordnen. ${ }^{812}$ Auch hier spielt also das Prinzip der Betriebsrisikolehre eine gewichtige Rolle. ${ }^{813}$ Zwar kann eine Zugehörigkeit zur betrieblichen Sphäre nicht mit einer tatsächlichen Organisations- und Herrschaftsgewalt des Arbeitgebers über die Risiken, denen der Beschäftigte ausgesetzt ist, begründet werden. ${ }^{814}$ Die Risiken im öffentlichen Verkehr sind nicht vom Arbeitgeber beeinflussbar; weder hat er die Anlage und Instandhaltung öffentlicher Straßen in der Hand, noch stehen ihm Präventionsmaßnahmen zur Verfügung, die etwa die Gefahr eines vom Beschäftigten unverschuldeten Verkehrsunfalls mindern würden. Zudem hängt die konkrete Strecke, die der Arbeitnehmer zurückzulegen hat, nicht nur von der örtlichen Lage der Betriebsstätte, sondern maßgeblich auch davon ab, wo sich seine Privatwohnung befindet, und er ist derjenige, der Verkehrsmittel und Arbeitsweg wählt. ${ }^{815}$ Es wurde daher bereits bei Einführung des Wegeunfallschutzes ${ }^{816}$, aber auch später der Vorwurf erhoben, dass kein innerer Zusammenhang des Wegeunfalls mit dem betrieblichen Unfallrisiko vorläge und die Risikoübertragung auf den Arbeitgeber daher nicht systemgerecht sei. ${ }^{817}$ Sie könne weder mit einer Organisationsherrschaft des Arbeitgebers noch mit einer Haftungsablösung be-

811 Ricke, in: Körner/Leitherer et al. (Hrsg.), Kasseler Kommentar zum Sozialversicherungsrecht, $\$ 8$ SGB VII Rn. 178.

812 BSG, Urt. v. 13.3.1956 - 2 RU 124/54 -, juris Rn. 17, 18.

813 Auch wenn die Versicherung der Wegeunfälle gelegentlich als Abkehr vom Grundsatz der Ablösung der Unternehmerhaftpflicht bezeichnet wird, vgl. Ricke, in: Körner/Leitherer et al. (Hrsg.), Kasseler Kommentar zum Sozialversicherungsrecht, $\mathbb{8} 8$ SGB VII Rn. 178; Keller, in: Hauck/Noftz/ders. (Hrsg.), SGB VII, $\$ 8$ Rn. 190.

814 In der Schweiz zahlen deswegen die Arbeitnehmer den Anteil an den Unfallversicherungsbeiträgen, der auf die Wegeunfallversicherung fällt, vgl. Art. 7 i.V.m. 8 und 91 des schweizerischen Unfallversicherungsgesetzes; vgl. dazu Müller/Hofer/ Stengel, AJP 2015, S. 564, 573 ff. Auf das Recht der Schweiz hinweisend auch Gitter, Schadensausgleich im Arbeitsunfallrecht, S. 38 ff.; Gitter/Nunius, in: Schulin (Hrsg.), Sozialversicherungshandbuch Band 2, $\$ 5$ Rn. 72.

815 Nußberger, in: Boecken/Hänlein/Kruse/Steinmeyer (Hrsg.), Öffentliche und private Sicherung gegen soziale Risiken, S. 193, 204.

816 Ausführlich zur Entwicklung des Wegeunfallschutzes im Einzelnen Kranig/ Aulmann, NZS 1995, S. 203, 205 ff.

817 Gitter, BB 1998, Beilage 6 S. 1, 5 f.; Zahnbrecher, Werkstorprinzip in der gesetzlichen Unfallversicherung, S. $114 \mathrm{ff}$. 
gründet werden, da eine solche für die Fälle des Wegeunfalls gerade nicht vorliege. ${ }^{818}$ Denn der Arbeitgeber ist am Arbeitsweg nicht in einem Maße beteiligt, der unter irgendwelchen denkbaren Umständen seine persönliche Haftung ermöglichen würde. ${ }^{819}$

Tatsächlich verlangt aber bereits die Betriebsrisikolehre eine Zuordnung des Arbeitsweges zum betrieblichen Risikobereich. ${ }^{820}$ Das Zurücklegen des Weges zum Ort der Tätigkeit ist eine eindeutig in betriebsdienlicher Intention durchgeführte Handlung. Sie ist gerade nicht eigenwirtschaftlicher Natur. Der Beschäftigte nimmt den Arbeitsweg nur deswegen auf sich, um seiner Arbeit nachgehen zu können, da seine Anwesenheit am Zielort arbeitsvertraglich geschuldet ist. ${ }^{821}$ Die zum Unfallzeitpunkt ausgeführte Verrichtung ist aber gerade der wesentliche Ansatzpunkt für die Zuordnung zur Betriebssphäre; das physische Sichbefinden im öffentlichen Raum muss dahinter zurücktreten. Im Regelfall wird sich der Arbeitnehmer zudem bereits auf dem Arbeitsweg innerlich auf anstehende berufliche Aufgaben vorbereiten; ebenso wird er auf dem Weg von der Arbeit zurück nach Hause noch damit zu tun haben, den Arbeitstag gedanklich zu bewältigen.

Darüber hinaus lassen sich auch Aspekte vorbringen, die dem Arbeitgeber wiederum eine gewisse Organisationsherrschaft über das bestehende Wegeunfallrisiko zueignen. So etwa ist der Unternehmer derjenige, der den Betriebsstandort wählt; liegt der Standort außerhalb einer Gegend, in der ausreichender und/oder bezahlbarer Wohnraum für Arbeitnehmer vorhanden ist, müssen die Beschäftigten längere und damit risikoreichere Anfahrtswege auf sich nehmen. ${ }^{822}$ Je nach Lage des Unternehmens ist das Wegeunfallrisiko danach höher oder geringer (Letzteres etwa, wenn eine gute

818 Römer, Reform der gesetzlichen Unfallversicherung, S. 30 ff.; vgl. auch Baldschun, Solidarität und soziales Schutzprinzip in der gesetzlichen Unfallversicherung, S. 76 ff.

819 Ricke bezeichnet den Einbezug des Wegeunfalls deswegen als sozialpolitische Entscheidung, vgl. ders., in: Körner/Leitherer et al. (Hrsg.), Kasseler Kommentar zum Sozialversicherungsrecht, $\mathbb{8} 8$ SGB VII Rn. 178.

820 Gitter, Schadensausgleich im Arbeitsunfallrecht, S. 85 ff.; Kranig/Aulmann, NZS 1995, S. 203, 208 ff.; Zahnbrecher, Werkstorprinzip in der gesetzlichen Unfallversicherung, S. $130 \mathrm{f}$.

821 Nußberger, in: Boecken/Hänlein/Kruse/Steinmeyer (Hrsg.), Öffentliche und private Sicherung gegen soziale Risiken, S. 193, 204; Krasney, NZV 1989, S. 369, 371.

822 Bernstein, Schadensausgleich bei Arbeitsunfällen, S. 60. 
Verbindung an den risikoärmeren öffentlichen Nahverkehr besteht). ${ }^{823}$ Der Versicherungsschutz spiegelt dabei die Mobilitätsanforderungen wieder, die an Beschäftigte gestellt werden. ${ }^{824}$ Weiterhin bestimmt der Arbeitgeber durch die Organisation der Arbeitsabläufe in seinem Betrieb, wann der Arbeitnehmer auf dem Gelände zu erscheinen hat, und damit zumindest mittelbar auch, wann er sich auf den Weg begeben muss; fällt diese Zeit in die sog. Rush Hour, erhöht der Arbeitgeber damit das Wegeunfallrisiko des Beschäftigten. ${ }^{825}$ Auch dann, wenn im Betrieb pünktliches Erscheinen gefordert ist, entsteht ein gefahrerhöhendes Moment in Form eines Zeitdrucks, der risikofreudigeres Verhalten wahrscheinlicher macht. Diesen Effekt könnte der Arbeitgeber etwa durch das Einführen einer Gleitzeitregelung verhindern. ${ }^{826}$

Zuletzt liegt es auch im Interesse der Erhaltung des Betriebsfriedens, auch Wegeunfälle miteinzubeziehen, um gerichtliche Auseinandersetzungen über die Folgen von im Zusammenhang mit der Arbeit erlittenen Unfällen zu vermeiden. ${ }^{827}$

2. Wegeabweichung zur Unterbringung von Kindern, $\mathbb{8} 8$ Abs. 2 Nr. 2 lit. a SGB VII

Für das Zurücklegen eines vom unmittelbaren Arbeitsweg abweichenden Weges, um Kinder von Versicherten, die mit ihnen in einem gemeinsamen Haushalt leben, wegen ihrer, ihrer Ehegatten oder ihrer Lebenspartner beruflichen Tätigkeit fremder Obhut anzuvertrauen, besteht seit dem Jahr 1971 eine spezielle Regelung. ${ }^{828}$ Sie findet sich inzwischen in $\$ 8$ Abs. 2 Nr. 2a SGB VII. Ausweislich der Gesetzesbegründung zum damaligen $\$ 550$ Abs. 2 Nr. 1 RVO wurden die sogenannten Kindergartenumwege unter den Wegeunfallschutz gefasst, um die Berufstätigkeit von Frauen zu fördern: Da diesen eine Berufstätigkeit nur möglich sei, wenn ihre Kinder

$823 \mathrm{Zu}$ den unterschiedlichen Risiken je nach Weg Schimmelpfennig, NZV 2015, S. 23, $23 \mathrm{ff}$.

824 Antwort der Bundesregierung v.27.6.2007 auf eine kleine Anfrage, BT-Drs. 15/1462, S. 13.

825 Schrader, ZSR 1958, S. 682, 687.

826 Kranig/Aulmann, NZS 1995, S. 203, 208.

827 Höcker, ZSR 1958, S. 604, 608.

828 Leube, NZV 2015, S. 275, 276. Vorher galten diese Wege als private Um- bzw. Abwege und waren nicht vom Schutzbereich umfasst, Ricke, in: Körner/Leitherer et al. (Hrsg.), Kasseler Kommentar zum Sozialversicherungsrecht, $₫ 8$ SGB VII Rn. 222. 
während der Arbeitszeit versorgt seien, bestehe angesichts der Tatsache, dass die Wirtschaft immer mehr auf eine Mitarbeit der Frauen angewiesen sei, stets ein betriebliches Interesse an der Unterbringung der Kinder. ${ }^{829}$ Inzwischen sind als Schutzzweck der Norm auch die Gleichbehandlung der Frauen im Arbeitsleben sowie die verbesserte Vereinbarkeit von Familie und Arbeit anerkannt. ${ }^{830}$ Zudem ist das Bedürfnis, Kinder in fremde Obhut zu geben, durch die inzwischen verstärkte Bedeutung der beruflichen Tätigkeit der Erziehungspersonen und den wachsenden gesellschaftlichen dahingehenden Druck gestiegen. ${ }^{831}$ Im SGB VII ist der Kindergartenumweg als Ausnahme zu dem Grundsatz, dass Um- und Abwege vom nach \8 Abs. 2 Nr. 1 SGB VII versicherten Arbeitsweg nicht unter Versicherungsschutz stehen, ausgestaltet. ${ }^{832}$ Aus dieser Gesetzessystematik und dem Wortlaut der Norm sowie der dies bestätigenden Gesetzesbegründung ergibt sich, dass der Weg zur Obhutsgewährung stets im Rahmen eines Weges zum oder vom Ort der Tätigkeit zurückgelegt werden muss. ${ }^{83}$

Wird das Kind wegen der beruflichen Tätigkeit des Versicherten in fremde Obhut gebracht, steht sein Verbringen dorthin nach $\$ 8$ Abs. 2 Nr. 2 lit. a SGB VII in sachlichem Zusammenhang mit der versicherten Tätigkeit. ${ }^{834}$ Dies bedeutet, dass die Obhutsverbringung mit der Handlungstendenz zu erfolgen hat, dass eine berufliche Tätigkeit ausgeübt werden kann. ${ }^{835}$ Dabei muss das Arbeitsverhältnis der sorgeberechtigten Person kausal im Sinne der Theorie der wesentlichen Bedingung für die Notwen-

829 Amtliche Begründung zum G. v. 18.3.1971, BT-Drs. VI/1333 S. 5 zu $₫ 2$ Nr. 1.

830 Ricke, in: Körner/Leitherer et al. (Hrsg.), Kasseler Kommentar zum Sozialversicherungsrecht, $\mathbb{8} 8$ SGB VII Rn. 222.

831 Krasney, WzS 2013, S. 67, 67.

832 Leube, NZV 2015, S. 275, 276. Zu den genaueren Voraussetzungen der „fremden Obhut", des „Kindes“ und der übrigen Tatbestandsvoraussetzungen vgl. ebd.

833 BSG, Urt. v. 20.3.2007 - B 2 U 19/06 R -, juris Rn. 16; Marschner, in: Rolfs/ Giesen et al. (Hrsg.), BeckOK Sozialrecht, $\mathbb{} 8$ SGB VII Rn. 61; Krasney, KrV 2014, S. 102, 111 f.; ders., WzS 2013, S. 67, 72; Schlaeger, NZS 2009, S. 559, 563.

834 BT-Drs. VI/1333, S. 5; Leube, NZV 2015, S. 275, 277. Streit besteht darüber, ob der Unfallversicherungsschutz nach den allgemeinen Grundsätzen des Wegeunfalls nur bis zur Eingangstür der Betreuungseinrichtung oder, dem Wortlaut und der Parallelregelung im BeamtVG entsprechend, bis zur tatsächlichen Übergabe des Kindes an die Betreuungsperson geht. Letzteres befürwortet Leube, a.a.O., S. 275, 278; a.A. Ricke, in: Körner/Leitherer et al. (Hrsg.), Kasseler Kommentar zum Sozialversicherungsrecht, $₫ 8$ SGB VII Rn. 223; Schlaeger, NZS 2009, S. 559, 559.

835 Keller, in: Hauck/Noftz/ders. (Hrsg.), SGB VII, $\$ 8$ Rn. 260 a. 
digkeit der Fremdbetreuung sein. ${ }^{836}$ Erforderlich ist, dass ohne Fremdbetreuung des Kindes die konkrete Berufstätigkeit des Versicherten unmöglich oder erheblich erschwert wäre. ${ }^{837}$ Die Betriebsbezogenheit des Weges zur Obhutsverbringung ergibt sich außerdem aus der Verknüpfung dieser Strecke mit dem Arbeitsweg. ${ }^{838}$

3. Das Verbringen von Kindern in fremde Obhut bei häuslicher Tätigkeit

a. Keine direkte oder analoge Anwendbarkeit des $\$ 8$ Abs. 2 Nr. 2 lit. a SGB VII

Teleheimarbeitnehmer jedoch, die ihre Kinder in eine Betreuungseinrichtung bringen, um anschließend im Homeoffice zu arbeiten, sind bei der Zurücklegung dieser Strecke nach allgemeiner Meinung nicht versichert: Literatur und Rechtsprechung stimmen überein, dass diese Wege nicht unter den Wortlaut des $\$ 8$ Abs. 2 Nr. 2 lit. a SGB VII zu fassen sind. ${ }^{839}$ Denn im Homeoffice beschäftigte Personen legen keinen Arbeitsweg im Sinne des $\mathbb{8} 8$ Abs. Nr. 1 SGB VII zurück. Ein solcher Weg kann innerhalb der Wohnung wegen des Grundsatzes, dass Wegeunfallschutz erst mit Durchschreiten der Außentür beginnt, nicht angenommen werden. Da damit schon kein versicherter Weg vorliegt, verbiete sich die Anwendung des $₫ 8$ Abs. 2 Nr. 2 lit. a SGB VII: Dieser stellt, wie oben dargelegt wurde, seinem Wortlaut und seiner systematischen Stellung nach nur solche Wege unter Schutz, die Abweichungen vom Weg von und zur Arbeit darstellen. ${ }^{840}$

836 Ziegler, in: Becker/Franke et al. (Hrsg.), SGB VII, $\mathbb{8}$ Rn. 253; Leube, NZV 2015, S. 275, 277. Dies liegt auch dann vor, wenn die sorgeberechtigte Person während der Betreuungszeiten zwar zu Hause wäre, aber etwa wegen Schichtdienstes vor oder nach der Arbeit besondere Ruhe benötigt, BSG, Urt. v. 26.3.1986 - 2 RU 7/85 -, juris Rn. 11; Keller, in: Hauck/Noftz/ders. (Hrsg.), SGB VII, $\$ 8$ Rn. 260a m.w.N.

837 Ricke, in: Körner/Leitherer et al. (Hrsg.), Kasseler Kommentar zum Sozialversicherungsrecht, $\mathbb{8} 8$ SGB VII Rn. 224b.

838 Krasney, WzS 2013, S. 67, 72.

839 Ricke, in: Körner/Leitherer et al. (Hrsg.), Kasseler Kommentar zum Sozialversicherungsrecht, $\ 8$ SGB VII Rn. 222a; Schlaeger, NZS 2009, S. 559, 560; Leube, NZV 2015, S. 275, 280; SG Hannover, Urt. v. 17.12.2015 - S 22 U 1/15 -, juris Rn. 17.

$840 \rrbracket 8$ Abs. 2 Nr. 2a SGB VII: „Versicherte Tätigkeiten sind auch [...] das Zurücklegen des von einem unmittelbaren Weg nach und von dem Ort der Tätigkeit abweichenden Weges, um [...] Kinder von Versicherten [...] wegen ihrer, ihrer 
Mangels Arbeitsweges kann der isoliert unternommene Weg zum Kindergarten auch nicht als Um- oder Abweg davon angenommen werden, sodass keine versicherte Tätigkeit im Sinne des $₫ 8$ Abs. 2 Nr. 2 lit. a SGB VII vorliegt. ${ }^{841}$

Mangels planwidriger Regelungslücke ${ }^{842}$ ist allerdings auch eine analoge Anwendung des $\mathbb{8}$ Abs. 2 Nr. 2 lit. a SGB VII auf Wege, die vom Homeoffice zur Betreuungseinrichtung und zurück führen, nicht möglich. ${ }^{843}$ Die detaillierte Regelung des $\$ 8$ Abs. 2 Nr. 2 SGB VII spricht dafür, dass der Gesetzgeber bewusst nur Wege, die unmittelbar im Zusammenhang mit versicherten Wegen im Sinne des $\$ 8$ Abs. 2 Nr. 1 SGB VII stehen, unter Versicherungsschutz stellen wollte. ${ }^{844} \mathrm{Im}$ Rahmen der ähnlichen Frage, ob $\$ 8$ Abs. 2 Nr. 2 lit. a SGB VII auf Betriebswege anwendbar ist, hat das Bundessozialgericht das Vorliegen einer planwidrigen Regelungslücke ebenfalls verneint. ${ }^{845}$

\section{b. Bedürfnis einer Korrektur}

Dieses Ergebnis ist jedoch weder mit dem Schutzzweck des $₫ 8$ Abs. 2 Nr. 2 lit. a SGB VII noch mit den Grundprinzipen der Unfallversicherung vereinbar und verstößt zudem gegen den allgemeinen Gleichheitssatz, Art. 3 Abs. 1 GG. ${ }^{846}$

Der sog. „Kindergartenumweg“ wurde mit dem Ziel in den Schutzbereich der Unfallversicherung einbezogen, Personen mit Kleinkindern die

Ehegatten oder ihrer Lebenspartner beruflichen Tätigkeit fremder Obhut anzuvertrauen $[\ldots]$...

841 SG Hannover, Urt. v. 17.12.2015 - S 22 U 1/15 -, juris Rn. 17; Leube, NZV 2015, S. 275, 280; Ricke, in: Körner/Leitherer et al. (Hrsg.), Kasseler Kommentar zum Sozialversicherungsrecht, $\mathbb{} 8$ SGB VII Rn. 223.

842 Vgl. zu den Voraussetzungen der analogen Anwendung einer Norm im öffentlichen Recht genauer Schmidt, VerwArch 2006, S. 139, $142 \mathrm{ff}$.

843 Schlaeger, NZS 2009, S. 559, 560; SG Hannover, Urt. v. 17.12.2015 - S 22 U 1/15 -, juris Rn. 19; Zahnbrecher, Werkstorprinzip in der gesetzlichen Unfallversicherung, S.96. Die Möglichkeit einer Analogie nicht kategorisch ausschlieBend Spellbrink, NZS 2016, S. 527, 531.

844 SG Hannover, Urt. v. 17.12.2015 - S 22 U 1/15 -, juris Rn. 19; Plagemann, ArbRAktuell 2016, S. 71, 71.

845 BSG, Urt. v. 12.1.2010 - B 2 U 35/08 R -, juris Rn. 24 ff.; teilw. kritisch dazu Ricke, in: Körner/Leitherer et al. (Hrsg.), Kasseler Kommentar zum Sozialversicherungsrecht, $\mathbb{} 8$ SGB VII Rn. 222b.

846 Ebenso Leube, NZV 2015, S. 275, 280. 
berufliche Tätigkeit zu erleichtern. ${ }^{847}$ Dasselbe Ziel verfolgen die meisten Unternehmer und Beschäftigten mit der Gestaltung des Arbeitsverhältnisses dahingehend, dass die geschuldete Leistung im Homeoffice erbracht werden kann. ${ }^{848}$ Beschäftigte, die in einem Haushalt mit betreuungsbedürftigen Kindern leben, arbeiten deswegen besonders häufig von zu Hause aus. ${ }^{849}$ Dass ausgerechnet diese Gruppe von Arbeitnehmern nicht von der Regelung des $₫ 8$ Abs. 2 Nr. 2 lit. a SGB VII profitiert, ist nicht nachvollziehbar. Ihnen wird durch den fehlenden Versicherungsschutz beim Verbringen ihrer Kinder in Fremdbetreuung die Ausübung ihrer beruflichen Tätigkeit gegenüber Betriebsbeschäftigten wesentlich erschwert, obwohl sie dieselbe berufliche Tätigkeit ausüben und, wie zu zeigen sein wird, auch die Betriebsrisikolehre für den Einbezug des Obhutsweges spricht. ${ }^{850}$ Das im Zusammenhang mit Teleheimarbeitern häufig vorgebrachte Argument, eine Wegeunfallversicherung sei für sie nicht gerechtfertigt, da sie gerade das Risiko des Sich-Begebens in den öffentlichen Verkehrsraum vermeiden würden, ${ }^{851}$ verfängt hier ebenfalls nicht.

Dies bestätigt auch die Anwendung der Betriebsrisikolehre. Denn mit der Unterbringung in einer Betreuungseinrichtung verfolgen Teleheimarbeitnehmer ebenfalls einen betriebsdienlichen Zweck. Um arbeiten zu können, sind auch im Homeoffice Tätige auf das Verbringen des Kindes in fremde Obhut angewiesen. Mitnichten wird das Kind in solchen Fällen ausschließlich aus privater Motivation in einer Betreuungseinrichtung untergebracht. ${ }^{852}$ Der sachliche Zusammenhang des Zurücklegens der Strecke bis zum Ort der fremden Obhut steht daher durchaus in sachlichem Zusammenhang mit der versicherten Tätigkeit. Dieser wird zwar nicht durch die Einbettung des Weges in einen Weg zu einem außer Haus liegenden Ort der Tätigkeit hergestellt, beruht aber - wie auch in den von $\$ 8$ Abs. 2 Nr. 2 lit. a SGB VII erfassten Fällen - auf der Tatsache, dass die Unterbringung mit betrieblicher Handlungstendenz erfolgt. ${ }^{853}$ Das Arbeitsverhältnis des Telearbeitnehmers ist ebenso kausal im Sinne der Theorie

847 S.o. Kap. 3 A. VII. 2.

848 S.o. Kap. 2 E I. sowie Kap. 3 A. I.

849 S.o. Kap. 3 A. I.

850 Leube, NZV 2015, S. 275, 280.

851 Spellbrink, NZS 2016, S. 527, 529.

852 So aber SG Hannover, Urt. v. 17.12.2015 - S 22 U 1/15 -, juris Rn. 16; zustimmend wohl Plagemann, ArbRAktuell 2016, S. 71, 71; Ricke, in: Körner/Leitherer et al. (Hrsg.), Kasseler Kommentar zum Sozialversicherungsrecht, $\mathbb{8} 8$ SGB VII Rn. 188.

853 A.A. SG Hannover, Urt. v. 17.12.2015 - S 22 U 1/15 -, juris Rn. 20. 
der wesentlichen Bedingung für die Fremdbetreuung des Kindes, wie dies bei Betriebsbeschäftigten der Fall ist. Das von Ricke vorgebrachte Argument, Kinder seien im Rahmen einer häuslichen Tätigkeit „weniger beschäftigungsfeindlich“, sodass ein Unfallversicherungsschutz für Wege zu Betreuungseinrichtungen nicht notwendig sei, ist nicht nachvollziehbar. ${ }^{854}$ Auch der im Homeoffice tätige Arbeitnehmer schuldet seinem Arbeitgeber schließlich eine qualitativ bestmögliche Arbeitsleistung. Er ist verpflichtet, seiner Arbeit vollumfänglich nachzugehen, und kann sich nicht nebenbei der Sorgearbeit widmen. Gerade die von der Norm hauptsächlich in den Blick genommenen Kleinkinder sind sehr betreuungsintensiv. Ein sorgfältiges und konzentriertes Arbeiten kann bei gleichzeitiger Verantwortung für das Wohlergehen anwesender Kinder nur gewährleistet werden, solange diese schlafen. Eine Betreuung der Kinder durch andere Personen ist deswegen auch für zu Hause arbeitende Arbeitnehmer unumgänglich. Sie dient dem Unternehmerinteresse. Der zurückzulegende Weg ist deswegen nach der Betriebsrisikolehre der beruflichen Sphäre zuzuordnen.

Zudem liegt in der Verweigerung des Unfallversicherungsschutzes für die Zurücklegung des Kindergartenweges eine ungerechtfertigte Ungleichbehandlung der Teleheimarbeitnehmer gegenüber Betriebsbeschäftigten, sodass ein Verstoß gegen Art. 3 Abs. 1 GG vorliegt. Dies wurde in Rechtsprechung und Literatur zwar mit dem Argument verneint, dass $₫ 8$ Abs. 2 Nr. 2 lit. a SGB VII nicht an den Arbeitsort der Beschäftigten, sondern an den Arbeitsweg anknüpft und damit ein sachlicher Grund für die Ungleichbehandlung vorliege; 855 die Obhutsgewährung sei, wenn sie nicht mit einem Arbeitsweg verbunden wäre, „von anderen Gegebenheiten geprägt“"856 Dies ändert jedoch nichts daran, dass Teleheimarbeitnehmer

854 Vgl. Ricke, WzS 2017, S.9, 14. Richtig an der zitierten Aussage ist lediglich, dass häusliche Tätigkeit und Sorgearbeit zu einer gegenüber außerhäuslicher Tätigkeit verbesserten Vereinbarkeit beider Verpflichtungen führt - gerade deswegen sollte der Unfallversicherungsschutz auf Wegen zur Fremdbetreuung den betroffenen Personen nicht verwehrt werden. Aus diesem, von ihm als „sozialpolitisch" deklarierten Grund befürwortet an anderer Stelle auch Ricke einen Einbezug dieser Wege in den Unfallversicherungsschutz, in: Körner/Leitherer et al. (Hrsg.), Kasseler Kommentar zum Sozialversicherungsrecht, $\mathbb{} 8$ SGB VII Rn. 222a.

855 SG Hannover, Urt. v. 17.12.2015 - S 22 U 1/15 -, juris Rn. 20; Plagemann, ArbRAktuell 2016, S.71, 71; Ricke, in: Körner/Leitherer et al. (Hrsg.), Kasseler Kommentar zum Sozialversicherungsrecht, $\$ 8$ SGB VII Rn. 222a.

856 Ricke, in: Körner/Leitherer et al. (Hrsg.), Kasseler Kommentar zum Sozialversicherungsrecht, $\mathbb{\int} 8$ SGB VII Rn. 222a. 
durch diese Regelung faktisch vom Unfallversicherungsschutz für die Obhutsverbringung ausgeschlossen sind, auch wenn und obwohl sie ihr Kind ebenso aus betrieblichen Gründen einer Fremdbetreuung übergeben und auf diese ebenso wegen ihrer beruflichen Tätigkeit angewiesen sind wie Personen, die am Unternehmenssitz arbeiten. ${ }^{857}$ Der innere Zusammenhang zur versicherten Tätigkeit besteht bei beiden Personengruppen in gleichem Maße, und auch das Schutzbedürfnis der Betroffenen ist angesichts der gleichen Risikolage im öffentlichen Verkehrsraum dasselbe. „Andere Gegebenheiten“ bestehen mithin lediglich im Hinblick auf das nach erfolgter Obhutsverbringung angesteuerte Ziel und sind auch insoweit unerheblich: Beide Beschäftigtengruppen setzen ihren Weg zu ihrem Ort der Tätigkeit fort. Dass sich dieser bei den einen am Unternehmenssitz, bei den anderen an ihrer Privatadresse befindet, kann für den zurückzulegenden Weg keinen Unterschied machen und stellt keinen sachlichen Grund für die Rechtfertigung einer Ungleichbehandlung dar. ${ }^{858}$ Denn zum einen sind beide physischen Orte vom Unternehmer als Arbeitsörtlichkeit bestimmte Räumlichkeiten und damit „Ort der Tätigkeit“ im Sinne des $\$ 8$ Abs. 2 Nr. 2 lit. a SGB VII; zum anderen ist Sinn und Zweck des $₫ 8$ Abs. 2 Nr. 2 lit. a SGB VII gerade die Unterschutzstellung der Tätigkeit des Verbringens des Kindes, wenn diese Unterbringung in sachlichem Zusammenhang mit der betrieblichen Tätigkeit steht. Dieses Kriterium ist ortsunabhängig.

Interessant ist in diesem Zusammenhang die Handhabung der entsprechenden Regelung im Unfallfürsorgerecht ${ }^{859}$ der Beamten. ${ }^{860}$ Hier ist anerkannt, dass auch im Homeoffice arbeitende Beschäftigte bei der Verbrin-

857 Leube, NZV 2015, S. 275, 280.

858 Vgl. VG Halle (Saale), Urt. v. 25.6.2014 - 5 A 136/11 -, juris Rn. 28.

859 Die Unfallfürsorge ist ein Teilbereich des Versorgungsrechts, welches das soziale Sicherungssystem der Beamten ist. Das Rechtsinstitut der Versorgung bezeichnet die Verantwortung des Staates, für seine Beamten zu sorgen, da diese aufgrund ihrer Verpflichtung, dem Staat treue Dienste zu leisten, selbst nicht für Unglücksfälle Vorsorge leisten können, indem sie etwa ein Beschäftigungsverhältnis eingehen, vgl. Steding, ZSR 1959, S. 89, 93. Kritisch zu sehen ist die Wahl des Begriffes der Fürsorge für die Unfallsicherung in $\$ 30$ Abs. 1 BeamtVG. Grundsätzlich unterscheidet sich Fürsorge von Versorgung dadurch, dass sie nicht aus rechtsstaatlichen, sondern aus sozialstaatlichen Motiven gewährt wird, vgl. ebd., S. 93. Die Leistungen der Unfallfürsorge hängen aber gerade nicht von der Bedürftigkeit des Betroffenen ab, sondern sind unabhängig von seiner Vermögenssituation als Folge von Dienstunfällen zu gewähren. Es wäre daher passender, von der Unfallversorgung der Beamten zu sprechen.

860 Leube, NZV 2015, S. 275, 280. Unabhängig von der Frage, ob die Ungleichbehandlung von Beamten und Beschäftigten im Rahmen ihrer Unfallsicherungsre- 
gung ihrer Kinder in Fremdbetreuung geschützt sind. Während $\$ 31$ Abs. 1 BeamtVG grundsätzlich Unfälle in den Schutzbereich der Unfallfürsorge einbezieht, die „in Ausübung des Dienstes“ eingetreten sind, gilt gemäß der Fiktion des $\$ 31$ Abs. 2 S. 1 BeamtVG der Weg zum Ort der Tätigkeit ebenfalls als Dienst. $\$ 31$ Abs. 2 S. 2 BeamtVG wiederum fingiert einen fortbestehenden Zusammenhang mit dem Dienst, wenn der Beamte wegen des Anvertrauens des Kindes an eine fremde Person „,on dem unmittelbaren Wege zwischen der Wohnung und der Dienststelle in vertretbarem Umfang abweicht“. Durch eine teleologische Auslegung dieser im Wortlaut mit $\mathbb{8}$ Abs. 2 Nr. 2 lit. a SGB VII vergleichbaren Norm gelangt die beamtenrechtliche Rechtsprechung zu dem Schluss, dass unabhängig davon, ob die Dienststelle in der privaten Wohnung des Beamten liegt oder nicht, jeder Weg zur Inobhutgabe des Kindes unter Unfallfürsorgeschutz steht, wenn sein Zurücklegen dem Zweck dient, die pflichtgemäße Erbringung des Dienstes zu ermöglichen. ${ }^{861}$ Auch hier kommt es also maßgeblich auf die objektivierte Handlungstendenz der betroffenen Person zum Unfallzeitpunkt an, was angesichts des Schutzzwecks des $\$ 8$ Abs. 2 Nr. 2 lit. a SGB VII und der Strukturprinzipien der Unfallversicherung auch das relevante Kriterium bei der Beurteilung des Kindergartenweges der Beschäftigten sein sollte.

Mangels der Möglichkeit einer analogen Anwendung des $₫ 8$ Abs. 2 Nr. 2 lit. a SGB VII ist zur Gewährleistung des Versicherungsschutzes für Wege zur Unterbringung von Kindern eine Anpassung des Gesetzestexts notwendig. Ein dahingehender Formulierungsvorschlag erfolgt sogleich, s.u. Kap. 3 D. II.

VII. Unfallversicherungsschutz bei Tätigkeit im Homeoffice und Diskriminierungsverbot

Angesichts der Erosion des Unfallversicherungsschutzes zum Nachteil von Beschäftigten, die von zu Hause arbeiten, ist es eine Überlegung wert, das

gimes verfassungsrechtlich rechtfertigbar ist, ist der Vergleich der beiden Personengruppen interessant, da sich anhand dessen andere Regelungsmöglichkeiten für ähnliche oder sogar gleiche Problemfelder aufzeigen lassen. Für eine Gleichbehandlung von Beamten und Beschäftigten im Unfallsicherungsrecht insgesamt Giesen, Arbeitsunfall und Dienstunfall, passim.

861 Mit lesenswerter Argumentation VG Halle (Saale), Urt. v. 25.6.2014 - 5 A 136/11 -, juris Rn. $26 \mathrm{f}$. 
Verbot der mittelbaren Diskriminierung 862 in die Diskussion um die Anpassung des Versicherungsschutzes der Teleheimarbeitnehmer an das $\mathrm{Ni}$ veau des Versicherungsschutzes Betriebsbeschäftigter einfließen zu lassen. In diesem Sinne wurde bereits argumentiert, dass ein geringerer Umfang des Unfallversicherungsschutzes für in häuslicher Arbeit tätige Personen eine mittelbare Diskriminierung von Frauen darstelle, da Letztere häufiger als Männer im Homeoffice tätig seien. ${ }^{863}$

Das Verbot der Benachteiligung wegen des Geschlechts nach Art. 3 Abs. 2 GG $^{864}$ greift dann, wenn die Ausgestaltung der Regelung Frauen nicht nur zufällig benachteiligt, sondern der Nachteil an Tatbestände anknüpft, die typischerweise überwiegend Frauen betreffen. ${ }^{865}$ Erforderlich ist für seine Anwendung eine geschlechtsneutral gehaltene Regelung, welche tatsächlich zu einer statistischen Diskriminierung einer Geschlechtergruppe führt. Dabei ist es nicht notwendig, dass die Abweichung zwischen den Geschlechtergruppen besonders hoch ist ${ }^{866}$ oder eine bestimmte prozentuale Grenze erreicht hat. ${ }^{867}$ Richtigerweise kommt es für die Feststellung eines Eingriffs lediglich darauf an, eine zufällige unterschiedliche Verteilung der Betroffenheit auszuschließen, sodass bereits ab statistischer Relevanz des Unterschieds - wenn also nach Herausrechnen der Fehlerwahr-

862 Zur Entwicklung der Rechtsfigur des Verbots der mittelbaren Diskriminierung durch den US-Supreme Court und seine Adaption durch den EuGH in der Richtlinie 79/7 EWG vgl. Pfarr/Bertelsmann, Gleichbehandlungsgesetz, S. 92 Rn. $191 \mathrm{ff}$;; Kalisch, Die Entwicklung des Verbots der mittelbaren Diskriminierung, S. 33 ff.; Bieback, Die mittelbare Diskriminierung wegen des Geschlechts, S. $20 \mathrm{ff}$. Zur Herleitung aus dem deutschen Verfassungsrecht Kalisch, ebd., S. 97 ff.; Bieback, ZIAS 1990, S. 1, 28 ff.; Bokeloh, DRV 2014, S. 88, 90 ff. Lesenswert zur möglichen Herleitung eines Gleichbehandlungsgebots im (Privat-)Versicherungsrecht bereits aus dem Versicherungsgedanken von Koppenfels-Spies, VersR 2004, S. 1085, $1086 \mathrm{ff}$.

863 Leube, NZV 2015, S. 275, 280; VG Halle (Saale), Urt. v. 25.6.2014 - 5 A 136/11 -, juris Rn. 31.

864 Zur Verortung des Verbotes der mittelbaren Diskriminierung in Art. 3 Abs. 2 GG vgl. Kalisch, Die Entwicklung des Verbots der mittelbaren Diskriminierung, S. 98 ff., 137.

865 Pfarr, NZA 1986, S. 585, 586.

866 So aber im Rahmen des europarechtlichen Diskriminierungsverbots der EuGH, welcher interpretationsoffen davon spricht, dass der Unterschied „wesentlich“ oder "erheblich“ zu sein habe bzw. ein „viel höherer Prozentsatz“ einer Geschlechtergruppe betroffen sein müsse, vgl. EuGH, Rs. 170/84, Bilka, Slg. 1986, S. 1607, 1626 Rn. 24; Rs. 171/88, Rinner-Kühn, Slg. 1989, S. 2743, 2760 Rn. 11; Rs. 33/89, Kowalska, Slg. 1990, I-2591, 2611 Rn. 13.

867 Kalisch, Die Entwicklung des Verbots der mittelbaren Diskriminierung, S. 142, fordert beispielsweise eine Differenz von 10 Prozent. 
scheinlichkeiten eine Differenz übrig bleibt - eine geschlechtsspezifische Wirkung im Sinne des Art. 3 Abs. 2 GG anzunehmen ist. ${ }^{868}$ Die festgestellte Ungleichbehandlung darf sodann nicht gerechtfertigt sein. Als Maßstab für die Rechtfertigung dient das Verhältnismäßigkeitsprinzip. Eine mittelbar diskriminierende Regelung ist dann gerechtfertigt, wenn die tatsächlichen Unterschiede der Lebenssachverhalte von Frauen und Männern sachgerecht mit in die Entscheidung einbezogen sind und sichergestellt ist, dass der Eingriff nicht unzumutbar ist. ${ }^{869}$ Die Maßnahme muss auf Faktoren beruhen, die objektiv gerechtfertigt sind und nichts mit einer Diskriminierung aufgrund des Geschlechts zu tun haben. ${ }^{870}$

Problematisch an der Argumentation mit dem Verbot der Benachteiligung wegen des Geschlechts im Zusammenhang mit dem Unfallversicherungsschutz von Telearbeitnehmern ist, dass - soweit ersichtlich - keine verlässlichen Zahlen darüber vorliegen, die beweisen, dass mehr Frauen als Männer im Homeoffice tätig sind. Für die Bejahung einer geschlechtsspezifischen Wirkung ist jedoch eine gesicherte empirische Basis unerlässlich. ${ }^{871}$ Es wird zwar weithin davon ausgegangen, dass Frauen häufiger zu Hause arbeiten als Männer: Das Interesse an der (teilweisen) Teleheimarbeit scheint bei Frauen signifikant höher zu sein als bei Männern, ${ }^{872}$ was damit zusammenhängen dürfte, dass die gesellschaftlich gängige Rollen-

868 Überzeugend Brauer, Das Verbot der mittelbaren Diskriminierung, S. 81 m.w.N.; Wisskirchen, Mittelbare Diskriminerung, S. 93 ff.; Fuchsloch, Geschlechtsdiskriminierung, S. 178 ff.; Bieback, Die mittelbare Diskriminierung wegen des Geschlechts, S. 87 f.

869 Kalisch, Die Entwicklung des Verbots der mittelbaren Diskriminierung, S. 152.

870 EuGH, Rs. 170/84, Bilka, Slg. 1986, S. 1607, Punkt 1 des Tenors; Rs. C-317/93, Nolte, Slg. 1995, I-4625 Rn. 28; Bieback, ZIAS 1990, S. 1, 8 f.

871 Brauer, Das Verbot der mittelbaren Diskriminierung, S. 25; Wisskirchen, Mittelbare Diskriminierung, S. 93.

872 Lott, Stressed despite or because of flexible work arrangements?, Hans Böckler Stiftung Working Paper Nb. 46/2017, S. 13; Arnold/Steffes/Wolter (Hrsg.), Mobiles und entgrenztes Arbeiten, S. 11 und 17. Zu diesem Ergebnis kommen außerdem verschiedene Umfragen, die allerdings von Interessenverbänden durchgeführt wurden. Nach einer Erhebung von ARIS im Auftrag des Interessenverbands Bitkom im Jahr 2009 seien 75 Prozent der befragten Frauen am Homeoffice interessiert, bei den Männern dagegen 63 Prozent, https://www.focus.de/fin anzen/karriere/berufsleben/home-office-frauen-bleiben-am-liebsten-zu-hause_aid _394435.html (Stand: 18.11.2018). Bei haufe findet sich eine von TeamViewer durchgeführte, wiederum marktforschungsorientierte Umfrage aus dem Jahr 2012, welche die Anteile bei den Frauen mit 49 Prozent, bei den Männern mit 39 Prozent beziffert, https://www.haufe.de/personal/hr-management/home-offic e-besonders-bei-frauen-und-aelteren-beliebt_80_128932.html (Stand: 18.11.2018). Auch aus einer Studie zur Attraktivität deutscher Arbeitgeber, die 
verteilung noch immer darin besteht, dass der größte Teil der Sorgearbeit von Frauen geleistet wird. ${ }^{873}$ So schreibt in diesem Zusammenhang etwa Leube, „gerade“ Frauen würden oft vormittags in Telearbeit arbeiten, um sich am Nachmittag ohne längere Arbeitswege um ihre Kinder kümmern zu können. ${ }^{874}$ Auch das Verwaltungsgericht Halle argumentierte in seiner Entscheidung zum Einbezug von Kindergartenwegen von im Homeoffice beschäftigten Beamten, dass nur so eine Diskriminierung von Frauen vermieden werden könne, da diese wegen ihrer Betreuungsverpflichtungen häufiger über Heimarbeitsplätze verfügten als Männer. ${ }^{875}$ Empirische Quellen nennen beide jedoch leider nicht. ${ }^{876}$ Eine Studie des Personaldienstleisters Randstad und des ifo-instituts, der sog. „Randstad ifo-flexindex“, kommt immerhin zu dem Ergebnis, dass 56 Prozent aller im Homeof-

das Meinungsforschungsinstitut Innofact 2017 im Auftrag der Targobank durchgeführt hat, geht hervor, dass Frauen örtliche und zeitliche Flexibilität im Beruf mehr schätzen als Männer, https:/www.personalwirtschaft.de/der-job-hr/arbeits welt/artikel/geschlechterunterschiede-bei-arbeitsplatzwuenschen-und-realitaet.ht $\mathrm{ml}$ (Stand: 18.11.2018).

873 Vgl. nur Statistisches Bundesamt, Pressemitteilung 077/17 vom 7.3.2017, abrufbar unter https:/www.destatis.de/DE/PresseService/Presse/Pressemitteilungen/2017/ 03/PD17_077_122pdf.pdf?_blob=publicationFile (Stand: 18.11.2018): Bei Paaren mit Kindern unter drei Jahren gingen im Jahr 2015 ca. 83 Prozent der Väter, jedoch nur 10 Prozent der Mütter einer Erwerbstätigkeit in Vollzeit nach; Pollmann-Schult, ZfF 22/2010, S. 350, 352. Häufig führt die Geburt von Kindern zu einer Retraditionalisierung: Während sich Frauen verstärkt um die Betreuungs- und Hausarbeit kümmern, wünschen Männer seltener eine Reduktion der Arbeitszeit und arbeiten weiterhin in Vollzeit, um das Defizit des fehlenden zweiten Einkommens sowie zusätzliche monetäre Belastungen zu kompensieren, vgl. ders., SW 2009, S. 163, 163. In einer von Ipsos durchgeführten Befragung gaben konsequenterweise 83 Prozent von über 18.000 befragten Personen an, dass der Vorteil der Telearbeit darin zu sehen sei, dass sie Frauen eine Weiterarbeit trotz Sorgeverpflichtungen ermögliche, vgl. Bundesregierung, https://ww w.erfolgsfaktor-familie.de/fileadmin/ef/data/mediathek/EF_Onlinepublikation_ Home-Office.pdf (Stand: 18.11.2018). Aus dem damit einhergehenden größeren Bedürfnis der Frauen, Erwerbs- und Sorgearbeit zu vereinbaren, erklärt sich auch die im Gegensatz zu männlichen Erwerbstätigen fünfmal so hohe Teilzeitquote der Frauen, Brenke, DIW Wochenbericht 8/2014, S. 131, 137 und 138.

874 Leube, NZV 2015, S. 275, 280.

875 VG Halle (Saale), Urt. v. 25.6.2014 - 5 A 136/11 -, juris Rn. 31.

876 Eine solche schuldig bleibt leider auch Vollmar, WzS 1992, S. 164, 164, der den Anteil der Frauen an allen in häuslicher Erwerbsarbeit tätigen Personen für das Jahr 1980 auf 90 Prozent beziffert. 
fice beschäftigten Personen Mütter sind. ${ }^{877}$ Mangels transparenter Methoden ist diese jedoch lediglich als Indiz wertbar. Zudem ist die Messung nicht im Sinne des Art. 3 Abs. 2 GG relevant: Um die geschlechtsspezifische Wirkung einer Maßnahme feststellen zu können, müsste der Anteil aller im Homeoffice arbeitenden Männer an allen beschäftigten Männern mit dem Anteil aller im Homeoffice arbeitenden Frauen an allen beschäftigten Frauen verglichen werden. ${ }^{878}$ Solche Daten finden sich, soweit ersichtlich, im Mikrozensus ${ }^{879} 2008$ und 2011 sowie im soep.v $31^{880}$. Überraschenderweise ergeben sie jedoch keinen größeren Frauenanteil, sondern befinden im Gegenteil sogar den Anteil der männlichen Arbeitnehmer als etwas höher: ${ }^{881}$ Die Daten des Mikrozensus 2011 ergeben, dass der Anteil der häuslich erwerbstätigen Frauen an allen erwerbstätigen Frauen 7,3 Prozent, der Anteil der häuslich erwerbstätigen Männer an allen erwerbstäti-

877 Hier sind allerdings die Zahl der befragten Personen („vierteljährlich mehr als 1.000 Personalleiter") und die Art der Stichprobenziehung unbekannt, sodass die Zahlen lediglich als Indiz gewertet werden sollten. Abrufbar unter https://w ww.randstad.de/workforce360/archiv/home-office-ueberzeugt-vor-allem-muetter (Stand: 21.1.2018). Zudem würden nach dieser Studie 39 Prozent der Väter im Homeoffice arbeiten. Wie viel Prozent dies von allen Teleheimarbeitnehmern ausmacht, wird allerdings nicht dargelegt, sodass die auf Mütter und Väter bezogenen Zahlen nicht vergleichbar sind.

878 Zur Notwendigkeit dieser Vorgehensweise Pfarr, NZA 1986, S. 585, 586; dies./ Bertelsmann, Gleichbehandlungsgesetz, S. 104 Rn. 217 ff.; Fuchsloch, Geschlechtsdiskriminierung, S. 168 ff.; Brauer, Das Verbot der mittelbaren Diskriminierung, S. 29 m.w.N.

879 Für den Mikrozensus wird 1 Prozent der Bevölkerung Deutschlands befragt, das sind ca. 830.000 Personen. Zu Rechtsgrundlagen und Methoden vgl. https://ww w.destatis.de/DE/ZahlenFakten/GesellschaftStaat/Bevoelkerung/Mikrozensus.ht $\mathrm{ml}$ (Stand: 18.11.2018).

880 Das Sozio-oekonomische Panel ist eine repräsentative Wiederholungsbefragung von über 20.000 Personen im Auftrag des DIW Berlin, die von TNS Infratest Sozialforschung durchgeführt wird und Daten aus den Jahren 1984 bis 2014 umfasst, vgl. https://www.diw.de/de/diw_01.c.519355.de/soep_v 31.html (Stand: 18.11.2018).

881 Eine mögliche Erklärung - welche aber eingehend empirisch zu überprüfen wäre - dafür könnte sein, dass Arbeitgeber Männern generell selbstständigeres und disziplinierters Arbeiten eher zutrauen und ihnen daher häufiger die Arbeit im Homeoffice erlauben. Zu diesem Ergebnis kommt jedenfalls die o.g. Studie zur Attraktivität deutscher Arbeitgeber, die das Meinungsforschungsinstitut Innofact 2017 im Auftrag der Targobank durchgeführt hat, https://www.personalwirts chaft.de/der-job-hr/arbeitswelt/artikel/geschlechterunterschiede-bei-arbeitsplatz wuenschen-und-realitaet.html (Stand: 18.11.2018). 
gen Männern dagegen 7,8 Prozent beträgt. ${ }^{882}$ Für das Jahr 2008 kommt der Mikrozensus auf einen Anteil von 8,8 Prozent bei den Frauen und einen Anteil von 9,7 Prozent bei den Männern. ${ }^{883}$ Nach den Ergebnissen des soep.v 31 arbeiteten im Jahr 201411 Prozent aller Arbeitnehmerinnen zu Hause, während es bei den Arbeitnehmern 13 Prozent waren. ${ }^{884}$ Auch wenn man danach fragt, wie viele Beschäftigte gerne zu Hause arbeiten würden, wenn sie dürften, ergibt sich nach dem soep.v 31 kein Unterschied hinsichtlich des Geschlechts: Innerhalb beider Geschlechtsgruppen würden 66 Prozent von denen, die bisher nicht zu Hause arbeiten, dies tun, wenn sie die Möglichkeit dazu hätten. Darüber hinaus liefern die genannten Zahlen auch keine Hinweise darauf, dass eine Geschlechtergruppe generell weniger die Möglichkeit zur Heimarbeit hätte: Sowohl 58 Prozent der Arbeitnehmerinnen als auch 58 Prozent der Arbeitnehmer gaben an, dass ihr Arbeitsplatz keine Arbeit zu Hause ermögliche.

Insgesamt können daher keine geschlechtsspezifische Wirkung der Benachteiligung von im Homeoffice beschäftigten Personen und somit auch keine mittelbare Diskriminierung angenommen werden. ${ }^{885}$

\section{B. Arbeitsunfall und mobile Arbeit}

\section{Begriff, Bedeutung und Umfang mobiler Arbeit}

Im folgenden Abschnitt wird die Reichweite des Unfallversicherungsschutzes bei der sogenannten "mobilen Arbeit" untersucht. Dieser Begriff meint die selbstbestimmte, örtlich flexible berufliche Tätigkeit. ${ }^{886}$ In Anlehnung an den Begriff des Homeoffice wird auch vom „Mobile Office“ gesprochen. ${ }^{887}$ Dass sie nicht auf einen Arbeitsplatz festgelegt ist, unterscheidet

882 Vgl. Brenke, DIW-Wochenbericht 8/2014, S. 136 f. Der Anteil der Frauen an allen Arbeitnehmern mit Heimarbeit beträgt nach diesen Zahlen 46,2 Prozent, während 53,8 Prozent Männer sind. Nach den Berechnungen Brenkes liegt dies nicht an der unterschiedlichen Berufsstruktur beider Geschlechtergruppen, ders., ebd. S. 137.

883 Vgl. Brenke, DIW-Wochenbericht 8/2014, S. 136 f. Der Anteil der Frauen an allen Arbeitnehmern mit Heimarbeit beträgt nach diesen Zahlen 44,6 Prozent, während 55,4 Prozent Männer sind.

884 Vgl. Brenke, DIW-Wochenbericht 5/2016, S. 100.

885 Dass eine solche mangels sachlichen Grunds nicht rechtfertigbar wäre, ergibt sich aus den Ausführungen des Kap. 3 A I.-VII.

886 Piele/Piele, Mobile Arbeit, S. 9.

887 Z.B. Oberthür, NZA 2013, S. 246, 246. 
diese Gestaltungsform von der soeben behandelten Arbeit im Homeoffice. Der Arbeitnehmer wählt frei und selbst, wo er seiner versicherten Tätigkeit nachgeht: ${ }^{88}$ Dies kann beispielsweise eine öffentliche Bibliothek, ein Café, ein Zugabteil oder auch der häusliche Küchentisch sein. ${ }^{889}$ Zumeist mithilfe von Internetkommunikationsmitteln verbinden sich die Beschäftigten von ihrem gewählten Ort aus mit ihrem Betrieb, ${ }^{890}$ aber auch „offline“ ist mobile Arbeit möglich. Die Anzahl sowohl der „Mobile Worker“ als auch der Unternehmen, die ihren Arbeitnehmern mobile Arbeit ermöglichen, ist in den letzten Jahren kontinuierlich gestiegen. ${ }^{891}$ Bei der Beschäftigtenbefragung der IG Metall im Jahr 2017 gaben 20 Prozent der Teilnehmer an, teilweise mobil arbeiten zu dürfen. ${ }^{892}$

Auch diese Form der Arbeit soll nach der Vorstellung der Unternehmer und der Beschäftigten dazu dienen, das Privatleben - insbesondere private Sorgearbeit - und berufliche Erwerbsarbeit besser miteinander vereinbaren zu können. ${ }^{893}$

Die unfallversicherungsrechtliche Einordnung von mobiler Arbeit ist noch ungeklärt; sie ist nicht unbesehen mit der Tätigkeit im Homeoffice gleichzusetzen, da - wie gesehen - der Arbeitgeber auf den häuslichen Arbeitsplatz in anderer Weise Einfluss zu nehmen vermag als auf einen frei gewählten und ständig wechselnden Arbeitsort. ${ }^{894}$ Zwar verpflichten $\$ 3$ Abs. 1 i.V.m. $\$ 4$ ArbSchG den Arbeitgeber auch bei mobiler Arbeit, den Arbeitsschutz durch geeignete technische, organisatorische oder persönliche Schutzmaßnahmen zu gewährleisten. ${ }^{895}$ Seine Einflussmöglichkeiten sind aber notwendigerweise schon deswegen geringer, da der Arbeitneh-

888 Dies unterscheidet die „mobile Arbeit“ von der ebenfalls an wechselnden Orten stattfindenden Tätigkeit bspw. von Handwerkern und Pflegediensten. Sie fällt daher auch nicht unter den Telearbeitsbegriff der ArbStättV, vgl. Wissenschaftliche Dienste des Deutschen Bundestags, Sachstand Telearbeit und Mobiles Arbeiten, WD 6 - 3000 - 149/16, S. 8.

889 Z.B. Oberthür, NZA 2013, S. 246, 246.

890 TBS NRW (Hrsg.), Mobile Arbeit, S. 25 f.

891 Vgl. die Zahlen bei Piele/Piele, Mobile Arbeit, S. 13 ff. Mobile Arbeit soll, so der Entwurf des Koalitionsvertrags zwischen CDU/CSU und SPD vom 7.2.2018, zukünftig weiter erleichtert und gefördert werden, abrufbar unter: https://www.sp d.de/fileadmin/Dokumente/Koalitionsvertrag/Koalitionsvertrag_2018.pdf (Stand: 23.2.2018), Rn. $1826 \mathrm{ff}$.

892 Ebd., S. 14.

893 Arnold/Steffes/Wolter, Mobiles und entgrenztes Arbeiten, S. 18; Piele/Piele, Mobile Arbeit, S. 26 m.w.N.; Raif/Nann, GWR 2016, S. 221, 221.

894 So aber Schramm/Hennig, AuA 2016, S. 504, 505.

895 Raif/Swidersky, GWR 2017, S. 351, 353; Oberthür, NZA 2013, S. 246, 247; Günther/Böglmüller, NZA 2015, S. 1025, 1029. 
mer den Ort seiner Tätigkeit selbst und nicht notwendigerweise täglich auf die gleiche Weise bestimmt. Immerhin wird auch hier häufig der Arbeitgeber die Arbeitsmittel bereitstellen. ${ }^{896}$

Da die unfallversicherungsrechtliche Einordnung von Arbeitsunfällen auf der Grenzziehung zwischen Privat- und Berufssphäre basiert, ist es sinnvoll, bei der folgenden Untersuchung des Schutzbereichs von mobiler Arbeit zwischen mobiler Arbeit im eindeutig der Privatsphäre zuzuordnenden Wohnbereich des Beschäftigten (II.) und mobiler Arbeit an anderen Orten (III.) zu unterscheiden.

\section{Mobile Arbeit im Wohnbereich des Beschäftigten}

\section{a. Meinungsstand in Literatur und Rechtsprechung}

Die Einordnung eines Unfalls, der sich weder am betrieblichen Arbeitsplatz noch am eingerichteten häuslichen Arbeitsplatz, sondern während einer versicherten Tätigkeit an einem anderen Ort in der Wohnung ereignet hat, ist bisher ungeklärt. Liest der Arbeitnehmer die von ihm zu bearbeitenden Personalakten etwa auf der Couch im privat genutzten Wohnzimmer und verunfallt währenddessen, wird die Frage nach dem sachlichen Zusammenhang in Literatur und Rechtsprechung nach unterschiedlichen Kriterien und mit unterschiedlichen Ergebnissen beantwortet. Dies ist auch der Tatsache geschuldet, dass bisher - anlässlich eines einschlägigen Urteils des BSG ${ }^{897}$ - eingehend lediglich die Problematik beleuchtet wurde, ob Unfälle, die im Zusammenhang mit dem Zurücklegen von Wegen innerhalb der Wohnung geschehen, als versichert anzusehen sind. ${ }^{898}$ Die hier angesprochene Frage des Erleidens eines Unfalls während der gezielten Arbeitstätigkeit in einem anderen Raum als dem Arbeitsbereich dagegen wurde in der Literatur und Rechtsprechung bisher nur gelegentlich berührt. Dabei lassen sich vier grundsätzliche Argumentationsrichtungen erkennen.

Zum einen wird vertreten, dass Unfälle im häuslichen Bereich außerhalb des Arbeitsraumes grundsätzlich keine Arbeitsunfälle sein können, z.B. von Schlaeger, der sich zum einen auf die Ansichten des BSG und des LSG

896 Oberthür, NZA 2013, S. 246, 248.

897 BSG, Urt. v. 5.7.2016 - B 2 U 5/15 R -, juris Rn. 27. Näheres zu diesem Urteil bei der Analyse der Betriebswege im häuslichen Bereich, s.o. Kap. 2 A. V. 1. a.

898 S.o. Kap. 2 A. V. 
München bezieht, nach denen der private Charakter der Umgebung im Wohnbereich so stark dominiere, dass Verrichtungen im Wohnbereich von vornherein nicht in sachlichem Zusammenhang mit der versicherten Tätigkeit stehen können. ${ }^{899}$ Zum anderen hält er diese räumliche Aufteilung der Risikosphären für notwendig, um eine klare rechtliche und praxistaugliche Abgrenzung vornehmen zu können. ${ }^{900}$

Ähnlich, aber weniger absolut wird in einer zweiten Ansicht vertreten, dass die Anforderungen an die Annahme eines sachlichen Zusammenhangs mit dem im privaten Bereich zu verortenden Unfall erhöht seien. Der II. Senat des LSG München erwähnt in einem Urteil, in dem es um das Zurücklegen eines Weges innerhalb der Wohnung ging, dass es bei Vorliegen eines räumlich getrennten Arbeitsbereichs innerhalb einer Wohnung den Aufenthalt außerhalb dieses Bereichs jedenfalls als gegen einen sachlichen Zusammenhang zu wertendes Indiz sieht. ${ }^{901}$ In diesem Sinne schreibt auch Jung, bei Unfällen innerhalb des Wohnbereichs des Beschäftigten sei eine restriktive Handhabung des Zuordnungsmechanismus zu empfehlen, da die Erbringung von Arbeit der Umgebung nicht den privaten Charakter nehme. ${ }^{902}$ Es sei zu bedenken, dass der Arbeitnehmer die Risiken seiner häuslichen Lebenssphäre selbst am besten beherrsche. Ein sorgloser Einbezug dieser Risiken in den Schutzbereich der Unfallversicherung würde eine grundlose Erweiterung der Unfallversicherung auf allgemeine Lebensrisiken bedeuten, welche dem Schutzzweck dieser widerspreche. ${ }^{903}$

Einer dritten Ansicht nach, die jüngst vor allem von Spellbrink geäußert wurde, besteht auch in dieser Konstellation kein Unterschied zu einer Beschäftigung auf einer Betriebsstätte. Es komme bei der Beurteilung des sachlichen Zusammenhangs ausschließlich darauf an, ob die zum Unfallzeitpunkt ausgeführte tatsächliche Verrichtung auf die Erfüllung des Tatbestandes der versicherten Tätigkeit gerichtet war. ${ }^{904}$ Dieser Ansicht war auch das Bundessozialgericht in einer älteren Entscheidung. ${ }^{905}$ Ebenso verlangt Eichenhofer die Bejahung des sachlichen Zusammenhangs, wenn die be-

899 Schlaeger, jurisPR-SozR 20/2017 Anm. 3.

900 Ebd.

901 LSG Muenchen, Urt. v. 5.4.2017 - L 2 U 101/14 -, juris Rn. 74.

902 Jung, SGb 2001, S. 398, 399.

903 Ebd.

904 Spellbrink, NZS 2016, S. 527, 528. So wohl auch Keller, in: Hauck/Noftz/ders. (Hrsg.), SGB VII, $\$ 8$ Rn. 116a.

905 BSG, Urt. v. 8.12.1994 - 2 RU 41/93 -, juris; zustimmend Wolber, SozVers 1997, S. 239, 239. 
triebliche Handlungstendenz dominiert. ${ }^{906}$ Auch Keller misst dem Unfallort in der Privatwohnung keine Bedeutung zu. ${ }^{907} \mathrm{Ganz}$ ähnlich äußert sich auch Leube, wenn er schreibt, dass es in privaten Räumen ebenfalls keine Vermutung gegen das Vorliegen eines Arbeitsunfalls gebe, und es auch bei dieser Fallgestaltung auf das Vorliegen einer vertraglich geschuldeten Arbeitsleistung ankommen lässt. ${ }^{908}$ Im Sinne dieser Autoren ist wohl auch Schwerdtfeger zu verstehen, der zunächst generell das Vorliegen eines sachlichen Zusammenhangs innerhalb des rein persönlichen, von den Betriebsräumen abgrenzbaren Lebensbereichs mit der beruflichen Tätigkeit verneint, um sodann Ausnahmen für den Fall zuzulassen, dass im privaten Bereich Arbeiten verrichtet werden, für die eine arbeitsvertragliche Verpflichtung besteht. ${ }^{909}$ Wird eine betriebsdienliche Verrichtung im häuslichen Bereich aufgenommen, erfasse die „räumliche Geltung des Versicherungsschutzes" auch diesen Bereich. ${ }^{910}$ Undeutlich ist die Aussage von Giesen, der zunächst betont, dass es im Unfallversicherungsrecht keine Bindung an einen bestimmten Arbeitsort gebe, dann aber ohne persönliche Wertung die Rechtsprechung des BSG dahingehend interpretiert, ein sachlicher Zusammenhang könne auch bei Vorliegen einer berufsbezogenen objektivierten Handlungstendenz in privaten Räumen nur bejaht werden, wenn ein Ausnahmefall der sofortigen Handlungsnotwendigkeit des Arbeitnehmers vorlag, und damit ein rein örtliches Kriterium wiederholt. ${ }^{911}$

Nach Ansicht von Ricke schließlich kommt es entscheidend weder auf den Unfallort noch auf die objektivierte Handlungstendenz des Beschäftigten, sondern maßgeblich auf die Art des sich im Unfall verwirklichenden Risikos an. Tätigkeitsbezogene Risiken seien versichert, da sie sich ortsunabhängig ereignen würden und die Tatsache, dass der Arbeitgeber auf das örtliche Umfeld keinerlei Einfluss habe, argumentativ nicht greife. Ein solches tätigkeitsbezogenes Risiko wäre etwa das Sichverletzen an der Heft-

906 Eichenhofer, SGb 2007, S. 742, 747.

907 Er weist darauf hin, dass im Gegensatz dazu im österreichischen Recht nur Unfälle, die aus besonderen betriebsbezogenen Gefahren resultieren, versichert sind, vgl. Keller, in: Hauck/Noftz/ders. (Hrsg.), SGB VII, $\$ 8$ Rn. 116a.

908 Leube, SGb 2012, S. 380, 381.

909 Schwerdtfeger, in: Lauterbach/Watermann/Breuer (Hrsg.), Unfallversicherung, $\mathbb{} 8$ Rn. 256. Er bezieht sich dabei auf die ältere Rechtsprechung des BSG, die insb. zu Selbstständigen ergangen ist, vgl. Nw. a.a.O.; siehe auch BSG, Urt. v. 8.12.1994 - 2 RU 41/93 -, juris Rn. 16.

910 Schwerdtfeger, in: Lauterbach/Watermann/Breuer (Hrsg.), Unfallversicherung, $\mathbb{} 8$ Rn. 256; zustimmend Holstraeter, in: Knickrehm/Kreikebohm/Waltermann (Hrsg.), Kommentar zum Sozialrecht, $\mathbb{8} 8$ SGB VII Rn. 15.

911 Giesen, Arbeitsunfall und Dienstunfall, S. 63 f. 
klammer der Akte. Verwirkliche sich im Unfall dagegen ein Risiko, das nicht eindeutig tätigkeitsbezogen ist, wird das Vorliegen eines sachlichen Zusammenhangs mit dem Argument verneint, dass der Unternehmer keinen Zugriff auf die dieses Risiko beeinflussenden Faktoren hat. ${ }^{912}$ So lässt sich auch das Urteil des BSG vom 5.7.2016 deuten, in welchem der entscheidende Senat darauf hinweist, dass wegen des Prinzips der Ablösung der Unternehmerhaftpflicht als tragendem Grundsatz der Unfallversicherung solche Risiken unversichert bleiben, die nicht in den Verantwortungsbereich des Unternehmers fallen. In der häuslichen Lebenssphäre vermöge sich kein betriebsbezogenes Haftungsrisiko verwirklichen, sodass kein sachlicher Zusammenhang mit der versicherten Tätigkeit vorliege. ${ }^{913}$ Denn zum einen beherrsche der Beschäftigte die seiner privaten Wohnung innewohnenden Risiken selbst am besten, da ihm sein Wohnbereich besser bekannt ist als anderen Personen, sodass er selbst für die mit seinem Wohnbereich einhergehenden Gefahren verantwortlich sei.914 Zum anderen könne der Arbeitgeber auf den häuslichen Bereich - abgesehen vom eingerichteten Arbeitsplatz - nicht durch präventive oder gefahrenreduzierende Maßnahmen einwirken. ${ }^{915}$ Auch nach diesem vielbeachteten Urteil ist jedoch nicht eindeutig zu beantworten, welche Unfälle innerhalb der Privatwohnung nach Ansicht des BSG generell nicht unter Unfallversicherungsschutz stehen können. ${ }^{916}$ Eindeutig verneint das BSG - in Übereinstimmung mit der früheren Rechtsprechung und Literatur - die Berufsbezogenheit von Unfällen, die die Verwirklichung von spezifisch in der Privatwohnung verankerten örtlichen Risiken darstellen. ${ }^{917}$ Vorstellbar wären in dieser Kategorie etwa das Ausgleiten auf einem besonders rutschigen Boden, das Durchbrechen einer maroden Treppenstufe oder das Erleiden eines Stromschlags durch Nutzung einer defekten Steckdose. Dagegen geht aus der Urteilsbegründung nicht hervor, ob auch Risiken, die sich ortsunabhängig stets verwirklichen können, innerhalb der Privatwohnung des Versicherten niemals dem Unternehmer zuzurechnen wären. Zu denken ist etwa an das anlasslose Stolpern oder Umknicken eines Fußes. Letztere

912 Ricke, in: Körner/Leitherer et al. (Hrsg.), Kasseler Kommentar zum Sozialversicherungsrecht, $\mathbb{8} 8$ SGB VII Rn. 130.

913 BSG, Urt. v. 5.7.2016 - B 2 U 5/15 R -, juris Rn. 27.

914 Ebd.

915 Ebd. Rn. 28.

916 Vgl. Ricke, in: Körner/Leitherer et al. (Hrsg.), Kasseler Kommentar zum Sozialversicherungsrecht, $\mathbb{8} 8$ SGB VII Rn. 130.

917 BSG, Urt. v. 5.7.2016 - B 2 U 5/15 R -, juris Rn. 27; ebenso Schwerdtfeger, in: Lauterbach/Watermann/Breuer (Hrsg.), Unfallversicherung, $\$ 8$ Rn. $259 \mathrm{~b}$. 
Unfälle basieren nicht auf tätigkeitsbezogenen Faktoren, sind aber genauso wenig der örtlichen Umgebung geschuldet.

Die von Ricke und Schlaeger im Dienste der Vermeidung von Abgrenzungsschwierigkeiten angebotenen Lösungsvorschläge - zum einen, jeder Unfall, der in der Wohnung, aber nicht im Arbeitszimmer stattfinde, sei ein Privatunfall, ${ }^{918}$ zum anderen, jeder Unfall, der kein tätigkeitsbezogenes Risiko verwirkliche, sei als privat einzuordnen ${ }^{919}$ - sind deswegen unbefriedigend, weil sie die virulente Frage nach einer sachgerechten Abgrenzung der Risikosphären von Unternehmer und Beschäftigtem durch rein pragmatische Erwägungen umgehen. Diese Frage sollte jedoch nicht beiseitegeschoben, sondern mit Blick auf die Grundprinzipien der Unfallversicherung gelöst werden. Sie spiegelt die Entgrenzung der räumlichen betrieblichen Strukturen auf der Ebene des Arbeitsunfalls und verlangt nach einer Rückbesinnung auf die Grundprinzipien der Unfallversicherung: die Garantie eines sozialen Schutzes für die Beschäftigten durch Absicherung gegen die aus der betrieblichen Sphäre herrührenden Gefahren sowie die Zuweisung des Betriebsrisikos an den Unternehmer sowie die Ersetzung deren zivilrechtlicher Haftung. ${ }^{920}$

b. Zurechnung zur Risikosphäre des Unternehmers aufgrund der Betriebsrisikolehre und des sozialen Schutzprinzips

Richtigerweise sind alle unternehmensdienlichen Tätigkeiten unabhängig vom physischen Aufenthaltsort des Beschäftigten in der Risikosphäre des Arbeitgebers zu verorten. Mit der Betriebsrisikolehre und dem sozialen Schutzprinzip sprechen beide Grundprinzipien der Unfallversicherung für diese Zuordnung. Zur Begründung ist auf die bereits oben bei der Prüfung von Unfällen auf Betriebswegen innerhalb der Privatwohnung entwickelten und ausführlich dargestellten Argumente zu verweisen.921 Was dort zur Fallgruppe der innerhäuslichen Betriebswege entwickelt wurde, gilt - da Betriebswege lediglich einen Spezialfall der versicherten Tätigkeit darstellen - notwendigerweise für jede Ausführung einer versicherten Tätigkeit zum Unfallzeitpunkt im privaten Wohnbereich. Weder die Betriebs-

918 Schlaeger, jurisPR-SozR 20/2017 Anm.3.

919 Ricke, in: Körner/Leitherer et al. (Hrsg.), Kasseler Kommentar zum Sozialversicherungsrecht, $₫ 8$ SGB VII Rn. 130 und Schlaeger, jurisPR-SozR 20/2017 Anm. 3.

920 Gitter/Nunius, in: Schulin (Hrsg.), Handbuch des Sozialversicherungsrechts Band 2, $\$ 5$ Rn. 4.

921 S.o. Kap. 2 A. V. 2. b. 
risikolehre noch das soziale Schutzprinzip verlangen eine räumliche Korrelation des Schadensereignisses mit unternehmenseigenem Gelände. Vielmehr kann es, um den Risikostrukturen der modernen Arbeitswelt gerecht zu werden, für die Beurteilung der Zugehörigkeit eines Unfalls zur Betriebssphäre entscheidend nur auf die ausgeführte Tätigkeit ankommen.

\section{Mobile Arbeit außerhalb der Privatwohnung des Beschäftigten}

Aus den obigen Ausführungen zum Unfallversicherungsschutz von Tätigkeiten, die im privaten Wohnbereich stattfinden, ergibt sich, dass auch die mobile Arbeit außerhalb der Wohnung immer dann zum Schutzbereich der Unfallversicherung zählt, wenn die objektivierte Handlungstendenz des Beschäftigten zum Unfallzeitpunkt auf die Erfüllung seiner versicherten Tätigkeit gerichtet war. Denn wenn diese Handlungen sogar dann zur Betriebsrisikosphäre des Unternehmers gezählt werden, wenn sie in einer Umgebung stattfinden, die der Arbeitnehmer zweifellos selbst am besten physisch beherrscht, so müssen sie erst recht dazu zählen, wenn der Arbeitnehmer weniger Einfluss darauf hat. Das von den Gegenansichten hauptsächlich eingesetzte Argument, der Arbeitnehmer habe die sich ergebenden Risiken dort allein in der Hand, ist bei mobilen Arbeitsstätten außerhalb der Wohnung in noch geringerem Maße gültig. Für die Bestimmung des sachlichen Zusammenhangs von Tätigkeiten im Mobile Office ist daher uneingeschränkt auf die zur Arbeit im häuslichen Bereich getätigten Überlegungen zu verweisen. Im Ergebnis kann es auch für ihre Zuordnung zum unfallversicherungsrechtlichen Schutzbereich nur auf die Handlungstendenz des Beschäftigten ankommen.

$\mathrm{Zu}$ bedenken ist auch hier, dass im weiteren Verlauf der Prüfung des Vorliegens eines Arbeitsunfalls im Rahmen der Unfallkausalität noch abgeklärt werden muss, ob versicherte Tätigkeit auch kausal im Sinne der Theorie der wesentlichen Bedingung für den Unfall war. Befindet der Arbeitnehmer sich während der Arbeitsausführung an einem unverantwortlichen, weil stark risikobelasteten Ort, kann die Einordnung des Unfalls als Arbeitsunfall mangels vorliegender Unfallkausalität verneint werden. Die Fokussierung auf die betriebsdienliche Intention stellt daher keinesfalls eine Einladung für Beschäftigte dar, sich während ihrer Arbeitserbringung wissentlich erhöhten Risiken auszusetzen. 


\section{Arbeitsunfall und ständige Erreichbarkeit}

I. Facetten der Erreichbarkeit: Begriffe und Relevanz

Auch die Frage, nach welchen Kriterien die Einordnung eines Unfalls als Arbeitsunfall in den Fällen der „ständigen Erreichbarkeit“ erfolgen sollte, stellt sich im Rahmen der zunehmenden Entgrenzung der Arbeitswelt. Dass Beschäftigte auch nach Ende ihrer Arbeitszeit für ihre Vorgesetzten, Kollegen und Kunden ansprechbar bleiben, ist inzwischen häufig. Dabei bestehen diverse Facetten der Verfügbarkeit, deren Unterscheidung für die unfallversicherungsrechtliche Behandlung relevant ist.

Unter Rufbereitschaft versteht man eine Zeit, während der sich Beschäftigte außerhalb ihrer üblichen Arbeitszeit für einen Arbeitseinsatz bereithalten. Dabei können sie sich an einem beliebigen Ort aufhalten, müssen aber jederzeit und unmittelbar erreichbar und in angemessener Frist reaktionsbereit sein. ${ }^{922}$ Dies bedeutet auch, dass sie sich in einer für den Arbeitszweck angemessenen Konstitution befinden müssen und daher während der Rufbereitschaft nicht alkoholisiert oder übermüdet sein dürfen. ${ }^{923}$ Als Arbeitszeit gelten dabei nur die tatsächlichen Einsätze während der Phase der Erreichbarkeit. Die Rufbereitschaft an sich wird dagegen arbeitszeitrechtlich als Ruhezeit im Sinne des $\mathbb{1 2}$ S. 1 AZV gesehen. In der Regel wird die tatsächlich geleistete Arbeit vergütet, die Rufbereitschaft dagegen pauschal abgegolten. ${ }^{924}$ Mit der Rufbereitschaft wird nicht die bessere Vereinbarkeit von Berufs- und Privatleben bezweckt. Sie ist vielmehr ein rein an den betrieblichen Interessen ausgerichtetes Konzept und wird herangezogen, um etwa Serviceleistungen rund um die Uhr garantieren zu können oder Produktionsausfälle abzufangen.925 Nach Zahlen des Verbundprojektes „RUF“ nutzen jedenfalls 43,8 Prozent aller in Deutschland ansässigen Betriebe Rufbereitschaft; in diesen Betrieben sind durchschnittlich 9 Prozent der Beschäftigten davon betroffen. ${ }^{926}$ Diese Zahlen erfassen jedoch lediglich die Betriebe, die Rufbereitschaft formell im Rahmen eines Individual- oder Tarifvertrags bzw. einer Betriebsvereinbarung ausgestaltet haben. Es wird davon ausgegangen, dass zusätzlich eine große

922 BAG, Urt. v. 24.10.2000 - 9 AZR 634/99 -, juris Rn. 21; v. 19.12.1991 - 6 AZR 592/89 -, juris Rn. 21.

923 Falder, NZA 2010, S. 1150, 1151.

924 Fietze/Keller/Friedrich/Dettmers, in: dies. (Hrsg.), Rufbereitschaft, S. 1, 2.

925 Tobsch/Schult/Fietze/Matiaske, in: Fietze/Keller/Friedrich/Dettmers (Hrsg.), Rufbereitschaft, S. 7.

926 Ebd., S. 12, 26. 
Anzahl kleinerer Betriebe informell auf Rufbereitschaft zurückgreift.927 Die BAuA-Arbeitszeitbefragung 2015 ergab, dass 8 Prozent der Beschäftigten mindestens einmal pro Monat Rufbereitschaft haben. ${ }^{928}$

Bereitschaftsdienst dagegen ist gemäß $\$ 2$ Abs. 1 ArbZG als Arbeitszeit zu qualifizieren. Umfasst ist der Zeitraum, während dem Arbeitnehmer sich an einem vom Arbeitgeber bestimmten Ort innerhalb oder außerhalb des Betriebes aufhalten müssen, um bei Bedarf die Arbeit kurzfristig aufnehmen zu können. ${ }^{929}$ Während dieses Zeitraums können die Beschäftigten jedoch auch privaten Tätigkeiten nachgehen. ${ }^{930}$ Bei der BAuA-Arbeitszeitbefragung 2015 gaben 7 Prozent der Beschäftigten an, mindestens einmal pro Monat Bereitschaftsdienst zu leisten. ${ }^{931}$

Arbeitsbereitschaft schließlich bezeichnet die nach $\$ 2$ Abs. 1 ArbZG ebenfalls als Arbeitszeit einzuordnende Zeitspanne, während derer Beschäftigte am Arbeitsplatz anwesend sein müssen, aber ihre gesamte Aufmerksamkeit nicht erforderlich ist und sie sich daher in „wacher Achtsamkeit im Zustand der Entspannung" befinden. ${ }^{932}$ Als Beispiele genannt werden Rettungsfachkräfte, die auf ihren Einsatz warten. ${ }^{933}$

Während die bereits genannten Erscheinungen nicht neu, sondern - abhängig von der betrachteten Branche - bereits längst etablierte Arbeitsformen sind, ist die ständige Erreichbarkeit im engeren Sinne ein mit der fortschreitenden Digitalisierung einhergehendes Phänomen der entgrenzten Arbeitswelt. ${ }^{934}$ Als ständige Erreichbarkeit wird bezeichnet, wenn Arbeitnehmer mittels Internetkommunikationsmitteln arbeitszeitunabhängig verfügbar sind, ohne dass Rufbereitschaft, Arbeitsbereitschaft oder Bereitschaftsdienst vereinbart sind. ${ }^{935}$ Sie betrifft die Verfügbarkeit des Beschäf-

927 Ebd., S. 12.

928 Bundesanstalt für Arbeitsschutz und Arbeitsmedizin (Hrsg.), Arbeitszeitreport 2016, https://www.baua.de/DE/Angebote/Publikationen/Berichte/F2398.pdf?_b lob=publicationFile\&v=20 (Stand: 18.11.2018), S. 72 .

929 Falder, NZA 2010, S. 1150, 1151.

930 Fietze/Keller/Friedrich/Dettmers, in: dies. (Hrsg.), Rufbereitschaft, S. 1, 3.

931 Bundesanstalt für Arbeitsschutz und Arbeitsmedizin (Hrsg.), Arbeitszeitreport 2016, https:/www.baua.de/DE/Angebote/Publikationen/Berichte/F2398.pdf?_b lob=publicationFile\&v=20 (Stand: 18.11.2018), S. 71 .

932 BAG, Urt. v. 25.10.1989 - 2 AZR 633/88 -, juris Rn. 35; Falder, NZA 2010, S. $1150,1151$.

933 Fietze/Keller/Friedrich/Dettmers, in: dies. (Hrsg.), Rufbereitschaft, S. 1, 3.

934 Vgl. Spellbrink, NZS 2016, S. 527, 531.

935 Schuchart, ArbuR 2016, S. 341, 341. Zur genauen arbeitsrechtlichen Einordnung und zur Problematik, ständige Erreichbarkeit wirksam arbeitsvertraglich zu vereinbaren, dies., in: Latzel/Picker (Hrsg.), Neue Arbeitswelt, S. 175, 179, 185 ff. 
tigten während der Zeit, die als Freizeit empfunden wird ${ }^{936}$ und arbeitszeitrechtlich als „Ruhezeit“ im Sinne des $\$ 5$ Abs. 1 ArbZG eingeordnet wird. ${ }^{937}$ Dazu gehört es etwa, wenn Beschäftigte faktisch erreichbar sind, weil sie ein und dasselbe mobile Endgerät für private und dienstliche Belange nutzen und so zu jeder Zeit über den Eingang einer beruflichen EMail informiert werden. ${ }^{938}$ Obwohl sie dies arbeitsvertraglich nicht schulden, lassen viele Arbeitnehmer ein derartiges Eindringen ihres Berufes in ihr Privatleben zu, weil sie befürchten, sonst wichtige Entwicklungen zu verpassen oder Nachteile im Arbeitsleben zu erleiden. ${ }^{939}$ Von Arbeitgebern wird dies zumeist jedenfalls geduldet. ${ }^{940}$ Die so definierte ständige Erreichbarkeit ist inzwischen für sehr viele Beschäftigte aller Qualifikationsebenen Alltag: 90 Prozent aller Arbeitnehmer sind außerhalb ihrer Arbeitszeit erreichbar. ${ }^{941} 22$ Prozent der befragten Beschäftigten gaben in der BAuA-Arbeitszeitbefragung 2015 an, dass in ihrem Umfeld erwartet würde, dass sie auch während der Freizeit zur Kontaktaufnahme zur Verfügung stehen. ${ }^{942}$ Ständige Erreichbarkeit ist, im Gegensatz zu Homeoffice und mobiler Arbeit, kein von den Beschäftigten gewünschtes Ziel, da es ihrer Meinung nach der Vereinbarkeit von Berufs- und Privatleben eher abträglich ist. ${ }^{943}$

Die genannten Gestaltungsformen der Erreichbarkeit verfügen allesamt über zwei konstituierende Teilaspekte. Zum einen gehört dazu die bloße

936 Bundesanstalt für Arbeitsschutz und Arbeitsmedizin (Hrsg.), Arbeitszeitreport 2016, https:/www.baua.de/DE/Angebote/Publikationen/Berichte/F2398.pdf?_b lob=publicationFile \&v=20 (Stand: 18.11.2018), S. 74. Dadurch ist sie von den bereits behandelten Phänomenen mobiler Arbeit und häuslicher Arbeit abgrenzbar, vgl. Schuchart, in: Latzel/Picker (Hrsg.), Neue Arbeitswelt, S. 175, 181.

937 Schuchart, ArbuR 2016, S. 341, 342.

938 Zur Nutzung von Endgeräten in beiden Sphären s.o. Kap. 2 E. 2.

939 Falder, NZA 2010, S. 1150, 1150; Schuchart, ArbuR 2016, S. 341, 341.

940 Ebd. Wird die Erreichbarkeit nicht nur geduldet, sondern gefordert, liegt keine ständige Erreichbarkeit im engeren Sinne, sondern Rufbereitschaft vor.

941 Schuchart, in: Latzel/Picker (Hrsg.), Neue Arbeitswelt, S. 175, 177 f. Zwei Drittel aller Beschäftigten werden zumindest gelegentlich in ihrer Freizeit beruflich kontaktiert, ebd.

942 Bundesanstalt für Arbeitsschutz und Arbeitsmedizin (Hrsg.), Arbeitszeitreport 2016, https://www.baua.de/DE/Angebote/Publikationen/Berichte/F2398.pdf?_b lob=publicationFile\&v=20 (Stand: 18.11.2018), S. 75 .

943 Ebd., S. 81. Aufgrund der mit ständiger Erreichbarkeit einhergehender Gesundheitsgefährdung wird bereits ein "Recht auf Unerreichbarkeit“ angenommen, vgl. Schuchart, ArbuR 2016, S.341, 343. Gelegentlich bringt die ständige Erreichbarkeit Beschäftigten wohl als Symbol ihrer Unentbehrlichkeit eine gewisse Gratifikation, vgl. dies., in: Latzel/Picker (Hrsg.), Neue Arbeitswelt, S. 175, 178. 
Verfügbarkeit, also das Sich-Befinden des Beschäftigten im sog. Stand-byModus (dazu siehe II.); die zweite Phase der Erreichbarkeit ist dann die tatsächliche Erbringung einer Arbeitstätigkeit (dazu III.). ${ }^{944}$ Für die unfallversicherungsrechtliche Einordnung der während dieser Zeiten erlittenen Unfälle ist es wegen der Maßgeblichkeit der tatsächlichen Verrichtung zum Unfallzeitpunkt für die Definition des Arbeitsunfalls notwendig, diese beiden Phasen zu unterscheiden.

\section{Unfall während der bloßen Verfügbarkeit}

\section{Rufbereitschaft}

Die schwierigere der genannten Konstellationen ist die Frage, wie ein Unfall einzuordnen ist, der sich während einer Zeit der Verfügbarkeit des Arbeitnehmers ereignet, ohne dass dieser zum Unfallzeitpunkt tatsächlich eine Arbeitsleistung im engeren Sinne erbracht hat.

Dies ist zunächst an einem Beispiel zur Rufbereitschaft zu erläutern: Ein Krankenpfleger in Rufbereitschaft führt im Stadtpark seinen Hund spazieren, stolpert und verletzt sich. Während der ganzen Zeit wurde er nicht beruflich kontaktiert. Der Unfall könnte dennoch als Arbeitsunfall anzusehen sein, wenn das Spazierenführen des Hundes wegen der bestehenden Rufbereitschaft als versicherte Tätigkeit anzusehen wäre. Einer Ansicht nach kann während einer Rufbereitschaft kein Versicherungsschutz bestehen, solange die Beschäftigte keine der grundsätzlich versicherten Tätigkeit dienende Verrichtung ausführt, Keller bezieht sich dabei auf das Altenpflegerinnen-Urteil des Bundessozialgerichts. ${ }^{945}$ Darin entschied das Gericht jedoch lediglich, dass das dienstliche Telefonat nicht bereits wegen der Rufbereitschaft als rechtlich wesentlich für den Unfall angesehen werden könne. ${ }^{946}$ Diese Aussage betrifft zunächst nur die Unfallkausalität und sagt noch nichts über die Bewertung des sachlichen Zusammenhangs aus. Für die Frage, ob überhaupt eine versicherte Tätigkeit vorliegt, ist allerdings lediglich der sachliche Zusammenhang maßgeblich. Erst wenn eine versicherte Tätigkeit bejaht wurde, ist in einem nächsten Schritt zu prüfen, ob

944 Vgl. Schuchart, in: Latzel/Picker (Hrsg.), Neue Arbeitswelt, S. 175, 180.

945 Keller, in: Hauck/Noftz/ders. (Hrsg.), SGB VII, $\$ 8$ Rn. 41 unter Bezugnahme auf BSG, Urt. v. 26.6.2014 - B 2 U 4/13 R -, juris Rn. 28; LSG Berlin-Brandenburg, Urt. v. 26.6.2014 - L 3 U 128/12 -, juris Rn. 34.

946 BSG, Urt. v. 26.6.2014 - B 2 U 4/13 R -, juris Rn. 28. 
diese kausal im Sinne der Theorie der wesentlichen Bedingung für den Unfalleintritt war. Aus der Rechtsprechung des Bundessozialgerichts ergibt sich deswegen keine Aussage über das Vorliegen einer versicherten Tätigkeit, wenn keine Kontaktaufnahme erfolgt ist.

Nach anderer Ansicht liegt allein durch die Rufbereitschaft bereits eine versicherte Tätigkeit vor. ${ }^{947}$ Dem ist zu folgen. Zwar scheint das bloße Warten auf Kontaktaufnahme zunächst keine Tätigkeit im Sinne einer aktiven Arbeitsleistung darzustellen. Unter dem Begriff einer Handlung versteht man zumeist ein proaktives Tätigwerden, während das Zurverfügungstehen als passiv qualifiziert werden könnte. Die Erreichbarkeit lässt sich allerdings dann unter den Begriff der Handlung fassen, wenn man bedenkt, dass zu ihrer Aufrechterhaltung aktive Vorkehrungen getroffen werden müssen. So ist beispielsweise das Diensthandy in funktionsfähigem $\mathrm{Zu}$ stand mitzuführen und regelmäßig auf Anrufe zu kontrollieren. Das Sichweisungsgemäß-zur-Verfügung-Halten ist daher als menschliches Tun und als tatsächliche Verrichtung qualifizierbar, die zweifellos auch mit betriebsdienlicher Handlungstendenz ausgeführt wird. Da die Beschäftigte für ihre Erreichbarkeit auch auf sichtbare Hilfsmittel - in der Regel ein Festnetzoder Mobiltelefon - angewiesen ist, kann die berufsdienliche Handlungstendenz auch von einem vorgestellten Dritten erkannt werden, sodass auch das Kriterium der Objektiviertheit (an welches ohnehin keine überhöhten Anforderungen zu stellen sind), erfüllt ist.

Da die Rufbereitschaft bereits tatsächliche, versicherte Verrichtung ist, kommen bei Unfällen während dieser in der Regel nur gemischte Tätigkeiten in Betracht. ${ }^{948}$ Denn in beinahe allen Fällen wird der Beschäftigte während seiner Erreichbarkeit einer zusätzlichen privaten Tätigkeit nachgehen. Diese Fallgestaltungen sind daher als gemischte Tätigkeiten im Rahmen der Unfallkausalität nach den anerkannten Grundsätzen zu lösen. Es ist danach zu fragen, ob der beruflich motivierte Teil der gemischten Tätigkeit also die Rufbereitschaft - auch wesentlich für den Unfalleintritt war. ${ }^{949}$ Es muss nach lebenspraktischen und naturwissenschaftlich-technischen Gesichtspunkten sowie im Rahmen einer Wertungsentscheidung beurteilt werden, ob die Rufbereitschaft einen derart gewichtigen Anteil an der Verursachung des Unfalls hatte, dass die konkurrierende private Ursache hier also das Spazierengehen - nicht als von überragender Bedeutung an-

947 Spellbrink, NZS 2016, S. 527, 531.

948 Nazarek, PersR 2016, S. 28, 29.

949 Zur Prüfung der gemischten Tätigkeit s.o. Kap. 1 D. III. 2. c. dd sowie Kap. 1 E. III. 3. b. 
zusehen ist. ${ }^{950}$ Im Rahmen dieser Entscheidung ist zu bedenken, dass Rufbereitschaft als Stressor für psychische Belastungen identifiziert ist, da das gefühlte ununterbrochene „Auf dem Sprung sein“ nicht zu einer mit tatsächlicher Freizeit vergleichbaren Erholung führt und es während der Zeiten der Erreichbarkeit deswegen leichter zu Unfällen kommen kann. ${ }^{951}$ Das soziale Schutzprinzip fordert aufgrund der dadurch erhöhten Schutzbedürftigkeit der betroffenen Arbeitnehmer einen großzügigen Maßstab zur Einordnung als Arbeitsunfall. Daraus kann zwar keine Vermutung dahingehend erfolgen, dass ein während der Zeiten der Erreichbarkeit erlittener Unfall stets als Arbeitsunfall zu qualifizieren ist. Es muss am Einzelfall überprüft werden, ob das im Unfall verwirklichte Risiko nach der Betriebsrisikolehre als beruflich eingeordnet werden kann. Richtig ist aber, mit Spellbrink keine zu strengen Anforderungen an die Wesentlichkeit zu setzen. ${ }^{952}$

\section{Arbeitsbereitschaft und Bereitschaftsdienst}

Zeiten der Arbeitsbereitschaft und des Bereitschaftsdienstes wiederum sind unproblematisch als versicherte Tätigkeiten zu qualifizieren. Sie stellen auch arbeitszeitrechtlich Arbeitszeit dar und werden als solche vergütet. Das Abwarten ist hier arbeitsvertraglich geschuldet und damit eindeutig eine auch von außen beobachtbare betriebsdienliche Handlung, sodass ein sachlicher Zusammenhang mit der versicherten Tätigkeit zu bejahen ist. ${ }^{953}$ Auch bei während dieser Zeiten erlittenen Unfällen kommt es daher auf die Unfallkausalität an, im Rahmen welcher eine Wertentscheidung nach den Grundsätzen der gemischten Tätigkeit zu erfolgen hat. Wenn die

950 Becker, SGb 2012, S. 691, 697.

951 Nazarek, PersR 2016, S. 28, 31; Spellbrink, NZS 2016, S. 527, 531.

952 Spellbrink, NZS 2016, S. 527, 531.

953 Schmitt, SGB VII, $\$ 8$ Rn. 16; Schaub, Arbeitsrechts-Handbuch, $\$ 61$ Rn. 13 f.; Ricke, in: Körner/Leitherer et al. (Hrsg.), Kasseler Kommentar zum Sozialversicherungsrecht, $\mathbb{S} 8$ SGB VII Rn. 47a. Der Arbeitnehmer schuldet im Rahmen des Arbeitsverhältnisses das Zurverfügungstellen seiner Arbeitskraft; wann und wie er tatsächlich tätig wird, ist Organisationssache des Arbeitgebers, vgl. MüllerGlöge, in: Säcker/Rixecker (Hrsg.), Münchner Kommentar zum BGB, $\$ 611$ Rn. 156. 
gleichzeitig getätigte private Verrichtung nicht von überragender Bedeutung für den Kausalverlauf ist, liegt ein Arbeitsunfall vor. ${ }^{954}$

\section{Ständige Erreichbarkeit im engeren Sinne}

Nicht als versicherte Tätigkeit zu qualifizieren ist dagegen die nicht vereinbarte „ständige Erreichbarkeit“ im engeren Sinne, die lediglich darauf beruht, dass der oder die Beschäftigte ein Mobiltelefon bei sich führt oder ein anderes Gerät nutzt, dessen Nummer seinem Arbeitgeber bekannt ist oder über das er dienstliche E-Mails empfangen kann. Denn obwohl auch diese Art der Erreichbarkeit nachgewiesenermaßen zu psychischen Belastungen des Arbeitnehmers führen kann, ginge eine Qualifizierung der gesamten Zeit der Mitführung eines mobilen Endgeräts als versicherte Tätigkeit zu weit. Obwohl den zitierten Studien zufolge ${ }^{955}$ in der heutigen Gesellschaft die ständige Erreichbarkeit in vielen Fällen Normalität geworden ist, kann sie nicht zulasten der Unternehmer als Betriebsrisiko gesehen werden, solange der Arbeitgeber diese nicht ausdrücklich oder konkludent fördert. Auch wenn argumentiert wird, dass Arbeitnehmer nicht wirksam auf Ruhezeit verzichten können und es deswegen im Verantwortungsbereich des Arbeitgebers liegt, für eine Einhaltung der arbeitsfreien Erholungszeiten zu sorgen, ${ }^{956}$ liegt es doch am Beschäftigten, die Weiterleitung der Kommunikation an seine Person zu unterbinden. Andernfalls fehlt es an der Zurechenbarkeit zum Arbeitgeber. ${ }^{957}$ Nur wenn im konkreten Fall die Erwartungshaltung des Arbeitgebers an den Beschäftigten derart manifest ist, dass von einer konkludenten Vereinbarung dahingehend auszugehen ist, dass der Arbeitnehmer sich zur Verfügung halten soll, liegt wiederum keine ständige Erreichbarkeit im engeren Sinne, sondern eine Rufbereitschaft vor, sodass nach dem soeben Gesagten eine versicherte Tätigkeit zu bejahen ist. Bei der Beurteilung der Frage, ob demnach eine ständige

954 Vgl. nur BSG, Urt. v. 26.6.2001 - B 2 U 30/00 R -, juris Rn. 17; Rolfs, in: MüllerGlöge/Preis et al. (Hrsg.), Erfurter Kommentar zum Arbeitsrecht, $\mathbb{} 8$ SGB VII Rn 6.

955 S.o. Kap. 3 C. I.

956 Falder, NZA 2010, S. 1150, 1152. Technisch kann der Arbeitgeber etwa dafür sorgen, dass über den betrieblichen Server eingegangene Kommunikation nur zu bestimmten Zeiten an außerhalb des Betriebs abrufbare Endgeräte weitergeleitet wird, vgl. ebd., S. $154 \mathrm{f}$.

957 Dieselbe Wertung, allerdings im Rahmen der Frage der Einordnung von ständiger Erreichbarkeit zur Arbeitszeit, trifft von Steinau-Steinrück, NJW-Spezial 6/2012, S. 178, 178. 
Erreichbarkeit oder eine Rufbereitschaft vorliegt, ist zu bedenken, dass der Arbeitgeber kraft seiner gegenüber dem Arbeitnehmer überlegenen Position zumeist in der Lage ist, Regelungen faktisch einseitig zu setzen und der Beschäftigte sich schnell zu einer Erreichbarkeit verpflichtet fühlen kann, weil er die Missbilligung des Arbeitgebers als seiner Karriere abträglich fürchtet. 958

III. Unfall bei tatsächlicher Arbeitserbringung während der Erreichbarkeit

Demgegenüber ist die Frage nach dem Unfallversicherungsschutz für alle Fallgruppen der Verfügbarkeit des Arbeitnehmers dann geklärt, wenn es während dieser Zeiten tatsächlich zur Arbeitserbringung kommt und sich der Unfall während einer solchen ereignet. Wird der Beschäftigte von sich aus oder nach Kontaktaufnahme im Rahmen seiner arbeitsvertraglichen Bedingungen in betriebsdienlicher Intention tätig, besteht ein sachlicher Zusammenhang zur versicherten Tätigkeit, sodass - soweit die weiteren Voraussetzungen des $₫ 8$ Abs. 1 SGB VII vorliegen - ein Arbeitsunfall vorliegt. Dies gilt auch dann, wenn der Arbeitnehmer ohne Verpflichtung erreichbar war und aus Anlass der Kontaktaufnahme freiwillig die Arbeit aufgenommen hat. Dieses als „Freizeittätigkeit“ bekannte Verhalten ist unproblematisch eine versicherte Tätigkeit, wenn sie vom Arbeitgeber akzeptiert ist. Unerheblich ist dabei, wie lange die unternehmensdienliche Tätigkeit andauert. Auch das kurze Überfliegen einer beruflichen E-Mail stellt eine versicherte Tätigkeit dar. ${ }^{959}$ Ihre kurze Dauer schadet nicht, da sie mit einer entsprechend kurzen Dauer des Versicherungsschutzes korrespondiert.

Insofern besteht für die Einordnung eines Unfalls als Arbeits- oder Privatunfall kein Unterschied zur oben behandelten mobilen Arbeit. Meist werden auch in diesen Fällen die bereits behandelten Grundsätze der gemischten Tätigkeit einschlägig sein, da der Beschäftigte bei Entgegennahme der dienstlichen Kontaktaufnahme zwangsläufig in den meisten Fällen zunächst einer privaten Tätigkeit nachgeht. So stellte sich der Sachverhalt in dem zur Rufbereitschaft hauptsächlich zitierten Urteil des Bundessozial-

958 Vgl. dazu Falder, NZA 2010, S. 1150, 1151 f.; zum Kräfteverhältnis zwischen Arbeitgeber und Arbeitnehmer s.o. Kap. 2. C. II.

959 Zur Qualifikation von kurzen beruflichen „Unterbrechungen“ der Freizeit als Arbeitszeit vgl. Raif/Swidersky, GWR 2017, S. 351, 353 m.w.N. 
gerichts zur Altenpflegerin, die bei Entgegennahme des dienstlichen Anrufs gerade ihren Hund spazieren führte und verunfallte, dar. ${ }^{960}$

\section{Lösungs- und Regelungsvorschläge zum Unfallversicherungsschutz bei entgrenzter Arbeit}

I. Fortentwicklung der Auslegungspraxis und klarstellende Ergänzung des Normtextes hinsichtlich der Bedeutung des Orts der Tätigkeit und der Rufbereitschaft

1. Festschreibung des Unfallorts als bloßes Indiz

Die Untersuchung hat gezeigt, dass die nach der bisherigen Rechtsprechungspraxis und weiten Teilen der Literatur vorgenommene Grenzziehung zwischen Arbeits- und Privatunfall im Homeoffice tätiger Beschäftigter gegen die Strukturprinzipien der Unfallversicherung verstößt. Indem in der Argumentation bei der Auslegung des $\$ 8$ Abs. 1 und 2 SGB VII der historisch als besonders maßgeblich angesehene Aspekt der „Betriebsherrschaft“ des Arbeitgebers über die örtliche Umgebung des Beschäftigten betont wird, werden die Implikationen der tatsächlichen Verhältnisse aktueller Arbeitsgestaltungen vernachlässigt. Das Abstellen auf den überwiegenden Nutzungszweck des Unfallorts als ausschlaggebendes Kriterium für die Bejahung des sachlichen Zusammenhangs mit der versicherten Tätigkeit und damit für die Zuordnung eines Unfalls zu Risikosphären ist angesichts entgrenzter Arbeitsgestaltung nicht mehr zeitgemäß. Verlässliches Kriterium zur Bestimmung eines Arbeitsunfalls kann grundsätzlich kein räumlicher Ansatz, sondern ausschließlich die objektive Handlungstendenz des Versicherten bei der zum Zeitpunkt des Unfalls ausgeführten Tätigkeit mit der versicherten Tätigkeit sein. Dies entspricht nicht nur den Schutzzwecken und der Systematik der Unfallversicherung, sondern auch dem tatsächlichen Wortlaut des $\mathbb{8} 8$ Abs. 1 SGB VII. Eine Anpassung des Unfallversicherungsschutzes von Teleheimarbeitnehmern im angestrebten Sinn ist daher bereits durch eine Änderung der Auslegungspraxis der Rechtsprechung zu erreichen. Die Gründe für diese wurden in Kapitel 3 ausführlich dargelegt, sodass hier lediglich eine Zusammenfassung der Ergebnisse erfolgt.

960 BSG, Urt. v. 26.6.2014 - B 2 U 4/13 R -, juris (zu dieser Entscheidung s.o. Kap. 1 D. III. c. dd.); Spellbrink, NZS 2016, S. 527, 531; Nazarek, PersR 2016, S. $28,29$. 
\8 Abs. 1 SGB VII ist dabei so auszulegen, dass Verrichtungen mit gespaltener Handlungstendenz auch wenn sie nicht am Unternehmenssitz erfolgen, immer dann als versicherte Tätigkeit einzuordnen sind, wenn sie hypothetisch auch dann vorgenommen worden wären, wenn ihre private Motivation nicht vorgelegen hätte. Gleichfalls sind Betriebswege im häuslichen Bereich oder im Bereich eines Mobile Office nach denselben Grundsätzen zu behandeln wie Betriebswege am Unternehmenssitz: Stellt das Zurücklegen der Strecke selbst bereits eine geschuldete Arbeitsleistung dar, sind sie versicherte Tätigkeit. Zudem sind sowohl innerhäusliche Wege zur Nahrungsaufnahme und zur Nutzung von Sanitäreinrichtungen als auch entsprechende vom häuslichen Bereich ausgehende Wege versicherte Tätigkeiten in dem Umfang, in dem dies für Wege zur Nahrungsaufnahme und zu Sanitäreinrichtungen vom Arbeitsplatz auf dem Betriebsgelände gilt. Für die mobile Arbeit gilt weiterhin, dass auch in ihrem Rahmen alle unternehmensdienlichen Tätigkeiten unabhängig vom physischen Aufenthaltsort des Beschäftigten in der Risikosphäre des Arbeitgebers zu verorten und daher nach den anerkannten Grundsätzen als versicherte Tätigkeiten zu qualifizieren sind.

Obwohl diese Ergebnisse schon durch eine Anpassung der Auslegungspraxis erreicht werden können, und sich eine solche Änderung in der Rechtsprechung des BSG in jüngster Zeit bereits angekündigt hat, ist es empfehlenswert, eine klarstellende Aussage in den Normtext zu übernehmen, um die Schlechterstellung von außerhalb des klassischen Betriebsgeländes tätigen Beschäftigten zu vermeiden und die Rechtssicherheit im Sinne der Normadressaten zu fördern. Vorgeschlagen wird daher, $\mathbb{8} 8$ SGB VII wie folgt zu verändern. ${ }^{961}$

In $₫ 8$ Abs. 1 wird S. 1 um einen Halbsatz ergänzt:

\section{$\$ 8$ Abs. 1 SGB VII}

Arbeitsunfälle sind Unfälle von Versicherten infolge einer den Versicherungsschutz nach $₫ 2,3$ oder 6 begründenden Tätigkeit (versicherte Tätigkeit), unabhängig davon, an welchem Ort sie sich ereignen. Unfälle sind zeitlich begrenzte, von außen auf den Körper einwirkende Ereignisse, die zu einem Gesundheitsschaden oder zum Tod führen die zu einem Gesundheitsschaden oder zum Tod führen.

Diese Formulierung macht deutlich, dass der Ort, an dem sich der Beschäftigte zum Zeitpunkt des Unfalls befindet, nicht das ausschlaggebende Kri-

961 Änderungs- und Ergänzungsvorschläge sind fett markiert. 
terium für die Beurteilung des Vorliegens eines Arbeitsunfalls ist. ${ }^{962}$ Eine Differenzierung nach der konkreten Nutzungshäufigkeit in privatem und beruflichem Kontext, wie sie bis jüngst in der Rechtsprechung der Sozialgerichte vorgenommen wurde, ist damit nicht mehr möglich; $\mathbb{} 8$ Abs. 1 S. 1 Hs. 2 SGB VII zeigt, dass der sachliche Zusammenhang zur versicherten Tätigkeit unabhängig ${ }^{963}$ von rein räumlichen Kriterien beurteilt werden muss. Vielmehr ist die Berücksichtigung des Ortes der Tätigkeit nur noch im Rahmen der Betrachtung der objektiven Umstände des Einzelfalls möglich und kann somit nur noch Indizwirkung entfalten, wie es der hier vertretenen Ansicht in allen Fällen entgrenzten Arbeitens und der jüngsten Rechtsprechung des Bundessozialgerichts zu innerhäuslichen Betriebswe-

962 Für das Einfügen eines Halbsatzes im Anschluss an die Legaldefinition der versicherten Tätigkeit spricht, dass so deutlich wird, dass es sich bei der Klarstellung nicht um eine Ergänzung der Definition der versicherten Tätigkeit handelt. Vielmehr soll der herrschenden Praxis, den in das Wort „infolge“ hineingelesenen notwendigen sachlichen Zusammenhang der Tätigkeit zum Unfallzeitpunkt mit der versicherten Tätigkeit vom überwiegenden Nutzungszweck des Unfallorts abhängig zu machen, eine Absage erteilt werden. Um zu vermeiden, dass die Ergänzung als Änderung der Definition der versicherten Tätigkeit verstanden wird, wird sprachlich nicht an den Ort der Tätigkeit, sondern an den Unfallort angeknüpft. Alternativ zu dieser Gestaltung hätte in $\mathbb{S} 8$ Abs. 1 SGB VII ein S. 3 mit dem Inhalt, der Unfallort sei nicht maßgeblich, neu eingefügt werden können. Um deutlich zu machen, dass sich ein solcher S. 3 auf S. 1 und nicht S. 2 bezieht, hätte in diesem jedoch genau bezeichnet werden müssen, in welchem Zusammenhang auf das Kriterium des Unfallorts verzichtet werden soll. Dies hätte eine deutlich umfangreichere Gesetzesergänzung notwendig gemacht, so dass die kürzere Variante des Einfügens eines achtwortigen Halbsatzes vorzuziehen ist.

963 Die Satzkonstruktion „unabhängig von“ wurde gewählt, weil dieses Adjektiv im Gegensatz zu einer Verbindung mit „gleichgültig, ob“ eine textlich weniger umfangreiche Gesetzesergänzung erfordert. Es ist nur die Ergänzung eines Präpositionalobjekts notwendig, während eine Konstruktion mit „gleichgültig, ob“ einen umfangreicheren Satzaufbau mit einem weiteren Nebensatz und mehreren Objekten notwendig gemacht hätte. So wird etwa auch in $₫ 5$ Abs. 1 BetrVG eine Satzkonstruktion mit „unabhängig von“ verwendet, der im weiteren Sinne ebenfalls den Ort der Tätigkeit zum Inhalt hat, wenn Arbeitnehmer im Sinne des Gesetzes definiert werden als „[...] Arbeiter und Angestellte [...], unabhängig davon, ob sie im Betrieb, im Außendienst oder mit Telearbeit beschäftigt werden.“ Ein Beispiel der umfangreicheren Satzkonstruktion mit „gleichgültig,

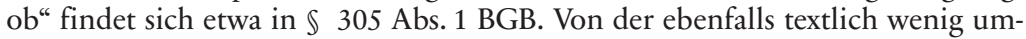
fangreichen Konstruktion des Satzes als ,gleichgültig, an welchem Ort sie sich ereignen" (so wird "gleichgültig“ etwa in $\$ 14$ Abs. 1 UStG verwendet) wurde abgesehen, da eine analoge Formulierung zu $\$ 5$ Abs. 1 BetrVG sinnvoll erscheint. 
gen von Unternehmern entspricht. Die vorgeschlagene Ergänzung unterstreicht die entscheidende Bedeutung der in S. 1 genannten Verrichtung, ohne zu verhindern, dass der Nutzungszweck der örtlichen Umgebung bei entsprechender Relevanz wertend in die Beurteilung des Vorliegens eines sachlichen Zusammenhangs einfließen kann - allerdings nur innerhalb der Definition der versicherten Tätigkeit bei der Frage danach, ob die Handlungstendenz des Versicherten durch die objektiven Umstände des Einzelfalls bestätigt wurde. Der Ansicht, bei einem Unfall innerhalb der häuslichen Umgebung wäre grundsätzlich ein Privatunfall zu vermuten, wird eine klare Absage erteilt.

\section{Klarstellung des Versicherungsschutzes der Rufbereitschaft}

Bereits das Zurverfügungstehen des Beschäftigten auf Abruf im Rahmen einer vereinbarten Rufbereitschaft stellt eine versicherte Tätigkeit dar. Richtigerweise ist bei Eintritt eines Unfalls während des Rufbereitschaftsdienstes daher immer ein Arbeitsunfall in Betracht zu ziehen, selbst wenn zum Unfallzeitpunkt keine weitere versicherte Tätigkeit in dem Sinne ausgeführt worden ist, dass die Arbeitskraft des Beschäftigten abgerufen worden ist. Es liegt, da bereits die Rufbereitschaft an sich versicherte Tätigkeit ist, jedenfalls eine gemischte Tätigkeit vor. Auch dieses Ergebnis kann durch Auslegung des $₫ 8$ Abs. 1 SGB VII erreicht werden. Ebenso wie bei der Frage nach der Maßgeblichkeit des Unfallortes ist es jedoch auch hier aufgrund divergierender Ansichten erstrebenswert, eine klarstellende Regelung in den Gesetzestext aufzunehmen.

In $\$ 8$ Abs. 2 erfolgt daher die Aufnahme eines neuen Katalogtatbestandes am Ende des Kataloges:

$\$ 8$ Abs. 2 SGB VII

Versicherte Tätigkeiten sind auch (...) 6. das vereinbarte Zurverfügungstehen an einem frei wählbaren Ort zur alsbaldigen Arbeitsaufnahme außerhalb der Arbeitszeit (Rufbereitschaft). 964

Dieser Formulierungsvorschlag bietet eine Legaldefinition der Rufbereitschaft. Während sie in Gesetzestexten bisher lediglich erwähnt, nicht aber legaldefiniert wurde (z.B. in $₫ 5$ Abs. 3 ArbZG), kann sie zukünftig sicher anhand $₫ 8$ Abs. 2 Nr. 6 SGB VII von der ständigen Erreichbarkeit im enge-

964 Der gesamte Text des $₫ 8$ SGB VII mit allen vorgeschlagenen Änderungen im Überblick s.u. Schluss B. 
ren Sinne abgegrenzt werden. Die gewählte Definition entspricht inhaltlich der allgemein anerkannten Bedeutungsumschreibung der Rufbereitschaft, wie sie durch die Rechtsprechung von EuGH und BAG, im wissenschaftlichen Diskurs und etwa in $\$ 7$ Abs. 4 des Tarifvertrags für den öffentlichen Dienst der Länder genutzt wird. ${ }^{965}$ Rufbereitschaft ist demnach die Zeit, während der sich Beschäftigte außerhalb ihrer üblichen Arbeitszeit für einen Arbeitseinsatz bereithalten. Dabei können sie sich an einem beliebigen Ort aufhalten, müssen aber jederzeit und unmittelbar erreichbar und in angemessener Frist reaktionsbereit sein. ${ }^{966}$

Von besonderer Relevanz für die hier vollzogene Risikoabgrenzung zwischen beruflicher und privater Risikosphäre ist dabei das Merkmal der Vereinbarung, welches den Unterschied zur von der Betriebsrisikolehre nicht erfassten ständigen Erreichbarkeit im engeren Sinne darstellt. Eine Vereinbarung hinsichtlich der Rufbereitschaft kann konkludent und ausdrücklich getroffen werden. Ihr Vorliegen ist im Anwendungsfall genau zu prüfen und hängt vom konkreten Einzelfall ab. Das Merkmal der Vereinbarung ist auch erfüllt, wenn der Arbeitgeber den Arbeitnehmer zur Rufbereitschaft gemäß $\$ 108 \mathrm{GewO}$ anweisen konnte, ohne dass es einer weiteren gegenseitigen Abrede bedurfte; in diesem Fall ist die generelle Möglichkeit von Rufbereitschaftsdienst bereits von der arbeitsvertraglichen Vereinbarung erfasst.

Durch die Begrenzung auf Zeiten außerhalb der Arbeitszeit in der Formulierung des $₫ 8$ Abs. 2 Nr. 6 SGB VII wird die Rufbereitschaft von Bereitschaftsdienst und Arbeitsbereitschaft abgegrenzt, welche, wie gesehen, bereits de lege lata vom Versicherungsschutz umfasst sind.

965 EuGH, Rs. C-303/98, Simap, Slg. 2000, I-7963 Rn. 50; BAG, Urt. v. 24.10.2000 9 AZR 634/99 -, juris Rn. 21; v. 19.12.1991 - 6 AZR 592/89 -, juris Rn. 21. Aus der Literatur vgl. Preis, in: Müller-Glöge/Preis et al. (Hrsg.), Erfurter Kommentar zum Arbeitsrecht, $\$ 611 \mathrm{a}$ BGB Rn.671; Wank, in: Müller-Glöge/Preis et al. (Hrsg.), Erfurter Kommentar zum Arbeitsrecht, $\mathbb{2}$ ArbZG Rn. 31; Kock, in: Rolfs/Giesen, et al. (Hrsg.), BeckOK Arbeitsrecht, $\$ 2$ ArbZG Rn. 10; Baeck/ Lösler, NZA 2005, S. 247, 249; von Steinau-Steinrück, NJW-Spezial 6/2012, S. 178, 178. Die Definition des Tarifvertrags für den öffentlichen Dienst der Länder lautet: „Rufbereitschaft leisten Beschäftigte, die sich auf Anordnung des Arbeitgebers außerhalb der regelmäßigen Arbeitszeit an einer dem Arbeitgeber anzuzeigenden Stelle aufhalten, um auf Abruf die Arbeit aufzunehmen.", $\mathbb{\$} 7$ Abs. 4 S. 1 TV-L v. 12.10.2006 i.d.F.d. Änderungstarifvertrages Nr. 9 v. 17.2.2017, abrufbar unter: http://www.tdl-online.de/fileadmin/downloads/rechte_Navigation/A._TV -L__2011_/01_Tarifvertrag/TV-L__i_d_F__des_\%C3\%84TV_Nr__9_VT.pdf (Stand: 18.11.2018).

966 Vgl. BAG, Urt. v. 24.10.2000 - 9 AZR 634/99 -, juris Rn. 21; v. 19.12.1991 - 6 AZR 592/89-, juris Rn. 21. 
II. Erweiterung des Unfallversicherungsschutzes auf Wege von und zu Kinderbetreuungsstätten bei häuslicher oder mobiler Arbeit durch Gesetzesänderung

Aus dem Telos des $₫ 8$ Abs. 2 Nr. 2 a SGB VII, aus dem Schutzzweck der Unfallversicherung insgesamt sowie aus ihren Strukturprinzipien und der Anwendung des Art. 3 Abs. 1 GG ergibt sich, dass auch Wege von im Homeoffice Beschäftigten, die vom häuslichen Arbeitsplatz aus zurückgelegt werden, um Kinder wegen ihrer beruflichen Tätigkeit fremder Obhut anzuvertrauen, als versicherte Tätigkeiten angesehen werden müssen. Dies ist jedoch nach dem aktuellen Gesetzestext nicht möglich. Um zu gewährleisten, dass der Versicherungsschutz den genannten Voraussetzungen entspricht, muss daher der Gesetzestext angepasst werden. Dabei ist am Kata$\log$ des $\$ 8$ Abs. 2 SGB VII anzusetzen. $\$ 8$ Abs. 2 Nr. 2 und 3 SGB VII lauten bisher:

„Versicherte Tätigkeiten sind auch [...]

2. das Zurücklegen des von einem unmittelbaren Weg nach und von dem Ort der Tätigkeit abweichenden Weges, um

a) Kinder von Versicherten ( $\$ 56$ des Ersten Buches), die mit ihnen in einem gemeinsamen Haushalt leben, wegen ihrer, ihrer Ehegatten oder ihrer Lebenspartner beruflichen Tätigkeit fremder Obhut anzuvertrauen oder

b) mit anderen Berufstätigen oder Versicherten gemeinsam ein Fahrzeug zu benutzen,

3. $[\ldots]$..

Es sind folgende Änderungen vorzunehmen. ${ }^{967}$

\8 Abs. 2 Nr. 2 wird ersetzt durch: „2a. das Zurücklegen eines Weges, um Kinder von Versicherten ( $\$ 56$ des Ersten Buches), die mit ihnen in einem gemeinsamen Haushalt leben, wegen ihrer, ihrer Ehegatten oder ihrer Lebenspartner beruflichen Tätigkeit fremder Obhut anzuvertrauen,

2b. das Zurücklegen des von einem unmittelbaren Weg nach und von dem Ort der Tätigkeit abweichenden Weges, um mit anderen Berufstätigen oder Versicherten gemeinsam ein Fahrzeug benutzen.“968

967 Der gesamte Text des $₫ 8$ SGB VII mit allen vorgeschlagenen Änderungen im Überblick s.u. Schluss B.

968 Im Interesse einer möglichst kontinuierlichen Bennenung der einzelnen Katalogbestände, um Rechtsanwendern den Umgang mit $₫ 8$ SGB VII zu erleichtern, 
Der vorgeschlagene $₫ 8$ Abs. 2 Nr. 2 SGB VII bildet die mit der Norm ursprünglich verfolgten Schutzzwecke ab. Dies wird erreicht, indem das Kriterium des Abweichens vom Arbeitsweg aus dem Normtext herausgenommen wird. Die so überarbeitete Norm fokussiert inhaltlich die berufsdienliche Intention der zurückgelegten Wegstrecke und verlangt neben einer Inobhutgabe des Kindes aus Gründen der beruflichen Tätigkeit die $\mathrm{Zu}$ rücklegung eines Weges, der diese Inobhutgabe zum Ziel hat. Die durch die Voraussetzung einer Abweichung vorhandene Differenzierung nach dem Arbeitsort entfällt. Damit werden alle Wege umfasst, die zur Fremdbetreuung des Kindes zurückgelegt werden, wenn die Betreuung durch die berufliche Tätigkeit veranlasst ist. Dagegen sind solche Wege nicht umfasst, die die Unterbringung des Kindes aus einem privaten Grund bezwecken. Der durch die Präposition „wegen“ verlangte Zusammenhang zwischen beruflicher Tätigkeit und Zurücklegen des Weges verhindert somit auch ohne die Notwendigkeit einer Einbettung des Weges in einen Arbeitsweg eine unsachgemäße Ausweitung des Versicherungsschutzes.

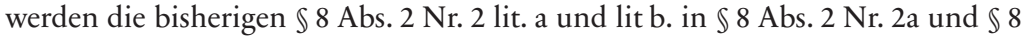
Abs. 2 Nr. 2b SGB VII umgewandelt. Da $\$ 8$ Abs. 2 Nr. 2 SGB VII gerade das für den Fall der Inobhutverbringung zu löschende Kriterium der Abweichung vom Arbeitsweg vor die Klammer der Tatbestände Nr. 2 a) und Nr. 2 b) stellt, hätte alternativ ebenfalls die gesamte Satzkonstruktion der Nr. 2 aufgelöst und die lit. a und b in zwei eigenständige Nummerierungen - Nr. 2 und Nr. 3 - umgewandelt werden müssen, was zwingend eine Verschiebung der bisherigen Nr. 35 in Nr. 4-6 erfordert hätte. Ein Erhalten des bisherigen $\$ 8$ Abs. 2 Nr. 2 lit. b SGB VII unter dieser Nummerierung war wegen dem vor die Klammer gesetzten Merkmal der Abweichung nicht möglich. Sprachlich wäre es zwar vorzugswürdig, wenn der Katalog ausschließlich aus ganzen Ziffern bestünde. Durch das Einführen der Nr. 2a und $2 b$ in den Katalog wird aber immerhin die Neubennenung der Nr. 3-5 vermieden. Gesetzestechnisch wurde eine solche Nummerierung von Katalogtatbeständen mithilfe von Buchstaben etwa auch in $\mathbb{1 9 9}$ Abs. 1 Nr. 3a BGB und $₫ 308$ Nr. 1a und 1b BGB gewählt. 


\section{Kapitel 4: „Umgekehrte“ Entgrenzung: private Tätigkeiten am Arbeitsplatz}

\section{A. Notwendigkeit einer Erweiterung des Unfallversicherungsschutzes auf nicht berufliche Tätigkeiten}

Die Entgrenzung der Arbeit geschieht nicht nur in eine Richtung: So wie durch Homeoffice, mobile Arbeit und ständige Erreichbarkeit das Berufliche in die Privatsphäre der Beschäftigten diffundiert, dringt umgekehrt immer mehr Privates in die Arbeitssphäre ein. ${ }^{969}$ Wie gesehen, werden Beschäftigte in vielen Fällen ermuntert, während der Arbeitszeit am Arbeitsort auch nicht berufliche Dinge zu tun. Selbst dort, wo das „Corporate Life“ nicht zum Firmenkonzept gehört, wächst angesichts der Verbreitung von Smartphones die soziale Akzeptanz von Unterbrechungen des Arbeitsflusses zugunsten der internetgestützten Kommunikation mit dem privaten Personenkreis. Das tägliche Verrichten privater Tätigkeiten am Arbeitsplatz gehört mittlerweile für einen großen Teil der Arbeitsverhältnisse zum Alltag. ${ }^{970}$

Dieses Phänomen ist nicht neu: Schon immer haben Arbeitnehmer während der Arbeitszeit Pausen eingelegt, sei es, um sich privat mit Kollegen zu unterhalten, um zu essen oder um zur Toilette zu gehen. Kaum jemand wird es schaffen, sich während der gesamten Dauer einer Arbeitsschicht ausschließlich auf die Arbeit zu konzentrieren. Menschen sind, um es in den Worten des Bundesverwaltungsgerichts auszudrücken, keine „Dienstleistungsautomaten“.971 In der Konsequenz gibt es umfangreiche Rechtsprechung und Literatur zum Themenbereich der sogenannten „eigenwirtschaftlichen Tätigkeiten“, deren Grundzüge oben bereits dargestellt und hier nur knapp wiederholt werden sollen: Private Handlungen stehen mangels sachlichem Zusammenhang mit der versicherten Tätigkeit grundsätzlich nicht unter Versicherungsschutz, es sei denn, sie stellen eine völlig

969 S.o. Kap. 2 E. II. 2.

970 Je nach Studie geben zwischen 50 und 82 Prozent der befragten Beschäftigten an, täglich Privates am Arbeitsplatz zu erledigen, s.o. Kap. 2 E. II. 2.

971 BVerwG, Urt. v. 24.10.1963, E 17, 59, 59 ff. Rn. 23. Zustimmend Kazmaier, in: Stegmüller/Schmalhofer et al. (Hrsg.), Beamtenversorgungsrecht, $\$ 31$ Rn. 65. 
unerhebliche Unterbrechung der Arbeit dar oder der erlittene Unfall wurde durch eine besondere Betriebsgefahr ausgelöst. ${ }^{972}$

Die Entwicklung der Entgrenzung macht eine neue Betrachtung der unfallversicherungsrechtlichen Einordnung nicht beruflicher Tätigkeiten notwendig. In dem Umfang, in dem Privates mit Erlaubnis, Billigung oder Duldung des Arbeitgebers während der Arbeit erledigt wird, sind auch diese Tätigkeiten vom Unfallversicherungsschutz zu umfassen. Dieser Gedanke erscheint angesichts der in den vorigen Kapiteln herausgearbeiteten Relevanz der ausgeführten Tätigkeit für die Zuordnung zur Berufssphäre zunächst radikal. Jede Fortentwicklung des Unfallversicherungsschutzes muss sich daran messen lassen, die mit ihr einhergehende kollektivierte Haftung der Arbeitgeber nicht systemwidrig auf das allgemeine - private Lebensrisiko der Beschäftigten auszudehnen. ${ }^{973}$ Mit Blick auf die Lebenswirklichkeit entgrenzt Beschäftigter ist es jedoch widersprüchlich, für die Bestimmung eines Unfalls als Arbeitsunfall grundsätzlich ausschließlich darauf abzustellen, ob die im Zeitpunkt des Unfalls durchgeführte Tätigkeit privater oder beruflicher Natur war. Denn wenn es als in der Natur des digitalisiert lebenden Beschäftigten angesehen wird, dass Privat- und Berufssphäre auf diese Art miteinander verwoben sind, so ist die Erledigung von privaten Tätigkeiten am Arbeitsplatz zur Arbeitszeit als dem Betriebsmittel „Mensch“ immanent anzusehen und damit dem Betriebsrisiko des Unternehmers zuzurechnen, der sich die Vorteile dieser Art des Arbeitens zunutze macht und daher auch die damit einhergehenden Nachteile zu tragen hat. Wird vom Arbeitgeber eine Integration des Privatlebens in das berufliche Umfeld gerade gewünscht und gefördert, so ist es sachgerecht, ihm auch das Risiko für die dadurch entstehenden Unfälle anzulasten. Gleiches gilt, wenn der Unternehmer sich dafür entschieden hat, im Rahmen seiner Betriebsorganisation private Verrichtungen in gewissem Umfang zu tolerieren. Die Reichweite des Unfallversicherungsschutzes muss, um dem sozialen Schutzprinzip und der Betriebsrisikolehre gerecht zu werden, die tatsächlichen Gegebenheiten der Arbeitswelt sowie die gesellschaftliche Realität reflektieren.

In diesem Kapitel wird der Frage nachgegangen, wie ein Einbezug erlaubter, gebilligter und geduldeter privater Tätigkeiten im Rahmen des Beschäftigungsverhältnisses in den Unfallversicherungsschutz erreicht wer-

972 S.o. Kap. 1 D. III. c.

973 Jung, SGb 2001, S.398, 399. Vgl. zur Eigenverantwortung der Betroffenen als Grund der Begrenzung der Übernahme allgemeiner Lebensrisiken Becker, Soziales Entschädigungsrecht, S. $116 \mathrm{f}$. 
den kann. Dazu wird der Zuordnungsmechanismus des $₫ 8$ Abs. 1 SGB VII zunächst mit den entsprechenden Regelungen der Unfallfürsorge der Beamten (dazu B.) verglichen, denn dort ist der Schutz nicht beruflicher Tätigkeiten gegeben. ${ }^{974}$ Ein Vergleich des Unfallversicherungsrechts mit den entsprechenden Regelungen des Unfallfürsorgerechts bietet daher Erkenntnisse über rechtliche Gestaltungsmöglichkeiten des Einbezugs privater Tätigkeiten in den Unfallversicherungsschutz. Die Analyse der Funktionsweise und Rechtsgründe dieser Zuordnungsprinzipien illustriert die Besonderheiten der Unfallsicherung der Beamten. Es wird herausgearbeitet, warum diese Personengruppe im Gegensatz zu Beschäftigten hinsichtlich eigenwirtschaftlicher Verrichtungen unterschiedlich behandelt wird. Im Ergebnis ist festzustellen, dass die Zuordnung zur Berufssphäre anhand räumlich-zeitlicher Komponenten, wie sie bei Beamten angewandt wird, nicht auf die Unfallversicherung von Beschäftigten übertragen werden kann (dazu C.). Auch sonst besteht keine Möglichkeit, den Versicherungsschutz privater Tätigkeiten in die bestehende Auslegungspraxis des $\mathbb{8} 8$ SGB VII einzufügen. Am Ende des Kapitels steht daher ein Vorschlag zur Fortentwicklung des Unfallversicherungsschutzes durch eine Gesetzesänderung.

\section{B. Beamtenrechtlicher Dienstunfall bei privater Tätigkeit im Vergleich}

\section{I. Überblick über die Dogmatik des $₫ 31$ Abs. 1 BeamtVG}

Während ausschließlich privat motivierte Tätigkeiten im Unfallversicherungsrecht de lege lata nur im Ausnahmefall - nämlich bei unerheblichen Unterbrechungen des Arbeitszusammenhangs sowie bei Verwirklichung einer besonderen Betriebsgefahr - in den Versicherungsschutz einbezogen werden, sind im Unfallfürsorgerecht der Beamten regelmäßig alle nicht beruflichen Tätigkeiten umfasst, die am Dienstort zur Dienstzeit erledigt wer-

974 Der Vergleich dieser Rechtsgebiete ist deswegen besonders fruchtbar, weil sie aufgrund ihrer Entstehungsgeschichte, ihrer Zweckbestimmung und der vergleichbaren Interessenlage der Rechtssubjekte eng miteinander verbunden sind, vgl. nur Pesch, DVB1. 1959, S. 43, 44 ff.; Kunze, VSSR 2005, S. 299, 300; Fieberg, ZTR 2007, S. 421 ff.; Leube, ZTR 2012, S. 682, 688; ders., NZV 2015, S. 275, 276; Giesen, Arbeitsunfall und Dienstunfall, S. 19. Zum Nutzen des Rechtsvergleichs insbesondere im Sozialrecht Becker, in: ders. (Hrsg.), Rechtsdogmatik im Sozialrecht I, S. 11, 19 ff. 
den. Die normative Basis des Zuordnungsmechanismus findet sich in den $\mathbb{S} \$ 30$ und 31 BeamtVG.

Unfallfürsorge gem. $\$ 30$ Abs. 1 S. 1 BeamtVG wird dann gewährt, wenn ein Beamter "durch einen Dienstunfall“ verletzt wird. $\$ 31$ Abs. 1 S. 1 BeamtVG definiert den Dienstunfall als ein „auf äußerer Einwirkung beruhendes, plötzliches, örtlich und zeitlich bestimmbares, einen Körperschaden verursachendes Ereignis, das in Ausübung des Dienstes eingetreten ist“.975 Die Prüfung des Vorliegens eines Dienstunfalls ähnelt der des Arbeitsunfalls. ${ }^{976}$ Nach der Feststellung des persönlichen Schutzbereichs ${ }^{977}$ wird das Vorliegen eines Unfallereignisses geprüft. Anders als im Unfallver-

975 Bis zum 10.1.2017 enthielt die Norm die Formulierung ,in Ausübung oder infolge des Dienstes“. Die Auslegung dieses Passus war umstritten, wobei die Ergebnisse der unterschiedlichen Auslegungen dieselben waren. Eine zeitliche Abgrenzung der Tatbestandsalternativen wurde in früheren Entscheidungen des BVerwG, z.B. Urt. v. 24.10.1963, E 17，59 Rn. 22; v. 17.3.1965, E 20, 347; v. 6.7.1965, E 21, 307; v. 18.1.1971, E 37, 139, 143; v. 12.2.1971, E 37, 203 sowie von Groepper/Tegethoff, in: Plog/Wiedow et al. (Hrsg.), Bundesbeamtengesetz, $\$ 31$ Rn. 48; Bauer, in: Stegmüller/Schmalhofer et al. (Hrsg.), Beamtenversorgungsrecht (81. Aufl.), Erl. 7 zu $₫ 31$ BeamtVG Rn. 2; Brinktrine, in: Kugele (Hrsg.), Kommentar zum Beamtenversorgungsgesetz, $\mathbb{} 31$ Rn. 15; Bulla, Der Dienst- und Arbeitsunfall als Institut des Allgemeinen Verwaltungsrechts, S. 185 sowie Giesen, Arbeitsunfall und Privatunfall, S.39, vertreten, während andere Autoren von einer sachlichen Differenzierung ausgingen, vgl. Schütz, DÖD 18 (1965), S. 161, 162; BVerwG, Urt. v. 29.8.2013 - 2 C 1/12 -, juris Rn. 13; Urt v. 29.10.2009, E 135, 176 Rn. 14 sowie Wilhelm, in: Franke/Weiß (Hrsg.), GKÖD, $\$ 31$ BeamtVG Rn 32. Einer dritten Ansicht nach besteht zwischen beiden Tatbestandsalternativen keinerlei Unterschied. So etwa BVerwG, Urt. v. 29.8.2013 - 2 C 1/12 -, juris Rn. 10; v. 24.10.1963, E 17, 59, 60; v. 15.11.2007 - 2 C 24.06 -, juris Rn.11; v. 25.2.2010 - 2 C 81.08 -, juris Rn. 17, aber auch bereits Urt. v. 4.6.1970, E 35, 234 ff. Rn. 31; Teutsch, in: Rohrbeck (Hrsg.), Beiträge zur Sozialversicherung, S. 305, 312; Reich, BeamtVG, $\$ 31$ Rn. 4; Schnellenbach, Beamtenrecht in der Praxis, S. 335; Minz/Leppek, Praxis-Handbuch Beamtenversorgungsrecht, Rn. 338; Brockhaus, in: Schütz/Maiwald (Hrsg.), Beamtenrecht, $\mathbb{3} 31$

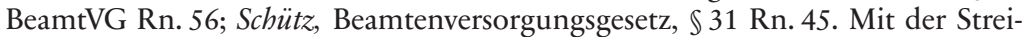
chung der Wörter „oder infolge“ durch Art. 3 des Gesetzes zur Änderung des Versorgungsrücklagegesetzes und weiterer dienstrechtlicher Vorschriften v. 5.1.2017, BGBl. I S. 17, gültig seit 11.1.2017, wurde dieser Streit nicht inhaltlich entschieden, sondern aufgrund seiner Bedeutungslosigkeit für die Praxis beendet, vgl. BT-Drs. 18/9532, S. 40.

976 So auch Leube, ZTR 2012, S. 682, 683. Anders als in der Unfallversicherung besteht in Literatur und Rechtsprechung zu $₫ 31$ BeamtVG kein übereinstimmendes Prüfungsschema, sodass Bezeichnungen und Reihenfolge der Prüfungsschritte variieren, vgl. Giesen, Arbeitsunfall und Dienstunfall, S. 30.

977 Gemäß $₫ 30$ Abs. 1 S. 1 BeamtVG sind, unabhängig von deren Status und der Frage, ob das Dienstverhältnis zum Zeitpunkt des Unfalls (noch oder schon) be- 
sicherungsrecht ist ein Ereignis nur dann als Unfall zu charakterisieren, wenn es plötzlich eingetreten ist. ${ }^{978}$ Zudem muss es nicht nur zeitlich begrenzt, sondern auch zeitlich und örtlich konkret bestimmbar sein. ${ }^{979}$ Als Primärschaden ist ein „Körperschaden“ erforderlich. ${ }^{980}$ Anders als in der

standen hat, alle Beamten des Bundes und deren Hinterbliebene erfasst. Es kommt also nicht darauf an, ob sie Beamte auf Lebenszeit oder auf Zeit sind. Auch Richter des Bundes und deren Hinterbliebene sind einbezogen $(\mathbb{S} 1$ Abs. 2 BeamtVG, $\mathbb{S} \mathbb{S} 46,71 \mathrm{a}$ DRiG). Ehrenbeamte des Bundes allerdings haben nur beschränkt Ansprüche aus Dienstunfällen ( $\$ 68$ BeamtVG). Näher zum persönlichen Schutzbereich Giesen, Arbeitsunfall und Dienstunfall, S. 28 m.w.N. Für Beamte der Länder gelten seit der Förderalismusreform I eigene Regelungen gleichen Inhalts, zu finden in $\$ 1$ bzw. Art. 1 des jeweiligen Landesbeamtenversorgungsgesetzes. Die für diese Untersuchung relevanten Regelungen, nämlich die einen Anspruch auf Unfallfürsorge begründenden Normen, sind inhaltlich gleich geblieben, vgl. ebenso Pflaum, RiA 2011, S. 198, 199. Unterschiedlich sind meist lediglich die Nummerierungen der Paragraphen bzw. Artikel. Der Einfachheit halber werden im Rahmen dieser Arbeit lediglich die Normen des BeamtVG zitiert.

978 Das Ereignis muss abrupt eingetreten sein und darf nur einen relativ kurzen Zeitraum andauern, Wilhelm, in: Franke/Weiß (Hrsg.), GKÖD, $\$ 31$ BeamtVG Rn. 12; Bauer, in: Geyer/Grunefeld et al. (Hrsg.), Beamtenversorgungsrecht des

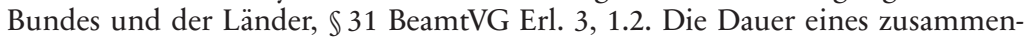
hängenden Tagesdienstes bildet die Höchstgrenze, vgl. BSG, Urt. v. 26.9.61, E 15, 112; Brinktrine, in: Kugele (Hrsg.), Kommentar zum Beamtenversorgungsgesetz, $\$ 31$ Rn. 20; Becker, SGb 2007, S. 721, 726; Brockhaus, in: Schütz/Maiwald (Hrsg.), Beamtenrecht, $\$ 31$ BeamtVG Rn. 23; Kunze, VSSR 2005, S. 299, 313. Näher zum Merkmal „plötzlich“ Pflaum, RiA 2011, S. 198, 199.

979 BVerwG, Urt. v. 9.11.1960, E 11, 229; Urt. v. 24.10.1962 - VI C 18.61 -, ZBR 63, S. 49; Beschl. v. 19.1.2006 - 2 B 46/05 -, juris. Zu den genauen Anforderungen vgl. Bauer, in: Geyer/Grunefeld et al. (Hrsg.), Beamtenversorgungsrecht des Bundes und der Länder, $\$ 31$ BeamtVG Erl. 3, 1.2; Summer/Baumgartner, Der Dienstunfall, Rn. 11 Fn. 53. So wird versucht, den Schutzbereich der Dienstunfallfürsorge festzulegen und das vom Dienstherrn zu tragende Risiko zu begrenzen, vgl. VG Münster, Urt. v. 20.5.2010 - 5 K 1066/09 -, juris. Sinn dieser aus der Sicht des Unfallversicherungsrechts zusätzlichen Anforderungen ist es, den Dienstherrn nur für solche Schadensereignisse haften zu lassen, die einem Nachweis zugänglich sind. Denn erst die eindeutige Bestimmung des Unfallereignisses macht es möglich, sicher festzustellen, ob bestehende pathologische Veränderungen des Gesundheitszustandes des Beamten auf einen Dienstunfall zurückzuführen sind, vgl. Biletzki, NVwZ 2010, S. 688, 689.

980 Dazu gehören schädliche Einwirkungen sowohl auf physischer als auch auf psychischer Ebene, vgl. näher Schwerdtfeger, in Lauterbach/Watermann/Breuer (Hrsg.), Unfallversicherung, $\$$ 8, Rn. 61 ff.; Schmitt, VSSR 2004, S. 349, 349 ff.; Kazmaier, in: Stegmüller/Schmalhofer et al. (Hrsg.), Beamtenversorgungsrecht, 
Unfallversicherung reicht dabei die Beschädigung orthopädischer oder anderer Hilfsmittel, die keine Körperersatzstücke sind, nicht aus. ${ }^{981}$

Sodann wird anhand des Tatbestandsmerkmals ,in Ausübung des Dienstes" ein Zusammenhang zwischen der Dienstausübung und dem Unfallereignis gefordert, womit funktional dem unfallversicherungsrechtlichen Kriterium der „Erfüllung des Versicherungstatbestands“ entsprochen wird. Auch im Rahmen des $₫ 31$ Abs. 1 BeamtVG muss dieser Zusammenhang finaler Art sein. ${ }^{982}$ Anders als im Unfallversicherungsrecht genügt es für die Bejahung dieses finalen Zusammenhangs allerdings, wenn der Unfall räumlich-zeitlich der Machtsphäre des Dienstherrn zuzuordnen ist. ${ }^{983} \mathrm{Da}$ durch sind auch Unfälle, die Beamte zum Zeitpunkt der Erledigung privater Verrichtungen am Dienstort zur Dienstzeit erleiden, Dienstunfälle im Sinne des Beamtenversorgungsrechts. ${ }^{984}$

Aus $₫ 30$ Abs. 1 S. 1 BeamtVG folgt schließlich die Notwendigkeit der Prüfung eines Ursachenzusammenhangs zwischen der geschützten Tätigkeit - dem Dienst - und dem Unfallereignis, denn er normiert, dass Unfallfürsorge gewährt wird, wenn die Verletzung „durch“ einen Dienstunfall eingetreten ist. $\mathrm{Zu}$ beachten ist, dass hierbei im Gegensatz zum Unfallversicherungsrecht nicht an die tatsächliche Verrichtung zum Unfallzeitpunkt, sondern an die Dienstausübung des Beamten an sich angeknüpft

\$31 Rn.36; Brinktrine, in: Kugele (Hrsg.), Kommentar zum Beamtenversorgungsgesetz, $\mathbb{} 31 \mathrm{Rn} .22$; Brockhaus, in: Schütz/Maiwald (Hrsg.), Beamtenrecht, $\$ 31$ BeamtVG Rn. 29; Minz/Leppek, Praxis-Handbuch Beamtenversorgungsrecht, Rn. 337.

981 Wilhelm, in: Franke/Weiß (Hrsg.), GKÖD, $\mathbb{3 1}$ BeamtVG Rn. 15; Schnellenbach, Beamtenrecht in der Praxis, S. 414; Groepper/Tegethoff, in: Plog/Wiedow et al. (Hrsg.), Bundesbeamtengesetz, $\mathbb{3} 31$ Rn. 46; Schütz, Beamtenversorgungsgesetz, $\$ 31$ Rn. 26; Brinktrine, in: Kugele (Hrsg.), Kommentar zum Beamtenversorgungsgesetz, $\mathbb{3 1}$ Rn. 22; Kazmaier, in: Stegmüller/Schmalhofer et al. (Hrsg.), Beamtenversorgungsrecht, $\mathbb{3} 31 \mathrm{Rn} .37$.

982 So die überwiegende Meinung, z.B. Schnellenbach, Beamtenrecht in der Praxis, S. 334; a.A. Brinktrine, in: Kugele (Hrsg.), Kommentar zum Beamtenversorgungsgesetz, $\mathbb{\$} 31$ Rn. 5, der einen Kausalzusammenhang für notwendig erachtet.

983 BVerwG, Beschl. v. 26.2.2008 - 2 B 135/07 -, juris; Urt. v. 24.10.1963, E 17, 59; v. 17.3.1965, E 20, 347; v. 28.1.1971, E 37, 139; v. 12.2.1971, E 37, 203; v. 12.7.1972, E 40, 220.

984 BVerwG, Urt. v. 25.2.2010 - 2 C 81.08 -, juris Rn. 18; v. 29.8.2013 - 2 C 1.12 -, juris Rn. 11; Giesen, Arbeitsunfall und Dienstunfall, S. 43; Groepper/Tegethoff, in: Plog/Wiedow et al. (Hrsg.), Bundesbeamtengesetz, $\mathbb{} 31$ Rn. 52. 
wird. ${ }^{985}$ Auch im Recht der Beamtenversorgung findet dabei als Kausalitätsprinzip die Theorie der wesentlichen Bedingung Anwendung. ${ }^{986}$ Ereignete sich der Unfall allerdings zur Dienstzeit am Dienstort des Beamten,

985 Giesen, Arbeitsunfall und Dienstunfall, S. 30. Dies hängt mit der geringen dogmatischen Durchdringung des Unfallfürsorgerechts zusammen. Zumeist wird nach Bejahung des Unfallereignisses ohne Differenzierung in Kausalitäts- und Finalitätsfragen versucht, einen direkten Zusammenhang zwischen der geschützten Tätigkeit - dem Dienst - und dem Unfallereignis herzustellen, ohne den im Unfallversicherungsrecht maßgeblichen „Umweg“ über die tatsächliche Verrichtung zu nehmen. Die tatsächliche Verrichtung spielt aber dennoch auch im Rahmen des Dienstunfalls eine wichtige Rolle, was sich daran zeigt, dass sie ohne feste Struktur zumeist auf kasuistischer Basis in die Prüfung der Wortfolge „,in Ausübung oder infolge des Dienstes“ hineingebracht wird, vgl. die Formulierungen nach dem Regel-Ausnahme-Verhältnis bei Wilhelm, in: Franke/Weiß (Hrsg.), GKÖD, $\$ 31$ BeamtVG Rn. 27 u. 31, oder die eine Definition der ausgeführten Tätigkeit vermeidende negative Abgrenzung bei Pflaum, RiA 2011, S. 198, 200. Diese Praxis führt zu einer schweren Durchdringbarkeit der Voraussetzungen für die Unfallfürsorge, die dadurch für den Rechtsadressaten verwirrend und in der Rechtsanwendung unsicher ist. Im Interesse einer systematischen Betrachtungsweise ist es indes hilfreich, auch in der Unfallfürsorge der Beamten trennscharf zwischen der Schutzbereichseröffnung und der Bestimmung der Kausalität von Unfall und ausgeführter Tätigkeit sowie zwischen Unfall und Primärschädigung zu unterscheiden. Wegen ihrer historischen und sachlichen Verwandtschaft zur gesetzlichen Unfallversicherung ist es sinnvoll, diese Systematisierung am Konstruktionsgerüst des Zuordnungsmechanismus der gesetzlichen Unfallversicherung auszurichten. Dies ermöglicht nicht nur den besseren Vergleich mit dem System der gesetzlichen Unfallversicherung, sondern führt vor allem zu einer abstrakteren, systematischeren Betrachtungsweise, was die Rechtssicherheit erhöht, vgl. auch Kunze, VSSR 2005, S. 299, 300; Köhler, SGb 2006, S. 9, 10 f.

986 Das Bundesverwaltungsgericht übernahm diese Theorie aus der Rechtsprechung der Sozialgerichte zur Unfallversicherung angesichts der Vergleichbarkeit des Begrenzungsinteresses der Risikosphären erstmalig, BVerwG, Urt. v. 20.5.1958, E 7, 48, 49 f., unter Bezug auf ihre Anwendung bereits durch das OVG Lüneburg, Urt. v. 15.1.1957 - II A 77/55 -, juris, und das OVG Münster, Besch. v. 22.3.1957, ZBR 1957, S. 292; Besch. v. 21.6.1957, ZBR 1958, S. 10. Mit ausführlicherer Begründung zur Übernahme der Theorie etwas später BVerwG, Urt. v. 20.4.1967, E 26, 332, 337; zur Anwendung der zur Unfallkausalität entwickelten Kasuistik im Beamtenrecht Wilhelm, in: Franke/Weiß (Hrsg.), GKÖD, $\$ 31$ BeamtVG Rn. 22, m.w.N. Damit wurde der bis zu diesem Zeitpunkt präferierten Adäquanztheorie eine Absage erteilt. Diese vertretend noch OVG Münster, Urt. v. 21.6.1957 - VIA 884/54 -, ZBR 58, 10. Vgl. zum Ringen um die passende Kausaltheorie für die Unfallfürsorge der Beamten Schlegel, DVBl. 1962, S. 8, 9; Schütz, DÖD 14 (1961), S. 101, 102; Reiff, NJW 1961, S. 630, 630 m.w.N.; Stich, ZBR 1958, S. 298, 301; Riedmaier, RiA 1979, S. 41, 43; Haueisen, JZ 1961, S. 9, $9 \mathrm{ff}$. 
wird das Vorliegen eines diesen Anforderungen genügenden Zusammenhangs ebenfalls unabhängig von der Natur der zum Unfallzeitpunkt ausgeführten Tätigkeit bejaht. ${ }^{987}$

Zuletzt muss, ebenso wie im Unfallversicherungsrecht, das Unfallereignis ursächlich im Sinne der Theorie der wesentlichen Bedingung für den Körperschaden gewesen sein. ${ }^{988}$

Wie unterschiedlich dadurch die Abgrenzung zwischen von der Unfallsicherung umfassten Berufsrisiken und privaten Risiken ausfällt, illustriert der folgende Vergleich der neueren Rechtsprechung der Sozial- und Verwaltungsgerichte. ${ }^{989}$

II. Illustration der unterschiedlichen Abgrenzung von Privat- und Berufssphäre anhand der aktuellen Rechtsprechung

1. Anhand der Schullandheim-Entscheidungen

a. Relevanz und Kontext der Entscheidungen

Im Jahr 2007 ergingen innerhalb eines Zeitraums von zwei Wochen unabhängig voneinander Entscheidungen sowohl in der Verwaltungs- als auch in der Sozialgerichtsbarkeit zu sehr ähnlich gelagerten Fällen. ${ }^{990}$ Sie hatten jeweils einen Unfall einer Lehrkraft zum Inhalt, der sich beim Duschen während eines Schullandheimaufenthalts ereignete. Die verbeamtete Lehrkraft duschte frühmorgens, die angestellte Lehrkraft spätabends; beide

987 Vgl. BVerwG, Urt. v. 29.8.2013 - 2 C 1/12 - juris Rn. 10; Urt. v. 15.11.2007 - 2 C 24/06 -, juris Rn. 11; Beschl. v. 26.2.2008 - 2 B 135/07 -, juris Rn. 6; Reich,

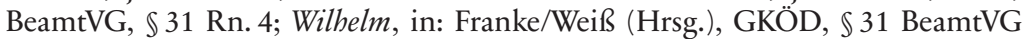
Rn. 48.

988 VGH Baden-Württemberg, Urt. v. 12.6.2012 - 4 S 1384/10 -, juris.

989 Dass Arbeits- und Dienstunfälle unterschiedlichen Gerichtsbarkeiten unterliegen, erklärt sich aus $₫ 40$ Abs. 1 S. 1 VwGO. Demnach ist für alle öffentlichrechtlichen Streitigkeiten der Verwaltungsgerichtsweg eröffnet, es sei denn, es liegt eine Sonderzuweisung zu einer anderen Gerichtsbarkeit vor. Eine solche Sonderzuweisung findet sich in $\$ 51$ Abs. 1 Nr. 3 SGG für den Arbeitsunfall der gesetzlichen Unfallversicherung; diese Materie ist den Sozialgerichten zugewiesen. Arbeitsunfälle wurden schon 1884 einer speziell für sie eingeführten Gerichtsbarkeit zugewiesen, vgl. Jung, in: Deutscher Richterbund (Hrsg.), Justiz und Recht im Wandel der Zeit, S. 253, 254.

990 VGH Baden-Württemberg, Urt. v. 28.9.2007 - 4 S 516/06 -, juris sowie LSG Sachsen-Anhalt, Urt. v. 13.9.2007 - L 6 U 13/04 -, juris. 
rutschten aus und zogen sich dabei Verletzungen zu. Der Verwaltungsgerichtshof Baden-Württemberg bejahte für die verbeamtete Lehrkraft das Vorliegen eines Dienstunfalls, während das Landessozialgericht SachsenAnhalt den Unfall der angestellten Lehrerin ihrer Privatsphäre zuordnete und einen Arbeitsunfall ablehnte. Beide Entscheidungen wurden angegriffen und führten im Wege der Beschwerde beziehungsweise der Revision zu letztinstanzlichen Urteilen, die die jeweiligen Entscheidungen ihrer Vorinstanzen aufrechterhielten. ${ }^{91}$ Die chronologisch zuletzt ergangene Entscheidung ist die des Bundessozialgerichts, das auf die Entscheidung der Verwaltungsgerichtsbarkeit ausdrücklich Bezug nimmt. Eine Analyse dieser Entscheidungen gibt daher Aufschluss über die unterschiedliche Interpretation der Reichweite der beruflichen Risikosphäre bei Beamten und bei Beschäftigten.

\section{b. Entscheidungsgründe im Vergleich}

Der Verwaltungsgerichtshof Baden-Württemberg sowie das Bundesverwaltungsgericht postulierten, dass eine verbeamtete Lehrkraft während eines Schullandheimaufenthalts 24 Stunden am Tag im Dienst sei, da sie stets die Aufsichtspflicht über die ihr anvertrauten Schülerinnen und Schüler habe und sich jederzeit zum Eingreifen bereit halten müsse. ${ }^{992}$ Das Schullandheim sei für die Dauer des Aufenthalts Dienstort der Lehrkraft, sodass diese sich im Banne des Dienstes befinde. ${ }^{993}$ Das Duschen habe in engem natürlichem Zusammenhang mit den der Lehrkraft übertragenen Dienstaufgaben gestanden, da ihre Dienstpflicht währenddessen fortbestand und sie deswegen gezwungen war, die sanitären Einrichtungen des Schullandheims zu nutzen. ${ }^{994}$ Der Dienstherr müsse das spezifische örtliche Risiko in Gebäuden tragen, in denen der Beamte zur Diensterfüllung übernachten müsse, da nach dem Normzweck des $\$ 31$ Abs. 1 S. 1 BeamtVG der

991 BVerwG, Beschl. v. 26.2.2008 - 2 B 135/07 -, juris respektive BSG, Urt. v. 18.11.2008 - B 2 U 31/07 R -, juris.

992 VGH Baden-Württemberg, Urt. v. 28.9.2007 - 4 S 516/06 -, juris Rn. 20; BVerwG, Beschl. v. 26.2.2008 - 2 B 135/07 -, juris Rn. 9.

993 VGH Baden-Württemberg, Urt. v. 28.9.2007 - 4 S 516/06 -, juris Rn.21.; BVerwG, Beschl. v. 26.2.2008 - 2 B 135/07 -, juris Rn. 7.

994 VGH Baden-Württemberg, Urt. v. 28.9.2007 - 4 S 516/06 -, juris Rn.20; BVerwG, Beschl. v. 26.2.2008 - 2 B 135/07 -, juris Rn. 9. 
Dienstort der räumlichen Risikosphäre des Dienstherrn zuzurechnen sei. ${ }^{995}$

Das Landessozialgericht Sachsen-Anhalt sowie das Bundessozialgericht dagegen entschieden, dass eine angestellte Lehrkraft während eines Schullandaufenthalts nicht ständig im Dienst sei, obwohl auch sie anerkennen, dass ihr die Aufsichtspflicht über die Schüler ohne Unterbrechung zugefallen war. ${ }^{996}$ Der Dienst würde aber durch höchstpersönliche Verrichtungen unterbrochen; eine solche höchstpersönliche Tätigkeit sei das Duschen. ${ }^{997}$ Dieses stünde nicht in sachlichem Zusammenhang mit der versicherten Tätigkeit, da es sich um eine Tätigkeit zur Erfüllung eines natürlichen Bedürfnisses eines jeden Menschen handele. ${ }^{998}$

In den Entscheidungstexten beider Gerichtsbarkeiten finden sich Überlegungen dazu, ob das Duschen eine private oder eine berufliche Verrichtung ist. Während das Bundessozialgericht der Ansicht ist, Duschen sei nur in zwei Ausnahmefällen nicht als privat zu qualifizieren (und zwar im Falle starker Verschmutzung durch die Arbeit oder im Falle starker Überhitzung, die dazu führen würde, dass ohne kühlende Dusche die Arbeitsschicht nicht zu Ende geführt hätte werden können), erklärt der Verwaltungsgerichtshof Baden-Württemberg, das Duschen stünde in einem engen natürlichen Zusammenhang mit den Dienstaufgaben, da ohne Körperpflege eine Dienstleistung auf längere Dauer nicht möglich sei. ${ }^{999}$

Auch zur Frage der Herrschaftssphäre des Dienstherrn beziehungsweise des Arbeitgebers finden sich auf beiden Seiten Aussagen. Das Bundesverwaltungsgericht ordnet das Schullandheim der Herrschaftssphäre des Dienstherrn zu, da sich die Lehrkraft auf Anweisung des Dienstherrn dort befindet und dort seine Dienstleistung zu erbringen hat. ${ }^{1000}$ Bauliche Besonderheiten und örtliche Risiken wie nasse Fliesen gehörten deswegen zur Risikosphäre des Dienstherrn. ${ }^{1001}$ Das Landessozialgericht Sachsen-An-

995 BVerwG, Beschl. v. 26.2.2008 - 2 B 135/07 -, juris Rn. 10; BSG, Urt. v. 18.11.2008 - B 2 U 31/07 R -, juris Rn. 15.

996 LSG Sachsen-Anhalt, Urt. v. 13.9.2007 - L 6 U 13/04 -, juris Rn. 25.

997 Ebenso nun im Fall einer ehrenamtlichen Begleitperson, die in der Dusche oder dem von ihr bewohnten Zimmer (der Unfallort war strittig) ausrutschte, da der Aufenthalt dort privat sei, LSG Nordrhein-Westfalen, Urt. v. 25.3.2014 L 15 U 243/12 -, juris.

998 LSG Sachsen-Anhalt, Urt. v. 13.9.2007 - L 6 U 13/04 -, juris Rn. 24.

999 VGH Baden-Württemberg, Urt. v. 28.9.2007 - 4 S 516/06 -, juris Rn. 21; das BVerwG bestätigt diese Aussage nicht explizit, widerspricht ihr aber auch nicht.

1000 BVerwG, Beschl. v. 26.2.2008 - 2 B 135/07 -, juris Rn. 9 f.

1001 Ebd. Rn. 10. 
halt dagegen deutet an, dass die Duschräume eines Schullandheims nicht mehr zur Einflusssphäre des Unternehmers gehören. ${ }^{1002}$ Die Art, wie die Dusche gebaut sei, und dass die Fliesen nass gewesen seien, gehören deswegen nicht zur Risikosphäre des Unternehmers, da sie in ähnlicher Art auch in anderen öffentlichen Duschräumen, etwa in Schwimmbädern, auffindbar seien. ${ }^{1003}$

Das Bundessozialgericht und der Verwaltungsgerichtshof Baden-Württemberg stimmen überein, dass private Tätigkeiten in fremder Berufsumgebung eher der beruflichen Sphäre zuzuordnen seien als dem häuslichen Bereich, da der Aufenthalt in einer fremden Umgebung beruflich beeinflusst ist. ${ }^{1004}$

Schließlich nimmt das Bundessozialgericht in seinen Entscheidungsgründen ausdrücklich auf das Urteil des Verwaltungsgerichtshofs BadenWürttemberg Bezug. An der Stelle, an der es vorbringt, auch während des Bestehens einer Aufsichtspflicht rund um die Uhr seien nur Tätigkeiten, die im sachlichen Zusammenhang mit der versicherten Tätigkeit stünden, versichert, schreibt es: „Die Auffassung des Verwaltungsgerichtshofs (VGH) Baden-Württemberg, ein beamteter Lehrer sei während der gesamten Dauer eines Schullandheimaufenthalts im Dienst, mithin 24 Stunden am Tag, es sei denn, er habe sich von diesem gelöst (VGH Baden-Württemberg vom 28. September 2007 - 4 S 516/06 - RdNr 20), ist auf die gesetzliche Unfallversicherung nach dem SGB VII nicht übertragbar. Zwischen der beamtenrechtlichen Unfallfürsorge und dem Unfallversicherungsschutz von Beschäftigten bestehen trotz gewisser Gemeinsamkeiten in der Ausgestaltung erhebliche strukturelle Unterschiede aufgrund der verschiedenen Inhalte von Beamtenverhältnis einerseits und Versicherungsverhältnis von Beschäftigten andererseits.“1005

\section{c. Folgerungen}

Aus den Schullandheim-Entscheidungen lassen sich bezüglich der unterschiedlichen Grenzziehung zwischen privater und beruflicher Risikosphäre im Rahmen der Unfallfürsorge der Beamten einerseits und der Unfall-

1002 LSG Sachsen-Anhalt, Urt. v. 13.9.2007 - L 6 U 13/04 -, juris Rn. 21.

1003 BSG, Urt. v. 18.11.2008 - B 2 U 31/07 R -, juris Rn. 25.

1004 Ebd. Rn. 15; VGH Baden-Württemberg, Urt. v. 28.9.2007 - 4 S 516/06 -, juris Rn. 21; das Bundesverwaltungsgericht widerspricht dem nicht.

1005 BSG, Urt. v. 18.11.2008 - B 2 U 31/07 R -, juris Rn. 13. 
versicherung der Beschäftigten andererseits zwei Schlussfolgerungen ziehen.

Zum einen gewichten die Gerichtsbarkeiten den Aspekt der Privatheit der Erfüllung eines Grundbedürfnisses unterschiedlich. Beide Gerichtsbarkeiten diskutieren die Frage der Natur der tatsächlichen Verrichtung des Duschens, das unter gleichen Bedingungen stattfand. Sie kommen zu unterschiedlichen Ergebnissen, weil das Bundessozialgericht den Zusammenhang mit der Berufsausübung durch das Duschen trotz fortdauernder Aufsichtspflicht unterbrochen sieht, da Körperhygiene seiner Meinung nach grundsätzlich privater Natur ist, während das Bundesverwaltungsgericht das Fortwirken der Dienstpflichten als Grund für eine ununterbrochene Dienstausübung anerkennt und die Tatsache, dass Körperhygiene ein privates Bedürfnis ist, dahinter zurücktreten lässt.

Zum anderen beurteilen die Gerichte die Frage anders, wann eine private Tätigkeit in den Schutzbereich des jeweiligen Unfallsicherungssystems fällt. Das Bundesverwaltungsgericht lässt eine Einordnung unter den Einflussbereich des Dienstherrn genügen und meint damit, dass es ausreichend ist, wenn der Dienstherr auch den Ort der Tätigkeit festgelegt hat. Das Bundessozialgericht bringt lediglich in seltenen Ausnahmefällen seine Fallgruppe der „betriebsbedingten Gefahr“" zur Anwendung, nach der private Tätigkeiten umfasst sind, wenn sich im Unfall ein spezifisches Risiko verwirklicht, das gerade nur aus dem Betrieb und nicht aus anderen Risikoquellen stammen kann.

Beide Gerichtsbarkeiten leiten ihre jeweilige Grenzziehung aus dem Schutzzweck ihres Unfallsicherungssystems ab. So schreibt das BSG, es könnten nicht alle Verrichtungen eines grundsätzlich versicherten Arbeitnehmers im Laufe eines Arbeitstags auf der Arbeitsstätte versichert sein, „weil nach dem Wortlaut des $₫ 8$ Abs. 1 S. 1 SGB VII nur Unfälle, infolge der versicherten Tätigkeit Arbeitsunfälle sind und es einen sogenannten Betriebsbann nur in der Schifffahrt ( $\mathbb{1 0}$ SGB VII), nicht aber in der übrigen gesetzlichen Unfallversicherung gibt“.1006 Die Grenze zwischen privater und beruflicher Sphäre sei wertend zu ermitteln. ${ }^{1007}$ Innerhalb dieser Wertung wird der Tätigkeit des Duschens hohe private Konnotation zugeteilt. Nach dem Bundesverwaltungsgericht hat die Grenzziehung zwischen privater und beruflicher Risikosphäre ebenfalls nach dem Normzweck zu erfolgen. In seiner Wertung kommt der Frage erhebliche Bedeutung zu, ob die Geschehnisse durch den Dienstherrn beherrschbar sind. Die höhere

1006 Edb. Rn. 11.

1007 Ebd. 
Gewichtung der Auswahl des Dienstortes durch den Dienstherrn im Beamtenrecht hat ihre Ursache nach Meinung des Bundessozialgerichts in den Unterschieden zwischen Beamten- und Arbeitnehmerstatus. ${ }^{1008}$

\section{Anhand der Garagenfälle}

Ein weiterer Aspekt der Divergenz der Reichweite der beruflichen Risikosphäre lässt sich an einer Fallgruppe zeigen, die zu den Wegeunfällen gehört. Findet der relevante Unfall in der privaten Garage des Geschädigten statt, so kommt es zu einer unterschiedlichen Behandlung von Beschäftigten und Beamten. Da das Bundessozialgericht generell die Außentüre des Wohnhauses als Grenze zwischen dem beruflichen und dem privaten Risikobereich ansieht, gehört der Bereich der privaten Garage grundsätzlich zum unfallversicherungsrechtlich geschützten Bereich. ${ }^{1009}$ Das Bundesverwaltungsgericht dagegen nimmt private Garagen aus dem Schutzbereich der Unfallfürsorge der Beamten aus, und zwar mit dem Argument, dass diese nicht vom Dienstherrn beeinflussbar seien, sich damit nicht in seiner Herrschaftssphäre befänden und Unfälle, die sich innerhalb der Garage ereignen, somit vom Schutzzweck der Unfallfürsorge nicht umfasst seien. ${ }^{1010}$ Aus der Grenzziehung des Bundessozialgerichts in diesen Urteilen lässt sich nichts in Bezug auf seine Grenzziehung in den hier allein interessierenden „normalen“ Arbeitsunfällen ableiten. Interessant ist an diesen Fällen aber die Tatsache, dass innerhalb der Verwaltungsgerichtsbarkeit ein für die Beamten ungünstigeres Ergebnis hingenommen wird, obwohl dies als Abweichung von der Rechtsprechung der Sozialgerichtsbarkeit erkannt und ausdrücklich angesprochen wird. ${ }^{1011}$ Begründet wird dies damit, dass

1008 Ebd. Rn. 13. Diese These wird im Folgenden verifiziert, s.u. Kap. 4 B. III. 3.

1009 BSG, Urt. v. 27.10.1976, E 42, 293 und v. 7.11.2000 - B 2 U 39/99 R -, juris m.w.N.

1010 BVerwG, Urt. v. 27.1.2005, E 122, 360, 363 Rn. 14. Vgl. auch BVerwG, Beschl. v. 26.1.2016 - 2 B 17/15 -, juris Rn. 14; Urt. v. 12.7.1972, E 40, 220, 225 Rn. 1.

1011 „Im Gegensatz zur Rechtsprechung des Bundessozialgerichts, das die Grenze der privaten und der öffentlichen Risikosphäre stets in der Außentür des Wohnhauses sieht, so dass auch der Innenraum einer Garage, unabhängig von deren Lage zum Wohnhaus des Arbeitnehmers, zum unfallversicherten Bereich zählt (vgl. Urteile vom 27. Oktober 1976 - 2 RU 247/74 - BSGE 42, 293 und vom 7. November 2000 - B 2 U 39/99 R - a.a.O.), sieht der erkennende Senat auch unter Berücksichtigung der Vorteile einer einheitlichen Rechtsprechung zum Unfallschutz der Beamten und Arbeitnehmer keine Notwendigkeit, das der gesetzlichen Regelung in $\$ 31$ Abs. 2 Satz 1 Halbsatz 1 BeamtVG 
der Beamte die Unfallgefahren in seiner privaten Garage selbst beherrsche. ${ }^{1012}$ Dies verdeutlicht die aus den Schullandheim-Entscheidungen gewonnene Erkenntnis, dass innerhalb der Verwaltungsgerichtsbarkeit dem Kriterium der „Herrschaftssphäre des Dienstherrn“ sehr große Bedeutung als Abgrenzungskriterium beigemessen wird. Das Bundesverwaltungsgericht erklärt dies damit, dass dieses Kriterium die Abgrenzung zwischen privater und beruflicher Risikosphäre vereinfache und zu sachgerechten Ergebnissen führe: „Die Beherrschbarkeit des Risikos ist ein unschwer zu konkretisierender unbestimmter Rechtsbegriff, der bei fallspezifischem Abgrenzungsbedarf durch eindeutige und sinngerechte Grenzziehungen, wie etwa durch das Abstellen auf Außentüren, ergänzt werden kann und geeignet ist, trotz sehr unterschiedlicher Wohnverhältnisse die unvermeidbare Kasuistik in Grenzen zu halten.“1013

\section{Zwischenergebnis}

Die vorstehende Untersuchung zeigt, dass die Gerichtsbarkeiten in den hier analysierten Entscheidungen den gleichen Zweck verfolgten, nämlich eine sachgerechte Grenzziehung zwischen privater und beruflicher Risikosphäre zu vollziehen. Dabei spielen in beiden Gerichtsbarkeiten dieselben Gesichtspunkte eine Rolle; in die Entscheidung einbezogen wird werden jeweils die Natur der Tätigkeit und die Frage danach, wie viel Einfluss der Dienstherr oder Arbeitgeber auf das im Unfall verwirklichte Risiko hatte. Dabei stellte sich heraus, dass die Verwaltungsgerichtsbarkeit größeren Wert auf die Frage nach dem Herrschaftsbereich des Dienstherrn legt, während es der Sozialgerichtsbarkeit in weiterem Umfang auf die Frage nach der Natur der tatsächlichen Verrichtung des Beschäftigten ankommt. Innerhalb der Zuordnung der spezifischen Tätigkeit zur beruflichen oder privaten Sphäre wiederum legt die Sozialgerichtsbarkeit einen strengeren Maßstab an. Das Bundessozialgericht stützt seine Rechtsprechung eher auf Kriterien, die nicht objektivierbar sind ${ }^{1014}$ - etwa die Betriebsdienlichkeit

nach Sinn und Zweck der Vorschrift zugrunde liegende Kriterium der Beherrschbarkeit des Risikos zu Lasten der öffentlichen Kassen auch dort zugunsten einer starren Grenzziehung aufzugeben, wo es für eine einfache Grenzziehung nicht erforderlich ist.“ BVerwG, Urt. v. 27.1.2005, E 122, 360, 363 Rn. 14).

1012 Ebd. Rn. 13.

1013 Ebd.

1014 Dies beobachtet auch Tomandl, in: v. Wulffen/Krasney (Hrsg.), FS 50 Jahre BSG, S. 557, 570 . 
und die Wesentlichkeit -, während das Bundesverwaltungsgericht faktische Grenzen wie die Zuordnung nach Ort und Zeit bevorzugt.

Das Bundesverwaltungsgericht erklärt seinen starken Fokus auf die Frage nach der Herrschaftssphäre des Dienstherrn mit pragmatischen Gründen: Kasuistik sei so weit als möglich zu vermeiden, weswegen auf objektive Kriterien abzustellen sei. ${ }^{1015}$ Das Bundessozialgericht benennt dagegen „strukturelle Unterschiede aufgrund der verschiedenen Inhalte von Beamtenverhältnis einerseits und Versicherungsverhältnis von Beschäftigten andererseits" als Grund dafür, dass es der Auslegung des Bundesverwaltungsgerichts nicht folgt. ${ }^{1016}$ Dass die inhaltlichen Unterschiede zwischen Beamten- und Beschäftigtenstatus die unterschiedliche Grenzziehung zwischen privater und beruflicher Risikosphäre im Rahmen der Unfallsicherungssysteme begründen, zeigt die folgende Untersuchung der Entwicklung der räumlich-zeitlichen Zuordnungskriterien der Unfallfürsorge.

III. Die Entwicklung des Dienstbanns aus den Grundprinzipien der Unfallfürsorge

1. Von tätigkeitsbezogenen zu räumlich-zeitlichen Zuordnungskriterien

a. Die Abgrenzung nach der tatsächlichen Verrichtung

Dass private Tätigkeiten im angegebenen Maße vom Unfallsicherungsrecht der Beamten umfasst sind, beruht auf dem Gedanken, dass sich Beamte „im Banne des Dienstes“ befinden, wenn sie sich zur Dienstzeit am Dienstort aufhalten. ${ }^{1017}$ Sie sind dann unabhängig von der Natur der konkreten verrichteten Tätigkeit zum Unfallzeitpunkt der beruflichen Sphäre zuzurechnen. ${ }^{1018}$ Anders als in der Unfallversicherung stützt sich die Zuord-

1015 BVerwG, Urt. v. 27.1.2005, E 122, 360, 363 Rn. 13; diese Tendenz wird auch von der Literatur beobachtet, vgl. Kugele, jurisPR-BVerwG 15/2008, Anm. 4.

1016 BSG, Urt. v. 18.11.2008 - B 2 U 31/07 R -, juris Rn. 13.

1017 VGH Baden-Württemberg, Urt. v. 28.9.2007 - 4 S 516/06 -, juris Rn.21.; BVerwG, Beschl. v. 26.2.2008 - 2 B 135/07 -, juris Rn. 7.

1018 Vgl. nur BVerwG, Urt. v. 29.8.2013 - 2 C 1/12 -, juris Rn. 11; Beschl. v. 26.2.2008 - 2 B 135/07 -, juris Rn. 6; Urt. v. 24.10.1963, E 17, 59 Rn. 23; v. 28.1.1971, E 37, 139, 139 ff. Rn. 20; v. 12.2.1971, E 37, 203, 203 ff. Rn. 14; Wilhelm, in: Franke/Weiß (Hrsg.), GKÖD, $\$ 31$ BeamtVG Rn. 33; Groepper/Tege-

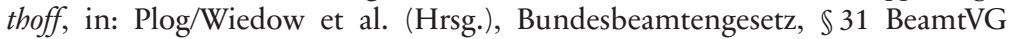
Rn. 52; Kazmaier, in: Stegmüller/Schmalhofer et al. (Hrsg.), Beamtenversorgungsrecht, $\mathbb{3} 31 \mathrm{Rn} .65$. 
nung eines Unfalls zur Dienstsphäre damit auf physische Abgrenzungskriterien, nämlich auf Zeit und Ort des Unfallereignisses. Dies war jedoch nicht immer der Fall. Auch in der verwaltungsrechtlichen Rechtsprechung kam es lange Zeit auf einen finalen Zusammenhang zwischen der ausgeübten Tätigkeit und der Dienstausübung an. ${ }^{1019}$ Die Grundsätze der $\mathrm{Zu}$ ordnung entsprachen dabei weitgehend denen, die sich noch heute in der Dogmatik des Unfallversicherungsrechts zu $₫ 8$ Abs. 1 SGB VII finden. Der Unfall musste sich nicht nur während der Dienstzeit am Dienstort, sondern auch während einer konkret dienstlichen Tätigkeit ereignet haben, um als Dienstunfall zu gelten. ${ }^{1020}$ Dabei ist vom Dienstbegriff des $₫ 61$ Abs. 1 S. 2 BBG auszugehen. ${ }^{1021}$ Dieser setzt voraus, dass der Geschädigte im Zeitpunkt der äußeren Einwirkung in einem gültigen Beamtenverhältnis steht, da er ansonsten keinen Dienst leisten kann. ${ }^{1022}$ Zum Dienst gehört sodann jede Tätigkeit, die dem Aufgabenkreis des Beamten zuzuordnen ist, welcher diesem durch Gesetz, Verordnung oder dienstliche Weisung übertragen worden ist. ${ }^{1023}$ Weiterhin zählen zum Dienst gemäß $\$ 31$ Abs. 1 S. 2 BeamtVG auch Dienstreisen und dienstliche Tätigkeiten am Bestimmungsort (Nr.1), die Teilnahme an dienstlichen Veranstaltungen (Nr. 2) sowie Nebentätigkeiten im öffentlichen Dienst oder in dem ihm gleichstehenden Dienst, zu deren Übernahme der Beamte gemäß $\$ 98$ BBG verpflichtet ist. Auch Nebentätigkeiten, deren Wahrnehmung von dem Beamten im Zusammenhang mit den Dienstgeschäften erwartet wird, sofern der Beamte hierbei nicht in der gesetzlichen Unfallversicherung versichert ist, gehören zum Dienst (Nr. 3). Durch $₫ 31$ Abs. 1 S. 2 BeamtVG wurde nicht der Begriff des Dienstes erweitert; die dort aufgeführten Tatbestandsmerkmale haben klarstellende Funktion. ${ }^{1024}$ Es soll deutlich gemacht werden, dass neben dem „eigentlichen“ Dienst auch dienstliche Veranstaltungen und Nebentätigkeiten zum Dienst gehören. Die Nr. 1 bis 3 enthalten Vermutungen einer dienstlichen Tätigkeit. ${ }^{1025}$ Als Dienst gilt auch das $\mathrm{Zu}$ rücklegen der mit dem Dienst zusammenhängenden Wege (Abs. 2).

1019 Giesen, Arbeitsunfall und Dienstunfall, S. 40.

1020 BVerwG, Urt. v. 28.1.1960, E 10, 128, 129; v. 12.2.1971, E 37, 203, 206 f; v. 17.10.1991, E 89, 117; Brinktrine, in: Kugele (Hrsg.), Kommentar zum Beamtenversorgungsgesetz, $\$ 31 \mathrm{Rn} .9$.

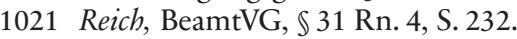

1022 BVerwG, Urt. v. 24.9.1959, E 9, 153; Groepper/Tegethoff, in: Plog/Wiedow et al. (Hrsg.), Bundesbeamtengesetz, $\$ 31$ Rn. 49.

1023 BVerwG, Urt. v. 28.1.1960, E 10, 128, 129; v. 12.2.1971, E 37, 203, 206 f.; Wilhelm, in: Franke/Weiß (Hrsg.), GKÖD, $\$ 31$ BeamtVG Rn. 27.

1024 Vgl. BVerwG, Urt. v. 19.4.1967 - 6 C 96.63 -, juris.

1025 Reich, BeamtVG, $\mathbb{3} 31$ Rn. 5. 
Als Ausnahme wurde ein Dienstunfall auch während einer eigenwirtschaftlichen Tätigkeit anerkannt, wenn diese den Zusammenhang mit dem Dienst nicht gelöst hatte, weil sie sehr kurzfristig oder geringfügig war. ${ }^{1026}$ Für die Bejahung des finalen Zusammenhangs kam es nicht darauf an, ob sie dem üblichen Aufgabengebiet des Beamten entsprach. Entscheidend war allein, ob der Beamte aufgrund seines Beamtenverhältnisses verpflichtet war, die Weisung zu befolgen, auf der die Verrichtung beruhte. ${ }^{1027}$ Ein Beamter befand sich demnach nur dann in Ausübung des Dienstes, wenn er sich „zur Zeit und am Ort des schädigenden Ereignisses auf Grund einer dienstlichen Weisung aufgehalten hat, deren Rechtsgrundlage das im Beamtenrecht geregelte Unterstellungsverhältnis war“. 1028

\section{b. Ort und Zeit des Unfalls als maßgebliche Kriterien}

Von dieser Sichtweise wandte sich das Bundesverwaltungsgericht erstmalig im Jahr $1963 \mathrm{ab}$, als es urteilte, der Beamte sei kein „Dienstleistungsautomat". 1029 Dass seine Gedanken gelegentlich abschweifen oder er einmal zur Kantine oder zur Toilette geht, sei natürlich und würde nicht bedeuten, dass er sich nicht mehr in Ausübung des Dienstes befinde. ${ }^{1030}$ Auch wenn er gerade keine ihm ausdrücklich angewiesene Tätigkeit ausführe, sei er, wenn er sich während der Dienstzeit am Dienstort aufhielte, ständig in

1026 Brinktrine, in: Kugele (Hrsg.), Kommentar zum Beamtenversorgungsgesetz, $\$ 31$ Rn. 9.

1027 BVerwG, Urt. v. 28.1.1960, E 10, 128, 129, wo sich diese Frage bei Polizeibeamten stellte, die während des Zweiten Weltkrieges zu Kampfhandlungen abgeordnet und dabei verletzt wurden. Das BVerwG schloss sich der engeren Auslegung des Bundesgerichtshofs ausdrücklich nicht an. Dieser hatte mit Urt. v. 20.9.1956 - III ZR 79.55 -, NJW 1957, S. 223 mit dem Zweck der gesetzlichen Dienstunfallfürsorge argumentiert und deswegen solche Unfälle ausgeschlossen, die im Rahmen einer zwar weisungsgemäß ausgeübten, nicht aber ihrem Wesen nach beamtenmäßigen Tätigkeit erfolgt sind und zudem anderweitig entschädigt werden. Dafür spreche, dass es einer Regelung zur besonderen Entschädigung nicht bedurft hätte, wenn auch Unfallfürsorge nach dem DBG gewährt würde. Das BVerwG folgt dem nicht mit dem Argument, dass der Gesetzgeber den Begriff „in Ausübung des Dienstes“ nicht so differenziert habe.

1028 BVerwG, Urt. v. 28.1.1960, E 10, 128, 129.

1029 BVerwG, Urt. v. 24.10.1963, E 17, 59, 59 ff. Rn. 23; Giesen, Arbeitsunfall und Dienstunfall, S. $41 \mathrm{f}$.

1030 BVerwG, Urt. v. 24.10.1963, E 17, 59, 59 ff. Rn. 23. 
Ausübung des Dienstes. ${ }^{1031}$ Eine Unterscheidung nach der Art der konkreten Tätigkeit entspreche nicht der Lebenswirklichkeit und sei daher nicht sachgerecht. ${ }^{1032}$ Im konkreten Fall hatte sich der Beamte auf dem Weg in die Kantine verletzt, wo er sich eine Flasche Milch kaufen wollte. Deutlich wird die Irrelevanz des subjektiven Kriteriums der Handlungstendenz auch in einem jüngeren Fall, in dem ein Beamter sich an der Türklinke des Dienstzimmers verletzte, als er hinauslief, um einer auf der Straße verunglückten Passantin zu helfen. ${ }^{1033}$ Dieses Handeln war weder auf Weisung geschehen, noch war es dazu bestimmt, dem Dienstherrn zu dienen. Das Bundesverwaltungsgericht bejahte dennoch einen Dienstunfall, da sich der Beamte während der Dienstzeit im Dienstgebäude verletzt hatte, und argumentierte, dass ihm dies auch jederzeit bei einer „echten dienstlichen Verrichtung" hätte zustoßen können. ${ }^{1034}$

Bei Aufenthalt am Dienstort zur Dienstzeit befindet sich der Beamte in einem vom Dienstherrn beherrschbaren räumlichen Risikobereich. Dienstort im dienstunfallrechtlichen Sinn ist der Ort, an dem der Beamte die ihm übertragenen Aufgaben zu erledigen hat. ${ }^{1035}$ Dies kann etwa das Dienstgebäude ${ }^{1036}$ oder ein räumlich abgrenzbarer Bereich außerhalb des Machtbereichs des Dienstherrn sein, soweit die Dienstleistung dort zu erbringen ist (vorübergehender Dienstort). ${ }^{1037}$ So ist zum Beispiel das Schullandheim Dienstort einer Lehrerin, die dort den Klassenausflug ihrer Schüler betreut. ${ }^{1038}$ Als Dienstzeit ist dagegen nicht nur die durch die Arbeitszeitregelung vorgegebene Zeit anzusehen, sondern es ist im Einzelfall mit Rücksicht auf das Berufsbild und die Gestaltungsmöglichkeiten des einzelnen Beamten bei der Bestimmung seiner Arbeitszeit zu entscheiden, ob gerade eine Dienstzeit vorlag. ${ }^{1039}$

Aus dem Schutzbereich fallen aber Unfälle, die sich zu Zeitpunkten ereignen, zu denen der Beamte mit Verrichtungen befasst war, die mit dessen dienstlichen Obliegenheiten schlechthin nicht in Zusammenhang ge-

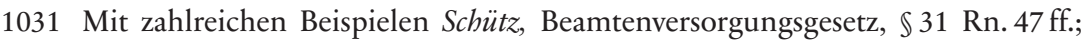
Brockhaus, in: Schütz/Maiwald (Hrsg.), Beamtenrecht, $\$ 31$ BeamtVG Rn. 61.

1032 BVerwG, Urt. v. 24.10.1963, E 17, 59, 59 ff. Rn. 23.

1033 BVerwG, Urt. v. 15.11.2007 - 2 C 24/06 -, juris Rn. 14.

1034 BVerwG, Urt. v. 15.11.2007 - 2 C 24/06 -, juris Rn. 14.

1035 BVerwG, Urt. v. 25.2.2010 - 2 C 81/08 -, juris Rn. 19.

1036 BVerwG, Urt. v. 15.11.2007 - 2 C 24/06 -, juris Rn. 12.

1037 BVerwG, Urt. v. 22.1.2009 - 2 A 3.08 -, juris Rn. 15; v. 25.2.2010 - 2 C 81/08 -, juris Rn. 19.

1038 BVerwG, Urt. v. 26.2.2008 - 2 B 135/07 -, juris Rn. 9.

1039 Bauer, in: Stegmüller/Schmalhofer et al. (Hrsg.), Beamtenversorgungsrecht des Bundes und der Länder, Erl. 6 zu $\$ 31$, Rn. 1.1.1. 
bracht werden können. ${ }^{1040}$ Dazu zählt etwa, wenn der Beamte seinen Aufgabenkreis offensichtlich und schwerwiegend überschreitet und dabei den wohlverstandenen Interessen des Dienstherrn erkennbar zuwiderhandelt ${ }^{1041}$ oder er gegen ein ausdrückliches Verbot des Dienstherrn verstößt, das die Tätigkeit selbst (nicht nur die Art und Weise ihrer Ausführung) betrifft $^{1042}$. Beispielsweise wurde durch das Oberverwaltungsgericht Koblenz als erkennbar dem Interesse des Dienstherrn widersprechend bewertet, dass ein Lehrer versuchte, sich gegen die auch körperliche Gewalt anwendenden Bediensteten des Sport- und Bäderamtes Zutritt zur Schwimmhalle zu verschaffen, um dort lehrplangemäß Schwimmunterricht für seine Schüler abzuhalten. ${ }^{1043}$ Denn obwohl der Schwimmunterricht seine dienstliche Aufgabe war, sei von einem Lehrer entsprechend seiner Vorbildfunktion zu erwarten, dass er im Dienst ,auch bei spannungsgeladenen Situationen im Umgang mit Dritten angemessen reagiert und sich selbst bei etwaigen Provokationen nicht zur Anwendung körperlicher Gewalt hinreißen lässt“.1044 Die Verletzungen, die der Lehrer davontrug, wurden daher nicht als in Folge eines Dienstunfalls eingetreten bewertet.

\section{c. Dogmatische Einordnung des „Dienstbanns“}

Die dogmatische Einordnung der in Anlehnung an den Begriff des „Betriebsbanns" und die Wortwahl des Bundesverwaltungsgerichts, der Beamte befände sich „im Banne des Dienstes“, als Dienstbann zu bezeichnenden räumlich-zeitlichen Zuordnungsregel ist unklar. Dies ist ersichtlich aus den uneinheitlichen und schwammigen Formulierungen, die für die Erklärung dieses Zusammenhangs genutzt werden. Die Rede ist von Dienstort und Dienstzeit als „prinzipiellen Abgrenzungskriterien“1045 und davon, dass bei Vorliegen der Kriterien von einer Zuordnung zum Dienst „auszu-

1040 BVerwG, Urt. v. 25.2.2010 - 2 C 81/08 -, juris Rn. 18; VGH Bayern, Urt. v. 24.4.2015 - 3 B 14.1141 -, juris Rn. 24; Wilhelm, in: Franke/Weiß (Hrsg.), GKÖD, $\mathbb{3} 31$ BeamtVG Rn. 33.

1041 BVerwG, Urt. v. 15.11.2007 - 2 C 24/06 -, juris Rn. $11 \mathrm{ff}$.

1042 Ebd. Rn. 13; vgl. auch Wilhelm, in: Franke/Weiß (Hrsg.), GKÖD, $\$ 31$ BeamtVG Rn. 64.

1043 OVG Rheinland-Pfalz, Beschl. v. 23.11.2004 - 2 A 11630/04 -, NVwZ-RR 2006, S. 199, 199.

1044 Ebd.

1045 Groepper/Tegethoff, in: Plog/Wiedow et al. (Hrsg.), Bundesbeamtengesetz, $\mathbb{} 31$ BeamtVG Rn. 3 und 4. 
gehen"1046 ist; diese Formulierungen suggerieren eine bloße Indizwirkung von Dienstzeit und Dienstort. In derselben Publikation wird an anderer Stelle dagegen konstatiert, bei einem Unfall während der Dienstzeit am Dienstort „handelt es sich um“1047 einen Dienstunfall. Letztere Wortwahl würde eine eindeutige Zuordnung bedeuten. Einerseits könnte die Wortfolge „in Ausübung des Dienstes“ im Gesetzeswortlaut als bedeutungsgleich mit „sich zur Dienstzeit am Dienstort befindend“ verstanden werden, andererseits die räumliche und zeitliche Beziehung zum Dienst lediglich eine Vermutung dafür begründen, dass sich der Beamte in Ausübung des Dienstes befand. Dienstort und Dienstzeit könnten in der Rechtsprechung des Bundesverwaltungsgerichts eine Fiktion oder eine Vermutung zum Tatbestandsmerkmal „in Ausübung des Dienstes“ bedeuten. Eine Fiktion unterstellt einen nicht vorliegenden Tatbestand und knüpft die an diesen Tatbestand gebundenen Rechtsfolgen an den fiktiven Sachverhalt. ${ }^{1048}$ Eine Vermutung dagegen legt fest, dass bei Vorliegen gewisser Voraussetzungen von dem Vorhandensein anderer Voraussetzungen auszugehen ist. ${ }^{1049}$ Ist die Vermutung widerleglich (praesumtio iuris tamtum) - wie es dem Normalfall entspricht, vgl. $\$ 292$ S. 1 ZPO - gilt das nur so lange, bis das Gegenteil bewiesen ist. Im Fall einer unwiderleglichen Vermutung (praesumtio iuris et de iure) dagegen ist kein Gegenbeweis möglich. Der Unterschied zur Fiktion besteht hier lediglich darin, dass es theoretisch möglich wäre, dass der notwendige Tatbestand erfüllt ist.

$\mathrm{Da}$ es immer möglich ist, dass die Tätigkeit tatsächlich dienstlicher Art war, handelt es sich bei der durch richterliche Rechtsfortbildung geprägten Zuordnungsregel des Dienstbannes um eine Vermutung. ${ }^{1050}$ Der Gedanke, der die dargestellte regelmäßige Zurechnung von sich am Dienstort zur Dienstzeit verwirklichenden Risiken an den Dienstherrn trägt, ist, dass dieser Risikobereich vom Dienstherrn beherrschbar ist. ${ }^{1051}$ Diese Rechtsprechung bezweckt eine Abgrenzung von Privat- und Dienstsphäre, die klar und einfach objektiv vorzunehmen ist. ${ }^{1052}$ Sie soll eine ausufernde Kasuis-

1046 Ebd.

1047 Ebd. Rn. 52.

1048 Bitter/Rauhut, JuS 2009, 289, S. 291.

1049 Musielak, JA 2010, S. 561, 566; Bitter/Rauhut, JuS 2009, S. 289, 91.

1050 Für eine widerlegliche Vermutung Reich, LKV 2014, S. 193, 194.

1051 BVerwG, Urt. v. 15.11.2007 - 2 C 24/06 -, juris Rn. 12; BVerwG, Urt. v. 26.2.2008 - 2 B 135/07 -, juris Rn. 7; Brinktrine, in: Kugele (Hrsg.), Kommentar zum Beamtenversorgungsgesetz, $\mathbb{3} 31 \mathrm{Rn} .10$.

1052 Groepper/Tegethoff, in: Plog/Wiedow et al. (Hrsg.), Bundesbeamtengesetz, $\mathbb{} 31$ BeamtVG Rn. 3. 
tik vermeiden, indem sie auf die Beherrschbarkeit des Risikos abstellt, das ein „unschwer zu konkretisierender unbestimmter Rechtsbegriff“" sei. ${ }^{1053}$ Dienstzeit und Dienstort, so arbeitete das Bundesverwaltungsgericht in seinem Urteil vom 24.10.1963 heraus, sind im Gegensatz zu einem Kriterium der Handlungstendenz einfacher zu bestimmende und objektive Abgrenzungsmomente, die mit großer Regelmäßigkeit zu gerechten Ergebnissen führen und Beweisprobleme vermeiden. ${ }^{1054}$ Beim Versuch, den Sachverhalt genau aufzuklären, würden sich Dienstherr und Beamte entzweien, da der Konflikt somit in der gesamten Einsatzstelle zum Tragen kommen würde. Engere Auslegungen der Dienstbezogenheit hielt das Gericht für impraktikabel, weil niemand beweisen könne, ob der Beamte gerade tatsächlich in Ausführung seiner Dienstaufgabe oder etwa gedanklich zu privaten Themen abgeschweift war. Zudem sei es lebensfremd, diese natürlichen $\mathrm{Zu}$ sammenhänge auseinanderzureißen. ${ }^{1055}$ Eventuelle Ungereimtheiten, die sich aus der regelmäßigen Zuordnung von Tätigkeiten, die in räumlichem und zeitlichem Zusammenhang mit dem Dienst ausgeführt werden, zum Dienst ergeben, seien im Gegenzug für diese Rechtssicherheit hinnehmbar, so lange die Unfallfürsorge dadurch nicht auf tatsächlich vom Beamten selbst beherrschbare Risiken ausgedehnt wird. ${ }^{1056}$ Dies bedeutet, dass eine Vermutung dahingehend besteht, dass sich ein Beamter zur Dienstzeit am Dienstort in Ausübung des Dienstes befindet. Eine Fiktion würde die Voraussetzungen nicht erfüllen, weil sonst der Dienstherr auch für solche Risiken einzustehen hätte, die sich aus Tätigkeiten ergeben, welche eindeutig seinen Interessen zuwiderlaufen. Die Vermutung ist aber nicht grundsätzlich widerleglich; denn wäre die Frage, ob der Beamte während der Dienstzeit am Dienstort auch in Ausübung des Dienstes war, stets dem Beweis zugänglich, würde dies zu den gerade erwähnten, von der Rechtsprechung zu vermeiden gesuchten Problemen führen.

1053 BVerwG, Urt. v. 15.11.2007 - 2 C 24/06 -, juris Rn. 14.

1054 BVerwG, Urt. v. 24.10.1963, E 17, 59, 59 ff. Rn. 24, 26, 27.

1055 Ebd. Rn. 24, 26, 28.

1056 VG Potsdam, Urt. v. 23.11.2011 - 2 K 2434/08 -, juris Rn. 17. 
2. Begründung des Fokus auf räumlich-zeitliche Kriterien als Konsequenz des Schutzzwecks der Unfallfürsorge

a. Sinn und Zweck der beamtenrechtlichen Unfallfürsorge

Als Grund für die dargestellte Abkehr von der tätigkeitsbezogenen Betrachtung und die Anwendung von Dienstzeit und Dienstort als maßgeblichen Zuordnungsregeln gab das Bundesverwaltungsgericht eine Rückbesinnung auf den Sinn und Zweck der beamtenrechtlichen Unfallfürsorge an. ${ }^{1057}$ Der Schutzzweck der Unfallfürsorge wird aus einer historischen Betrachtung ihrer Entwicklung deutlich.

Das Unfallfürsorgerecht der Beamten wurde eingeführt, um sie vor den negativen Folgen von im Zusammenhang mit ihrem Dienst erlittenen Unfällen zu schützen. ${ }^{1058}$ Dieser Schutz sollte noch über die allgemeine Fürsorge des Dienstherrn hinausgehen und die Risiken umfassen, die in dem Gefahrenbereich eintreten, in dem der Beamte aufgrund der Anforderungen des Dienstes tätig wird, und die daher der dienstlichen Risikosphäre zuzuordnen sind. ${ }^{1059}$ Dies erklärt sich aus der rechtlichen Situation der Beamten vor der Einführung der Unfallfürsorge. Genau wie die Arbeitnehmer waren sie im Fall eines schädigenden Ereignisses vor Einführung der Unfallversicherungsvorschriften faktisch schutzlos. ${ }^{1060}$ Im ersten Dienstrecht der Beamten, dem Reichsbeamtengesetz von $1873^{1061}$, war keine Entschädigung für während des Dienstes erlittene Verletzungen vorgesehen. Es bestand lediglich eine Bevorteilung im Zusammenhang mit Pensionsansprüchen für Beamte, die im Zusammenhang mit dem Dienst ohne eigenes Verschulden einen Unfall erlitten hatten und dadurch dauerhaft dienstunfähig waren. Im Gegensatz zu Beamten, die aus anderem Grund dienstunfähig wurden, war bei den während der Dienstausübung verunfallten Beamten keine zehnjährige Mindestamtszeit notwendig, um einen Anspruch auf eine lebenslange Pension auszulösen ( $\left.\mathbb{S} \int 35,36 \mathrm{RBG}\right)$. Die Kosten der Heilbehandlung, die der Unfall verursachte, trug der betroffene

1057 BVerwG, Urt. v. 29.8.2013 - 2 C 1.12 -, juris Rn. 11; Giesen, Arbeitsunfall und Dienstunfall, S. 42.

1058 Ebd.

1059 BVerwG, Urt. v. 29.8.2013 - 2 C 1.12 -, juris Rn. 11; Biletzki, NVwZ 2010, S. $688,689$.

1060 Bulla, Der Dienst- und Arbeitsunfall als Institut des Allgemeinen Verwaltungsrechts, S. 129; Teutsch, in: Rohrbeck (Hrsg.), Beiträge zur Sozialversicherung, S. 305, 314; Hueck, RdA 7 (1954), S. 251, 251.

1061 RGBl. S. 61. 
Beamte allerdings selbst; seine Situation entsprach insoweit der des Arbeiters vor 1884. ${ }^{1062}$ Auch Beamte waren darauf verwiesen, eventuell bestehende Schadensersatzansprüche in einem aufwendigen und kostenintensiven Verfahren zivilrechtlich geltend zu machen. Die Grundsätze der Verschuldenshaftung mit ihren hohen Beweisanforderungen galten auch hier, sodass die Durchsetzung selbst eines bestehenden Anspruchs den Beamten in vielen Fällen unmöglich war. ${ }^{1063}$

Mit dem Erlass des Unfallversicherungsgesetzes 1884 besserte sich die Situation für diejenigen Beamten, die unter 2000 Mark im Jahr verdienten und in Betrieben arbeiteten, für die das Unfallversicherungsgesetz galt. Denn diese sog. Betriebsbeamten waren von der gesetzlichen Unfallversicherung umfasst ( $\$ 1$ UVG). Dies führte dazu, dass Betriebs-, Reichs-, Staats- und Kommunalbeamte eine ungleiche Behandlung erfuhren, was als ungerecht empfunden wurde. ${ }^{1064} \mathrm{Um}$ die Ungleichbehandlung zwischen den Beamtengruppen zu beseitigen, wurde am 15.3.1886 das Gesetz betreffend die Fürsorge für Beamte und Personen des Soldatenstandes in Folge von Betriebsunfällen (UFG) ${ }^{1065}$ erlassen. Dieses Gesetz normierte in seinem $₫ 1$ einen Pensionsanspruch für jeden Beamten, der durch einen Betriebsunfall während der Ausübung des Dienstes ganz oder teilweise dienstunfähig wurde. Auch Ansprüche auf Ersatz der Heilungskosten, auf Sterbegeld und Hinterbliebenenrente wurden festgeschrieben $(\mathbb{S} \mathbb{S} 1,2$ UFG). Wie im UVG wurden auch im UFG alle Schadensersatzansprüche des Beamten gegen seinen Dienstherrn ausgeschlossen, außer, der Dienstherr hatte vorsätzlich gehandelt ( $\$ \$ 8,10$ UFG). Beamte waren aus der gesellschaftlichen Situation heraus ebenso schutzbedürftig wie die Arbeiter es vor Einführung des UVG gewesen waren. ${ }^{1066}$ Aus der Lektüre der Protokolle der Beratungen des Reichstags zum Unfallfürsorgegesetz ergibt sich, dass den Abgeordneten vornehmlich die Gleichstellung der Beamten mit den Arbeitnehmern bei Dienstunfällen wichtig war. ${ }^{1067}$ Damit waren nun

1062 Bulla, Der Dienst- und Arbeitsunfall als Institut des Allgemeinen Verwaltungsrechts, S. 21.

1063 Ebd., S. 21 f., 129.

1064 Ebd., S. 21.

1065 RGBl. S. 53.

1066 Bulla, Der Dienst- und Arbeitsunfall als Institut des Allgemeinen Verwaltungsrechts, S. 129; Teutsch, in: Rohrbeck (Hrsg.), Beiträge zur Sozialversicherung, S. 305, 314; Hueck, RdA 7 (1954), S. 251, 251.

1067 Stenographische Berichte des Reichstags, Protokolle, 6. Legislaturperiode, II. Session 1885/1886, 39. Sitzung v. 4.2.1886, S. $873 \mathrm{ff}$. sowie 47. Sitzung v. 15.2.1886, S. 1087 ff., abrufbar unter http://www.reichstagsprotokolle.de (Stand: 18.11.2015). 
nicht nur die Betriebsbeamten, sondern alle Beamten geschützt; allerdings nur gegen solche Unfälle, die sich in einem Betrieb ereigneten, der dem Schutzbereich des Unfallversicherungsgesetzes unterlag. Mit solchen Betrieben kamen manche Beamte im Rahmen der staatlichen oder polizeilichen Beaufsichtigung in Kontakt. Alle anderen Beamten verfügten noch immer über keinerlei Absicherung gegen Unfälle, die sie im Zusammenhang mit ihrer Dienstausübung erlitten.

Erst mit dem Deutschen Beamtengesetz vom 26.1.1937, ${ }^{1068}$ welches das Unfallfürsorgegesetz außer Kraft setzte, trat eine Verbesserung der Rechtsstellung der Beamten ein. Es regelte die Unfallfürsorge in den $\$ \mathbb{S} 107 \mathrm{ff}$. für alle Beamten. Nun löste jeder „in Ausübung oder infolge des Dienstes” erlittene Unfall - unabhängig davon, ob es sich um einen Betriebsunfall handelte - Ansprüche des verletzten Beamten sowie seiner Hinterbliebenen zum Beispiel auf Erstattung der Heilungskosten aus. ${ }^{1069}$ Diese Situation wurde als zufrieden stellend empfunden. ${ }^{1070}$ Das Bundesbeamtengesetz $^{1071}$, welches im Jahr 1953 das Deutsche Beamtengesetz ablöste, behielt dessen Regelungen zur Unfallfürsorge im Wesentlichen bei $(\mathbb{S} 134 \mathrm{ff}$. BBG). Durch ein Änderungsgesetz ${ }^{1072}$ wurden im Jahr 1941 schließlich Berufskrankheiten sowie Wegeunfälle in den Schutzbereich der Unfallfürsorge einbezogen.

\section{b. Unzulänglichkeiten dieses Begründungsansatzes}

Die genannten Schutzzweckerwägungen allein vermögen allerdings nicht zu erklären, wieso das Bundesverwaltungsgericht die Abgrenzung zwischen Dienst- und Privatunfall allein anhand rein objektiver Kriterien vollzieht. ${ }^{1073}$ Denn obwohl mit dem Unfallversicherungsrecht dasselbe Ziel verfolgt wird, wird dort die Abgrenzung von Privat- und Berufssphäre richtigerweise gerade nicht nach rein räumlich-zeitlichen Aspekten, sondern in Anknüpfung an die zum Unfallzeitpunkt verrichtete Tätigkeit erreicht. Ka-

1068 RGBl. I, S. 39.

1069 Amtliche Begründung zu $\$ \mathbb{S} 107-125$ DBG, Deutscher Reichsanzeiger und Preußischer Staatsanzeiger, 1937 Nr. 22, S. 4.

1070 Bulla, Der Dienst- und Arbeitsunfall als Institut des Allgemeinen Verwaltungsrechts, S. 21.

1071 BGBl. I, S. 551.

1072 3. Gesetz zur Änderung des Deutschen Beamtengesetzes vom 21.10.1941, RGBl. I, S. 646.

1073 BVerwG, Urt. v. 29.8.2013 - 2 C 1.12 -, juris Rn. 11. 
pitel 2 und 3 dieser Arbeit haben gerade gezeigt, dass die Konzentration auf die tatsächliche Handlung für eine sachgerechte Definition des Arbeitsunfalls unerlässlich ist.

In diesem Sinne ist die Rechtsprechungspraxis des Bundesverwaltungsgerichts auch von Biletzki kritisiert worden. ${ }^{1074}$ Die Einordnung eines jeden zur Dienstzeit am Dienstort erlittenen Unfalls sei auch angesichts des Zwecks der Unfallfürsorge nicht sachgerecht und überschreite „den Rubikon des Zumutbaren“. ${ }^{1075}$ Der Beamte solle gegen Risiken geschützt werden, die aus seiner speziellen dienstlichen Tätigkeit heraus entstammen und ihn im Vergleich zur übrigen Bevölkerung einem gesteigerten Risiko aussetzen. Dies sei aber nicht bei jedem beliebigen Unfall am Dienstort, sondern nur bei für seine spezielle Dienstaufgabe typischen, signifikant gefährdenden Tätigkeiten der Fall. ${ }^{1076}$ Es müsse deswegen darauf ankommen, Unfallrisiken, die der rein privaten Risikosphäre entstammen, von solchen abzugrenzen, die sich aus einem spezifischen Gefahrenbereich ergeben, dem der Beamte aufgrund der besonderen und typischen Anforderungen gerade seines Dienstes ausgesetzt ist. ${ }^{1077}$ Eine reine räumlich-zeitliche Betrachtung könne dies nicht leisten. Der Dienstherr müsse das Risiko also auch tatsächlich beherrschen können; es dürfe sich nicht um ein nur bei Gelegenheit des Dienstes realisiertes, auch vom Dienstherrn nicht beherrschbares Unfallereignis handeln. Nur wenn sich ein gesteigertes Risiko realisiere, das sich aus dem spezifischen Gefahrenbereich ergibt, dem der Beamte aufgrund der besonderen und typischen Anforderungen seines Amtes ausgesetzt wird, sei der Zurechnungszusammenhang zu bejahen. ${ }^{1078}$

1074 Biletzki, NVwZ 2010, S. 688 ff.

1075 Ebd., S. 688, 690.

1076 Ebd.

1077 Ebd.

1078 Ebd., S. 688, 690 f. Im Fall einer verbeamteten Lehrerin, die während eines Schullandheimaufenthalts von einer Zecke gebissen wurde und anschließend an Borreliose erkrankte, verneint diese Ansicht daher das Vorliegen eines Dienstunfalls, während das BVerwG, Urt. v. 25.2.2010 - 2 C 81/08 -, juris, einen solchen bejahte. Biletzki argumentierte, eine (verbeamtete) Lehrerin unterläge nicht einer größeren Gefahr, einen Zeckenbiss zu erleiden, als ein beliebiger anderer Bürger. Deswegen dürfe sie auch nicht bevorzugt behandelt werden; das Risiko ihres Zeckenbisses der Allgemeinheit aufzubürden, würde sie ungerechtfertigt besserstellen. Dieser Kritik ist entgegenzuhalten, dass die meisten Geschädigten bei Anwendung dieser Grundsätze in Beweisschwierigkeiten kommen würden. Lediglich Beamte, die typischerweise in der freien Natur ihren Dienst verrichteten, könnten wohl den Beweis führen, dass ein Zusammenhang mit ihrem Tätigkeitsfeld vorliegt. Es ist aber nicht einzusehen, 
Für die Abgrenzung allein nach räumlich-zeitlichen Kriterien ist deswegen eine über den Schutzzweck der Unfallfürsorge hinausgehende Begründung notwendig. Dass es tatsächlich sachgerecht ist, Ort und Zeit des Unfalls die maßgebliche Bedeutung für die Einordnung eines Unfalls als Dienstunfall zuzuweisen, ergibt sich erst aus den Rechtsgründen, auf denen die Zuordnung zur dienstlichen Sphäre im Beamtenversorgungsrecht basiert (dazu 3.). Denn obwohl diese ähnlich wie im Unfallversicherungsrecht sowohl im Fürsorgeprinzip (3. c.) als auch im Grundsatz der Betriebsrisikolehre (3. d.) wurzeln, offenbart ihre Analyse die Notwendigkeit, die Risikosphären nicht tätigkeitsbezogen abzugrenzen. Der Grund dafür liegt, wie der folgende Abschnitt zeigt, in den unterschiedlichen, den beiden Unfallsicherungsregimes zugrunde liegenden Rechtsverhältnissen (3.a).

3. Begründung des Fokus auf räumlich-zeitliche Kriterien als Konsequenz der Rechtsgründe der Unfallfürsorge und des Beamtenstatus

a. Die Besonderheiten des Beamtenstatus gegenüber dem Arbeitsverhältnis

Dass Fürsorgeprinzip und Betriebsrisikolehre im Unfallfürsorgerecht zu anderen Abgrenzungskriterien führen als im Unfallversicherungsrecht, erklärt sich aus den gegenüber dem Arbeitsverhältnis bestehenden Besonderheiten des Beamtenstatus. Das Arbeitsverhältnis und das Beamtenverhältnis bilden die Rechtsverhältnisse, an die beide Unfallsicherungssysteme anknüpfen. Dies überrascht nicht, da die Unfallsicherungssysteme gerade zum Zweck haben, Risiken, die sich aus diesen Rechtsbeziehungen ergeben, abzufangen. Gemeinsame Elemente beider Rechtsverhältnisse sind Unterordnung und Fremdbestimmtheit der arbeits- oder dienstleistenden Person gegenüber dem Arbeitgeber oder Dienstherrn. Im Beschäftigungsverhältnis ist der Beschäftigte vom Arbeitgeber persönlich abhängig und damit weisungsgebunden im Sinne des $\$ 106$ GewO; im Dienstverhältnis schuldet der Beamte seinem Dienstherrn Treue und Gehorsam ( $\$ \$ 4,62$ BBG, 35 BeamtStG). Sowohl Beamte als auch Beschäftigte wenden also

wieso ein Beamter, der weisungsgebunden eine atypische Dienstleistung zu erbringen hat, bei dieser nicht genau so geschützt sein soll wie Beamte, die diese Tätigkeit regelmäßig zu erbringen haben. So auch Wilhelm, in: Franke/Weiß (Hrsg.), GKÖD, $\$ 31$ BeamtVG Rn. 33. 
ihre Arbeitskraft auf, um mit der Erfüllung ihrer Aufgaben ihren Lebensunterhalt zu verdienen. ${ }^{1079}$ Beide Personengruppen begeben sich deswegen in von ihrem Dienstherrn geschaffene Gefahrenbereiche. Ihre soziale Stellung ist daher - in engen Grenzen - vergleichbar. ${ }^{1080}$

Gravierende Unterschiede bestehen jedoch in der Rechtsnatur der Beziehungen. Das Arbeitsverhältnis ist ein privatrechtliches Schuldverhältnis zwischen Arbeitgeber und Arbeitnehmer, dessen Bedingungen nach den Regeln der Privatautonomie ausgehandelt werden können. Das Beamtenverhältnis ist dagegen öffentlich-rechtlicher Natur und wird durch einseitigen Hoheitsakt des Dienstherrn begründet. Sein Inhalt ist gesetzlich vorgeschrieben; Separatvereinbarungen zwischen Dienstherrn und Beamten auf vertraglicher Grundlage sind unzulässig. ${ }^{1081}$ Dies ist ein eklatanter Gegensatz zu dem das Arbeitsvertragsrecht durchdringenden Prinzip der Privatautonomie. Zwar ist deren Reichweite nicht unbegrenzt, sondern durch zwingendes Recht (genannt werden können etwa Mindestlohn-, Kündigungsschutz- oder Mutterschutzgesetz), zivilrechtliche Generalklauseln und die Betriebs- und Tarifautonomie eingeschränkt. Die Verhandlungsfreiheit der Parteien bleibt dennoch Kernprinzip des Arbeitsrechts. ${ }^{1082} \mathrm{Im}$ Beamtenverhältnis gelten allgemeine zivilrechtliche Rechtsgrundsätze dagegen aufgrund ihres öffentlich-rechtlichen Charakters nicht. ${ }^{1083}$

Auch die Zweckrichtung der Rechtsverhältnisse ist verschiedenartig. Das Arbeitsverhältnis dient dem Bestehen und der Weiterentwicklung eines auf Erwerb gerichteten wirtschaftlichen Unternehmens. ${ }^{1084}$ Das Beamtenverhältnis dagegen dient der Erledigung von hoheitlichen und sonstigen öffentlichen Verwaltungsaufgaben. ${ }^{1085}$

Während im Arbeitsverhältnis aus dem Arbeitsvertrag nur eine inhaltlich bestimmte und nach Zeit und Umfang begrenzte Arbeitsleistung geschuldet wird, erwächst Beamten unmittelbar aus dem Gesetz im Rahmen ihres Dienst- und Treueverhältnisses die Pflicht, ihrem Dienstherrn ihre ge-

1079 Teutsch, in: Rohrbeck (Hrsg.), Beiträge zur Sozialversicherung, S. 305, 306.

1080 Ebd.

1081 Werres, in: Brinktrine/Schollendorf (Hrsg.), BeckOK Beamtenrecht Bund, $\mathbb{} 4$ BBG Rn. 4; Walter/Böhm, in: Paulssen/Richter et al. (Hrsg.), Sozialpolitik, Arbeits- und Sozialrecht, S. 181, 198; Hense, in: Epping/Hillgruber (Hrsg.), BeckOK GG, Art. 33 Rn. 44; historisch vgl. Brand, Das Beamtenrecht, S. 46 f.

1082 Boemke, NZA 1993, S. 532, 533 f.; Kempen, NZA-Beil. 2000, S. 7, 13.

1083 Es sei denn, sie sind als Ausdruck allgemeiner Rechtsgrundsätze zugleich Bestandteil des öffentlichen Rechts, Battis, BBG, $\mathbb{} 4$ Rn. 32.

1084 Walter/Böhm, in: Paulssen/Richter et al. (Hrsg.), Sozialpolitik, Arbeits- und Sozialrecht, S. 181, 198.

1085 Ebd. 
samte Arbeitskraft zur Verfügung zu stellen. ${ }^{1086}$ Der Arbeitgeber schuldet aus dem schuldrechtlichen Synallagma die Entlohnung des Arbeitnehmers als Gegenleistung, der Dienstherr dagegen hat die Pflicht, dem Beamten amtsangemessene Besoldung und Versorgung für sich und seine Familie zu gewähren. ${ }^{1087}$ Diese Treuepflichten - aufseiten des Beamten Treuepflicht, aufseiten des Dienstherrn Alimentations- und Fürsorgepflicht genannt basieren zwar auf Gegenseitigkeit, stehen aber nicht im Synallagma. ${ }^{1088}$ Ihre inhaltliche Ausgestaltung obliegt vielmehr dem Gesetzgeber. ${ }^{1089}$ Anders als ein Arbeitnehmer ist ein Beamter nicht an einen einzelnen Dienstherrn, sondern an die öffentliche Gewalt als solche gebunden, was ihm den Wechsel zwischen einzelnen Gebieten ermöglicht. Die öffentliche Gewalt ist für den Beamten das, was für den Arbeitnehmer der Betrieb ist. ${ }^{1090}$

Der Inhalt des beamtenrechtlichen Dienst- und Treueverhältnisses geht weit über das in arbeitsvertraglich geregelten Beschäftigungsverhältnissen zulässige Maß hinaus. ${ }^{1091}$ So ist die Gehorsamspflicht des Beamten ( $\mathbb{S} 33$ ff. BeamtStG) strenger als die Bindung des Arbeitnehmers an Weisungen seines Arbeitgebers. ${ }^{1092}$ Der Beamte hat sich mit seinem gesamten dienstlichen und privaten Verhalten zur freiheitlichen demokratischen Grundordnung im Sinne des Grundgesetzes zu bekennen und für deren Erhaltung einzutreten. ${ }^{1093}$ Er hat die Pflicht, sein Amt uneigennützig, gemeinwohlorientiert und unparteilich zu führen. ${ }^{1094}$ Seine Persönlichkeit ist vollumfänglich an die dienstliche Aufgabe gebunden. ${ }^{1095}$ Der Beamte ist auch in seiner Ausübung von Grundrechten beschränkt, soweit sie mit der Erfüllung der Pflichten aus dem besonderen Dienst- und Treueverhält-

1086 Vgl. nur BVerfG, Beschl. v. 24.11.1998, E 99, 300, 317.

1087 Ebd. Rn. 42; Badura, in: Maunz/Dürig et al. (Hrsg.), Grundgesetz, Art. 33 Rn. 72.

1088 Battis, BBG, $\$ 4$ Rn. 4; Laubinger, in: König/Laubinger et al. (Hrsg.), Öffentlicher Dienst, S. 89, 110; Lecheler, AöR 1978, S. 349, 373; BVerwG, Urt. v. 27. 6.1961, E 12, 273, 273.

1089 Hense, in: Epping/Hillgruber (Hrsg.), BeckOK GG, Art. 33 Rn. 42.

1090 Walter/Böhm, in: Paulssen/Richter et al. (Hrsg.), Sozialpolitik, Arbeits- und Sozialrecht, S. 181, 192.

1091 Werres, in: Brinktrine/Schollendorf (Hrsg.), BeckOK Beamtenrecht Bund, $\mathbb{} 4$ BBG Rn. 5.

1092 Teutsch, in: Rohrbeck (Hrsg.), Beiträge zur Sozialversicherung, S. 305, 306.

1093 Badura, in: Maunz/Dürig et al. (Hrsg.), Grundgesetz, Art. 33 Rn 59.

1094 Hense, in: Epping/Hillgruber (Hrsg.), BeckOK GG, Art. 33 Rn. 42 m.w.N.

1095 Walter/Böhm, in: Paulssen/Richter et al. (Hrsg.), Sozialpolitik, Arbeits- und Sozialrecht, S. 181, 193. 
nis unvereinbar sind. ${ }^{1096}$ Beispielsweise muss er Einschränkungen der Berufsfreiheit des Art. 12 Abs. 2 GG hinnehmen, wenn ihm die Möglichkeit einer Nebentätigkeiten durch Gesetz beschränkt wird, oder seine Religionsfreiheit aus Art. 4 Abs. 1, 2 GG muss hinter die Gehorsamspflicht zurücktreten. ${ }^{1097}$ Die Stärke der Bindung des Beamtenverhältnisses zeigt auch, dass es im Gegenzug zum kündbaren Arbeitsverhältnis auf Lebenszeit ausgelegt ist. ${ }^{1098}$

\section{b. Maßgeblichkeit der Statusunterschiede bei der Einführung der Unfallfürsorge}

Die unterschiedliche Abgrenzung zwischen Berufs- und Privatsphäre im Unfallsicherungsrecht der Beamten und Beschäftigten kann nur dann maßgeblich auf den Statusunterschieden beruhen, wenn diese auch bei Einführung der Sicherungssysteme eine Rolle spielten. Dies ist der Fall. Zwar scheint das Unfallversicherungsgesetz von 1884 zunächst dafür zu sprechen, dass dessen Gesetzgeber Beamte und Arbeiter für gleich hielt und auch gleich behandeln wollte. $\$ 1$ UVG lautet: „Alle in Bergwerken, Salinen, Aufbereitungsanstalten, Steinbrüchen, Gräbereien (Gruben), auf Werften und Bauhöfen, sowie in Fabriken und Hüttenwerken beschäftigten Arbeiter und Betriebsbeamten, letztere sofern ihr Jahresarbeitsverdienst an Lohn oder Gehalt zweitausend Mark nicht übersteigt, werden gegen die Folgen der bei dem Betriebe sich ereignenden Unfälle nach Maßgabe der Bestimmungen dieses Gesetzes versichert.“ Betriebsbeamte mit geringerem Jahreseinkommen als 2000 Mark, die ohne festes Gehalt und ohne Pensionsansprüche beschäftigt wurden, wurden dadurch gleich behandelt wie Arbeiter, nicht jedoch die Reichs-, Staats- und Kommunalbeamten. Diese waren gem. $\$ 4$ UVG ausdrücklich ausgeschlossen: „Auf Beamte, welche in Betriebsverwaltungen des Reichs, eines Bundesstaates oder eines Kommunalverbandes mit festem Gehalt und Pensionsberechtigung angestellt sind, findet dieses Gesetz keine Anwendung.“ Diese Pensionsberechtigung war jedoch minderwertig gegenüber den Leistungen des UVG. Be-

1096 Werres, in: Brinktrine/Schollendorf (Hrsg.), BeckOK Beamtenrecht Bund, $\mathbb{} 4$ BBG Rn. 4.

1097 Zum Beispiel kann verbeamteten Lehrerinnen das Tragen von Kopftüchern aus religiösen Gründen schulaufsichtlich untersagt werden, vgl. Badura, in: Maunz/Dürig et al. (Hrsg.), Grundgesetz, Art. 33 Rn 59.

1098 Walter/Böhm, in: Paulssen/Richter et al. (Hrsg.), Sozialpolitik, Arbeits- und Sozialrecht, S. 181, 194. 
triebsbeamte ohne Pensionsansprüche standen jedoch in einem Beamtenverhältnis, während sich Arbeiter in einem Beschäftigungsverhältnis befanden; dieser Unterschied in der rechtlichen Ausgestaltung hat den Gesetzgeber 1884 nicht davon abgehalten, beide Personengruppen gleich zu behandeln. Denn diese Gruppe der Beamten als auch die Arbeiter waren in seinen Augen in gleichem Maße schutzbedürftig. ${ }^{1099}$

Es handelt sich jedoch nur um eine Teilgruppe der Beamtenschaft, die, da ohne festes Gehalt und Pensionsansprüche beschäftigt, die klassischen Vorteile des Beamtenstatus gerade nicht genoss. Der maßgebliche Unterschied zwischen der rechtlichen Ausgestaltung des Arbeits- und des Dienstverhältnisses ist aufseiten des Dienstleistenden gerade, dass das Dienstverhältnis den Dienstherrn zu Unterhalt und Versorgung des Beamten auf Lebenszeit verpflichtet. Liegt ein Dienstverhältnis vor, das diese Versorgung im Sinne von festem Gehalt und Pensionsberechtigungen ausschließt, so kann von einem Statusunterschied zwischen diesem Beamten und einem Arbeiter nur noch formell, nicht aber materiell gesprochen werden. Dass diese Teilgruppe der Beamten im UVG von 1884 mit den Arbeitern gleich behandelt worden ist, zeigt deswegen nicht, dass der damalige Gesetzgeber die unterschiedliche rechtliche Ausgestaltung von Arbeits- und Dienstverhältnissen für unerheblich hielt. ${ }^{1100}$

Die materiell unterschiedliche Behandlung von Unfällen der Beamten und Beschäftigten ist auch nicht rein auf historisch begründete Zufälligkeiten zurückzuführen. Als im Jahr 1885 allen Beamten eine Unfallfürsorge zuteilwerden sollte, wurde zwar durchaus darüber diskutiert, statt eines eigenen Unfallsicherungssystems für Beamte das bereits bestehende UVG der Arbeiter auf die Beamten auszudehnen. ${ }^{1101}$ Es wird in diesem Zusammenhang auch vertreten, dass man sich aus rein pragmatischen Gründen dafür entschieden hätte, die Unfallfürsorge der Beamten getrennt zu regeln. ${ }^{1102}$

1099 Bulla, Der Dienst- und Arbeitsunfall als Institut des Allgemeinen Verwaltungsrechts, S. 254; Hueck, RdA 7 (1954), S. 251, 251; Teutsch, in: Rohrbeck (Hrsg.), Beiträge zur Sozialversicherung, S. 305, 308.

1100 A.A. wohl Bulla, Der Dienst- und Arbeitsunfall als Institut des Allgemeinen Verwaltungsrechts, S. 129.

1101 Vgl. die zweite Beratung des Gesetzesentwurfs betr. die Fürsorge für Beamte und Personen des Soldatenstandes in Folge von Betriebsunfällen, Stenographische Berichte des Reichstags, Protokolle, 6. Legislaturperiode, II. Session 1885/1886, 39. Sitzung v. 4.2.1886, S. 873 ff., abrufbar unter http://www.reichst agsprotokolle.de (Stand: 18.11.2018).

1102 Bulla, Der Dienst- und Arbeitsunfall als Institut des Allgemeinen Verwaltungsrechts, S. $130 \mathrm{ff}$. 
Dagegen spricht aber bereits die amtliche Begründung zum Unfallfürsorgegesetz, aus der deutlich wird, dass der Gesetzgeber sich für die eigenständige Regelung der Unfallsicherung der Beamten entschied, weil er dadurch der „besonderen Stellung des Beamten“ gerecht werden wollte. ${ }^{1103}$ In der Gesellschaft des 19. Jahrhunderts hielt man Beamte für sozial erheblich bedeutender als Industriearbeiter. Beamte trugen nach allgemeiner Sichtweise die größere Verantwortung und hatten daher auch eine angesehenere Stellung als Arbeiter inne. ${ }^{1104}$ Aus diesem andersartigen gesellschaftlichen und rechtlichen Status wurden auch unterschiedliche Anforderungen an eine Unfallversicherung abgeleitet. Da der Beamte gesteigerte Pflichterfüllungspflichten hatte, sollte nicht nur eine vorsätzliche Herbeiführung des Unfalls, sondern auch ein Verschulden, das so stark war, dass es die Dienstentlassung rechtfertigte, den Unfallschutz verhindern. ${ }^{1105}$

Die Statusunterschiede von Beamten und Arbeitern wurden also durchaus als relevant für die Unfallsicherung angesehen. ${ }^{1106}$ Die Unfallversorgung der Beamten beruht auf der spezifischen Natur ihres Dienstverhältnisses. ${ }^{1107}$

1103 Amtliche Begründung zum Unfallfürsorgegesetz, Stenographische Berichte des Reichstages, 6. Legislaturperiode, II. Session 1885/1886, Drucks. Nr. 5, S. 52; vgl. auch die zweite Beratung des Gesetzesentwurfs betreffend die Fürsorge für Beamte und Personen des Soldatenstandes in Folge von Betriebsunfällen, Stenographische Berichte des Reichstags, Protokolle, 6. Legislaturperiode, II. Session 1885/1886, 39. Sitzung v. 4.2.1886, S. 873 ff., 874, abrufbar unter www.reic htsagsprotokolle.de (Stand: 18.11.2018).

1104 Bulla, Der Dienst- und Arbeitsunfall als Institut des Allgemeinen Verwaltungsrechts, S. 130 m.w.N.

1105 Ebd.

1106 Pesch, DVBl. 1959, S. 43, 45.

1107 Ebenso Becker, Soziales Entschädigungsrecht, S. 122. Da die Unterschiede in der unfallsicherungsrechtlichen Behandlung zwischen Beschäftigten und Beamten auf die dargestellten Statusunterschiede zurückzuführen sind, stimmt es nachdenklich, dass gerade die Besonderheiten des beamtenrechtlichen Status durch kontemporäre Entwicklungen in Frage gestellt werden, vgl. ebd., S. 119, Fn. 634. Insbesondere durch die anhaltende Diskussion um ein Streikrecht für Beamte dürfte eine Neubeurteilung des Beamtenverhältnisses erforderlich werden. Denn das Streikverbot hängt in besonders enger Weise mit dem Kern dessen zusammen, was den Statusunterschied zwischen Beschäftigten und Beamten ausmacht, vgl. Battis/Grigoleit, et al., NVwZ 2016, S. 194, 197 m.w.N.; Lorse, ZBR 2015, S. 109, 113 f. Die oben herausgearbeitete fundamentale Differenz in den Status findet ihren Grund in der gegenüber dem Arbeitsverhältnis stark gesteigerten Treuepflicht des Beamten gegenüber seinem Dienstherrn. Gerade aus dieser Treuepflicht folgt zwingend das beamtenrechtliche Streikverbot: Ein Beamtenstreik als Arbeitskampfmaßnahme würde, da ein Streik stets auf die 


\section{c. Die Fürsorgepflicht des Dienstherrn im Gegensatz zur Fürsorgepflicht des Arbeitgebers}

Im Gegenzug für die dargestellte umfassende Dienst- und Treuepflicht obliegt dem Dienstherrn die Fürsorgepflicht für den Beamten. Diese Fürsorgepflicht bildet den rechtlichen Grund der Risikoübertragung für die Folgen von Dienstunfällen auf den Dienstherrn in $\$ \$ 30,31$ BeamtVG. ${ }^{1108}$ Wegen des stärkeren Unterwerfungsverhältnisses im öffentlich-rechtlichen Dienstverhältnis kommt der Fürsorgepflicht hier eine größere Rolle als Rechtsgrund als im Unfallversicherungsrecht zu. ${ }^{1109}$ Sie geht inhaltlich

Unterdrucksetzung des Verhandlungspartners durch die Zufügung wirtschaftlicher Nachteile gerichtet ist, darauf zielen, dem Dienstherrn zu schaden. Eine solche Absicht der Schadenszufügung steht in eklatantem Widerspruch zu der Treuepflicht des Beamten, Bitsch, ZTR 2012, S. 78, 81. Gerade mit dem Beamtenstatus unterscheidet das deutsche Recht zwischen Staatsbediensteten, die wegen ihres besonderen Dienst- und Treueverhältnisses eine durchgehend loyale und treue Amtsausübung schulden von Angestellten, die aufgrund eines privatautonomen Vertrags beschäftigt werden und von denen in Konsequenz gerade keine durchgehend loyale und treue Amtsausübung verlangt wird, Bitsch, a.a.O. Das Streikverbot ist dem Beamtenverhältnis dadurch immanent, dass man sich einen Beamtenstatus mit Streichrecht kaum vorstellen kann, Widmaier/Alber, ZEuS 2012, S. 387, 415. Ein Rütteln am Streikverbot erschüttert das gesamte Wesen des Beamtenverhältnisses und lässt zu Recht die Frage danach laut werden, ob die Fürsorgepflicht, da sie im Gegenzug zur Treuepflicht besteht, noch in ihrer jetzigen Form Geltung beanspruchen kann, Michaelis, JA 2015, S. 121, 124. Treffend formuliert es Di Fabio: „Das Gesamtverhältnis der Gegenseitigkeit einer besonderen Loyalitätsbeziehung [...] wäre aufgekündigt, wenn man als Beamtin oder Beamter die Bedingungen des Dienstes und der Besoldung durch Dienstverweigerung durchsetzen könnte. [...] Man kann nicht das Streikverbot aufweichen, ohne das Beamtenverhältnis insgesamt preiszugeben., ders., Das beamtenrechtliche Streikverbot, S. 56. Die Anerkennung eines Streikrechts für Beamte würde deswegen eine Neubeurteilung der Unfallsicherungssysteme notwendig machen.

1108 Schütz, Beamtenversorgungsgesetz, $\mathbb{3 0}$ Rn. 2 m.w.N.; Brockhaus, in: Schütz/ Maiwald (Hrsg.), Beamtenrecht, $\$ 30$ BeamtVG Rn. 3; Bulla, SGb 2007, S. 653, 656; Voitl/Luber, Das neue Dienstrecht in Bayern, S. 108; Reich, LKV 2014, S. 193, 193; Leppek, Beamtenrecht, S. 218; Reich, BeamtVG, $\mathbb{3 1}$ Rn. 1; Wolff, Verwaltungsrecht III, S. 149; Pflaum, RiA 2011, S. 198, 198; Pesch, DVBl. 1959, S. 43, 46; Walter/Böhm, in: Paulssen/Richter et al. (Hrsg.), Sozialpolitik, Arbeitsund Sozialrecht, S. 181, 195; Badura, in: Maunz/Dürig et al. (Hrsg.), Grundgesetz, Art. 33 Rn. 71.

1109 Bulla, Der Dienst- und Arbeitsunfall als Institut des Allgemeinen Verwaltungsrechts, S. 125; Fischer, ZBR 1960, S. 148, 148. 
weit über die Fürsorgepflicht eines Arbeitgebers hinaus, da die Pflichten des Beamten ebenso weit die Pflichten eines Arbeitnehmers übersteigen.

Die Fürsorgepflicht des Dienstherrn war schon bei Einführung des Unfallfürsorgegesetzes 1886 Konsens. Heute ist sie im Grundgesetz in Art. 33 Abs. 4 GG normiert. ${ }^{1110}$ Als hergebrachter Grundsatz des Beamtentums fällt sie unter die Garantie des Art. 33 Abs. 5 GG und ist damit in ihrem Wesensgehalt unantastbar. ${ }^{1111}$ Eine generalklauselartige Definition der Fürsorgepflicht findet sich in $\$ 78$ BBG sowie wortgleich in $\ 45$ BeamStG; demnach hat der Dienstherr "für das Wohl der Beamtinnen und Beamten und ihrer Familien, auch für die Zeit nach Beendigung des Beamtenverhältnisses, zu sorgen“.

Im Rahmen der Fürsorgepflicht hat der Dienstherr den Beamten und seine Familie lebenslang amtsangemessen zu alimentieren und ihnen Fürsorge und Schutz zu gewähren. ${ }^{112} \mathrm{Er}$ ist dazu verpflichtet, die Beamten vor Bedrohungen ihres Lebens und ihrer Gesundheit im Arbeitsumfeld zu bewahren, sogar ihr Eigentum, ihre Ehre und ihr Ansehen zu schützen, sie wohlwollend zu fördern und zu beraten. ${ }^{1113}$ Unter diese Pflicht zu Schutz und Fürsorge ist die Pflicht, den Beamten bestmöglich vor den Folgen eines Dienstunfalls zu schützen, zu subsumieren. ${ }^{1114}$ Mit der Fürsorgepflicht korrespondiert der verfassungsrechtliche Anspruch des Beamten auf ihre Erfüllung. ${ }^{1115}$

1110 Vgl. nur Becker, in: WEISSER RING e.V. (Hrsg.), Moderne Opferentschädigung, S. 71; Schütz, Beamtenversorgungsgesetz, $\$ 30$ Rn. 3; Pesch, DVBl. 1959, S. 43, 43; Werres, Beamtenverfassungsrecht, S. 32; BVerwG, Urt. v. 11.10.1962 II C 156.60 -, juris; BGH, Urt. v. 9.3.1965, Z 43, 178, 178.

1111 Battis, BBG, $\$ 78$ Rn. 2.

1112 BVerfG, Beschl. v. 30.3.1977, E 44, 249, 264 ff.; v. 23.6.1981, E 58, 68, 77; Badura, in: Maunz/Dürig et al. (Hrsg.), Grundgesetz, Art. 33 Rn. 71.

1113 Werres, Beamtenverfassungsrecht, S. $33 \mathrm{ff}$.

1114 So auch BVerwG, Urt. v. 11.10 .1962 - II C 156.60 -, juris; BGH, Urt. v. 9.3.1965, Z 43, 178, 178 sowie Bulla, SGb 2007, S. 653, 656 ff.; Brockhaus, in: Schütz/Maiwald (Hrsg.), Beamtenrecht, $\$ 30$ BeamtVG Rn. 2 f. m.w.N.; Bulla, SGb 2007, S.653, 656; Pesch, DVBl. 1959, S. 43, 46; Voitl/Luber, Das neue Dienstrecht in Bayern, S. 108; Reich, LKV 2014, S. 193, 193; Reich, BeamtVG, \31 Rn. 1; Wolff, Verwaltungsrecht III, S. 149; Pflaum, RiA 2011, S. 198, 198; Leppek, Beamtenrecht, S.218; Schütz, Beamtenversorgungsgesetz, $\$ 30$ Rn. 2 m.w.N.

1115 Ist Erfüllung unmöglich, kann der Beamte einen Amtshaftungsanspruch auf Schadenersatz geltend machen (Art. 34 S. 1 GG i.V.m. $\$ 839$ BGB), vgl. Werres, Beamtenverfassungsrecht, S. 35 . 


\section{d. Der Betriebsrisikogedanke im Beamtenrecht}

Auch der für die Legitimation der gesetzlichen Unfallversicherung konstitutive Gedanke des Betriebsrisikos ist im Recht der Beamten als Rechtsgrund für die Übertragung des Unfallrisikos auf den Dienstherrn vorhanden. Denn die dem Prinzip des Betriebsrisikos inhärenten Wertungsgesichtspunkte der Beherrschbarkeit des Risikos und der wirtschaftlichen Interessenverteilung treffen auch auf das Beamtenverhältnis zu und waren bei Einführung der Unfallfürsorge maßgeblich.

Zunächst folgt aus dem Beamtenverhältnis für den Beamten die Pflicht, seine Arbeitskraft voll im Dienst für seinen Dienstherrn einzusetzen. Dies beinhaltet auch die Verpflichtung, die für den Dienst typischen Berufsrisiken zu übernehmen. ${ }^{1116}$ Daraus entsteht eine Berufsgefahr für den Beamten, die unmittelbar auf das Beamtenverhältnis zurückzuführen ist. Ebenso wie der Unternehmer die Risikoquelle der Betriebsunfälle schafft, setzt der Dienstherr zweifellos den Anlass für die Ausübung des Dienstes. ${ }^{1117}$ Weiterhin liegt es am Dienstherrn, durch Handlungsvorgaben und Verhaltensvorschriften den Ablauf der Dienstausführung zu organisieren. Wie der Unternehmer auch, ist der Dienstherr damit herrschend über die zu erledigenden Abläufe. Daraus ergibt sich ein Funktionsgefüge, in das der Beamte sich einfügen muss - genau so, wie der Arbeitnehmer im wirtschaftlichen Sinne in den Betriebsablauf eingegliedert ist. Beide, sowohl abhängig Beschäftigter als auch Beamter, haben Tätigkeiten auszuführen, die ihnen aufgetragen sind, und handeln nicht selbstbestimmt (bei beiden Personengruppen in unterschiedlichen Graden, abhängig von ihrer hierarchischen Stellung). ${ }^{1118}$ In den Fällen, in denen der Dienstherr die Risiken steuern kann oder veranlasst hat, greift das Dienstunfallrecht ein. ${ }^{1119}$ Dies entspricht dem Beherrschbarkeitsargument des Zurechnungsgrundes des Betriebsrisikos, sodass das Argument bei Beamtenverhältnissen genauso greift wie bei Arbeitsverhältnissen.

Auch das Wirtschaftlichkeitsargument ist auf die Unfallfürsorge der Beamten übertragbar. Es besagt, dass derjenige das Risiko zu tragen hat, der auch die mit dem Risiko verbundenen Gewinnchancen für sich beansprucht. Zwar ist staatliches Handeln dem Grunde nach nicht an ökonomischen Grundsätzen ausgerichtet, da es nicht vornehmlich nach Profit

1116 Wilhelm, in: Franke/Weiß (Hrsg.), GKÖD, O vor $\$ 30$ BeamtVG, Rn. 1.

1117 Pflaum, RiA 2011, S. 198, 198.

1118 Reinhardt, Die dogmatische Begründung der Haftungsbeschränkung des Arbeitnehmers, S. $196 \mathrm{ff}$.

1119 Pflaum, RiA 2011, S. 198, 198. 
strebt, sondern die Förderung des Gemeinwohls zum Ziel hat. ${ }^{1120}$ Da beide - Beamter und Dienstherr - dies verwirklichen wollten, wurde argumentiert, es ließen sich zwischen ihnen keine eigenen Risikosphären abgrenzen. ${ }^{1121}$ Nutznießen kann aber auch im nicht-finanziellen Sinne verstanden werden: Der Staat als Dienstherr profitiert von der Arbeit der Beamten, da diese für ihn seine Aufgaben übernehmen und erledigen. ${ }^{1122}$ Das Argument, dass derjenige für ein Risiko einzustehen hat, der den damit entstandenen Nutzen haben soll - cuius commodum, eius periculum greift unabhängig davon, ob dieser Nutzen unmittelbar finanziell ausgedrückt werden kann. ${ }^{1123}$ Dies zeigt sich auch daran, dass in der gesetzlichen Unfallversicherung Beschäftigte von Unternehmen mit gemeinnütziger oder öffentlicher Ausrichtung versichert sind, obwohl diese überhaupt keine Gewinnorientierung vorweisen. ${ }^{1124}$

Sowohl das Beherrschbarkeitsargument als auch das Gewinnerzielungsargument können also für das Beamtenverhältnis fruchtbar gemacht werden. $\mathrm{Zu}$ diesem Ergebnis kommt auch Bulla, der die Unfallfürsorge als Fall der Gefährdungshaftung nennt, da sie darauf beruhe, dass sich eine vom Dienstherrn zu verantwortende Gefahrenlage verwirkliche. ${ }^{1125}$ Dass Fürsorgepflicht und Betriebsrisiko als Rechtsgründe nebeneinander bestehen können, zeigt Pflaum, wenn er schreibt, die Regelungen zum Dienstunfall seien Ausdruck besonderer Fürsorge des Dienstherrn, welche eingreift, weil sich Risiken verwirklichen, die dem Dienstherrn zuzurechnen sind, da er sie steuern kann oder er veranlasst hat, dass der Beamte sich ihnen aussetzt. ${ }^{1126}$ Damit ist die Berufsgefahr der Beamten zum „Betriebsrisiko“ der Verwaltung zu zählen. ${ }^{1127}$ Die Betriebsrisikolehre ist damit der zweite maßgebliche Rechtsgrund der Unfallfürsorge.

1120 Fischer, ZBR 1960, S. 148, 149.

1121 Ebd.

1122 Heine, Die schadensgeneigte Arbeit des Beamten, S. 126 ff.; Kohte, Arbeitnehmerhaftung und Arbeitgeberrisiko, S. 221.

1123 Eingehend Heine, Die schadensgeneigte Arbeit des Beamten, S. 157 ff. m.w.N.

1124 Schelp, Die Haftungsbelastung des Arbeitnehmers bei Schädigung Dritter, S. 45.

1125 Bulla, Der Dienst- und Arbeitsunfall als Institut des Allgemeinen Verwaltungsrechts, S. 140.

1126 Pflaum, RiA 2011, S. 198, 198.

1127 Wilhelm, in: Franke/Weiß (Hrsg.), GKÖD, O vor $₫ 30$ BeamtVG, Rn. 2. 
e. Zwischenergebnis: Die physische Gefahrherrschaft des Dienstherrn als ausreichendes Zuordnungskriterium

Da der Beamte anders als der Arbeitnehmer gerade nicht vornehmlich die Erbringung einer Leistung - nämlich die versicherte Tätigkeit - schuldet, sondern insgesamt zur Treue verpflichtet ist, kann im Beamtenverhältnis nicht auf die tatsächliche Handlung zum Unfallzeitpunkt angeknüpft werden. Damit würde der Natur des Beamtenverhältnisses nicht genüge getan. Der Beamte gibt im Rahmen seines hoheitlichen Dienstverhältnisses einen großen Teil seiner Privatsphäre in die Hände des Dienstherrn. Die gegenseitige Loyalitätsbeziehung gilt nicht nur für die Dauer des Arbeitslebens, sondern darüber hinaus. Dies ist nicht an die tatsächliche Ausübung seiner angewiesenen Tätigkeit gebunden. Zugespitzt lässt sich dies wie folgt ausdrücken: Das Arbeitsverhältnis wird von der geschuldeten Tätigkeit des Arbeitnehmers geprägt; das Beamtenverhältnis dagegen durch die Unterordnung des Beamten unter den Dienstherrn. Deswegen kommt es im Unfallfürsorgerecht verstärkt auf die Beherrschbarkeit durch den Dienstherrn, im Unfallversicherungsrecht dagegen auf die geschuldete Tätigkeit an. ${ }^{1128}$

Es ist deswegen folgerichtig, einen Zusammenhang im Sinne der $\mathbb{S} 30$, 31 BeamtVG zur Dienstausübung über den Schutz bei konkreter Diensttätigkeit hinaus auch dann zu bejahen, wenn der Beamte sich in der Einflusssphäre des Dienstherrn befindet, was jedenfalls dann der Fall ist, wenn er sich zur Dienstzeit am Dienstort befindet. Der höhere Umfang der Fürsorgepflicht des Dienstherrn gegenüber der Fürsorgepflicht des Arbeitgebers verlangt eine umfangreichere Definition des beruflichen Risikobereichs. Dass der Dienstherr den örtlichen und zeitlichen Verbleib des Beamten zum Unfallzeitpunkt zu verantworten hat, genügt deswegen für eine Einordnung des schädigenden Ereignisses als Dienstunfall.

1128 Ähnlich Leube, ZTR 2012, S. 682, 686 f. Daraus erklärt sich im Übrigen, wieso für das Recht der Beamten im Rahmen der Dienstunfallfürsorge ursprünglich die Adäquanztheorie als der Theorie der wesentlichen Bedingung vorzuziehende Kausalitätslehre vorgeschlagen wurde, vgl. Reiff, NJW 1961, S.630, 630 m.w.N., Schütz, DÖD 14 (1961), S. 101; ebenso OVG Münster, Urt. v. 21.6.57 VIA 884/54 -, ZBR 58, 10. Die Adäquanztheorie stellte in ihrer ursprünglichen Form entscheidend auf den Urheber der schädigenden Ursache ab und hätte damit die Bedeutung der Risikobeherrschung des Dienstherrn ins Zentrum gerückt. Im Recht der Dienstunfallfürsorge steht direkt der Dienstherr für die Folgen einer schädigenden Ursache ein, sodass er mit einem Unternehmer vergleichbar sei - jedenfalls mehr als der Unfallversicherungsträger, der für Schäden von Arbeitsunfällen im Rahmen der Unfallversicherung der Arbeitnehmer einsteht. 


\section{Regelungsvorschlag: Vertragskonformes privates Verhalten während der Arbeit als versicherte Tätigkeit}

I. Keine Möglichkeit der Bewältigung mithilfe anerkannter Zuordnungskriterien

\section{Keine Subsumtion unter den Versicherungstatbestand}

Es ist nicht möglich, vertragskonforme private Tätigkeiten nach geltendem Recht in den Versicherungsschutz der Beschäftigten einzubeziehen. Eine Subsumtion unter das Tatbestandsmerkmal der versicherten Tätigkeit gelingt nicht. Diese ist nach der inzwischen als gefestigte Rechtsprechung zu bezeichnenden neuen Definition des Bundessozialgerichts jede Tätigkeit des Beschäftigten, die erstens zumindest dazu ansetzt und darauf gerichtet ist, eine eigene objektiv bestehende Haupt- oder Nebenpflicht aus seinem Beschäftigungsverhältnis zu erfüllen, oder zweitens eine objektiv nicht geschuldete Handlung darstellt, der Beschäftigte aber damit bezweckte, eine vermeintliche Pflicht aus dem Beschäftigungsverhältnis zu erfüllen, oder drittens, eine Ausübung eigener, unternehmensbezogener Rechte aus der Beschäftigung darstellt. ${ }^{1129}$ Eigenwirtschaftliche Tätigkeiten während der Arbeit können auch dann nicht als geschuldete Haupt- oder Nebenpflicht bezeichnet werden, wenn ihre Durchführung vom Arbeitgeber erlaubt, gebilligt oder geduldet und damit arbeitsvertragskonform ist. Die Pflichten des Arbeitnehmers im Rahmen des Arbeitsverhältnisses umfassen die Zurverfügungstellung der Arbeitskraft als Hauptleistungspflicht sowie Kooperations-, Schutz- und Rücksichtnahmepflichten als Nebenleistungspflichten. ${ }^{1130}$ Zwar wird der betriebliche Pflichtenkreis im Rahmen der unfallversicherungsrechtlichen Beurteilung weit verstanden, und so wird etwa auch die freiwillige Auseinandersetzung mit betrieblichen Inhalten wie der Austausch unter Kollegen zu betrieblichen Themen nach Arbeitsschluss als versicherte Tätigkeiten angesehen. ${ }^{131}$ Private Anrufe, Shopping oder Surfen im Internet lassen sich jedoch nicht mehr vom Wortsinn erfassen. Sie stellen vielmehr eine Ausübung von Rechten dar. Diese ist laut der Definition des Bundessozialgerichts dann versicherte Tätigkeit, wenn es

1129 Z.B. BSG, Urt. v. 15.5.2012, E 111, 37 Rn. 27.

1130 Müller-Glöge, in: Säcker/Rixecker (Hrsg.), Münchner Kommentar zum BGB, \611 Rn. 156 m.w.N.; zu den Pflichten im Einzelnen Brötzmann, öAT 2017, S. $73,73 \mathrm{f}$.

1131 BSG, Urteil vom 14.11.2013 - B 2 U 27/12 R -, juris Rn. 13; Schafhausen/ Schäfer-Kuczinksi, ArbRAktuell 2017, S. 612, 612. 
sich um „unternehmensbezogene“ Rechte handelt. Die Bedeutung dieser Einschränkung ist bisher nicht erschöpfend behandelt worden. Als Beispiele für unternehmensbezogene Rechte werden die Teilnahme an einer Betriebswahl oder die Tätigkeit als Betriebsrat genannt. ${ }^{132}$ Da private Tätigkeiten per definitionem gerade nicht mit der beruflichen Tätigkeit im $\mathrm{Zu}$ sammenhang stehen, sind sie jedenfalls nicht unter den Begriff „unternehmensbezogen" zu subsumieren.

2. Keine Anwendbarkeit von räumlich-zeitlichen Koinzidenzregeln im Sinne eines Betriebsbanns

Ein Einbezug privater Tätigkeiten am Arbeitsplatz in den Versicherungsschutz kann weiterhin nicht damit begründet werden, dass der Arbeitgeber die tatsächliche Herrschaft über Arbeitszeit und Arbeitsort innehat, da es für das Vorliegen eines Arbeitsunfalls auf die Betriebsbezogenheit der ausgeführten Verrichtung zum Unfallzeitpunkt ankommt. Die Darstellung in Kapitel 3 sowie der Vergleich mit dem Unfallfürsorgerecht der Beamten haben gezeigt, dass die alleinige Anwendung von Koinzidenzregeln räumlich-zeitlicher Natur im Unfallversicherungsrecht nicht sachgerecht ist.

Etwas anderes ergibt sich auch nicht daraus, dass mit $\$ 10$ SGB VII auch in der Unfallversicherung eine Regelung besteht, die die Zuordnung eines Unfalls zur Betriebssphäre hauptsächlich von räumlich-zeitlichen Kriterien abhängig macht. Die „Betriebsbann“ genannte Regelung des $₫ 10$ SGB VII stellt eine Schutzbereichserweiterung zu $\$ 8$ SGB VII dar. ${ }^{1133}$ Sie gilt ausdrücklich nur für die See- und Binnenschifffahrt. ${ }^{1134}$ In sachlicher Hin-

1132 Schlaeger, jurisPR-SozR 24/2012, Anm. 3.

1133 Keller, in: Hauck/Noftz/ders. (Hrsg.), SGB VII, $\$ 7$ Rn. 3a. $\$ 10$ Abs. 1 SGB VII lautet: „In der See- und Binnenschifffahrt sind Versicherungsfälle auch Unfälle infolge von Elementarereignissen (1.), der einem Hafen oder dem Liegeplatz eines Fahrzeugs eigentümlichen Gefahren (2.), der Beförderung vom Land zum Fahrzeug oder vom Fahrzeug zum Land (3.).“ Das Wort „auch“ bezieht sich dabei auf die Regelung des $\$ 7$ Abs. 1 SGB VII, nach dem Versicherungsfälle Arbeitsunfälle und Berufskrankheiten sind.

1134 Die See-Unfallversicherung hatte sich aus historischen Gründen zunächst getrennt zur allgemeinen Unfallversicherung entwickelt. Es bestanden bereits seit dem 17. Jahrhundert sog. Schiffergesellschaften, die die Fürsorge für Berufsgenossen privat übernahmen; im 18. Jahrhundert kam eine gesetzlich auferlegte „Reederfürsorge“ hinzu, vgl. Dieter, in: Schimmelpfennig (Hrsg.), Grundsatzfragen der sozialen Unfallversicherung, S.119, 120, bevor am 13.7.1987 das See-Unfallversicherungsgesetz in Kraft trat (RGBl 1887, 182). 
sicht werden alle Unfälle umfasst, die sich innerhalb des örtlich festgelegten Bereichs der Nr. 1 und 2 ereignen und zusätzlich durch eine betriebsspezifische Gefahr bedingt wurden. ${ }^{1135} \$ 10$ Abs. 1 Nr. 1 SGB II definiert auch höhere Gewalt als betriebsspezifische Gefahr, da Beschäftigte auf einem Schiff oder am Hafen dieser in hohem Maße ausgesetzt sind. ${ }^{1136} \mathrm{Im}$ Anwendungsbereich von $\ 8$ SGB VII sind durch solche Elementarereignisse ausgelöste Unfälle dagegen nicht als Arbeitsunfälle zu sehen, da sie allgemeines Lebensrisiko darstellen und nicht durch die zum Unfallzeitpunkt ausgeführte versicherte Tätigkeit wesentlich verursacht wurden. ${ }^{1137}$

Dass in der See- und Binnenschifffahrt ein weiterer Schutzbereich als in der allgemeinen Unfallversicherung besteht, begründet sich aus der engen Verbindung von Arbeit, Wohnen und Freizeit, die die Berufswelt der in diesen Branchen Beschäftigten prägt. ${ }^{1138}$ Das Schiff ist Arbeits- und Wohnstätte zugleich, die Lebens- und Arbeitsverhältnisse sind wegen des Platzmangels sehr beengt. ${ }^{1139}$ Der Betriebsbann soll den spezifischen Risiken gerecht werden, denen diese Beschäftigten ausgesetzt sind. ${ }^{1140}$ Diese spezifischen Risiken ragen wegen der Verquickung von Arbeits- und Wohnstätte in den privaten Bereich der dort Beschäftigten hinein. ${ }^{1141}$ Außerdem befindet sich der Beschäftigte aufgrund seines Berufes auch in seiner arbeitsfreien Zeit ständig in Arbeitsbereitschaft: Auch wer gerade seine eigentlich ar-

Zur Entstehungsgeschichte dieses Gesetzes vgl. Wickenhagen, Geschichte der gewerblichen Unfallversicherung, S. $54 \mathrm{ff}$. Die "See- und Binnenschifffahrt“ ist dabei im Gesetz nicht definiert. Sie ist vom Schutzzweck des $₫ 10$ SGB VII her zu bestimmen und damit dann zu bejahen, wenn es sich bei der Beschäftigung des grundsätzlich Versicherten um eine im Bereich des Betriebes von Seeschiffen handelt, gleichgültig, in welchem Fahrgebiet oder zu welchem Zweck das Schiff betrieben wird, Marschner, in: Rolfs/Giesen et al. (Hrsg.), BeckOK Sozialrecht, $\mathbb{S} 10$ SGB VII Rn.3. Zu näheren Voraussetzungen der Seefahrt vgl. Schmitt, SGB VII, $\mathbb{\$} 10$ Rn. 4.

1135 Schmitt, SGB VII, $\mathbb{1} 10$ Rn. 7; Rapp, in: Becker/Franke et al. (Hrsg.), SGB VII, $\$ 10$ Rn. 8.

1136 Schmitt, SGB VII, $\mathbb{S} 10$ Rn. 5; Rapp, in: Becker/Franke et al. (Hrsg.), SGB VII, $\$ 10$ Rn. 5 f.

1137 Schmitt, SGB VII, $\mathbb{1} 10$ Rn. 5.

1138 Marschner, in: Rolfs/Giesen et al. (Hrsg.), BeckOK Sozialrecht, $\$ 10$ SGB VII vor Rn. 1.

1139 Keller, in: Hauck/Noftz/ders. (Hrsg.), SGB VII, $\mathbb{1} 10$ Rn. 1.

1140 Marschner, in: Rolfs/Giesen et al. (Hrsg.), BeckOK Sozialrecht, $\mathbb{} 10$ SGB VII Rn. 2; Holstraeter, in: Knickrehm/Kreikebohm/Waltermann (Hrsg.), Kommentar zum Sozialrecht, $\mathbb{S} 10$ SGB VII Rn. 1; BSG, Urt. v. 14.12.1967 - 2 RU 55/64 -, juris Rn. 20.

1141 Rapp, in: Becker/Franke et al. (Hrsg.), SGB VII, $\mathbb{1} 10$ Rn. 1. 
beitsfreie Zeit unter Deck verbringt, muss bei Bedarf sofort einspringen. ${ }^{1142}$ Die hier angesprochene und der Anwendung des Betriebsbanns zugrunde gelegte Untrennbarkeit von Arbeit, Wohnen und Freizeit ist zwar vergleichbar mit den dargestellten Folgen der Entgrenzung der Arbeit. Die „Work-Life-Integration“ bzw. das „Corporate Life“ und das Phänomen der ständigen Erreichbarkeit kommen in ihrer Bedeutung aber nicht an die Situation von Beschäftigten wie Seeleuten heran, denen ein Verlassen des Arbeitsplatzes von vornherein nicht möglich ist. Ein Seefahrer ist, soweit sich sein Schiff auf See befindet, örtlich sehr stark eingeschränkt und den Verhältnissen an Bord vollkommen ausgeliefert. Solche Verhältnisse sind am ehesten noch mit denen von Beschäftigten vergleichbar, die auf ihrer Betriebsstätte tatsächlich auch wohnen, wie etwa Hausangestellte oder Berufskraftfahrer. Selbst auf diese ist der Betriebsbann jedoch nicht weiterentwickelt worden, da die Relevanz der versicherten Tätigkeit nicht überwunden werden konnte. ${ }^{1143}$ Für sie besteht auch keine Notwendigkeit, da die Fälle der betriebsspezifischen Gefahr durch die anerkannte Fallgruppe der „besonderen Betriebsgefahr“ bereits abgedeckt sind. ${ }^{1144}$

2. Betriebssport, Gemeinschaftsveranstaltungen, unerhebliche Unterbrechungen

Auch die im Rahmen des $₫ 8$ Abs. 1 SGB VII anerkannten Fallgruppen, nach denen eigentlich als nicht beruflich zu qualifizierende Tätigkeiten unter Unfallversicherungsschutz stehen, ermöglichen keine Subsumtion der genannten privaten Tätigkeiten. Das Surfen im Internet oder die private Kommunikation ist weder mit den Fällen des Betriebssports noch der Gemeinschaftsveranstaltungen vergleichbar und zumeist auch nicht als unerhebliche Unterbrechung zu qualifizieren.

1142 BSG, Urt. v. 25.5.1961, E 14, 197, 200 ff. Rn. 18 m.w.N.

1143 Vollmar, Sozialversicherung 1963, S. 184, 185. Einen „Versicherungsbann“ für Berufskraftfahrer hatte das LSG Baden-Württemberg, Urt. v. 28.3.1962 - L 2 Us 1270/61 -, juris (Kurztext), Breith. Slg. 51 (1962), 974 ff. (Langtext) für die gesamte Ausdehnung des Straßenbereichs angenommen. Auch Hausangestellte stehen nicht im gesamten Arbeitsbereich unter Versicherungsschutz, wie das BSG anhand des Falles einer Haushälterin eines Klerikers entschied, die auf dem Weg zum Füttern ihrer eigenen Hunde und des Hundes des Arbeitgebers verunfallt war, Beschl. v. 5.8.1993 - 2 BU 37/93 -, juris Rn. 5.

1144 S.o. Kap. 1 D. III. 2. C. ee. 
Unfälle, die sich während des Betriebssports ereignen, sind dann Arbeitsunfälle, wenn die sportliche Aktivität entweder selbst betrieblichen Belangen oder dem Ausgleich von betrieblichen Belastungen dient. ${ }^{1145}$ Maßgeblich zur Zuordnung des Betriebssports zum Unternehmensrisiko ist damit zum einen, dass das Unternehmen von der Ausübung des Sportes profitiert, da der Arbeitnehmer dadurch ausgeglichener wird, und zum anderen, dass die Arbeit für das Unternehmen gerade erst den körperlichen Ausgleichsbedarf geschaffen hat. ${ }^{1146}$ Diese Schutzzwecke werden von privaten Tätigkeiten am Arbeitsplatz nicht erfüllt.

Soweit die Teilnahme an einer betrieblichen Gemeinschaftsveranstaltung versichert ist, erfolgt die Zurechnung zum Betriebsrisiko des Arbeitgebers aufgrund des unternehmensdienlichen Zwecks der Veranstaltung, die Verbundenheit der Beschäftigten untereinander oder der Beschäftigten mit der Unternehmensleitung zu fördern. ${ }^{1147}$ Private Tätigkeiten am Arbeitsplatz sind nur insofern vergleichbar, als die Akzeptanz oder ausdrückliche Erlaubnis zur Durchführung nicht beruflicher Handlungen die Verbundenheit mit dem Betrieb als solchen stärken kann, weil die Arbeitsbedingungen als gut empfunden werden. Eine solche Stärkung der Verbundenheit zum Betrieb ist aber von den Fallgruppen der Gemeinschaftsveranstaltung gerade nicht umfasst, wie die Rechtsprechung zu sog. IncentiveVeranstaltungen zeigt. ${ }^{1148}$

Eine unerhebliche Unterbrechung stellt die private Tätigkeit nur dann dar, wenn sie "quasi im Vorbeigehen" und "ganz nebenher" zur eigentlichen beruflichen Tätigkeit geschieht, wobei ihre zeitliche Dauer nicht einmal fünf Minuten betragen darf. ${ }^{1149} \mathrm{Zu}$ den vom Arbeitgeber erlaubten privaten Tätigkeiten am Arbeitsplatz dürften im Normalfall aber auch sol-

1145 BSG, Urt. v. 2.7.1996 - 2 RU 32/95 -, juris Rn. 18; v. 26.10.2004 - B 2 U 38/03 R -, juris Rn. 15; Legerlotz, ArbRB 2011, S. 350, 351; Schlaeger/Lenger, DB 2010, S. 2280, 2280 m.w.N.; Marschner, in: Rolfs/Giesen, et al. (Hrsg.), BeckOK Sozialrecht, $\$ 8$ SGB VII Rn. 18; Krasney, KrV 2014, S. 104, 106 f.; Köhler, VSSR 2016, S.31, 35. Eingehend zur Betriebsdienlichkeit der Teilnahme von Beschäftigten am Betriebssport Trasker, Betriebssport, S. $10 \mathrm{ff}$.

1146 BSG, Urt. v. 99.8.1973 - 2 RU 52/71 -, juris Rn. 22; Ricke, in: Körner/Leitherer et al. (Hrsg.), Kasseler Kommentar zum Sozialversicherungsrecht, $\mathbb{8} 8$ SGB VII Rn. 61 m.w.N. Zu den Grenzen des Betriebssports Bieresborn, SGb 2007, S. 472, $476 \mathrm{f}$.

1147 S.o. Kap. 1 D. IV.3.

1148 BSG, Urt. v. 7.12.2004 - B 2 U 47/03 -, juris Rn. 15.

1149 Eine genaue Zeitgrenze besteht nicht, fünf Minuten ist aber das maximale, was als noch unerheblich vertreten wird. Genauer zur Fallgruppe der unerheblichen Unterbrechung s.o. Kap. 1 D. III. 2. c. bb. 
che Handlungen gehören, die etwas länger andauern. Die sozial akzeptierten Anrufe bei der Familie etwa, das Durchführen von Online-Shopping oder das Nutzen von Social-Media-Portalen dürften zumeist nicht in unter fünf Minuten zu erledigen sein. Unpassend ist die Fallgruppe zudem vor allem dann, wenn der Arbeitgeber private Tätigkeiten am Arbeitsplatz ausdrücklich wünscht, um die Attraktivität seines Unternehmens zu steigern.

\section{Regelungsvorschlag: Privates Verhalten während der Arbeit als versicherte Tätigkeit}

Vertragskonforme private Tätigkeiten am Arbeitsplatz müssen, da sie nicht mithilfe bereits anerkannter Kasuistik erfasst werden können, durch eine Erweiterung des Schutzbereichs in die Unfallversicherung einbezogen werden. Mangels einer Möglichkeit, sie mit den bisher zur Verfügung stehenden Zuordnungsmechanismen dem Versicherungsschutz zu unterstellen, ist eine Änderung des Gesetzestexts angezeigt. Um den Grundprinzipien der Unfallversicherung gerecht zu bleiben, muss diese Fortentwicklung an die tatsächliche Verrichtung zum Unfallzeitpunkt anknüpfen, sodass nicht auf eine räumlich-zeitliche Koinzidenzregel zurückgegriffen werden kann. Da eigenwirtschaftliche Handlungen gerade nicht den Versicherungsschutz begründende Tätigkeiten sind, ist es sinnvoll, sie nicht in die Definition der versicherten Tätigkeit des $₫ 8$ Abs. 1 SGB VII einzufügen, sondern sie als zusätzlichen Spezialfall in den Katalogtatbestand in $\$ 8$ Abs. 2 SGB VII zu verorten. ${ }^{1150}$ Bei der Formulierung der umfassten Tätigkeiten ist darauf zu achten, dass der Unfallversicherungsschutz nicht über Gebühr ausgedehnt wird. Einerseits können nicht alle privaten Tätigkeiten am Arbeitsplatz erfasst werden, da die Zuordnung sonst faktisch allein auf den Ort der Ausübung gestützt würde, was einem systemwidrigen Betriebsbann gleichkäme. ${ }^{1151}$ Andererseits müssen alle Verrichtungen umfasst sein, deren Durchführung aufgrund ihrer sozialen Akzeptanz im Allgemeinen und der des Arbeitgebers im Besonderen, vergleichbar dem gedanklichen Abschweifen oder der Erfüllung von Grundbedürfnissen, zum Wesen des Nutzens der menschlichen Arbeitskraft gehört. Ansatzpunkt muss daher

1150 Zur Einordnung der Katalogtatbestände als Spezialfälle Marschner, in: Rolfs/ Giesen et al. (Hrsg.), BeckOK Sozialrecht, $\mathbb{\$} 8$ SGB VII Rn. 46; Giesen, Arbeitsunfall und Dienstunfall, S. 113 m.w.N.

1151 Vgl. nur Giesen, Arbeitsunfall und Dienstunfall, S. 32 m.w.N.; Becker, SGb 2007, S. 721, 722. 
sein, dass die privaten Tätigkeiten nicht über das konkludent oder ausdrücklich erlaubte, gebilligte oder geduldete Maß hinausgehen.

$\$ 8$ Abs. 2 SGB VII ist daher wie folgt zu ergänzen: ${ }^{1152}$

\$ 8 Abs. 2 SGB VII:

Versicherte Tätigkeiten sind auch (1.-6.)

7. private Verrichtungen, die die versicherte Tätigkeit im Sinne des Absatz 1 unterbrechen, soweit ihre Durchführung im konkreten Arbeitsverhältnis erlaubt, gebilligt oder geduldet ist.

Auf die Unterbrechung der eigentlichen versicherten Tätigkeit in Abs. 1 wird dabei Bezug genommen, um zu verhindern, dass Unfallversicherungsschutz für private Tätigkeiten bereits durch die Anwesenheit am Arbeitsplatz ausgelöst werden kann, auch wenn keinerlei unternehmensdienliche Tätigkeit intendiert ist. Der Einbezug von Erlaubnis, Billigung und Duldung ist notwendig, um alle Formen der Übereinkunft von Arbeitgeber und Arbeitnehmer zu umfassen. Während die Erlaubnis ein im Vorhinein gegebenes Einverständnis meint, stellt eine Billigung eine im Nachhinein gegebene Genehmigung dar. Beide Formen können sowohl stillschweigend als auch ausdrücklich auftreten. Genauso müssen private Tätigkeiten versichert sein, von denen der Arbeitgeber weiß und die er hinnimmt, ohne Vorkehrungen zu treffen, dass sie zukünftig unterbleiben. ${ }^{1153} \mathrm{Da}$ die Formulierung den Ort der Tätigkeit nicht benennt, sind derartige private Tätigkeiten unabhängig davon versichert, ob der Beschäftigte gerade am Betriebssitz, am häuslichen oder am mobilen Arbeitsplatz tätig ist.

Dieser Regelungsvorschlag führt dazu, dass zukünftig auch die Nutzung der Toilette sowie die Aufnahme von Nahrung dem Versicherungsschutz unterstehen. Dies widerspricht der bisher herrschenden Ansicht, nur die Wege dorthin, nicht aber die Vornahme der Handlungen selbst seien versichert, da Letztere originär privater Natur seien. ${ }^{154}$ Dennoch folgt der Schutz auch dieser Verrichtungen aus den dargestellten Überlegungen zu den Strukturprinzipien und Schutzzwecken der Unfallversicherung. Wege zur Nahrungsaufnahme bzw. zur Toilette stehen, wie gesehen, aus zwei

1152 Ergänzungsvorschläge sind fett markiert.

1153 Zu den Begriffen der Billigung und der Duldung Schaub, Arbeitsrechts-Handbuch, $\mathbb{} 69 \mathrm{Rn} .18 \mathrm{f}$. Der Unfallversicherungsschutz für private Tätigkeiten der Beschäftigten bleibt damit noch immer hinter dem der Beamten zurück, nach deren Unfallsicherungsregime private Tätigkeiten am Dienstort zur Dienstzeit nur dann keine Einordnung als Dienstunfall auslösen, wenn sie den Interessen des Dienstherrn erkennbar widersprechen, s.o. Kap. 4 B. I.

1154 S.o. Kap. 3 A. VI. 
Gründen unter dem Schutz der Unfallversicherung: zum einen, weil die Nahrungsaufnahme bzw. die Verrichtung der Notdurft für die Erhaltung der Arbeitsfähigkeit notwendig und deswegen betriebsdienlich ist; zum anderen, weil der räumliche Verbleib des Beschäftigten dabei durch seine Eingliederung in den Betrieb geprägt ist. ${ }^{1155}$ Vor diesem Hintergrund leuchtet es schlichtweg nicht ein, wieso die Essensaufnahme in der Betriebskantine und die Verrichtung der Notdurft auf der betriebseigenen Toilette nicht unter Unfallversicherungsschutz stehen. ${ }^{1156}$

Da Nahrungsaufnahme und Nutzung der Sanitäreinrichtung dem Aufrechterhalten der Arbeitskraft dienen, deren Zurverfügungstellung Hauptleistungspflicht des Beschäftigten im arbeitsvertraglichen Synallagma ist, sind sie zumindest auch unternehmensdienlich. Da der Umfang der betriebsdienlichen Intention bereits genügt, um sogar den Weg zur Erreichung des Handlungsziels unter Versicherungsschutz zu stellen, muss sie erst recht ausreichend sein, um einen sachlichen Zusammenhang der Handlung selbst mit der versicherten Tätigkeit herzustellen.

Wenn die Wege zu diesen Orten versichert sind, weil sich der Arbeitnehmer in die vom Arbeitgeber kontrollierte Risikosphäre begeben hat und sich nur deshalb, weil er in einen fremden Betrieb und dessen Organisation eingegliedert ist, auf diesen Wegen befindet, so gilt dasselbe erst recht für den Aufenthalt in den jeweiligen Räumen selbst. ${ }^{1157} \mathrm{Da}$ Telos des Einbezugs in den Schutzbereich in diesem Fall gerade ist, den Arbeitnehmer vor den Risiken zu schützen, die sich aus seinem Verbleib in den vom Arbeitgeber beherrschten Räumlichkeiten ergeben, so ist ein Schutz aller sich dort ereignenden Unfälle die natürliche Konsequenz aus der Umsetzung dieses Schutzzwecks. Aus dem Schutz der Zurücklegung der Strecke wegen ihres angestrebten Ziels folgt a minori ad maius auch der Schutz der intendierten Handlung selbst.

Ebenso wie fahrlässiges und sogar verbotswidriges Verhalten des Beschäftigten einen Arbeitsunfall nicht ausschließen, weil Unachtsamkeit und sogar vorsätzliches Missachten von Regeln zu den als normal angesehenen menschlichen Eigenschaften gehören und deswegen als dem Betriebsmittel Mensch immanentes Risiko anzusehen sind, umfasst die Betriebsrisikolehre auch die Erfüllung von Grundbedürfnissen und das Abschweifen des Ar-

1155 Ebd.

1156 Für eine Ausweitung des Unfallversicherungsschutzes auf den Aufenthalt in der Kantine bereits Ricke, in: Körner/Leitherer et al. (Hrsg.), Kasseler Kommentar zum Sozialversicherungsrecht, $\$ 8$ SGB VII Rn. 74 m.w.N.; Schmitt, SGB VII, $₫ 8$ Rn. 79.

1157 So z.B. Spellbrink, NZS 2016, S. 527, 530. 
beitnehmers von der geschuldeten Tätigkeit in sozial akzeptiertem Maße. Der Einbezug in den Schutzbereich der gesetzlichen Unfallversicherung durch einen neu einzuführenden $\$ 8$ Abs. 2 Nr. 7 SGB VII ist daher sachgerecht. 


\section{Schluss}

\section{A. Zusammenfassung der Ergebnisse}

Die Reichweite des Unfallversicherungsschutzes de lege lata entspricht nicht den Anforderungen der Lebenswirklichkeit der Beschäftigten und Unternehmer, da die zu ihrer Bestimmung notwendige Abgrenzung zwischen Arbeits- und Privatsphäre der Beschäftigten anhand von Kriterien vorgenommen wird, die die moderne Arbeitswelt nicht abzubilden vermögen.

Im Rahmen des Zurechnungsprinzips der Betriebsrisikolehre wird bei der Auslegung des $₫ 8$ SGB VII die Frage nach der Beherrschbarkeit des Unfallrisikos durch den Unternehmer ins Zentrum gestellt. Damit ist in den meisten Fällen gemeint, ob der Arbeitgeber die physischen Eigenheiten des Unfallortes sowie die zeitlichen Rahmenbedingungen des schädigenden Ereignisses de facto kontrollieren konnte. Diese Auslegungspraxis wurzelt in der Entstehungsgeschichte der Unfallversicherung. Die Überlegungen zu ihrer Einführung waren darauf gerichtet, den fordistisch-tayloristischen „Normalarbeiter" der Industrialisierung vor den Folgen der sehr reellen Gefahren zu schützen, die sich aus dessen Umgang mit teilweise unausgereiften und störungsanfälligen Maschinen in den Fabriken der Epoche ergaben. Die erlebte Welt dieses in den Blick genommenen Beschäftigtentypus war weitestgehend problemlos in einen privaten und einen beruflichen Bereich zu trennen. Der Arbeitgeber herrschte über den Betrieb, indem er für die Auswahl, Anschaffung und Wartung der Maschinen, die Gestaltung des Arbeitsbereichs, die Einteilung der Arbeiter, die Einführung von Sicherheitsmaßnahmen, das Bestimmen über den organisatorischen Ablauf der Produktion sowie die Einteilung und Unterweisung der Arbeiter verantwortlich war; der Beschäftigte dagegen war Herr seiner Privatsphäre. Darüber hinaus befand sich der Arbeitnehmer im Regelfall nur während seiner Arbeitszeit im Dienst; die Zeit davor und danach blieb von Arbeit frei. Ein Verschwimmen der Grenzen zwischen beiden Bereichen kam nicht in einem Maße vor, das die generelle Möglichkeit der Abgrenzung nach tatsächlichen Herrschaftssphären infrage gestellt hätte.

Anderes gilt inzwischen für eine Vielzahl von Beschäftigungsverhältnissen, die im Rahmen der Tertiarisierung und der Digitalisierung dem Prozess der Entgrenzung unterlagen und noch unterliegen. Geistige Arbeit drängt manuelle Leistungserbringung zurück. Sie findet häufig nicht 
mehr auf dem eigentlichen Betriebsgelände, sondern entweder im Homeoffice oder im Rahmen von mobiler Arbeit an einem nicht vom Arbeitgeber, sondern vom Beschäftigten bestimmten Ort statt, den der Arbeitgeber eventuell nicht einmal kennt und über den er daher auch keine Verfügungsgewalt ausübt. Auch die zeitliche Trennung der beiden Lebensbereiche ist weitgehend aufgehoben, da die meisten Arbeitnehmer zum einen auch außerhalb ihrer regulären Arbeitszeiten für berufliche Aufgaben zur Verfügung stehen und zum anderen auch während der Arbeitszeit privat kommunizieren. Dies hat zur Folge, dass die Anwendung der bestehenden Abgrenzungsparameter eine Erosion des Unfallversicherungsschutzes der Beschäftigten bedeutet. So ist nach geltendem Recht der Weg zur Unterbringung eines Kindes in fremde Obhut wegen der beruflichen Beschäftigung der Sorgeperson nicht geschützt, wenn diese Person ihren Ort der Tätigkeit in ihrer Wohnung hat, von der aus sie das Kind in die Betreuung bringt. Auch Wege zur Nahrungsaufnahme, die - gehen sie vom Betriebsgelände aus - stets unter Unfallversicherungsschutz stehen, werden bei entgrenzt arbeitenden Beschäftigten nach herrschender Ansicht zum Privatrisiko gezählt. Insofern verstößt der aktuelle Rechtsstand nicht nur gegen die Grundprinzipien der Unfallversicherung, sondern wegen der ungerechtfertigten Ungleichbehandlung von im Homeoffice tätigen Personen auch gegen Art. 3 Abs. 1 GG. Darüber hinaus war bisher nicht geklärt, ob Tätigkeiten mit gespaltener Handlungstendenz im Homeoffice oder an einem selbstbestimmten Arbeitsort im Gegensatz zu solchen am arbeitgeberbestimmten Ort der Tätigkeit überhaupt versicherte Tätigkeiten sein können. Gleiches gilt für Betriebswege innerhalb des häuslichen Bereichs, die nach bis jüngst überwiegender Ansicht nur dann versicherte Tätigkeiten sein sollten, wenn sie sich an überwiegend beruflich genutzten Orten ereigneten. In Fällen mobiler Arbeit wird noch immer weithin eine Einschränkung des Versicherungsschutzes aufgrund mangelnder Beherrschbarkeit durch den Arbeitgeber vertreten. Zudem müsste die vereinbarte Rufbereitschaft schon allein wegen ihres aus beruflichen Gründen erhöhten Risikopotenzials versicherte Tätigkeit sein, ist aber nach derzeitiger allgemeiner Meinung der Privatsphäre zuzurechnen.

Dem Schutzzweck der gesetzlichen Unfallversicherung wird durch diese Einschränkung des Versicherungsschutzes entgrenzt Beschäftigter nicht genüge getan. Ihr Ziel ist es, Beschäftigte vor den Folgen von Unfällen zu schützen, die sie im Zusammenhang mit ihrer beruflichen Tätigkeit erleiden. In dem Maße, in dem sich die Risikostrukturen der Beschäftigungsverhältnisse verändert haben, muss auch der Unfallversicherungsschutz angepasst werden. Dies entspricht sowohl den Interessen der zu schützenden Arbeitnehmer als auch denen des Arbeitgebers, da dieser seine Beschäftig- 
ten nur bei angemessenem Unfallversicherungsschutz zu den für ihn vorteilhaften entgrenzten Arbeitsgestaltungen motivieren können dürfte. Die aus entgrenzten Arbeitsverhältnissen entstehenden Unfallrisiken müssen umfasst sein, um dem Telos der Unfallversicherung zu entsprechen. Dies bedeutet, dass die Abgrenzung zwischen Arbeits- und Privatunfall nicht mehr an örtlichen und zeitlichen Faktoren festgemacht werden darf, sondern sich zum einen verstärkt an der Natur der zum Unfallzeitpunkt ausgeführten Tätigkeit orientieren muss, zum anderen aber im Rahmen der Betriebsrisikolehre die Besonderheiten des Einsatzes von im Wesentlichen geistiger menschlicher Arbeitskraft als Betriebsmittel betont werden müssen. In der Folge müssen - entgegen der gegenwärtigen Lage - auch private Tätigkeiten, die vom Arbeitgeber erlaubt, gebilligt oder geduldet sind, zum Betriebsrisiko des Unternehmers gerechnet und dem Versicherungsschutz der Unfallversicherung unterstellt werden.

Um eine teleologisch sachgerechte, verfassungskonforme und den Anforderungen der gesellschaftlichen Realität gerecht werdende Abgrenzung von Arbeits- und Privatunfall zu gewährleisten, ist in $\mathbb{8} 8$ SGB VII festzuschreiben, dass dem Unfallort bei der Prüfung des Vorliegens eines Arbeitsunfalls lediglich Indizwirkung zukommen darf, Wege zur Inobhutgabe von Kindern auch dann versichert sind, wenn sie keine Unterbrechung eines Arbeitsweges darstellen, sowie dass Rufbereitschaft und vertragskonforme private Tätigkeiten, welche die Ausübung der eigentlichen Arbeit unterbrechen, als versicherte Tätigkeiten anzuerkennen sind.

\section{B. Vorschlag zur Neufassung des $\ 8$ SGB VII}

Unter Berücksichtigung der oben vorgebrachten Regelungsvorschläge zur Fortentwicklung des Unfallversicherungsschutzes zur Bewältigung der Herausforderungen zunehmender entgrenzter Arbeit ergibt sich folgender neuer Gesamttext des $\$ 8$ SGB VII: 1158

$\$ 8$ SGB VII Arbeitsunfall

(1) Arbeitsunfälle sind Unfälle von Versicherten infolge einer den Versicherungsschutz nach $\$ 2,3$ oder 6 begründenden Tätigkeit (versicherte Tätigkeit), unabhängig davon, an welchem Ort sie sich ereignen. Unfälle sind zeitlich begrenzte, von außen auf den Körper einwirkende Ereignisse, die zu einem Gesundheitsschaden oder zum Tod führen.

1158 Die vorgeschlagenen Ergänzungen und Änderungen sind fett markiert. 
(2) Versicherte Tätigkeiten sind auch

1. das Zurücklegen des mit der versicherten Tätigkeit zusammenhängenden unmittelbaren Weges nach und von dem Ort der Tätigkeit,

2a. das Zurücklegen eines Weges, um Kinder von Versicherten ( $\$ 56$ des Ersten Buches), die mit ihnen in einem gemeinsamen Haushalt leben, wegen ihrer, ihrer Ehegatten oder ihrer Lebenspartner beruflichen Tätigkeit fremder Obhut anzuvertrauen,

2b. das Zurücklegen des von einem unmittelbaren Weg nach und von dem Ort der Tätigkeit abweichenden Weges, um mit anderen Berufstätigen oder Versicherten gemeinsam ein Fahrzeug zu benutzen,

3. das Zurücklegen des von einem unmittelbaren Weg nach und von dem Ort der Tätigkeit abweichenden Weges der Kinder von Personen ( $\$ 56$ des Ersten Buches), die mit ihnen in einem gemeinsamen Haushalt leben, wenn die Abweichung darauf beruht, dass die Kinder wegen der beruflichen Tätigkeit dieser Personen oder deren Ehegatten oder deren Lebenspartner fremder Obhut anvertraut werden,

4. das Zurücklegen des mit der versicherten Tätigkeit zusammenhängenden Weges von und nach der ständigen Familienwohnung, wenn die Versicherten wegen der Entfernung ihrer Familienwohnung von dem Ort der Tätigkeit an diesem oder in dessen Nähe eine Unterkunft haben,

5. das mit einer versicherten Tätigkeit zusammenhängende Verwahren, Befördern, Instandhalten und Erneuern eines Arbeitsgeräts oder einer Schutzausrüstung sowie deren Erstbeschaffung, wenn diese auf Veranlassung der Unternehmer erfolgt,

6. das vereinbarte Zurverfügungstehen zur alsbaldigen Arbeitsaufnahme außerhalb der Arbeitszeit (Rufbereitschaft),

7. private Verrichtungen, die die versicherte Tätigkeit im Sinne des Absatz 1 unterbrechen, soweit ihre Durchführung im konkreten Arbeitsverhältnis erlaubt, gebilligt oder geduldet ist.

(3) Als Gesundheitsschaden gilt auch die Beschädigung oder der Verlust eines Hilfsmittels. 


\section{Literaturverzeichnis}

Albrecht, Rüdiger, Die Einrichtung von Tele- und Außenarbeitsplätzen - Rechtliche und personalpolitische Anforderungen, NZA 1996, S. 1240-1245.

Andersen, Arne, Vom Glück, einen Unfall zu erleiden. Unfallversicherung und arbeitsbedingte Erkrankungen in der Chemieindustrie, in: Machtan, Lothar (Hrsg.), Bismarcks Sozialstaat, Beiträge zur Geschichte der Sozialpolitik und zur sozialpolitischen Geschichtsschreibung, Frankfurt 1994, S. 207-258.

Angermaier, Alexander, Unfallversicherungsschutz bei häuslicher Arbeitsstätte. Anmerkung zu BSG v. 05.07.2016 B 2 U 5/15 R, jM 2017, S. 69-71.

Arnold, Daniel/Steffes, Susanne/Wolter, Stefanie (Hrsg.), Monitor - Mobiles und entgrenztes Arbeiten: Aktuelle Ergebnisse einer Betriebs- und Beschäftigtenbefragung, ZEW-Gutachten und Forschungsberichte, 2015.

Aumann, Annemarie/Hack, Melanie, Wahlarbeitszeit und Arbeitszeitflexibilisierung. Modelle einer selbstbestimmten Erwerbsbiografie in Deutschland und Norwegen, ZESAR 2016, S. 266-276.

Ayaß, Wolfgang, Wege zur Sozialgerichtsbarkeit: Schiedsgerichte und Reichsversicherungsamt bis 1945, in: Masuch, Peter/Spellbrink, Wolfgang/Becker, Ulrich/Leibfried, Stephan (Hrsg.), Grundlagen und Herausforderungen des Sozialstaats. Denkschrift 60 Jahre Bundessozialgericht. Eigenheiten und Zukunft von Sozialpolitik und Sozialrecht, Band 1, Berlin 2014, S. 265-282.

Badura, Bernhard/Ducki, Antje/Schröder, Helmut/Klose, Joachim/Meyer, Markus (Hrsg.), Fehlzeiten-Report 2012, Schwerpunktthema: Gesundheit in der flexiblen Arbeitswelt: Chancen nutzen, Risiken minimieren, Berlin 2012.

Baeck, Ulrich/Loesler, Annette, Neue Entwicklungen im Arbeitszeitrecht, NZA 2005, 247-250.

Baldschun, Katie, Solidarität und soziales Schutzprinzip in der gesetzlichen Unfallversicherung, Die Anwendbarkeit des Europarechts auf mitgliedstaatliche Systeme der sozialen Sicherung am Beispiel der Berufsgenossenschaften, Berlin 2008.

Balke, Rüdiger, Die Beurteilung der Unfallkausalität eines Bandscheibenvorfalls, SVR 2009, S. 58-63.

Bamberger, Heinz-Georg/Roth, Herbert/Hau, Wolfgang/Poseck, Roman (Hrsg.) Beck'scher Online-Kommentar zum BGB, 43. Ed., Stand: 15.6.2017, München 2017 (zitiert als: Bearbeiter, in: Bamberger/Roth/Hau/Poseck (Hrsg.), BeckOK BGB.).

Barta, Heinz, Kausalität im Sozialrecht, Entstehung und Funktion der sogenannten Theorie der wesentlichen Bedingung: Analyse der grundlegenden Judikatur des Reichsversicherungsamtes in Unfallversicherungssachen (1884-1914). Der Weg vom frühen zivilen, industriell/gewerblichen Haftpflichtrecht zur öffentlichrechtlichen Gefährdungshaftung der Arbeiter(unfall)versicherung, Berlin 1983. 
Battis, Ulrich, BBG: Bundesbeamtengesetz Kommentar, 4. Aufl., München 2009 (zitiert als: Battis, BBG).

Battis, Ulrich/Grigoleit, Klaus Joachim/Hebeler, Timo, Entwicklung des Beamtenrechts in den Jahren 2010--2015, NVwZ 2016, S. 194-201.

Bauer, Wilhelm/Rief, Stefan/Kelter, Jörg/Haner, Udo-Ernst, Arbeitswelten 4.0, Wie wir morgen arbeiten und leben = Working environments 4.0, Stuttgart 2012 (zitiert als: Bauer/Rief et al., Arbeitswelten 4.0).

Becker, Harald/Franke, Edgar/Molkentin, Thomas (Hrsg.), SGB VII, Lehr- und Praxiskommentar, 3. Aufl., Baden-Baden 2011.

Becker, Peter, Der Arbeitsunfall, SGb 2007, S. 721-729.

Becker, Peter, Die wesentliche Bedingung - aus juristischer Sicht, MedSach 2007, S. 92-97.

Becker, Peter, Unterschiedliche Kausalitätsprüfungen im Zivilrecht und im Sozialrecht am Beispiel neuerer BGH-Urteile zum Sudeck-Syndrom, MedSach 2011, S. 32-36.

Becker, Peter, Zur Unfallkausalität, SGb 2012, S. 691-698.

Becker, Peter/Burchardt, Klaus/Krasney, Otto Ernst/Kruschinsky, Michael, Gesetzliche Unfallversicherung (SGB VII), 13. Aufl., Sankt Augustin 2016.

Becker, Ulrich, Der Finanzausgleich in der gesetzlichen Unfallversicherung. Zur Lastenverteilung zwischen den gewerblichen Berufsgenossenschaften und ihrer Reform, Baden-Baden 2004.

Becker, Ulrich, The Challenge of Migration to the Welfare State, in: Benvenisti, Eyall Nolte, Georg (Hrsg.), The Welfare State, Globalization, and International Law, Berlin 2004, S. 1-31.

Becker, Ulrich (Hrsg.), Rechtsdogmatik und Rechtsvergleich im Sozialrecht I, Baden-Baden 2010.

Becker, Ulrich, Rechtsdogmatik und Rechtsvergleich im Sozialrecht, in: ders. (Hrsg.), Rechtsdogmatik und Rechtsvergleich im Sozialrecht I, Baden-Baden 2010, S. 11-63.

Becker, Ulrich, Das Opferentschädigungsgesetz im europäischen Kontext, in: WEISSER RING e.V. (Hrsg.), Moderne Opferentschädigung, Baden-Baden 2012, S. 7182.

Becker, Ulrich, Organisation und Selbstverwaltung der Sozialversicherung, in: von Maydell, Bernd/Ruland, Franz/Becker, Ulrich (Hrsg.), Sozialrechtshandbuch, 5. Aufl., Baden-Baden 2012, \$13, S. 673-694.

Becker, Ulrich, Unfallversicherungsrecht, in: Ehlers, Dirk/Fehling, Michael/Pünder, Herrmann (Hrsg.), Besonderes Verwaltungsrecht Band 3, Kommunalrecht, Haushalts- und Abgabenrecht, Ordnungsrecht, Sozialrecht, Bildungsrecht, Recht des öffentlichen Dienstes, 3. Aufl., Heidelberg 2013, \$78, S. 958-1036.

Becker, Ulrich, Soziales Entschädigungsrecht. Bestand, Grundsätze, Neuordnung, Baden-Baden 2018.

Beckers, Markus, Die Außenhaftung des Arbeitnehmers, Konstanz 1996. 
Benz, Manfred, Der Versicherungsschutz der gesetzlichen Unfallversicherung bei Unfällen aus innerer Ursache, BB 1982, S. 1860-1866.

Benz, Manfred, Anmerkung zum Urteil des BSG vom 5.5.1998 - B 2 U 40/97 R -, SGb 1999, S. 83-85.

Benz, Manfred, Der Arbeitsunfall im Sinne des $\$ 8$ SGB VII - Notwendigkeit und Grenzen der Kasuistik, SGb 2001, S. 220-225.

Benz, Manfred, Der Betriebsweg nach $\$ 8$ Abs. 1 Satz 1 SGB VII, SGb 2003, S. 12-20.

Bereiter-Hahn (Begr.), Werner/Mehrtens, Gerhard, Gesetzliche Unfallversicherung, Berlin 2010.

Bernet, Brigitte, Vom „Berufsautomaten“ zum „flexiblen Mitarbeiter“. Die Krise der Organisation und der Umbau der Personallehren um 1970, in: Dietz, Bernhard/ Neuheiser, Jörg (Hrsg.), Wertewandel in der Wirtschaft und Arbeitswelt, Berlin 2017, S. 31-54.

Bernstein, Herbert, Schadensausgleich bei Arbeitsunfällen, Karlsruhe 1963.

Bieback, Karl-Jürgen, Mittelbare Diskriminierung der Frauen im Sozialrecht - nach EG-Recht und dem Grundgesetz -, ZIAS 1990, S. 1-33.

Bieback, Karl-Jürgen, Die mittelbare Diskriminierung wegen des Geschlechts. Ihre Grundlagen im Recht der EU und ihre Auswirkungen auf das Sozialrecht der Mitgliedstaaten, Baden-Baden 1999.

Bieresborn, Dirk, Schach, Kartfahren, Freeclimbing und Drachenfliegen - Versicherter Betriebssport in der gesetzlichen UV? - Ein Beitrag zu den Grenzen, SGb 2007, S. 472-482.

Biletzki, Gregor, Der Zeckenstich als Dienstunfall nach $₫ 31$ I 1 BeamtVG: Dienstliches oder privates Risiko?, NVwZ 2010, S. 688-691.

von Birkmeyer, Karl, Über Ursachenbegriff und Kausalzusammenhang im Strafrecht, Rectoratsrede geh. zur Feier d. 28. Febr. 1885, Rostock 1885.

Bitsch, Christian, Streikrecht für Beamte?, ZTR 2012, S. 78-85.

Bitter, Georg/Rauhut, Tilman, Grundzüge zivilrechtlicher Methodik, JuS 2009, S. 289-298.

Boecken, Winfried/Hänlein, Andreas/Kruse, Jürgen/Steinmeyer, Heinz-Dietrich (Hrsg.), Öffentliche und private Sicherung gegen soziale Risiken, Baden-Baden 2000 (zitiert als: Bearbeiter, in: Boecken/Hänlein/Kruse/Steinmeyer (Hrsg.), Öffentliche und private Sicherung gegen soziale Risiken).

Boemke, Burkhard, Privatautonomie im Arbeitsvertragsrecht, NZA 1993, S.532538.

Boemke, Burkhard/Ankersen, Per, Das Telearbeitsverhältnis - Arbeitsschutz, Datenschutz und Sozialversicherungsrecht, BB 2000, S. 1570-1573.

Boewer, Dietrich/Gaul, Björn, Aktuelles Arbeitsrecht 2016, Köln 2016.

Bokeloh, Arno, Die mittelbare Diskriminierung im Europäischen Sozialrecht - Eine Rechtsfigur mit vielen Facetten und Unschärfen -, DRV 2014, S. 88-100.

Boll, Christina, Die Arbeitsteilung im Paar. Theorien, Wirkungszusammenhänge, Einflussfaktoren und exemplarische empirische Evidenz. Expertise für den zweiten Gleichstellungsbericht der Bundesregierung, 2017. 
Brand, Arthur, Das Beamtenrecht, Die Rechtsverhältnisse der preußischen unmittelbaren und mittelbaren Staatsbeamten, 1. Aufl., Berlin 1914.

Brauer, Bianca, Das Verbot der mittelbaren Diskriminierung und seine Anwendung auf die gesetzliche Rentenversicherung, Baden-Baden 2004 (zitiert als: Brauer, Das Verbot der mittelbaren Diskriminierung).

Brenke, Karl, Heimarbeit: Immer weniger Menschen in Deutschland gehen ihrem Beruf von zu Hause aus nach, DIW Wochenbericht Nr. 8/2014, S. 131-139.

Brenke, Karl, Homeoffice - Möglichkeiten werden bei weitem nicht ausgeschöpft, DIW Wochenbericht Nr. 5/2016, S. 95-105.

Breuer, Joachim, $\mathbb{\$} 1$ Geschichtliche Entwicklung, in: Schulin, Bertram (Hrsg.), Handbuch des Sozialversicherungsrechts Band 2: Unfallversicherungsrecht, München 1996, S. 1-46.

Brink, Hans-Jürgen, Nächtlicher Heimweg vom Abendessen in einem Restaurant trotz betriebsdienlicher Tätigkeiten nicht unfallversichert. Anmerkung zu BSG, Urteil vom 18.06.2013 - B 2 U 7/12 R -, ArbRAktuell 2013, S. 632.

Brink, Hans-Jürgen, LSG Baden-Württemberg: Versicherungsschutz für Freiberufler im Home Office ist nur in engen Grenzen gewährleistet, FD-SozVR 2015, 368217.

Brinktrine, Ralf/Schollendorf, Kai (Hrsg.), Beck'scher Online-Kommentar Beamtenrecht Bund, 2. Ed., München 2016 (zitiert als: Brinktrine/Schollendorf (Hrsg.), BeckOK Beamtenrecht Bund).

Brock, Gabriele, SGB VII, in: Doetsch, Werner (Hrsg.), Handbuch zum Sozialrecht, Neuwied 2014.

Brötzmann, Ulrich, Die Nebenpflichten im Arbeitsverhältnis, öAT 201, S. 73-76.

Buchner, Herbert/Becker, Ulrich, Mutterschutzgesetz und Bundeselterngeld- und Elternzeitgesetz, Kommentar, 8. Aufl., München 2008.

Bulla, Eckart, Der Dienst- und Arbeitsunfall als Institut des Allgemeinen Verwaltungsrechts, Hamburg 1970.

Bulla, Eckart, Die gesetzliche Unfallversicherung der Arbeitnehmer. Rechtssystematische Rahmenbedingungen für eine Änderung ihrer Rentenstruktur, SGb 2007, S. 653-667.

Bulla, Gustav, Schadensausgleich im Arbeitsverhältnis, DAR 1942, S. 19-22.

Bultmann, Stefan, Neue Ansätze bei der Theorie der wesentlichen Bedingung im Sozialrecht, SGb 2016, S. 143-148.

Bundesministerium für Arbeit und Soziales, Arbeit weiter denken. Grünbuch Arbeiten 4.0, Berlin 2015 (zitiert als: BMAS, Grünbuch Arbeiten 4.0).

Bundesministerium für Arbeit und Soziales, Mobiles und entgrenztes Arbeiten. Aktuelle Ergebnisse einer Betriebs- und Beschäftigtenbefragung, Berlin 2015 (zitiert als: BMAS, Mobiles und entgrenztes Arbeiten).

Bundesministerium für Arbeit und Soziales, Wertewelten Arbeiten 4.0, Berlin 2016.

Bundesministerium für Arbeit und Soziales, Arbeit weiter denken. Weißbuch Arbeiten 4.0, Berlin 2017 (zitiert als: BMAS, Weißbuch Arbeiten 4.0). 
Burmann, Michael/Jahnke, Jürgen, Gesetzliche Unfallversicherung - Soziale Sicherung und Haftpflichtabsicherung, NZV 2014, S. 5-11.

Canaris, Claus-Wilhelm, Risikohaftung bei schadensgeneigter Tätigkeit in fremdem Interesse, RdA 19 (1966), S. 41-51.

Dahm, Dirk, Die Bedeutung der vorsätzlichen Straßenverkehrsgefährdung für die Entschädigung eines Wegeunfalls in der gesetzlichen Unfallversicherung, NZV 2014, S. 114-115.

Däubler, Wolfgang, Entgrenzung der Arbeit - ein Problem des Arbeitsrechts?, SR 2014, S. 45-65.

Deutsche Gesetzliche Unfallversicherung (Hrsg.), 125 Jahre gesetzliche Unfallversicherung - Streiflichter, Berlin 2010.

Deutscher Sozialgerichtstag (Hrsg.), Rechtsfortbildung durch die sozialgerichtliche Rechtsprechung, Verhandlungen des deutschen Sozialgerichtsverbandes, 1. Deutscher Sozialgerichtstag am 30. und 31. Mai 1972 in Kassel, Schriftenreihe des Deutschen Sozialrechtsverbandes (SDSRV), Band 10, Bonn 1973 (zitiert als: Deutscher Sozialgerichtstag (Hrsg.), SDRSV Bd. 10).

Deutschmann, Christoph, Der Weg zum Normalarbeitstag. Die Entwicklung der Arbeitszeiten in der deutschen Industrie bis 1918, Frankfurt a.M. 1985.

Devetzi,Stamatia/Janda, Constanze (Hrsg.), Freiheit - Gerechtigkeit - Sozial(es) Recht. Festschrift für Eberhard Eichenhofer, Baden-Baden 2015.

Di Fabio, Udo, Das beamtenrechtliche Streikverbot, München 2012.

Diem, Martin, Die theoretischen Grundlagen der Beschränkung der Haftung des Arbeitnehmers gegenüber dem Arbeitgeber bei schadensgeneigter Arbeit, Tübingen 1969.

Dieter, Bernd, Die historischen, tatsächlichen und rechtlichen Besonderheiten der See-Unfallversicherung, in: Schimmelpfennig, Heinrich (Hrsg.), Grundsatzfragen der sozialen Unfallversicherung, Festschrift für Dr. Herbert Lauterbach zum 60. Geburtstag, Mannheim 1961, S. 119-127.

Dietz, Bernhard/Neuheiser, Jörg (Hrsg.), Wertewandel in der Wirtschaft und Arbeitswelt, Berlin 2017, S. 31-54.

djb-Kommission für Arbeits-, Gleichstellungs- und Wirtschaftsrecht, Konzept für ein Wahlarbeitszeitgesetz, djbZ 2015, S. 121-128.

Dunkel, Wolfgang/Kratzer, Nick/Menz, Wolfgang, „Permanentes Ungenügen“ und "Veränderung in Permanenz" - Belastungen durch neue Steuerungsformen, WSI-Mitteilungen 2010, S. 357-365.

Edenfeld, Stefan, Die Fürsorgepflicht des Arbeitgebers bei Auslandseinsätzen, NZA 2009, S. 938-943.

Eich, Rolf-Achim, Der Wandel der BAG-Rechtsprechung zur Haftung des Arbeitnehmers bei gefahrgeneigter Arbeit, NZA 1984, S. 65-72.

Eichendorf, Walter, Arbeiten 4.0 und die Rolle der gesetzlichen Unfallversicherung, Sicherheitsingenieur 2016, S. 20-22.

Eichenhofer, Eberhard, Unfall im Eigenheim, Anmerkung zu BSG, Urt. v. 12.12.2006 - B 2 U 28/05 R -, SGb 2007, S. 742-748. 
Endemann, Wilhelm, Das Reichsgesetz über Haftpflicht der Eisenbahnen, Bergwerke usw., Vierteljahresschrift für Volkswirtschaft und Kulturgeschichte Bd. 36, 9. Jahrg., Berlin 1872.

Epping, Volker/Hillgruber, Christian (Hrsg.), Beck'scher Online-Kommentar GG, 35. Ed., München 2017 (zitiert als: Bearbeiter, in: Epping/Hillgruber (Hrsg.), BeckOK GG).

Erlenkämper, Arnold, „Wesentliche Bedingung“ und „Gelegenheitsursache“ - Zum Problem der Ausgrenzung von Unfallfolgen durch den Begriff der Gelegenheitsursache in der gesetzlichen Unfallversicherung, Brennpunkte des Sozialrechts 1996, S. 1-34.

Erman, Walter, Der Sphärengedanke als Gesichtspunkt für die Verteilung der Preisgefahr beim Werkvertrag, JZ 1965, S. 657-661.

Esser, Josef, Grundlagen und Entwicklung der Gefährdungshaftung, 2. Aufl., München 1969.

Falder, Roland, Immer erreichbar - Arbeitszeit- und Urlaubsrecht in Zeiten des technologischen Wandels, NZA 2010, S. 1150-1157.

Faude, Michael, Selbstverantwortung und Solidarverantwortung im Sozialrecht. Strukturen und Funktionen der sozialrechtlichen Relevanz des Selbstverschuldens des Leistungsberechtigten, Bonn 1983.

Fieberg, Christian, Arbeits- und Dienstunfallversorgung der Arbeitnehmer und Beamten im öffentlichen Dienst - ein Vergleich, ZTR 2007, S. 421-428.

Fietze, Simon/Keller, Monika/Friedrich, Niklar/Dettmers, Jan, Rufbereitschaft und das Projekt „Flexibilität und Verfügbarkeit durch Arbeit auf Abruf“ (RUF), in: dies. (Hrsg.), Rufbereitschaft. Wenn die Arbeit in der Freizeit ruft, München 2014.

Fietze, Simon/Keller, Monika/Friedrich, Niklar/Dettmers, Jan (Hrsg.), Rufbereitschaft. Wenn die Arbeit in der Freizeit ruft, München 2014.

Fischer, W., Beamtenrecht und innerbetrieblicher Schadensausgleich bei besonders gefahrbehafteter Tätigkeit, ZBR 1960, S. 148-150.

Fishback, Price V./Kantor, Shawn Everett, A Prelude to the Welfare State, The Origins of Workers' Compensation, Chicago 2000.

Franke, Ingeborg/Weiß, Hans-Dietrich (Hrsg.), Gesamtkommentar Öffentliches Dienstrecht, Berlin 2014 (zitiert als: Bearbeiter, in: Franke/Weiß (Hrsg.), GKÖD).

Franzen, Martin, Folgen von Industrie 4.0 für die Betriebsverfassung - Betriebsbegriff und Vereinbarungen nach $\$ 3$ BetrVG, in: Giesen, Richard/Junker, Abbo/Rieble, Volker (Hrsg.), Industrie 4.0 als Herausforderung des Arbeitsrechts, München 2016, S. 107-126.

Frerich, Johannes/Frey, Martin, Handbuch der Geschichte der Sozialpolitik in Deutschland. Band 1: Von der vorindustriellen Zeit bis zum Ende des Dritten Reiches, München 1993.

Frey, Erich, Die Gemeinschaft als anspruchsvernichtendes Element bei der Schadenshaftung von Arbeitnehmern untereinander, AuR 7 (1959), S. 193-205.

Friauf, Karl-Heinrich, Arbeitgeberbeiträge zu einer sozialen Pflegeversicherung?, DB 1991, S. 1773-1779. 
Friauf, Karl-Heinrich/Höfling, Wolfram (Hrsg.), Berliner Kommentar zum Grundgesetz, Berlin 2017 (zitiert als: Bearbeiter, in: Friauf/Höfling (Hrsg.), GG).

Fuchs, Maximilian, Die Legitimation der gesetzlichen Unfallversicherung, SGb 1995, S. 421-426.

Fuchsloch, Christine, Das Verbot der mittelbaren Geschlechtsdiskriminierung. Ableitung, Analyse und exemplarische Anwendung auf staatliche Berufsausbildungsförderung, Baden-Baden 1995 (zitiert als: Fuchsloch, Geschlechtsdiskriminierung).

Gamillscheg, Franz/Hanau, Peter, Die Haftung des Arbeitnehmers, 2. Aufl., Karlsruhe 1974.

Geyer, Kristin/Grunefeld, Hans Ulrich/Kazmaier, Michaela/Leihkauff, Werner/Weinbrenner, Sarab/Wittmer, Gerhard/Zahn, Ulrich/Gerke, Michael (Hrsg.), Beamtenversorgungsrecht des Bundes und der Länder, Kommentar, München, Loseblatt, Stand: Mai 2015 (zitiert als: Bearbeiter, in: Geyer/Grunefeld et al. (Hrsg.), BeamtVG).

Giesen, Gerd, Arbeitsunfall und Dienstunfall. Zur Reichweite des Unfallschutzes von Arbeitnehmern und Beamten nach $₫ 8$ SGB VII und $\$ 31$ BeamtVG, Berlin 2016.

Giesen, Richard/Junker, Abbo/Rieble, Volker (Hrsg.), Industrie 4.0 als Herausforderung des Arbeitsrechts, München 2016.

Giesen, Richard/Kersten, Jens, Arbeit 4.0. Arbeitsbeziehungen und Arbeitsrecht in der digitalen Welt, München 2018.

Gitter, Wolfgang, Schadensausgleich im Arbeitsunfallrecht, Tübingen 1969.

Gitter, Wolfgang, Der Weg zur Unfallversicherung aus rechtswissenschaftlicher Sicht, Der Prozeß der Herauslösung aus dem allgemeinen Schadensrecht, in: Hauptverband der gewerblichen Berufsgenossenschaften e.v./Bundesverband der landwirtschaftlichen Berufsgenossenschaften e.V./Bundesverband der Unfallversicherungsträger der öffentlichen Hand e.V. (Hrsg.), 100 Jahre gesetzliche Unfallversicherung, Wiesbaden 1985, S. 22-31.

Gitter, Wolfgang, Grundlagen der gesetzlichen Unfallversicherung im Wandel der Zeit, SGb 1993, S. 297-303.

Gitter, Wolfgang, Die gesetzliche Unfallversicherung nach der Einordnung ins Sozialgesetzbuch - ein Versicherungszweig ohne Reformbedarf?, BB 1998, Beilage 6 zu Heft 22, S. 1-19.

Gitter, Wolfgang/Nunius, Volker, Grundgedanken und Prinzipien, in: Schulin, Bertram (Hrsg.), Handbuch des Sozialversicherungsrechts Band 2: Unfallversicherungsrecht, München 1996, $\$ 5$, S. 98-133.

Gitter, Wolfgang/Thieme, Wolfgang/Zacher, Hans F. (Hrsg.), Im Dienst des Sozialrechts, Festschrift für Georg Wannagat, Köln 1981.

Gontard, Alexander, Der Verkehrsunfall als Arbeits- und Dienstunfall, in: Deutsche Akademie für Verkehrswissenschaft (Hrsg.), 19. Deutscher Verkehrsgerichtstag 1981, Hamburg 1981, S. 274-283. 
Gottschall, Karin/Voß, G. Günter (Hrsg.), Entgrenzung von Arbeit und Leben. Zum Wandel der Beziehung von Erwerbstätigkeit und Privatsphäre im Alltag, 2. Aufl., München 2005 (zitiert als: Gottschall/Voß (Hrsg.), Entgrenzung von Arbeit und Leben).

Gottschall, Karin/Voß, G. Günter, Entgrenzung von Arbeit und Leben. Zur Einleitung, in: dies. (Hrsg.), Entgrenzung von Arbeit und Leben. Zum Wandel der Beziehung von Erwerbstätigkeit und Privatsphäre im Alltag, 2. Aufl., München 2005, S. 11-36.

Greiner, Stefan, Das Beschäftigungsverhältnis im Sozial- und Arbeitsrecht, in: Rolfs, Christian (Red.), Schriftenreihe des Deutschen Sozialrechtsverbandes (SDSRV), Band 62: Selbständigkeit und Abhängigkeit der Dogmatik des Sozialrechts, 12. Sozialrechtslehrertagung am 23./24. Februar 2012 in Hannover, Berlin 2012, S. 9-28 (zitiert als: Greiner, SDRSV Bd. 62).

Grimm, Detlef, Veränderungsbedarf in Bezug auf Arbeitszeit und -ort, Arbeitnehmerbegriff, Betriebsverfassung sowie Beschäftigtendatenschutz, ArbRB 2015, S. 336-339.

Günther, Jens/Böglmüller, Matthias, Arbeitsrecht 4.0 - Arbeitsrechtliche Herausforderungen in der vierten industriellen Revolution, NZA 2015, S. 1025-1031.

Häfner-Beil, Nadja/Nacke, Philipp, Sind Beschäftigte bei einem Arbeitsunfall im Homeoffice genauso versichert wie Beschäftigte im Betrieb?, Gute Arbeit 11/2016, S. 4.

Hanau, Hans, Schöne digitale Arbeitswelt?, NJW 2016, S. 2613-2617.

Hänlein, Andreas/Tennstedt, Florian, $\mathbb{} 2$ Geschichte des Sozialrechts, in: von Maydell, Bernd/Ruland, Franz/Becker, Ulrich (Hrsg.), Sozialrechtshandbuch, 5. Aufl., Baden-Baden 2012, S. 67-111.

Hauck, Karl/Noftz, Wolfgang/Keller, Wolfgang (Hrsg.), Sozialgesetzbuch SGB VII: Gesetzliche Unfallversicherung, Berlin, Loseblatt, Stand Januar 2016 (zitiert als: Bearbeiter, in: Hauck/Noftz/Keller(Hrsg.), SGB VII).

Haueisen, Fritz, Die Theorie der wesentlichen Bedingung - eine wichtige Ursachenlehre, JZ 1961, S. 9-12.

Heine, Bernd, Die schadensgeneigte Arbeit des Beamten, München 1969.

Heinze, Meinhard, Möglichkeiten der Fortentwicklung des Rechts der Sozialen Sicherheit zwischen Anpassungszwang und Bestandsschutz, in: Ständige Deputation des deutschen Juristentages (Hrsg.), Verhandlungen des fünfundfünfzigsten deutschen Juristentages, Hamburg 1984, München 1984, Gutachten E.

Heinze, Meinhard/Ricken, Oliver/Giesen, Richard, Neue Brille nach Arbeitsunfall, BSG, vom 11.9.2001 - B 2 U 38/00 R -, NZA 2001, S. 1376.

Heise, Dietmar, Sozialversicherungspflicht in der agilen Arbeitswelt, NZA 2017, S. 1571-1577.

Held, Susanne, Die Verfassungsmäßigkeit der Arbeitgeberbeiträge zu den Sozialversicherungen, Hamburg 2011.

Herschel, Wilhelm, Das Unternehmerwagnis im Arbeits- und Wirtschaftsrecht, Iherings Jahrbücher für die Dogmatik des bürgerlichen Rechts 90 (1943), S. 145188. 
Hillmann, Karl-Heinz, Wörterbuch der Soziologie, Stuttgart 2007.

Hippel, Eike von, Haftungsersetzung durch Versicherungsschutz - Rechtsvergleichendes Generalreferat, in: Fleming, John G./Hellner, Jan/Hippel, Eike von (Hrsg.), Haftungsersetzung durch Versicherungsschutz, Länderberichte zum anglo-amerikanischen Recht und zum schwedischen Recht zusammen mit einem rechtsvergleichenden Generalbericht und einem Bericht über die Diskussion. Verhandlungen d. Fachgruppe für Zivilrechtsvergleichung auf der Tagung für Rechtsvergleichung in Lausanne vom 12.-15. September 1979, Frankfurt am Main 1980, S. 40.

Höcker, Heinz, Zur Reform der gesetzlichen Unfallversicherung, ZSR 1958, S. 604629.

Höller, Edlyn, Die gesetzliche Unfallversicherung in der Rechtsprechung des Bundessozialgerichts, in: Udsching, Peter/Rolfs, Christian (Hrsg.), Jahrbuch des Sozialrechts. Gesetzgebung - Verwaltung - Rechtsprechung - Literatur. Nachschlagewerk für Wissenschaft und Praxis, Band 34. Dokumentation für das Jahr 2012, Berlin 2013, S. 211-217.

Hollo, Dierk F., Unfallversicherungsschutz im Home-Office. Anmerkung zu BSG 2. Senat, Urteil vom 05.07.2016 - B 2 U 5/15 R -, jurisPR-SozR 10/2017 Anm. 4.

Hohmann-Dennhardt, Christine, Der Sozialstaat - ein Auslaufmodell?, AuR 54 (2006), S. 77-81.

Hromadka, Wolfgang, Zukunft des Arbeitsrechts, NZA 1998, S. 1-9.

Hübner, Jürgen, Schadenszurechnung nach Risikosphären. Eine Studie zur schadensgeneigten Arbeit selbständig Tätiger am Beispiel der privatrechtlichen Haftung des Seelotsen, Berlin 1974.

Hueck, Götz, Schadenersatz und Regress bei Dienst- und Arbeitsunfällen, RdA 7 (1954), S. 251-257.

Hume, David, Untersuchung in Betreff des menschlichen Verstandes, Berlin 1869.

Isenhardt, Tilman, Homeoffice: Einrichtung und Ausgestaltung, DB 2016, S. 14991502.

Isensee, Josef, Umverteilung durch Sozialversicherungsbeiträge. Eine finanzverfassungsrechtliche Studie über den Solidarausgleich in der Gesetzlichen Krankenversicherung, Berlin 1973.

Jacobs, Matthias, Reformbedarf im Arbeitszeitrecht, NZA 2016, S. 733-737.

Jarass, Hans/Pieroth, Bodo, Grundgesetz für die Bundesrepublik Deutschland, 14. Aufl., München 2016 (zitiert als:Jarass/Pieroth, GG).

Jørgensen, Stig, Recht und Gesellschaft, Göttingen 1971.

Jorns, Dieter, Das Betriebsrisiko unter besonderer Berücksichtigung der Rechtsprechung nach dem zweiten Weltkriege, Heidelberg 1957.

Jung, Eberhard, Anmerkung zu BSG, Urt. v. 7.11.2000 - B 2 U 39/99 R -, SGb 2001, S. 398-399.

Jung, Eberhard, Probleme bei der Ermittlung der Finalität bzw. des inneren/sachlichen Zusammenhangs bei der Feststellung eines Arbeitsunfalls, WzS 2011, S. 227-231. 
Jung, Eberhard, Private, betriebliche und gemischte Tätigkeiten, BPUVZ 2013, S. 623-624.

Jung, Eberhard, Warten, Pausieren und privates Telefonieren als versicherte Tätigkeiten, BPUVZ 2014, S. 202-204.

Jung, Eberhard, Anmerkung zu BSG, Urt. v. 5.7.2016 - B 2 U 5/15 R -, SGb 2017, S. 408-415.

Jung, Hans-Peter, Die Sozialgerichtsbarkeit als besonderer Gerichtszweig in der Bundesrepublik Deutschland, in: Deutscher Richterbund (Hrsg.), Justiz und Recht im Wandel der Zeit, Festgabe 100 Jahre Deutscher Richterbund, Köln 2009, S. 253264.

Kahrs, Hans Jürgen, Kausalität und überholende Kausalität im Zivilrecht, Hamburg 1969.

Kalisch, Ines, Die Entwicklung des Verbots der mittelbaren Diskriminierung wegen des Geschlechts im Sozialrecht, Frankfurt a.M. 1999 (zitiert als: Kalisch, Die Entwicklung des Verbots der mittelbaren Diskriminierung).

Kaltenborn, Markus, Die Sozialgesetzgebung des Reichskanzlers Fürst Otto von Bismarck, JZ 53 (1998), S. 770-773.

Kater, Horst/Leube, Konrad, SGB VII, Kommentar, München 1997.

Keller, Wolfgang, Der Zurechnungszusammenhang bei Unfällen von Beschädigten und Arbeitsunfallopfern nach dem Versorgungsrecht und dem Recht der gesetzlichen Unfallversicherung - Besprechung des Urteils des BSG vom 20.5.1992 (9a RV 28/90) -, NZS 1994, S. 161-166.

Keller, Wolfgang, Rechtsfragen des Versicherungsschutzes nach dem Recht der gesetzlichen Unfallversicherung bei Unfällen aus innerer Ursache während einer versicherten Tätigkeit, NZS 1995, S. 58-63.

Keller, Wolfgang, Anmerkung zu BSG, Urt. v. 29.11.2011 - B 2 U 10/11 R -, SGb 2012, S. 668-671.

Kelsen, Hans, Kausalität und Zurechnung, Österreichische Zeitschrift für öffentliches Recht und Völkerrecht 1953/55, S. 125-151.

Kempen, Otto Ernst, Kollektivautonomie contra Privatautonomie: Arbeitsvertrag und Tarifvertrag, NZA-Beil. 2000, S. 7-14.

Kempny, Simon/Reimer, Philipp, Die Gleichheitsrechte - Versuch einer übergreifenden dogmatischen Beschreibung ihres Tatbestands und ihrer Rechtsfolgen, Tübingen 2012.

Kilian, Wolfgang/Heussen, Benno (Hrsg.), Computerrechts-Handbuch, 33. Aufl., München 2017.

Kleemann, Frank, Zur Re-Integration von Arbeit und Leben in Teleheimarbeit, in: Gottschall/Voß (Hrsg.), Entgrenzung von Arbeit und Leben. Zum Wandel der Beziehung von Erwerbstätigkeit und Privatsphäre im Alltag, 2. Aufl., München 2005, S. 59-86.

Knickrehm, Sabine (Hrsg.), Gesamtes soziales Entschädigungsrecht, Handkommentar, Baden-Baden 2012. 
Knickrehm, Sabine/Kreikebohm, Ralf/Waltermann, Raimund (Hrsg.), Kommentar zum Sozialrecht, 4. Aufl., München 2015.

Knörr, Saskia, Die Entstehung einer eigenständigen Sozialgerichtsbarkeit unter besonderer Berücksichtigung Bayerns, Regensburg 2007.

Koch, Zum Entwurf des Unfallversicherungsgesetzes, ZSR 1956, S. 207-209.

Köhler, Karl Friedrich, Anmerkung zu BSG, Urt. v.26.10.2004 - B 2 U 24/03 -, SGb 2005, S. 655-659.

Köhler, Karl Friedrich, Die versicherte Tätigkeit der „grundsätzlich“ versicherten Person als haftungsbegründende Ursache im Recht der gesetzlichen Unfallversicherung. Eine terminologische Klarstellung vor dem Hintergrund der neuesten BSG-Rechtsprechung, SGb 2006, S. 9-20.

Köhler, Karl Friedrich, Zeichnet sich eine neue Kausalitätsformel im Recht der gesetzlichen Unfallversicherung ab? Literatur und Rechtsprechung auf dem Weg zu einer Neudefinition der haftungsbegründenden und haftungsausfüllenden Kausalität, SdL 2006, S. 185-205.

Köbler, Karl Friedrich, Handlungstendenz, Handlungsmotiv, gemischte Tätigkeit und gemischte Motivationslage, WzS 2011, S. 203-208.

Köhler, Karl Friedrich, Die „Theorie der wesentlichen Bedingung“ im Recht der gesetzlichen Unfallversicherung, ZfSH/SGB 2012, S. 383-394.

Köbler, Karl Friedrich, Die Gelegenheitsursache im Recht der gesetzlichen Unfallversicherung, VSSR 2013, S. 47-74.

Köhler, Karl Friedrich, Der Zusammenhang zwischen Betriebssport und unfallversicherter Tätigkeit - Ein Erbe nationalsozialistischer Soziologie und Weltanschauung?, VSSR 2016, S. 31-57.

Köhler, Karl Friedrich, Hat der versicherungsökonomische Begriff des „Moral Hazard" im Sozialversicherungsrecht, insbesondere im Recht der gesetzlichen Unfallversicherung, ein semantisches Potential?, VSSR 2017, S. 111-147.

Kohte, Hans-Wolfhard, Arbeitnehmerhaftung und Arbeitgeberrisiko, Königstein/Ts. 1981.

Kohte, Wolfhard, Die Bedeutung der Unfallversicherung für das Recht der Arbeitnehmerhaftung, AuR 31 (1983), S. 229-238.

Kohte, Wolfhard, Anordnungen im Arbeitszeitrecht. Anmerkung zu VG Ansbach 4. Kammer, Urteil vom 25.01.2017 - AN 4 K 15.00907 -, jurisPR-ArbR 17/2017 Anm. 2.

Koenig, Christian, Die gesetzgeberische Bindung an den Gleichheitssatz - Eine Darstellung des Prüfungsaufbaus zur Rechtssetzungsgleichheit, JuS 1995, S.313318.

Königlich-Preussische Akademie der Wissenschaften (Hrsg.), Kant's gesammelte Schriften, Berlin 1900.

Koppenfels-Spies, Katharina von, Der Gleichbehandlungsgrundsatz im Versicherungsrecht, VersR 2004, S. 1085-1090. 
Koppenfels-Spies, Katharina von, Kongruenzen und Inkongruenzen im sozial- und privatrechtlichen Haftungsrecht, in: Rolfs, Christian (Red.), Schriftenreihe des Deutschen Sozialrechtsverbandes (SDSRV), Band 62: Selbständigkeit und Abhängigkeit der Dogmatik des Sozialrechts, 12. Sozialrechtslehrertagung am 23./24. Februar 2012 in Hannover, Berlin 2012, S. 87-106 (zitiert als: von Koppenfels-Spies, SDRSV Bd. 62).

Korenke, Thomas, Der Versicherungsfall des Arbeitsunfalls im Recht der gesetzlichen Unfallversicherung - dogmatische Grundstrukturen und deren Bedeutung in der sozialgerichtlichen Praxis, in: Bontrup, Heinz-Josef/Korenke, Thomas/Wienbracke, Mike (Hrsg.), Arbeit - Personal - Soziales, Festschrift zum 65. Geburtstag von Peter Pulte, Hamburg 2012, S. 161-177.

Körner, Anne/Leitherer, Stephan/Mutschler, Bernd (Hrsg.), Kasseler Kommentar zum Sozialversicherungsrecht, 2015.

Kranig, Andreas/Aulmann, Heinz, Das Wegeunfallrisiko als Gegenstand der gesetzlichen Unfallversicherung (Teil 1), NZS 1995, S. 203-210.

Krasney, Otto Ernst, Rechtsfortbildung durch Richterrecht in der gesetzlichen Unfallversicherung, in: Wannagat, Georg (Hrsg.), Entwicklung des Sozialrechts, Aufgabe der Rechtsprechung: Festgabe aus Anlaß des 100jährigen Bestehens der sozialgerichtlichen Rechtsprechung, Köln 1984, S. 355-373.

Krasney, Otto Ernst, Der Verkehrsunfall als Arbeitsunfall, NZV 1989, S. 369-376.

Krasney, Otto Ernst, Abgrenzung der Risiken in der gesetzlichen Unfallversicherung, Fragen und Vorschläge zu einem SGB VII, VSSR 1993, S. 81-117.

Krasney, Otto Ernst, Die Handlungstendenz als Kriterium für die Zurechnung in der gesetzlichen Unfallversicherung - Herrn Präsidenten des Bundessozialgerichts a.D. Prof. Dr. jur. Heinrich Reiter zum 70. Geburtstag -, NZS 2000, S. 373-379.

Krasney, Otto Ernst, Die gesetzliche Unfallversicherung und ihr Einfluss auf den Betriebsfrieden, AuR 49 (2001), S. 423-428.

Krasney, Otto Ernst, Die Handlungstendenz als Kriterium für die Zurechnung in der gesetzlichen Unfallversicherung, NZS 2013, S. 681-685.

Krasney, Otto Ernst, Familienbezogener Unfallversicherungsschutz, WzS 2013, S. 67-73.

Krasney, Otto Ernst, Grenzziehungen bei Wegeunfällen, SGb 2013, S. 313-319.

Krasney, Otto Ernst, Schutz in der gesetzlichen Unfallversicherung. Eine begrenzte Übersicht über die Rechtsprechung des Bundessozialgerichts, KrV 2014, S. 102113.

Krasney, Otto Ernst, Zum Irrtum im Unfallversicherungsrecht, NZS 2014, S.607612.

Krasney, Otto Ernst, Wahl des Arbeitsstils - Auswirkungen im Recht der gesetzlichen Unfallversicherung, in: Devetzi, Stamatia/Janda, Constanze (Hrsg.), Freiheit - Gerechtigkeit - Sozial(es) Recht. Festschrift für Eberhard Eichenhofer, 2015, S. 389-400 (zitiert als: Krasney, in: Devetzi/Janda (Hrsg.), FS Eichenhofer).

Kratzer, Nick, Arbeitskraft in Entgrenzung, München 2003. 
Kratzer, Nick/Sauer, Dieter, Entgrenzung von Arbeit. Konzept, Thesen Befunde, in: Gottschall, Karin/Voß, G. Günter (Hrsg.), Entgrenzung von Arbeit und Leben, 2. Aufl., München 2005, S. 87-123.

Krause, Rüdiger, Digitalisierung der Arbeitswelt - Herausforderungen und Regelungsbedarf, Gutachten B zum 71. Deutschen Juristentag, in: Ständige Deputation des deutschen Juristentages (Hrsg.), Verhandlungen des 71. deutschen Juristentages, Essen 2016, München 2016, B 7-B 112. München 2016 (zitiert als: Krause, Digitalisierung der Arbeitswelt, Gutachten B zum 71. DJT, B.).

Krause, Rüdiger, Herausforderung Digitalisierung der Arbeitswelt und Arbeiten 4.0, NZA-Beilage 2017, S. 53-59.

Krohn, Johannes, Die Soziale Unfallversicherung im System des Rechts, in: Schimmelpfennig, Heinrich (Hrsg.), Grundsatzfragen der sozialen Unfallversicherung, Festschrift für Dr. Herbert Lauterbach zum 60. Geburtstag, Mannheim 1961, S. 23-34.

Kruschinsky, Michael, Unfallversicherungsschutz für gemischte Tätigkeiten, Anmerkung zu BSG 2. Senat, Urteil vom 26.6.2014 - B 2 U 4/13 R -, jurisPR-SozR 6/2015, Anm. 4.

Kugele, Dieter, Dienstunfall im Schullandheim, jurisPR-BVerwG 15/2008, Anm. 4.

Kugele, Dieter (Hrsg.), Kommentar zum Beamtenversorgungsgesetz, 2. Aufl., Münster 2011.

Kümper, Boas, Risikoverteilung im Staatshaftungsrecht, Tübingen 2011.

Kunze, Thomas, Kausalität in der gesetzlichen Unfallversicherung, VSSR 2005, S. 299-334.

Landmann, Robert von/Rohmer, Gustav (Begr.), Gewerbeordnung und ergänzende Vorschriften. Loseblatt-Kommentar, 76. Aufl., München 2017 (zitiert als: Bearbeiter, in: Landmann/Rohmer, Gewerbeordnung, $\mathbb{\$}$ Rn.).

Latzel, Clemens/Picker, Christian, Vorwort, in: dies. (Hrsg.), Neue Arbeitswelt, 3. Assistententagung im Arbeitsrecht 2013 in München, Tübingen 2014, S. V-VII.

Laubinger, Hans-Werner, Die Treuepflicht des Beamten im Wandel der Zeiten, in: König, Klaus/Laubinger, Hans-Werner/Wagener, Frido (Hrsg.), Öffentlicher Dienst. Festschrift für Carl Hermann Ule zum 70. Geburtstag am 26. Februar 1977, Köln 1977, S. 89-110.

Lauterbach, Herbert/Watermann, Friedrich/Breuer, Joachim (Hrsg.), Unfallversicherung - Sozialgesetzbuch VII, Stuttgart, Loseblatt, Stand Juli 2015 (zitiert als: Bearbeiter, in: Lauterbach/Watermann/Breuer (Hrsg.), Unfallversicherung).

Lecheler, Helmut, Die „hergebrachten Grundsätze des Berufsbeamtentums“ in der Rechtsprechung des Bundesverfassungsgerichts und des Bundesverwaltungsgerichts, AöR 103 (1978), S. 349-382.

Legerlotz, Christoph, (Arbeits-)Unfälle bei betrieblich organisierter Freizeit. Die Vermischung von Arbeit und Freizeit als Haftungsproblem im Unfallversicherungsrecht, ArbRB 11/2011, S. 350-352.

Lepa, Meike, Haftungsbeschränkungen bei Personenschäden nach dem Unfallversicherungsrecht. Eine kritische Analyse der Neuregelung in $\$ \$ \$ 104 \mathrm{ff}$. SGB VII, Berlin 2004. 
Leppek, Sabine, Beamtenrecht, 12. Aufl., Heidelberg 2015.

Leube, Konrad, Arbeitsunfall und Dienstunfall auf dem Arbeitsweg - Entsprechungen und Abwandlungen, ZTR 2012, S. 682-688.

Leube, Konrad, Häusliche Telearbeit und gesetzliche Unfallversicherung, SGb 2012, S. 380-385.

Leube, Konrad, Anmerkung zu Urt. des BSG v. 4.7.2013 - B 2 U 12/12 R -, SGb 2014, S. 392-398.

Leube, Konrad, Gesetzliche Unfallversicherung: Zweifelsfragen beim „Kindergartenumweg“ ( $\$ 8$ II Nr. 2a SGB VII), NZV 2015, S. 275-280.

Lorse, Jürgen, Das Streikverbot für Beamte im Lichte der aktuellen Rechtsprechung des Bundesverwaltungsgerichts zu Artikel 11 EMRK, ZBR 2015, S. 109-120.

Lott, Yvonne, Selbstorganisiertes Arbeiten als Ressource für Beschäftigte nutzen, Forschungsförderung Policy Brief 03, Hans Böckler Stiftung Juli 2017.

Lott, Yvonne, Stressed despite or because of flexible work arrangements? Flexible work arrangements, job pressure and work-to-home conflict for women and men in Germany, Forschungsförderung Working Paper Nb. 046, Hans Böckler Stiftung August 2017.

von Mangoldt, Hermann/Klein, Friedrich/Starck, Christian (Hrsg.), Kommentar zum Grundgesetz, Band 1: Präambel, Artikel 1 bis 19, 6. Auflage, München 2010 (zitiert als: Bearbeiter, in: v. Mangoldt/Klein/Starck (Hrsg.), GG).

Marton, Géza, Versuch eines einheitlichen Systems zivilrechtlicher Haftung, AcP 162 (1963), S. 1-87.

Maschmann, Frank, Die mangelhafte Arbeitsleistung, NZA-Beil. 2006, S. 13-21.

Masuch, Peter/Spellbrink, Wolfgang/Becker, Ulrich/Leibfried, Stephan (Hrsg.), Grundlagen und Herausforderungen des Sozialstaats. Denkschrift 60 Jahre Bundessozialgericht. Eigenheiten und Zukunft von Sozialpolitik und Sozialrecht, Band 1, Berlin 2014.

Mataja, Victor, Das Recht des Schadenersatzes vom Standpunkte der Nationalökonomie, Leipzig 1888.

Maunz, Theodor/Dürig, Günter/Herzog, Roman/Scholz, Rupert/Herdegen, Matthias/ Klein, Hans (Hrsg.), Grundgesetz Kommentar, München 2015 (zitiert als: Bearbeiter, in: Maunz/Dürig et al. (Hrsg.), GG).

Maydell, Bernd Baron von, Empfiehlt es sich, die Zuweisung von Risiken und Lasten im Sozialrecht neu zu ordnen?, NJW 1992, S. 2195-2200.

Meder, Stephan, Schuld, Zufall, Risiko. Untersuchungen struktureller Probleme privatrechtlicher Zurechnung, Frankfurt am Main 1993.

Meik, Frank, Herausforderung Digitale Transformation und Arbeit 4.0, NZA-Beilage 2017, S. 43-45.

Mestitz, Franz, Probleme der Geschichte des Arbeitsrechts, ZNR 1980, S. 47-65.

Michaelis, Lars Oliver, Das beamtenrechtliche Streikverbot, JA 2015, S. 121-125.

Minz, Hubert/Leppek, Sabine, Praxis-Handbuch Beamtenversorgungsrecht. Eine systematische Darstellung mit aktueller Rechtsprechung, 4. Aufl., Regensburg 2015 . 
Moll, Wilhelm, Münchener Anwaltshandbuch Arbeitsrecht, 3. Aufl., München 2012.

Möller, Iris, Finanzielle Mitarbeiterbeteiligung. Noch viel Platz für Ausbau, IAB-Forum 2013, S. 48-53.

Möller, Ralf, Finanzierung der Gesetzlichen Unfallversicherung. Jüngste Rechtsprechung des Bundessozialgerichts (BSG) zur Gestaltung eines Gefahrtarifs der gewerblichen Berufsgenossenschaften (BG), SGb 2014, S. 435-441.

Mühlheims, Laurenz, Spazierengehen und dienstlich telefonieren - zwei Tätigkeiten, ein Arbeitsunfall?, DGUV Forum 2014, S. 41.

Müller, Roland/Hofer, Céline/Stengel, Manuel, Arbeitsort und Arbeitsweg, AJP 2015, S. 564-578.

Müller-Erzbach, Rudolf, Gefährdungshaftung, AcP 106 (1910), S. 309-476.

Müller-Glöge, Rudi/Preis, Ulrich/Schmidt, Ingrid (Hrsg.), Erfurter Kommentar zum Arbeitsrecht, 16. Aufl., München 2016.

Musielak, Hans Joachim, Die sog. tatsächliche Vermutung, JA 2010, S. 561-566.

Mutschler, Bernd, Arbeitsunfälle verursacht durch Angriffe am Arbeitsplatz oder auf Wegen, SGb 2011, S. 684-691.

Mutschler, Bernd, Versicherungsschutz gegen Angriffe Dritter in der gesetzlichen Unfallversicherung, NZS 2014, S. 647-653.

Mütze, Wolfgang, Unfallversicherungsschutz außerhalb des häuslichen Arbeitszimmers im selbstgenutzten Wohnhaus. Anmerkung zu SG Karlsruhe 4. Kammer, Urteil vom 30.09.2010, S 4 U 675/10, jurisPR-SozR 25/2010, Anm. 5.

Nazarek, Robert, Ständige Erreichbarkeit und Unfallversicherung, PersR 2016, S. 28-31.

Negt, Oskar, Arbeit und menschliche Würde, Göttingen 2001.

Nebls, Jürgen, Kausalität innerhalb der versicherten Tätigkeit nach der Rechtsprechung des Bundessozialgerichts oder Finalität?, BG 1983, S. 227-233.

Nußberger, Angelika, Die Versicherung gegen Arbeitsunfälle und Berufskrankheiten - ein traditionsreiches Modell auf dem Prüfstand, in: Boecken, Winfried/Hänlein, Andreas/Kruse, Jürgen/Steinmeyer, Heinz-Dietrich (Hrsg.), Öffentliche und private Sicherung gegen soziale Risiken, Baden-Baden 2000, S. 193-212.

Oberthür, Nathalie, Die Arbeitssicherheit im Mobile Office, NZA 2013, S. 246-249.

Osing, Saskia, Sehr enger Bezug von vor- und nachbereitenden Handlungen zur Haupttätigkeit für Versicherungsschutz erforderlich, Anmerkung zu BSG 2. Senat, Urteil vom 13.11.2012 - B 2 U 27/11 R -, jurisPR-SozR 2013, Anm. 3.

Pattar, Andreas/Sartorius, Ulrich, Rechtsprechungs- und Literaturübersicht zum Sozialrecht - 2. Halbjahr 2016, ZAP Fach 18, S. 1503-1518.

Peltzer, Gerhard, Die Theorie der wesentlichen Bedingung, Köln 1964.

Pesch, Karl, Der Ursachenzusammenhang beim Dienstunfall des Beamten, DVBl. 1959, S. 43-48.

Pfarr, Heide, Mittelbare Diskriminierung von Frauen. Die Rechtsprechung des EuGH, NZA 1986, S. 585-589. 
Pfarr, Heide/Bertelsmann, Klaus, Gleichbehandlungsgesetz. Zum Verbot der unmittelbaren und der mittelbaren Diskriminierung von Frauen im Erwerbsleben, Wiesbaden 1985.

Pflaum, Ulrich, Unfallereignis und Kausalität im Dienstunfallrecht, RiA 2011, S. 198-203.

Piele, Christian/Piele, Alexander, Mobile Arbeit. Eine Analyse des verarbeitenden Gewerbes auf Basis der IG Metall-Beschäftigtenbefragung 2017, Fraunhofer-Institut für Arbeitswirtschaft und Organisation, Stuttgart 2017.

Plagemann, Herrmann, Kein Wegeversicherungsschutz beim Homeoffice, ArbRAktuell 2016, S. 71.

Plagemann, Hermann/Radtke-Schwenzer, Kerstin, Aktuelle Entwicklung im Recht der gesetzlichen Unfallversicherung, NJW 2013, S. 1924-1929.

Planck, Max, Der Kausalbegriff in der Physik, 2. Aufl., Leipzig 1937.

Planck, Max, Der Kausalitätsbegriff in der Physik, in: Max-Planck-Gesellschaft (Hrsg.), Max Planck, Vorträge und Reden, Braunschweig 1958, S. 19-50.

Plog, Ernst/Wiedow, Alexander/Lemböfer, Bernt/Groepper, Michael/Schmidt, Hans-Jochen/Tegethoff, Carsten (Hrsg.), Bundesbeamtengesetz, Stuttgart, Loseblatt, Stand Januar 2016 (zitiert als: Bearbeiter, in: Plog/Wiedow et al. (Hrsg.), BBG).

Pollmann-Schult, Matthias, Arbeitszeitwunsch und -wirklichkeit im Familienkontext: Eine Analyse der Diskrepanzen zwischen präferierter und tatsächlicher Arbeitszeit, Soziale Welt 2009, S. 163-178.

Pollmann-Schult, Matthias, Wenn Männer Väter werden: über die Auswirkungen der Vaterschaft auf Freizeit, Lebenszufriedenheit und familiäre Beziehungen, ZfF 22/2010, S. 350-369.

Prütting, Hanns, Gegenwartsprobleme der Beweislast. Eine Untersuchung moderner Beweislasttheorien und ihrer Anwendung insbesondere im Arbeitsrecht, München 1983.

Raif, Alexander/Nann, Philipp, Arbeitsrecht 4.0 - Möglichkeiten und Hürden in der digitalen Arbeitswelt, GWR 2016, S. 221-224.

Raif, Alexander/Swidersky, Manuel, Arbeit 4.0 - Typische Fehler in der digitalen Arbeitswelt vermeiden, GWR 2017, S. 351-354.

Reich, Andreas, BeamtVG Kommentar, München 2013.

Reich, Andreas, Dienstunfall bei strafbarer Handlung auf dem Heimweg, LKV 2014, S. 193-198.

Reichsversicherungsamt (Hrsg.), Handbuch der Unfallversicherung Band 1, 3. Aufl., Leipzig 1909.

Reiff, Eduard, Die Lösung des ursächlichen Zusammenhangs zwischen Unfall und Tätigkeit in der Unfallversicherung der RVO, Münster 1960.

Reiff, Eduard, Begriff der Kausalität in der Unfallversicherung, NJW 1961, S. 630633.

Reinhardt, Uwe, Die dogmatische Begründung der Haftungsbeschränkung des Arbeitnehmers. Zugleich ein Beitrag zum Zivilrechtlichen Verschuldensbegriff, Göttingen 1977. 
Ricke, Wolfgang, Kausale und finale Aspekte der versicherten Tätigkeit, BG 1984, S. 351-357.

Ricke, Wolfgang, Von Babylon, Pfingsten sowie dem inneren, sachlichen und ursächlichen Zusammenhang. Eine Urteilsanmerkung, BG 1994, S. 360-361.

Ricke, Wolfgang, Entschädigung von Arbeitsunfällen und Berufskrankheiten im Vereinigten Königreich (UK) ZESAR 2008, S. 13-21.

Ricke, Wolfgang, Abels Tod - der erste Arbeitsunfall?, NZS 2011, S. 213-215.

Ricke, Wolfgang, Neue Worte - neue Wahrheiten? Zur jüngeren Rechtsprechung des BSG in Sachen Arbeitsunfall, WzS 2013, S. 241-246.

Ricke, Wolfgang, Anmerkung zu BSG, Urt. v. 7.12.2015 - B 2 U 8/14/R -, SGb 2016, S. 705-710.

Ricke, Wolfgang, „Gebrochenes Bein im Home Office ist kein Arbeitsunfall“ - Vermischtes zum Urteil des 2. Senats des BSG vom 5.7.2016 -, WzS 2017, S. 9-15.

Ricken, Oliver, Unfallversicherung bei Vor- oder Nachbereitungshandlungen Sportklettern eines Profi-Eishockeyspielers, BSG, Urt. v. 13.11.2012 - B 2 U 27/11 R -, NZS 2013, S. 774.

Ricken, Oliver, Unfallversicherungsschutz bei Telearbeit, BSG, Urt. v. 5.7.2016 - B 2 U 5/15 R -, NZA 2017, S. 371.

Riedmaier, Karl, Zur Kausalität im Dienstunfallrecht, RiA 26, 1979, S. 41-48.

Ritter, Gerhard A./Tenfelde, Klaus, Arbeiter im Deutschen Kaiserreich. 1871 bis 1814, Bonn 1992.

Roemer, Heinrich, Die Auslegungsmaßstäbe im Versorgungsrecht, ZfS 1962, S. 305360.

Robrbeck, Walter, Der Begriff der Sozialversicherung und ihre Abgrenzung zur Versorgung und Fürsorge, in: Bogs, Walter (Hrsg.), Gegenwartsfragen sozialer Versicherung. Vorträge und Aussprachen auf der sozialversicherungswissenschaftlichen Tagung der Hochschule für Arbeit, Politik und Wirtschaft vom 5. bis 8. Juli 1950, Heidelberg 1950, S. 17-32.

Rolfs, Christian (Red.), Schriftenreihe des Deutschen Sozialrechtsverbandes (SDSRV), Band 62: Selbständigkeit und Abhängigkeit der Dogmatik des Sozialrechts, 12. Sozialrechtslehrertagung am 23./24. Februar 2012 in Hannover, Berlin 2012.

Rolfs, Christian/Giesen, Richard/Kreikebohm, Ralf/Udsching, Peter (Hrsg.), Beck'scher Online-Kommentar Arbeitsrecht, 38. Ed., München 2015 (zitiert als: Bearbeiter, in: Rolfs/Giesen et al. (Hrsg.), BeckOK Arbeitsrecht).

Rolfs, Christian/Giesen, Richard/Kreikebohm, Ralf/Udsching, Peter (Hrsg.), Beck'scher Online-Kommentar zum Sozialrecht, 46. Ed., München 2017 (zitiert als: Bearbeiter, in: Rolfs/Giesen et al. (Hrsg.), BeckOK Sozialrecht).

Römer, Michael, Reform der gesetzlichen Unfallversicherung, Berlin 2004.

Rönnau, Thomas/Faust, Florian/Fehling, Michael, Durchblick: Kausalität und objektive Zurechnung, JuS 2004, S. 113-118.

Rümelin, Gustav, Dienstvertrag und Werkvertrag, Tübingen 1905.

Rümelin, Max, Der Zufall im Recht. Akademische Antrittsrede, Freiburg i.B. 1896. 
Rüthers, Bernd/Fischer, Christian/Birk, Axel, Rechtstheorie mit Juristischer Methodenlehre, 9. Aufl., München 2016.

Sachs, Michael, Zur dogmatischen Struktur der Gleichheitsrechte als Abwehrrechte, DÖV 1984, S. 411-419.

Sachs, Michael (Hrsg.), Grundgesetz, 7. Aufl., München 2014 (zitiert als: Bearbeiter, in: Sachs (Hrsg.), GG).

Säcker, Franz Jürgen/Rixecker, Roland (Hrsg.), Münchner Kommentar zum BGB, 6. Aufl., München 2013.

Sandbiller, Eva, Alkoholkonsum und Versicherungsschutz in der gesetzlichen Unfallversicherung bei Wegeunfällen, SGb 2012, S. 576-584.

Sauer, Dieter, Entgrenzung - Chiffre einer flexiblen Arbeitswelt - Ein Blick auf den historischen Wandel von Arbeit, in: Badura, Bernhard/Ducki, Antje/Schröder, Helmut/Klose, Joachim/Meyer, Markus (Hrsg.), Fehlzeiten-Report 2012, Schwerpunktthema: Gesundheit in der flexiblen Arbeitswelt: Chancen nutzen, Risiken minimieren, Berlin 2012, S. 3-13.

Schaer, Roland, Die unendliche Geschichte oder der Leidensweg der Jurisprudenz bei Schleudertraumata und psychischen Erwerbsunfähigkeiten, Teil I, SVR 1993, S. 25-28.

Schafhausen, Martin/Schäfer-Kuczinski, Jana, Gesetzliche Unfallversicherung: Kein Versicherungsschutz bei eigenwirtschaftlicher Tätigkeit, ArbRAktuell 2017, S. 612-615.

Schaub, Günter, Arbeitsrechts-Handbuch. Systematische Darstellung und Nachschlagewerk für die Praxis, 15. Aufl., München 2013.

Schelp, Ira, Die Haftungsbelastung des Arbeitnehmers bei Schädigung Dritter. Eine rechtsvergleichende Untersuchung mit dem Regelungsentwurf für ein europäisches Modellgesetz, Berlin 2004.

Schimmelpfennig, Karl-Heinz, Unfallrisiko Landstraße, NZV 2015, S. 23-26.

Schirmer, Uwe, Selbstbestimmtes Arbeiten in der digitalen Arbeitswelt, NZA-Beilage 2016, S. 85-89.

Schlaeger, Tobias, Wegeunfälle im Zusammenhang mit der Unterbringung von Kindern wegen Berufstätigkeit, NZS 2009, S. 559-563.

Schlaeger, Tobias, Unfallversicherungsschutz von Beschäftigten. Anmerkung zu BSG 2. Senat, Urt. v. 15.5.2012 - B 2 U 8/11 R -, jurisPR-SozR 24/2012, Anm. 3.

Schlaeger, Tobias, Unfallversicherungsschutz für Teilnahme an privater Geburtstagsfeier am Arbeitsplatz? Anmerkung zu LSG Chemnitz 6. Senat, Urt. v. 7.10.2015 - L 6 U 183/13 -, jurisPR-SozR 23/2015 12.11.2015, Anm. 3.

Schlaeger, Tobias, Unfallversicherungsschutz im Home-Office, Anmerkung zu LSG München 2. Senat, Urt. v. 05.04.2017 - L 2 U 101/14 -, jurisPR-SozR 20/2017 Anm. 3.

Schlaeger, Tobias/Lenger, Norman, Betriebssportverein - Sozialversicherungsschutz oder steuerliche Gemeinnützigkeit?, DB 2010, S. 2280-2283.

Schlaeger, Tobias/Voelzke, Thomas/Brandenburg, Stephan, SGB VII, Saarbrücken 2009. 
Schlegel, Helmut, Zur Beurteilung des ursächlichen Zusammenhangs bei Dienstunfällen, DVBl. 77, 1962, S. 8-11.

Schlegel, Rainer, Sozialleistungen lösen Versprechen unserer Verfassung ein, SGb 2016, S. 605-607.

Schlegel, Rainer/Voelzke, Thomas/Brandenburg, Stephan (Hrsg.), SGB VII, juris PraxisKommentar, 2. Aufl., Saarbrücken 2014.

Schmidt, Thorsten Ingo, Die Analogie im Verwaltungsrecht, VerwArch 2006, S. 139164.

Schmitt, Jochem, SGB VII, Gesetzliche Unfallversicherung, Kommentar. Umfassend berücksichtigt: das neue Unfallversicherungsmodernisierungsgesetz (UVMG), 4. Aufl., München 2009.

Schmitt, Jochem, Unfallversicherung, in: Maydell, Bernd Baron von/Ruland, Franz/ Becker, Ulrich (Hrsg.), Sozialrechtshandbuch, 5. Aufl., Baden-Baden 2012, $\$ 16$, S. 802-850.

Schmitt, Walter, Der lange Abschied von der sozialrechtlichen Ursachenlehre, VSSR 2004, S. 349-369.

Schnapp, Friedrich E., Die arbeitsrechtliche Fürsorgepflicht - Legitimation für den Arbeitgeberanteil in der gesetzlichen Rentenversicherung?, in: Söllner, Alfred/ Gitter, Wolfgang/Waltermann, Raimund $u$. a. (Hrsg.), Gedächtnisschrift für Meinhard Heinze, München 2005, S. 815-830.

Schnellenbach, Helmut, Beamtenrecht in der Praxis, 7. Aufl., München 2011.

Schöpf, Udo, Multikausale Schäden in der gesetzlichen Unfallversicherung. Gedanken zur systemgerechten Zuweisung arbeitsbedingter Gesundheitsrisiken, Berlin 1995.

Schrader, Wolfgang, Zur Reform der gesetzlichen Unfallversicherung. Eine Entgegnung, ZSR 1958, S. 682-692.

Schramm, Michael/Hennig, Jörg, Das Mobile Office, AuA 2016, S. 504-507.

Schuchart, Uta, Ständige Erreichbarkeit im Arbeitsverhältnis - Ist das Arbeitszeitgesetz noch zeitgemäß?, in: Latzel, Clemens/Picker, Christian (Hrsg.), Neue Arbeitswelt, 3. Assistententagung im Arbeitsrecht 2013 in München, Tübingen 2014, S. $175-210$.

Schuchart, Uta, Ständige Erreichbarkeit - Arbeitszeit light vs. Recht auf Unerreichbarkeit, ArbuR 2016, S. 341-343.

Schudt, Ernst-Rainer, Kausalitätsprobleme im Verwaltungsrecht, Hamburg 1971.

Schuffelen, Andreas, Die verschuldensunabängige Haftung des Arbeitgebers, Münster 2016.

Schulin, Bertram, Der natürliche - vorrechtliche - Kausalitätsbegriff im zivilen Schadensersatzrecht, Berlin 1976.

Schulin, Bertram, Der Versicherungsfall „Arbeitsunfall“, in: Schulin, Bertram (Hrsg.), Handbuch des Sozialversicherungsrechts Band 2: Unfallversicherungsrecht, München 1996, S. 550-636.

Schulin, Bertram (Hrsg.), Handbuch des Sozialversicherungsrechts Band 2: Unfallversicherungsrecht, München 1996. 
Schur, Oliver, Gesetzlicher Unfallversicherungsschutz auf dem Weg zwischen einem Restaurant und einem Home-Office, Anmerkung zu BSG v. 18.06.2013 - B 2 U 7/12 -, jM 2014, S. 249-251.

Schur, Oliver/Spellbrink, Wolfgang, Unfallversicherungsschutz auf dem Weg von und nach der Arbeitsstelle. Neue Wege in der Rechtsprechung des BSG zu Wegeunfällen?, SGb 2014, S. 589-596.

Schuster, Doris-Maria, Die rechtliche Stellung des mehrköpfigen Betriebsrats, Münster 2000.

Schütz, Erwin, Ursachenzusammenhang und Beweislast im Dienstunfallrecht, DÖD 14 (1961), S. 101-105.

Schütz, Erwin, In Ausübung oder infolge des Dienstes. Zur Auslegung dieser Begriffsmerkmale im Dienstunfallrecht, DÖD 18 (1965), S. 161-164.

Schütz, Erwin, Beamtenversorgungsgesetz, Heidelberg 1980.

Schütz, Erwin (Begr.)/Maiwald, Joachim/Schachel, Jens (Hrsg.), Beamtenrecht des Bundes und der Länder, München, Loseblatt, Stand März 2016 (zitiert als: Bearbeiter, in: Schütz/Maiwald (Hrsg.), Beamtenrecht).

Schweigler, Daniela, Das Recht auf Anhörung eines bestimmten Arztes ( $\mathbb{1 0 9}$ SGG). Dogmatische Einordnung und sozialgerichtliche Praxis eines umstrittenen Prozessinstruments, Baden-Baden 2013.

Schwirz, Karl Heinz, Kein Versicherungsschutz beim Gang in die Küche, BGHM Aktuell 1/2017, S. 31.

Seel, Henning-Alexander, Krankheitsbedingte Arbeitsunfähigkeit - Rechte und Pflichten von Arbeitgeber und Arbeitnehmer, JA 2009, S. 131-136.

Siefert, Jutta, NZS-Jahresrevue 2016 - Unfallversicherung, NZS 2017, S. 766-770.

Söllner, Alfred, „Ohne Arbeit kein Lohn“, AcP 167 (1967), S. 132-147.

Spellbrink, Wolfgang, Gemischte Tätigkeit und gemischte Motivationslage bei der Feststellung von Arbeitsunfällen, WzS 2011, S. 351-354.

Spellbrink, Wolflgang, Unfallversicherungsschutz bei Tätigkeiten im Home Office und bei Rufbereitschaft, NZS 2016, S. 527-531.

Spellbrink, Wolfgang, Gibt es eine neue BSG-Rechtsprechung zur Kausalitätsprüfung in der Gesetzlichen Unfallversicherung?, SGb 2017, S. 1-5.

Sprang, Friedrich, Kausaler, innerer, sachlicher Zusammenhang. Was ist los mit der Rechtsprechung des BSG?, BG 1989, S. 144-147.

Steding, Erich, Verwirrung über das Wesen der Kriegsopferversorgung, ZSR 1959, S. 89-99.

Stegmüller, Manfred/Schmalhofer, Rudolf/Bauer, Erwin (Hrsg.), Beamtenversorgungsrecht des Bundes und der Länder, Heidelberg, Loseblatt, Stand Januar 2008 (zitiert als: Bearbeiter, in: Stegmüller/Schmalhofer et.al. (Hrsg.), Beamtenversorgungsrecht (81. Aufl.)).

Stegmüller, Manfred/Schmalhofer, Rudolf/Bauer, Erwin (Hrsg.), Beamtenversorgungsrecht des Bundes und der Länder, Heidelberg, Loseblatt, Stand Mai 2015 (zitiert als: Bearbeiter, in: Stegmüller/Schmalhofer et.al. (Hrsg.), Beamtenversorgungsrecht). 
Stegmüller, Wolfgang, Wissenschaftliche Erklärung und Begründung. Probleme und Resultate der Wissenschaftstheorie und Analytischen Philosophie, Bd. 1, Berlin 1969.

Stegner, Stefan, Zwischen Souveränität und Ökonomie: Die Konstruktion von Zugehörigkeit im modernen Staat. Eine Geschichte sozialversicherungsrechtlicher Beziehungen zwischen Deutschland und Polen 1918-1945, Baden-Baden 2018.

von Steinau-Steinrück, Robert, Smartphone versus Arbeitsrecht, NJW-Spezial 6/2012, S. 178-179.

Stich, Rudolf, Ursachen- und Beweisfragen beim Dienstunfall, ZBR 1958, S. 298303.

Stolleis, Michael, Geschichte des Sozialrechts in Deutschland, Stuttgart 2003.

Summer, Rudolf/Baumgartner, Josef, Der Dienstunfall. Grundriß zum Dienstunfallrecht. Dargestellt für die Zwecke der Verwaltungspraxis und Ausbildung, 2. Aufl., München 1966.

Tamm, Marina, Die rechtliche Behandlung der Rufbereitschaft und die Notwendigkeit der Regelung des Morbus Blackberry-Phänomens, ZfPR 2011, S. 67-71.

Technologieberatungsstelle beim DGB NRW e.V. (Hrsg.), Mobile Arbeit. Das allmähliche Verschwinden der Trennung von Arbeit und Freizeit, Reihe Arbeit, Gesundheit, Umwelt, Technik, Heft 63, Oberhausen 2005 (zitiert als: TBS NRW (Hrsg.), Mobile Arbeit).

Tennstedt, Florian, Sozialgeschichte der Sozialpolitik in Deutschland, Göttingen 1981.

Tennstedt, Florian, Die Ablösung privater Haftpflicht durch öffentlich-rechtliche Pflichtversicherung gegen Unfälle: die Folgen für die private Unfallversicherung - ein historischer Rückblick aus aktuellem Anlass, in: Hänlein, Andreas/Roßnagel, Alexander (Hrsg.), Wirtschaftsverfassung in Deutschland und Europa, Festschrift für Bernhard Nagel, Kassel 2011, S. 483-494.

Tennstedt, Florian/Winter, Heidi, „Der Staat hat wenig Liebe - activ wie passiv“, Die Anfänge des Sozialstaats im Deutschen Reich von 1871. Ergebnisse archivalischer Forschungen zur Entstehung der gesetzlichen Unfallversicherung, ZSR 1993, S. 362-392.

Teutsch, August, Das Beschäftigungsverhältnis als Grundlage des gesetzlichen Unfallversicherungsschutzes und des Haftungssonderrechts der Unternehmer, Tübingen 1953.

Teutsch, August, Probleme der beamtenrechtlichen Unfallfürsorge, in: Robrbeck, Walter (Hrsg.), Beiträge zur Sozialversicherung, Festgabe für Johannes Krohn zum 70. Geburtstag, Berlin 1954, S. 305-324.

Thüsing, Gregor, Digitalisierung der Arbeitswelt - Impulse zur rechtlichen Bewältigung der Herausforderung gewandelter Arbeitsformen, SR 2016, S. 87-108.

Tiemann, Susanne, Die Bedeutung der Prävention im System sozialer Sicherung, SGb 1996, S. 405-410. 
Tobsch, Verena/Schult, Mandy/Fietze, Simon/Matiaske, Wenzel, Rufbereitschaft aus betrieblicher Sicht: Bedeutung, Ausgestaltung und Erfahrung, in: Fietze, Simon/ Keller, Monika/Friedrich, Niklar/Dettmers, Jan (Hrsg.), Rufbereitschaft. Wenn die Arbeit in der Freizeit ruft, München 2014, S. 7-28.

Tomandl, Theodor, Der Wegunfall in der österreichischen und deutschen Unfallversicherung - zugleich ein Beitrag zur Theorie der wesentlichen Bedingung, Sozialversicherung 1975, S. 137-172.

Tomandl, Theodor, Der Arbeitsunfall. Ein Rechtsprechungsvergleich: BSG und österreichischer OGH, in: Wulffen, Matthias von/Krasney, Otto Ernst (Hrsg.), Festschrift 50 Jahre BSG, München 2004, S. 557-570.

Trasker, Britta, Soll Betriebssport künftig weiterhin unter dem Schutz der gesetzlichen Unfallversicherung stehen?, Hamburg 2010 (zitiert als: Trasker, Betriebssport).

Triebel, Matthias, Einführung in das Recht der gesetzlichen Unfallversicherung, JURA 2007, S. 521-531.

Udsching, Peter/Rolfs, Christian (Hrsg.), Jahrbuch des Sozialrechts. Gesetzgebung Verwaltung - Rechtsprechung - Literatur. Nachschlagewerk für Wissenschaft und Praxis, Band 34. Dokumentation für das Jahr 2012, Berlin 2013 (zitiert als Bearbeiter, in: Udsching/Rolfs (Hrsg.), Jahrbuch des Sozialrechts 34 (2013)).

Uffmann, Katharina, Digitalisierung der Arbeitswelt. Wie gestalten wir die notwendigen Veränderungen?, NZA 2016, S. 977-984.

Uffmann, Katharina, Möglichkeiten und Grenzen erfolgsabhängiger Vergütung, in: Giesen, Richard/Junker, Abbo/Rieble, Volker (Hrsg.), Industrie 4.0 als Herausforderung des Arbeitsrechts, München 2016, S. 31-53.

Uffmann, Katharina, Digitalisierung der Arbeitswelt. Eine kritische Analyse der Gestaltungsvorschläge befristete Teilzeit und Wahlarbeitszeit aus methodisch-gestalterischer Perspektive, NZA-Beilage 2017, S. 45-53.

Vitu ${ }^{\circ}$ Dana, Die verfassungsrechtlichen Grenzen der Abgabenbelastung des Arbeitgebers. Dargestellt am Beispiel des Sozialversicherungsbeitrags, Tönning 2008.

Voitl, Alexander/Luber, Michael, Das neue Dienstrecht in Bayern. Bayerisches Beamtenrecht, München 2011.

Vollmar, Kai, Ist es gerechtfertigt, den Begriff „Versicherungsbann“ für den Unfallversicherungsschutz der Berufskraftfahrer einzuführen?, Sozialversicherung 1963, S. 184-186.

Vollmar, Karl, Unfallversicherungsschutz für Heimarbeiter - wieder im Aufwärtstrend?, WzS 1992, S. 264-266.

Wagner, Gundolf, Beginn und Ende des Unfallversicherungsschutzes beim Zurücklegen von Betriebswegen, Anmerkung zu BSG 2. Senat, Urteil vom 12.12.2006 B 2 U 1/j06 R -, jurisPR-SozR 23/2007 Anm. 5.

Wallerath, Maximilian, Zurechnung und Kausalität im Versorgungsrecht, VSSR 1974, S. 233-258.

Wallerath, Maximilian/Rühr, Christian, Besonderheiten und Grenzen kausaler Zusammenhänge im Recht der gesetzlichen Unfallversicherung, NZS 2007, S. $63-$ 69. 
Walser, Martin, Homeoffice in den Niederlanden, ArbuR 2016, S. 338-341.

Walter, Gerhard/Böhm, Norbert, Arbeitsverhältnis und Beamtenverhältnis, in: Paulssen, Hans Constantin/Richter, Willi/Freitag, Walter u. a. (Hrsg.), Sozialpolitik, Arbeits- und Sozialrecht, Festschrift für Friedrich Sitzler zu seinem 75. Geburtstag, Stuttgart 1956, S. 181-201.

Waltermann, Raimund, Auswirkungen des Sozialrechts, insbesondere des Unfallversicherungsrechts, auf die privatrechtliche Schadensersatzpflicht, in: Wulffen, Matthias von/Krasney, Otto Ernst (Hrsg.), Festschrift 50 Jahre BSG, München 2004, S. 571-589.

Waltermann, Raimund, Zu rechtlichen Rahmenbedingungen einer Fortentwicklung der sozialen Unfallversicherung, VSSR 2005, S. 103-127.

Waltermann, Raimund, Unfallversicherungsschutz bei Weihnachtsfeier. Anmerkung zu BSG, Urt. v. 5.7.2016 - B 2 U 19/14 R -, NZS 2017, S. 25-28.

Wank, Rolf, Die Auslegung von Gesetzen, 6. Aufl., München 2015.

Wannagat, Georg, Rechtsfortbildung durch die sozialgerichtliche Rechtsprechung, in: Deutscher Sozialgerichtstag (Hrsg.), Rechtsfortbildung durch die sozialgerichtliche Rechtsprechung, Verhandlungen des deutschen Sozialgerichtsverbandes, 1. Deutscher Sozialgerichtstag am 30. und 31. Mai 1972 in Kassel, Schriftenreihe des Deutschen Sozialrechtsverbandes (SDSRV), Band 10, Bonn 1973, S. 17-39.

Wannagat, Georg (Hrsg.), Entwicklung des Sozialrechts, Aufgabe der Rechtsprechung: Festgabe aus Anlaß des 100jährigen Bestehens der sozialgerichtlichen Rechtsprechung, Köln 1984.

Watermann, Friedrich, Kausalität und Finalität im Recht der gesetzlichen Unfallversicherung, in: Schimmelpfennig, Heinrich (Hrsg.), Grundsatzfragen der sozialen Unfallversicherung, Festschrift für Dr. Herbert Lauterbach zum 60. Geburtstag, Mannheim 1961, S. 129-153.

Watermann, Friedrich, Die Ordnungsfunktionen von Kausalität und Finalität im Recht, unter besonderer Berücksichtigung des Rechts der gesetzlichen Unfallversicherung, Berlin 1968.

Wedde, Peter, Telearbeit. Arbeitsrecht - Sozialrecht - Datenschutz, München 2002.

Wehler, Hans-Ulrich, Deutsche Gesellschaftsgeschichte. Dritter Band: Von der „Deutschen Doppelrevolution“ bis zum Beginn des Ersten Weltkrieges, 1849-1914, München 1995.

Weingarten, Helmut, Die Beitragspflicht der Arbeitgeber und Arbeitnehmer zur sozialen Pflegeversicherung unter grundrechtlichem Blickwinkel, Frankfurt am Main 1996.

Welskop-Deffaa, Eva, Aufgaben für Gewerkschaften und Sozialpartner in der Arbeitswelt 4.0, NZA-Beilage 2017, S. 60-62.

Werres, Stefan, Beamtenverfassungsrecht. Systematische Darstellung des Berufsbeamtentums auf Grundlage der verfassungsrechtlichen Vorschriften, Heidelberg 2011.

Wicke, Julia, Soziale Sicherung bei Arbeitsunfällen und Berufskrankheiten. Rechtsvergleichende Untersuchung der deutschen und tschechischen Unfallversicherung, Baden-Baden 2000. 
Wickenhagen, Ernst, Geschichte der gewerblichen Unfallversicherung. Wesen und Wirken der gewerblichen Berufsgenossenschaften, München 1980.

Widmaier, Ulrich/Alber, Siegbert, Menschenrecht auf Streik auch für deutsche Beamte?, ZEuS 2012, S. 387-416.

Wiebauer, Bernd, Arbeitsschutz und Digitalisierung, NZA 2016, S. 1430-1436.

Wiese, Günther, Personale Aspekte und Überwachung der häuslichen Telearbeit, RdA 1999, S. 344-353.

Wiedemann, Herbert, Das Arbeitsverhältnis als Austausch- und Gemeinschaftsverhältnis, Heidelberg 1966.

Winter, Martin, Versicherungsschutz bei Unfällen infolge Mitwirkung besonderer Betriebsgefahren, Sozialversicherung 2003, S. 317-319.

Winter, Martin, Tod durch Blitzschlag - gesetzlich unfallversichert?, NZS 2009, S. 199-202.

Wirtz, Markus/Strohmer, Janina (Hrsg.), Lexikon der Psychologie, 17. Aufl., Bern 2014.

Wissenschaftlicher Rat der Dudenredaktion (Hrsg.), Duden. Das große Wörterbuch der deutschen Sprache in zehn Bänden, 3. Aufl., Mannheim 1999.

Wisskirchen, Christine, Mittelbare Diskriminierung von Frauen im Erwerbsleben: die Rechtsprechung des Bundesarbeitsgerichts, des Europäischen Gerichtshofes und des U.S. Supreme Court, Berlin 1994 (zitiert als: Wisskirchen, Mittelbare Diskriminierung).

Woitowitz, Hans-Joachim/Krasney, Otto Ernst, Antwort auf Leserfrage: „Was ist eine wesentliche Ursache in der Unfallversicherung bei polyätiologischen (multikausalen) Krankheiten?‘, Der Betriebsarzt 1996, S. 209-212.

Wolber, Kurt, Telearbeit zu Hause und Schutz der gesetzlichen Unfallversicherung, SozVers 1997, S. 239-240.

Wolff, Hans Julius, Verwaltungsrecht III, Ordnungs- und Leistungsrecht, Verfahrensund Prozessrecht, München 1966.

Wulffen, Matthias von/Krasney, Otto Ernst (Hrsg.), Festschrift 50 Jahre Bundessozialgericht, München 2004 (zitiert als: von Wulffen/Krasney (Hrsg.), FS 50 Jahre BSG).

Zacharias, Franzjosef, Wesen und Rechtsnatur der Ansprüche der Versicherten aus der gesetzlichen Unfallversicherung, Köln 1956.

Zacher, Hans F. (Hrsg.), Die Rolle des Beitrags in der sozialen Sicherung, Colloquium: Papers and discussions, Berlin 1980.

Zacher, Hans F., Verschulden im Sozialrecht - Eine Skizze -, ZfS 1983, S. 171-178.

Zacher, Hans F., Arbeit, Arbeitslosigkeit und Sozialleistungssysteme in globaler Perspektive, in: Gitter, Wolfgang/Schulin, Bertram/Zacher, Hans F. (Hrsg.), Festschrift für Otto Ernst Krasney zum 65. Geburtstag am 16. Dezember 1997, München 1997, S. 807-845 (zitiert als: Zacher, in: Gitter/Schulin/ders. (Hrsg.), FS Krasney).

Zänker, Stefan, Die Haftung der Unternehmer, der Unternehmensangehörigen und anderer Personen in der gesetzlichen Unfallversicherung, Karlsruhe 2004. 
Zahnbrecher, Patrick, Werkstorprinzip in der gesetzlichen Unfallversicherung? Eine kritische Betrachtung des Versicherungsfalls Wegeunfall, Frankfurt am Main 2014.

Zippelius, Reinhold, Juristische Methodenlehre, 11. Aufl., München, 2012. 
